\title{
BTS/BLF Young Investigators Prize Symposium
}

\section{T1 LYMPHOCYTIC BRONCHOALVEOLITIS IN IDIOPATHIC CHRONIC COUGH}

S.S. Birring, C.E. Brightling, F.A. Symon, S. Barlow, A.J. Wardlaw, I.D. Pavord. Institute For Lung Health, Glenfield Hospital, Leicester

We have recently reported an excess of cases of organ specific autoimmune diseases amongst patients with idiopathic chronic cough and have suggested that the cough may be due to homing of activated lymphocytes from the primary site of autoimmune inflammation to the lung. We tested this hypothesis in comparative immunopathology study of 19 patients with idiopathic chronic cough recruited over a two year period (mean age $54 y, 79 \%$ female, mean duration of cough 2y), 11 healthy subjects and 14 patients with explained chronic cough of similar severity. Organ specific autoimmune disease was present in six of idiopathic cough patients $(32 \%)$ but no normals/ explained cough subjects. All subjects had a bronchoscopy, bronchoalveolar lavage (BAL) and bronchial biopsy using standard techniques.

We obtained cytospins from the BAL for a differential cell count and studied BAL T-cell status (CD4/8), activation (CD 103, CD25, CD49a, HLA-DR) and chemokine receptors (CCR3,5,6,11, CXCR3) using 3 -colour flow cytometry. Bronchial biopsies were embedded in glycolmethacrylate and immunohistochemistry for $C D 3,4,8$ (lymphocytes), CD14 (monocytes), CD45 (leukocytes), CD56 (NK cells), EG2 (eosinophils), Neutrophil Elastase (neutrophils), AAl (mast cells), Interferon- $\gamma$, IL5, 3H4(IL4) was performed. The mean (SEM) BAL differential lymphocyte count was $6.8(1.3) \%$ in normals, $15.1(2.6) \%$ in idiopathic cough (mean difference from normals 8.3\%; $95 \%$ Confidence Interval 1.6 to $15.0 ; p<0.02)$ and $7.0(1.7) \%$ in explained cough. The proportion of BAL T-cells expressing CD4 was similar in all three groups and there were no differences in activation status of T-cells or chemokine receptor expression. CD56 and IFN $\gamma$ expression in biopsies (cells $/ \mathrm{mm}^{2}$ submucosa) were reduced in subjects with idiopathic cough compared to normals and subjects with explained cough ( $p=0.03$ and 0.047: Kruskal-Wallis). EG2 and $3 \mathrm{H} 4$ expression were raised in explained cough $(p=0.02 \& 0.03)$ but there were no differences in expression of CD3 and other markers between groups. In conclusion, we have made a novel observation of lymphocytic bronchoalveolar inflammation in patients with idiopathic chronic cough. The association with organ specific autoimmune disease suggests that this might be due to homing of activated lymphocytes from primary sites of autoimmune inflammation or a hitherto unrecognised autoimmune bronchitis. Further studies are required to investigate the interaction between T-cells and the cough reflex.

\section{T2 TGF- $\beta$ ACTIVATION IS DIMINISHED FOLLOWING BLEOMYCIN-INDUCED LUNG INJURY IN MICE LACKING NEUTROPHIL ELASTASE}

F. Chua', S.E. Dunsmore', A.W. Segal ${ }^{2}$, J. Roes' ${ }^{2}$, G.J. Laurent' . 'Centre for Respiratory Research; ${ }^{2}$ Department of Medicine, Royal Free and University College London School of Medicine, London WCIE 6JJ

Transforming growth factor-beta (TGF- $\beta$ ) is widely implicated in the pathogenesis of pulmonary fibrosis. We have previously reported that mice lacking neutrophil elastase $\left(\mathrm{NE}^{-}\right)$are resistant to pulmonary fibrosis induced by bleomycin instillation. We hypothesised that decreased TGF- $\beta$ activation may contribute to the resistance of these null animals against fibrosis. Active TGF- $\beta$ was quantitated in bronchoalveolar lavage fluid (BALF) and lung tissue from wild type (WT) and $\mathrm{NE} \%$ mice seven days following instillation of 0.05 unit bleomycin or saline. Levels of active TGF- $\beta$ in bleomycin-treated WT BALF averaged $0.36 \pm 0.02 \mathrm{mg} / \mathrm{ml}$, two-fold greater than in saline controls $(p<0.001)$. In contrast, active TGF- $\beta$ levels in bleomycin-treated $\mathrm{NE}^{\prime}$ BALF $(0.23 \pm 0.01 \mathrm{mg} / \mathrm{ml}, p<0.001 \vee W T$ values) were not different from saline controls. Conversely, a greater amount of TGF- $\beta$ was detected in lung tissue from bleomycin-treated $\mathrm{NE}^{-}$mice $(\mathrm{p}<0.05 \mathrm{v}$ WT). No differences in inflammatory cellularity, alveolar-capillary leak, TGF- $\beta_{1}$ or TGF- $\beta_{3}$ mRNA expression were apparent between the two genotypes at this time point. Furthermore, in bleomycin-treated WT lungs, immunocytochemical staining for active TGF- $\beta$ (LCl-30 antibody, Dr. K. Flanders, NIH) was widespread, with prominent localization to areas of inflamed or damaged alveoli. In contrast, staining for active TGF- $\beta$ in bleomycin-treated NE $\%$ lungs was minimal, and limited to peribronchial and perivascular locations. In conclusion, TGF- $\beta$ activation was diminished in alveolar fluid from bleomycintreated $\mathrm{NE} \%$ mice and correlated with decreased staining for active TGF- $\beta$ in lung tissue. These data provide the first in vivo evidence that neutrophil elastase may play a critical role in modulating TGF- $\beta$ activation. In particular, this study suggests that neutrophil elastase inhibitors may have a therapeutic potential in abrogating TGF- $\beta$-mediated fibrotic lung disease.

Supported by the Wellcome Trust, MRC and the British Lung Foundation.

\section{T3 THE COST EFFECTIVENESS OF AN ASTHMA MANAGEMENT STRATEGY DIRECTED AT NORMALISING THE INDUCED SPUTUM EOSINOPHIL COUNT}

R.H. Green, C.E. Brightling, S. McKenna, B. Hargadon, D. Parker, P. Bradding, A.I. Wardlaw, I.D. Pavord. Institute for Lung Health, Glenfield Hospital, Leicester, UK

We have recently shown that a management strategy directed at normalising the induced sputum eosinophil count reduces asthma exacerbations and hospital admissions compared to a traditional approach. We now report the results of a concurrent health economic evaluation designed to determine the cost effectiveness of this sputum management strategy. Seventy four subjects with moderate to severe asthma recruited from hospital clinics were randomised into two groups: one managed by standard British Thoracic Society asthma guidelines (BTS management group) and one managed using an algorithm aimed at normalising the induced sputum eosinophil count as well as minimising symptoms (sputum management group). Patients were seen nine times over 12 months and on each occasion sputum was induced and processed, but the results were not disclosed in the BTS guidelines group. Throughout the study patients completed daily diary cards recording medication use, days off work, emergency GP or hospital visits and hospital admissions. The overall cost of each management strategy was calculated using our estimates of the cost of sputum induction and processing, the 2001 Unit Costs of Health and Social Care, the Department of Health 2001 reference costs and the British National Formulary. Total costs for each patient were calculated as the sum of the costs of hospital out-patient appointments, primary care visits, hospital admissions and medication use throughout the 12 months, with the addition of the costs of sputum induction and processing for patients in the sputum management group only. Patients in the sputum management group experienced significantly fewer severe asthma exacerbations than patients in the BTS management group $(35 \vee 109, p=0.01)$ and significantly fewer patients were admitted to hospital with asthma (1 $v 6, p=0.047)$. Amongst the $49(24 \vee 25)$ patients in regular employment, the mean (SEM) days off work was $2.2(0.8)$ in the sputum management group and $8.3(2.1)$ in the BTS management group $(p=0.01)$. The estimated annual total mean (SEM) cost per patient was (£1755 (119) in the sputum management group and £1954 (164) in the BTS management group $(p=0.30)$. A treatment strategy directed at normalising the induced sputum eosinophil count reduces asthma exacerbations and hospital admissions without incurring additional costs to the health service. In addition, by reducing work days lost due to asthma, this approach may result in significant cost savings to society.

T4 THE $\alpha$ V $\beta 5$ INTGERIN INDUCES ANOIKIS IN SQUAMOUS CELL CARCINOMA (SCC) CELLS BY ACTIVATING THE INTRINSIC AND EXTRINSIC DEATH PATHWAYS AND INHIBITING AN AKT/PKB SURVIVAL SIGNAL

S.M. Janes, F.M. Watt. Cancer Research UK, 44 Lincoln's Inn Fields, London, WC2A 3PX, UK

Introduction: Focal or extensive loss of $\alpha v \beta 5$ is a feature of the most poorly differentiated SCCs, while an increase in $\alpha v \beta 6$ is associated 
with invasiveness and metastatic spread (Watt. Dev Supp/ 1993:18592). Epithelial cells normally undergo apoptosis on detachment from their extracellular matrix (anoikis), but transformed cells do not. Hence failure to express a particular integrin may render tumour cells "deaf" to specific signals determining apoptosis. We hypothesised that expression of $\alpha \mathrm{v}$ on $\mathrm{H} 357$ cells (which completely lack $\alpha$ ) and SCC4 cells (which have low expression) would restore their ability to undergo anoikis.

Methods: $\alpha v$ or $\alpha 4$ integrin subunits were transfected into H357 and SCC4 cells using the pBabepuro retroviral vector giving high expressing polyclonal populations. Anoikis was assessed by FACS analysis for sub-G1 cells and by TUNEL staining. Signalling pathways were investigated using MEK, p38MAPK, and PI3K inhibitors, and a constituitively active Akt construct. The apoptotic pathway was investigated using specific caspase inhibitors, Western blotting for activated pro and anti apoptotic molecules and a dominant negative FADD construct. Anoikis activation was examined using a chimeric molecule with the cytoplasmic domain of $\beta 5$ attached to the extracellular domain of $\beta 6$.

Results: The $\alpha v$ subunit formed a functional heterodimer with $\beta 5$ as measured by FACS and adhesion to vitronectin. Anoikis was dramatically increased in the $\alpha \mathrm{v}$ infected cells (both H357s and SCC4s) compared to the parental populations, empty vector controls and $\alpha 4$ infected cells at 48 and 72 hours $(p<0.01)$. Anoikis was not increased in $\alpha v \beta 6$ expressing cells. The pathways involved in $\alpha v \beta 5$ induced anoikis include a suppression of activation of the survival factor Akt/ PKB, possibly via the cytoplasmic domain of $\beta 5$ and both the intrinsic (mitochondrial) and extrinsic (death receptor mediated) cell death pathways.

Conclusion: Re-introduction of the $\alpha v$ integrin subunit increased SCCs ability to undergo anoikis via the $\alpha v \beta 5$ heterodimer. Both the intrinsic and extrinsic apoptotic pathways are required, and a cell survival mechanism of activating $\mathrm{Akt} / \mathrm{PKB}$ is suppressed.

\section{T5 NASAL MUCOCILIARY CLEARANCE IS NORMAL IN CHILDREN WITH CF: EVIDENCE AGAINST A PRIMARY CFTR-RELATED MECHANISM IN THE UPPER AIRWAY}

D. McShane, J.C. Davies, T. Wodehouse, A. Bush, D. Geddes, E. Alton. Departments of Paediatrics and Gene Therapy, Royal Brompton Hospital, London, UK

Studies in adult CF patients have reported impaired mucociliary clearance (MCC) in both the nose and lungs, which has been implicated in the pathogenesis of airway plugging and bacterial lung infection. We studied young children to explore a primary versus secondary cause of impaired nMCC. Saccharin clearance times were measured in 18 children with CF (median age 11 y (9.5B 15y)) and 21 non-CF children (median age 12y (9.5B16y)). Eleven children with Primary Ciliary Dyskinesia (PCD) served as positive controls (median age 12y 19 B 14.7y)l. A $2 \mathrm{~mm}$ piece of saccharin was placed on the inferior turbinate and the time taken to taste measured up to 30 mins. Nasal lavage (NL) was performed from the other nostril with $4 \mathrm{ml}$ sterile PBS. No difference was seen in $\mathrm{nMCC}$ times between the CF and non-CF groups (CF median (IQR) $11(8 ; 15)$ min; non-CF median $11(9 ; 16)$ $\mathrm{min})$, whereas those in the PCD group all had nMCC times of $>30$ minutes ( $p<0.05$ for both CF \& non-CF). There was no evidence of excess inflammation in the CF NL samples as assessed by levels of the neutrophil chemoattractant, IL-8 (CF $52(3 ; 75) \mathrm{pg} / \mathrm{ml}$ NLF;non-CF 37 $(5 ; 108) \mathrm{pg} / \mathrm{ml} \mathrm{NLF})$. Interestingly, the children with PCD had significantly raised levels of IL-8 $(377(244 ; 598) \mathrm{pg} / \mathrm{ml} \mathrm{NLF} ; \mathrm{p}<0.01$ compared with both CF \& non-CF). We next measured nMCC in adult CF patients with and without chronic sinus (CS) disease (CS+ or CS-). $\mathrm{CS}+\mathrm{CF}$ patients had significantly longer clearance times than CSsubjects $(C S+n=12: 18(11 ; 24)$ mins;CS- $n=16: 14(6 ; 16)$ mins; $\mathrm{p}<0.05)$. IL-8 was significantly higher in the CF CS + group than in the healthy adult group (CF CS+45 $(15 ; 76) \mathrm{pg} / \mathrm{ml} \mathrm{NLF}$; healthy adults: $10(1 ; 64) \mathrm{pg} / \mathrm{ml} \mathrm{NLF} ; \mathrm{p}=0.01)$ and there was a trend with regards to the CF CS-group $(15(1 ; 39) \mathrm{pg} / \mathrm{ml} \mathrm{NLF} ; \mathrm{p}=0.06)$. In conclusion, the delayed MCC reported in the CF nose was not seen in a group of young children, but became apparent in adult patients with chronic sinus disease. This suggests a role for mucosal inflammation in the aetiology of delayed MCC in the upper and proximal airways but not distal airways where the ciliated epithelium is more sparse, and low volume ASL may be more detrimental on the clearance process.

\section{T6 PHOSPHODIESTERASE 4 ISOFORMS DIFFERENTIALLY REGULATE LPS STIMULATED MACROPHAGE FUNCTION: THE ROLE OF PDE4B2.}

M.C. Shepherd' 2 , N.C. Thomson' ${ }^{2}$ M.D. Houslay'. ${ }^{1}$ Institute of Biological and Life Sciences, University of Glasgow, Glasgow, UK; ${ }^{2}$ Department of Asthma research, Medicine and Therapeutics, Gartnavel General Hospital Glasgow UK

Phosphodiesterase4 (PDE4), a family of cAMP hydrolysing enzymes is widely expressed in immune cells. Four genes encode PDE4A, PDE4B, PDE4C, and PDE4D. Differential mRNA splicing produces variability within these families. Structural differences between isoforms suggest specific roles in cell regulation. I have shown PDE4 isoform expression regulation with macrophage differentiation. Such monocytes lose PDE4D expression, but gain PDE4A and PDE4B isoforms. I hypothesised important roles for PDE4 isoforms in macrophage behaviour.

Methods: RAW 264.7 cells were treated with $10 \mathrm{ng} / \mathrm{ml} \mathrm{lps} \mathrm{+/-} \mathrm{the}$ PDE4 inhibitor rolipram, PDE3 inhibitor cilostamide or signal transduction inhibitors. Cyclo-oxygenase 2 (COX-2) and inducible nitric oxide synthase (iNOS) expression, PDE4 isoform activity or ERK 1/2 activity was measured. ERK activity was assessed by the phospho-ERK/Total ERK ratio on western blot. Total PDE4, PDE3, and PDE4 isoform activity from immunoprecipitates were assessed, TNF $\alpha$ and prostaglandin E2 (PGE2) production were measured in growth medium by ELISA. RAP-1A G-protein activity mutants were used to investigate rolipram's action.

Results: Rolipram, not cilostamide caused indomethacin sensitive increase in iNOS expression, but indomethacin resistant increase in COX-2 expression in lps stimulated macrophages. PGE2 production was dose dependently increased by rolipram while TNF $\alpha$ production was inhibited in an indomethacin resistant fashion. Rolipram caused an increased and early activation of ERK 2 . Lps led to a MEK dependent increase in PDE 4 activity by $40 \%$ and PDE 4 B by $42 \%$. Transfected RAP-1A activity mutants did not influence inflammatory mediator production.

Discussion: Rolipram increases the expression of COX-2, PGE2, and iNOS while inhibiting TNF $\alpha$. TNF $\alpha$ inhibition is not due to increased PGE2 production. Complex crosstalk between the cAMP and MAPKinase signal cascades suggests increased PDE4B activity acts to regulate pro-inflammatory signal propagation. Rolipram alters the response of ERK 2 to lps, promoting a "proliferative" response. The G-protein Rap-1A did not mediate the interaction between cAMP and MAPKinase. 


\section{Spoken sessions}

\section{Lung cancer outcomes}

\section{S1 CARBOPLATIN AND VINORELBINE AS FIRST LINE OUTPATIENT TREATMENT IN NSCLC}

J. Maguire, N. Smith, V. Kelly, M. Ledson, M. Walshaw. Liverpool Lung Cancer Unit, Cardiothoracic Centre, Thomas Drive, Liverpool, UK

Outpatient chemotherapy offers advantages for the Health Service in terms of resource utilisation and expense compared to the patient treatment, and is preferred by the vast majority of patients and their families.

We have treated 130 patients ( 84 male, mean age 65.8 years, 46 female, mean age 62.2 years) with stage III or Stage IV NSCLC and performance status $0-2$ with outpatient chemotherapy using Carboplatin and Vinorelbine using the following regimen:

Carboplatin AUC 5 day 1,

Vinorelbine $25 \mathrm{mg} / \mathrm{m}^{2}$ day 1 , day 8

The intended duration of treatment was 3 or 4 courses of chemotherapy using a 21 day treatment cycle. The mean number of courses given was 3.2 (range 1-6)

With 102 patients so far having completed treatment, response rates are as follows: Complete Responses 2\%, Partial Responses $34.8 \%$, Stable Disease 32\%, and Progressive Disease $17.1 \%$. Overall median survival is 9.1 months.

Treatment was generally tolerated. Toxicity included neutropenic sepsis in $22 \%$ and anaemia requiring blood transfusion in $29 \%$. There were $8(7.8 \%)$ treatment related deaths. Patients with performance status 2 at commencement of treatment were at significantly greater risk of serious morbidity and treatment related death compared to patients with performance status $0-1$. Patients aged $>70$ were more likely to require blood transfusions but were not at increased risk of neutropenic sepsis or treatment related death. We conclude that Carboplatin and Vinorelbine is a relatively safe and effective regimen for the outpatient treatment of patients with NSCLC.

\section{S2 A COMPARISON OF ACE AND CE CHEMOTHERAPY IN SCLC}

N. Diar Bakerley, N.P. Keaney, K. Sridharan, I.K. Taylor, H.W. Clague. Chest Diseases Unit, Sunderland Royal Hospital, Sunderland SR4 7TP, UK

Background: Chemotherapy plays an important role in the treatment of Small Cell Lung Cancer (SCLC). Several standard regimens are in existence but there have been few direct comparisons with regard to toxicity. In this retrospective audit we compare the toxicity of ACE (Adriamycin, Cyclophosphamide and Etoposide) with CE (Carboplatin and Etoposide).

Methods: Over a 12 month period from April 200028 patients with SCLC received chemotherapy with either ACE or CE chemotherapy. Toxicity was evaluated by a retrospective audit of case notes looking in particular at neutropenia, neutropenic sepsis, clinically significant anaemia, in hospital duration of stay and toxic death.

Results: Eleven patients (39.2\%) received $A C E, 12$ patients $(42.8 \%)$ received $C E$ and five patients $(17.8 \%)$ received both. In total 63 ACE and 80 CE chemotherapy cycles were given.

Severe neutropenia (neutrophils $0-0.5 \%$ ) complicated $21(33.3 \%)$ ACE compared with 11 (13.7\%) CE chemotherapy sessions $(p=0.005)$. Neutropenic sepsis occurred in $14(22.2 \%)$ ACE sessions compared with $2(2.5 \%)$ CE sessions ( $p=0.0002)$. Thirty $(46.8 \%)$ ACE sessions were complicated with clinically significant anaemia compared to $18(22.5 \%)$ in the CE group ( $p=0.002)$. The average in-hospital stay per patient was 19.3 days for patients receiving $A C E$ and 3.6 days for patients receiving CE. Toxic death happened in 1 patient in the ACE group and 1 patient in the CE group $(p=0.9)$.

Conclusion: This retrospective audit indicates that in our experience ACE chemotherapy for SCLC is associated with greater toxicity than the CE regimen. This led to a greater use of resources in terms of antibiotic therapy, blood transfusion, and hospital bed usage and has led us to adopt the CE regimen as first choice chemotherapy for this group of patients.

\section{S3 PROLONGED SURVIVAL IN INOPERABLE NSCLC WITH CONCURRENT CHEMO-RADIOTHERAPY}

J. Maguire, R. Page, V. Kelly, N. Smith, M. Ledson, M. Walshaw. Liverpool Lung Cancer Unit, Cardiothoracic Centre, Thomas Drive, Liverpool, UK

Synchronous chemotherapy and radiotherapy is a highly effective treatment modality for a number of solid tumours including inoperable Non-Small Cell Lung Cancer, and has been adopted as a standard therapy for lung cancer in the United States. However, experience with this form of treatment for lung cancer in the UK is limited.

We have treated 54 patients with locally advanced, inoperable NSCLC (8 Stage IIB, 46 Stage IIIB) with concurrent cisplatinum and radical radiotherapy using a tumour dose of 52.5 Gy given in 20 daily fractions over four weeks, together with cisplatinum $20 \mathrm{mg} / \mathrm{m}^{2}$ concurrent with fractions $1-5$ and 16-20. Thirty four patients received 2-4 courses of chemotherapy after concurrent chemo-radiation. Toxicity was acceptable, with three cases of severe but self-limiting oesophagitis, a $70 \%$ incidence of mild to moderate oesophagitis and no treatment related deaths.

One, two, and three year survival rates for patients with Stage IIIB disease are $74 \%, 35 \%$, and $32 \%$ respectively. Patients with Stage IIIB disease treated with concurrent chemo-radiotherapy followed by systemic chemotherapy have a median survival of 25 months, 3 year survival of $49 \%$, and a local control rate of $89.5 \%$.

Concurrent chemo-radiotherapy is a highly effective treatment modality for patients with locally advanced inoperable NSCLC, but this form of treatment is only suitable for patients who have good performance status (PS 0-1), minimal co-morbidity and disease which can be encompassed within a radical radiotherapy treatment volume.

\section{S4 OUTCOME OF PATIENTS OVER SEVENTY YEARS OLD WITH SMALL CELL LUNG CANCER TREATED WITH CHEMOTHERAPY}

P. Temblett', J. Baker', F. MacBeth', A.P. Smith'. 'Section of Respiratory Medicine, University of Wales College of Medicine, Llandough Hospital, Cardiff, UK CF64 2XX, UK; 'Velindre Hospital, Whitchurch, Cardiff CF14 2JL, UK

Introduction: Small cell lung cancer (SCLC) accounts for $25 \%$ of all newly diagnosed cases of lung cancer over seventy years. No standard treatment is defined for elderly patients with SCLC, and prognosis remains poor.

Methods: We performed a retrospective review of all SCLC cases over 70 years treated with carboplatin and etoposide between $01 / 01 / 2000$ and $31 / 12 / 2001$. Prophylactic cranial irradiation $(P C I)$, chest and palliative radiotherapy were used when indicated.

Results: 29 patients were treated, median age 73.7 years, four patients over 80 years. $52 \%$ had limited disease. $7 \%$ had performance status (PS) $=0,41 \% \mathrm{PS}=1,35 \% \mathrm{PS}=2,17 \% \mathrm{PS}=3$. The average number of cycles given was $3.27,55 \%$ receiving a dose reduction. $\mathrm{PCl}$ and chest radiotherapy was used in 4 patients, 5 patients received palliative radiotherapy. The overall response rate was $65 \%$. Actuarial median survival was 30 weeks $195 \% \mathrm{Cl}$ $22.1-37.91,1$ year survival $=23 \%, 18$ month survival $=18 \%, 2$ year survival $=10 \%$. Four patients are still alive (range 33 B 121.4 weeks) Febrile neutropenia occurred in $17 \%$ and neutropenic death in $10 \%$. $21 \%$ of patients received blood transfusions. There were $5(17 \%)$ early deaths (<21 days), 3 of which were neutropenic.

Conclusions: This review confirms that the treatment of unselected SCLC elderly patients with chemotherapy causes significant risks with lower response rates and survival than previously reported. Despite this palliation is achieved in a significant number with prolongation of survival. Co-morbidity and quality of life issues should be carefully considered when treating such patients. 
S5 WHAT PERCENTAGE OF PATIENTS WITH LUNG CANCER PRESENT WITH POTENTIALLY CURABLE DISEASE?

P.M. Donnelly, W.J.A. Anderson on behalf of the Northern Board Lung Cancer Group. Regional Cancer Center for the Northern Board, Antrim Hospital, Bush Rd, Antrim BT4 1 2QB, UK

Background: Since 1995 there has been concern that the 5 year survival of patients suffering from lung cancer in the UK (6-7\%) was significantly less than that quoted for America and other western countries (14-15\%). This might represent differences in recording, differences in disease or differences in treatment. The aim of this audit was to define the number of patients with curable disease at presentation.

Method: We prospectively filled in audit sheets based on the diagnostic module of he RCP (London) lung cancer data sheets from 1/1/ 2001-1/5/2002. At the end of the audit period we checked the hospital databases in the catchment area for any additional patients recorded as having lung cancer and retrospectively collected data for these patients.

Results: 175 patients were identified. Patients had a mean age of 70 years (range 43-94). 133 had a pathological diagnosis - 27 had small cell tumours and 106 had non small cell tumours. 59 patients were excluded from radical treatment by performance status, a further 62 by stage of disease, a further 15 by inadequate pulmonary function and 8 by other co-morbidity. Thirty-one (16\%) remained and were potentially suitable for radical treatment

Conclusion: In this study only $16 \%$ of patients were potentially curable at presentation. On the basis of these figures targets for surgical resection rates around $20 \%$ seem unrealistic, although with current resection rates running around $10 \%$ in the UK there may be some subjects who are suitable for surgery to whom it is not offered. Offering surgery or radical radiotherapy to these patients might result in some improvement in survival.

\section{COPD: Cellular activation and inflammation}

\section{S6 RELATIONSHIP BETWEEN BRONCHIAL BIOPSY INFLAMMATORY CELL POPULATIONS AND PHYSIOLOGICAL PARAMETERS IN COPD}

I.S. Patel', N.J. Roberts', R.J. Sapsford', M. Sheaff', J.A. Wedzicha'. 'Academic Unit of Respiratory Medicine; ${ }^{2}$ Department of Morbid Anatomy, Barts and the London NHS Trust, UK

This study was designed to evaluate the relationships between patterns of bronchial biopsy inflammation and physiological parameters in COPD. Bronchoscopic biopsies were taken from 36 patients with COPD (mean (SD) age 69.5 (8.75) yrs, FEV, 1.36 (0.59) I, FVC 2.6 (0.88) I, $\mathrm{FEV} \%$ predicted 54.5 (16.2)\%, $\mathrm{PaO}_{2} 8.8$ (1.2) $\mathrm{kPa}, \mathrm{PacO}_{2}$ $5.4(0.65) \mathrm{kPa}, 46.2$ (31.8) pack years of smoking, MRC dyspnoea score 3.06 (1.24), daily inhaled steroid dosage 747 (810) $\mu g, 16$ current smokers). Samples were wax embedded and stained for CD3, CD4, CD8, CD68, and EG2 positive inflammatory cells by immunohistochemistry. Intraepithelial CD3 positive cells (per 100 epithelial cells) were related to daily inhaled steroid dosage ( $r h o=0.675$, $\mathrm{p}=0.046$ ) and inversely related to the $\mathrm{PaO}_{2}(\mathrm{rho}=-0.714, \mathrm{p}=0.047$ ). Patients with symptoms of daily dyspnoea had significantly higher numbers of intraepithelial CD3 cells than those without (median (IQR) CD3 cells $/ 100=17(8.5)$ versus 9.5 (6.75), $p=0.016)$. Numbers of intraepithelial CD68 positive cells/100 were related to daily inhaled steroid dosage ( $r h o=0.731, p=0.016$ ) and the MRC dyspnoea score (rho $=0.815, p=0.007$ ). Patients with symptoms of daily dyspnoea also had significantly higher numbers of intraepithelial CD68 cells than those without (median (IQR) CD68 cells/100 = 9 (7) versus 4 (3), $\mathrm{p}=0.027)$. Intraepithelial CD4 positive cells/100 were related to inhaled steroid dosage ( $r h o=0.850, p=0.032$ ). An inverse relationship was seen between numbers of intraepithelial EG2 positive cells/ 100 and the $\mathrm{FEV} \%$ predicted ( $\mathrm{rho}=-0.753, \mathrm{p}=0.031$ ). Numbers of EG2 cells per high-powered field also increased with increasing daily inhaled steroid dosage ( $r h o=0.442, p=0.076$ ). No effect on cell numbers was seen with current smoking.

Bronchial wall inflammation in COPD increases with increasing indices of disease severity, as measured by $\mathrm{PaO}_{2}, \mathrm{FEV}_{1} \%$ predicted, inhaled steroid dosage and the symptoms of daily dyspnoea.

Supported by the Joint Research Board, St. Bartholomew's Hospital.
Abstract S7 Mean (SE) PMN-endothelial cell interation results

\begin{tabular}{llll}
\hline & NS & HS & COPD \\
\hline Adherent PMN & $303.2(22.7)$ & $324(26.7)$ & $449(70.8)$ \\
Transmigrated PMN & $199.2(33.8)$ & $191.1(32.1)$ & $256(52.0)$ \\
Total interacting PMN & $502(31.2)$ & $515.8(35.5)$ & $706(62.3)^{*}$ \\
\hline${ }^{*}$ p $<0.05 v$ HS and NS. & & &
\end{tabular}

\section{S7 NEUTROPHILS FROM PATIENTS WITH CHRONIC OBSTRUCTIVE PULMONARY DISEASE DEMONSTRATE ENHANCED ENDOTHELIAL CELL INTERACTIONS}

I.S. Woolhouse, N. Carrabino, D. Bayley, P.F. Lalor', D.H. Adams' ${ }^{1}$, R.A. Stockley. Lung Investigation Unit; 'Liver Research Laboratories, Queen Elizabeth Hospital, Birmingham B15 2TH, UK

Chronic obstructive pulmonary disease (COPD) is usually caused by smoking, yet only 10-15\% of smokers develop clinically significant disease. Given that neutrophil (PMN) recruitment, and subsequent inflammation, is central to the development of COPD, it is possible that PMN from smokers who develop the disease are primed to migrated from the bloodstream into the lung. The aim of this study was to compare endothelial cell interactions, under flow conditions, and adhesion molecule expression of PMN from non-smokers (NS), smokers without COPD (HS) and patients with COPD.

PMN were isolated from $8 \mathrm{NS}, 8 \mathrm{HS}$ and 10 COPD patients. The three groups were age and sex matched, and the mean (SD) FEV \% predicted were 101.8 (10.9), 97.4 (12.9), and 45.9 (20.9), respectively. Pack year smoking history was similar in the HS and COPD groups (32.1 (10.0) $\vee 47.0$ (19.3), $p=n s)$. To assess endothelial adhesion and migration, PMN were perfused at a physiological flow rate over interleukin-1 $\beta$ stimulated human umbilical vein endothelial cells cultured in microslides. Adherent and migrated PMN were counted by phase contrast microscopy. CDI 1b/CD18 (Mac-1) and CD62l (L-selectin) expression were assessed by flow cytometry. Mean (SE) PMN-endothelial cell interaction results are tabulated in the table.

There were no significant differences in PMN expression of Mac-1 and L-selectin between the three groups.

The data suggest that PMN from smokers who develop COPD are primed to adhere to, and migrate across, vascular endothelium. This appears to be independent of Mac-1 or L-selectin expression. Further work is needed to clarify the mechanism, but the PMN priming seen here in COPD provides a potential target for new therapy.

\section{S8 DIFFERENCES IN AIRWAY NEUTROPHIL NUMBERS, ACTIVATION AND TLR-2 EXPRESSION IN SUBJECTS WITH COPD AND AGE-MATCHED CONTROLS}

M.I. Allenby, B. Sempasa, M.W. Lethbridge, J.C. Ratoff, M.W. Jensen, B.J. O'Connor. Department of Respiratory Medicine and Allergy, King's College London, UK

Rationale: Subjects with COPD have higher numbers of airway neutrophils and these numbers correlate with airflow limitation (Lacoste, et al 1993). The activation status of these neutrophils, and the effect of smoking on this, is unknown. The toll-like receptor family is responsible for innate immune responses against a wide variety of bacterial molecules. Their presence on airway neutrophils has not previously been investigated.

Methods: 15 subjects with COPD and 18 healthy age-matched controls underwent flexible bronchoscopy for collection of bronchoalveolar lavage fluid (BALF). Both groups contained a mix of smokers and ex/non-smokers. A BALF differential cell count was performed. The neutrophils were labelled with fluorochrome-conjugated antibodies against surface markers of activation (CD63, CD14) and the tolllike receptor 2 (TLR-2), and analysed using flow cytometry. The percentage of cells expressing the antibodies was calculated. Statistical analysis was performed using an unpaired $t$ test.

Results: All data are expressed as means (SD). There was an increase in the percentage of neutrophils within the BALF of subjects with COPD when compared with controls (1.644 (1.717) v 0.343 $(0.084) ; p=0.0037)$ irrespective of smoking status. However, the percentage of CD63+ve neutrophils was significantly lower in current smokers when compared to ex/non-smokers (14.78 (17.06) v 45.64 (22.86); $p<0.0001)$ regardless of airflow limitation. The same was true for CD14+ve neutrophils (11.18 (15.65) v 40.98 (25.78); 
$\mathrm{p}=0.0002$ ). Similarly, the percentage of TLR2 expression was reduced in current smokers (10.57 (1 1.20) v 38.41 (23.80); $p=0.0004)$.

Conclusions: Although airway neutrophils are increased in the lungs of COPD subjects, their activation is related to the smoking status of the subject. The fall in the level of neutrophil activation in smokers may represent an immune suppression or increased recruitment of neutrophils from the circulation.

Work supported by a grant from GKT School of Medicine.

\section{S9 SPUTUM BACTERIAL COLONISATION IN STABLE COPD: ITS INFLUENCE ON AIRWAY INFLAMMATION, HEALTH STATUS, AND FIBRINOGEN}

D. Baneriee' 2, D. Honeybourne'2, O.A. Khair'. 'Department of Respiratory Medicine, City Hospital NHS Trust, Dudley Road, Birmingham, UK; ${ }^{2}$ Department of Respiratory Medicine, Birmingham Heartlands Hospital, Bordesley Green East, Birmingham, UK

Introduction and aims: It is unclear if the presence of potential pathogenic micro-organisms (PPMs) in the sputum of patients with moderate to severe COPD influences health status and blood fibrinogen levels during the stable clinical state. This study aimed to determine if health status, fibrinogen and bronchial airway inflammation in those harbouring PPMs differ from those who do not (Non-PPMs).

Methods: moderate to severe patients with no recent exacerbations in the last 6 weeks were recruited. Saline sputum induction was performed and markers of airway inflammation (total cell count, neutrophil count, IL-8, LTB4, TNF- $\alpha$, neutrophil elastase (NE), and neutrophil chemotaxis (NC)-Boyden chamber technique), sputum bacterial culture, health status (SGRQ and SF-36) and blood fibrinogen were measured.

Results: 67 patients were recruited, $69 \%$ male, mean age (SD) $66.7(7.9)$ years and $27(40 \%)$ were current smokers. $27(40 \%)$ of patients grew PPMs, total number of bacterial isolates $38 ; H$ influenzae (14), M catarrhalis (10), S pneumoniae (9) and others (5). There was no significant difference in age, spirometry or smoking pack years between the PPMs and Non-PPMs groups. Those with PPMs had a worse health status score, mean (SD): Total SGRQ 58.7 (14.7) v 47.4 (15.7), $p=0.004, S G R Q$ impact 46.1 (16.1) v 34.1 (17.3), $\mathrm{p}=0.005, \mathrm{SGRQ}$ symptoms 70.3 (14.8) v 60.5 (21.4), $\mathrm{p}=0.03$, SGRQ activity $76.8(17) \vee 63.2(20.4), \mathrm{p}=0.004$. SF-36 vitality and physical role limitation were also worse in this group. The PPMs group had an exaggerated airway inflammatory response, mean (SD): sputum supernatant $\log$ IL-8 $(\mathrm{nM}) 0.01(0.35) v-0.29$ (0.47) $p=0.001, \log$ LTB4 (nM) $0.32(0.33) \vee 0.01$ (0.40), $\mathrm{p}=0.008), \log$ TNF- $\alpha(\mathrm{pM})-0.15(0.78) v-0.78(0.56), \mathrm{p}=0.001$ NE $(\mu M)-1.27(1.01) v-2.04(0.52), p=0.001$ and $\mathrm{NC}(\% \mathrm{fMLP})$ 70.4 (17.1) v 54.6 (19.0), $p=0.001$. There were increased numbers of sputum neutrophils (absolute count) but this was not statistically significant $(p=0.058)$. Fibrinogen level $(g / l)$ was greater in the PPMs group: $3.21(0.87)$ v $2.85(0.64), p=0.05$.

Conclusion: clinically stable moderate to severe COPD patients with sputum PPMs have a worse health status, exaggerated bronchial airway inflammation and a higher blood fibrinogen level.

\section{S10 RELATIONSHIP BETWEEN BRONCHIAL BIOPSY CELL POPULATIONS, EXACERBATION FREQUENCY, AND BACTERIAL COLONISATION IN COPD}

I.S. Patel', N.J. Roberts', R.J. Sapsford', M. Sheaff', J.A. Wedzicha'. 'Academic Unit of Respiratory Medicine; ${ }^{2}$ Department of Morbid Anatomy, Barts and the London NHS Trust, UK

The effect of exacerbation frequency on bronchial wall inflammation in COPD, and the effect of bacterial colonisation in this context are unknown. Bronchoscopic biopsies were taken from 36 patients with COPD (mean (SD) age 69.5 (8.75) yrs, FEV 1.36 (0.59) I, FVC 2.6 (0.88) l, $\mathrm{FEV} \%$ predicted $54.5(16.2) \%, \mathrm{PaO}_{2} 8.8(1.2) \mathrm{kPa}, \mathrm{PacO}_{2}$ $5.4(0.65) \mathrm{kPa}, 46.2(31.8)$ pack years of smoking, MRC dyspnoea score 3.06 (1.24), daily inhaled steroid dosage 747 (810) $\mathrm{\mu g}, 16$ current smokers, median (IQR) 2 (3) exacerbations per year). Samples were wax embedded and stained for CD3, CD4, CD8, CD68, and EG2 positive cells by immunohistochemistry. Lower airway bacterial colonisation was quantitatively assessed by means of protected specimen brushings in 30 patients, of whom 14 were colonised by a potential pathogen. Patients were defined as frequent ( $\geqslant 3$ per year) or infrequent ( $\leqslant 2$ per year) exacerbators. Total numbers of CD3 positive cells per section were related to exacerbation frequency (rho $=0.512$, $\mathrm{p}=0.013$ and the number of exacerbations in the previous year (rho=0.459, $\mathrm{p}=0.011$ ). Total CD8 positive cells per high-powered field were related to exacerbation frequency ( $\mathrm{rho}=0.454, \mathrm{p}=0.03$ ) and number (rho=0.443, $\mathrm{p}=0.034$ ). CD4 positive cells per high-powered field also increased with exacerbation number (rho=0.573, p=0.016). CD68 positive cells per high-powered field were related to exacerbation frequency (rho=0.655, $p=0.002$ ) and number (rho $=0.584, p=0.007$ ), as were numbers of activated eosinophils (rho $=0.623, p=0.003$ and $r h o=0.555, p=0.009$ respectively). Exacerbation frequency was related to colonisation with a potential pathogen (rho=0.383, $\mathrm{p}=0.041$ ). The total bacterial count was related to numbers of CD3 cells per high-powered field (rho=0.443, $\mathrm{p}=0.06$ ) and worsening MRC dyspnoea score (rho=0.597, $p=0.007$ ).

Patients with frequent COPD exacerbations show increased numbers of lymphocytes, macrophages and eosinophils in their bronchial biopsies when stable. These cells may contribute to the increased airway inflammation seen in patients with frequent COPD exacerbations, which may also be modulated by the presence of lower airway bacterial colonisation.

Supported by the Joint Research Board, St. Bartholomew's Hospital.

\section{Smoking cessation, attitudes, and examples}

\section{S1 1 A SURVEY OF PUBLISHED CURRICULUM CONTENT ON SMOKING AND SMOKING CESSATION IN UK MEDICAL SCHOOLS}

E.L. Jones, P.J. Rubin, J. Britton. University of Nottingham, UK

Rationale: Cigarette smoking is the single most important avoidable cause of respiratory disease in the UK, and smoking cessation interventions are amongst the most cost-effective interventions available in medicine. We have assessed the extent to which UK medical schools recognise and address smoking as a medical problem in their training programmes by a search of published data on curriculum content.

Methods: We searched printed and electronic information on course content published by UK medical schools for teaching on smoking and smoking cessation, using the keywords "smoking", "smoking cessation", "tobacco", "tobacco control", and "nicotine" in electronic searches. Content was assessed according to previously defined criteria.'

Results: Of 23 UK medical schools with current students, 9 (40\%) make no references to smoking or smoking cessation in their published curriculum material. Most of the references made in the remaining 14 schools relate to the importance of taking a smoking history, and to the occurrence of tobacco-related diseases. Four medical schools (17\%) offer optional modules in smoking related issues, four include management of nicotine addiction as part of psychology or public health modules, one included a module on smoking within respiratory medicine, and one included a role-playing smoking cessation session within a Primary Care module. No references were found to teaching on the pharmacology of nicotine addiction.

Conclusions: This study suggests that teaching on the pharmacology and determinants of nicotine addiction, and practical training in the delivery of effective smoking cessation interventions, receive little attention in UK undergraduate medical curricula.

The Department of Health funded this research.

1. Ferry $L H$, Grissino LM, Runfola PS. Tobacco dependence curricula in US undergraduate medical education. JAMA 1999;282:825-9.

\section{S12 ATTITUDES OF GENERAL PRACTITIONERS AND PATIENTS TOWARDS SMOKING CESSATION ADVICE IN PRIMARY CARE}

J. Cleland, M. Thomas, H. Pinnock, T. Van der Molen. University of Aberdeen, UK

Despite compelling evidence for the effectiveness of smoking cessation advice from a GP, rates of provision of such advice remain persistently sub-optimal (Freeth S. Smoking-related behaviour and attitudes, 1997: a report on research using the ONS Omnibus Survey produced on behalf of the Department of Health. London: The Stationery Office, 1998). Why are GPs not providing routine opportunistic smoking cessation intervention? Previous research on provision of smoking cessation advice has focused mainly on the professional's perspective. It may be that smokers have quite clear ideas about what approach and/or content they believe would be effective (Butler CC, et al. BM $1998 ; 316: 1878-81)$. Is there is an area of common ground between 
GP and patient attitudes where opportunistic interventions could be based, thus maximising the chance of such an intervention being received positively?

This qualitative study aimed to identify and explore barriers to the routine provision of smoking cessation advice by GPs, GP and patient attitudes towards such advice. Individual interviews and focus groups were carried out separately with GPs, smokers, and ex-smokers.

Our results indicate that both GPs and patients think that primary care smoking cessation interventions should be: (a) pertinent to the consultation rather than population-based advice, (b) personalised, linked to specific health benefits for that particular individual, and linked to the individual's personal timetable of change, (c) positive, emphasising the positive benefits of quitting seems to be the preferred approach, and (d) practical, which includes GPs prescribing NRT and bupropion.

The importance and potential value of linking smoking to the presenting complaint needs to be further researched. Ways of linking information about the relationship of smoking to the patient's own illness need to be explored. There needs to be rapid access at appropriate times for individuals who may be at a window of opportunity in the cycle of change. The recent development of problem-oriented guidelines for smoking cessation in primary care may encourage GPs to provide such advice and are likely to be more acceptable to patients.

\section{S13 "IF SOMEONE COULD WAVE A MAGIC WAND I'D NEVER SMOKE AGAIN..."-BARRIERS AND MOTIVATORS TO ACCESSING SMOKING CESSATION SERVICES AMONGST SMOKERS IN DEPRIVED AREAS OF NOTTINGHAM}

E.L. Jones, A.W.P. Molyneux, M. Anotoniak, J.R. Britton, S.A. Lewis. Division of Respiratory Medicine, City Hospital, University of Nottingham

Rationale: Smoking is the main factor responsible for inequalities in health between rich and poor.' Although smoking cessation services have been developed in the UK as part of the Tobacco White Paper, ${ }^{2}$ these services have traditionally failed to reach many of the most deprived smokers. ${ }^{3}$ Qualitative research methods are an appropriate way to explore the views of smokers who live in deprived areas who have made an attempt to stop smoking but who have not accessed the local smoking cessation services.

Methods: We conducted a postal survey of the most deprived households in Nottingham, and invited respondents who had made an unsuccessful attempt to stop smoking in the last year without using the Nottingham NHS Smoking Cessation Service, to attend focus groups to explore attitudes, experiences, and knowledge of smoking and stopping smoking, attitudes to smoking cessation services and interventions, and barriers and motivators to the access of such services. Group discussions were recorded and transcribed and a line-byline analysis was undertaken to allow themes and categories to emerge from the data.

Results: Most participants started smoking in their teens and felt highly addicted to nicotine. All were aware of the risks of smoking and had tried to quit smoking, many on multiple occasions, but knowledge of smoking cessation interventions and their effectiveness was poor. Barriers to the access of smoking cessation services - such as cost, timing, childcare, lack of appropriate information, perceived ineffectiveness, and negative publicity-were explored. Novel approaches to the management of nicotine addiction were discussed, including parallels with drug and alcohol addiction treatments, brain surgery, in-patient quit attempts, "staining" cigarettes and government subsidy for complementary therapies.

Conclusions: Deprived smokers are highly addicted, have a poor perception of the availability and efficacy of smoking cessation interventions and are unlikely to access services unless these barriers are broken down.

Funded by the New Leaf, Nottingham NHS Smoking Cessation Service.

1. Acheson D. Independent inquiry into inequalities in health. London: The Stationery Office, 1998.

2. Department of Health. Smoking kills: a white paper on tobacco. London, 1998.

3. Jarvis M. The tobacco epidemic. Basel: Karger, 1997:151-64.

\section{S14 EXAMPLE OF AN EFFECTIVE SMOKING CESSATION SERVICE WITHIN A PRIMARY AND SECONDARY CARE TRUST}

\section{A. Burgoyne, A. Graham, M. Babores. East Cheshire NHS Trust, UK}

Smoking is the nation's single greatest cause of preventable illness and early death. More than 120000 people a year in the UK die from smoking related diseases and it costs the NHS 1.7 billion each year. In April 2000, funding was allocated from South Cheshire Health Authority which supported the development of a locally based smoking cessation service in east Cheshire for the following two years $-£ 9000$ for year one and $£ 18500$ for year two.

A multidisciplinary team was responsible for the co-ordination and development of the smoking cessation service in east Cheshire with representatives reporting to the Eastern Cheshire Primary Care Group (Trust) and the NSF CHD Local Implementation Team. These services were based in the Primary and Secondary health care settings delivering a standard rolling 6-8 weeks programme. This included one to one or group support, counselling from a trained smoking cessation adviser, provision of Nicotine Replacement Therapy and Bupropion where appropriate.

Targets set were 650 smokers setting quit dates over two years. Actual targets achieved doubled with 1190 smokers setting a quit date. Of those $612(52 \%)$ were not smoking at 4 weeks with CO validation, $244(20 \%)$ relapsed and 334 were lost to follow up $(28 \%)$. Twelve months follow up rates are to be reported in August 2002.

Monitoring data supports the continuation of a smoking cessation service in east Cheshire. Planning for long term funding needs to be identified. Maintaining smoking cessation as a priority for health interventions is vital. Continued development and co-ordination of smoking cessation services will be required to meet increasing public and professional demand.

\section{S15 12 MONTH QUIT RATES OF A "SPECIALISED" HOSPITAL BASED SMOKING CESSATION SERVICE}

S. Brown, R.M. Angus, I. Davies. Aintree Chest Centre, University Hospital Aintree, Liverpool L9 7AL, UK

It is reported that intensive behavioural support plus Nicotine Replacement Therapy (NRT) or Bupropion enables about $20 \%$ of smokers to stop long term, compared to $5 \%$ from brief advice from a General Practitioner.' Quit rates in those with established smoking related diseases are surprisingly lower than those in "healthy smokers". We established a hospital-based smoking cessation service for smokers with respiratory disease. Patients are offered one-to-one counselling with frequent structured advice and regular support. NRT and/or Bupropion can be prescribed as required. All quit results were validated by expired air CO (carbon monoxide). From April 2001 to March 2002, 337 patients were referred into the service. Fifty nine $(16 \%)$ did not attend their first appointment and $40(12 \%)$ were not motivated to quit at the time of referral. Two hundred and thirty eight $(71 \%)$ set quit dates and the following are the results of these 238 patients. Mean (SD) age 57 (9) years, 135 (57\%) female. Two hundred and nine $(88 \%)$ had previously tried to quit. Diagnoses were as follows: COPD $107(45 \%)$, asthma $58(24 \%)$, bronchiectasis 14 $(6 \%)$, lung cancer $13(5 \%)$ and "other" $46(19 \%)$. Patients used the following pharmacological support: NRT patches $107(45 \%)$, Bupropion 41 (17\%), NRT inhalator 37 (16\%), NRT gum $10(4 \%)$, and NRT lozenge $7(3 \%)$. Thirty six $(15 \%)$ used willpower alone. Of 238 patients $147(62 \%)$ reached a 4 week quit, 81 (55\%) female. Thirty nine (16\%) patients were lost to follow up. Quit results at 6 months were $49 / 102(48 \%)$, with $19(19 \%)$ lost to follow up and at 12 months were $18 / 24(75 \%)$ with $3(13 \%)$ lost to follow up. Of the 18 patients that were continuously abstinent at 12 months, 11 (61\%) were female and products used were Bupropion 7 (39\%), NRT patches $7(39 \%)$, and willpower alone $4(22 \%)$. These results compare favourably to recent Department of Health figures for smoking cessation services in the UK, where it was quoted that quit rates of around $20 \%$ at the 12 month follow up should be expected. ${ }^{2}$ This demonstrates the value of targeting thoracic patients in specialist smoking cessation services in the UK.

1. World Health Organisation. The case for commissioning smoking cessation services. London, 2001.

2. Department of Health. New NHS Smoking Cessation Services. London: Stationery Office, 1999. 


\section{Sleep: New outlooks}

\section{S16 THE UPPER AIRWAY IN PREGNANCY AND PRE-ECLAMPSIA}

B. Izci', R.L. Riha', S.E. Martin', M. Vennelle', W.A. Liston², K. Dundas², A. Calder $^{2}$, N.J. Douglas'. 'Edinburgh Sleep Centre, University of Edinburgh, UK; ${ }^{2}$ Department of Reproductive and Developmental Sciences, University of Edinburgh, UK

Background: Snoring is common in pregnancy and snoring pregnant women have increased rates of pre-eclampsia. Patients with pre-eclampsia have an increased rate of upper airways (UA) narrowing during sleep which may contribute to their blood pressure elevation.

Aims: To compare upper airway dimensions in pregnant and nonpregnant women and in patients with pre-eclampsia.

Method: 50 women in the 3rd trimester of pregnancy and 37 women with pre-eclampsia were recruited consecutively from the antenatal service and matched with 50 non-pregnant women. UA dimensions were measured using acoustic reflection. Comparisons were by analysis of variance and Student-Newman-Keuls tests.

Results: The pregnant, pre-eclamptic, and non-pregnant women did not differ in terms of age or height, or in pre-pregnant weight or body mass index. $14 \%$ of non-pregnant, $28 \%$ of pregnant, and $75 \%$ of pre-eclamptic women reported they snored $(p<0.001)$. Oropharyngeal junction area (OPJ) in the supine position was narrower in pregnant than non-pregnant women (1.0 SD 0.1, 1.1 SD $0.1 \mathrm{~cm}^{2} ; p=0.05$ ) and smaller yet in pre-eclamptics $\left(0.8 \mathrm{SD} 0.1 \mathrm{~cm}^{2} ; p<0.05\right)$. Seated OPJ was narrower $(p<0.05)$ in the pre-eclamptics $\left(1\right.$ SD $\left.0.1 \mathrm{~cm}^{2}\right)$ than either controls $\left(1.2 \mathrm{SD} 0.1 \mathrm{~cm}^{2}\right)$ or pregnant women $\left(1.3 \mathrm{SD} 0.1 \mathrm{~cm}^{2}\right)$.

Conclusions: Upper airways are narrower during the third trimester of pregnancy, and women with pre-eclampsia have further airway narrowing. This could result from a combination of FRC reduction due to the pregnancy and generalised oedema. These changes could contribute to the increased snoring in pregnancy, and to the upper airways resistance episodes during sleep in pre-eclampsia which may further increase blood pressure.

Study supported by the Cunningham Trust.

\section{S17 BRAIN FMRI ACTIVATION AND EVOKED POTENTIALS DURING VISUAL STIMULATION AND VISUO-MOTOR TRACKING IN NORMAL SUBJECTS BEFORE AND AFTER SLEEP DEPRIVATION}

G.V. Robinson', R.C. Miall', P.M. Matthews ${ }^{3}$, E.M. Jones', J. Winter ${ }^{2}$, J. $S_{\text {Stein }}^{2}$, J.R. Stradling', R.J.O. Davies'. 'Oxford Centre for Respiratory Medicine, Churchill Hospital, Oxford, UK; ${ }^{2}$ University Laboratory of Physiology, Oxford, UK; ${ }^{3}$ FMRIB Centre, John Radcliffe Hospital, Oxford, UK

Patients sleep deprived by obstructive sleep apnoea (OSA) have a 4-7 fold increased motor accident rate. This is associated with impairment on tasks that test vision and tracking, including simulated steering tests (Thorax 2001;56(Suppl 3):28). The neural explanation for this is unknown.

Methods: Using a previously developed paradigm (Nature Neurosci 2001;4:638), we have assessed the brain functional magnetic resonance imaging (fMRI) activation patterns in normal subjects during pure visual stimulation and during performance of coordinated hand/eye tracking manoeuvres, before and after 28-32 hours total sleep deprivation (TSD). Evoked potentials to a potent visual stimulus were also recorded from the occipital cortex.
After pre-training, 10 normal subjects (7M, 3F, mean age 27) underwent FMRI imaging, while looking at an alternating chequerboard (pure visual stimulus) and while performing the visual tracking paradigm (visuo-motor stimulus). Subjects were assessed at baseline after normal sleep. Further assessments were performed, in random order, after normal sleep and 28-32 hours of actigraphy monitored TSD and stimulant abstinence. 4 subjects also had occipital visual evoked potentials (VEPs) recorded.

Results: Following normal sleep, the expected brain areas were activated on $\mathrm{FMRI}$ by both tasks. Tracking performance was significantly impaired by TSD (mean tracking error 114.3 SD 49.1 after normal sleep, 219.7 SD 47.6 after TSD, $p<0.0001$, paired $t$ test). During the chequerboard (pure visual stimulus), TSD increased brain activation in the primary visual cortex, precuneus and left prefrontal areas, but decreased activation in the extra striate visual cortex. The VEP studies confirmed a neural basis for this reduced brain activation. During the tracking (visuo-motor) task, TSD lead to reduced cerebellar and premotor activation, with unchanged primary motor cortex activation.

Conclusion: TSD in normal subjects impairs tracking. This is associated with activation of neural centres that may be associated with vigilance maintenance (prefrontal cortex and precuneus) and depression of visual association areas and secondary motor centres.

\section{S18 PLATELET ACTIVATION IS INCREASED IN OBSTRUCTIVE SLEEP APNOEA AND DOES NOT FALL WITH CPAP TREATMENT}

G.V. Robinson', J.C.T. Pepperell', H. Segal'2, B. Langford', R.J.O. Davies', J.R. Stradling'. 'Oxford Centre for Respiratory Medicine, Churchill Hospital, Oxford, UK; ${ }^{2}$ Oxford Haemophilia and Thrombosis Unit, Churchill Hospital, Oxford, UK

Obstructive sleep apnoea (OSA) is an independent risk factor for hypertension and arterial thrombotic disease (Am J Resp Crit Care Med 2002;166:159). The increased cardiovascular risk is probably multifactorial, and related to insulin resistance and endothelial dysfunction, in addition to hypertension (the increased incidence of which in OSA is now well established). Platelets have a central role in the pathogenesis of acute cardiovascular syndromes, and platelet activation is associated with increased cardiovascular risk in normals (Thromb Haemost 2001;85:584). The influence of OSA on platelet function is not clear.

Methods: 94 male subjects mean (SD) age 48 (11) with OSA defined as $\geqslant 10$ oxygen saturation dips $\geqslant 4 \%$ per hour (actual dip rate 42.5 (23.0)) and an Epworth sleepiness score (ESS) $\geqslant 10$ (actual ESS 15.7 (3.0)) were randomised to one month's treatment with therapeutic or sub-therapeutic $(-1 \mathrm{~cm}$ water pressure) continuous positive airways pressure (CPAP) treatment. Plasma levels of soluble P-selectin (sP-sel), a marker of chronic platelet activation, were measured by ELISA before and after treatment. 22 unmatched normal subjects were used to establish a normal range.

Results: $\mathrm{SP}$-sel was higher in the untreated OSA patients than in the normal subjects (OSA patients $55.4 \mathrm{ng} / \mathrm{ml}(37.3)$, normal subjects $29.5 \mathrm{ng} / \mathrm{ml}$ (10.5), $p<0.0001$, unpaired $t$ test). No significant fall in sP-sel was seen following one month's treatment with either therapeutic or sub-therapeutic CPAP: therapeutic CPAP, pre treatment 58.3 $\mathrm{ng} / \mathrm{ml}(41.9)$, post treatment $54.0 \mathrm{ng} / \mathrm{ml}(35.5), \mathrm{p}>0.4$, paired $t$ test; sub-therapeutic CPAP, pre treatment $52.5 \mathrm{ng} / \mathrm{ml}$ (32.1), post treatment $45.4 \mathrm{ng} / \mathrm{ml}(22.7), \mathrm{p}>0.1$, paired $t$ test.

Conclusion: OSA may cause increased platelet activation, which does not fall with one month's CPAP treatment. This is likely to be a further contributor to the increased vascular morbidity of OSA, which is not improved with one months standard OSA treatment.

\begin{tabular}{llrl}
\hline Abstract S19 Table 1 & \multicolumn{3}{l|}{} \\
\hline & Baseline & Post CPAP & $\mathrm{p}$ Value \\
\hline Epworth Score & $14(0-20)$ & $8(0-20)$ & $<0.005$ \\
MFI-20, General Fatigue & $17(8-20)$ & $12(4-20)$ & $<0.005$ \\
MFI-20, Physical Fatigue & $16(4-20)$ & $13(4-20)$ & $<0.01$ \\
MFI-20, Reduced Activity & $10(4-17)$ & $8(4-20)$ & $<0.05$ \\
MFI-20, Mental Fatigue & $13(4-20)$ & $7(4-20)$ & $<0.005$ \\
SF-36, Physical Limitations & $25(0-10)$ & $75(0-10)$ & $<0.005$ \\
SF-36, Vitality & $30(0-85)$ & $55(0-10))$ & $<0.001$ \\
SF-36, Mental Health & $68(4-100)$ & $84(28-100)$ & $<0.005$ \\
\hline
\end{tabular}


Abstract S19 Table 2 MFI-20, $\triangle M F I-20, R A$ $=1.59+0.5 \Delta \mathrm{ESS}$ $=0.80+0.31 \Delta \mathrm{ESS}$ $=0.40+0.27 \Delta \mathrm{ESS}$

$\begin{array}{rl}r^{2}=0.30 & p<0.001 \\ r^{2}=0.13 & p<0.01\end{array}$

$p<0.01$

$r^{2}=0.12 \quad p<0.05$
S19 THE MULTIDIMENSIONAL FATIGUE INVENTORY (MFI-20) IN OBSTRUCTIVE SLEEP APNOEA (OSA): EFFECTS OF NASAL CONTINUOUS POSITIVE AIRWAY PRESSURE (CPAP)

N. Wiltshire, F. Buchanan, A. Harper, J.R. Catterall, A.H. Kendrick. Sleep Unit, Department of Respiratory Medicine, Bristol Royal Infirmary, Bristol, UK

We have shown that the MFI-20 provides useful additional information to the Epworth Score (ESS) and SF-36 in patients with OSA, and separates sleepiness and fatigue (Kendrick et al. Thorax 2001;56/Suppl III):46).

Aim: To assess the response of the MFI-20 in a CPAP naive patients and to compare this with data from the ESS and the dimensions of the SF-36.

Methods: Patients were given the ESS, SF-36 and MFI-20 questionnaires before and at the end of a 4 week trial of CPAP as part of our clinical management of patients with OSA. Data are given as median (range).

Results: 50 patients (6F), age $54.5 \mathrm{yr}$ (27-80) and Body Mass Index $31.8 \mathrm{~kg} \cdot \mathrm{m}^{-2}$ (22.8 to 52.6 ) were studied. The results are summarised in table 1 .

At the end of the trial and using a cut-off of 10 for each MFI-20 dimension and for ESS, 16/50 had a MFI-20 GH >10, 17/50 had an MFI-20 PH > 10 and 20/50 had an MFI-20 RA > 10 indicating significant fatigue problems remain in the absence of daytime hypersomnolence. The relation between changes in ESS and MFI-20 are shown in table 2 .

Conclusion: The MFI-20 is a simple self-completion questionnaire that provides useful additional information to that obtained from the ESS and the SF-36 and separates out sleepiness and fatigue pre and post CPAP.

\section{S20 MODAFINIL IMPROVES WAKEFULNESS AND OVERALL CLINICAL CONDITION AS ADJUNCTIVE THERAPY FOR RESIDUAL EXCESSIVE SLEEPINESS IN OBSTRUCTIVE SLEEP APNEA: A 12 WEEK RANDOMISED TRIAL}

A. Williams and the Modafinil OSA study group. Sleep Disorders Centre, St. Thomas's Hospital NHS Trust, UK

Objective/Methods: A 12 week, randomised, double-blind, placebo-controlled study was conducted to assess the efficacy and safety of modafinil as adjunctive therapy for residual excessive sleepiness in patients with obstructive sleep apnea (OSA) treated with nasal continuous positive airway pressure (nCPAP). Patients were randomized to receive placebo, modafinil $200 \mathrm{mg}$ once daily, or modafinil $400 \mathrm{mg}$ once daily. Modafinil was initiated at $100 \mathrm{mg} / \mathrm{d}$ and titrated to 200 or $400 \mathrm{mg} / \mathrm{d}$ within 1 week. Key outcomes included changes from baseline in objective (Maintenance of Wakefulness Test [MWT]) and subjective (Epworth Sleepiness Scale [ESS]) measures of wakefulness and patients' overall clinical condition (Clinical Global Impression of Change [CGI-C]) at Week 12. Secondary efficacy measures included changes from baseline in MWT, ESS, and CGI-C at Weeks 4 and 8. nCPAP use and adverse events were monitored.

Results: A total of 323 randomised patients received treatment (placebo: $\mathrm{n}=108$; modafinil $200 \mathrm{mg} / \mathrm{d}$ : $\mathrm{n}=109$; modafinil $400 \mathrm{mg} / \mathrm{d}$ : $\mathrm{n}=106$ ). Modafinil significantly improved patients' ability to sustain wakefulness on the MWT at Week 12 (increases of $1.6 \mathrm{~min}$ for modafinil $200 \mathrm{mg} / \mathrm{d}$ and $1.5 \mathrm{~min}$ for modafinil $400 \mathrm{mg} / \mathrm{d}$ ) compared with placebo (decrease of $1.1 \mathrm{~min} ; \mathrm{p}<0.0001)$. Modafinil treatment also significantly improved wakefulness as measured by the ESS scores: a 4.5-point decrease in ESS score was observed with modafinil compared with a 1.9-point decrease with placebo at Week 12 ( $p<0.0001)$. Modafinil significantly improved patients' overall clinical condition at Week 12, as measured by the CGI-C $(p<0.0001)$. Modafinil had no effect on nCPAP use or nighttime sleep. Modafinil was associated with significant improvements in wakefulness and clinical condition at Weeks 4, 8, and 12. Headache $112 \%$;
$26 \%)$, nausea $(2 \% ; 11 \%)$, and anxiety $(2 \% ; 8 \%)$ were the most common adverse events occurring in the placebo and modafinil treatment groups, respectively.

Conclusions: Adjunct modafinil therapy significantly improves wakefulness and overall clinical condition and is well tolerated in patients with OSA who have residual sleepiness despite treatment with nCPAP.

\section{Pulmonary hypertension: Clinical issues}

\section{S21 PULSE PRESSURE PREDICTS MORTALITY IN PULMONARY ARTERIAL HYPERTENSION}

J.D. Chalmers', R. Syyed' ${ }^{2}$, V. Impey', A. Peacock'. 'University of Glasgow; ${ }^{2}$ Scottish Pulmonary Vascular Unit, Western Infirmary, Glasgow, UK

Introduction: It is known that mean pulmonary artery pressure predicts survival in pulmonary hypertension. Following the Framingham heart study, it was shown that pulse pressure (PP) outperformed mean arterial, systolic and diastolic pressures as predictors of mortality in systemic hypertension. We wished to discover if this held true for the pulmonary circulation.

Methods: We retrospectively reviewed all the patients with Pulmonary hypertension who had been studied between 1996 and January 2002. 80 patients had Pulmonary hypertension (PHT). Of the 80 patients 19 ( 11 female, 8 male) had PPH, 18 ( 10 female, 8 male) had thromboembolic disease, 17 (13 female, 4 male) had connective tissue disease, 10 ( 8 female, 2 male) had COPD, 10 ( 8 female, 2 male) had Eisenmengers, 3 ( 2 female, 1 male) had porto-pulmonary hypertension, 2 (2 female) had valvular heart disease and 1 ( 1 female) had Sarcoidosis. Haemodynamic variables as well as performance status and medical history were examined. Kaplain Meier Survival curves and regression analysis were used to determine correlation between variables and survival.

Results: PP against survival produced a correlation coefficient ( $r$ ) of $-0.76(p<0.001)$ and Kaplain Meier survival curves revealed a $100 \%$ probability of survival at 2 years for $\mathrm{PP}<30 \mathrm{mmHg}$ compared to $85 \%$ probability of survival for PP $30-40$ and $46 \%$ for PP greater than 40 . No other variable predicted survival with this accuracy.

Conclusion: Pulse pressure is a powerful predictor of mortality in all causes of pulmonary hypertension. No other variables proved superior to pulse pressure, regardless of underlying diagnosis.
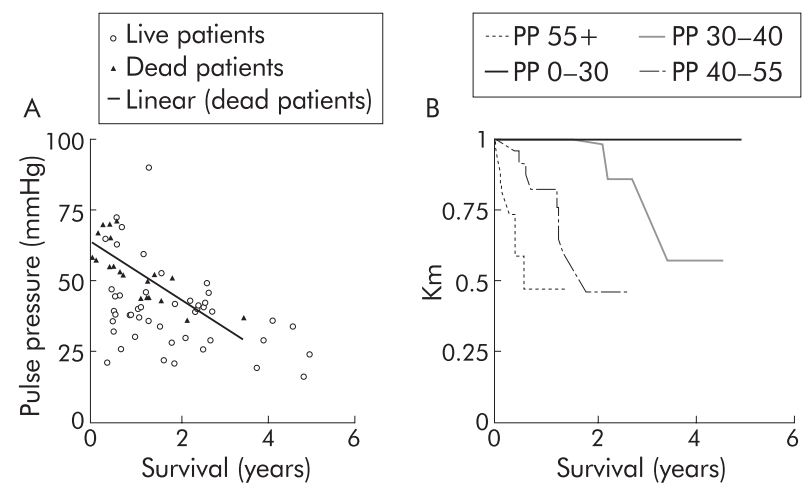

Abstract S21 (A) Pulse pressure and survival. (B) Kaplan Meier survival curves, pulse pressure and survival.

\section{S22 LONG DURATION OF BREATHLESSNESS AT DIAGNOSIS IS ASSOCIATED WITH LONGER SURVIVAL IN PPH: A CLINICAL PARADOX}

V. Impey, A. Peacock'. University of Glasgow, Glasgow G12 8QQ, UK; 'Scottish Pulmonary Vascular Unit, Western Infirmary, Glasgow G1 1 6NT, UK

Introduction: Previous studies have shown that breathlessness predicts survival. It is widely believed that a longer duration of breathlessness is related to a worse prognosis, probably due to slower time to diagnosis. We expected to confirm this supposition in our data 

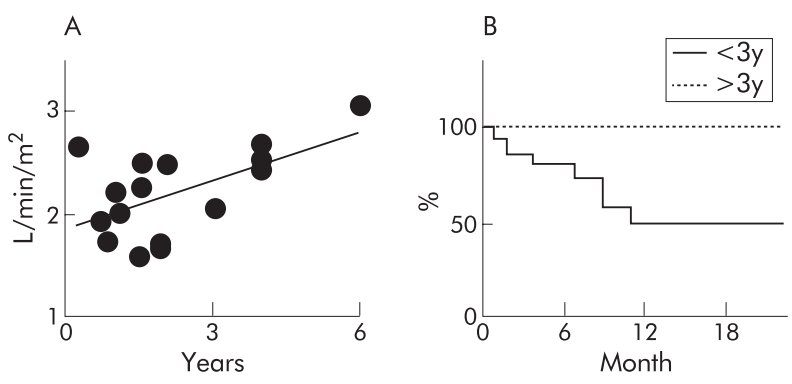

Abstract S22 (A) Breathlessness and cardiac index. (B)

Kaplan-Meier curve: breathlessnes.

series but found the reality to be the opposite. We have previously found cardiac index $(\mathrm{Cl})$ to be an important predictor of survival so we correlated duration of breathlessness and $\mathrm{Cl}$.

Methods: We examined admission data in all our patients with $\mathrm{PPH}$. We included demographic variables (sex, age) medical history (duration of breathlessness, NYHA class), haemodynamic data (mean pulmonary artery pressure, cardiac index). Kaplan-Meier (KM) survival curves and regression analysis were used to examine the correlation between these variables and survival.

Results: For our 25 patients with PPH, the probability of survival at 12 months after diagnosis was $100 \%$ for those 8 patients with a history of breathlessness $>3$ years and $51 \%$ for those with breathlessness $<3$ years (13 patients). There was also a positive correlation coefficient of 0.6 between cardiac index and duration of breathlessness (we excluded 2 patients with breathlessness longer than 6 years as outliers).

Conclusion: A longer duration of symptoms at diagnosis may represent a slower progression of disease and better survival prospects, reflected by the improved cardiac index.

\section{S23 SCREENING FOR EARLY PULMONARY ARTERIAL HYPERTENSION IN PATIENTS WITH SYSTEMIC SCLEROSIS (SSCPAH): RATIONALE FOR AN INVASIVE APPROACH}

D. Mukeriee, D. St George, J. Davar, C.M. Black, J.G. Coghlan. Departments of Rheumatology and Cardiology, Royal Free Hospital, Pond Street, London NW3 2QG, UK

Aims: To document the accuracy of echocardiography to identify early pulmonary hypertension (PHT) in a high-risk Systemic Sclerosis (SSc) cohort, we identified 140 patients who have had echo and cardiac catheter within \pm 3 months of either investigation. Previous studies have documented under-estimation of echo-estimated tricuspid gradient (TG) compared to catheter results, with only a few studies reporting echo over-estimation of this parameter.

Method: Echo-estimated TGs and the catheter-measured TGs were correlated and a linear regression was derived to predict catheter-measured mean Pulmonary Artery Pressures (mPAPs) from echo estimated TGs.

Results: $N=140,(F: M=119: 21)$, mean age $=56$ (7) years. Haemodynamic parameters: $\mathrm{mPA}$ Systolic Pressure $=47 \mathrm{mmHg}$, mPA diastolic Pressure $=18 \mathrm{mmHg}, \mathrm{mPAP}=34 \mathrm{mmHg}, m R A P=4 \mathrm{mmHg}$, mean echo estimated TG $=42 \mathrm{mmHg}$. Echo-estimated TG showed a positive correlation with catheter-measured TGs in 140 patients $(\mathrm{r} 2=0.44$, $\mathrm{p}<0.005)$. By linear regression, echocardiography was sufficiently accurate at moderate/ high pressures (echo TG $>45 \mathrm{mmHg}$ ) identifying $>80 \%$ of patients correctly. In contrast, at lower pressures where early diagnosis is critical, echo was less accurate-it both under-estimated the catheter-measured mPAPs in 30\% and overestimated the catheter-measured mPAPs in $32 \%$ of cases. The latter inaccuracy may lead to a potential for over-diagnosis in unaffected patients.

Conclusions: Though echo accurately identified a poor prognostic group of PHT patients (mPAP $>35 \mathrm{mmHg}$ on cardiac catheter), reliance on the present technique to accurately identify early PHT in an "at risk" SSc cohort is inadequate. In its current form, echo-estimated TGs demonstrate a tendency to both under and over-estimation at the lower end of the spectrum of pulmonary artery pressures - with the subsequent risk of exposing a patient with either no or mildly elevated pulmonary pressures to over-investigation. This latter finding is particularly relevant when applied to low prevalence PHT populations where the false positive rates are proportionately higher and in such scenarios the accuracy of echo is further diminished.

\section{S24 THE UTILITY OF D-DIMERS AND OTHER PREDICTORS OF PULMONARY EMBOLI IN A LARGE SERIES FROM A DISTRICT GENERAL HOSPITAL}

S.A. Evans, S.C. Ward, M. Jarral, R.J. Clark, J. Langham-Brown, T. Fail, P. Featherstone, P. Schmidt. Portsmouth Hospitals Trust, UK

Previous studies suggest that a negative D-dimer can have a negative predictive value (NPV) for objectively diagnosed pulmonary emboli (PE) of $99 \%$ (Egermeyer et al. Thorax 1998;53:830-4). A pilot study in our hospital comparing bed side and laboratory SimpliRED and a ELISA D-dimer assays for DVT gave NPV=s of between $69 \%$ and $76 \%$.

We have used 4 parameters to assess the pre-test probability of PE: (a) D-dimers (SimpliRED, performed on the ward), (b) Respiratory rate (RR) $>20 /$ minute (c) $\mathrm{PaO}_{2}$ on air of $<10.7 \mathrm{kPa}$, (d) Important risk factors from the history i.e. surgery/trauma, malignancy, cardiovascular disease, previous PE/DVT, post-partum, immobilisation, and hereditary thrombotic disorders.

These were prospectively evaluated in 521 patients who were investigated for suspected PE. Assessment was incomplete in 101 cases, leaving 420 cases for analysis. Those with a normal CXR and no chronic lung disease initially had a perfusion (Q) scan $(n=297)$. Leg dopplers ( $n=95$ ) were performed on those with an abnormal CXR, chronic lung disease or an indeterminate $Q$ scan. CT pulmonary angiograms were requested on those with negative or indeterminate leg dopplers $(n=51) .27$ patients had features of a massive PE and were investigated with urgent $\mathrm{CT}$ angiogram or an echocardiogram.

PE was confirmed in 131 patients, excluded in 289. The NPV of a D-dimer was $83 \%$, a RR $<2090 \%$, a $\mathrm{PaO}_{2}>10.7 \mathrm{kPa} 83 \%$, and absent clinical risk factor(s) $81 \%$. Logistic regression gave RR high odds ratios and suggested that $\mathrm{PaO}_{2}$ added little to the other 3 parameters. A combination of no risk factor and a $R R<20$ gave a risk of objectively confirmed $\mathrm{PE}$ of $<5 \%$ irrespective of the $\mathrm{D}$-dimer result and the $\mathrm{PaO}_{2}$.

We conclude that negative D-dimer results are best used in conjunction with other predictors to exclude PE.

\section{S25 OUTCOME AFTER PULMONARY THROMBOENDARTERECTOMY FOR CHRONIC THROMBOEMBOLIC PULMONARY HYPERTENSION}

F. Reichenberger, J. Pepke Zaba, D. Hodgkins, A. Vuylsteke, K. McNeill, J. Dunning. Papworth Hospital, Cambridge, UK

Pulmonary thromboendarterecomy (PTE) is the treatment of choice in chronic thromboembolic pulmonary hypertension (CTEPH) with proximal vascular obstructions. However PTE is associated with a significant perioperative risk. Over the last 5 years, 100 patients $(50$ male, 50 female, mean age 53 (18-81) years) underwent PTE at Papworth Hospital. 71 patients survived the procedure, whereas 29 died postoperatively: 10 due to reperfusion oedema, ARDS, or bronchopulmonary infections despite significant improved haemodynamics, 12 had incomplete clearance of vascular obstruction with additional peripheral vascular obstructions, 3 were misdiagnosed preoperatively (PPH, peripheral CTEPH, advanced pulmonary artery sarcoma). Four patients developed surgical complications. The quartile distribution of the outcome is shown below. Evaluating preoperative factors that influenced perioperative outcome, the surviving patients had a significantly higher 6 minute walking distance (268 v 194 metres, $p<0.01)$, and higher cardiac index $(1.8 \mathrm{v} 1.5 \mathrm{l} / \mathrm{min} / \mathrm{qm}, \mathrm{p}<0.01)$ compared to the non-survivors, whereas right atrial and pulmonary artery pressures

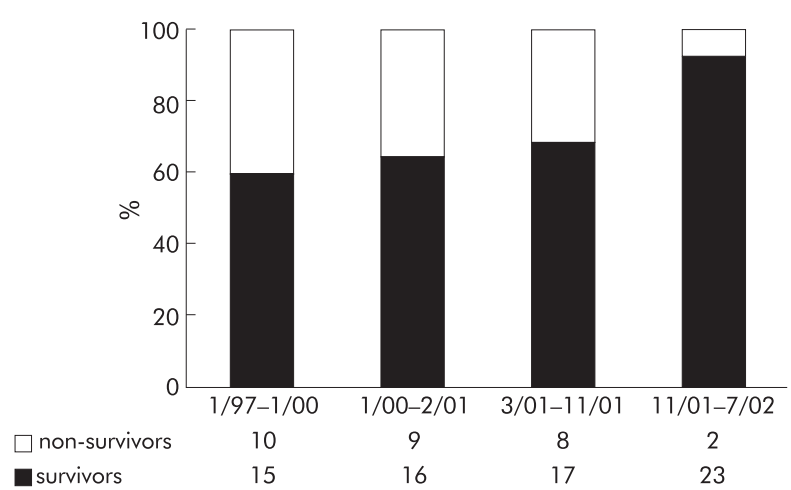

Abstract S25 
were similar in both groups. At our institution, experience within the recently established PTE programme shows a learning curve associated with a significant improvement in the postoperative outcome in this high risk patient population.

\section{Asthma therapeutics}

\section{S26 THE USE OF NEBULISED ISOTONIC MAGNESIUM SULPHATE AS AN ADJUVANT TO SALBUTAMOL IN THE TREATMENT OF SEVERE ASTHMA IN ADULTS}

R.J. Hughes' ${ }^{3}$, A.L. Goldkorn², M. Masoli ${ }^{3}$, M. Weatherall', C. Burgess², C.R.W. Beasley ${ }^{3}$ ' $G$ reenlane Hospital; ${ }^{2}$ Wellington School of Medicine; ${ }^{3}$ Medical Research Institute of New Zealand, New Zealand

Background: Intravenous magnesium has been shown to cause significant bronchodilation in the treatment of severe asthma, however its effect by the nebulised route is uncertain. In this study we assessed the efficacy of isotonic magnesium sulphate as an adjuvant to nebulised salbutamol in severe asthma.

Methods: We enrolled 52 subjects with severe exacerbations of asthma ( $F E V,<50 \%$ predicted) presenting to the Emergency Departments at two hospitals in New Zealand. In this randomised double-blind placebo-controlled trial subjects received nebulised salbutamol $(2.5 \mathrm{mg})$ mixed with either $2.5 \mathrm{~mL}$ of isotonic magnesium sulphate or isotonic saline on three occasions at 30 minute intervals. The primary outcome measures were $\mathrm{FEV}$, at 90 minutes and requirement for admission.

Results: The mean $\mathrm{FEV}$ in both groups at randomisation was similar (1.24 litres, $31.9 \%$ predicted $v 1.20$ litres, $31.8 \%$ predicted, $p=0.73)$. Subjects who received nebulised salbutamol with the magnesium adjuvant achieved a greater improvement in $\mathrm{FEV}_{1}(0.72 \mathrm{v}$ 0.35 litres, difference 0.37 litres, $p=0.004$ ) when compared with nebulised salbutamol with the saline adjuvant. A corresponding reduction in requirement for admission (relative risk 0.61 , confidence interval 0.37 to 0.99 ) was demonstrated. The greatest difference between the two regimens occurred in those individuals presenting with life-threatening exacerbations, defined by an $\mathrm{FEV}$, of less than $30 \%$ predicted (increase in $\mathrm{FEV}, 0.83 \vee 0.18$ litres, difference 0.75 litres, $p<0.0001$ ).

Conclusions: The use of isotonic magnesium as an adjuvant to nebulised salbutamol results in an enhanced bronchodilator response in the treatment of severe asthma.

\section{S27 GLUCOCORTICOID RECEPTOR ACTIVATION IN INDUCED SPUTUM FOLLOWING INHALED LONG-ACTING 32 -AGONIST AND GLUCOCORTICOID TREATMENT}

O.S. Usmani, K. Maneechotesuwan, I.M. Adcock, P.J. Barnes. Department of Thoracic Medicine, National Heart and Lung Institute, Imperial College School of Medicine, London, UK

The clinical evidence is well established for the complementary effects of inhaled long-acting $\beta 2$-agonists (LABA) and glucocorticoids (GC) on asthma control. Recent in vitro data suggest the molecular mechanisms may involve enhanced glucocorticoid receptor (GR) nuclear translocation, as LABA have been shown to induce ligand-independent GR translocation. GC activate GR which translocate to the nucleus and bind to DNA to regulate the expression of GC-sensitive target genes, or to coactivators to switch off inflammatory genes. The aim of our research was to develop a model using cells relevant to airway disease, to test the hypothesis that inhaled LABA were able to modulate the intracellular partitioning of GR in vivo.

We previously described a semi-quantitative method to identify GR subcellular expression in induced sputum cells using immunocytochemistry, and showed ligand-induced GR activation in this model. Six healthy subjects inhaled beclomethasone dipropionate $(800 \mu \mathrm{g})$ once, and sputum was induced at $0,30,60,120$ mins post-inhalation. We observed significant GR translocation $(71 \%)$ at $60 \mathrm{mins}$ post GC inhalation compared to baseline $(30 \%)(p<0.05)$. Using this information on the optimal time point for GR activation, we describe here the effects of $L A B A$ and $G C$ in asthmatics.

Seven steroid-naïve asthmatics inhaled single doses of fluticasone

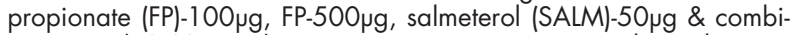
nation FP/SALM 100/50 pg on separate visits. Dose dependent GR activation was observed following FP; FP-100 (47\%), FP-500 (61\%) where the higher dose was significant $v$ placebo $(31 \%) \quad(p<0.05)$.
SALM alone achieved $43 \%$ GR translocation, but as combination therapy, SALM was able to augment the action of FP on GR translocation $(54 \%)(p<0.05)$.

We have shown it is possible to use induced sputum to investigate the molecular effects of inhaled drug therapy. Our data support the proposition that GR nuclear translocation may underlie the complementary effects of LABA and GC. The precise signal transduction mechanisms remain unknown, but LABA may prime inactive GR through phosphorylation, and subsequently $G R$ may require less $G C$ for nuclear translocation. We now aim to research this hypothesis, as it may identify biochemical targets for future therapeutic modulation.

\section{S28 DOSE-RESPONSE RELATION OF INHALED BUDESONIDE IN ADOLESCENTS AND ADULTS WITH ASTHMA}

M. Masoli, S. Holt, M. Weatherall, R. Hughes, R. Beasley. Medical Research Institute of New Zealand.

Objective: To examine the dose-response relation of inhaled budesonide in adolescents and adults with asthma.

Design: Meta-analysis of placebo controlled, randomised clinical trials that presented data on at least one outcome measure of asthma and that used at least two doses of budesonide, delivered by turbuhaler twice daily.

Setting: Medline, Embase, and Astra-Zeneca's internal clinical study registers.

Main outcome measures: $\mathrm{FEV}_{1}$, morning and evening peak expiratory flow, $\beta$-agonist use, withdrawals and exacerbations of asthma leading to withdrawal.

Results: Three studies of 1013 adolescents and adults with moderately severe asthma, met the inclusion criteria for the meta-analysis. Only one study examined doses $>800 \mu \mathrm{g} /$ day and no studies examined doses $>1600 \mu \mathrm{g} /$ day. A negative exponential model for the data, without meta-analysis, indicated that $80 \%$ of the benefit at 1600 $\mu \mathrm{g} /$ day was achieved at doses of $250-350 \mu \mathrm{g} /$ day and $90 \%$ by 350-500 $\mathrm{\mu g} /$ day. A quadratic meta-regression showed that the maximum effect was obtained with doses of around $1000 \mu \mathrm{g} /$ day. Comparison of the standardised difference in $\mathrm{FEV}$, for an inhaled dose of $400 \mathrm{\mu g} /$ day against higher doses showed a difference in FEV 0.03 of a standard deviation $(-0.153$ to 0.213$)$. It was not possible to undertake a meaningful statistical analysis of withdrawals, however examination of individual study data indicated that most of the benefit with respect to reduction in asthma exacerbations leading to withdrawal was achieved with a dose of $400 \mu \mathrm{g} /$ day.

Conclusions: Determination of the dose-response relation of budesonide was limited by the lack of individual patient data, the paucity of studies reporting the effect of doses of $>800 \mu \mathrm{g} /$ day and insufficient withdrawal data. However, utilising the available published data, most of the therapeutic benefit of budesonide delivered by the turbuhaler device was achieved with a total daily dose of 250-500 $\mathrm{\mu g} /$ day, and the maximum effect at around 1000 $\mu \mathrm{g} /$ day, in adolescents and adults with asthma. These findings are consistent with the recently determined dose-response relation of fluticasone propionate, assuming a potency ratio of $1: 2$. We recommend that national and international consensus guidelines and formularies are modified to ensure that they are consistent with the published data from which the therapeutic dose-response range of inhaled corticosteroids has been derived.

\section{S29 DOSE-RESPONSE RELATION OF INHALED} FLUTICASONE PROPIONATE IN CHILDREN WITH ASTHMA-A SYSTEMATIC REVIEW OF ITS EFFICACY AND ADRENAL EFFECTS

M. Masoli, M. Weatherall, S. Hold, R. Beasley. Medical Research Institute of New Zealand

Objective: To examine the dose-response relation of inhaled fluticasone propionate for both efficacy and adrenal function in children with asthma.

Design: Analysis of placebo-controlled randomised clinical trials of fluticasone in children of at least 4 weeks duration, that used at least one dose of fluticasone, and that presented data on at least one clinical outcome measure of asthma or at least one sensitive measure of adrenal function

Setting: EMBASE and Medline.

Main outcome measures: $\mathrm{FEV}_{1}$, morning and evening peak expiratory flow, night awakenings, $\beta$-agonist use, major exacerbations leading to withdrawal, 12 or 24 hour urinary cortisol, peak plasma cortisol post-stimulation. 
Results: Five studies of 1150 children with asthma met the inclusion criteria for efficacy, with no studies examining doses $>200 \mu g$ per day. The dose-response curve for each outcome measure suggested that the response began to plateau between a dose of 100 and $200 \mathrm{\mu g}$ per day. The odds ratio for patients remaining in a study at a dose of $100 \mu \mathrm{g}$, compared with $200 \mu \mathrm{g}$ was $0.8(95 \% \mathrm{Cl} 0.46$ to 1.37 )

Three studies of 523 children with asthma met the inclusion criteria for assessment of adrenal function with no studies examining doses $>200 \mu \mathrm{g}$ per day. A meta-analysis could not be undertaken as the data was not presented in an appropriate format. The largest study of 437 children reported no difference in 24 hour urinary cortisol between placebo and fluticasone at doses of 100 and $200 \mu \mathrm{g}$ per day. However, the two smaller studies demonstrated evidence of a reduction in urinary cortisol at these doses.

Conclusions: There is insufficient data to determine the dose-response relation of fluticasone in children at doses $>200 \mu \mathrm{g}$ per day. The dose-response curve for fluticasone appears to plateau between 100 and $200 \mu \mathrm{gg}$ per day for efficacy; there was weak evidence of adrenal suppression at these doses. Pending formal studies which determine the dose-response relation of fluticasone in greater detail, we recommend that fluticasone should be routinely prescribed in children with asthma in doses of up to $200 \mu \mathrm{pg}$ per day.

\section{S30 STEPPING DOWN INHALED CORTICOSTEROIDS IN ASTHMA: A RANDOMISED CONTROLLED TRIAL}

G. Hawkins', A.D. McMahon', S. Twaddle ${ }^{3}$, S.F. Wood', I. Ford ${ }^{2}$, N.C. Thomson'. 'Department of General Practice, University of Glasgow, UK ${ }^{2}$ Robertson Centre for Biostatistics, University of Glasgow, UK; ${ }^{3}$ Stobhill Hospital, Glasgow, UK; ${ }^{4}$ Department of Respiratory Medicine, University of Glasgow, UK

Background: In an attempt to optimise the therapeutic action of inhaled corticosteroids whilst minimising their side effects, asthma management guidelines recommend that a reduction in the dose of inhaled corticosteroid is undertaken when asthma is stable.' We aimed to determine whether a $50 \%$ reduction in the dose of inhaled corticosteroid could be undertaken in patients with chronic stable asthma, whilst maintaining control.

Methods: We recruited 259 adult asthmatics receiving regular treatment with high-dose inhaled corticosteroids (mean daily dose $=$ 1430 mcg beclomethasone dipropionate ) to a one-year, randomised, double-blind, parallel group trial. Patients were allocated to receive either no alteration to their inhaled corticosteroid dose (control) or a $50 \%$ reduction in their dose if they met criteria for stable asthma (stepdown). We compared asthma exacerbation rates, asthma related general practice and hospital visits, quality of life measures and corticosteroid dose between the two groups.

Findings: We found no significant difference in the asthma exacerbation rate between the two groups (step-down $=31 \%$, control $=26 \%$, $p=0.354$ ). Similarly, there were no significant differences in the numbers of general practice or hospital visits, or in disease specific and generic measures of health status over the one-year period. On average, the step-down group received $348 \mathrm{mcg}(95 \% \mathrm{Cl} 202$ to 494$)$ of beclomethasone dipropionate less per day than the controls with no difference in the annual dose of oral corticosteroids between the two treatment regimes.

Interpretation: Our study provides convincing evidence that it is possible to step down high dose inhaled corticosteroids in patients with chronic stable asthma, without compromising asthma control.

1. The British Guidelines on Asthma Management: 1995 Review and Position Statement. Thorax 1997;52(suppl 1):S1-S21.

\section{S31 A PLACEBO CONTROLLED COMPARISON OF FORMOTEROL, MONTELUKAST OR HIGHER DOSE OF INHALED CORTICOSTEROIDS IN SUBJECTS WITH SYMPTOMATIC ASTHMA DESPITE TREATMENT WITH LOW DOSE INHALED CORTICOSTEROIDS}

R.H. Green, C.E. Brightling, S. McKenna, B. Hargadon, D. Parker, A.J. Wardlaw, I.D. Pavord. Institute for Lung Health, Glenfield Hospital, Leicester, UK

An important number of patients with asthma remain symptomatic despite treatment with low dose inhaled corticosteroids. There is relatively little data from placebo controlled studies directly comparing the different treatment options for this group of patients. We have performed a randomised, double blind, four way cross-over study comparing the effects of one months treatment with higher dose budesonide $(400 \mathrm{mcg} \mathrm{bd})$, additional formoterol $(12 \mathrm{mcg} \mathrm{bd})$ and additional montelukast $(10 \mathrm{mg}$ od) with placebo in patients with asthma who remain symptomatic despite low dose inhaled corticosteroids (budesonide $100 \mathrm{mcg}$ bd). Patients were seen before and 12 hours after each treatment phase which was separated by a one month washout period during which they took budesonide $100 \mathrm{mcg}$ bd and prn salbutamol only. At each visit exhaled nitric oxide (NO), spirometry, methacholine $\mathrm{PC}_{20}$, visual analogue symptom scores (VAS), the Juniper Asthma Quality of Life questionnaire and induced sputum were performed. Patients recorded twice daily peak expiratory flow (PEF) throughout and the mean morning PEF was calculated for the final week of each treatment and washout period. 49 patients with symptoms consistent with asthma and objective evidence of variable airflow obstruction despite low dose inhaled corticosteroids were recruited. High dose budesonide was the most efficacious treatment resulting in significant improvements in global VAS $(-21.3 \mathrm{~mm} ; 95 \% \mathrm{Cl}-40.4$ to -2.3$)$ morning PEF $(16.5 \mathrm{l} / \mathrm{min}$; $95 \% \mathrm{Cl} 2.3$ to 30.7$), \mathrm{FEV}(0.14 \mathrm{l} ; 95 \% \mathrm{Cl} 0.0$ to 0.28$)$ and exhaled $\mathrm{NO}$ (fold reduction $1.9 ; 95 \% \mathrm{Cl} 1.1$ to 3.1 ) compared to placebo. Formoterol was the next most efficacious treatment with similar improvements in morning PEF $(17.5 \mathrm{l} / \mathrm{min}, 95 \% \mathrm{Cl} 4.0$ to 31.0$)$. However the change in sputum eosinophil count with formoterol $12.4 \%$ to $3.8 \%$; fold reduction $0.6,95 \% \mathrm{Cl} 0.5$ to 0.9 ) differed significantly from the change seen with placebo $(2.8 \%$ to $2.5 \%$; fold reduction 1.1 , $95 \% \mathrm{Cl} 0.7$ to $1.6 ; \mathrm{p}=0.03$ ) and high dose inhaled corticosteroids $(2.7 \%$ to $1.6 \%$; fold reduction $1.6,95 \% \mathrm{Cl} 1.2$ to $2.2 ; \mathrm{p}<0.001)$. We conclude that treatment given in addition to low dose inhaled corticosteroids results in modest benefits. Despite similar effects on morning peak flow, long acting $\beta_{2}$-agonists and high dose inhaled corticosteroids differ in their effects on eosinophilic airway inflammation.

\section{Paediatric airways disease}

\section{S32 THE BRAVELUNG STUDY: BRONCHIOLITIS RSV AUDIT INTO VACCINATING FOR EARLY LUNGS}

J. McCormick, M. Almaghrabi. Tayside Institute of Child Health, Ninewells Hospital, Dundee, UK

Background: Each winter season there are pervasive outbreaks of respiratory syncytial virus (RSV) bronchiolitis. Premature infants with CLD who are re-admitted with RSV utilise greater health service resources in the first two years of life after birth.' Palivizumab, the humanised RSV monoclonal antibody has been shown to cause a relative reduction in re-admission rates in infants born at $<35$ weeks group by $55 \% .^{2}$

Objectives: To audit current protocols and practice into Palivizumab prescribing and Palivizumab funding in Scotland and Northern Ireland over the winter 2001-2002 season.

Design: Retrospective postal questionnaire.

Methods: Targeted questionnaire to lead neonatal or paediatric consultants in 27 units in Scotland and Northern Ireland following the end of the RSV season in late March/early April 2002.

Results: Response rate was $100 \%$ from units responsible for an estimated 72400 births. 200 babies received a course of Palivizumab over the winter season ( 1 per 362 births) at an estimated cost of $£ 490$ 000. Important regional variations were demonstrated; 1 in 179 babies born in Northern Ireland compared to 1 in 538 babies born in Scotland receive RSV prophylaxis. Six units using agreed guidelines prescribe $49 \%$ of the palivizumab in Scotland. Only $63 \%$ of respondents had an agreed protocol for palivizumab administration. Inclusion criteria for receiving palivizumab included; chronic lung disease (76\%), home oxygen (71\%), congenital cardiac abnormality $(59 \%)$, specific gestation $(53 \%)$, and consultant discretion $(35 \%)$. The source of funding was described as Trust $(50 \%)$, Health Board $(32 \%)$, Directorate $(14 \%)$ and $4 \%$ from winter pressures money. There were three reports of "breakthrough" RSV infection (3\% of treated babies) following immunisation.

Discussion: Palivizumab is prescribed 3 times more in Northern Ireland than Scotland. Should UK guidelines for palivizumab administration (like the US) now be the goal?

1. Greenough A, et al. Arch Dis Child 2001;85:463-8.

2. The IMpact-RSV Study Group. Pediatrics 1998;102:531-7. 


\section{S33 THE COMPLEX RELATIONSHIP BETWEEN ASTHMA AND AIRWAY RESPONSIVENESS THROUGHOUT CHILDHOOD}

S.W. Turner, L.J. Palmer, P.J. Rye, N.A. Gibson, P.K. Judge, S. Young, J. Goldblatt, L.I. Landau, P.N. Le Souëf. Princess Margaret Hospital for Children, Perth, Australia

Introduction: The relationship between childhood asthma and increased airway responsiveness (AR) remains uncertain.

Aims: To investigate whether AR tracks from infancy through to childhood; to determine which factors influence $A R$ and assess interactions with asthma.

Methods: From a cohort of 253 individuals, longitudinal assessments of AR and atopy were made at one, six and 12 months and at six and 11 years of age. AR was expressed as a dose response slope (DRS) or graded on a scale of 0 to 2 .

Results: DRS was measured in 203 individuals aged one month, 174 at six months, 147 at 12 months, 103 at six years and 176 at 11 years of age. There were 22 asthmatics at six and 27 at 11 years of age. There was a positive relationship between the DRS in infants aged one month and in children who were not atopic aged 11 years $(r=0.24, n=65, p=0.05)$. Atopy at six years of age was positively associated with grade of $A R \chi_{2}^{2}=6.2, n=93, p<0.05$ ). Atopy at six months and at 11 years of age was positively associated with the grade of AR aged 11 years $\chi_{2}^{2}=6.8, n=150, p=0.03$ and $\chi_{2}^{2}=18.7$, $\mathrm{n}=175, \mathrm{p}<0.001$, respectively). The DRS at six, but not 11 , years of age was positively related to the urinary cotinine concentration at 12 months of age $(r=0.45, n=32, p<0.001)$ and also the number of cigarettes currently smoked by parents $(r=0.01, n=83, p=0.04)$. The DRS at 11 , but not six, years of age was increased in the presence of lower respiratory tract infection (LRTI) in the first six months $(n=79, p<0.001)$ but not the second six months of life. Adjusting for the presence of asthma, the DRS at six years of age was related to the urinary cotinine concentration aged 12 months $(p=0.001)$ and current atopy $(p=0.03)$; and the DRS at 11 years of age was related to atopy aged six months $(p=0.001)$ and LRTI before six months of age $(p=0.001)$.

Conclusions: The data suggested that the level of AR in childhood was determined in early infancy and then influenced in later infancy by factors that included atopy, tobacco smoke exposure and lower respiratory tract infections. Factors present in infancy are associated with increased childhood AR and these appear to act independently of asthma.

\section{S34 TUMOUR NECROSIS FACTOR GENE POLYMORPHISMS AND CHILDHOOD WHEEZING}

H. Bilolikar' 2, A. Rodriguez $\mathrm{Nam}^{3}$, M. Rosenthal', D.C. Henderson'2, I.M. Balfour-Lynn' 2. 'Dept. Paediatric Respiratory Medicine, Royal Brompton Hospital; ${ }^{2}$ Depts. Paediatrics and Immunology, Chelsea \& Westminster Hospital; ${ }^{3}$ Dept. Clinical Immunology, Hammersmith Hospital, London, UK

Background: TNF $\alpha$ secretion is influenced by single nucleotide gene polymorphisms within the TNF gene cluster. Higher constitutive and inducible production of TNF $\alpha$ is associated with allele 2 of TNF-308 and allele 1 of an Ncol polymorphism of the LT $\alpha$ gene. Since TNF $\alpha$ is associated with infant wheezing and asthma, a genetic predisposition to produce TNF $\alpha$ may be important. We compared the TNF $\alpha$ and LT $\alpha$ polymorphisms in controls with a group of infant wheezers, and childhood asthmatics of differing severity. We also measured nasal TNF $\alpha$ levels in the infants with acute wheezing to determine whether an association between phenotype (in vivo production) and genotype existed.

Methods: Asthmatic patients were identified in clinic with respiratory paediatrician-diagnosed asthma, which was defined as severe if they were on inhaled steroids $>800 \mathrm{mcg} /$ day (budesonide equivalent). Infant wheezers were inpatients with acute wheezing. Controls were school children with no asthma. Nasal lavage was performed in the infants using the inulin method to account for dilution, and nasal TNF $\alpha$ was measured by commercial ELISA. Genomic DNA was extracted from buccal smears using the Nucleon extraction kit. After amplification by PCR, the DNA was sized by electrophoresis.

Results: There were 88 asthmatics (mean age 6.1 yrs, 52 boys), 27 severe asthmatics (mean age 12.8 years, 17 boys), 55 wheezy infants (mean age 6.5 months, 35 boys) and 156 controls (mean age 10.2 years, 77 boys). All data were compared to the controls. In the presence of TNF1 homozygosity, the risk $(95 \% \mathrm{Cl})$ of being a wheezy infant was $3.2(2-5)$ greater if they were also LT $\alpha$ AA than if one or two $\mathrm{LT} \alpha \mathrm{G}$ genes were present. For all asthma the risk was 2.0 (1.23.2) and for severe asthma risk was 2.4 (1.3-4.5). In the presence of 1 or 2 TNF2 genes, the co-presence of LT $\alpha$ AA gave a 12.6 (1.6-98) risk of being a wheezy infant than if there was a $L T \alpha G$ gene present. The risk for all asthma was 18 (2.4-128) and for severe asthma 13 (1.5-114). Although the positive predictive values were high, the sensitivity of the testing was relatively low. The haplotype TNF 12/LT $\alpha$ AA gives a positive predictive value for any wheezing of $96 \%$, with sensitivity of $17 \%$.

In the wheezy infants, nasal TNF $\alpha$ levels were not influenced by RSV status. Levels were significantly lower in the presence of a TNF2 gene $(p=0.006)$, which was the opposite of that expected, and there was a trend $(p=0.08)$ to higher levels if the LT $\alpha A$ gene was present (as expected).

Conclusions: The TNF2 allele is associated with wheezing whereas the LT $\alpha G$ gene had a protective effect. Clearly the TNF genotype influences the development of childhood wheezing. The results of nasal TNF $\alpha$ needs further elucidation.

\section{S35 DEVELOPMENTAL CHANGES IN VENTILATION DISTRIBUTION IN HEALTH AND CF LUNG DISEASE}

P. Aurora, H. Jungberg, C. Oliver, P. Gustafsson², J. Stocks. 'Portex Respiratory Unit, Institute of Child Health, London, UK; ${ }^{2}$ Queen Silvia Children's Hospital, Göteborg, Sweden

Longitudinal monitoring of airway function through childhood is often complicated by the need to correct results for age and body size.

Aims: In this study we investigated the relationship between age and lung clearance index (LCI), an index of ventilation inhomogeneity derived from multiple breath inert gas washout (MBW) (Larsson et al. J Appl Physiol 1988;65:2030-9), in cystic fibrosis (CF) and healthy control children aged from 0 to 18 years.

Methods: 131 children (64 with CF) were tested. Infants were measured whilst asleep, older children whilst awake. All performed 3 $\mathrm{SF}_{6} \mathrm{MBWs}$, and mean $\mathrm{LCl}$ was calculated for each child.

Results: $\mathrm{LCl}$ remained constant throughout childhood in healthy controls, but became progressively elevated with increasing age among those with CF. See table and figure.

Conclusion: The $\mathrm{LCl}$ can be used to assess airway function from infancy to adulthood and has potential for monitoring CF lung disease.

Abstract $\mathrm{S} 35 \mathrm{LCl}$ by age group expressed as mean and (SD)

\begin{tabular}{llcc}
\hline & $\begin{array}{l}\text { Infants } \\
(0-2 \text { yrs })\end{array}$ & $\begin{array}{l}\text { Pre-school } \\
(3-5 \text { yrs })\end{array}$ & $\begin{array}{l}\text { School-age } \\
(6-18 \text { yrs })\end{array}$ \\
\hline Healthy & $6.47(0.33)(n=23) 6.49(0.51)(n=22) 6.50(0.54)(n=22)$ \\
CF & $8.05(1.41)(n=24) 8.92(1.74)(n=19) 11.48(3.01)(n=21)$ \\
\hline
\end{tabular}

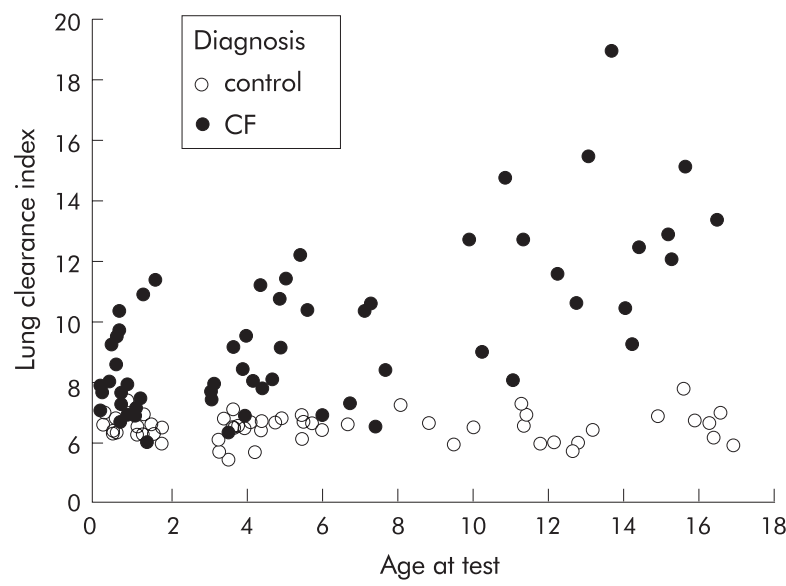

Abstract S35 Lung clearance index as a function of age in healthy controls and CF. 
Abstract S36

\begin{tabular}{lccc}
\hline & Term (SD) & Preterm (SD) & Control (SD) \\
\hline MIP-1 $\alpha(\mathrm{pg} / \mathrm{ml})$ & $409(493)$ & $208(230)$ & $74(58)$ \\
RANTES $(\mathrm{pg} / \mathrm{ml})$ & $243(483)$ & $407(1268)$ & $28(69)$ \\
Eotaxin $(\mathrm{pg} / \mathrm{ml})$ & $123(249)$ & $81(148)$ & $288(312)$ \\
MCP-1 $(\mathrm{pg} / \mathrm{ml})$ & $1268(1779)$ & $474(679)$ & $1134(1189)$ \\
\hline
\end{tabular}

\section{S36 CHEMOKINE PRODUCTION IN SEVERE RSV BRONCHIOLITIS}

P.S. McNamara, G. Jeffers, B.F. Flanagan, P. Newlands, P.C. Ritson, C.A. Hart, R.L. Smyth. Institute of Child Health, Alder Hey Children's Hospital, Liverpool, UK

Introduction: Respiratory syncytial virus (RSV) bronchiolitis is one of the most important causes of death and morbidity in infants worldwide. Factors that predispose to severe disease include prematurity. Neutrophils are the predominant cell-type within upper and lower respiratory tract secretions from infants with bronchiolitis and reach the airways along chemoattractant gradients. Our aim was to compare the pulmonary chemoattractant protein response in term and preterm infants ventilated with RSV bronchiolitis with that from a control group.

Subject/Methods: We collected non-bronchoscopic bronchoalveolar lavage (BAL) samples from 48 infants $(25$ born at term $1 \geqslant 37$ weeks) and 23 born preterm (<37 weeks)) ventilated for RSV bronchiolitis. We also collected BAL samples from 13 "control" patients ventilated for non-respiratory causes. All samples were collected within 24 hours of being intubated. BAL protein concentrations were measured using ELISAs (R\&D) according to the manufacturers instructions.

Results: Mean ages on admission were: term infants, $6.7 \mathrm{wks}$; preterm, 16.0 wks; control, 5.5 wks. Mean weights on admission were: term, $4.3 \mathrm{~kg}$; preterm, $3.5 \mathrm{~kg}$; control, $4.2 \mathrm{~kg}$. Mean gestational ages at birth were: term, $38.6 \mathrm{wks}$; preterm, $30.1 \mathrm{wks}$; control, $38.8 \mathrm{wks}$. Preterm infants were ventilated for twice as long as term infants $(4.4 \mathrm{v}$ 9.0 days, $\mathrm{p}=0.02$ ).

MIP-1 $\alpha$, RANTES and Eotaxin concentrations all differed significantly between the three groups.

Conclusions: We have identified differences in the immunological response in the lungs of infants ventilated for RSV bronchiolitis compared to a control group. Our data also highlight for the first time differences in this response between term and preterm infants with RSV bronchiolitis which may relate to disease severity.

Study funded by Action Research

\section{S37 SALMETEROL/FLUTICASONE COMBINATION (SFC) IS ASSOCIATED WITH IMPROVED COMPLIANCE IN CHILDREN COMPARED WITH INHALED CORTICOSTEROID (ICS) ALONE, OR CONCURRENT SALMETEROL + ICS}

T.P. McCarthy, L. Rice, C.A. Blair. GlaxoSmithKline, Uxbridge UB 11 1BT, UK

Introduction: Paediatric compliance with regular asthma medication is a concern, with levels of 55-58\% being reported (Coutts J. Arch Dis Child 1991; Milgrom H. J Allergy Clin Immunol 1996). It has been suggested that a combination of a long-acting $\beta_{2}$ agonist (LAB) and an

Abstract S37

\begin{tabular}{lllll}
\hline & SFC & F & S & BDPI \\
\hline No patients & 80 & 662 & 242 & 47 \\
Mean p (SD) & $8.7(4.9)$ & $6.2(4.7)$ & $6.5(4.1)$ & $3.3(2.2)$ \\
Compliance (\%) & 72.5 & 51 & 54 & 27.5 \\
Treatment difference & & 2.4 & 2.1 & 5.3 \\
( $v$ SFC) & & 1.3 to 3.5 & 1.0 to 3.23 .8 to 6.8 \\
95\% confidence interval & $<0.0001$ & $=0.0004$ & $<0.0001$ \\
p Value (SFC $v$ comparators) & \multicolumn{2}{l}{ < } \\
\hline
\end{tabular}

inhaled steroid in one inhaler-SFC (Seretide ${ }^{T M}$ ) may appeal to children in terms of convenience; and the $L A B$, providing a rapid improvement in lung function which will wear off after 24 hours, may serve as a reminder that the medication should be taken.

Method: To evaluate compliance in children < 16 using prescribing data as a surrogate marker, DIN-LINK Data (CompuFile Ltd) were used to analyse prescription collection/year (P) from 100 GP practices, for SFC, fluticasone (F), salmeterol (S) and beclometasone (BDPI (dry powder inhalers)|.

Results: 1031 asthma patients identified who had been prescribed SFC, F, S or BDPI over the 12 months January-December 2001. 99\% of patients prescribed $S$ for asthma were prescribed an ICS. Accordingly $S$ may be taken to represent concurrent $L A B(S)+I C S$ ( $p$ value SFC $\vee S$ unchanged).

Discussion: Although prescribing data are only a surrogate for compliance, as patients may not collect or use all their prescriptions, this study found compliance with $\mathrm{F}$ and $\mathrm{S}$ in keeping with other studies. In contrast SFC achieved compliance that was significantly greater versus $F, S$, and $B D P I$. As $99 \%$ of patients on $S$ are concurrently on ICS, SFC achieved significantly greater compliance compared with concurrent LAB and ICS therapy. In this study SFC was associated with greater compliance with treatment than is usually found in children on regular inhaled therapy for asthma.

\section{Pulmonary rehabilitation}

\section{S38 CAN ALL COPD PATIENTS COPE WITH PULMONARY REHABILITATION?}

E. Roberts', J. Goldman', W.H. Williams', D Egan'. 'Clinical Health Psychology, Torbay Hospital, Torquay TQ2 7AA, UK; ${ }^{2}$ School of Psychology, University of Exeter, Exeter EX4 4QG, UK

It has been suggested that hypoxemia may be responsible for intellectual difficulties observed in COPD patients. Research to date is inconclusive as to the nature of these difficulties and at what stage of disease they occur. This investigation aimed to establish what the difficulties are, which disease factors are involved and whether they are mediated by emotional well-being. Forty patients participated. Inclusion criteria were an established diagnosis of COPD; $\mathrm{FEV}_{1}<80 \%$ predicted, and an $\mathrm{FEV}_{1} / \mathrm{FVC}$ ratio of $<70 \%$. Those who were receiving LTOT, had suffered a severe head injury in the past, who had a learning disability or who had other medical conditions known to affect intellectual functioning were excluded. A control group of 22 healthy volunteers was also recruited. Blood gases and lung function measures were taken from the patients; oxygen saturation of haemoglobin was measured for all participants. A range of intellectual assessments measured verbal fluency, memory, attention processes and other skills associated with the frontal lobes of the brain. The HAD assessed anxiety and depression. Mean $\mathrm{PaO}_{2}$ was 8.6 (.97), mean $\mathrm{PaCO}_{2} 5.2$ (.63). Oxygen saturation at rest was $93.4 \%(3.29)$ in patients. Percentage of predicted $\mathrm{FEV}_{1} / \mathrm{FVC}$ ratio was $45.8 \%$ (12.4). Independent t-tests showed that COPD patients performed significantly worse than controls on speed of information processing $(t=-2.99$, $\mathrm{p}<0.05)$, immediate memory $(t=-3.24, \mathrm{p}<0.01)$, divided attention $(t$ $=2.60, p<0.01)$ and verbal fluency $(t=-2.58, p<0.05)$. Significant correlations were obtained between these measures and some physiological measures: speed of information processing and \% of predicted $\mathrm{FEV}_{1} / \mathrm{FVC}(\mathrm{r}=-0.33, \mathrm{p}<0.05)$; and immediate memory with $\mathrm{PaO}_{2}(\mathrm{r}$ $=0.33, \mathrm{p}<0.05)$; divided attention with $\mathrm{PaCO}_{2}(\mathrm{r}=-0.34, \mathrm{p}<0.05)$ and with FVC $(r=-0.27, p<0.05)$. Independent $t$ tests showed patients scored significantly higher than controls on both anxiety and depression ( $t=-3.89, \mathrm{p}<0.001$ and $t=4.63, \mathrm{p}<0.001$ respectively) but were not correlated with any of the intellectual measures. These findings suggest that mildly hypoxemic patients show impairments in intellectual performance. There is some evidence for the contribution of severity of illness, although other factors related to COPD or chronic illness in general may be implicated. Future work could assess whether severe hypoxemia is accompanied by more intellectual deterioration. The presentation of rehabilitation programmes can be tailored to take account of patients' difficulties.

\section{S39 RESPONDER ANALYSIS OF PULMONARY REHABILITATION}

R. Garrod', K. Dix, C. Daly, M. Howard, P.W. Jones. 'School of Physiotherapy St George's Hospital and Kingston University; St George's Hospital NHS Trust, UK

Introduction: Research has shown improvements in exercise tolerance (ET) and health related quality of life (HRQOL) after 
pulmonary rehabilitation. However there are few reports of the effects of "real life" rehabilitation, in particular descriptors of response and non response behavior. This study reports on data from a 7 week, $2 \times$ weekly exercise and education programme run at St George's Hospital, London.

Methods: Outcome measures were spirometry, Shuttle walk test (SWT), HRQoL using SGRQ and mood state using the Hospital Anxiety and Depression Scale (HAD). 76 patients were admitted to the programme, 67 were available for follow up.

Results: There was a statistically significant improvement in SWT mean change; $52 \mathrm{~m}(84.4)(p=0.0001)$ and in SGRQ; 5.2 (10.6) $(n=67)$. Responder analysis, based on a change $>30 \mathrm{mt}$ for SWT and $>4$ points for $S G R Q$, showed that 37 patients were responders (R) for ET and 33 for HRQoL. 18 patients were characterised as non responders (NR) in both HRQoL and ET. 12 patients were NR for exercise tolerance but $\mathrm{R}$ for HRQoL, 17 patients were classified as $\mathrm{R}$ for SWT but NR for HRQoL and only 20 patients were classified as $R$ in both aspects. In these 20 patients the change in SWT and SGRQ was large; 124 (71.4)m and 12 (6.2) respectively. There was a significant difference for SGRQ between R and NR; 57 (14.6) and 47 (14.4), p $=0.04$ with Rs showing poorer QoL, similarly for depression 7.5 (3.2), $5.6(2.4), \mathrm{p}=0.04$. Rs showed a larger initial SWT compared to NR; 270 (139.2) and 217 (94.6) $\mathrm{m}$ although the difference was not significant $(p=0.18)$. Baseline spirometry showed no significant difference, $R$ and NR, mean (SD): $1.1(0.5) \mathrm{I}(\mathrm{n}=17)$ and $1.1(0.5) \mathrm{l}$ $(n=15)$, respectively.

Conclusion: These data generate the testable hypothesis that greater exercise tolerance and poor HRQoL are associated with better improvements in pulmonary rehabilitation. Prospective randomised trials are warranted.

\section{S40 SYMPTOMS LIMITING EXHAUSTIVE WALKING AND CYCLING EXERCISE IN COPD}

J. Gearing, W.D-C. Man' , S.G. Radford, R.E. Atkins, A.J. Rossell, B.Gray', M.I. Polkey', J. Moxham'. Department of Physiotherapy and 'Respiratory Medicine, King's College Hospital; ${ }^{2}$ Royal Brompton Hospital, London, UK

Background: Previous studies have shown that leg fatigue is a common symptom following exhaustive cycling in COPD patients. However cycling is not familiar to many COPD patients, and walking field tests may be more representative of everyday activities. We hypothesised that the symptoms limiting exhaustive exercise in COPD is dependent on the type of exercise performed.

Method: 50 stable patients with COPD (27M:23F, mean age 68.6 yrs, mean FEVI 0.95) were recruited. After an initial familiarisation period, each patient performed to exhaustion an incremental shuttle walk (ISW), an endurance shuttle walk (ESW), incremental cycle ergometry (ICE), and endurance cycle ergometry (ECE), on four separate visits. Patients were asked to name the predominant symptom limiting further exercise: shortness of breath (SOB), leg fatigue (LF), an equal combination of $\mathrm{SOB}$ and $\mathrm{LF}$, or other symptoms.

Results: See table.

Discussion: $\mathrm{SOB}$ is by far the most common limiting symptom following exhaustive walking exercise, but is less important following exhaustive cycling exercise when LF becomes more prominent. It may be more appropriate to use walking tests to assess the effects of therapeutic interventions on exercise-induced dyspnoea.

WDCM is a Clinical Research Training Fellow of the MRC (UK).

\begin{tabular}{lllll}
\multicolumn{5}{l}{ Abstract $\mathrm{S} 40$} \\
\hline \multirow{4}{l}{$\%$ Total number of patients } \\
\cline { 2 - 5 } Test & SOB & LF & SOB/LF & Other \\
\hline ISW & 84 & 6 & 4 & 6 \\
ESW & 62 & 12 & 14 & 12 \\
ICE & 36 & 40 & 22 & 2 \\
ECE & 30 & 40 & 30 & 0 \\
\hline
\end{tabular}

\section{S41 ASSESSING THE DETERMINANTS OF INCREMENTAL SHUTTLE WALKING DISTANCE}

S. Dogan', B. Watson', W. Cochrane' 2, G. Dovey-Pearce', G. Afolabi', M. Heatley', C. McAlpine', G. Oliver'. 'Northumbria Healthcare NHS Trust, UK; ${ }^{2}$ Northumbria University, UK

Introduction: The shuttle test is a widely used outcome measure for pulmonary rehabilitation to assess a person's exercise tolerance. To better understand what this outcome means we examined the relationship between incremental shuttle test walking distance and biomedical and psychological dimensions in people with COPD. These findings derive from the baseline data of a trial comparing pulmonary rehabilitation with solely exercise and psychological interventions.

Participants: All 218 participants had stable COPD. 60\% reported MRC Dyspnoea grade 3,29\% grade 4, and $11 \%$ grade $5.44 \%$ were male and mean $(95 \% \mathrm{Cl})$ age was $68.8(67.8-69.7)$ years.

Methods: Incremental shuttle walking test was carried out. Spirometric and anthropometric measurements were recorded including Forced Expiratory Volume $\left(\mathrm{FEV}^{-1}\right)$, weight, and four site skinfold thickness. Perceived health status, personal cognitions of illness and affect were assessed using the Chronic Respiratory Disease Questionnaire (CRDQ), the Illness Perceptions Questionnaire (IPQ) and the Hospital Anxiety and Depression Scale (HADS) respectively. Stepwise multiple linear regression was carried out with incremental shuttle walking test distance as the dependent variable.

Results: Mean (SD) incremental shuttle walking distance $=202.5$ (111.3) meters. Independent variables included: predicted FEV (mean $(S D)=52.7$ (15.6)); fat free mass index (mean (SD) 17.6 (2.6)); CRDQ (mastery (median (IQR) = 19.0 (9.0)), fatigue (median $(I Q R)=14.0(8.0)$ ), emotional function (median (IQR) = $32.0(12.8)$ ), dyspnoea (median (IQR) = 14.0 (7.8)); IPQ (timeline (median (IQR) = $21.0(3.0)$ ), consequences (median (IQR) = 19.5 (5.0)), personal control (median $(I Q R)=20.0(5.0))$, treatment control (median $(I Q R)=$ 16.0 (4.0)), illness coherence (median (IQR) = 12.0 (6.0)); and HADS (anxiety (median $(I Q R)=18.0(5.0))$, depression $($ median $(I Q R)=$ 15.0 (3.0)). In a model controlling for participant age and gender, and explaining $27.7 \%(p<0.001)$ of the variance in the dependent variable, fatigue (beta $=4.6(\mathrm{p}=0.004))$ and mastery (beta $=3.5$ $(p=0.02))$, as measured by the $C R D Q$, were the only significant variables entered.

Conclusion: In this population, these findings demonstrate that a person's perception of their fatigue and their degree of control and confidence in managing their condition, play an important and independent role in promoting exercise performance. This suggests it is important to directly address these factors when providing pulmonary rehabilitation.

\section{S42 EVALUATION OF MAINTENANCE PROGRAMMES AFTER PULMONARY REHABILITATION IN THE COMMUNITY SETTING}

K. Dix, C. Daly, M. Howard, E. Porter, C.F.J. Rayner. St George's Hospital NHS Trust, Tooting, London, UK

Introduction: The role of maintenance programmes (MP) in pulmonary rehabilitation is at present unknown. This data reports on the outcomes of a community based MP provided after a 7 week $2 \times$ weekly out patient programme (OP) as part of "real life" clinical service.

Methods: After the 7 week out patient programme, patients self selected to either attend a 6 month $M P(n=10)$ or not $(n=15)$. The MP was provided once weekly in a local leisure centre. Assessments of Shuttle Walk Test (SWT) and St George's Hospital Respiratory Disease Questionnaire (SGRQ) were made at the end of the 7 week out patient and after the 6 month period $(n=25)$.

Results: Descriptive data are provided, based on an improvement in SWT >30mt and >4 points for SGRQ. Of those who attended, 4 improved SWT, 5 stayed the same and 1 deteriorated; mean change post $\mathrm{OP}$ rehabilitation, $17 \mathrm{~m}$. Of those who did not, 7 improved, 6 stayed the same and 2 got worse, mean change $45 \mathrm{~m}$. For SGRQ in group attendees, 1 improved, 5 stayed the same and 4 got worse. In the non attendees, 6 improved SGRQ, 6 stayed the same and 4 got worse. There were no significant differences between the groups for SWT or SGRQ.

Conclusion: This early report of a clinical community MP suggests that patients who choose to attend the MP do as well as those who do not attend for exercise and health related quality of life. It is unknown whether these patients would maintain improvements without the opportunity of a maintenance programme. Long term studies are indicated.

\section{S43 A FOCUS GROUP STUDY ON THE IMPACT OF THE DIFFERENT COMPONENTS OF PULMONARY REHABILITATION}

M. Gray', R.C.M. Jones', M. Hyland ${ }^{2}$, J. Goldman ${ }^{3} .{ }^{\prime}$ Respiratory Research Unit, University of Plymouth, Devon; ${ }^{2}$ Department of Psychology, University of Plymouth, Devon, UK; ${ }^{3}$ The Heart and Lung Unit, Torquay Hospital, Torquay, Devon, UK

Background: Multi-professional pulmonary rehabilitation (PR) has been shown to improve exercise tolerance and quality of life. It is not 
known which aspects of PR (e.g. exercise versus education versus social support) are found to be most helpful by patients, and existing quality of life tools do not explore this issue.

Methods: Six focus groups were held 3 months after PR with patients recruited from 2 programmes. One being a typical intensive, hospital-based scheme, (Torquay), the other a short, once weekly programme based in various locations in the community (Plymouth).

Results: Perceived effects of education included reduced fear of dyspnoea, improved use of benefit system and improved drug compliance; perceived effects of social context included encouragement during exercise and smoking cessation, and new social activities amongst group members; exercise in a safe environment increased confidence in activity and also reduced fear of dyspnoea, leading to new activities (e.g. holidays, shopping trips etc.) Patients judged PR to be more helpful then medical interventions. There appeared to be more extracurricula social contact in the community group.

Conclusions: Patients reported benefits of PR can be attributed to exercise, education, social context, supporting the use of multiprofessional, multi-component PR programmes. Peer group support in both programmes appears to be an important factor in behavioral change.

\section{Interstitial lung disease: From diagnosis to treatment}

\section{S44 AN ASSESSMENT OF REPRODUCIBILITY OF DIAGNOSIS IN DIFFUSE PARENCHYMAL LUNG DISEASES}

A.G. Nicholson, A.R. Gibbs, B.J. Addis, S. Stewart, C.A. Clelland, N.B. Ibrahim, W.A. Wallace, P.S. Hasleton, B. Corrin, H. Bharucha, K.M. Kerr (UK-ILD panel), A.U. Wells. Departments of Histopathology, Royal Brompton, Llandough, Southampton, Papworth, John Radcliffe, Frenchay, Edinburgh, Wythenshawe, Royal Victoria Hospital, Belfast, Aberdeen Royal Hospitals, and Department Of Medicine, Royal Brompton Hospital, UK

There are very few inter-observer studies of histologic patterns of diffuse parenchymal lung disease (DPLD), and the reproducibility of the consensus ATS/ERS classification for interstitial pneumonias has not yet been tested. This study assesses inter-observer variation in the diagnosis of DPLDs, both for interstitial pneumonias and orphan lung diseases (OLDs). Cases referred for clinical assessment of DPLD between Jan 1996 and Dec 1997, in which a surgical lung biopsy was taken, were retrieved and H\&E slides were circulated to 7 reviewers, with knowledge only of age and sex of patient and site of biopsy. As well as histologic patterns in the consensus classification, follicular bronchiolitis (FB), extrinsic allergic alveolitis (EAA), sarcoidosis, end-stage lung, normal, non-diagnostic, unclassifiable and "other" for OLDs were permitted. Reviewers provided a first choice diagnosis with a confidence rating of $1(>95 \%), 2(70-95 \%)$ or $3(30-65 \%)$ for each lobe $(n=133)$. The differential diagnosis for each lobe was also recorded along with its percentage likelihood, censored at $5 \%$. The same procedure was applied for the gestalt diagnosis if patients $(n=83)$ had more than one biopsy. Statistical analysis was performed using STATA software (CA, USA). A confidence level of $>95 \%$ for diagnosis was made in $37 \%$ (range 22-47) of cases and $>70 \%$ in $64 \%$ of cases (range 54-73). The overall kappa coefficient for first choice lobar diagnoses was 0.35 . Examples for more commonly found patterns of interstitial pneumonias were UIP, 0.43 ; NSIP, 0.24 ; DIP, 0.51; OP, 0.53; DAD, 0.52; EAA, 0.32; sarcoidosis, 0.70. In cases with a high degree of confidence $(n=79)$, the overall kappa value was 0.49 , whilst for low confidence diagnoses $(n=54)$ this was only 0.19. For UIP, the weighted kappa for lobar diagnosis was 0.55 rising to 0.59 for the gestalt diagnoses. Selected examples of weighted kappas for gestalt diagnosis were NSIP, 0.34; OP, 0.58 ; EAA, 0.50. These data suggest that the ATS/ERS consensus classification is sufficiently reproducible when used by pathologists with a specialist interest in pulmonary pathology.

\section{S45 INCREASED SERUM LEVELS OF MUCIN KL-6 SURFACTANT PROTEIN-D(SP-D) AND ANTIBODY TO DIETRY ANTIGENS SUGGEST ALTERED MUCOSAL PERMEABILITY AMONG PIGEON FANCIERS}

C. McSharry, K. Anderson, T. Ismail, F. Boyd, J. Burgon, K.B.M. Reid, G. Boyd. Department of Respiratory Medicine and Immunology, North Glasgow University Hospitals NHS Trust

Background: Increased clearance of inhaled 99mTc-DTPA in pigeon fanciers has been observed irrespective of symptoms. The aim of this study was to use serum levels of lung-epithelium-derived KL-6 and SP-D to assess lung epithelial permeability and antibody to dietary antigens to assess gut epithelial permeability in pigeon fanciers.

Methods: Serum KL-6, SP-D, antibody to inhaled avian antigens and to common dietary antigens was quantified by enzyme immunoassay in 60 pigeon fanciers.

Results: The serum KL-6 levels in pigeon fanciers was (median, I-q range) $=422(244-616)$ units $/ \mathrm{ml}$ and the serum SP-D level was (mean (SD) $=201$ (141) $\mathrm{ng} / \mathrm{ml}$ ). Both of these were significantly higher than normal. These levels were not significantly higher in those with EAA, but there was a significant correlation between the KL-6 levels and the $\lg G$ antibody to inhaled avian antigen $(r=0.435$, $p=0.001)$ and between SP-D level and the $\lg G$ antibody $(p=0.005)$. There were significantly higher than normal titres of $\lg G$ antibody to common dietary antigens among the pigeon fanciers suggesting increased gut permeability, but these did not correlate with either symptom category or antibody titre to avian antigens.

Conclusion: Increased lung mucosal permeability reflects local inflammation which could be the cause or the effect of antibodyassociated events. The increased gut permeability in pigeon fanciers suggests either an inflammatory reaction in the gut to avian antigens in the diet or a pre-existing generalised increase in mucosal permeability.

\section{S46 MACROPHAGE MIGRATION INHIBITORY FACTOR INDUCES PROLIFERATION AND HAS PRO-FIBROTIC EFFECTS IN PRIMARY HUMAN PULMONARY FIBROBLASTS}

K.M.A. O'Reilly', J.A. Baugh', B.J. Plant', M.X. Fitzgerald', R. Bucala ${ }^{2}$ S.C. Donnelly'. 'Department of Medicine and Therapeutics, University College Dublin, Ireland; ${ }^{2}$ School of Medicine, Yale University, USA

Macrophage Migration Inhibitory Factor (MIF) is an important pro-inflammaory cytokine which has been linked to the development of fibro-proliferative or "chronic" acute respiratory distress syndrome (ARDS). ${ }^{1}$ This finding lead you to postulate that MIF might have direct pro-fibrogenic effects on fibroblasts, and therefore be an important effector in the development of pulmonary fibrosis from a variety of causes.

Primary human pulmonary fibroblasts (CCD-19Lu) were transiently transfected with MIF, RNA extracted and an RNase protection assay (RPA) performed. Levels of transforming growth factor (TGF)- $\beta$, a cytokine known to be highly pro-fibrogenic, were found to be significantly up-regulated (336\% above control levels $(n=3))$. Similarly, when the fibroblasts were stimulated with recombinant MIF, levels of secreted TGF- $\beta$ (measured by ELISA) were increased by $295 \%$ compared to controls $(n=3)$. In order to assess whether MIF had any direct effects on fibroblast proliferation, MIF was co-incubated with the primary pulmonary fibroblasts and cellular proliferation assessed by $\left(\mathrm{H}^{3}\right)$-thymidine incorporation. Fibroblast proliferation was increased by $210 \%$ over controls $(n=9)$.

These data identify MIF as a cytokine which has the capacity to both significantly up-regulate TGF- $\beta$ production and induce fibroblast proliferation; both key parameters which have the capacity to drive an exaggerated pathological fibro-proliferative response.

This work is supported by the Wellcome Trust.

\section{S47 COLLAGENASE 1 (MATRIX METALLOPROTEINASE 1 MMP1) IS INVOLVED IN THE DEVELOPMENT OF CRYPTOGENIC FIBROSING ALVEOLITIS}

A. Walne, P. Lympany, K. Welsh, R. du Bois. National Heart and Lung Institute, Imperial College of Science, Technology and Medicine, London

Cryptogenic Fibrosing Alveolitis (CFA) is a relentlessly progressive diffuse lung disease of unknown aetiology, characterised by fibroblast proliferation and extracellular matrix (ECM) accumulation. It is the most common of the diffuse lung diseases, affecting up to 10 adults per 100000 in the UK and has a median survival time of only 3 years from diagnosis. Even though the existence of familial CFA suggests there may be a genetic component, there have been few large scale studies showing an association between any genetic marker and the development of CFA. The genetic component of CFA is likely to be complex, involving several genes each with a variable effect, acting in combination to determine the predisposition to lung fibrosis. In CFA, the pathological process is characterised by ECM accumulation and abnormal remodeling that may be due to a relative deficit in proteolysis. 


\begin{tabular}{|c|c|c|c|c|c|c|}
\hline \multicolumn{7}{|c|}{ Abstract S49 } \\
\hline \multirow[b]{2}{*}{ Test } & \multicolumn{3}{|c|}{ Placebo median (SD) } & \multicolumn{3}{|c|}{ Septrin median (SD) } \\
\hline & Pre & $3 \mathrm{mths}$ & Post rehab & Pre & $3 \mathrm{mths}$ & Post rehab \\
\hline $\begin{array}{l}\text { Shuttle }(\mathrm{m}) \\
\mathrm{PO}_{2}(\mathrm{kPa})\end{array}$ & $\begin{array}{l}200(57) \\
9.7(1.7)\end{array}$ & $\begin{array}{l}210(48) \\
8.9(1.4)\end{array}$ & $220(85)$ & $\begin{array}{l}200(56) \\
9.9(1.2)\end{array}$ & $\begin{array}{l}360(73)^{*} \\
11.3(1.5)\end{array}$ & $350(86)^{*}$ \\
\hline FVC (L) & $1.8(0.6)$ & $1.8(0.7)$ & $1.8(0.6)$ & $1.8(0.6)$ & $2.0(0.8)$ & $2.3(1.0)$ \\
\hline TLC & 3.75 & 3.51 & & 3.73 & 4.22 & \\
\hline DLCO & 4.81 & 4.18 & & 4.78 & 5.13 & \\
\hline MRC $5 \mathrm{Pt}$ & 3 & 3 & 3 & 3 & 2 & 2 \\
\hline
\end{tabular}

In this study we fine mapped across collagenase-1, the gene coding for the main enzyme involved in type I collagen degradation. We examined 12 single nucleotide polymorphisms (four promoter, two $3=U T R$, one intron 1 /exon 1 boundary and five intronic) in 50 CFA patients and 225 Caucasian controls. The genotype, allele and allele carriage frequencies for the intron/exon boundary polymorphism $(C / T)$ were significantly different between patients and controls (genotype $p_{c}=0.01$, allele $p_{c}=0.03$, allele carriage for $C p_{c}=0.006$ ). There were differences in the $\mathrm{T}$ allele carriage for the intron/exon polymorphism $(p=0.016)$; in genotype frequency for one of the intronic polymorphisms (MMP1-3 $p=0.036$ ) and in allele carriage for one of the $3=$ UTR polymorphisms $(G, p=0.045)$, but these differences became trends when corrected for the total number of polymorphisms studied.

The finding of an association between collagenase-1 polymorphisms and CFA may indicate a role for this enzyme in the pathogenesis of this disease, but at present, the functional consequences of these polymorphisms are unknown. They may affect mRNA splicing or stability; alternatively, they may be markers for other unidentified polymorphisms in this or other genes in the MMP cluster on chromosome $11 \mathrm{q} 23$. We conclude that a susceptibility marker for CFA maps to this MMP region.

\section{S48 THE DEVELOPMENT OF LUNG FIBROSIS IN TRANSGENIC TGF $\beta 1$ MICE}

Y. Haider, M.W.J. Ferguson, J. Egan. CID, Stopford Building, University of Manchester, Oxford Road, UK

TGF $\beta 1$ has been shown to be an important growth factor in the pathogenesis of lung fibrosis. It has been hypothesised that human genetic predisposition mediated via TGF $\beta 1$ may place some individuals at risk of developing lung fibrosis (Anscher et al. NEJM 1993;328:1593-8). We previously studied the natural history of a transgenic mouse colony that expressed high circulating plasma levels of active TGF $\beta 1$ and found that lung fibrosis was not present irrespective of the age of the mouse. This was despite histological evidence of liver fibrosis.

We proceeded to study the potential susceptibility of these mice to the development of lung fibrosis using a known chemical injury (bleomycin) and a suspected environmental pathogen (a herpes virus).

We bred two lines of transgenic mouse and confirmed their phenotype $(\mathrm{Tr}+$ ) qualitatively (by tail DNA analysis) and quantitatively (by plasma TGF $\beta 1$ bioassay). We then analysed their lungs histologically after bleomycin injection (3000 IU intraperitoneal)( $(n=8)$, and after administration of herpesvirus $\left(4 \times 10^{5}\right.$ pfu of Murine gammaherpesvirus- 68 intranasal)( $n=8)$. Mice were killed after 6 weeks. A control population received the same treatment $(n=8)$. Lung fibrosis was graded $0-3$, based on a previously published grading system (Ashcroft et al. J Clin Pathol 1988;41:467-70).

The $\mathrm{Tr}+$ mice were confirmed to have higher circulating levels of active TGF $\beta 1$ compared to controls $(p<0.05)$. Prior to bleomycin exposure, the lungs of the $\mathrm{Tr}+$ and control mice were histologically normal. After bleomycin, control lung showed fibrosis (mean score 1.4) and Tr+ lung showed more severe and extensive fibrosis (mean score 2.7) $(p<0.05)$. Prior to herpesvirus innoculation, the lungs of the $\mathrm{Tr}+$ and control mice were normal. After herpesvirus, there was no evidence of lung fibrosis in either group. In conclusion plasma TGF $\beta 1$, while not causing de novo lung fibrosis, appears to predispose to lung fibrosis when exposed to an exogenous injury (e.g bleomycin). This may be a consequence of a compartmental disruption allowing passage of circulating TGF $\beta 1$ into lung tissue. Therefore lung injury may be of primary importance and individual susceptibility a secondary phenomenon.
S49 A DOUBLE BLIND RANDOMISED PLACEBO CONTROLLED PILOT STUDY OF SEPTRIN IN THE TREATMENT OF CRYPTOGENIC FIBROSING ALVEOLITIS

V. Varney, H. Parnell, D. Salisbury, S. Ratneepan, R. Tayar. Department of Respiratory Medicine, St Helier Hospital, Wrythe Lane, Carshalton, Surrey SM5 IAA, UK

Background: 14 patients with end stage pulmonary fibrosis were openly treated with oral Septrin. All made a dramatic clinical recovery and were discharged home. This prompted a double blind randomised placebo controlled pilot study in earlier stages of the disease when cough, dyspnoea and oxygen desaturations on exercise were present

Method: 20 patients with pulmonary fibrosis (aged 49-84 years) underwent a detailed assessment of arterial blood gases, lung function, progressive shuttle walking tests with oxygen saturation monitoring, quality of life and diary cards etc. Randomisation was to Septrin or identical placebo for 3 months followed by 6 weeks of pulmonary rehabilitation (rehab) before decoding. 2 weekly reassessments of shuttle walk (etc) were made throughout the study.

Results: The first 16 cases have been decoded $(8$ Septrin, 8 placebo). The remaining patients will complete by September 2002.

Conclusion: Septrin produced a significant improvement in shuttle walking distance with improved arterial oxygen values, lung volumes and quality of life assessments. A fully decoded study by October 2002.

\section{Acute respiratory distress syndrome}

\section{S50 CHARACTERISATION OF A MURINE MODEL OF OLEIC ACID-INDUCED ACUTE LUNG INJURY}

K. Ulrich', M. Stern', M. Goddard², D. Geddes', M. Takata² E. Alton'. 'Department of Gene Therapy, Faculty of Medicine at the National Heart and Lung Institute, Imperial College, London, UK; ${ }^{2}$ Department of Anaesthetics and Intensive Care, Faculty of Medicine at the Chelsea and Westminster Hospital, Imperial College, London, UK

We are developing a programme of gene therapy for acute respiratory distress syndrome (ARDS). Oleic acid-induced lung injury has been suggested as the optimal animal model, but has not been characterised in mice. Current gene therapy studies focus on this species, and we have therefore developed a mouse model of oleic acid-induced lung injury. BalbC mice were anaesthetised, ventilated and injected with either oleic acid $(\mathrm{OA}, 0.2$ or $0.4 \mathrm{ml} / \mathrm{kg}$ body weight) or saline. One hour after OA administration $(0.2 \mathrm{ml} / \mathrm{kg})$, mouse lungs had significantly $(p<0.01)$ higher wet-to-dry weight ratios (6.9 (0.3) compared to $4.9(0.04))$. Analysis of BAL fluid from treated animals demonstrated significant $(\mathrm{p}<0.005)$ increases in total protein $(2.9(0.4)$ and $5.9(0.6) \mathrm{\mu g} / \mathrm{\mu l}$ respectively for 0.2 and $0.4 \mathrm{ml} / \mathrm{kg} \mathrm{OA}$ compared to $0.2(0.03)$ in the PBS control group). Albumin was also detected in BALF from all OA-treated animals $(0.2(0.0) \mathrm{\mu g} / \mu \mathrm{l})$. Total cell numbers $\left(1.4(0.3) \times 10^{6}\right.$ and $1.9(0.3) \times 10^{6} / \mathrm{ml}$ for the two doses compared to $0.4(0.1) \times 10^{6} / \mathrm{ml}$ in control) and the marker of cell damage, lactate dehydrogenase (LDH) activity (156 (40) and 446 (1 17) $\mathrm{U} / \mathrm{l}$ compared to control 4.9 (1.3) $\mathrm{U} / \mathrm{l}$ ) were also significantly increased. MIP-2, isolated from lung homogenates, revealed a significant $(p=0.005)$ increase in mice treated with the lower dose of $O A$ 
compared to control. OA treatment resulted in substantial hypoxemia $\left(\mathrm{PaO}_{2}\right.$ at $\mathrm{FlO}_{2} 1.0$ decreased from $425(28)$ to $\left.68(6) \mathrm{mmHg}\right)$ with decreased respiratory system compliance $(69.7 \quad(5.6) \%, n=4)$. Electron microscopy demonstrated the presence of intra-alveolar fibrin and haemorrhage, type I alveolar epithelial cell necrosis and destruction of alveolar architecture. Histological quantification of the above lung damage parameters revealed a dose dependent increase in patchy lung damage with treated mice having 34.7 (7) and 48.9 (3.6) \% damage compared to 14.9 (2.9) \% in control animals, $p=0.05$ and 0.005 respectively). Correlation of these physiological, histological and clinically relevant parameters in the OA mouse provides a model for further investigation of treatments for ARDS.

\section{S51 REGULATION OF NEUTROPHIL FATE BY HYPOXIA}

S.R. Walmsley, A. Cowburn, J. Deighton, K.I. Mecklenburgh, E.R. Chilvers. Respiratory Medicine Division, Department of Medicine, University of Cambridge School of Clinical Medicine, Addenbrooke's and Papworth Hospitals, Cambridge CB2 2QQ, UK

Introduction: Neutrophil apoptosis represents a major mechanism involved in the resolution of acute inflammation. In contrast to many other cell types, we have previously shown that hypoxia (0-3.5 kPa $\mathrm{O}_{2}$ ) has the ability to inhibit apoptotic cell death in neutrophils when aged in vitro. This may be of physiological importance given the significant drop in local oxygen tension at sites of inflammation. We therefore sought to elucidate the oxygen sensing pathways involved in this regulation of neutrophil apoptosis.

Methods: Human neutrophils were purified from the peripheral blood of healthy volunteers by dextran sedimentation followed by centrifugation through discontinuous plasma-Percoll gradients. Cells were cultured in supplemented MDM +/- test reagents in normoxic (19 kPa), hypoxic $(3 \mathrm{kPa})$ or anoxic $(0 \mathrm{kPa})$ environments. Apoptosis was assessed by cell morphology and flow cytometry with annexin $\mathrm{V}$ and propidium iodide. Cytokine release was measured by ELISA, and cytosolic and nuclear protein expression by western blotting.

Results: Oxygen deprivation profoundly inhibited constitutive apoptosis in human neutrophils from $59 \%$ to $29 \%$ ( $p=0.0025)$. Conditioned medium from oxygen deprived neutrophils also induced cell survival in normoxia but this was independent of GM-CSF, IL-1 $\beta$, IL-6 and TNF $\alpha$. Furthermore, the hypoxic inhibition of neutrophil apoptosis was unaffected by the PI3 kinase inhibitors LY294002 (10 $\mu \mathrm{M})$ or wortmannin (100 $\mathrm{nM})$. The hypoxic survival of neutrophils previously shown to be mimicked by the iron chelators desferrioxamine and hydroxypyridone (Mecklenburgh KI et al. Blood 2002; in press) was replicated with the novel prolyl hydroxylase inhibitor dimethyl oxaloylglycine at HIF stabilising concentrations (1 mM).

Conclusion: Our results indicate that neutrophils have a ferro-protein oxygen sensing mechanism involving prolyl hydroxylase domain (PHD) containing proteins which can regulate the hypoxic inhibition of neutrophil apoptosis. This survival effect is independent of the PI3-kinase pathway. Moreover neutrophils cultured under hypoxic conditions secrete an apparently novel factor that has a profound survival effect on neutrophils cultured in normoxic conditions.

Research funded by the MRC, and Sackler studentship.

\section{S52 TOLL-LIKE RECEPTOR EXPRESSION IN ACUTE RESPIRATORY DISTRESS SYNDROME (ARDS): A ROLE FOR TLR-2?}

L. Armstrong, A.R.L. Medford, K.M. Uppington, A.B. Millar. Lung Research Group, University of Bristol Division of Medicine, UK

ARDS is a condition commonly associated with sepsis. Recently the toll-like receptor family has been identified on the basis of homology with the type I IL-1 receptor. 10 members have been described, but only 3 of them have a known function: TLR- 4 is a component of the LPS receptor and is essential for gram negative responses, TLR-2 is a receptor for gram positive lipoproteins but may also cross-signal in response to LPS with TLR-4. TLR-9 recognises CpG bacterial motifs. We have looked at the expression of TLR-2 and TLR- 4 on monocytes from patients with gram negative $(n=20)$ and gram positive sepsis $(n=20)$, compared to non-septic ITU controls $(n=15)$. TLR expression was also determined on alveolar neutrophils and macrophages. We also looked at the relationship between TLR expression and the development of ARDS. Monocytes were isolated from whole blood by density gradient centrifugation and adherence. Total RNA was isolated after 2 hours in culture and RT-PCR was performed for TLR-2, TLR-4 and GAPDH transcripts. TLR protein expression was determined by flow cytometry on whole blood, dual stained with CDI4-FITC and specific TLR-2 and TLR-4 monoclonal antibodies, or on ungated macrophages and neutrophils. Expression of both TLR-2 and TLR-4 mRNA (\% GAPDH) was significantly increased in sepsis $(126$ and $87.5 \%$ respectively) versus non-sepsis controls (96 and $68 \%$ respectively) $(p<0.05)$. This was reflected at the protein level; TLR-2 expression (above isotype control) on monocytes was 15.44 versus 0.13 for nonsepsis controls $(p<0.05)$. TLR-4 expression was 3.77 versus 0.59 nonsepsis controls. Both TLR mRNAs significantly correlated with TNF- $\alpha$ mRNA suggesting increased function. In ARDS subjects there was no significant differences in TLR-4 expression. However, monocytes from ARDS subjects had significantly lower levels of TLR-2 mRNA (83\% of GAPDH for ARDS subjects vs $126 \%$ for non-ARDS subjects, $\mathrm{p}<0.001$ ). Similarly TLR-2 mRNA was lower in patients who died (103\%) versus survivors (144\%). In the lung, alveolar neutrophils and macrophages both express high levels of TLR-2 and TLR- 4 . We hypothesise that reduced levels of TLR-2 may influence development of ARDS in sepsis populations by increasing the availability of LPS for TLR-4 receptor ligation and signalling.

\section{S53 ELEVATION OF THIOREDOXIN CONCENTRATIONS IN PLASMA AND BRONCHO-ALVEOLAR LAVAGE FLUID FROM PATIENTS WITH THE ACUTE RESPIRATORY DISTRESS SYNDROME}

M.E.J. Callister', A. Burke-Gaffney', G.J. Quinlan', H. Nakamura², J. Yodoi ${ }^{2}$, T.W. Evans'. 'Unit of Critical Care, National Heart and Lung Institute, Imperial College Faculty of Medicine, Royal Brompton Hospital, London, UK; ${ }^{2}$ Department of Biological Responses, Institute of Virus Research, University of Kyoto, Japan

Introduction: The acute respiratory distress syndrome (ARDS) is characterised by refractory hypoxaemia secondary to alveolar derecruitment and disordered ventilation-perfusion matching. Oxidative stress and inflammation are key features that contribute to the pathogenesis of ARDS. Thioredoxin (Trx) is an intracellular redox active protein involved in maintaining cellular redox balance, and is actively secreted into the extracellular space in response to clinical conditions associated with oxidative stress and inflammation. Extracellular Trx may have profound implications for the inflammatory response by virtue of its reported chemokine/cytokine properties. We therefore measured Trx levels in plasma and broncho-alveolar lavage fluid (BALF) from 30 patients with ARDS and 18 healthy controls.

Results: Trx levels were significantly elevated in ARDS patients $v$ controls in plasma $(45.9(27.9) \mathrm{ng} / \mathrm{ml} \vee 23.6(13.4) \mathrm{ng} / \mathrm{ml}$, $\mathrm{p}<0.002)$ and BALF (103.8 (116.0) ng/ml $\vee 17.4(9.6) \mathrm{ng} / \mathrm{ml}$, $p<0.0001)$. There were significant positive correlations between $\operatorname{Trx}$ concentration and IL-8 concentrations ( $p<0.001, r=0.631)$ and IL-1 $\beta$ concentrations $(p<0.05, r=0.448)$ in BALF from the ARDS population. BALF Trx concentrations were higher in patients with ARDS of pulmonary compared to extrapulmonary aetiology $(132.3$ (129.2) ng/ml v $39.7(26.9) \mathrm{ng} / \mathrm{ml}, \mathrm{p}<0.01)$. BALF from patients with pulmonary ARDS also had significantly higher concentrations of IL-8 $(p<0.05)$ and a greater percentage neutrophil count $(p<0.05)$ compared to BALF from patients with extrapulmonary ARDS. Plasma and BALF Trx levels were not significantly different between surviving and non-surviving patients and there was no relationship between Trx levels and the sequential organ failure assessment (SOFA) score when all patients were considered. However, in cases of pulmonary ARDS, there was a significant association between SOFA score and plasma Trx levels $(p<0.05, r=0.482)$.

Discussion: These results demonstrate that concentrations of Trx are increased in BALF and plasma in patients with ARDS. Trx has been shown to have profound effects on the inflammatory response and the correlation between levels of $\operatorname{Trx}$ and pro-inflammatory cytokines suggests a link between Trx and the inflammatory response in this condition, although the exact nature of the role of increased extracellular Trx in ARDS remains to be evaluated.

Research Funded by the Wellcome Trust.

\section{S54 PLASMA VASCULAR ENDOTHELIAL GROWTH FACTOR (VEGF) LEVELS AND THE VEGF + $936 \mathrm{C} / \mathrm{T}$ POLYMORPHISM IN ACUTE RESPIRATORY DISTRESS SYNDROME (ARDS)}

A.R.L. Medford', L.J. Keen², D.R. Thickett', K.J. Hunter', A.B. Millar'. 'Lung Research Group, University of Bristol Division of Medicine, Southmead Hospital, Bristol, UK; ${ }^{2}$ Department of Pathology and Microbiology, University of Bristol, Homeopathic Hospital Site, Bristol, UK

Previous work in our laboratory suggested a role for VEGF in the pathogenesis of ARDS (Thickett DR et al. Am J Resp Crit Care Med 
$2001 ; 164: 1601-5)$. There is considerable interindividual variation in plasma VEGF levels and the $+936 \mathrm{C} / \mathrm{T}$ polymorphism (CT or TT genotypes) is associated with low plasma VEGF levels (Renner W et al. J Vasc Res 2000;37:443-8). Because of the possible functional role of VEGF, we hypothesised that the frequency of this polymorphism would be altered in ARDS. DNA was extracted from healthy subjects $(n=50)$ as well as ARDS patients $(n=45)$ and ventilated at risk patients in intensive care $(n=38)$. A 120 base pair fragment of the VEGF gene containing the polymorphism was amplified and cross-matched with an internal heteroduplex generator containing a polyA insert adjacent to the polymorphism. Artificial heteroduplex formation was analysed on a $15 \%$ polyacrylamide gel. ELISA was performed to measure VEGF levels in matched plasma samples from the ARDS and at risk groups. The abnormal (CT or TT) genotypes occurred in $31.3 \%$ of at risk patients, $28.9 \%$ of ARDS patients and $24 \%$ of normal subjects. In ARDS patients, those with an abnormal genotype had a significantly higher 30 day mortality than those with a normal genotype. In the at risk groups, plasma VEGF levels were lower in those with abnormal genotypes compared to normal genotype $(129 \mathrm{pg} / \mathrm{ml}$ versus 305 $\mathrm{pg} / \mathrm{ml})$ but this was not observed in ARDS $(337 \mathrm{pg} / \mathrm{ml}$ versus 309 $\mathrm{pg} / \mathrm{ml}$ ). The $+936 \mathrm{C} / \mathrm{T}$ polymorphism occurs more frequently in at risk and ARDS patients than normal subjects indicating a possible functional significance in intensive care patients. The normal relationship between plasma VEGF level and genotype appears to be disrupted in ARDS suggesting additional regulatory mechanisms may be important in these patients.

\section{Asthma mechanisms I}

\section{S55 EXPRESSION OF TRANSFORMING GROWTH FACTOR ISOFORMS IN HUMAN AIRWAY SMOOTH MUSCLE AND NORMAL LUNG TISSUE}

B. Islam, D. Bradbury, L. Corbett, I. Soomro, J. Ronan, A. Knox. 'Division of Respiratory Medicine; ${ }^{2}$ Histopathology, Nottingham City Hospital, Hucknall Road, Nottingham NG5 IPB, UK

Introduction: transforming growth factor beta (TGF- $\beta$ ) is an immunomodulatory cytokine regulating the proliferation and differentiation of various cell types. It also contribute to the maintenance of tissue architecture by influencing the production of extracellular matrix components. In this study we examined the expression of TGF- $\beta$ isoforms in human airway smooth muscle cells (HASMC) and normal lung tissues by rt-PCR, bioassay and immunohistochemical staining.

Methods: HASMC were obtained from post-mortem samples of human lung and studied at passage 6. Normal lung tissues were obtained from lung biopsy samples and thoracic surgery. Mink lung epithelial cells (Mv1Lu) were obtained commercially. Recombinant TGF- $\beta-1$ and neutralising antibodies to TGF- $\beta-1,-2,-3$ were obtained from R\&D Systems. TGF $\beta$ isoforms mRNA were measured by rt-PCR using primers designed from the human sequences of TGF $\beta-1,-2,-3$ genes and GAPDH as control. The presence of bioactive TGF- $\beta$ in HASM-conditioned medium was determined using a bioassay that is based on the ability of TGF- $\beta$ to inhibit proliferation of the Mv 1 LU cells. Immunohistochemical localisation of TGF- $\beta-1,-2,-3$ in normal lung sections was assessed using avidin-biotin-peroxidase and antibody to the TGF $\beta$ Bisoforms.

Results: Messenger RNA transcripts for TGF- $\beta-1,-2,-3$ were all expressed in the HASMC. The conditioned medium shows presence of bioactive TGF- $\beta$ in the acid treated sample and by using the neutralising antibodies it demonstrated that all three isoforms are present in the conditioned medium. Very little TGF $\beta$ was present in the non-acid treated medium, implying that majority of the TGF $\beta$ secreted by the HASMC is in the biologically non-active form. The TGF- $\beta$ bioactivity was significantly abrogated by panspecific antibody to TGF- $\beta$. Immunohistochemistry showed that all three isoforms of TGF $\beta$ were detected in the epithelial cells, smooth muscle cells, and macrophages.

Conclusion: This study demonstrates the expression of TGF- $\beta$ isoforms in the lung, and suggest that autocrine/paracrine release of TGF- $\beta$ by HASMC could play an important role in the remodelling of airway smooth muscle in asthma.

\section{S56 IL-4 EXPRESSION IS INCREASED AND CO-LOCALISED TO MAST CELLS WITHIN THE AIRWAY SMOOTH MUSCLE IN ASTHMA}

C.E. Brightling, F.A. Symon, S.T. Holgate', A.J. Wardlaw, I.D. Pavord, P. Bradding. Division of Respiratory Medicine, Institute for Lung Health, Leicester, UK; 'University of Southampton, Southampton General Hospital, UK

Airway smooth muscle infiltration by mast cells is a key feature of asthma and not eosinophilic bronchitis (Brightling CE, et al. NEJM
2002;346:1699-705). In asthma Th2 cytokines have been implicated as playing a critical role in the development of airway inflammation, but whether inflammatory cells within the airway smooth muscle release these cytokines is unknown.

We have undertaken a comparative immunohistochemical study in bronchial biopsies from 14 subjects with asthma, 10 with eosinophilic bronchitis and 8 normal controls recruited from two centres.

The median cells $/ \mathrm{mm}^{2}$ smooth muscle were significantly higher in the subjects with asthma than eosinophilic bronchitis and normal controls for IL-4 $(3 \mathrm{H} 4)+$ cells $(2.4,0,0$ respectively; $\mathrm{p}=0.001)$, and IL-4 $(4 D 9)+$ cells $(1.6,0,0$ respectively; $p=0.02)$. There were significant differences in the median (range) cells $/ \mathrm{mm}^{2}$ smooth muscle IL-5+ cells in the subjects with asthma $0(0-1.7)$, eosinophilic bronchitis 0 $(0-1.4)$ and normal controls $0(0-0.3)(p=0.31)$. 94\% of the cells expressing IL-4 $(3 \mathrm{H} 4)$ and $92 \%$ of those expressing IL-4+ (4D9) in the airway smooth muscle were mast cells. $55 \%$ of the mast cells within the airway smooth muscle co-localised to IL-4 (3H4) and $29 \%$ to IL-4 (4D9).

In asthma mast cells localised within the airway smooth muscle express IL-4 but not IL-5, suggesting that IL-4 may play an important role in mast cell-airway smooth muscle interactions.

Supported by the National Asthma Campaign.

\section{S57 EFFECTS OF LONG ACTING $\beta$ AGONISTS AND STEROIDS ON CYTOKINE EXPRESSION}

H. Yanagawao, I.M. Adcock', A.L. Hewitt', H. Buck ${ }^{2}$ National Heart and Lung Institute, Imperial College London, UK; ${ }^{2}$ Innovata Biomed Ltd, St Albans, UK

Rationale: In bronchial asthma treatment, combination therapy with inhaled corticosteroids and long acting $\beta$ agonists (LA $\beta A$ ) can improve disease control. Since research suggests that ligand-independent activation of the glucocorticoid receptor (GR) by $L A \beta A$ may aid steroid anti-inflammatory action, the functional consequence of GR nuclear translocation was examined via the effect of LA $\beta A$ and steroids on cytokine production.

Methods: U937 cells were incubated with fluticasone, budesonide, formoterol and salmeterol, alone and in combination $\left(10^{-6} \mathrm{~B} 10^{-11} \mathrm{M}\right)$, for 1 hour prior to lipopolysaccharide (LPS) stimulation. Secreted cytokines (GM-CSF, TNF- $\alpha$, IL-1 ra and IL-10) were quantified by ELISA.

Results: Addition of formoterol or salmeterol to steroid gave greater suppression of LPS-induced GM-CSF and TNF- $\alpha$ release compared with that observed with budesonide or fluticasone alone, although neither $L A \beta A$ altered the steroid concentration-response curve. Additionally, both LA $\beta A$ prevented steroid-induced repression of IL-1 ra release. The actions of the LABA differed with IL-10, where formoterol, in combination with fluticasone, enhanced production, whilst salmeterol did not increase LPS-induced IL-10 production compared with fluticasone alone.

Conclusions: These data indicate that the added benefit of formoterol may relate to the anti-inflammatory gene-inducing action of steroids rather than in enhancing the repressive functions of steroids towards inflammatory cytokines.

This study was sponsored by Innovata Biomed Ltd.

\section{S58 LEUKOTRIENE RECEPTORS ON HUMAN EOSINOPHILS}

H. Rupani, I. Hao, A.P. Sampson. Respiratory Cell and Molecular Biology Division University of Southampton School of Medicine

Cysteinyl-leukotrienes are potent bronchoconstrictor mediators and eosinophilotaxins released by mast cells and eosinophils within the asthmatic airway. These actions are thought to be mediated by CysLT receptors, with vascular effects mediated by $\mathrm{CysLT}_{2}$ receptors. CysLT, expression on human eosinophils may be regulated by cytokines, but the effects of asthma therapies including corticosteroids and methylxanthines are unknown. Recently, eosinophils were reported to transcribe larger amounts of mRNA for CysLT, than for CysLT, (Mita, et al. Clin Exp Allergy 2001;31:1714-23). We investigated firstly whether CysLT, on eosinophils is modulated by dexamethasone or theophylline, and secondly whether eosinophils also express CysLT receptors on their cell surface. Immunomagnetically-purified human blood eosinophils of normal and mild atopic volunteers were cultured in HEPES-buffered RPMI 1640 with 10\% FCS for 22 hours in the pres-

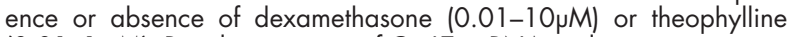
(0.01-1 mM). Basal expression of CysL $T_{1}$ mRNA and protein was confirmed by RT-PCR, flow cytometry, immunocytochemistry, and immunoblotting. Flow cytometric expression of cell-surface CysLT, declined 
during 22 hours culture from a median fluorescence intensity (MFI) of $4.96(0.50)$ to $2.87(0.23)$ arbitrary units $(p<0.009 ; n=9)$. This decline was not observed in the presence of dexamethasone $(1 \mathrm{\mu M})$, with an MFI value of $4.01(0.36)$ at 22 hours ( $p=0.044 v$ untreated). Theophylline $(0.1 \mathrm{mM})$ provided similar protection but this did not achieve statistical significance $(\mathrm{p}=0.056)$. In contrast to Cys $\mathrm{LT}_{1}$, no Cys $\mathrm{LT}_{2}$ expression was detectable by flow cytometry on the surface of live eosinophils, although these cells transcribed mRNA for CysLT ${ }_{2}$. Flow cytometry readily detected surface expression of CysLT and the chemokine receptor CCR-3 on eosinophils, while CysLT ${ }_{2}$ surface expression was detected on autologous blood monocytes and T-cells. We conclude firstly that dexamethasone may upregulate eosinophil expression of CysLT, receptors, a paradoxical effect also observed for the LT-synthesising enzymes (Cowburn, et al. J Immunol 1999;163:456-65). Secondly, while CysLT 2 mRNA may be transcribed, the receptor protein does not appear to be expressed on the surface of blood eosinophils from healthy donors.

\section{S59 CAT-213, AN ANTI-EOTAXIN (CCL1 1) ANTIBODY, REVEALS A ROLE FOR EOTAXIN IN THE EOSINOPHIL CHEMOTACTIC ACTIVITY OF ASTHMATIC SPUTUM}

G. Dent', C. Hadjicharalambous', J. Ward', T. Yoshikawa', G. Angco, D.E. Davies', R. Louis², R.L.C. Handy ${ }^{3}$, J. Powell', I.K. Anderson ${ }^{3}$, R. Dukanović'. 'Respiratory Cell \& Molecular Biology, University of Southampton School of Medicine, Southampton; ${ }^{2}$ Department of Pneumonology, University of Liège, Liège, Belgium; ${ }^{3}$ Cambridge Antibody Technology, Melbourn, Cambridgeshire, UK

To understand the mechanisms underlying eosinophilic airways inflammation in asthma, it is necessary to characterise the chemoattractants mediating eosinophil recruitment. The CC chemokines eotaxin (CCL1 1), eotaxin-2 (CCL24), RANTES (CCL5) and monocyte chemotactic proteins 2,3 and 4 (CCL8, CCL7, CCL13) are potent eosinophil chemoattractants and are believed to play an important role in eosinophilic inflammation (Kita \& Gleich. J Exp Med $1996 ; 183: 2421)$. Of these, eotaxin and eotaxin-2 act exclusively via the CC chemokine receptor CCR3 upon a restricted set of cells: namely eosinophils, basophils and Th2 lymphocytes. We have investigated the role of eotaxin in the eosinophil chemotactic activity of asthmatic airway secretions using a specific anti-eotaxin antibody, CAT-213.

Sputum samples were collected from 60 volunteers: 11 healthy controls, 12 mild asthmatics, 12 stable moderate asthmatics, 12 unstable moderate asthmatics and 13 severe asthmatics. Sputum was processed by thorough mixing with 4 volumes of phosphate-buffered saline (PBS) containing protease inhibitors. Cells and mucus were precipitated by centrifugation and supernatants were assayed for immunoreactive eotaxin by ELISA and for eosinophil chemotactic activity in a fluorescence-based modified Boyden chamber assay.

Sputum eotaxin concentration differed significantly among the subject groups ( $p=0.0096$, ANOVA); group mean eotaxin concentration showed a significant linear trend $v$ group number when groups were sorted by disease severity $(\mathrm{P}=0.0006)$, rising from 3.71 (1.07) pmoll ${ }^{-1}$ (mean (SEM)) in healthy controls to 35.9 (1 1.4) pmol $\mathrm{H}^{-1}$ in severe asthmatics. Eosinophil chemotactic activity also differed among groups $(P=0.0073$, Kruskal-Wallis) and showed a significant increase over healthy control in both moderate groups $(P<0.05$, Dunn=s test) and the severe asthmatic group $(P<0.01)$. There was a significant linear correlation between chemotactic index and eotaxin concentration $(P=0.008)$. CAT-2 $13(100 \mathrm{~nm})$ caused significant inhibition of sputum chemotactic activity in the unstable moderate and severe asthmatic groups $(\mathrm{P}<0.001$, paired ANOVA and Dunnett's test), with a mean inhibition of $80(13) \%$ in the severe asthmatic group.

We conclude that eotaxin contributes significantly to the eosinophil chemotactic activity of asthmatic sputum. This contribution increases with disease severity. CAT-213 may provide therapeutic benefit in the treatment of severe asthma.

\section{S60 DIFFERENTIAL ACTIONS OF PHOSPHATIDYINOSITOL 3-KINASE INHIBITORS ON HUMAN EOSINOPHIL CHEMOTACTIC RESPONSES TO PLATELET-ACTIVATING FACTOR AND EOTAXIN (CCL11)}

R. Mishra, B.C. Gray, R. Djukanović, G. Dent. Respiratory Cell \& Molecular Biology, University of Southampton School of Medicine, Southampton, UK

The signal transduction pathways that lead to chemotaxis of human eosinophils are incompletely understood but are believed to involve protein tyrosine kinase(s) and MAP kinase(s) but not protein kinase $C$ (Schweizer et al. J Leukoc Biol 1996;59:347; Kampen et al. Blood 2000;95:1911). As MAP kinase activation by eotaxin in human eosinophils has been shown to occur downstream of phosphatidylinositol 3-kinase (PI-3K) activation (Miike et al. J Leukoc Biol 2000;67:117), we investigated a possible role for PI-3K in the induction of eosinophil chemotaxis using the inhibitors wortmannin and LY294002 in human eosinophils stimulated with platelet-activating factor (PAF) or eotaxin (CCLI 1).

Eosinophils were isolated from the blood of atopic, non-asthmatic donors and chemotaxis was evaluated in a fluorescence-based 96-well blind-chamber assay. Calcein-labelled eosinophils $\left(2 \mathrm{H}^{1} \mathrm{O}^{5}\right)$ were pre-incubated for $30 \mathrm{~min}$ at $37 \mathrm{EC}$ in the absence and presence of wortmannin (0.1-100 $\mathrm{nM}$ ) or LY294002 (0.1-100 $\mathrm{\mu M})$ and placed in the upper wells of the chemotaxis chamber. Migration through 8-um pore-size PVP-coated polycarbonate filters in response to PAF or eotaxin (both at $30 \mathrm{nM}$ in the lower wells) was measured after $1 \mathrm{~h}$ as cell-associated fluorescence $\left(\lambda_{\mathrm{em}}=485 \mathrm{~nm}, \lambda_{\mathrm{ex}}=530 \mathrm{~nm}\right)$ in the lower wells.

PAF and eotaxin caused chemotaxis of eosinophils (geometric mean $19,140(14,060-26,060)$ and $27,010(15,310-47,640)$ cells well $^{-1}$, respectively, above the background level of migration; $n=12$ ) Wortmannin caused concentration-dependent inhibition of PAFinduced eosinophil chemotaxis $\left(\mathrm{IC}_{50}=1.24 \mathrm{nM}(0.092-16.6 \mathrm{nM})\right.$; $n=6)$, with significant inhibition observed at $1 \mathrm{nM}(\mathrm{P}<0.0001)$, while responses to eotaxin were inhibited substantially less potently $\left(I C_{50}=17.6 \mathrm{nM}(1.06-292 \mathrm{nM}) ; \mathrm{n}=6\right)$, with significant inhibition observed only at $100 \mathrm{nM}(\mathrm{P}<0.05)$. LY294002 also inhibited PAF-induced chemotaxis $\left(I C_{50}=0.76 \mu M(0.026-22.1 \mu M) ; n=6\right)$ but had no effect on responses to eotaxin $(<50 \%$ inhibition at $100 \mu \mathrm{M}$; $n=6$ ).

We conclude that, although PAF and eotaxin both induce MAP kinase activation and chemotaxis in human eosinophils, PAF-induced chemotaxis is dependent upon the activation of PI-3K while the response to eotaxin involves PI- $3 \mathrm{~K}$ to a much lesser extent. This may indicate alternative pathways of activation of MAP kinases and chemotactic responses.

\section{Ongoing management issues in cystic fibrosis}

\section{S61 STENOTROPHOMONAS MALTOPHILIA ACQUISITION IN CYSTIC FIBROSIS; A COMPLICATION OF ASPERGILLUS FUMIGATUS?}

A. Equi, V. Marchac, C. Le Bihan-Benjamin, M. Hodson, A. Bush. Department of Respiratory Paediatrics and Department of Cystic Fibrosis Royal Brompton Hospital, London, UK; Service de Pneumologie et D'Allergologie et Bio-statistique et Informatique Medicale, Hopital Necker- Enfants Malades, Paris, France

Stenotrophomonas maltophilia (SM) is a Gram negative bacillus which is resistant to many antibiotics. Its role as a pulmonary pathogen in $\mathrm{CF}$ is still being defined.

Methods: The aim of this study was to use a case control design to describe the characteristics of CF patients isolating SM in sputum, to assess risk factor for acquisition of SM and to assess clinical outcomes. Data were collected on patients and controls between 1991 and 1999. A control patient had never isolated SM in sputum and was matched for age, sex and $\mathrm{FEV},( \pm 10 \%)$. The following data were collected: FEV 1, FVC; height and weight; sputum culture and antibiotic sensitivity; antibiotic use; oral steroid use.

Results: 63 patients isolated SM at least once. Controls were found for 52 patients. Prevalence of SM rose from 3.3 to $15 \%$ by 1999. Isolation of Aspergillus fumigatus (AF) was much more frequent in SM patients than controls, $51 \%$ v $8.9 \%(p=0.001$, OR 5.9 (95\% Cl 2.017.8). The effect of AF was independent of steroid use. Post acquisition there was no difference between the two groups in mortality, antibiotic use, lung function or nutritional status. Antibiotic treatment had no impact on future isolation of SM.

Conclusions: This is the first documentation of an association of $\mathrm{SM}$ with $\mathrm{AF}$ isolation. We also can reassure patients that $\mathrm{SM}$ acquisition is unlikely to cause accelerated decline in clinical status. 


\begin{tabular}{|c|c|c|c|c|c|c|c|}
\hline \multicolumn{8}{|l|}{ Abstract S64 } \\
\hline \multirow[b]{2}{*}{ Results: $n=17$ in all groups } & \multicolumn{2}{|c|}{ Group A } & \multicolumn{2}{|c|}{ Group B } & \multicolumn{2}{|c|}{ Group C } & \multirow{2}{*}{$\begin{array}{l}\text { - } \mathrm{p} \text { Value } \mathrm{A} v \\
\mathrm{~B}, \& \mathrm{C}\end{array}$} \\
\hline & mean & SD & mean & SD & mean & SD & \\
\hline Packed red cells (units) & 5.8 & 4.9 & 3.6 & 1.7 & 4.5 & 3.3 & $p<0.05$ \\
\hline Platelets (pools) & 0.9 & 1.2 & 0.6 & 0.9 & 0.3 & 0.5 & NS \\
\hline \multirow[t]{2}{*}{ Fresh frozen plasma (units) } & 2.7 & 2.5 & 1.9 & 2 & 1.6 & 1.5 & NS \\
\hline & \multicolumn{2}{|c|}{ number } & \multicolumn{2}{|c|}{ number } & \multicolumn{2}{|l|}{ number } & \\
\hline Re-exploration for bleed & 2 & & 0 & & 1 & & NS \\
\hline 30 day mortality & 0 & & 0 & & 0 & & NS \\
\hline Dense adhesions & 8 & & 0 & & 0 & & $p<0.01$ \\
\hline No adhesions & 1 & & 7 & & 13 & & $p<0.01$ \\
\hline
\end{tabular}

\section{S62 GENOTYPING PSEUDOMONAS AERUGINOSA IN CYSTIC FIBROSIS CLINICS: IMPLICATIONS FOR SEGREGATION}

F.P. Edenborough, H.R. Stone, S.J. Kelly, P. Zadik', J.R.W. Govan². Adult Cystic Fibrosis Unit and 'Public Health Laboratory, Northern General Hospital, Sheffield, UK; ${ }^{2}$ Department of Medical Microbiology, University of Edinburgh, UK

We were concerned that the antiobiograms from patients harbouring Pseudomonas aeruginosa appeared to show increasing resistance, despite segregation policies initiated early in 1999. Genomic finger printing was performed on $P$ aeruginosa isolates from 40 patients attending the clinic.

Twenty three $(57 \%)$ had unique strains, $10(25 \%)$ had strains indistinguishable from the "Liverpool" epidemic strain and 7 (17\%) shared a common strain not previously seen, henceforth referred to as the "Sheffield" epidemic strain. 4/10 patients with the Liverpool strain had multiresistant (MR) organisms, compared to all 7 patients with Sheffield strain and only 1 patient $(4 \%)$ out of the 23 with a unique strain. One patient harboured both Sheffield and Liverpool MR strains.

Those with the Sheffield MR strain were younger with poorer FEV and weight together with a higher proportion of ${ }^{~} \mathrm{~F} /{ }^{\wedge} \mathrm{F}$ genotype, pancreatic insufficiency, diabetes mellitus and liver disease vs unique strains. Consequently numbers of clinic visits, days in hospital and time on intravenous antibiotics were increased. Comparison of Liverpool MR with Liverpool non-MR showed a similar pattern.

9/10 with Liverpool strains had been transferred from the paediatric unit since July 2000. MR strains being detected on the sputum sample taken when or in some cases before the patient first attended the CF Unit. Four of 7 Sheffield MR patients had also been transferred but all have been inpatients in the adult centre at the same time as subjects who were found to have Sheffield MR strains already (including 1 sibling pair). We suspect we have "imported" the Liverpool strain, which is known to become multiresistant from the paediatric unit, but the Sheffield strain is our own and is transmissible. If MR confers poor prognosis we believe these groups should be segregated from each other and those with unique strains, both as inpatients and in the outpatient clinic and have acted accordingly.

\section{S63 RE-AUDIT OF THE SCREENING PROTOCOL FOR CYSTIC FIBROSIS RELATED DIABETES (CFRD) IN AN ADULT CENTRE}

A. Verma, A. Claridge, T. Havelock, J. Biesty, D. McMenna, A.K. Webb. Manchester Adult Cystic Fibrosis Centre, Wythenshawe Hospital, Manchester M23 9LT, UK

Introduction: The average age of onset of CFRD is 18-21 years and is associated with a 6-fold increase in mortality and associated co-morbidity.' Yung et al described annual oral glucose tolerance testing (OGTT) as being time and resource consuming for patients and staff. ${ }^{2}$ An earlier audit at our centre, using these selective screening criteria for OGT testing found that, the identified incidence of $1.9 \%$ was significantly less than the expected $(4-6 \%)$ and concluded that only $52 \%$ of patients with CFRD would have been identified. ${ }^{3}$ Hence all patients were offered routine annual OGT testing.

Aims: To assess whether all patients received annual OGTT between 3.00-3.01. To assess the true incidence of CFRD at the Centre. To identify the CFRD patients who would have been "missed" if the old screening protocol were still in place. To calculate the sensitivity and specificity of the selection criteria in our population
Method: A retrospective review of case notes using the OGTT and $\mathrm{HbAlc}$ databases. 242 patients were identified but 91 patients excluded due to pre-existing CFRD at 01/03/2000 ( $n=52)$, referred after $01 / 03 / 00(n=13)$, care transferred to other centre $(n=18)$, posttransplant $(n=7)$, not CF $(n=1)$.

Results: 89 OGTT were performed on 151 patients. The incidence was found to be $4.4 \%$ and the prevalence $26.9 \%$. Using the previous selective screening protocol, 6 patients with CFRD and 10 patients with impaired glucose tolerance would have been missed. The total sensitivity and specificity of the screening criteria would only have been $70 \%$ and $51 \%$ respectively.

Conclusions: In view of the need for early diagnosis and treatment of CFRD, annual OGT testing is essential for all CF centres especially for adult patients. Selective screening does not identify all patients who need to be tested.

1. Moran et al. Diabetes Res Clin Pract 1999;45:61-73.

2. Yung et al. J R Soc Med 1999;92:35-40.

3. Verma et al. J of CF 2001;PS138.

\section{S64 LUNG TRANSPLANTATION OUTCOME IN CYSTIC FIBROSIS PATIENTS WITH PREVIOUS PLEURAL PROCEDURES}

H.J. Curtis, S.J. Bourke, P.A. Corris, J.H. Dark. Cardiothoracic Centre, Freeman Hospital, University of Newcastle upon Tyne, UK

Background: High post-operative mortality secondary to haemorrhage from pleural adhesions as a consequence of previous invasive pleural procedures (IPP) was reported in the early experience of lung transplantation. This observation led to IPP becoming a relative/ absolute contra-indication to transplantation in some centres.

Aim: Comparison of post-operative outcome in patients with and without previous IPP, undergoing single sequential lung transplant (SSLT).

Method: Retrospective review of 3 groups of patients undergoing SSLT at this centre from 1989-2002. Group A: 17 cystic fibrosis (CF) patients with a history of previous pneumothorax (PTX) +/- IPP. Group B: $17 \mathrm{CF}$ patients with no history of PTX. Group C: 17 non-CF/non-bronchiectatic (emphysema, fibrosing alveolitis or obliterative bronchiolitis) patients with no history of PTX. Patients were matched for year of transplantation to allow for changes in surgical technique. Measured outcomes included: pre- and post-operative haemoglobin; blood products given intra-operatively; operation and cardiopulmonary bypass times; post-op haemorrhage; times to extubation, ITU discharge and hospital discharge; FEV , at 6 months; 30 day mortality; and adhesions scored descriptively via pathology reports

Conclusion: Patients with CF and previous PTX +/- IPP undergoing SSLT have more dense pleural adhesions and increased requirement for blood transfusion. However this does not significantly affect surgical outcome. Patients with emphysema, fibrosing alveolitis or obliterative bronchiolitis were significantly more likely to be free of pleural adhesions at operation B suggesting that the inflammatory/chronic infective component of CF independently contributes to the increased pleural adhesions. Previous IPP for PTX should not be considered a contra-indication in the assessment of suitability for lung transplantation. 
S65 PULMONARY ARTERY PRESSURE AND RIGHT VENTRICULAR FUNCTION IN CYSTIC FIBROSIS ADULTS

R.J. Bright-Thomas', M.I. Burgess' ${ }^{2}$, M.E. Dodd', S.G. Ray², A.K. Webb' ${ }^{\prime}$ Adult Cystic Fibrosis Centre; ${ }^{2}$ Department of Cardiology, Wythenshawe Hospital, Manchester, UK

Introduction: Pulmonary hypertension has been reported as a poor prognostic marker in adult CF patients with severe disease. We evaluated the association between clinical status, oxygen status, pulmonary artery pressure and right ventricular (RV) function in a cross-section of CF adults.

Methods: CF adults and healthy volunteers were studied. Demographic and clinical data collected. Patients were stable at the time of study. All subjects underwent echocardiographic examination by a trained operator (RBT). Pulmonary artery systolic pressure (sPAP) was measured from the peak velocity of tricuspid regurgitant jets. Systolic function of the RV assessed via measurement of RV dimensions and tricuspid long axis motion (TLAM) and diastolic function via measurements of transtricuspid flow profile.

Results: 65 CF adults age, mean (SD), 26.1(7.1) years and 25 healthy controls age 27.7 (8.8) years were studied. Partial pressure of oxygen $\left(\mathrm{PO}_{2}\right)(\mathrm{mmHg}) 68.8$ (12.2) v 90.1 (9.7) (p<0.001) and FEV percentage predicted $\left(\mathrm{FEV}_{1} \%\right) 47.0$ (21.7) v 98.1 (9.8) $(\mathrm{p}=<0.001)$ respectively. sPAP $(\mathrm{mmHg})$ was 35.8 (9.7) in CF patients and $21.6(3.2)$ in controls $(p<0.001) .36$ patients and 0 controls had $\mathrm{sPAP}>30 \mathrm{mmHg}$. There was no significant difference in RV dimensions between groups. TLAM $(\mathrm{cm})$ was reduced in CF patients compared to controls $2.1(0.4) \vee 2.5(0.3)(p<0.001)$. There was a significant difference in the diastolic variables $A$ wave velocity and $E / A$ ratio between patients and controls; values (CF $v$ control): Avel $(\mathrm{cm} / \mathrm{sec}) 45$ (13) v 29 (6) $(p<0.001), E / A$ ratio $1.4(0.5) \vee 1.8$ (0.3) $(p<0.001)$. Pearson correlation of CF patient data identified significant correlations between both $\mathrm{PO}_{2}$ and $\mathrm{FEV} \%$ with $\operatorname{sPAP}(\mathrm{r}=-0.516$, $p<0.001 ; r=-0.432, p=0.001)$ and TLAM ( $r=0.459, p<0.001$; $r=0.620, p<0.001$ ) respectively. An association was also found between diastolic variables and $\mathrm{PO}_{2}$ and $\mathrm{FEV} \%$ : Avel $(\mathrm{r}=-0.311$, $p=0.012 ; r=-0.420, p=0.001), E / A$ ratio $(r=0.302, p=0.015$; $\mathrm{r}=0.412, \mathrm{p}=0.001$ ) respectively.

Conclusion: Pulmonary artery pressure is raised in adult CF patients. RV dimensions are normal in CF but there is evidence of deranged RV systolic and diastolic function. Both $\mathrm{PO}_{2}$ and $F E V_{1} \%$ correlate with SPAP and measures of RV function. Whether the abnormal RV function is due to the effect of hypoxia on the RV or due to pulmonary hypertension is unclear.

\section{S66 INCREASED ENERGY COSTS DURING UPPER AND LOWER LIMB ACTIVITIES IN ADULTS WITH CYSTIC FIBROSIS}

A.A. Jonescu, T.D. Mickleborough', K. Chatham, M. Lindley', R. Colasanti $^{2}$, I.S. Nixon, C.E. Bolton, D.J. Shale. 'Section of Respiratory Medicine, School of Sports Science, University of Wales Institute Cardiff and School of Applied Sciences; ${ }^{2}$ University of Glamorgan, University of Wales College of Medicine, Cardiff, UK

Patients with Cystic Fibrosis (CF) have increased resting energy expenditure, which may be associated with altered body composition. We hypothesised that adults with CF have increased energy costs during skeletal muscle work. Twelve patients were studied after 2 weeks treatment for an exacerbation (to assess them with their best lung function); mean $(95 \% \mathrm{Cl})$ age $24.6(21.4,27.8)$ years, $\mathrm{FEV}, 51.3$ (36.3, $66.2) \%$ predicted, body mass index $21.5(20.8,22.2) \mathrm{kg} / \mathrm{m}^{2}$. Fat free mass index (FFMI, $\mathrm{kg} / \mathrm{m}^{2}$ ) was assessed by anthropometrics. Ten healthy subjects were studied, age 32.1 (27.9, 36.1) years. Energy expenditure (EE, kJoule $(\mathrm{kJ}) / \mathrm{kg} / \mathrm{min}$ ) was calculated from breath by breath $\mathrm{O}_{2}$ uptake and $\mathrm{CO}_{2}$ output measured with a mask (K4b, Cosmed). Subjects completed the following: 10 handgrips (HG, force in

Abstract S66 Energy expenditure ( $\mathrm{J} / \mathrm{kg} / \mathrm{min})$ for $\mathrm{kJ}$ work performed (means, 95\% Cl)

\begin{tabular}{llll}
\hline & Stepping & Lifting & Handgrip $(\times 10)$ \\
\hline Patients & 0.55 & 3.27 & 0.13 \\
& $(0.45 \text { to } 0.65)^{* *}$ & $(2.61$ to 3.92$)$ & $(0.11 \text { to } 0.17)^{*}$ \\
Healthy & $0.37(0.31$ to 0.44$)$ & 2.99 & 0.09 \\
& & $(1.94$ to 4.04$)$ & $(0.07$ to 0.11$)$ \\
\hline
\end{tabular}

${ }^{*} p<0.05 ;{ }^{*} p<0.01$ $\mathrm{cm} \mathrm{H} \mathrm{H}_{2} \mathrm{O}$ ), 1/second; 10 steps (20 cm high), 1/second, and 10 lifts of a $1 \mathrm{~kg}$ weight through $80 \mathrm{~cm}$ height, levery 2 seconds. EE ratio to the work performed and the recovery time after each activity were calculated.

EE ratio to the work performed during HG and stepping, but not during lifting were greater in patients (table). The recovery time was greater for patients than healthy subjects for HG $(46.4$ (29.9 to 62.9) and 21.6 (10.9 to 32.3) seconds, $p=0.02)$ and stepping $(70.7$ (47.2 to 94.2 ) and 28.9 (16.6 to 41.2 ), $p=0.002$ ), but not lifting (35.9 (26.7 to 45.1$)$ and 28.6 (17.7 to 39.4$), p=0.3)$. FFMI was inversely related to $E E$ during $H G$, $(r=-0.75, p=0.005)$.

Adults with CF have greater energy expenditure for upper and lower limb muscle work. This excess energy expended for tasks similar to those of daily living suggests physical activity in such patients adds to the potential for negative energy balance and weight loss.

Supported by the Cystic Fibrosis Trust UK.

\section{Issues in paediatric lung disease}

\section{S67 EFFECT OF $\beta$-2 ADRENOCEPTOR ( $\beta 2$-AR) POLYMORPHISMS ON NEONATAL LUNG FUNCTION AND ASTHMA IN SCHOOL CHILDREN}

N.M. Wilson', J.C.W. Mak², J. Lamprill, H. Bilolikar, J.R. Clarke, A. Bush, M. Silverman'. 'Department of Paediatrics and '2Respiratory Medicine, Royal Brompton Hospital, Imperial School of Medicine, London, UK; ${ }^{3}$ Department of Child Health, University of Leicester, Leicester, UK

An association between neonatal bronchial responsiveness (BR) and lung function at $11 \mathrm{yr}$, related to $\beta-2 A R$ gene influence, has been reported (Turner, et al. ER 2001;18(suppl 33);2S). Polymorphisms of $\beta 2-A R$ at amino-acid (aa) 27 and aal6 have also been shown to be related to bronchial BR and childhood asthma. To assess the effect of these polymorphisms on the development of wheeze, lung function and $B R$, we genotyped from blood or buccal cells 41 children at risk of atopy, in whom maximal flows at FRC (VmaxFRC) and BR had been measured in the first month of life using the "squeeze" technique. They were followed prospectively and reviewed at age $10 \mathrm{yr}$ (SD 0.8), when lung function $\left(F E V_{1}\right)$ and $B R$ were repeated. We also genotyped 166 local school children from buccal cells

Neonatal BR correlated significantly with FEV, $(p=0.03)$ but not BR at 10 yr. VmaxFRC varied according to aa27 genotype (ANOVA $\mathrm{p}=0.023$ ); it was significantly increased in those homozygous for glutamate at aa27 ( $\mathrm{p}=0.01)$, but had no effect on history of wheeze. No effect of aa 16 was found on lung function or BR at either age, but homozygous glycine at aal6 was seen more frequently in those wheezing beyond 4 years, when compared to local school children (Fischer's exact test $\mathrm{p}=0.05$ ).

We have shown for the first time an effect of 32 -AR polymorphism at aa27 on neonatal lung function, and confirmed the association of B2-AR polymorphism at aal6 with childhood asthma, as wheeze beyond 4 years was strongly related to atopy and increased BR at $10 \mathrm{yr}$.

\section{S68 PNEUMOCOCCAL SEROTYPES IN CHILDREN WITH CULTURE NEGATIVE EMPYEMA}

K.M. Eastham', R. Freeman², A. Kearns², G. Eltringham², J.P. Leeming ${ }^{3}$, J. Clark', D.A. Spencer' . 'Freeman Hospital, Newcastle Upon Tyne, ${ }^{2}$ Newcastle Public Health Laboratory, ${ }^{3}$ Bristol Public Health Laboratory, ${ }^{4}$ Newcastle General Hospital, Newcastle Upon Tyne, UK

Background: Streptococcus pneumoniae serotype 1 accounts for up to $50 \%$ of pneumococcus culture positive childhood empyema in the USA.' This organism has been a relatively uncommon cause of invasive pneumococcal disease in the under 5 age group in England and Wales. ${ }^{2}$

Aim: To describe the pneumococcal serotype distribution and penicillin susceptibility of consecutive cases of parapneumonic effusion and empyema presenting to a Tertiary Referral Centre over a 4.5 year period.

Method: 43 pleural fluid specimens, negative for pneumococcus on routine culture, were analysed for pneumococccal DNA by real-time polymerase chain reaction (PCR). Penicillin susceptibility was determined by a complementary PCR assay. Capsular serotype specific antigen detection was by Enzyme Immuno-Assay (EIA) using monoclonal antibodies to types $1,3,4,5,6 \mathrm{~A}, 6 \mathrm{~B}, 7 \mathrm{~F}, 9 \mathrm{~V}, 14,18 \mathrm{C}$, $19 \mathrm{~A}, 19 \mathrm{~F}$, and $23 \mathrm{~F}$. 


\begin{tabular}{lll} 
Abstract S68 & & \\
\hline & $\begin{array}{l}\text { Pneumococcal DNA } \\
\text { +ve (32) }\end{array}$ & $\begin{array}{l}\text { Pneumococcal DNA } \\
\text {-ve (11) }\end{array}$ \\
\hline Serotype 1 & 17 & 0 \\
Serotype 3 & 3 & 0 \\
Serotype 14 & 4 & 1 \\
Negative & 3 & 7 \\
Insufficient & 5 & 3 \\
\hline
\end{tabular}

Results: The median age was 5.6 years (0.6-16.9 years). All pneumococcal DNA positive specimens were penicillin sensitive.

Conclusions: Pneumococcus is the major pathogen in childhood empyema, accounting for $75 \%$ of culture negative cases. Capsular antigen detection indicates that $63 \%$ of are serotype 1, paralleling culture-based studies.' These data have implications for vaccine development strategy, as the new 7-valent pneumococcal vaccine does not protect against serotype 1. Penicillin resistance does not appear to be a problem in our population.

1. Byington CL, et al. CID 2002;34:434-40.

2. George AC, Melegaro A. CDR Weekly 2001;11:21.

\section{S69 RESPIRATORY DISEASE IN CHRONIC GRANULOMATOUS DISEASE}

L. Jones', T.J. Flood'2, P. McGrogan', L. Morton ${ }^{3}$, L. Parker', D. Goldblatt', A. Thrasher ${ }^{3}$, A.J. Cant'. 'Department of Child Health, University of Newcastle; ${ }^{2}$ Department of Paediatric Immunology, Newcastle General Hospital; ${ }^{3}$ Institute of Child Health, Great Ormond Street Hospital, University College London, UK

Chronic Granulomatous Disease (CGD) is a rare primary immunodeficiency due to defective phagocytic cell oxidative burst, rendering patients susceptible to severe recurrent bacterial and fungal infections. Recurrent respiratory infection is a common presenting feature.

Objectives: To describe the common pathogens causing pneumonia in these patients in the UK and review their lung function.

Methods: The first national survey of CGD patients was started in 2000 and aimed to characterise the epidemiological and clinical features of CGD.

Results: Of 82 patients analysed to date, aged 0-60 years, 39 $(48 \%)$ have suffered from pneumonia and $7(9 \%)$ from lung abscesses. 85 episodes of probable pneumonia were recorded with $x$ ray changes documented in $65(77 \%)$ of these. Organisms were isolated in 37 cases $(44 \%)$. However Staphylococcus aureus and Burkholderia, respiratory pathogens previously reported in other series, ${ }^{1}$ only accounted for 5 cases. Aspergillus was isolated in 10 and suspected in a further 12 cases. Of the 11 documented cases of lung abscess, Staphylococcus species and Aspergillus species were isolated in 4 cases, however Streptococcus milleri was also isolated from 2 patients. 4 patients suffered from their first episode of pneumonia before the age of 1 year, a further 5 before the age of 5 years and a further 14 before the age of 15. Lung function is documented in 15 patients who had pneumonia $(39 \%)$ and in an additional 7 patients. Of the 16 patients for whom heights are available, 3 demonstrated a restrictive pattern, 3 an obstructive pattern and 10 had normal lung function.

Conclusion: Pneumonia is the most common infection encountered in patients with CGD across all age groups and is typically caused by Staphylococcus aureus, Burkholderia cepacia, and Aspergillus species. Surprisingly Burkholderia accounted for only 2 cases in this series. Monitoring lung function is essential in CGD and further prospective studies to delineate the extent of occult pulmonary morbidity are indicated.

1.Winkelstein JA, et al. Medicine 2000,79:155-69.

\section{S70 EXERCISE LIMITATION IN CF AND NON-CF BRONCHIECTASIS}

I. Narang, E. Edwards, A. Li, D. Hansell, M. Rosenthal, A. Bush. Department of Paediatric Respiratory Medicine, Royal Brompton Hospital, Sydney Street, London SW3 6NP, UK

Non-CF bronchiectasis $(\mathrm{Bx})$ is an orphan disease; little is known about exercise physiology.
Aims: (1) To compare factors limiting exercise in CF and non-CF Bx compared to normals; (2) To establish whether chest CT is a useful indicator of functional capacity in either disease.

Methods: Clinical assessment, spirometry, chest CT and incremental exercise test using cycle ergometry and respiratory mass spectrometer were performed at a time of disease stability. We measured effective pulmonary blood flow (Qeff), oxygen consumption $\left(\mathrm{VO}_{2}\right)$, effective stroke volume (SV), alveolar ventilation (VA), transfer factor (DLCO), and functional residual capacity (FRC). Heart rate (HR) and oxygen saturations $\left(\mathrm{SaO}_{2}\right)$ were measured by continuous pulse oximetry. The CT scans were scored using a modification of the Bhalla system.

Results: We compared 18 children with CF $(7$ males; median age 13 years (range 10.7-17) median $\mathrm{FEV}_{1} 76 \%$ predicted (range 40-95\%), and 17 children with non-CF Bx (7 males; median age 13 years (range 10.6-17.1) median $\mathrm{FEV}_{1} 74 \%$ predicted (range 47-90\%). Data are expressed as mean $z$ scores derived from normal values at rest and at maximum exercise (MEX) in 106 normal children:

Conclusions: In both groups Qeff was abnormally low at rest and did not increase normally during exercise in spite of an increased heart rate, due to SV limitation during exercise. A low Qeff with a high VA and a low FRC is evidence of significant mismatching during exercise. There was no correlation between CT and any exercise parameter in either group. Since the haemodynamic and functional impairment is similar in non CF BX as in CF. More attention should be paid to this neglected disease.

\begin{tabular}{|c|c|c|c|c|}
\hline \multicolumn{5}{|c|}{ Abstract S70 } \\
\hline Parameter & CF rest & CF MEX & Bx rest & Bx MEX \\
\hline Qeff & -0.11 & $-1.08^{*}$ & -0.1 & $-0.95^{*}$ \\
\hline SV & $-0.6^{*}$ & $-1.0^{*}$ & -0.71 * & -0.94 \\
\hline $\mathrm{HR}$ & $1.1^{*}$ & $0.85^{*}$ & $0.9 *$ & $1.29 *$ \\
\hline VA & 0.15 & $1.33^{*}$ & 0.08 & $1.0^{*}$ \\
\hline DLCO & -0.11 & $-0.82^{*}$ & 0.64 & -0.32 \\
\hline FRC & 0.4 & $-1.11^{*}$ & $1.12^{*}$ & -0.6 \\
\hline
\end{tabular}

*Statistically significant $(p<0.05)$ compared to normal.

\section{S71 RESPIRATORY SYMPTOMS IN CHILDREN WITH DOWN'S SYNDROME}

N. John', K. Williams2', I.J.M. Doull'. 'Cystic Fibrosis/Respiratory Unit, Department of Child Health, University Hospital of Wales, Cardiff, UK; ${ }^{2}$ University of Leeds, UK

Background: Children with Down's syndrome (DS) are at increased risk of hospitalisation due to respiratory disorders. Although anecdotally they report frequent respiratory symptoms, objective evidence is lacking.

Aims: To compare parental reported respiratory symptoms of children with DS with normal controls.

Methods: Questionnaire survey of all children with DS under 12 years of age in the Bro Tâf Health Authority. Families completed questionnaires for any unaffected sibling(s) as controls. The questionnaire was based on the ISAAC core questions, with additional sections on upper airway and cardiac disease.

Results: Questionnaires were received on 65 children with DS 138 male) and 35 controls (24 male). There was no significant difference in mean age between the 2 groups: DS mean (SD) 6.5 (2.8) years (range 1.4-11.1); controls 5.6 (2.9) years (range 0.3-10.8). Children with DS had significantly greater ever wheeze (OR 10.1; $95 \% \mathrm{Cl} 3.8-26.6$ ); wheeze in the preceding 12 months (OR 10.2; $95 \% \mathrm{Cl} 3.5-29.9$ ); wheeze during exercise (OR $4.2 ; 95 \% \mathrm{Cl}$ 1.1-15.6); use of anti asthma medication (OR 3.5; 95\% Cl 1.3-9.6), but were no more likely to be labelled asthmatic. Children with DS had significantly greater chest congestion or phlegm with (OR4.1;95\% Cl 1.7-10.0) or without (OR 18.3; 95\% Cl 2.3-143) a cold, were receiving nasal medications (OR $8.3 ; 95 \% \mathrm{Cl} 2.6-26)$ but were no more likely to be labelled rhinitic. Twenty-one DS and 1 control reported a cardiac condition. After excluding these cases, most relationships with wheeze, chest congestion, and coughing up phlegm with or without a cold persisted.

Conclusions: Children with DS have significantly greater respiratory symptoms than normal-wheeze, chest congestion, and coughing up phlegm with or without a cold. The aetiology of symptoms is unclear, but do not appear related to cardiac conditions. 
S72 Table of results

$\mathrm{HC}(\mathrm{n}=20) \quad \mathrm{CF}(\mathrm{n}=21) \quad 95 \% \mathrm{Cl}: \mathrm{CF}-\mathrm{HC}$

$\mathrm{FRC}_{\text {mbw }}$ mean (SD) $\quad 19.4(3.5) \quad 20.3(4.4) \quad-1.6 ; 3.5$

$\begin{array}{llll}\text { FRC mean }(\mathrm{SD}) & 19.2(3.1) & 25.4(7.4) & 2.6 \% 9.8\end{array}$

$\begin{array}{lll}95 \% \mathrm{Cl}_{\mathrm{F}} \mathrm{FRC}_{\mathrm{p}} \mathrm{FRC}_{\mathrm{mbw}} & -0.8 ; 0.4 & 2.3 ; 7.9\end{array}$

All values expressed as $\mathrm{mL} / \mathrm{kg}$ body weight

\section{S72 ESTIMATES OF PLETHYSMOGRAPHIC FRC EXCEED THOSE BY GAS DILUTION IN INFANTS WITH CYSTIC FIBROSIS (CF) BUT NOT IN HEALTHY CONTROLS}

H. Ljungberg ${ }^{2}$, G Hulskamp², A.-F. Hoo ${ }^{2}$, J. Pillow², S. Lum², P. Gustafsson², J. Stocks'. 'Portex Respiratory Unit, Institute of Child Health, London, UK' ${ }^{2}$ Queen Silvia Children's Hospital, Göteborg, Sweden

Involvement of the peripheral airways is an early feature of CF lung disease and may result in pathological gas trapping. This is reflected by the difference between plethysmographic and gas dilution estimates of functional residual capacity (FRC) in older children and adults.

Aim: To compare paired measures of FRC using plethysmography ( $F R C_{p}$ ) and multiple breath inert gas washout ( $F R C_{\text {mbw }}$ ) in infants with recently diagnosed CF and healthy control infants.

Methods: 21 infants with CF, median age (range) 36 (10-83)w, and 20 healthy control $(\mathrm{HC})$ infants 42 (5-91)w were studied during quiet sleep. $\mathrm{FRC}_{\text {mbw }}$ was measured using a respiratory mass spectro-meter with $\mathrm{SF}_{6}$ as the tracer gas, and determined from the cumulative volume of expired gas divided by the difference between end-tidal gas concentration at start and at the end of the washout. FRC was measured immediately afterwards according to ERS/ATS guidelines (ERI $2001 ; 17: 302-12$ ) using commercially available equipment (Jaeger MasterScreen BabyBodyplethysmograph).

Results: The mean within-subject coefficient of variation for FRC and $\mathrm{FRC}$ was $3.0 \%$ and $2.9 \%$ respectively.

Summary: In healthy infants, there was no difference in FRC as measured by plethysmography and inert gas washout, and there was no difference in $\mathrm{FRC}_{\text {mbw }}$ when comparing $\mathrm{HC}$ and $\mathrm{CF}$. In infants with $C F$, however, the $\mathrm{FRC}_{\mathrm{p}}$ was significantly elevated not only compared to $\mathrm{HC}(p=0.001)$, but also compared to $\mathrm{FRC}_{\mathrm{mbw}}$ in the $\mathrm{CF}$ group itself $(p=0.01)$.

These findings may be explained by pathological gas trapping. Paired measurements of FRC using these two techniques may provide a sensitive and useful method for early detection of pulmonary changes in CF.

Henrik Ljungberg is supported by an ERS Long Term Research Fellowship.

\section{Mechanisms of cough}

\section{S73 RELATIONSHIP BETWEEN CAPSAICIN COUGH SENSITIVITY AND QUALITY OF LIFE IN PATIENTS WITH CHRONIC COUGH}

S.S. Birring, B. Prudon, I.D. Pavord. Institute for Lung Health, Respiratory Medicine, Glenfield Hospital, Leicester, UK

Most patients with a chronic cough have a heightened cough reflex. Little is known about how this relates to patients perception of cough severity or the degree to which the cough impacts on quality of life. We have assessed the relationship between capsaicin cough sensitivity, cough visual analogue score and quality of life in 26 patients with chronic cough presenting to our outpatient clinic. All patients had (1) capsaicin cough reflex sensitivity measured using a dosimeter with the results expressed as the concentration of capsaicin (imol/L) causing 2 (C2) and 5 (C5) coughs, (2) cough visual analogue score (VAS; $0-100 \mathrm{~mm}$ ), (3) cough specific quality of life measurement using the Leicester Cough Questionnaire (LCQ). The LCQ is a 19-item self-administered health related quality of life questionnaire for patients with chronic cough that has 3 domains (physical, psychological and social; domain score range 1-7, total score 3-21; higher score indicated better quality of life) and a 7-point Likert response scale. We have previously shown that the $L C Q$ is a fully validated, reliable, repeatable and responsive instrument that is brief and easy to administer. The patients were $62 \%$ female, mean (SEM) age 57 (3) years and had a mean (SEM) cough duration of 69 (23) months. The mean (SEM) cough VAS score was 53 (6) mm; logC2: 0.57 (0.08); logC5 1.41 (0.18) $\mathrm{mmol} / \mathrm{L}$; physical domain score: 4.7 (0.3); psychological domain score: 4.8 (0.3); social domain score: 4.8 (0.3); LCQ total score: $14.4(0.8)$. There was no correlation between logC2 and $\log C 5$ and the LCQ total score ( $r=0.19$ and 0.23 respectively), the individual LCQ domain scores or the cough VAS score $(r=-0.32$ and B0.34 respectively). In conclusion, we found no relationship between quality of life or symptom scores and capsaicin cough reflex sensitivity in patients with chronic cough. Our results suggest factors other than a heightened cough reflex are important in how a patient perceives the severity of their cough, or how it impacts on their quality of life. These measures assess different aspects of cough and therefore may provide complimentary information.

Supported by the British Lung Foundation and University Hospitals of Leicester NHS Trust.

\section{S74 REPEATABILTY OF CAPSAICIN COUGH REFLEX SENSITIVITY MEASUREMENT}

B. Prudon, S.S. Birring, D.D. Vara, I.D. Pavord. Institute for Lung Health, Department of Respiratory Medicine, Glenfield Hospital, Leicester, UK

Although widely used in clinical practice and research, little is known about the validity and repeatability of capsaicin cough sensitivity in healthy adults and patients with chronic cough. We measured capsaicin cough reflex sensitivity in 134 healthy subjects and 85 patients with an isolated chronic cough using a KoKo Digidoser with an inspiratory flow regulator valve $(0.5 \mathrm{~L} / \mathrm{s})$. Two-week repeatability was assessed in 15 healthy subjects and 15 patients with chronic cough. Healthy subjects (mean age 42 years (range 20-78), female 62\%) had a wide variation of capsaicin cough sensitivity (mean log capsaicin concentration that causes 2 coughs C2 (SD) $1.2(0.8)$ and 5 coughs (C5) 2.7 (1.8) $\mathrm{mmol} / \mathrm{L})$. There was no correlation between age and $\log C 2(r=-0.1)$ or $\log C 5(r=-0.1)$. Females had significantly raised capsaicin cough sensitivity compared to males $(\log C 2: 1.0 \mathrm{v}$ 1.4 ; mean difference $0.4 ; 95 \%$ confidence interval 0.1 to 0.8 ; $\mathrm{p}=0.01$ and $\log \mathrm{C} 5: 2.3 \vee 3.3$; mean difference $1.0 ; 95 \% \mathrm{Cl} 0.2$ to $1.8 \mathrm{~mol} / \mathrm{L} ; \mathrm{p}=0.011$. Patients with chronic cough were significantly more sensitive than healthy subjects (mean age 59 (range 27-83), female $64 \%$; $\log C 2$ : 0.5 v 1.2 ; mean difference $0.7 ; 95 \% \mathrm{Cl} 0.5$ to $0.9 ; \mathrm{p}<0.001$ and $\log \mathrm{C} 5: 1.3 \vee 2.7 ;$ mean difference $1.4 ; 95 \% \mathrm{Cl}$ 0.9 to $1.8 ; p<0.001)$. The capsaicin cough reflex sensitivity measurement was repeatable over 2 weeks in both healthy subjects (mean of difference (within subject SD): $\log C 2-0.2(0.2), \log C 5-0.4(0.5)$ $\mathrm{\mu mol} / \mathrm{L}$; intraclass correlation coefficients 0.9 and 0.9 respectively), and patients with chronic cough /mean of difference (within subject $\mathrm{SD})$ : $\log C 2 \mathrm{BO} .1(0.2), \log \mathrm{C} 5 \mathrm{BO} .3(0.5)$; intraclass correlation coefficients 0.6 and 0.7 respectively). We have shown a wide variation of capsaicin cough reflex sensitivity in health which potentially limits the use of this method as a discriminatory test. We have also demonstrated an effect of gender but not age on capsaicin cough reflex sensitivity measurement highlighting the importance of matching subjects when making comparisons using this test. Capsaicin cough reflex sensitivity measurement is a repeatable test over 2 weeks (C2 more than (5) in both healthy subjects and patients with chronic dry cough. These data would be useful for powering clinical studies of antitussive drugs.

Supported by BLF and PPP Healthcare Trust.

\section{S75 UPPER AIRWAY SENSITIVITY IN SUBJECTS WITH A CHRONIC NON-PRODUCTIVE COUGH}

B. Prudon', S.S. Birring', A.P. Hall', J.P. Thompson², I.D. Pavord'. 'Institute for Lung Health, Respiratory Medicine, Glenfield Hospital, Leicester, UK; ${ }^{2}$ Department of Anaesthesia, Leicester Royal Infirmary, Leicester, UK

Subjects suffering from a chronic non-productive cough usually have a heightened cough reflex compared to healthy subjects. Whether this phenomenon is confined to the cough reflex, or whether other upper airway reflexes are also abnormal is unknown. We measured the sensitivity of the "Glottic-stop reflex" (a reflex closure of the vocal cords in response to inhaled irritants), and the capsaicin cough reflex sensitivity in 16 healthy subjects and 14 subjects with a chronic cough /cough subjects were predominantly female $(81 \%)$ and the causes of cough were idiopathic chronic cough (11), cough variant asthma (1), rhinitis (1) and rhinitis and gastro-oesophageal reflux (1). Glottic stop sensitivity was measured using a previously validated non-invasive technique in which subjects inhaled single breaths of increasing concentrations of ammonia and adduction of the vocal cords was detected using a 
pneumotachograph. Cough sensitivity was measured at the same time of day on a different occasion greater than 1 week apart with a single-breath dosimeter method, using capsaicin as the tussive agent and results expressed as the log of concentration ( $\mu \mathrm{mol} / \mathrm{L})$ required to cause 2 coughs (C2). Cough subjects had a significantly more sensitive glottic stop reflex and capsaicin cough reflex sensitivity compared to age and sex matched healthy subjects (mean glottic reflex sensitivity threshold: 483 v 1029 ppm; mean difference 546ppm, 195\% Confidence Interval 137 to 954$], p=0.01 ; \log 22: 0.3 \vee 1.1 ;$ mean difference $0.7,(95 \% \mathrm{Cl} 0.4$ to 1.1), $p<0.001)$. Glottic stop reflex sensitivity correlated significantly with cough reflex sensitivity $(r=0.5$, $p=0.006)$. These results suggest that the cough reflex and the glottic stop reflex share a common pathway, or that subjects who have a chronic cough have a global abnormality of upper airway reflexes. Further investigation of possible common mechanisms sensitising these two reflexes may lead to a greater understanding of chronic cough, and lead to new targets for antitussive medications.

1. Langton JA, et al. Br J Anaesth 1993;70:126-30.

\section{S76 ASSOCIATION BETWEEN COUGH AND REFLUX EVENTS IN PATIENTS WITH CHRONIC COUGH WITH AND WITHOUT GASTRO-OESOPHAGEAL REFLUX}

J.A. Kasterlik, I.P.A. McGarvey, S.A. Mulrennan, B. Johnston, J.C. Ojoo', A.E. Redington', A.H. Morice'. 'Academic Department of Medicine, University of Hull, Castle Hill Hospital, Cottingham, UK; ${ }^{2}$ Department of Medicine, Queen's University Belfast, UK

Background: Gastro-oesophageal reflux (GOR) is a common cause of chronic cough, but the mechanisms of GOR related cough are not fully understood.

Aim: To determine the association between cough and reflux events in patients with chronic cough due to GOR and due to causes other than GOR.

Methods: 60 patients with undiagnosed chronic cough aged mean (IQR) 55 (49-62) yr with cough duration $8(1.5-10)$ yr underwent 24-h $\mathrm{pH}$ monitoring during which cough and reflux events were recorded. GOR related cough was diagnosed when $\mathrm{pH}<4$ was recorded for $>4 \%$ of total time and when cough improved with subsequent antireflux therapy. A reflux event was defined as a drop of $\mathrm{pH}<4$ for $>12 \mathrm{sec}$. Cough was considered associated with reflux when it occurred within 5 min from reflux.

Results: 29 patients had GOR related cough. The other 31 patients had normal 24-pH monitoring, and cough was either due to asthma or rhinosinusitis or was idiopathic. The total number of coughs did not differ significantly $(\mathrm{p}=0.85)$ between patients with GOR $(9(5-17) / 24$ $\mathrm{hr}$ ) and without GOR (8 (6-16)/24hr). Patients with GOR related cough had $20(10-31) \%$ of coughs associated with reflux, compared with $0(0-7) \%$ in patients without GOR $(p<0.001)$. In patients with GOR related cough, $7(1-10) \%$ of total reflux events occurred within 5 minutes before cough, compared with $1(0-2) \%$ in patients without GOR ( $p<0.001)$. In patients with GOR related cough $6(4-6) \%$ of reflux events occurred within 5 min after cough, compared with 3 $(0-2) \%$ in patients without GOR $(p=0.002)$.

Conclusions: In GOR related chronic cough, reflux and cough events are frequently associated. In patients with other causes of chronic cough, in contrast, this association is infrequent.

\section{S77 SMOKING, SALBUTAMOL, AND THE COUGH REFLEX}

S.A. Mulrennan', J.C. Ojoo', J.A. Kastelik', C. Wright', R.H. Thompson', A.E. Redington', P. Goustas², A.H. Morice'. 'Academic Department of Medicine, University of Hull, Castle Hill Hospital, Cottingham, UK; ${ }^{2}$ GlaxoSmithKline, Stockley Park West, Uxbridge, Middlesex, UK

Background: Smokers have an increased prevalence of chronic cough. Patients frequently complain of exacerbation of cough when attempting smoking cessation.

Hypothesis: Do cigareftes affect cough in smokers and does salbutamol have antitussive properties in smoking related cough?

Methods: A randomised double blinded crossover study to determine the efficacy of $400 \mu \mathrm{g}$ salbutamol via MDI plus spacer versus placebo on cough before and after the first cigarette of the day in healthy adult smokers. Medication was given at time 0 minutes. Number of coughs were recorded from $0-10$ and $10-20$ min using a voice activated analogue tape recorder. Coughs following a cigarette at 20 minutes were observed at $20-40$ and $40-60 \mathrm{~min}$. Citric acid cough challenge was performed at 60 minutes after medication.

Results: 44 healthy smoking subjects ( 13 females), mean age 34 (range 20-61) were recruited. Cigarettes significantly reduced cough in those on placebo. Mean cough frequency per minute 20 minutes pre cigarette was 0.3 compared to 0.2 post cigarette $(p=0.02)$. Cough frequency did not reduce significantly pre and post cigarette in those taking salbutamol. Mean 0.23 versus 0.19 respectively $(p=0.116)$. The citric acid concentration causing two coughs $(C 2)$ at 60 minutes increased on salbutamol. Geometric mean 278.8 compared to $190.4 \mathrm{mM}$ on placebo $(\mathrm{p}=0.03)$.

Conclusions: Cough is reduced by the morning cigarette and this corroborates smokers observations of increased cough on smoking cessation and the "beneficial" effect of smoking on that symptom. The absence of a significant difference in cough before and after smoking a cigarette and the increase in C2 suggests that $\beta$ agonists may be useful in cough associated with cigarette abstinence. The effect of salbutamol is however small and other more potent and longer acting agents may prove more effective.

Funded by GlaxoSmithKline.

\section{S78 CHOCOLATE, CHILLI PEPPER, AND COUGH}

O.S. Usmani, H.J. Patel, M.G. Belvisi, P.J. Barnes. Department of Thoracic Medicine, National Heart and Lung Institute, Imperial College School of Medicine, London, UK

Cough is the most common respiratory symptom for which medical attention is sought. It may become debilitating and socially distressing, and a recent meta-analysis showed that most OTC remedies were ineffective against cough. Opiods are often used, but have unpredictable efficacy and side-effects. There is great need for effective therapies. Capsaicin, derived from chilli peppers, is a well-established, reproducible, tussive stimulant widely used in clinical research to test the efficacy of new cough treatments. We describe theobromine, a methylxanthine derivative present in cocoa and chocolate, as an effective anti-tussive agent able to elevate the capsaicin induced cough threshold in healthy volunteers.

Ten healthy non-smoking subjects participated in a randomised double blind cross-over study. The anti-tussive effect of a single-dose of theobromine $(1000 \mathrm{mg})$ was compared to codeine phosphate $(60 \mathrm{mg})$ and placebo. The main outcome measure was the capsaicin concentration required to induce five coughs (C5). Subjects had their screening C5 value established, and at each treatment visit their C5 threshold was determined 2 hours post-dosing. The mean $\mathrm{C} 5$ values between treatments were compared using two-way ANOVA.

Theobromine was markedly able to increase the cough threshold when compared to codeine phosphate $(p=0.07)$ and placebo $(p<0.01)$. There was no significant difference between codeine and placebo $(p=0.25)$. The $C 5$ variability was similar between the placebo and screening values, supporting the reproducibility of the capsaicin challenge in this subject group. No adverse effects, particularly CVS or CNS, were observed.

Although the anti-tussive mechanisms remain unknown, preliminary data have shown direct inhibition of sensory nerve depolarisation in isolated guinea-pig vagus. Theobromine has been shown to possess anti-angiogenic properties through its action on adenosine (A2) receptors, and it also has weak phosphodiesterase inhibiting activity. It may act on the capsaicin vanilloid receptor (VR1). Elucidation of the molecular targets using in vitro and animal models, will help development of novel therapeutic entities.

In conclusion, the data suggest a significant anti-tussive effect of theobromine in healthy subjects when compared to placebo. Further studies are needed in select patient categories to see whether these phase I effects are borne out.

\section{Issues in non-invasive ventilation}

\section{S79 A SURVEY OF HOME MECHANICAL VENTILATION IN THE UK}

S.J. Lloyd-Owen', M.W. Elliott' ${ }^{2}$ A.K. Simonds' ${ }^{3}$, J.A. Wedzicha'. European Union Concerted Action on Home Respiratory Ventilation, 'St Bartholomew's Hospital, London; ${ }^{2}$ St James's University Hospital, Leeds; ${ }^{3}$ Royal Brompton Hospital, London

As part of a European-wide Project, a survey of custom and practice in Home Mechanical Ventilation (HMV) was carried out in the UK. 80 known and possible HMV centres were targeted with initial requests, of which 65 responded. 13 of these did not have any HMV users. Full surveys were received from 47 centres (at least $70 \%$ response rate). 7 of these were paediatric centres. The answers reflected the situation on the 1st July 2001. A total of $2842 \mathrm{HMV}$ users were identified in the 
UK by the 47 centres. The median size of the units was 18 users (IQR 6.5 to 46.5 ), with 7 centres having more than 100 users. $96 \%$ of the users had their HMV initiated at that centre with 13\% having some form of shared care between units. 27 centres described themselves as University hospitals (57\%) with 18 non-University hospitals (38\%) and 1 Outpatient facility (no data for 1 centre). 26 of the centres took referrals, either regional only (18) or regional and national (8). The median year of each centre starting HMV was 1992 (IQR 1987 to 1996). The users' characteristics were: $55 \%$ male, $45 \%$ female; ages: 16 years or less: $7 \%, 17$ to 25 years: $7 \%, 26$ to 65 years: $59 \%, 66$ years or more: $27 \% .21 \%$ had been on HMV for less than 1 year, $52 \%$ for 1 to 5 years, $23 \%$ for 6 to 10 years and $4 \%$ for more than 10 years. The conditions requiring ventilation were categorised into 3 groups with lung and airways diseases (LA) in $26 \%$, thoracic cage (TC) problems in 35\% and 39\% neuromuscular (NM). Pressure preset positive pressure ventilators (PP) were used most often ( $92 \%$ overall) with relatively more volume preset ones (VP) in the TC and NM groups (LA: $95 \%$ PP, 5\% VP; TC: $91 \%$ PP, 9\% VP; NM: $89 \%$ PP, $11 \%$ VP). Nasal (N) and facial (F) masks were predominant in all groups with tracheostomy (T) used more frequently in the NM group (LA: $77 \% \mathrm{~N}$, $22 \% \mathrm{~F}, 1 \% \mathrm{~T}$; TC: $86 \% \mathrm{~N}, 13 \% \mathrm{~F}, 1 \% \mathrm{~T}$; NM: $65 \% \mathrm{~N}, 18 \% \mathrm{~F}, 17 \%$ T). 24 hour ventilation was also commoner in the NM group (12\% compared to $1 \%$ for LA and TC). Concomitant oxygen use was more frequent in the LA group (LA: 61\%; TC: 16\%; NM: 8\%). Indications for ventilation and tracheostomy use and investigations were consistent across centres, but there was variability in the provision of follow up, training and homecare.

Funded by the European Commission.

\section{S80 RESPIRATORY FAILURE: EARLY NON-INVASIVE VENTILATION (NIV) USE IN A DISTRICT GENERAL}

R. Rabanni, W. Heberlein, J. Wilson, R. Hayton', M. Al-Jibry, H. Moudgil. Departments of Respiratory Medicine and 'Clinical Audit, Princess Royal Hospital NHS Trust, Telford, UK

Severe respiratory acidosis $\left(\left[\mathrm{H}^{+}\right]>55 \mathrm{mmol}, \mathrm{pH}<7.26\right)$ predicts mortality in exacerbation of chronic obstructive pulmonary disease (COPD). Non-invasive ventilation (NIV) is an effective alternative to intubation and mechanical ventilation and is justified in milder respiratory acidosis $(\mathrm{pH} 7.35$ to 7.26$)$ associated with exacerbation of COPD. To assess our use in the first year since introduction (to December 2001) we have retrospectively audited our management of patients with hospital discharge codes for COPD or respiratory failure. Where results from arterial blood gas (ABG) samples were available $(\mathrm{n}=239,46 \%), 60$ had type 2 respiratory failure complicated by acidosis. Of the others, 74 had type 1 respiratory failure, 48 had compensated type 2 respiratory failure, 10 had metabolic acidosis, 2 had hyperventilation, and 45 had no respiratory failure. Of those with respiratory acidosis $(n=60), 15$ were excluded from NIV; reasons included post-arrest, respiratory depression with drugs, pneumothorax, brainstem stroke, and immediate self-correction of acidosis. Of the remaining 45, 24 had a review by the anesthetists (ITU) who further excluded four as inappropriate, offered NIV to 17 patients and intubation with mechanical ventilation to three. Of those initiated on NIV, 11 satisfied criteria for mild acidosis with six others more acidotic (range 7.0 to 7.24 ). Of the 17 offered NIV, 10 had successful correction of acidosis with mean duration of assisted therapy over 3.3 days (range 1 to 9 days); one subsequently required re-ventilation and one died. Of the seven who failed a trial of NIV, length of time on NIV was at a mean of 0.7 days (range 0 to 2 days); three had been intubated within two hours and three died. Of the original group with respiratory acidosis $(n=45)$ but not given additional ventilatory support ( $n=25$ ), no ITU input was requested for 21 and only one set of $A B G$ s had been requested on 15 (with 14/15 patients within the mild category for acidosis); five in this group had contraindications to NIV, and in total five died. We find that (1) not all patients are being assessed by formal $A B G$ s, (2) not all patients satisfying the criteria for NIV were being offered that provision, and (3) there has been inappropriate use of NIV in some patients with more severe acidosis who possibly should have been intubated as a first measure.

\section{S81 TITRATION OF NON-INVASIVE VENTILATION IN COPD}

\section{J.M. Tuggey, M.W. Elliott. St James's University Hospital, Leeds, UK}

Introduction: The settings used in establishing a patient on NIV are often somewhat arbitrary, often defined by patient comfort and improvement in arterial blood gas settings. There is contention that higher levels of IPAP simply result in greater leak with no improvement in minute ventilation. The aim of this study was to identify the relationship between set pressure, delivered minute ventilation (MVe) and mask interface leak.

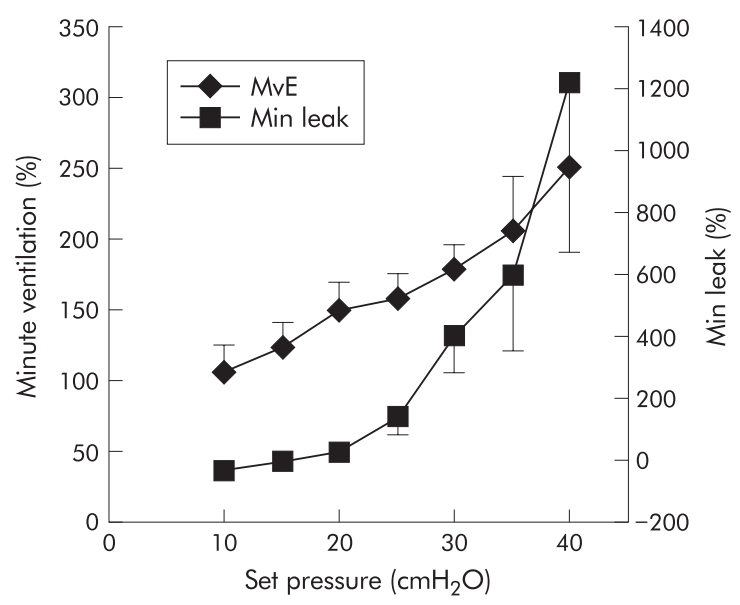

Abstract S8 1

Method: We measured the effects of varying IPAP levels in 9 patients (mean age 60, mean FVC 1.7 I) with COPD already using NIV at home. Using a pneumotachometer connected adjacent to the nasal mask, measurements of delivered tidal volume (Vti), expired tidal volume (Vte) and respiratory rate (fR) were made once Vte was stable for each pressure setting. Minute leak (Min leak) was measured as the difference between inspiratory and expiratory tidal volume multiplied fR. Recordings were repeated at least three times for each combination of settings.

Results: $M V e$ is expressed as percentage of spontaneous ventilation. Leak varies considerably from patient to patient depending on mask fit. To allow for this, leak is expressed as change from that measured at $15 \mathrm{cmH}_{2} \mathrm{O}$ in each subject. The data from 9 subjects is presented in the figure.

Conclusion: Whilst minute ventilation increases linearly with set pressure, minute leak increases exponentially, particularly above $20 \mathrm{cmH}_{2} \mathrm{O}$. Some patients will benefit from increased minute ventilation at higher pressures, but may suffer intolerable leak.

JT is funded by NHS Northern \& Yorkshire Executive.

\section{S82 FACTORS WHICH MIGHT INFLUENCE MEDIUM TERM PROGNOSIS IN SUBJECTS WITH ACUTE VENTILATORY FAILURE TREATED WITH NON-INVASIVE VENTILATION}

S. Nadeem, P. George, M. Healy, M.J. Doherty. Dudley Group Of Hospitals, Dudley, West Midlands, UK

Subjects treated with NIV for acute ventilatory failure have been shown to have a reasonable medium term prognosis. We examined if any easily attainable data help to suggest a good medium term prognosis. Survival data were obtained on a group of 47 consecutive patients treated with NIV (Mean age 72 (7) years, 24 males, 38 main diagnosis COPD, mean pre NIV arterial blood gases $\mathrm{pH} 7.23$ (0.09), $\mathrm{PaO}_{2} 8.2(2.4), \mathrm{PaCO}_{2} 11.8(2.5)$, bic 35 (8). This was collected 2 to 3 years after the initial illness by review of the case notes and contact with the relevant GP. Data on severity of ventilatory failure, stable \% predicted FEV , reported exercise tolerance, diagnosis, etc were compared between those still alive and those dead at two time points, 2-3 years post treatment and one year post treatment, using Wilcox an and $\chi^{2}$. Survival at one year was $60 \%$. There was no difference between survivors and non-survivors in terms of age 71 (8) v 73 (7) $p=0.3$, sex (53 v 54\% males), mean \% predicted FEV1 45 (21) v 35 (16) $\mathrm{p}=0.08$ or severity of initial ventilatory failure $\mathrm{pH} 7.24(0.1) \mathrm{v}$ $7.23(0.08) p=0.22$ or by diagnosis (33 of those still alive $v 66 \%$ of dead subjects did not have COPD as the cause of their ventilatory failure, $p=0.08$ ). The only factor which significantly varied between the two groups was reported exercise tolerance 150 (230) v 54 (79) metres, $p=0.016$. At the end of a mean follow up of 26 months $45 \%$ of subjects were still alive. There was no difference between those who survived and those that did not in terms of sex or initial blood gases $\mathrm{pH}(\mathrm{p}=0.22), \mathrm{PaCO}_{2}(\mathrm{p}=0.14)$, bic $(\mathrm{p}=0.5)$. However the difference almost reached significance in terms of age 70(6.7) $\vee 73.5$ (7) $p=$ 0.057 and \% predicted FEV 145 (17) v 39 (7) $p=0.058$. There were significant differences in terms of reported exercise tolerance 180 (255) $\vee 57$ (84) metres, $p=0.022$ and diagnosis, all patients who did not have COPD being dead at the end of follow up compared to $45 \%$ of patients with COPD being dead. In summary in the medium term survival in this group was related to the subjects usual chronic state 
rather than the severity of ventilatory failure at presentation. Reported exercise tolerance was the only factor which varied between survivors and non survivors at both time points.

\section{S83 PREDICTORS OF BENEFIT FROM, AND COMPLIANCE WITH, NON-INVASIVE VENTILATION IN MOTOR NEURONE DISEASE}

\section{S.C. Bourke, G.J. Gibson. University Of Newcastle, UK}

Background: In motor neurone disease (MND), there is considerable variation in the reported tolerance of, and benefit from, non-invasive ventilation (NIV).

Methods: NIV was initiated in 11 subjects with MND and orthopnoea due to respiratory muscle weakness. Maximum inspiratory pressure $\left(\mathrm{P}_{1} \mathrm{max}\right), \mathrm{PaCO}_{2}$, bulbar score (amyotrophic lateral sclerosis functional rating scale), limb and axial muscle score and quality of life (QoL; SF-36) were assessed at baseline. Subsequently, the SF-36 was completed every two months until death in all subjects. Relations between 1) survival, and 2) the duration the SF-36 mental component summary (SF-36 MCS) was maintained above baseline, and each of: NIV compliance, age, gender, $\mathrm{P}$ max, $\mathrm{PacO}_{2}$, bulbar score, and limb and axial muscle score were evaluated by univariate and multivariate analysis. Relations between compliance and subject characteristics at initiation of NIV were also assessed. Variables were included in the multivariate analysis only if they showed a relation with the dependent variable on univariate analysis $(p<0.1)$.

Results: Duration of survival correlated with NIV compliance $(r=0.70 p=0.016)$ only. In univariate analysis, duration of QoL benefit (SF-36 MCS) correlated with NIV compliance $(r=0.86, p<0.001)$ and age $(r=-0.61, p=0.048)$, however in multivariate analysis, NIV compliance was the only independent predictor of QoL benefit. In univariate analysis, NIV compliance correlated with age $(r=-0.62 p=$ $0.042)$ and upper limb muscle score $(r=0.67 p<0.05)$, and showed a trend towards correlation with ALSFRS bulbar score $(r=0.58 p=0.06)$ and $P_{\max }(r=-0.56 p=0.07)$. In multivariate analysis the only independent predictors of compliance were age and upper limb muscle score (adjusted $r^{2}=0.76$ ).

Conclusions: In MND subjects with symptomatic respiratory compromise, NIV compliance was the sole independent predictor of survival and duration of QoL benefit. Younger patients with relatively preserved upper limb function (more likely to be able to fit and remove their mask independently), were more likely to comply with, and benefit from, NIV.

\section{S84 AN AUDIT OF NON-INVASIVE VENTILATION DELIVERED BY A CRITICAL CARE OUTREACH TEAM IN A DISTRICT GENERAL HOSPITAL}

C.D. Shee, M. Green, H. Hackwood, A. Cook. Queen Mary's Sidcup NHS Trust, Sidcup, Kent DA14 6LT, UK

In March the British Thoracic Society published guidelines on non-invasive ventilation (NIV) in acute respiratory failure (Thorax 2002;57:192-211) and recommended regular audit. We have audited NIV used in medical patients in our hospital in the three months prior to publication of the guidelines. We have excluded patients where NIV was started on the intensive care unit (ICU) or on surgical wards. The NIV service is available 24 hours per day and is supervised by a critical care outreach team led by a nurse consultant who is supported by five clinical nurse specialists, ICU senior house officers and the senior respiratory physiotherapist. Patients at risk are notified to the team by the accident and emergency department (A\&E), ward nurses, doctors or physiotherapists.

In the three months, 35 patients (18 male) received NIV. The mean age was 72 years (range 18-86) with $13(37 \%)$ over 80 years. Geriatricians looked after 17 patients $(49 \%)$, chest physicians 7 , and other physicians 11 . Only 14 patients $(40 \%)$ were treated for an exacerbation of COPD (mean arterial pH 7.25; mean $\mathrm{PaCO}_{2} 11.2 \mathrm{kPa}$ ). The other groups were: pulmonary oedema, $n=10\left(\mathrm{pH} 7.22, \mathrm{PaCO}_{2} 9.0\right)$; neuromuscular, $\mathrm{n}=4$ (pH 7.34, $\mathrm{PacO}_{2}$ 6.2); bronchopneumonia, $\mathrm{n}=7$ $\left(\mathrm{pH} 7.26, \mathrm{PaCO}_{2} 6.3\right)$. NIV was started on admission in 17 patients, and in 18 on a mean of nine days post-admission. NIV was started in $A \& E$ in five patients and on general wards in 30 patients. Most patients were documented as not for intubation/ventilation $(n=26$; $74 \%$ ), of whom $12(46 \%)$ survived. Seven of nine patients $(78 \%)$ for full active resuscitation survived. The overall survival figures for COPD were 8/14 (57\%); for pulmonary oedema 7/10 (70\%); for neuromuscular problems $3 / 4(75 \%)$, but for bronchopneumonia (including three patients with lymphoma) only $1 / 7$ (14\%).
Although NIV was probably life-saving in some patients, in many it could be considered a "palliative" intervention to help symptoms. Provision of a comprehensive hospital-wide NIV service is feasible when run by a critical care outreach team. However, patients referred to such a team are likely to be older and frailer and with a wider range of diagnoses, and less likely to be for intubation/resuscitation than the usual patients treated by a respiratory-led NIV team.

\section{Neutrophil and epithelial cell biology}

\section{S85 EFFECT OF NEUTROPHIL PRIMING AND ACTIVATION ON ${ }^{18} \mathrm{~F}$-DEOXYGLUCOSE ('8 FDG) UPTAKE IN VITRO}

J.F. White, J.R. Ballinger, K.C. Cadwallader, H.A. Jones, A.M. Peters, E.R. Chilvers. Departments of Medicine and Nuclear Medicine, University of Cambridge School of Clinical Medicine, Addenbrooke's and Papworth Hospitals, Cambridge CB2 2QQ, UK

Priming describes up-regulation of the neutrophils response to a secretogogue agonist following prior exposure to a priming agent. ${ }^{18} \mathrm{FDG}$ is used in PET to identify areas of infection and inflammation. This study addresses the mechanisms underlying ${ }^{18} \mathrm{FDG}$ accumulation in vitro in human neutrophils and monocytes. Time courses of ${ }^{18} \mathrm{FDG}$ accumulation for both reversible priming agent platelet activation factor (PAF) and priming/activating agents GM-CSF and FMLP were undertaken. ${ }^{18} \mathrm{FDG}$ uptake by neutrophils and monocytes was compared.

Methods: Isolated human neutrophils and monocytes were resuspended in PBS with calcium and magnesium and incubated with $0.1 \mathrm{MBq}{ }^{18} \mathrm{FDG}$ and $200 \mathrm{U} / \mathrm{ml} \mathrm{TNF}-\alpha, 100 \mathrm{ng} / \mathrm{ml}$ GM-CSF, $100 \mathrm{nM}$ FMLP and 1 nM PAF. Reactions were terminated by addition of iodoacetic acid and cold PBS. Differential counts in the cell pellet and supernatant were compared.

Results: Neutrophils took up more ${ }^{18} \mathrm{FDG}$ than monocytes. In neutrophils incubated for 35 mins ${ }^{18} \mathrm{FDG}$ accumulation increased from 20.2 (3.7) \% (mean (SEM)) in control conditions to 49.3 (3.1) \% when primed with TNF- $\alpha(p<0.05)$ and $48.2(4.9) \%$ when fully activated with TNF- $\alpha$ followed by FMLP $(p<0.05)$. Uptake by monocytes increased from 2.8 (1.0) \% at basal conditions to 13.5 (4.3) \% when fully activated. ${ }^{18}$ FDG uptake in cells treated with either GM-CSF or FMLP peaked at 60 mins with accumulations of 60.6 (1.9) \% and 59.9 (3.5) \% respectively. Neutrophils incubated with PAF showed faster initial uptake of ${ }^{18} \mathrm{FDG}$ although the final uptake was less at only 13.3 (2.7) \%.

Conclusions: Our data correlate well with in vivo autoradiography studies where neutrophils rather than monocytes take up ${ }^{18} \mathrm{FDG}$ at sites of inflammation/infection (Jones et al. Am J Respir Crit Care Med 1994;149:1635-9). Time courses of ${ }^{18}$ FDG uptake mirror previously documented shape change responses and priming of the oxidative burst. Priming, in isolation from superoxide anion release or degranulation, requires glucose uptake. Furthermore agents vary in the rate and amount of ${ }^{18} \mathrm{FDG}$ accumulation in line with their demonstrated priming efficacy.

\section{S86 DIMINISHED NEUTROPHIL RESPONSES IN MICE LACKING THE REGULATORY SUBUNIT OF PHOSPHOINOSITIDE 3-HYDROXYKINASE $\gamma$ (PI3K $\gamma$ )}

A.M. Condliffe, ${ }^{1,2}$, G.J. Ferguson ${ }^{1,2}$, E.R. Chilvers'2, P.T. Hawkins', L.R. Stephens'. 'Babraham Institute, Babraham, Cambridge CB2 4AT, UK; 'Department of Medicine, Addenbrookes' Hospital, Cambridge CB2 2QQ, UK

Protection from infection is conferred by the coordinated and regulated response of the non-specific and specific arms of the immune response. Excessive or dysregulated immune responses may cause tissue injury. Neutrophils, recruited rapidly and in large numbers to inflammatory foci, have been implicated in the pathogenesis of a number of diseases, including the acute respiratory distress syndrome (ARDS) and bronchiectasis. Neutrophil-mediated tissue injury is produced by release of reactive oxygen species e.g. superoxide $\left(\mathrm{O}_{2}\right)$ and histotoxic enzymes (e.g. elastase), both processes requiring the enzyme phosphoinositide 3-hydroxykinase (PI3K). The isoform $\mathrm{PI} 3 \mathrm{~K} \gamma$, activated by G-protein linked agonists such as activated complement $(\mathrm{C} 5 \mathrm{a})$ and bacterial formylated peptides (fMLP), is thought to mediate aspects of neutrophil activation, with a possible role for its unique regulatory subunit, p101.

To clarify these issues, we have engineered mice lacking p101 (p101\% mice). These mice are healthy and fertile, with normal expression of the $\mathrm{pl} 10 \gamma$ catalytic subunit of $\mathrm{PI} 3 \mathrm{~K} \gamma$. Analysis of 
$\beta$-galactosidase (introduced by the targeting process) confirmed that p101 is expressed predominantly by cells of the myeloid lineage. Bone marrow-derived neutrophils displayed reduced formation of phosphatidylinositol 3,4,5-trisphosphate (the second messenger product of PI3K) when stimulated by $\mathrm{C} 5 \mathrm{a}(36 \pm 3 \%$ and $52 \pm 6 \%$ of wild type values for 5 and $100 \mathrm{nM} \mathrm{C5}$ a respectively, $n=3$ at 10 seconds). Activation of protein kinase $B$ was also substantially reduced $(27 \pm 5 \%$ and $23 \pm 8 \%$ of wild type values for 5 and $100 \mathrm{nMC} 5 \mathrm{a}, \mathrm{n}=4$ at 5 minutes), but activation of mitogen-activated protein kinase and c-jun $\mathrm{N}$-terminal kinase were unaffected. $\mathrm{p} 101 \%$ neutrophils exhibited reduced chemotaxis towards several G-protein coupled agonists, but equivalent formation of reactive oxygen species compared to wild type controls. Baseline rates of apoptosis were unaffected, but $\mathrm{C} 5 \mathrm{a}$, which did not affect apoptosis in wild-type bone marrow-derived neutrophils, was pro-apoptotic when applied to p101\% cells. We conclude that p101 enhances the catalytic activity of PI3K $\gamma$ in vivo, and that this reaction is important in fundamental neutrophil responses.

\section{S87 THE EFFECT OF SALBUTAMOL ON NEUTROPHIL ADHESION MOLECULE EXPRESSION}

G.D. Perkins', W. Rea', F. Gao', D.R. Thickett'. 'Birmingham Heartlands Hospital, Birmingham B9 5SS, UK; ${ }^{2}$ Queen Elizabeth Hospital, Birmingham B15 2TH, UK

Background: Neutrophil activation and recruitment to the alveolar space are important steps in the development of the Acute Respiratory Distress Syndrome (ARDS). Neutrophil emigration from the pulmonary circulation has been shown to occur by CD11/18 dependent and independent pathways. Furthermore blockade of the CD1 1/18 pathway can reduce lung injury and improve survival in animal models of ARDS. $\beta$-Agonists have already been proposed as a potential therapy for ARDS through their ability to accelerate alveolar fluid clearance. These drugs also affect a variety of neutrophil functions including chemotaxis. The aim of this study was to investigate the effects of in vitro incubation of whole blood with physiological doses of salbutamol on neutrophil adhesion molecule expression.

Methods: Whole blood from 10 healthy volunteers was incubated with RPMI or salbutamol $\left(10^{-5} \mathrm{M}\right.$ or $\left.10^{-9} \mathrm{M}\right)$ at room temperature for 15 minutes. Unstimulated or FMLP $\left(10^{\circ} \mathrm{M}\right)$ stimulated samples were then stained with CD $11 \mathrm{~b}, \mathrm{CD} 18, \mathrm{CD} 62(\mathrm{~L})$ or Mab 24 (an activation dependent epitope of (FA-1). Samples were analysed by flow cytometry and data collected as median flouresence intensity and analysed using Wilcoxon signed rank test.

Results: See table.

Conclusions: The effects of $\beta$-agonists on neutrophil chemotaxis and adhesion may be unrelated to changes in the adhesion molecule expression (CD 11b, CD18, Mab 24). L-selectin expression. which falls with neutrophil activation, was significantly reduced by salbutamol only at the higher end of the physiological dose range, suggesting that these agents may have some role in reducing neutrophil activation.

Abstract $\mathrm{S} 87$

\begin{tabular}{lllll}
\hline $\begin{array}{l}\text { Median } \\
\text { (IQR) }\end{array}$ & Unstim & FMLP & $\begin{array}{l}\text { FMLP/Salb } \\
\left(10^{-9} \mathrm{M}\right)\end{array}$ & $\begin{array}{l}\text { FMLP/Salb } \\
\left(10^{-5} \mathrm{M}\right)\end{array}$ \\
\hline CD 11b & $25(14-31)$ & $42(27-72)^{*}$ & $42(30-81)^{*}$ & $45(28-75)^{*}$ \\
CD 18 & $36(23-47)$ & $57(42-70)^{*}$ & $48(31-69)^{*}$ & $50(45-71)^{*}$ \\
CD 62(L) $49(22-57)$ & $16(4-26)^{*}$ & $14(4-31)^{*}$ & $26(6-37)^{\dagger}$ \\
MAb 24 $15(5-33)$ & $40(36-54)^{*}$ & $48(28-57)^{*}$ & $43(31-55)$
\end{tabular}

${ }^{*} p<0.05$ compared to unstimulated; ${ }^{\dagger} p<0.05$ compared to FMLP stimulated.

\section{S88 CO-LOCALISATION OF MMP-9 WITH NEUTROPHILS IN STABLE LUNG TRANSPLANT RECIPIENTS-A POTENTIAL ROLE IN BRONCHIOLITIS OBLITERANS}

I.A. Forrest', C. Ward', G. Pritchard', A.D. Rowan², T.E. Cawston², P.A. Corris'. ' Lung Biology and Transplantation Group; ${ }^{2}$ Department of Rheumatology, University of Newcastle-upon-Tyne and The Freeman Hospital, Newcastle-upon-Tyne NE7 7DN, UK

Background: Chronic rejection of lung allografts is manifest by Bronchiolitis Obliterans Syndrome (BOS), occurring in up to $50 \%$ of lung transplant recipients by 2 years. This is an inflammatory/fibrotic process resulting in collagen deposition, luminal obliteration of airways and resultant fall in lung function. Increased airway neutrophilia is recognised as a predictive feature of those at risk of BOS. Matrix Metalloproteinases (MMPs) and their inhibitors (TIMPs) tightly regulate the turnover of the extracellular matrix. There has been recent interest in the role of MMPs in airway diseases characterised by remodelling, such as asthma. The MMP/TIMP system may offer novel therapeutic targets.

Hypothesis: MMPs are effector mechanisms in the remodelling of the airway associated with BOS and other airway diseases.

Methods: Stable lung transplant recipients $(n=27)$, more than three months post transplant and without evidence of acute rejection, infection or $\mathrm{BOS}$ underwent standardised bronchoalveolar lavage (BAL $3 \times 60 \mathrm{mls}$ ) and large airway endobronchial biopsy.

Results: BAL showed a significant neutrophilia $(4 \% \pm 0.8) \mathrm{com}-$ pared to normal controls $(n=34)(1.6 \% \pm 0.2)$ (Mean \pm SEM, $p<0.005)$ Immunohistochemistry (Mouse anti-human MMP-9) of endobronchial biopsies demonstrated apparent cellular localisation of MMP9 to neutrophils. Migrating neutrophils were demonstrable within bronchial epithelium as well as the sub basement membrane area. Gelatin zymography performed on lavage supernatant confirmed the presence of significant activity at $92 \mathrm{kDa}$ with lesser activity at $72 \mathrm{kDA}$, suggesting pro-MMP-9 to be the predominant gelatinase. In addition a $125 \mathrm{kDa} \mathrm{mw}$ band was apparent, likely relating to a complex of MMP9 and Neutrophil Gelatinase Associated Lipocalin.

Conclusions: Neutrophils are present in significant numbers in apparently stable lung transplant recipients and are the primary source of MMP-9. The local balance of MMPs/TIMPs in the pericellular space is likely integral to airway remodelling. An ongoing prospective longitudinal study relating architectural changes of the airway and clinical outcomes will help clarify the role of MMPs in BOS.

\section{S89 DETERMINANTS OF CYTOTOXICITY OF RESIDUAL OIL FLY ASH (ROFA) ON BRONCHIAL EPITHELIAL CELLS}

P.A.B. Wark, S.M. Puddicombe, A.J. Frew, S.T. Holgate, D.E. Davies Brooke Laboratories, School of Medicine, University of Southampton, UK

Rationale: Epidemiologic studies have demonstrated associations between particulate air pollution and increased asthma symptoms. Epithelial damage is a pathological feature of asthma and is associated with pro-inflammatory responses. Residual oil fly ash (ROFA) is an emission particle with in vitro toxicity to epithelial cells.' We hypothesised that less confluent epithelial cell cultures would be more prone to injury by ROFA.

Methods: $16 \mathrm{HBE}$ bronchial epithelial cells were cultured and treated for up to 48 hours with concentrations of ROFA 10 and $100 \mu \mathrm{g} / \mathrm{mL}$ at $80 \%$ confluence or at $60 \%$ culture confluence. Cells were exposed in the absence or presence of TNF- $\alpha 10 \mathrm{ng} / \mathrm{ml}$. Cells were photographed using phase contrast microscopy, cultures were then fixed and the supernatant removed. Cell biomass was measured using a methylene blue assay, cell necrosis by lactate dehydrogenase (LDH) activity and interleukin (IL)-8 release by ELISA.

Results: Cells treated with either dose of ROFA showed a significant increase in IL- 8 secretion by 8 hours, peaking at 24 hours. Cells at $80 \%$ confluence treated with high dose ROFA showed a $40 \%$ reduction in cell number as compared with controls $(p=0.03)$, while those treated at $60 \%$ confluence had a $70 \%$ reduction compared to controls $(p=0.004)$. An increase in LDH activity was seen at both $80 \%$ and $60 \%$ confluence by 24 hours. In contrast confluent cells treated with low dose ROFA showed reduced LDH activity even at 48 hours while those also treated with TNF had an increase in cell biomass of $16 \%$ above controls $(p=0.03)$. This effect was not seen in cells treated with ROFA $10 \mu / \mathrm{ml}$ at $60 \%$ confluence.

Conclusion: Exposure of $16 \mathrm{HBE}$ cells to ROFA leads to an early dose dependent inflammatory response with IL-8 release and dose and time dependent cell necrosis which is more pronounced in less confluent cell cultures, suggesting damage to cells is cumulative and related to pre-existing cell numbers. The addition of TNF to confluent cells exposed to low dose ROFA appears to be partially protective to the cytotoxic effects of ROFA and appears to be associated with cell proliferation.

NHMRC (Australia) and British Medical Association.

1. Jiang, et al. Toxical Appl Pharmacol 2000;163:221-30. 
S90 DOXYCYCLINE INHIBITS CIGARETTE SMOKE STIMULATED IL-8 RELEASE FROM NCI-H292 CELLS

T. Howell, A. Richter, S.T. Holgate, D.E. Davies. Division of Infection, Inflammation and Repair, Brooke Laboratory, Level F, South Block (888), Southampton General Hospital, Southampton SO 16 6YD, UK

Cigarette smoke (CS) has been shown to cause phosphorylation and activation of the epidermal growth factor receptor (EGFR) leading to upregulation of mucin expression in bronchial epithelial cells. It has been suggested that this occurs via an oxidant-mediated, ligandindependent mechanism (Takeyama, et al. Am J Physiol Lung Cell Mol Physiol 2001;280:L165-L172). However, we have shown recently that CS stimulated the transcription and release of EGFR ligands from $\mathrm{NCl}-\mathrm{H} 292$ bronchial epithelial cells (Richter, et al. Am J Resp Cell Mol Biol 2002; 27:85-90). Moreover, CS also induced the production of IL-8 from H292 cells and this response was mediated via the EGFR. Therefore upregulation of EGFR signalling may underlie some of the long-term effects of CS on bronchial epithelium such as chronic inflammation and goblet cell hyperplasia.

EGFR ligands are cleaved from transmembrane precursors by $\mathrm{Zn}^{2+}$ dependent metalloproteinases (MP) of the A-Disintegrin and Metalloprotease (ADAM) family to generate active soluble peptides. The antibiotic doxycycline has been reported to inhibit MP activity independently of its antimicrobial properties (Golub, et al. Crit Rev Oral Biol 1991;2:297-322). Therefore we tested its ability to reduce EGFR ligand shedding and inhibit IL-8 production in NCI-H292 cells exposed to CS. Firstly, we determined the toxicity of doxycycline to in vitro cultures of $\mathrm{H} 292$ cells. We then exposed the cells to an aqueous extract of cigarette smoke (CSE) in the presence or absence of sub-toxic doses of doxycycline. We used enzyme-linked immunoassay to determine the concentration of EGFR ligands and IL-8 in the culture medium 6 and $24 \mathrm{hr}$ post exposure. We measured their level of mRNA expression $6 \mathrm{hr}$ post exposure by quantitative real-time PCR.

CSE stimulated the release of EGFR ligands and IL-8 and these responses were inhibited by doxycycline in a dose dependent manner. Doxycycline also blocked CSE-induced ligand and IL-8 mRNA transcription. We propose that CSE acts initially by promoting the shedding of EGFR ligands. This causes autocrine activation of the EGFR and, subsequently, increases the gene transcription of EGFR ligands and IL-8. Doxycycline, by acting as an MP inhibitor, prevents the shedding of EGFR ligands hence and blocks EGFR activation by CSE.

\section{Infections: From bench to bedside}

\section{S91 THE MAIN SITE OF INOS ACTIVITY INDUCED BY GRAM POSTIVE STAPHYLOCOCCUS AUREUS MAY BE BLOOD VESSELS AND NOT MACROPHAGES: COMPARISONS WITH GRAM NEGATIVE ESCHERICHIA COLI}

E. Belcher, T.W. Evans, S. Sriskandan, J.A. Mitchell. Unit of Critical Care Medicine, Royal Brompton Hospital, NHLI Imperial College School of Medicine, Dovehouse Street, London SW3 6LY, UK

Sepsis and septic shock caused by either Gram positive or Gram negative bacteria is associated with a mortality rate of $40-70 \%$. We, and others have hypothesised that such adverse outcomes result from an unchecked immune response mounted initially by neutrophils and macrophages in order to kill the invading pathogen. As sepsis develops, endothelium and vascular smooth muscle become activated to express inducible (i) inflammatory genes such as that encoding for nitric oxide synthase (iNOS), leading to the production of large amounts of NO, rendering the vessel hyporesponsive to constrictor agents. This contributes significantly to the profound decline in blood pressure that typifies septic shock. However, the ability of bacteria to induce iNOS activity in immune cells (e.g. macrophages) versus vascular tissue remains unclear. Secondly, a comparison between the ability of Gram positive and Gram negative bacteria to induce iNOS in these tissues has not been made. We therefore assessed the ability of heat killed $S$ aureus or $E$ coli to induce iNOS (indexed by the ability of tissue to release nitrite, measured by Griess assay) in murine macrophages (J774.2) versus murine blood vessel (aorta) in cell and organ culture respectively. $E$ coli induced concentration-dependent increases in NO release in $\mathbf{J 7 4}$ macrophages, to a maximum of 24FM. By contrast, $S$ aureus induced only low levels of $N O$ release (2FM). E coli induced significant NO release from aorta, but similar in magnitude to that produced by $S$ aureus (figure).

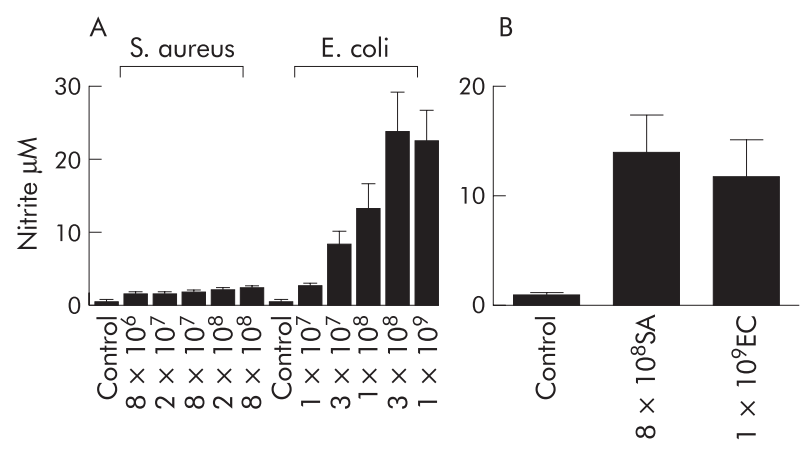

Abstract S91 Effects of heat killed $S$ aureus and $E$ coli on NO release by $(A)$ segments $(2-3 \mathrm{~mm})$ of intact mouse aorta $(\mathrm{n}=4)$ and (B) 7774.2 macrophages $(n=6)$ incubated in 96 well plates containing $200 \mu$ medium for 24 hours

$E$ coli induced $\mathrm{NO}$ release from both immune modulating cells (macrophages) and vascular tissue. By contrast, $S$ aureus caused NO release only in aorta. The inflammatory response to bacterial infection may be dictated by the inflammatory stimulus.

Work supported by the MRC.

\section{S92 THE PRESENCE OF QUORUM SENSING SIGNAL MOLECULES IN CLINICALLY STABLE LUNG ALLOGRAFT RECIPIENTS SUGGESTS BACTERIAL BIOFILM BIOLOGY}

C. Ward, M. Cámara', I. Forrest, B. Rutherford, G. Pritchard, M. Daykin', A. De Soyza, A.J. Fisher, P. Williams' 2, P.A. Corris. Lung Biology and Transplantation Group, University of Newcastle upon Tyne and The Freeman Hospital, 'School of Pharmaceutical Sciences; ${ }^{2}$ Institute of Infections and Immunity, University Park, University of Nottingham, UK

Introduction: Infection with bacteria such as Pseudomonas is common in lung allograft recipients, particularly during chronic rejection. Analysis of sputum samples from CF patients infected with Pseudomonas aeruginosa or Burkholderia cepacia have indicated the presence of the bacterial $\mathrm{N}$-acylhomoserine lactones (AHLs) quorum sensing signaling molecules. AHLs not only control the expression of bacterial virulence genes but are also involved in stimulating the maturation of antibiotic resistant biofilms.

Hypothesis: AHL activity may be detected even in clinically stable lung transplant recipients free of clinical infection or rejection.

Methods: A standardised $3 \times 60 \mathrm{~mL}$ Bronchoalveolar lavage (BAL) taken from 9 stable, non smoking lung transplant recipients, 3 B 12 months post transplant. Detection of AHLs was carried out on dichloromethane extracted supernatants, using the bioluminescence-based AHL reporter plasmid pSB 1075. This responds to AHLs with long acyl chains (10-14C), generating light. Synthetic AHLs were included as +ve controls.

Results: From the 9 BAL supernatants, 5 exhibited AHL activity, suggesting the presence of long chain AHLs. There was no correlation between the levels of AHLs detected $(+,++,+++)$, or their absence, BAL microbiology or pre transplant diagnosis.

Discussion: We provide the first evidence of $\mathrm{AHL}$ quorum sensing signals in human lung allograft recipients, with activity even in subjects with no rejection or infection. Longitudinal studies are required of $\mathrm{AHL}$ levels, to elucidate potential links with infection, rejection, and allograft deterioration.

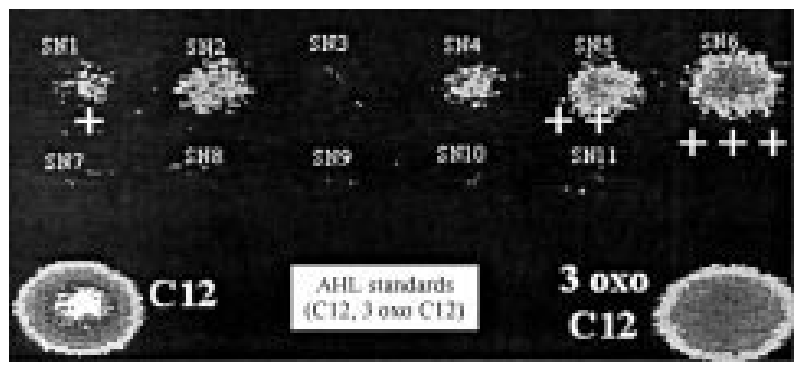

Abstract S92 Representative bioluminescence photon capture. 


\section{S93 HEME OXYGENASE INDUCTION IN ISOLATED NEUTROPHILS STIMULATED WITH LPS, OR FROM PATIENTS WITH SEPSIS DUE TO NOSOCOMIAL PNEUMONIA}

R.L. Upton, J.H. Cranshaw, G.J. Quinlan, T.W. Evans. Unit Of Critical Care, National Heart And Lung Institute, Dovehouse Street, London SW3 6LY, UK

Rationale: Evidence suggests that macrophages are a source of Heme Oxygenase $(\mathrm{HO})$ protein and activity during inflammatory processes. $\mathrm{HO}$ enzymes have anti oxidant potential and may confer anti-inflammatory protection. However, few data relate to the induction of this enzyme in other inflammatory cell types, such as neutrophils, which are recruited initially to sites of infection or injury. We therefore evaluated the potential for human neutrophils to produce $\mathrm{HO}$ under contrasting inflammatory conditions.

Methods: Neutrophils were isolated from whole human blood and stimulated with LPS $(10 \mathrm{ug} / \mathrm{ml})$ in 6 well plates at a concentration of 5 $\times 10^{6}$ cells/well for 8,16 and 24 hours, harvested and lysed. HO-1 and HO-2 protein expression was measured by Western blot. Stimulated cells were compared to un-stimulated neutrophils at time zero, 8, 16 and 24 hours. Blood (with cycloheximide $1 \mathrm{mg} / \mathrm{ml}$ ) was also taken from patients with Gram-negative septic shock $(n=6)$ and from healthy volunteers $(n=5)$. In four of the six patients with septic shock microbiological culture identified the lung as a site of infection. Neutrophils were isolated and $5 \times 10^{6}$ cells were harvested and lysed. HO- 1 and $\mathrm{HO}-2$ protein expression was measured as before.

Results: In vitro, HO-1 levels were elevated 8 hours after LPS by comparison to baseline and unstimulated cells $(199.2 \% \pm 42 \%, n=9$, $\mathrm{p}<0.05)$. HO-1 levels in cells 16 and 24 hours after LPS were not significantly different from levels in untreated cells because of non-specific induction of $\mathrm{HO}-1$ in the unstimulated cells $(115.8$ (21.19), $n=9$ and 94.94 (12.58), $n=9$ respectively). HO-2 levels were not significantly altered at any of the timepoints between the LPS stimulated and non-stimulated cells.

By contrast, in vivo, $\mathrm{HO}-1$ protein was lower in septic patients compared to normal controls $(60.97$ (24.5), $n=6$ and 125.7 (34.4), $n=5$ respectively). HO-2 levels were not changed between the two groups.

Conclusion: HO-1 production can be induced by LPS in neutrophils, and is also present in neutrophils from patients with sepsis. However, HO-1 levels in septic patients are lower than controls. The anti oxidant potential of HO-1 may therefore be reduced in the neutrophils of these patients, which has implications for neutrophil mediated tissue damage in this population.

This work was supported by The British Lung Foundation and The Dunhill Trust.

\section{S94 THE EFFECT OF AIRWAY BACTERIAL LOAD ON EXACERBATION SEVERITY IN PATIENTS WITH COPD}

T.M.A. Wilkinson, I.S. Patel, A.C. Whiley, M. Wilks, J.A. Wedzicha. Academic Unit of Respiratory Medicine, St Bartholomew's and the Royal London School of Medicine and Dentistry, London, UK

COPD exacerbations are an important cause of morbidity and mortality. In the majority of these episodes infective agents including both bacteria and viruses can be identified. However bacteria can also be isolated from the lower airway of many stable COPD patients and the type and number of these bacteria affects the level of airway inflammation in these colonised patients in the stable state. The changes between bacterial load and type seen at exacerbation and at baseline and how these changes may affect the deterioration in lung function seen at exacerbation are poorly described.

75 patients (mean (SD) FEV 1.00 (0.38) I, FEV \% predicted 38 (16.8), FVC $2.57\left(0.93\right.$ ) I, $\mathrm{PaO}_{2} 10.2$ (1.1) kPa, $\mathrm{PaCO}_{2} 6.22$ (0.9) kPa, age 67.4 (1 1.8) yrs, m 40, 28 current smokers) completed daily diary cards of respiratory symptoms were followed up for 12 months and reported symptoms of exacerbation to our study team. Sputum was initially sampled in the stable state and later within 48 hours of the onset of exacerbation (before antibiotic treatment was commenced) and analysed for quantitative and qualitative bacteriology. Contemporaneous lung function measurement was performed by spirometry. 55 satisfactory paired samples were obtained, the relative frequency of bacterial isolates being: Haemophilus influenzae in 12\% of stable samples (1) and $31 \%$ of exacerbation samples (2), Streptococcus pneumoniae ((1) 9\%, (2) 11\%), Haemophilus parainfluenzae ((1) $12.5 \%$, (2) 2\%), Branhamella catarrhalis ((1) 7\%, (2) 13\%) and Pseudomonas aeruginosa ((1) $5 \%$, (2) $5 \%$ ) with Non specific growth in (1) $48 \%$ and (2) $25 \%$. The mean (SD) stable bacterial load was $10^{7.49}$ $\left(10^{1.26}\right) \mathrm{log} \mathrm{cfu} \mathrm{ml}^{-1}$ rising to $10^{8.00}\left(10^{1.02}\right) \mathrm{cfu} \mathrm{m}^{-1}$ at exacerbation $\mathrm{p}=0.0001$. The mean (SD) stable $\mathrm{FEV}_{1}$ was $0.99(0.38)$ I falling to
$0.90(0.31)$ I at exacerbation. The percentage fall in $\mathrm{FEV}_{1}$ at exacerbation was related to the rise in airway bacterial load rho=0.411 $p=0.018$. Linear regression analysis confirmed that a rise in bacterial load contributed to a fall in FEV, $\mathrm{p}=0.034,95 \% \mathrm{Cl}(0.006-0.141)$. Both airway bacterial load and the prevalence of potentially pathogenic organisms increase at exacerbation. Exacerbation severity as measured by the acute deterioration in $\mathrm{FEV}_{1}$ is directly related to the rise in airway bacterial load seen at exacerbation.

Supported by The Joint Research Board, St Bartholomew's Hospital.

\section{S95 HOW USEFUL ARE THE BTS COMMUNITY ACQUIRED PNEUMONIA (CAP) GUIDELINES IN MANAGING URBAN ELDERLY PATIENTS}

J. Harbison, R. Shepherd, S.K. Bansal. Dept of Geriatric Medicine, Sunderland Royal Hospital, UK

Introduction: In 2001 the BTS published guidelines for the recognition and management of adult community acquired pneumonia (CAP) in which it is stated that elderly patients "more frequently present with non-specific symptoms" and "are less likely to have a fever specific symptoms and pyrexia" with CAP. However both presence of specific symptoms and pyrexia are included in the clinical diagnostic criteria in the guidelines. SRH has an age related admissions policy, patients $\geqslant 70$ years being admitted under Geriatric physicians and the BTS guidelines being used in their care.

Purpose of study: We sought to determine the utility of the BTS CAP guidelines in the management of consecutive admissions with a primary diagnosis of lower respiratory tract infection. Subjects with carcinoma, tuberculosis or non-CAP were excluded.

Results: 81 subjects (average age 78yrs, 39 F) were admitted over the 6-week study period. $55(68 \%)$ had a previous diagnosis of COPD or Asthma. 17 subjects $(21 \%(\mathrm{Cl} 12-30 \%))$ had CAP according to the guidelines' clinical diagnostic criteria. In contrast, $48159 \%, 95 \% \mathrm{Cl}$ $54-64 \%$ ) had pneumonia identified on admission radiograph. Radiographic and clinical diagnoses agreed in only 38 cases (Pneumonia agreed present in 11 , absent in 27 . Kappa 0.041 , very poor agreement).

Core adverse prognostic features used for severity assessment in the guidelines (new confusion, Urea $\geqslant 7 \mathrm{mmol} / \mathrm{l}$, respiratory rate $\geqslant 30$, Systolic BP $<90 \mathrm{mmHg}$ or diastolic $\leqslant 60 \mathrm{mmHg}$ ) were recorded in the 48 subjects with radiographic evidence of CAP. 13 subjects had no features, 31 had one and 3 had two. As guideline advice for those $\geqslant 50$ years of age with no or one feature is to "Use Clinical Judgement" with regard to admission and specific management, the guidelines directed management in only 3 patients $(6 \% \mathrm{Cl} 0-13 \%)$ Of note $26 \%(54 \%)$ of subjects had an elevated Urea and $7(15 \%)$ had evidence of hypotension, the causes of which were multifactorial.

Conclusions: The clinical diagnostic criteria for community acquired pneumonia in the BTS guidelines are unsuitable for use in an urban elderly population. This could be expressed more explicitly in the text. The severity assessment flow chart in the guidelines is of limited use in the majority of elderly subjects with community acquired pneumonia. Consideration should be given to the development of specific guidelines for the management of lower respiratory tract infection and pneumonia in the elderly.

\section{Procedures in respiratory medicine}

\section{S96 BTS GUIDELINES FOR BRONCHOSCOPY: PHYSICIANS APPROACH TO THE NON-EVIDENCE BASED RECOMMENDATIONS}

A. Mirakhur, M. Al-Aloul, D. Russell, M.J. Ledson, M.J. Walshaw, C. Smyth. Liverpool Lung Cancer Unit, The Cardiothoracic Centre, Liverpool, UK

In March 2001 the BTS published guidelines for the practice of diagnostic flexible bronchoscopy. However, it was apparent that many of the recommendations were not evidence based (27 of $68(40 \%)$ were SIGN Grade C), and we had the impression that they were not routinely practiced by chest physicians within the UK. We therefore compared the SIGN Grade C recommendations in these guidelines with the routine practice of chest physicians as described by a postal questionnaire sent to 548 UK chest physicians in 2000. Three hundred and twenty eight questionnaires $(60 \%)$ were returned. Based on these, prior to bronchoscopy 227 physicians $(69 \%)$ routinely measured spirometry and $117(37 \%)$ pulse oximetry. Whilst only 7 
physicians (2\%) measured blood gases in all patients, $70(21 \%)$ did so depending upon spirometry, $48(15 \%)$ upon the patient's clinical status, and $35(11 \%)$ when the $\mathrm{SpO} 2<93 \%$. Overall, 122 physicians $(37 \%)$ did not measure blood gases prior to bronchoscopy under any circumstances. For patients theoretically at risk from bacteraemia during bronchoscopy, only 192 physicians $(59 \%)$ routinely gave prophylactic antibiotics. Prior to transbronchial biopsy, 281 physicians (86\%) checked the platelet count, but only $273(83 \%)$ checked clotting parameters. Two hundred and eighty operators $(85 \%)$ always used prophylactic venous access, and $26(8 \%)$ occasionally. Thus, 14 physicians $(7 \%)$ carried out bronchoscopy with no routine venous access. Nearly all physicians $(324,99 \%)$ monitored pulse oximetry during bronchoscopy, but $180(56 \%)$ did not monitor ECG or blood pressure. In 19 centres $(6 \%)$, the physician carried out bronchoscopy with only 1 endoscopy assistant and in $2(1 \%)$ no trained nurse was present. In terms of operator safety, for routine bronchoscopy, $(315,96 \%)$ wore gloves, but only $197(60 \%)$ wore gowns and very few goggles (45, $14 \%)$ or a facemask $(80,24 \%)$. However, for "high risk" bronchoscopy, these figures improved to 321 (98\%), 290 (88\%), 228 $(69 \%)$, and $290(88 \%)$, respectively. Thus, based upon this postal survey, it is apparent that there are wide discrepancies between current practice and the guideline recommendations for diagnostic flexible bronchoscopy that are not evidence based.

\section{S97 PROSPECTIVE RANDOMISED TRIAL OF ABRAM'S PLEURAL BIOPSIES VERSUS CT GUIDED CUTTING NEEDLE BIOPSIES IN UNDIAGNOSED PLEURAL EFFUSIONS}

N.A. Maskell, F.V. Gleeson, R.J.O. Davies. Oxford Centre for Respiratory Medicine, Churchill Hospital, Oxford, UK

Introduction: Approximately 180000 people in the U.K., develop a pleural effusion each year, of which 40000 will turn out to be due to malignancy. Unfortunately, cytological examination alone has a sensitivity for malignancy of only $60 \%$, necessitating further more invasive investigations in many cytology negative pleural effusions. With the rising incidence of mesothelioma (where track invasion by fumour is common), the minimum number of invasive investigations to obtain a diagnosis is desirable. We have assessed whether CT guided pleural biopsy is more sensitive than blind Abram's biopsy in these patients.

Methods: 50 consecutive patients with undiagnosed exudative effusions, negative pleural fluid cytology, but possible pleural malignancy, were recruited. All received a contrast enhanced CT thorax, with the degree of pleural thickening recorded. Patients were randomised to blind Abram's pleural biopsy or CT guided cutting needle biopsy. The Abram's pleural biopsy was performed by NAM without knowledge of the CT findings. The CT guided biopsy was performed by FVG.

Results: Of the 50 patients, 3 did not have a biopsy 11 withdrew consent, 1 pleural effusion resolved and 1 had deranged blood clotting). There was one chest wall haemtoma in the Abram's group. The median age was 72 (range 25-88) in the CT group and 73 (41-85) in the Abram's group. The maximal pleural thickening on CT was $<5 \mathrm{~mm}$ in $17 / 25$ patients in the radiology group and in $17 / 25$ of the Abram's group. The final diagnosis was confirmed with a minimum follow up of 1 year. Abram's blind biopsy gave the true positive (TP) diagnosis of malignancy in $8 / 17$ cases, Sensitivity $47 \%$, Specificity $100 \%$ and Negative predicted value (NPV) $44 \%$. In the CT group, TP rate for malignancy was higher at $13 / 15\left(p=0.02, \chi^{2}\right)$, Sensitivity $87 \%$, Specificity $100 \%$, NPV $80 \%$. 7 of the 8 cases of mesothelioma in the radiology group were established by biopsy, Sensitivity $88 \%$, Specificity $100 \%$, NPV 94\%. This compared with only 6 of the 11 cases in the Abram's group, Sensitivity 55\%, Specificity 100\%, NPV $72 \%$.

Conclusion: In cytology negative pleural effusions CT guided pleural biopsy has a significantly higher sensitivity for malignancy and should be the biopsy method of choice in patients unable to tolerate thoracoscopy.

\section{S98 THE COMPLICATION RATE OF PERCUTANEOUS IMAGE-GUIDED CHEST DRAIN INSERTION}

J.C. Hillier', C.L. Davies', R.J.O. Davies², Z.C. Traill², F.V. Gleeson². 'Department of Radiology, Chelsea and Westminster Hospital, 369 Fulham Road, London SW 10 9NH, UK; ${ }^{2}$ Department of Radiology, Churchill Hospital, Oxford Radcliffe Hospitals, Old Road Headington, Oxford OX3 7IJ, UK

Purpose: The aim of this study was to assess prospectively the success and complication rate associated with image-guided percutaneous pleural drainage.
Materials/Methods: A prospective single institution study of all image-guided chest drains placed over a 36-month period was carried out. All drains were inserted using the Seldinger technique. Data collected included, indication for drain insertion, size of drain, type of image-guidance used (CT or ultrasound), demographic data, nature and amount of fluid aspirated, success rate, duration of drainage and complications.

Results: 352 drains were inserted into 273 patients. 121 patients were male and 152 female. 149 were inserted on the left and 203 on the right. The majority of drains were 12 French, (256), other drain sizes were 8 French (90), 10 French (2) to 14 French (3) 16 French (1). 333 drains were placed under ultrasonic guidance and 19 under CT. Indications included 236 malignant effusions 94 empyemas and 22 benign non-infective effusions. 350 drains were successfully inserted. Two patients suffered significant immediate complications of haemorrhage during drain placement due to intercostal artery laceration. Both drains were inserted posteriorly. Both patients underwent percutaneous artery embolisation. One procedure was successful; unfortunately one procedure was unsuccessful and the patient died within 24 hours of drain insertion of intrathoracic haemorrhage. Both of these patients had drain insertion for a current empyema, had a history of chronic renal failure and a prior empyema.

Conclusion: Percutaneous image-guided drain placement is successful in the majority of patients (99\%). Posteriorly inserted drains may have a higher and more significant complication rate compared to other drain position. Patients with renal impairment appear at greater risk.

\section{S99 TALC BUT NOT TETRACYCLINE PLEURODESIS INDUCES HYPOXIA AND INCREASED DTPA CLEARANCE OF THE} CONTRA-LATERAL LUNG

N.A. Maskell, Y.C.G. Lee, E. Jones, F.V. Gleeson, R.J.O. Davies. Oxford Centre for Respiratory Medicine, Churchill Hospital, Oxford, UK

Introduction: Talc slurry is the most commonly used and effective pleurodesis agent in the UK. There are concerns about its safety and it is associated with at least 34 reported cases of severe lung inflammation leading to Adult Respiratory Distress Syndrome (ARDS). It is not known if milder generalised lung inflammation is a consistent feature of talc pleurodesis. $99 \mathrm{mTc}$-DTPA lung clearance is a very sensitive marker of lung inflammation. This study assesses whether DTPA clearance from the lung contra-lateral to a pleural effusion is changed by talc or tetracycline pleurodesis used to control the effusion.

Methods: 31 patients with recurrent symptomatic effusion consented to the study. 11 were excluded (5 with trapped lung, 3 unable to perform baseline DPTA, 1 M.I. pre pleurodesis, 2 chest tube displacements). 20 were randomised to $4 \mathrm{~g}$ talc slurry (10) or $20 \mathrm{mg} / \mathrm{Kg}$ tetracycline (10) balanced by type of malignancy and pleural $\mathrm{pH}$. Oxygen saturation breathing air, serum $\mathrm{C}$-reactive protein (CRP), limited HRCT and DTPA scan were performed before and 48 hours after pleurodesis. Pleural fluid IL-8 was measured at before and 24 hours after pleurodesis.

Results: DTPA $t_{1 / 2}$ of the contra-lateral lung fell significantly after talc but rose after tetracycline. Talc 64.4 minutes (90\% range 40-111.1) to 59.5 (35.3-84.8) difference B8.95 (-35.2-12.4), tetracycline 60.1 (26.1-119) to $83.6(27.8-189.2)$ difference 2.85 (-16.3-75.1), $\mathrm{p}<0.03$ Mann-Whitney. No changes were detected on limited HRCT. CRP rise was significantly higher in the talc than tetracycline group. Talc 198 (SD 79) $v$ Tetracycline 74 (SD 79) $p=0.004 . \mathrm{SaO}_{2}$ also fell significantly more in the talc group. Talc $95.5(2.3)$ to 92.2 (2.6) difference 3.2 (SD 1.6), tetracycline 94.0 (4.7) to 93.3 (4.9) diff 0.7 (SD 1.5) $p=0.003$. There was also a trend towards a greater increase in pleural fluid IL-8 levels in the talc group: Talc 12618 (258-2991 1) vs Tetracycline 50 (-1657-29919) $\mathrm{p}=0.13$

Conclusion: Talc pleurodesis induces a greater systemic inflammatory response than tetracycline quantified by CRP. Increased aerosol DTPA clearance from the contra-lateral lung and greater hypoxaemia are induced by talc but not by tetracycline. This suggests that occasional severe ARDS induced by talc is the severe extreme of a spectrum and not an idiosyncratic adverse reaction.

\section{S100 TRANSBRONCHIAL NEEDLE ASPIRATION (TBNA)-AN ADDITIONAL TOOL TO INCREASE THE DIAGNOSTIC YIELD OF BRONCHOSCOPY}

J. Mills, P. Nuttall, S. Kearney, J. Edwards, M. Munavvar. Royal Preston Hospital, Preston, Lancashire, UK

Introduction: In patients with essentially extrinsic or submucosal disease on Bronchoscopy, the positive diagnostic rate of conventional 
sampling with endobronchial biopsy, brush and wash (CS) remains relatively low (Dasgupta, et al. Chest 1999; 1 15). TBNA is reported to increase the pick up rate in this group, and also in necrotic endobronchial tumours, but is not widely used (Mehta. Clin Chest Med 1999;20:39-51).

Methods: We prospectively recorded the results of TBNA in addition to appropriate conventional sampling in 23 patients over a 12 month period. All the sampling was done by a single operator, and during regular bronchoscopy lists. 22G Olympus and Millrose-Wang needles were used.

12 patients had submucosal/ extrinsic disease and 7 had necrotic endobronchial lesions. Bronchoscopy was normal in 4 patients. CT scans were performed prior to the bronchoscopy in 13 patients.

Results: TBNA was positive in $21 / 23$ patients $(91 \%)$, whereas conventional samples were positive in $16 / 22$ patients $(73 \%)$. Biopsy was positive in $13 / 15$, Brush in $12 / 15$ and wash in $9 / 22$ patients respectively. TBNA was the only positive diagnostic test in 5 patients $(22 \%)$. The diagnosis was non-small cell malignancy in 15 and small cell carcinoma in 5 patients. One patient presented with isolated subcarinal adenopathy, and acid fast bacilli were demonstrated on the FNA sample. There were no significant complications during or after the procedure.

In short, with the addition of TBNA, the diagnostic yield of bronchoscopy went up from $73 \%$ to $91 \%$.

Conclusions: TBNA is a cost-effective and safe technique to improve the diagnostic yield of bronchoscopy, particularly in patients with (a)extrinsic/submucosal disease (b) normal bronchoscopy but CT scan evidence of peribronchial disease or medistinal abnormality and (c) necrotic, haemorrhagic endobronchial lesions. It can also be used to diagnose benign disease. TBNA can be performed during a standard flexible bronchoscopy list, and could potentially reduce the need for a surgical biopsy.

\section{S101 FLUORESCENCE BRONCHOSCOPY IN CLINICAL PRACTICE}

A.K. Baneriee,' P.H. Rabbitts', P.J.M. George 2. 'Molecular Oncology Group, University of Cambridge, UK; ${ }^{2}$ The Middlesex Hospital, London, UK

Introduction: Autofluorescence bronchoscopy (AFL) uses the fluorescence properties of bronchial mucosa to enhance the real-time detection of abnormal endobronchial lesions. This study assesses the efficacy of the Storz bronchoscope in a UK population of patients.

Methods: Patients with suspected lung cancer attending for diagnostic bronchoscopy underwent AFL during the same procedure. Conventional white light followed by AFL was performed. Biopsies of all abnormal areas and control biopsies from bronchoscopically normal areas were obtained. The bronchoscopic and histological findings were compared.

Results: 53 patients have undergone AFL (41 male), mean age $63.8 \mathrm{yrs}$ (range 35-79 yrs). Controls: 106 areas were biopsied as controls. 3.7\% showed high grade pre-invasive lesions (carcinoma-insitu \& severe dysplasia). The remaining areas showed no abnormality or low-grade pre-invasive lesions. Bronchoscopically abnormal areas: The table shows the histology and bronchoscopic findings of the 133 areas biopsied. Compared toconventional white light bronchoscopy, autofluorescence improved the detection of high-grade pre-invasive

\begin{tabular}{lll}
\hline Abstract S101 & \\
\hline Biopsy result & $\begin{array}{l}\text { No areas +ve } \\
\text { white-light }\end{array}$ & $\begin{array}{l}\text { No areas +ve } \\
\text { AFL }\end{array}$ \\
\hline Normal \inflammation & 13 & 35 \\
Low grade pre-invasive & 10 & 16 \\
High grade pre-invasive & 35 & 48 \\
Squamous carcinoma & 27 & 34 \\
\hline
\end{tabular}

lesions. 7 early-stage microinvasive carcinomas were detected by AFL alone. The false negative rate for AFL detection of high-grade pre-invasive lesions was $3.7 \%$ and the false positive rate $38.3 \%$.

Conclusions: The addition of AFL to conventional bronchoscopy improves the sensitivity of high-grade pre-invasive lesion and importantly invasive carcinoma detection. Detection of microinvasive carcinoma at a radiologically occult stage allows intervention with potentially a $90 \% 5$ year survival. The dilemma of pre-invasive lesion management is also raised. Not all pre-invasive lesions become invasive carcinomas, and so intervention is not justified for all lesions. Further information on the natural history of such lesions is needed. The high false positive rate is similar to that found in other studies and suggests that abnormal findings must be confirmed histologically and that approaches to improve specificity are required.

\section{Lung cancer targets}

\section{S102 AUDIT IMPROVES DIAGNOSTIC DELAYS IN LUNG CANCER MANAGEMENT}

J. Walker, J.S. Sarvesvaran, M. Patel, A. Coote, K.R. Patel. Department of Respiratory Medicine, Gartnavel General Hospital, Glasgow, UK

Aim: Delays in diagnosis are often causes of distress to patients, and may have a detrimental effect on therapeutic options and overall prognosis. The aim was to prospectively audit the time taken to diagnose patients with suspected lung cancer in a large teaching hospital with reference to established national guidelines of best practice.

Method: From January 1999, all available data required for the Scottish Intercollegiate Guidelines Network (SIGN) and Royal College of Physicians (RCP) minimum dataset for lung cancer, was collected from case notes of patients managed by the lung cancer team at Gartnavel hospital. This was prospectively entered into Microsoft access and SPSS databases.

Results: 518 patients were identified between 1999 and 2001. Histological evidence of lung or other malignancy was obtained in $474(91.5 \%)$ patients. The time taken to see a respiratory physician, for the investigations to be performed and to obtain a definitive diagnosis is presented below, with the p-values for differences between the time taken in 1999 and 2001.

Conclusion: Simple organisational changes following clinical audit have significantly reduced the time taken for CT imaging of thorax, and have improved diagnostic delays, in patients with lung cancer.

\begin{tabular}{|c|c|c|c|c|}
\hline \multicolumn{5}{|l|}{ Abstract S102 } \\
\hline Time taken (days): & 1999 & 2000 & 2001 & $\begin{array}{l}t \text { test } 1999 \\
\text { to } 2001\end{array}$ \\
\hline To see respiratory physician from referral & $\begin{array}{l}n=155 \\
\text { mean }=8.3 \\
S D=13.3\end{array}$ & $\begin{array}{l}n=213 \\
\text { Mean }=6.6 \\
S D=8.6\end{array}$ & $\begin{array}{l}n=150 \\
\text { Mean }=7.3 \\
S D=10.8\end{array}$ & NS \\
\hline For bronchoscopy from clinic review & $\begin{array}{l}n=116 \\
\text { Mean=9.9 } \\
S D=24.2\end{array}$ & $\begin{array}{l}n=163 \\
\text { Mean }=11 \\
S D=22.8\end{array}$ & $\begin{array}{l}n=125 \\
\text { Mean=9.3 } \\
S D=25.8\end{array}$ & NS \\
\hline For CT scan of thorax from clinic review & $\begin{array}{l}\mathrm{n}=122 \\
\text { Mean }=28.8 \\
\mathrm{SD}=45.9\end{array}$ & $\begin{array}{l}n=170 \\
\text { Mean=21.0 } \\
S D=38\end{array}$ & $\begin{array}{l}n=131 \\
\text { Mean }=18.2 \\
S D=30.0\end{array}$ & $p=0.03$ \\
\hline For definitive diagnosis from clinic review & $\begin{array}{l}n=155 \\
\text { Mean }=33.1 \\
S D=90.6\end{array}$ & $\begin{array}{l}n=211 \\
M e a n=29.3 \\
S D=57.1\end{array}$ & $\begin{array}{l}n=150 \\
\text { Mean }=16.4 \\
S D=59.7\end{array}$ & $p=0.06$ \\
\hline
\end{tabular}




\section{S103 THERAPEUTIC DELAYS IN LUNG CANCER MANAGEMENT}

A. Coote, K.R. Patel, J.S. Sarvesvaran, J. Walker, M. Patel. Department of Respiratory Medicine, Gartnavel General Hospital, Glasgow, UK

Aim: As lung cancer is such an aggressive disease with poor prognosis, following the diagnosis, an appropriate member of the multidisciplinary team should initiate treatment quickly. To identify any therapeutic delays in lung cancer management, a prospective audit was set up in Jan 1999

Method: A SPSS database was constructed and all available data required for the Scottish Intercollegiate Guidelines Network(SIGN) and Royal College of Physicians (RCP) minimum dataset entered prospectively by a part time data manager

Results: During the period Jan 1999 to Dec 2001, the lung cancer team at Gartnavel General hospital managed 518 patients. 96 $(18.5 \%)$ patients were diagnosed with small cell lung cancer (SCLC), $335(64.7 \%)$ were non-small cell lung cancer (NSCLC). The times taken for patients to receive the different modalities of lung cancer treatment are shown in the table.

Conclusion: Chemotherapy for SCLC is prescribed primarily by respiratory physicians and is administered promptly. Similarly patients with NSCLC who are fit for surgery have the procedure within the recommended period. Although oncology review of patients is rapid, patients receiving chemotherapy for NSCLC, face a slight delay. This may be due in part to patients requiring time to consider therapeutic options but administrative delays due to treatment being administered at another hospital site cannot be excluded. The significant delay particularly for radical radiotherapy is of concern as it may have a detrimental effect on overall survival.

\begin{tabular}{lll} 
Abstract S103 & & \\
\hline & \multicolumn{2}{l}{$\begin{array}{l}\text { Time taken in days from } \\
\text { referral for treatment during } \\
\text { Jan 1999-Dec 2001 }\end{array}$} \\
\hline SCLC chemotherapy & $\mathrm{n}=88$ & median $=6$ \\
NSCLC chemotherapy & $\mathrm{n}=47$ & median $=23$ \\
NSCLC surgery & $\mathrm{n}=55$ & median $=18$ \\
Oncology review & $\mathrm{n}=338$ & median $=6$ \\
Radical XRT & $\mathrm{n}=29$ & median $=49$ \\
High dose palliative XRT & $\mathrm{n}=38$ & median $=32$ \\
Palliative XRT & $\mathrm{n}=150$ & median $=18$ \\
\hline
\end{tabular}

\section{S104 MEETING GOVERNMENT LUNG CANCER TARGETS: DOES LUNG CANCER PRESENTATION HAVE A SEASONAL VARIATION?}

A. Bastin, A.G. Davison, D. Eraut, A. Hutchings, A.S. Haque, A. Lamont, C. Trask. Southend Associate University Teaching Hospital, Southend on Sea, Essex SSO ORY, UK

Currently the Government target for seeing a patient with suspected lung cancer is within 2 weeks of referral and from 2005 the target time for starting treatment is to be within one month of diagnosis. It is known that exacerbations of COPD vary seasonally and are more common in winter months. COPD is very common in patients with lung cancer. The knowledge of any seasonal variation in lung cancer presentation is important for service planning and to allow Government targets to be met.

We have analysed the 2127 new cancer cases presenting over a ten year period from 1990 to 1999 from the Southend Lung Cancer Study. This includes every case in a well defined population of 325 000. Winter (W) months are defined as December, January, and February, and Summer (S) months are June, July, and August. The number presenting in S (561) was slightly higher than W (494). There was a lack of evidence that presence of cough at diagnosis differed by season $(66 \%$ had a cough in $W$, compared to $70 \%$ in $S)(p=0.14)$. There was a lack of evidence that dyspnoea at diagnosis differed by season $(59 \%$ in $\mathrm{W}$, compared to $63 \%$ in $S)(p=0.25)$. There was a lack of evidence that the presence of COPD in those presenting with lung cancer lusing the BTS COPD Guidelines criteria for diagnosis and severity) differed by season (74\% in W compared to $71 \%$ in S), nor that there was any difference in the severity of COPD at presentation according to season $(p=0.6)$. Similar results were found when
Abstract $\mathrm{S} 105$

\begin{tabular}{llllllll}
\hline Stage & la & lb & Ila & Ilb & Illa & IIlb & IV \\
\hline$\%$ & $5.1 \%$ & $13.2 \%$ & $0.3 \%$ & $7.7 \%$ & $13.9 \%$ & $21.3 \%$ & $39 \%$ \\
Mountain & $13 \%$ & $23 \%$ & $1 \%$ & $7 \%$ & $10 \%$ & $20 \%$ & $27 \%$ \\
\hline
\end{tabular}

those presenting in the 6 months of October through to March were compared to those presenting in the 6 months of April through to September.

Conclusion: There is no increase, unlike COPD, in the presentation of lung cancer in the $\mathrm{W}$ months. Even patients with severe COPD and lung cancer do not present more commonly in $W$. There is no difference in the respiratory symptoms in lung cancer patients presenting in the $\mathrm{W}$ or $\mathrm{S}$. These results have important implications for the planning of lung cancer services. Diagnostic services, e.g. outpatient 2 week lung cancer slots, MDTs, bronchoscopy, CT scanning, histology etc, and treatment services, surgery, radiotherapy, and chemotherapy need to be provided on a continual basis throughout the year. In particular in order to meet Government targets they will need to be maintained in the summer, the traditional holiday period for staff.

\section{S105 LUNG CANCER: ARE THE NSF TARGETS FOR RESECTION AND RADICAL RADIOTHERAPY ACHIEVABLE?}

N.J. Ali', G.A. Cox', A. Hastings' ${ }^{1}$, S. Brauer' ${ }^{1}$, C. Butcher', D. Fyfe ${ }^{2}$, D. Beggs', M. Curtis', J. Morley'. 'Kings Mill Hospital, Sutton in Ashfield, UK; ${ }^{2}$ Nottingham City Hospital, UK

The NSF for Lung Cancer has set targets for histology and resection rates for lung cancer against which services can be judged. One report from a tertiary referral centre suggested that resection rates of $25 \%$ were achievable (Laroche, et al. Thorax 1999;53:445-9). We set out to review the demographics and staging of lung cancer patients diagnosed at our DGH (Kings Mill Hospital) to examine the reasons for not obtaining a pathological diagnosis and not operating on patients with stage I and II disease. We have prospectively collected the full BTS/RCP audit data set on all patients diagnosed with lung cancer at our hospital since April 1999. These results are presented.

Over three years between 04/99 and 03/02 we diagnosed 441 cases of lung cancer. The rate of histological confirmation overall was $83.5 \%$. More than $85 \%$ had their management discussed at the weekly Multi Disciplinary Meeting, which included respiratory physicians, a thoracic surgeon and an oncologist.

Small cell lung cancer (SCLC) accounted for $14 \%$ of all cases.

Of the 379 remaining patients 310 had confirmed Non Small Cell Lung Cancer (NSCLC). 299 of these (96\%) were formally staged. The stage distributions are shown below and compared with those reported by Mountain (Mountain. Chest 1997;111:1710-17).

Of those with stages I and II, the surgical resection rate was $42 \%$ and radical radiotherapy rate $28 \%$. Co-morbidity prevented the remaining $30 \%$ from receiving potentially curative treatment. The resection rate for pathologically confirmed NSCLC was 32/ $310=10.3 \%$ or $32 / 379=8.4 \%$ if those without pathologically confirmed disease are included. Our results show that at our hospital patients with lung cancer present with later stage disease than those reported from tertiary referral centers. Selection bias may explain the differences between our results and those previously reported (see above). Even if all those with stage I and II disease underwent surgery we would barely achieve $15 \%$ resection rate.

\section{S106 SURGICAL REFERRAL AND RESECTION RATE FOR NSCLC AT QUEEN ELIZABETH HOSPITAL OVER A 5 YEAR PERIOD: WHY IS IT SO LOW?}

J.I. Whitehouse, N. Jahan, J.R. Webb, T.C. Stokes. Department of Respiratory Medicine, Queen Elizabeth Hospital, Stadium Road, Woolwich, London SE $184 \mathrm{QH}$, UK

Aim: to analyse the referral rate for surgery in stage I and II NSCLC in Queen Elizabeth Hospital Woolwich, from September 1997 up to April 2002

A total of 746 patients were diagnosed with lung cancer between 1997 and 2002. Of these, 458 had histologically proven non-small 


\begin{tabular}{llll} 
Abstract S106 & & & \\
\hline Stage & Not referredReferred & $\begin{array}{l}\text { Resection (\% of stage } \\
\text { group) }\end{array}$ \\
\hline $\begin{array}{l}\text { Stage I } \\
52\end{array}$ & 19 & 33 & $23(44 \%)$ \\
$\begin{array}{l}\text { Stage II } \\
55\end{array}$ & 28 & 27 & $13(24 \%)$ \\
$\begin{array}{l}\text { Stage IIIA } \\
50\end{array}$ & 34 & 16 & $1(2 \%)$ \\
$\begin{array}{l}\text { Total } \\
157 / 458(34.3 \%)\end{array}$ & 81 & 76 & $37 / 458(8 \%)$ \\
\hline
\end{tabular}

cell lung cancer (NSCLC). All were staged and discussed at a multidisciplinary meeting, which does not have the input of a surgeon. Reasons for non-referral of stage I and II patients included poor lung function (13), age (10), WHO health status (5) patient refusal (5) other diseases (3) or a combination of the above (11). The median age for those referred relative to those not referred was 66 and 76 , respectively ( $p<0.0001$, Wilcoxon rank sum). Of the 60 stage I and II patients referred for surgery, $40 \%$ were deemed to be inoperable. Reasons were upstaging by PET scanning (9), age/lung function (3), upstaging at mediastinoscopy/thoracoscopy (5) and surgical opinion (7).

Conclusions: the surgical referral and resection rates for patients with operable cancer on staging criteria remains low. Despite the initiation of multidisciplinary meetings in 1997, fewer than half of the potentially operable patients (stages I, II and IIIA) were referred for surgery. However, nearly half of all stage I and II patients referred for a surgical opinion were refused, predominantly after upstaging. The introduction of a telemedicine link with cardiothoracic surgeons may improve referral rates.

\section{S107 WHAT'S IN I.T. FOR US? USE OF AN I.T. SYSTEM TO INFORM AND IMPROVE A LUNG CANCER SERVICE}

J.M. Hill, A. Lally, P.B. Anderson, B.J. Hutchcroft, P.M. Fisher, B. Entwistle. Chest Clinic, Northern General Hospital, Herries Road, Sheffield S5 7AU, UK

Background: An audit of 100 lung cancer case records in our institution in 1999 demonstrated that only 18\% of cases had an MDT discussion and decision recorded in the medical records, and no regular outcome data were available.

Process: During 2 timeout sessions in 1999 and 2000 lead clinicians in the lung cancer services (diagnostics, thoracic surgery, oncology) mapped the lung cancer care pathway and clinical processes, and identified key stages of the patients care journey. This work was influenced by the need for performance target data and clinical minimum data sets.

In 2000 the Infoflex ${ }^{\mathrm{TM}}$ system was chosen to support the lung cancer services. The implementation required clinicians to define information requirements and outputs.

Outcome: The use of the Infoflex system coincided with the development of a weekly combined lung cancer clinic (Respiratory Medicine, Clinical Oncology and Palliative Care). Prior to the weekly Lung Cancer clinic ( $t=0$ days) a first visit proforma and faxable immediate GP letter are printed, incorporating patient demographics from the hospital PAS system. Data from the proforma are entered on to the Infoflex system immediately after the clinic. Bronchoscopy findings are entered live onto the Infoflex system by clinicians and a bronchoscopy report, GP letter, and histopathology request form are printed. Prior to the MDT meeting ( $t=10$ days) the results of investigations are entered onto the system and an MDT summary sheet is produced. The summary sheet aids decision making in the MDT meeting and serves as a referral letter between specialities. A second clinic visit proforma and immediate faxable GP letter are prepared for the second clinic visit ( $\mathrm{t}=14$ days).

The initial 12 months of complete data demonstate that $92 \%$ of patients diagnosed with lung cancer have an MDT discussion and decision recorded. Reports for any time frame are readily accessible, and present a realtime position of all suspected lung cancer referrals, their progress and outcome. Future plans include the use of projection equipment in the MDT meeting to view the discussion sheet and record decisions realtime, and the use of Infoflex for comparison of data with other units, primarily to ascertain reasons for differences in resection rate.

\section{The genetics of respiratory disease}

\section{S108 AGE-SPECIFIC EFFECT OF THE CYSTIC FIBROSIS MODIFIER GENE, MBL-2}

J.C. Davies' ${ }^{2}$, M. Johnson ${ }^{3}$, C. Booth ${ }^{3}$, K. Fidler ${ }^{3}$, A. Bush', D.M. Geddes², E.W.F.W. Alton ${ }^{2}$, M.W. Turner ${ }^{3}$, N. Klein ${ }^{3}$. 'Department of Paediatric Respiratory Medicine, Royal Brompton Hospital, UK; ${ }^{2}$ Dept of Gene Therapy, Imperial College, UK; ${ }^{3}$ Immunobiology Unit, Institute of Child Health, University College, London, UK

The variable severity of cystic fibrosis lung disease, even in subjects with identical CFTR mutations, has led to the search for non-CFTR modifier genes. Mannose-binding lectin (MBL) is involved in innate defence, both through direct opsonic activity and complement activation. Low-expressing MBL-2 haplotypes were reported in two studies of older CF children and adults to lead to a poor outcome. We have looked for correlations with clinical status in 260 paediatric CF patients. Genomic DNA was analysed for structural mutations (designated 0 ; wild-type $\mathrm{A}$ ) and the low $(\mathrm{X})$ or high $(\mathrm{Y})$ expressing promoter polymorphisms. Patients were grouped based on haplotype which correlated closely with MBL levels (measured by ELISA) into group 1 $(\mathrm{A} / \mathrm{A}, 62.9 \%)$, group $2(\mathrm{YA} / \mathrm{O}, 24 \%)$ and group 3 (XA/O or $\mathrm{O} / \mathrm{O}$, $13.1 \%)$. At the earliest time point available (7.0 (6.9-7.4) years), children in group 3 had a significantly $(p<0.05)$ higher $F E V_{1}$ than those in either of the other groups lgp 3: median [IQR] 105.5 $(83 ; 113) \%$; gp 2: $85.5(78 ; 103) \%$; gp 1: 91 (76;105)\%). At the age of 9 , a similar trend was seen $(p=0.055)$. At approximate ages of 11 , 13 and 15 years, no difference was seen in either parameter between groups. Annual rate of decline was not affected by MBL status (gp 3: $-3.4(-6.7 ; 0.3) \%$; gp 2 : $-3.5(-5.9 ;-0.8 \%$ gp $1:-3.0(-6.4$; $0.7) \%)$. Infection with $P$ aeruginosa increased the rate of decline, but affected each group equally. In contrast to adult studies, low MBL is not detrimental in childhood CF. The surprising finding that children with the lowest $M B L$ levels in fact have higher lung function early in life may relate to the complex role of this protein in the inflammatory response. The loss of effect later is likely due to the increased protease activity on the airway surface with advanced disease. The most obvious difference between our group and those previously reported relates to year of birth, other groups having been born up to 20 years earlier. Over this time, CF treatment has progressed rapidly, possibly reducing the importance of certain host factors. These results highlight the importance of considering such environmental and treatment factors when studying modifier genes in CF.

\section{S109 POLYMORPHISMS IN THE HYDROPHILIC SURFACTANT PROTEINS A AND D ARE MODIFIERS OF LIVER INVOLVEMENT BUT NOT LUNG DISEASE IN CHILDREN WITH CYSTIC FIBROSIS}

J.C. Davies' ${ }^{3}$, P. Pantelidis' ${ }^{2}$ A. Lagan², R. Du Bois' ${ }^{2}$, K. Welsh'2, D.M. Geddes $^{3}$, E.W.F.W. Alton ${ }^{3}$. 'Dept of Gene Therapy and ${ }^{2}$ Clinical Genomics Unit, Imperial College, UK; ${ }^{3}$ Dept of Paediatric Respiratory Medicine, Royal Brompton Hospital, London, UK

The surfactant proteins $A$ and $D$ are members of the collectin family involved in the innate immune system. Polymorphisms within SP-A have been associated with infant respiratory distress syndrome and severe RSV bronchiolitis. We have explored a modifying effect of these proteins on disease phenotype in children with CF. A sequence specific primer-PCR methodology was employed which enabled the identification of all known allelic variants on SP-A1, SP-A2 and SP-D genes directly from genomic DNA samples. Clinical data collected included lung function at defined ages, infection with common CF pathogens, and the presence of liver disease on ultrasound. Data were avaliable on 241 children at a mean (SEM) age of 8.5 (0.3) years. No correlation was seen between any haplotype and lung function at any age, risk of infection, use of IV antibiotics or age at diagnosis. However, in children with liver disease $(n=19)$ both the SPA-1 allele $6 \mathrm{~A}^{2}$, and the SP-A2 allele, $1 \mathrm{~A}^{\circ}$, were significantly overrepresented $16 \mathrm{~A}^{2} / 6 \mathrm{~A}^{2} 68 \%$ v $33 \%, 6 \mathrm{~A}^{2} /$ non- $6 \mathrm{~A}^{2} 32 \%$ v $47 \%$, non $6 A^{2} /$ non $6 A^{2} 0 \%$ vs $19 \%, p<0.01 ; 1 A^{0} / 1 A^{0} 63 \% \vee 34 \%$, 
$1 \mathrm{~A}^{0} /$ non $1 \mathrm{~A}^{0} 32 \% \vee 47 \%$, non $1 \mathrm{~A}^{0} /$ non $1 \mathrm{~A}^{0} 5 \%$ v $\left.18 \%, \mathrm{p}<0.05\right)$. A significant but less pronounced relationship was seen with the SP-D group possessing both the $11 \mathrm{~T}$ and $160 \mathrm{~A}$ polymorphisms $(\mathrm{p}<0.05)$. The lack of an association between these proteins and lung disease may either reflect redundancy in the host defence system, or enzymatic destruction of these proteins after release. The association with liver disease was unexpected and is interesting. Inflammation has been described in CF liver disease, although whether this is primary or secondary remains uncertain. The surfactant proteins have recently been identified in many extra-pulmonary sites including the the gastrointestinal tract, to date, only SP-D has been found in the biliary tree. These data suggest a role for inflammation and host defence proteins in the development of CF liver disease, and if further work is confirmatory, may allow identification of a subgroup at risk of this complication.

\section{S1 10 PRELIMINARY IDENTIFICATION OF GENETIC LOCI ASSOCIATED WITH HIGH ALTITUDE PULMONARY HYPERTENSION BY ASSOCIATION MAPPING}

L. Long, A.A. Aldashev, A. Hensiek, S. Eddahibi, S. Adnot, R.C. Trembath, M.R. Wilkins, N.W. Morrell. University of Cambridge School of Clinical Medicine, Department of Medicine, Addenbrookes' and Papworth Hospitals, Cambridge CB2 2QQ, UK

Hypoxia-induced pulmonary hypertension is observed in residents at high altitudes and in patients with hypoxic lung diseases, such as chronic obstructive pulmonary disease. Well documented differences between individuals, and between high altitude populations, in susceptibility to pulmonary hypertension in low oxygen environments, suggest that genetic factors may play a role. To identify genes conferring susceptibility to hypoxia-induced pulmonary hypertension in native highlanders, we performed an ECG survey of the inhabitants (age 16 to 75, $n=741$ ) of 3 villages in Kyrgyzstan, at an altitude of 2800 to $3100 \mathrm{~m}$ above sea level. Subjects with and without ECG signs of cor pulmonale underwent echocardiography to define groups of highlanders with and without pulmonary hypertension (defined by mean pulmonary arterial pressure $>25 \mathrm{mmHg}$ ). DNA samples were obtained from 30 cases and 30 controls. We used a DNA pooling technique and performed a whole genome screen using 811 microsatellite markers (Applied Biosystems high density linkage mapping set LMS-HD5), allowing a resolution of 5-10cM across the genome. Association mapping identified alleles that occured with significantly different frequency between cases and controls. Fiffeen markers showed significantly different frequencies $(p<0.05)$ on chromosomes $1,3,5,6,7,8,15,17$, and 20. An initial search for candidate genes identified the locus for the serotonin transporter (5HTT) lying in close proximity $(2 \mathrm{mB})$ to the marker on chromosome 17. A polymorphism in the promoter of the $5 \mathrm{HTT}$ gene $(\mathrm{L} / \mathrm{S})$ is known to alter the expression of $5 \mathrm{HTT}$ and is associated with primary pulmonary hypertension. The frequency of the LL genotype, which increases $5 \mathrm{HTT}$ expression and activity, was $24 \%$ in the group with high altitude pulmonary hypertension compared with $12 \%$ in the controls. These results require confirmation in a larger cohort of cases and controls, and refinement of the chromosomal loci to aid in the identification of further candidate genes. Nevertheless, this study provides a novel approach to the identification of genes that confer susceptibility to hypoxia-induced pulmonary hypertension.

Funding: Wellcome Trust and British Heart Foundation.

\section{S111 SMOKING RELATED EMPHYSEMA AND SMALL AIRWAYS DISEASE HAVE INDEPENDENT GENETIC RISK FACTORS IN COPD}

B.D. Patel, A. Tasker, N. Screaton, W. Anderson, S. Sharma, E.K. Silverman, D.A. Lomas. Departments of Medicine and Radiology, University of Cambridge, UK; GlaxoSmithKline, North Carolina, USA; Channing Laboratory, Brigham and Women's Hospital, Boston, USA

We previously have shown that COPD clusters within families suggesting that shared genetic factors predispose some smokers to airflow obstruction. It is unknown whether these genetic factors contribute to the airway disease and/or emphysema components of COPD. This has been addressed as part of the GSK COPD Genetic International Network designed to identify the genes that predispose smokers to COPD. The network has recruited probands aged 45-65 with an FEV $<60 \%$ predicted and $>5$ pack years smoking history in the absence of $\mathrm{PiZ} \alpha_{1}$-antitrypsin deficiency. Siblings of the proband with $a>5$ pack year smoking history were screened by spirometry for airflow obstruction. An HRCT scan was conducted on all consenting probands and siblings and the extent and distribution of emphysema was quantified by two independent radiologists. To date the network has recruited approximately 2400 individuals from over 800 families. 350 individuals have been recruited from 105 families by the Cambridge centre and this cohort has been used to define the familial risk of airways disease and emphysema. Family phenotype was determined by the extent of airways disease or emphysema in the proband. A stronger correlation was found between pack years smoked and $\mathrm{FEV}_{1} \%(\mathrm{r}=-0.62, \mathrm{p}=0.003)$ in the 20 siblings from families in whom the proband had predominantly airways disease $(<5 \%$ emphysema) than in the 84 sibs from families where the proband had significant emphysema $(r=-0.32, p=0.0001)$. The familial risk for emphysema was assessed in 89 sibs ( 47 men and 42 women) from 49 families in whom the proband and at least 1 sibling had an HRCT. The siblings of probands with $10-25 \%$ emphysema had an increased risk for significant $(>5 \%$ ) emphysema (unadjusted OR $4.64, p=0.05$ ) when compared to siblings of probands with $<10 \%$ emphysema. This risk was independent of pack years smoked but was significantly greater in women (OR 3.58, $p=0.03$ ). In conclusion, our data show that individuals with COPD have a genetic susceptibility to airways disease that is dependent on the pack years smoked. However emphysema develops in genetically susceptible individuals who smoke in a manner that is less dose dependent than airways disease. Finally women may be more susceptible to emphysema than men if they smoke.

\section{S1 12 IMMUNOGENETICS OF LABORATORY ANIMAL ALLERGY}

H. Jeal, A. Draper, M. Jones, J. Harris, K. Welsh', A. Newman Taylor, P. Cullinan. Department of Occupational and Environmental Medicine and 'Interstitial Lung Disease Unit, Imperial College (NHLI), London, UK

Laboratory animal allergy is a common occupational health problem affecting approximately $30 \%$ of the exposed population. Allergic reactions to rats or mice are most common, probably because these animals are most frequently used in experimental studies. HLA class II molecules are involved in the presentation of allergen to the T cell and are therefore likely candidates for controlling the immune response. We hypothesised that HLA class II molecules might be associated with sensitisation to rat urinary protein among individuals exposed to laboratory animals.

We undertook a cross sectional study of 741 employees in contact, at work, with laboratory rats at 6 pharmaceutical sites across the UK. 109 cases (defined as having a skin prick test wheal $\geqslant 3 \mathrm{~mm}$ to rat urine and/or a rat urine RAST >2\%binding) and 397 non-sensitised referents were HLA typed for DRBI and DQBI loci. Participants were asked to complete a questionnaire enquiring into symptoms, exposure, and job history.

After adjustment for independent risk factors, HLA-DR7 was found to be associated with sensitisation (OR $1.99 \mathrm{Cl}$ 1.91-3.27), workrelated chest symptoms $(O R 2.98 \mathrm{Cl} 1.66-5.36)$ and sensitisation with symptoms (OR $4.81 \mathrm{Cl} 2.29-10.13)$. HLA-DR3 was protective against sensitisation (OR $0.55 \mathrm{Cl} 0.31-0.98$ ). Atopy and exposure proved to be more strongly associated with sensitisation and sensitisation with symptoms than HLA.

Amino acid analysis of the associated HLA molecules provides a biologically plausible explanation for these associations.

\section{S1 13 MATERNAL BUT NOT PATERNAL GENETIC VARIATION IN GSTPI IS ASSOCIATED WITH ASTHMA PHENOTYPES IN CHILDREN}

F. Child', W. Lenney', S. Clayton', S. Davies', P.W. Jones², J. Alldersea ${ }^{3}$ R.C. Strange ${ }^{3}$, A.A. Fryer ${ }^{3}$. 'Academic Department of Paediatrics, North Staffordshire Hospital, Stoke on Trent, UK; ${ }^{2}$ Department of Statistics, University of Keele, UK; ${ }^{3}$ School of Medicine, Keele University, North Staffordshire Hospital, Stoke on Trent, UK

Background: Maternal factors including atopy and smoking during pregnancy are associated with the risk of developing asthma in childhood. Suggested mechanisms include transmission of specific maternal alleles to the child and maternal influences on the intrauterine environment. We have previously shown that polymorphism in glutathione S-transferase, GSTP1 is associated with asthma, airway hyperresponsiveness (AHR) and atopy in adults. We now hypothesise that GSTP1 genotypes in the both mother and child, but not the father, mediate asthma phenotypes in the child.

Methods: 145 Caucasian families were recruited via an asthmatic proband aged 7-18 years. Atopy and asthma were assessed using a 
questionnaire, skin prick testing, total serum lgE, spirometry and methacholine challenge testing. Phenotypic data were analysed as continuous and categorical variables. Bronchial challenge data were corrected for age, gender, height and baseline lung function. GSTP 1 genotyping was determined using PCR.

Results: GSTP1 $\mathrm{Val}^{105} / \mathrm{Val}^{105}$ genotype in the child was weakly associated with a reduced risk of atopy $(p=0.038)$ and AHR $(p=0.069)$. In mothers $(p=0.026)$ but not fathers $(p=0.407)$, GSTP 1 $\mathrm{Val}^{105} \mathrm{Nal}^{105}$ was associated with a reduced risk of AHR in the child. This was independent of the child's genotype, maternal and child atopic status, maternal smoking during pregnancy, and transmission of parental GSTP1 alleles.

Conclusion: For the first time, we have shown an association between maternal genotype and the child's asthma phenotype that is not due to transmission of specific maternal alleles to the child. This suggests an in utero effect of maternal genotype on the child and adds new insights into the mechanisms by which maternal factors may influence the development of asthma in childhood.

\section{Asbestos and the pleura}

\section{S114 DIFFUSE ASBESTOS-RELATED PLEURAL FIBROSIS; A POOR GUIDE TO HEAVY DUST EXPOSURE?}

\section{McGavin, K Smith, L Sykes. Derriford Hospital, Plymouth, UK}

There has been an assumption that diffuse pleural fibrosis (DPF) is an indicator of heavy dust exposure. 'This has considerable implications in the decision as to whether a given lung cancer has been caused by asbestos. The UK Industrial Injuries Advisory Council ${ }^{2}$ consider that lung cancer in the presence of DPF is an industrial tumour, but not in the presence of pleural plaque (PP).

Method: We have tested the hypothesis that DPF is a marker of heavy exposure by comparing estimated asbestos burden in 192 workers from the Devonport Dockyard, 96 with PP and 96 with DPF (43 bilateral). Dust burden was calculated from previously published data of exposure to asbestos by individual trades within the Yard multiplied by years spent in that trade prior to 1972. Detailed occupational histories were taken by one experienced observer who had also read all the radiographs (CMcG).

Results: There were no differences between the groups in terms of age or time since first exposure. There were no differences in estimated dust burden between men with PP and DPF (independent samples $t$ test, $D F$ adjusted for unequal variances, $t=1.045, D F=179$, $\mathrm{p}=0.3$, nor between PP and unilateral and bilateral DPF analysed separately, using logs of asbestos burden because of unequal variances $(F 2,189=2.56, p=0.08)$. However there was evidence that men with bilateral DPF had had more exposure than those with unilateral $(t=2.86, D F=88, p=0.004$, t-test adjusted for unequal variances).

Conclusion: We found no evidence that men with DPF have had a greater exposure to asbestos than men with PP, and conclude that there is no justification for using the presence of DPF as an indicator of heavy exposure, for example in qualifying a lung cancer for industrial status. Bilateral DPF suggests a heavier dust burden than unilateral.

1. International Expert Meeting on Asbestos, Asbestosis \& Cancer: the Helsinki Critera. Scand J Work Environ \& Health 1997;23:311-16.

2. IIAC. Asbestos-related disease without Asbestosis. HMSO 1984, C9184.

\section{S1 15 TRANSFORMING GROWTH FACTOR BETA (TGF $\beta$ ) INDUCES MITOGEN-ACTIVATED PROTEIN KINASE (MAPK) SIGNALLING IN MALIGNANT MESOTHELIOMA CELLS}

M.K. Wood, K.S. Abayasiriwardana, G.J. Laurent, S.E. Mutsaers, R.J. McAnulty. Centre for Respiratory Research, University College London, London WCIE 6JJ, UK

Malignant Mesotheliomas ( $M M)$ are fibrous tumours containing abundant collagen. MM cells produce collagen as well as TGF $\beta$, which is a potent inducer of collagen synthesis. We have shown that antibody neutralisation of TGF $\beta$ or inhibition of collagen synthesis using a proline analogue inhibits collagen production in $M M$ cells and reduces tumour growth. TGF $\beta$ signals predominantly via the Smad pathway, but also through the MAPKs. Over-expression of Smad7, the natural inhibitor of the Smad pathway, blocks collagen production in fibroblasts. However, in MM cells, we demonstrated enhanced collagen production and tumour growth following transfection with Smad7. From these findings we hypothesised that TGF $\beta$-induced collagen production by $M M$ cells occurs via MAPK signalling. To assess this hypothesis we performed Western blotting to determine if TGF $\beta$ stimulated activation of two components of the MAPK pathway, ERK1/2 and p38 kinase, in $M M$ cells. The effect of blocking these pathways,

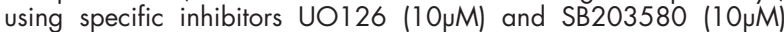
respectively, on collagen production was assessed by measuring hydroxyproline levels using HPLC (nmol hydroxyproline/10 cells \pm SEM)

Results: Exogenous TGF $\beta$ activated both ERK 1/2 and p38 kinase in $M M$ cells within $5 \mathrm{~min}$. Blocking ERK $1 / 2$ activity increased procollagen production basally (control $0.26 \pm 0.01, \mathrm{UO} 126 \quad 0.49 \pm 0.02$, $\mathrm{p}<0.01$ ) and after TGF $\beta$ stimulation (control $0.58 \pm 0.004$, UO126 $0.72 \pm 0.02, p<0.01)$. However the fold increase in collagen production in response to TGF $\beta$, was 2.3 and 1.5 times basal levels in control and $\mathrm{UO} 126$ treated groups respectively, suggesting a decrease in the response to TGF $\beta_{1}$. Blocking p38 kinase activity had a small inhibitory effect on collagen production basally (control $0.48 \pm 0.02$ SB203508 $0.39 \pm 0.02, p<0.01$ ) and after TGF $\beta$, (control 0.59 \pm 0.01 , SB203508 0.47 $\pm 0.04, p<0.02$ ).

Conclusions: (1) the ERK1/2 and p38 kinase MAPK pathways are rapidly activated by TGF $\beta$ in these cells, which may be important in MM tumorigenesis; (2) MAPKs appears to have a role in TGF $\beta$ stimulated collagen production by MM cells but do not appear to be the major route of signalling in this response. Further elucidation of the TGF $\beta$ signalling pathways involved in the regulation of collagen synthesis and the specific roles of the MAPKs in MM may identify unique pathways in this tumour, which might be exploited as potential therapeutic targets.

This work was funded by Cancer Research UK and British Lung Foundation.

\section{S116 IMMUNOHISTOCHEMICAL PROGNOSTIC MARKERS IN MALIGNANT MESOTHELIOMA}

N. Chaudhuri, I. Cawkwell, M.E. Cowen, A.P. Campbell, M.J. Lind. Academic Depts. of Oncology and Surgery, Castle Hill Hospital, Cottingham, UK

Objective: A nihilistic attitude exists towards malignant mesothelioma of the pleura (MMP) as most people die within a year of diagnosis regardless of treatment or palliation. Some patients do survive longer, multimodality treatment has increased survival in some centres and there are promising novel therapies on the horizon. Immunohistochemical markers have shown promise in predicting survival in other solid tumours and they could be used to select patients for further treatment in MMP.

Methods: Archival specimens were identified from a pathological database. Immunostaining was carried out with p53, proliferative markers-MIBI and PCNA and apoptotic markers-Bcl2 and BAX. Up to 90 blocks analysed from 74 patients (M:F-66:8, median age 67.7 years) diagnosed with malignant mesothelioma between 1997 and January 2001

Results: Median and mean survivals were 218 and 314 (SD331) days respectively. Patients were divided into two groups. See table.

\begin{tabular}{|c|c|c|c|}
\hline & $\begin{array}{l}\text { Survival } \\
\geqslant 10 \text { mths } \\
\text { Mean (SD) }\end{array}$ & $\begin{array}{l}\text { Survival } \\
<10 \text { mths } \\
\text { Mean (SD) }\end{array}$ & $\mathrm{p}$ Value \\
\hline Age (years) & $64.1(10.2)$ & $68.5(9.8)$ & 0.021 \\
\hline MIB + ve stain & 43.8 (14.9) & $78.3(12.2)$ & $<0.001$ \\
\hline PCNA + ve stain & $79.4(14.4)$ & 75.5 (14.4) & NS \\
\hline BAX score & $4.7(1.6)$ & $4.7(1.1)$ & NS \\
\hline $\mathrm{Bcl} 2$ score & $0.9(1.4)$ & $1.2(1.3)$ & NS \\
\hline P53 score & $3.8(1.3)$ & $4.0(1.3)$ & NS \\
\hline P53 stain & $57.0(18.3)$ & $51.8(19.4)$ & NS \\
\hline $\begin{array}{l}\text { Histological } \\
\text { subtype }\end{array}$ & $\begin{array}{l}\text { Mean Survival } \\
\text { (days) }\end{array}$ & SD & $\mathrm{p}$ Value \\
\hline Epithelial & 425.8 & 277.2 & $<0.001$ \\
\hline Biphasic & 212.3 & 162.5 & \\
\hline Sarcomatous & 127.8 & 113.2 & \\
\hline
\end{tabular}


Conclusions: Age and histological subtype influence survival in malignant mesothelioma as has been shown in other studies. In our study we found MIBI could predict long-term survival in patients with MMP.

\section{S117 SURVIVAL IN SURGICALLY DIAGNOSED PATIENTS WITH MALIGNANT MESOTHELIOMA IN CURRENT PRACTICE}

C. Tan', S. Swift', C. Gilham ${ }^{3}$, S. Shaefi', W. Fountain ${ }^{2}$, J. Peto ${ }^{3}$, T. Treasure'. 'Thoracic Unit, Guy's Hospital, London, UK; ${ }^{2}$ Thoracic Unit, Harefield Hospital, Harefield, UK; Institute of Cancer Research, London, UK

Objective: There is an epidemic of malignant mesothelioma in Europe that presents a challenge to thoracic surgeons. The median survival is quoted as five months ( 150 days) but reports are from ten or more years ago. To evaluate results of new treatments we set out to establish survival statistics for current practice.

Methods: We searched back five years in the pathology database of our two hospitals for all pleural biopsies in which mesothelioma was diagnosed.

Results: We found a total of 426 cases and report on 409 where we know vital status (table). Survival was 174 days (82\%) longer at one hospital than the other. This difference is not explained by histological type, sex, or age. Epithelioid type and age (younger) were associated with better survival as is consistently shown.

Conclusion: Claims for improved treatments are made with reference to historical data for the natural history of the disease. Not only are survival times (from diagnosis) longer than those quoted but the difference between two hospitals is far greater than the likely gain from any novel or more radical therapy. The possible explanations include lead time bias due to earlier referral in Harefield as opposed to Guy's or differences in histological diagnostic threshold. Whatever the explanation it illustrates the need for a contemporaneous control group and our data emphasise the case for a randomised controlled trial.

\section{S1 18 ACTIVATION OF PROTEASE ACTIVATED RECEPTOR-2 IN MESOTHELIAL CELLS: A NOVEL MECHANISM OF PLEURAL INFLAMMATION}

Y.C.G. Lee, D.A. Knight, K.B. Lane, D.S. Cheng, M.A. Koay, I.R. Teixeira, P.J. Thompson, R.W. Light. Vanderbilt University and St. Thomas Hospital, Nashville, TN, USA; Wellcome Trust Centre for Human Genetics, Oxford University, UK; University of Sao Paulo, Brazil; Asthma and Allergy Research Institute and University of Western Australia, Australia.

Pleural inflammation underlies a vast range of pleural diseases, but its mechanism remains poorly understood. Protease activated receptor (PAR)-2 is a novel seven-transmembrane $G$ protein-coupled receptor. We recently showed that PAR-2 are present in abundance on human pleural mesothelial cells, and stimulation of PAR-2 in vitro resulted in significant release of inflammatory cytokines (AJRCCM 2002; 165:A606)

We hypothesised that PAR-2 in mesothelial cells has a functional role in pleural inflammation in vivo.

$\mathrm{C} 57 \mathrm{BL} / 6$ mice were given a single intrapleural injection of $10 \mathrm{mg} / \mathrm{kg}$ of SLIGRL-NH2 (a specific PAR-2 activating peptide), or $10 \mathrm{mg} / \mathrm{kg}$ of LSIGRL-NH2 (control peptide), or the vehicle, phosphatebuffered saline (PBS). At 4 hours, the mice were sacrificed. Cytokines were measured with ELISA. Neutrophils were counted on cytospin slides (Geimsa stains).

Pleural fluid MIP-2 levels were significantly higher in mice injected with SLIGRL-NH2 $(2710 \pm 165 \mathrm{pg} / \mathrm{mL})$ than in those given control peptide $(880 \pm 357 \mathrm{pg} / \mathrm{mL})$ or PBS $(88 \pm 46 \mathrm{pg} / \mathrm{mL}), p<0.001$. Similarly, pleural fluid TNF $\alpha$ was significantly higher in the SLIGRL-NH2 group. In the SLIGRL group, the neutrophil counts in the pleural fluid were significantly higher than the control peptide (by 40 fold) and the PBS groups (by 26 fold). The MIP-2 and TNF $\alpha$ levels were 15- and 4-fold higher in the pleural fluid than in serum, consistent of local pleural production. There were no differences in the serum levels of these cytokines among the three groups. The MIP-2 and TNF $\alpha$ levels were strongly correlated in the pleural fluids $(r=0.92, p<0.00001)$ and in serum $(r=0.76, p<0.01)$. There were no differences in the pleural fluid volume or in its VEGF concentrations among the three groups.

This study is the first to show a functional role for PAR-2 in the pleura. Our results confirmed that activation of PAR-2 in mesothelial cells in vivo resulted in significant production of pro-inflammatory cytokines with resultant neutrophil recruitment into the pleural cavity.

\section{Asthma mechanisms II}

\section{S119 INCREASED SUB-EPITHELIAL PROTEOGLYCANS IN THE AIRWAYS OF MICE FOLLOWING OVALBUMIN SENSITISATION AND CHALLENGE}

A.K. Reinhardt, S.E. Bottoms, G.J. Laurent, R.J. McAnulty. Centre for Respiratory Research, University College London, London WCIE 6JJ, UK

The sub-epithelial thickening of asthmatic airways is manifested by increased fibroblast/myofibroblast proliferation and deposition of extra-cellular matrix components including collagens and proteoglycans. The degree of proteoglycan deposition has been correlated with airway responsiveness. Existing murine models of asthma, including our own, have demonstrated increased amounts of airway sub-epithelial collagen following ovalbumin sensitisation and challenge. However, we are not aware of any studies examining altered airway proteoglycan deposition in an asthma model. In this study we have investigated changes in proteoglycan deposition in sections of murine lung using a selective histological stain and computer-assisted image analysis.

Wild-type SV129/C57BL/6 mice were sensitised by intraperitoneal injection of $10 \mu \mathrm{g}$ of ovalbumin in $0.1 \mathrm{ml}$ saline on two occasions 10 days apart. 21 days after the second sensitisation mice were challenged with $400 \mu g$ of ovalbumin in $50 \mu$ l saline by intra-tracheal instillation daily for 6 days. Control mice were sham sensitised/sham challenged. 12 days after the final challenge mice were killed. Lungs were inflated with a 1:3 embedding matrix:saline mixture at a pressure of $25 \mathrm{~cm}$ water, set in embedding matrix and frozen in liquid nitrogen for histological analysis. $7 \mu \mathrm{m}$ frozen sections were stained overnight using cupromeronic blue and a critical electrolyte concentration of $250 \mathrm{mM}$ magnesium chloride. Sub-epithelial proteoglycan staining was quantitated using a computer-assisted image analysis system. Airways were selected using pre-defined criteria and each airway was examined using separate colour thresholding for lumen and sub-epithelial proteoglycans. Results were expressed as amount of proteoglycan per unit airway lumen perimeter.

Lung sections from a total of 19 animals were examined (10 ovalbumin sensitised/challenged and 9 controls). A total of 52 ovalbumin sensitised/challenged and 32 control airways were analysed. The mean area of proteoglycan/ $\mathrm{mm}$ airway perimeter was $5.46 \pm 0.39 \mu \mathrm{m}^{2}$ in the ovalbumin sensitised and challenged group and $4.13 \pm 0.44 \mathrm{um}^{2}$ in the sham sensitised/sham challenged group $(p=0.03)$. This represents a mean increase of $32 \%$ in sub-epithelial proteoglycan deposition following ovalbumin sensitisation and challenge. Interestingly, in view of the association between proteoglycans and collagen fibril assembly, sub-epithelial collagen is increased by $33 \%$ using the same sensitisation/challenge protocol. We conclude that the increase in sub-epithelial proteoglycan and collagen deposition found in the airways of asthmatics can be reproduced in mice following ovalbumin sensitisation and challenge and that this may be a useful model to assess the mechanisms regulating sub-epithelial airway remodelling.

Work funded by the Wellcome Trust.

Abstract $\mathrm{S} 117$

\begin{tabular}{llllllll}
\hline Hospital & $\begin{array}{l}\text { Number of survival } \\
\text { (days) }\end{array}$ & $\begin{array}{l}\text { Dases to } \\
\text { cas\% dead }\end{array}$ & $\begin{array}{l}\text { Days to } \\
\text { 75\% dead }\end{array}$ & $\begin{array}{l}\text { Age median } \\
\text { (IQR) }\end{array}$ & Males & $\begin{array}{l}\text { Epithelioid } \\
\text { histology }\end{array}$ \\
\hline Guy's Hospital & 175 & 213 & 93 & 478 & $67(59-74)$ & $80 \%$ & $54 \%$ \\
Harefield Hospital & 234 & 387 & 162 & - & $68(62-74)$ & $83.4 \%$ & $58 \%$ \\
\hline
\end{tabular}




\section{S120 WNT SIGNAL TRANSDUCTION IN ADULT BRONCHIAL EPITHELIAL CELLS}

M.D. Steel, D.E. Davies, S.M. Puddicombe, S.T. Holgate, J.E. Collins. Division of Inflammation, Infection and Repair, School of Medicine, University of Southampton, Southampton, SO16 6YD, UK

Wnts are a highly conserved family of secreted glycoproteins that play a fundamental role in cell fate determination and tissue morphogenesis during embryonic development. 'Through binding to members of the Frizzled (Fzd) receptor family, class- 1 Wnts induce the accumulation of hypophosphorylated $\beta$-catenin by inhibiting the GSK-3 $\beta$ /axin/ APC destruction complex. Consequent translocation of $\beta$-catenin to the nucleus gives rise to activation of TCF/LEF-1 transcription factors, leading to expression of genes involved in cell migration (CD44, MMP 7 and proliferation (c-myc, cyclin-D1). In mammalian embryonic lung, activation of this "canonical" pathway in airway epithelial cells has been implicated in the process of branching morphogenesis, and Wnt secretion by underlying mesenchymal cells is thought to play a key role. ${ }^{2}$ Despite reports that several WNT and FZD genes are expressed in adult human lung tissue, very little is known about which cells are involved, and their functional significance remains unclear. Using an RNAse protection assay, we have identified expression of FZD $-2,-3,-5$ and -6 in both H292 and primary bronchial epithelial cells. In addition, these cells also express the gene encoding secreted Frizzled receptor protein-1 (SFRP1), the product of which is capable of modulating Wnt signals in the extracellular compartment. Postulating that human airway epithelial cells retain the ability to transduce a canonical Wnt signal in adult life, we employed RT-PCR and observed expression of TCF-4 mRNA in primary cells, with weaker expression of TCF-3, but no detectable message for TCF-1 or LEF-1. Using a TCF reporter construct (TOPFLASH) in $\mathrm{H} 292$ cells, we demonstrate repression of TCF transcription at baseline, with activation induced by stimulation with lithium, an inhibitor of GSK-3 $\beta$ and mimicker of class-1 Wnt activity. Our data supports our hypothesis, and we speculate that reactivation of this morphogenetic pathway in adult human lung may play an important role in airway epithelial regeneration, as well as remodelling in airways disease.

This study is funded by: Medical Research Council (UK) G84/5708

1. Wodarz, et al. Ann Rev Cell Dev Biol 1998;14:59-88.

2. Tebar, et al. Mech Dev 2001;109:437-40.

\section{S121 FACTORS INFLUENCING CROSS SECTIONAL AND LONGITUDINAL ASSOCIATIONS BETWEEN EXHALED NITRIC OXIDE AND INDUCED SPUTUM EOSINOPHIL COUNT IN ADULTS WITH ASTHMA}

M.A. Berry, R.H. Green, A.J. Wardlaw, I.D. Pavord. Glenfield Hospital, Groby Road, Leicester LE3 9QP, UK

There is increasing evidence that a management approach that includes monitoring airway inflammation in asthma leads to an improved outcome. Induced sputum eosinophil count and exhaled nitric oxide (NO) concentration are both potential non-invasive measures of airway inflammation, although exhaled $\mathrm{NO}$ is more suited to serial measurements. Little is known about the relationship between these two measurements and the factors which influence it. We have investigated the relationship in 246 non-smoking and 75 smoking adults with stable asthma of variable severity who had both exhaled $\mathrm{NO}$ and induced sputum eosinophil counts measured on the same visit. We have also examined the relationship between change in sputum eosinophil count and exhaled NO in 75 patients with moderately severe asthma who were participating in a prospective longitudinal study and had 9 paired measurements over the period of one year. We found a significant positive correlation between exhaled NO and sputum eosinophil count in non-smokers $(r=0.461, p<0.001)$ and between change in the variables $(r=0.262, p<0.001)$. There was no significant correlation between the two measurements in smokers. Within the non-smoking group the correlation was stronger in: males $(r=0.637, p<0001)$ than females $(r=0.387, p<0.001)$, in those not on inhaled steroids $(r=0.497, p<0.001)$ compared to those who were $(r=0.385, p<0.001)$ and in atopic $(r=0.605, p<0.001)$ compared to non-atopic patients $(r=0.366, p<0.001)$. The relationship between change in sputum eosinophil count and exhaled $\mathrm{NO}$ was also stronger in males $(r=0.303, p<0.001)$ than females $(r=0.183, p=0.016)$. Smoking, gender, atopy and inhaled steroid use have a significant impact on the relationship between exhaled NO and sputum eosinophil counts in cross sectional analysis. In both cross sectional and longitudinal analysis there is a marked difference in the relationship between exhaled NO and sputum eosinophil count in males and females. This could be due to the effect of female sex hormones on NO-synthase. The relationship between the variables is much closer in cross sectional study than between change in the variables, suggesting that they identify a common airway abnormality but are regulated differently by factors that alter airway inflammation.

\section{S122 SIMVASTATIN HAS AN ANTI-INFLAMMATORY EFFECT IN A MURINE MODEL OF ALLERGIC ASTHMA}

A. McKay' 2 , B.P. Leung', I.B. Mclnnes', S. Culshaw', N.C. Thomson², F.Y. Liew'. 'Departments of immunology and ${ }^{2}$ Respiratory Medicine, Western Infirmary and University of Glasgow, UK

Introduction: Asthma is an eosinophilic inflammatory airways disease. There is increasing evidence that statins, such as simvastatin, have anti-inflammatory properties which are unrelated to their lipid-lowering activity. We therefore wished to study the effect of simvastatin in a murine model of asthma.

Methods: BALB/c mice primed with ovalbumin (OVA) were re-challenged with OVA on three consecutive days. Simvastatin $40 \mathrm{mg} / \mathrm{kg}$ or $4 \mathrm{mg} / \mathrm{kg}$ or vehicle control were given intraperitoneally (i.p.) at the time of these challenges. Analysis was done one day after the last challenge.

Results: Simvastatin treatment at a dose of $40 \mathrm{mg} / \mathrm{kg}$ i.p. resulted in a significant reduction in bronchoalveolar lavage (BAL) total cellularity (mean \" SD: simvastatin 17.9 \" $5.53 \times 10^{4} / \mathrm{ml} v$ vehicle control $39.4(15.2) \times 10^{4} / \mathrm{ml}, \mathrm{p}<0.01$ ) and eosinophilia (simvastatin $5.59(3.17) \times 10^{4} / \mathrm{ml} v$ vehicle control $19.4(9.92) \times 10^{4} / \mathrm{ml}, \mathrm{p}<$ $0.01)$. Both high and low dose i.p. simvastatin treatment were associated with a reduction in BAL interleukin (IL)- 4 and IL-5 levels and also in OVA-induced IL-4 and IL-5 production in thoracic lymph node (LN) cultures. See table.

Reduced inflammation was observed in lung histology in the simvastatin-treated mice. Serum OVA-specific lgG1, lgG2a, and total IgE levels were unaltered by simvastatin treatment.

Conclusion: These results demonstrate that simvastatin has anti-inflammatory effects in this murine model of allergic asthma.

\begin{tabular}{llll} 
Abstract S122 & & \\
\hline Mean (SD) & $\begin{array}{l}\text { Simvastatin } \\
40 \mathrm{mg} / \mathrm{kg} \text { i.p. }\end{array}$ & $\begin{array}{l}\text { Simvastatin } \\
\text { 4mg/kg i.p. }\end{array}$ & Vehicle control \\
\hline BAL & & & \\
II-4 (pg/ml) & $13.2(21.9)^{* *}$ & $26.0(20.2)^{*}$ & $52.6(32.2)$ \\
LL-5 (pg/ml) & $57.8(30.2)^{* *}$ & $90.2(34.5)^{* *}$ & $219.3(52.2)$ \\
LN & & & \\
IL-4 (pg/ml) & $55.1(15.1)^{* *}$ & $99.8(18.3)^{*}$ & $160.9(11.8)$ \\
IL-5 (pg/ml) & $510(40)^{* *}$ & $1096(76.5)^{* *}$ & $1747(239)$ \\
\hline i.p., intraperitoneally. & & \\
\hline
\end{tabular}

\section{S123 A CASE-CONTROL STUDY OF MEMBRANE FATTY ACIDS IN ASTHMA}

E. Broadfield, A. Whitehead, N. Lawson, J. Britton, A. Fogarty. University of Nottingham, UK

Rationale: As substrates for eicosanoid production, it is hypothesised that omega-6 polyunsaturated fatty acids (PUFA) may increase the prevalence and/or severity of asthma. Furthermore as competitive antagonists of this process, omega- 3 fatty acids may have a protective role. This study was designed primarily to explore whether asthmatics have increased levels of erythrocyte membrane omega-6 PUFA compared to non-asthmatics, and secondarily whether there are differences in the levels of the other main fatty acids between asthmatics and controls.

Method: Fasting blood samples were taken from 89 asthmatics on inhaled steroids and 89 community controls, matched for age, sex, and area of residence. Percentage levels of the 8 most abundant erythrocyte membrane fatty acids were measured using gas chromatography, and the levels in cases and controls compared using the paired $t$ test.

Results: The levels of two PUFA (palmitoleic and eicosapentaenoic acids) were too small to be measured and were therefore excluded from the analysis. Cases were found to have significantly lower erythrocyte membrane levels of the omega- 6 fatty acid linoleic acid and higher levels of the saturated fatty acid stearic acid. See table. 
Abstract S123

\begin{tabular}{lccccc}
\hline & \multicolumn{3}{c}{ \% composition } & & \\
\cline { 2 - 4 } & Asthmatic & Controls & Mean difference & Standard error & $\mathrm{p}$ Value \\
\hline Palmitic acid & 38.2 & 37.5 & 0.678 & 1.003 & 0.50 \\
Stearic acid & 20.1 & 18.0 & 2.090 & 0.478 & 0.00 \\
Oleic acid & 13.1 & 13.0 & 0.060 & 0.443 & 0.89 \\
Linoleic acid & 7.4 & 8.6 & -1.197 & 0.347 & 0.00 \\
Arachidonic acid 15.4 & 16.3 & -0.936 & 0.533 & 0.08 \\
$\begin{array}{l}\text { Docosahexcae } \\
\text { noic acid }\end{array}$ & 5.8 & 6.3 & -0.535 & 0.612 & 0.38 \\
\hline
\end{tabular}

Conclusion: These findings are consistent with the hypothesis that dietary omega-6 PUFA may be involved in the aetiology of asthma, but not with a protective role for omega-3 fatty acids. The unexpected finding of increased levels of the erythrocyte membrane saturated fatty acid stearic acid warrants further investigation.

\section{Drug therapy in cystic fibrosis}

\section{S124 SERUM AND SPUTUM CONCENTRATIONS FOLLOWING THE ORAL ADMINISTRATION OF LINEZOLID IN ADULT PATIENTS WITH CYSTIC FIBROSIS}

D. Saralaya, D. Peckham, B. Hulme, C. Tobin, S. Conway. Regional Adult Cystic Fibrosis Unit, Seacroft Hospital, Leeds LSI 4 6UH, UK

Introduction: Linezolid is a new antibiotic with efficacy against methicillin resistant Staphylococcal aureus (MRSA). Although licensed for the treatment of respiratory tract infections, there are, as yet, no published trials of its use in cystic fibrosis (CF).

Aim: The objective of the study was to evaluate the absorption and sputum penetration of oral Linezolid in with CF patients.

Methods: 10 ( 5 male \& 5 female) adult CF patients were recruited over a 3 month period. The mean (range) age, BMl and $\%$ predicted FEV1 were 25.4 years (19-36), $20(2.2)$ and $47.8 \%(22-90)$ respectively. Inclusion criteria included the absence of MRSA infection or significant liver disease. Treatment was administered under nursing supervision. Subjects received $600 \mathrm{mg}$ of Linezolid orally every 12 hours for 6 doses. Serum and sputum drug levels were measured before and at 2 hours after the final dose of Linezolid. A further serum level was measured at 4 hours. Serum and sputum levels were measured by High Performance Liquid Chromatography.

Results: Mean (SD) serum Linezolid levels were $2.3 \mathrm{mg} / \mathrm{l}(1.5)$ at 12 hours following the 5th dose and $13.5 \mathrm{mg} / \mathrm{l}(4.3)$ and $8.1 \mathrm{mg} / \mathrm{l}$ (3.3) at 2 and 4 hours following the 6th dose. High sputum concentrations were obtained with mean (SD) levels of $3.6 \mathrm{mg} / \mathrm{l}(2.1)$ and 17.3 (6.9) at 2 and 4 hours following drug administration. Good sputum penetration was observed with mean sputum to plasma ratio of 1.4 at 2 hours. There was a significant variation in peak serum levels within the studied population. However, even the lowest peak concentration exceeded the MIC 90 for MRSA (2-4 mg/l). Serum levels in this study are slightly lower than levels obtained in non-CF historical controls.

Conclusion: The administration of 12 hourly, $600 \mathrm{mg}$ oral Linezolid to CF patients results in sputum levels that exceed the MIC90 of MRSA for almost the whole dosing period. Further clinical trials are needed to assess the efficacy of Linezolid against MRSA in this patient group.

\section{S125 A PROSPECTIVE, DOUBLE-BLIND, RANDOMISED, PLACEBO CONTROLLED, CROSSOVER TRIAL OF AZITHROMYCIN IN PAEDIATRIC CYSTIC FIBROSIS}

A. Equi, A. Bush, I.M. Balfour-Lynn, M. Rosenthal. Royal Brompton and Harefield NHS Trust, London, UK

Following significant improvements in lung function in a pilot study of seven patients with cystic fibrosis who received azithromycin daily for at least three months, ${ }^{1}$ we now report a prospective, double blind, placebo controlled, double blind, crossover trial of AZM in paediatric CF patients. $^{2}$

Methods: $41 \mathrm{CF}$ children aged 8 to 18 years, median expired volume in one second $\left(\mathrm{FEV}_{1}\right) 61 \%$ (range 33 to $80 \%$ ) participated in a 15 month randomised double-blind placebo controlled crossover trial receiving either Azithromycin (body weight <40kg: $250 \mathrm{mg}$ daily, >40kg: $500 \mathrm{mg}$ daily) or placebo for 6 months. Following 2 months washout, the treatments were crossed over. Spirometry, sputum cultures, sputum interleukin 8 and neutrophil elastase, exercise testing, quality of life, antibiotic usage and pulmonary exacerbation rates were outcome measures. Side effects were assessed by pure tone audiometry and liver function tests.

Results: The median relative difference in $\mathrm{FEV}_{1}$ between azithromycin and placebo was $+5.4 \%(95 \% \mathrm{Cl} 0.8$ to $10.5 \%)$. $13 / 41$ subjects improved by $>13 \%$ and $5 / 41$ deteriorated by $>13 \%(p=0.059)$. The median relative difference between azithromycin and placebo was $+11.5 \%$ (5.3 to 16.5) when not receiving concurrent rhDNAse $(n=26)$ and $-3.6 \%(-22$ to +3.9$)$ for the 15 receiving rhDNAse (Mann Whitney $p=0.003$ ). There was no significant overall change in forced vital capacity or mid expiratory flow rates but the effect of rhDNAse usage was similar for these measurements. Overall, 17/41 subjects had 24 fewer oral antibiotic courses when on azithromycin compared with placebo and 5 subjects had 6 extra courses $(p<0.005)$. Of the $12 / 15$ children on rhDNase, $11 / 15$ needed intravenous antibiotics whilst on azithromycin compared with $6 / 15$ when on placebo $(p<0.05)$. There were no changes in sputum bacterial densities, inflammatory markers exercise tolerance or subjective well-being. There were no significant side effects.

Conclusions: A four to six month trial of azithramycin is justified in children with CF not responding to conventional therapy.

1. Jaffe $A$, et al. Long-term azithromycin may improve lung function in children with cystic fibrosis. Lancet 1998;351:420

2. Equi A, Balfour-Lynn I, Bush A, et al. Long term azithromycin in children with cystic fibrosis: a randomised, placebo-controlled crossover trial. Lancet 2002;360:978

\section{S126 A DOUBLE-BLIND PLACEBO CONTROLLED CLINICAL TRIAL TO DETERMINE THE SAFETY OF ICODEXTRIN AND ITS EFFECT ON MUCOCILIARY CLEARANCE IN CYSTIC FIBROSIS}

M. Stern ${ }^{1}{ }^{4}$, T. Wodehouse ${ }^{1}{ }^{4}$ A. Hasani ${ }^{2}$, J. Davies' ${ }^{4}$, D. Hansell', M. Parry-Billings ${ }^{3}$, E. Peers ${ }^{3}$, M. Shott ${ }^{3}$, J. Agnew ${ }^{2}$, D.M. Geddes ${ }^{1}{ }^{4}$, E.W.F.W Alton' ${ }^{4}$. 'Dept Gene Therapy, Imperial College at the NHLI; ${ }^{2}$ Dept Medical Physics, Royal Free Hospital \& Medical School; ${ }^{3}$ Innovata Biomed Ltd, St Albans, Hertfordshire, UK; ${ }^{4}$ UK Gene Therapy Consortium

Cystic fibrosis (CF) may best be managed by therapy directed at restoring the volume of airway surface liquid (ASL). We tested a strategy to increase ASL, and hence mucociliary clearance, by providing an osmotic stimulus to the lower airways using icodextrin, a high molecular weight glucose polymer used in peritoneal dialysis to produce colloidal osmosis but never tested in the human lung. In a double-blind, placebo-controlled crossover study, $10 \mathrm{CF}$ patients ( $\geqslant 18 \mathrm{yr}, \mathrm{FEV}, \geqslant 60 \%$ predicted) were randomised to receive either nebulised (Pari LC-65) icodextrin (10 ml, $200 \mathrm{mg} / \mathrm{ml}$ in normal saline) or placebo (10 ml normal saline). The second treatment was administered one week later. Following each treatment, measurements of lung function (spirometry and oximetry) made daily for 7 days as well as 2 serial high resolution CT scans of the lungs showed no evidence of icodextrin-associated pulmonary toxicity. There were no significant changes detected in serial full blood counts, ESR, CRP, renal and liver function tests, serum IL-6 and IL-8, supporting systemic safety. Baseline mucociliary clearance (MCC) was determined for a 4 hour period using a radioaerosol/gamma camera technique and $5 \mathrm{~m}$ ${ }^{99 m T c}$ polystyrene particles. The same scanning protocol was repeated after each treatment. Compared to baseline (443.5 $\pm 7.8 \%$ /hour), icodextrin (401.5 $\pm 11.8 \%$ /hour), but not placebo $(417.8 \pm 10.2$ 
$\% /$ hour), was associated with a significant ( $p=0.01)$ increase in MCC as expressed by the area under the whole lung radioaerosol retention curve. The effect of icodextrin was most marked on tracheobronchial clearance rate after 150 and 180 minutes respectively when significantly $(p=0.03)$ less radioaerosol $(26.4 \pm 2.4 \%$ and $21.8 \pm 2.0 \%)$ was retained compared to baseline $(43.2 \pm 4.8 \%$ and $34.2 \pm 4.1 \%)$. Whilst compared to placebo, icodextrin was associated with increased tracheobronchial radioaerosol clearance, the difference between icodextrin and placebo did not reach statistical significance at any time point measured. In conclusion, nebulised icodextrin appears to improve MCC in CF patients but further optimisation of dose and delivery may still be required to determine whether it offers any significant advantage over nebulised saline.

\section{S127 INTERACTION BETWEEN AEROSOL DELIVERY SYSTEM AND BRONCHODILATORS IN CF PATIENTS TAKING COLISTIN}

M.E. Dodd', S. Conway', R.J. Marsden ${ }^{3}$, P.H. Weller ${ }^{4}$ 'Wythenshawe Hospital, Manchester, UK; ${ }^{2}$ St. James's Hospital, Leeds, UK; ${ }^{3}$ Profile Therapeutics, UK; ${ }^{4} T$ he Birmingham Children's Hospital, Birmingham, UK

Chest tightness is a recognised side effect of nebulised antibiotics (AB). We describe a sub group of patients who nebulised colistin in a clinical trial. Patients using nebulised $A B$ and $D$ Nase for $>90$ days were randomised to use the HaloLite $A A D$ system (AAD) or a conventional high output nebuliser (NEB) over a 182 day period (study MAL 25-70). All patients used bronchodilators, some of the patients in each nebuliser group used a pMDI or DPI (INHL) others used a solution (SLN) form through the study device. This abstract reports preliminary analysis of \% predicted FEVI mean change from baseline to day 28 and to day 182 for each combination of nebuliser (AAD or NEB) and bronchodilator (SLN or INHL).

Results: 189 of 259 patients used colistin. See table.

Two way anaysis of variance demonstrated a significant interaction between device type and bronchodilator type for change in FEV $(p=0.001)$.

Conclusions: The use of a bronchodilator prior to nebulising antibiotics is recommended practice to prevent inhaled antibiotic induced chest tightness in adult and paediatric patients. These data suggest that the use of a bronchodilator solution with colistin in patients using $A A D$ has a positive effect on maintaining both short and long-term FEV 1 . This effect was not seen in the NEB group, nor was it evident in patients using $\mathrm{INHL}$.

This study was sponsored by Profile Therapeutics, UK.

\section{S128 THE UTILITY OF SALIVARY LEVELS FOR MONITORING ONCE DAILY INTRAVENOUS AMINOGLYCOSIDES IN CHILDREN WITH CYSTIC FIBROSIS}

H. Spencer, P. Webber, M. Chadwick, S. Makhechas, J. Kerr, J.C. Davies. The Royal Brompton Hospital, London, UK

First line intravenous antibiotic treatment for children with pulmonary exacerbations of cystic fibrosis (CF) includes an aminoglycoside (AG) such as gentamicin or tobramycin. The nephro and ototoxic side effect of these drugs make monitoring of levels mandatory. Two previous studies have reported the use of salivary trough levels to monitor once daily AGs in patients without CF. Although a correlation was shown between saliva and serum values, there was no confirmation that the method could reliably detect toxic levels. In view of this, and the fact that CF saliva is known to be abnormal, we have assessed the utility of this approach in children with CF. CF children prescribed once daily AGs (10-12 mg/kg) were eligible for inclusion if they were old enough to produce saliva, and if they and their parent consented to the study. 28 patients (21 gentamicin, 7 tobramycin, median (range) age9.97 years(3.89 to 16.75 ) had simultaneous serum and saliva samples immediately prior to the 3 rd dose of drug. In the majority $(n=25)$, a few crystals of citric acid were placed on the tongue to stimulate saliva production, up to $2 \mathrm{mls}$ of which was collected into a sterile polystyrene container. Blood samples were taken by peripheral venepuncture. Saliva collection was well tolerated in all cases. 27/28 patients had a serum level of $<1$ (mg/L). In 24 of these ( $89 \%$ ), the salivary level was also $<1$, but in 3 patients higher levels were obtained $(8.96,4.98,4.3)$. Only one patient had a toxic serum level of 1.6 ; this patient also had a high salivary level (7.7).

This study demonstrates that both gentamicin and tobramycin can be detected in saliva. The salivary measure confirmed a safe serum level $(<1 \mathrm{mg} / \mathrm{L})$ in $89 \%$ of children, but in a minority, salivary levels were spuriously high. With regards to safety of salivary monitoring, only one patient had a serum trough level that was considered toxic at $>1$ ( $\mathrm{mg} / \mathrm{L})$. In this child, the saliva sample detected this and was also abnormally high. Although these data are promising, confirmation of safety will require evidence from further children that salivary levels are consistently raised in the presence of toxic serum levels.

\section{S129 ALTERED TISSUE DISTRIBUTION IN ADULTS WITH CYSTIC FIBROSIS}

C.E. Bolton, A.A. lonescu, W.D. Evans, R. Pettit, D.J. Shale. Sections of Respiratory Medicine and Medical Physics, University of Wales College of Medicine, Llandough Hospital, Penarth, Cardiff CF64 2XX, UK

We studied the distribution of altered body composition in 51 adults with cystic fibrosis (CF) colonised with Pseudomonas aeruginosa and 18 age and sex matched healthy subjects. Using DEXA scanning we derived height indices for total fat mass (FMI), fat free mass (FFMI) and bone mineral content (BMCI) of the right arm, leg and trunk. Spirometry, height, weight, and physical activity (METS) data were determined.

In patients, FFMl of the arm and leg were less $(p<0.05)$. The truncal FFMI difference was not significant (possibly due to group diversity or that viscera are classed as FFM). Amongst the patients, there was a greater deficit (compared with the mean control) of FFMI in the leg than arm than the trunk $(18.19 \%, 14.86 \%,+0.09 \%, \mathrm{p}<0.02)$.

For patients, FFMI for arm, leg and trunk were less $(p<0.05)$ in the severe $(\mathrm{FEV},<45 \%)$ than the mild $(\mathrm{FEV},>65 \%)$ disease group. Amongst the severe group, there was $(p<0.02)$ greater deficit of FFMI in leg $(28.68 \%)$ than arm $(23.76 \%)$ than trunk $(3.93 \%)$. See table.

Patients with a normal BMI, low total FFM $(n=10)$ had a lower $(p<0.01)$ arm, leg and trunk FFMI than those with a normal BMI, normal total FFM ( $n=13)$.

The $\mathrm{BMCl}$ of the arm, leg, and trunk of patients were less (all $p<0.001$ ) than the controls. There was a lesser $\mathrm{BMCl}$ in the severe than the mild $(p<0.03)$ disease. Patients with a normal BMI, low total FFM had a lower $(p<0.03)$ arm, leg, and trunk $B M C l$ than those with a normal BMI, normal total FFM. In all cases, there was no significant difference in the deficit of $\mathrm{BMCl}$ between arm, leg, and trunk. Fat mass in patients was not reduced.

\begin{tabular}{|c|c|c|c|c|c|}
\hline \multicolumn{6}{|l|}{ Abstract S127 } \\
\hline $\begin{array}{l}\text { Device } \\
\text { bronchodilator } \\
\text { combination }\end{array}$ & $n$ & $\begin{array}{l}\text { Baseline age } \\
\text { median (IQR) }\end{array}$ & $\begin{array}{l}\text { Baseline FEV } \\
\% \text { predicted } \\
\text { mean (CI) }\end{array}$ & $\begin{array}{l}\% \text { Change in } \mathrm{FEV}_{1} \% \\
\text { predicted at } 28 \text { days } \\
\text { mean (CI) }\end{array}$ & $\begin{array}{l}\% \text { Change in } \mathrm{FEV}_{1} \% \\
\text { predicted at } 182 \text { days } \\
\text { mean (CI) }\end{array}$ \\
\hline AAD SLN & 51 & $\begin{array}{l}19 \\
(13 \text { to } 26)\end{array}$ & $\begin{array}{l}50 \\
(44 \text { to } 56)\end{array}$ & $\begin{array}{l}6.6 \\
(1.0 \text { to } 12.1)\end{array}$ & $\begin{array}{l}0.5(n=48) \\
(-6.6 \text { to } 7.7)\end{array}$ \\
\hline NEB SLN & 43 & $\begin{array}{l}19 \\
\text { (14 to } 28)\end{array}$ & $\begin{array}{l}53 \\
(47 \text { to } 60)\end{array}$ & $\begin{array}{l}-3.9 \\
(-8.6 \text { to } 0.8)\end{array}$ & $\begin{array}{l}-3.7(n=41) \\
(-11.7 \text { to } 4.3)\end{array}$ \\
\hline AAD INHL & 43 & $\begin{array}{l}13 \\
(10 \text { to } 18)\end{array}$ & $\begin{array}{l}65 \\
\text { (59 to } 70)\end{array}$ & $\begin{array}{l}-7.3 \\
(-12.5 \text { to }-2.1)\end{array}$ & $\begin{array}{l}-7.0(n=39) \\
(-12.1 \text { to }-2.0)\end{array}$ \\
\hline NEB INHL & 41 & $\begin{array}{l}15 \\
\text { (13 to } 18)\end{array}$ & $\begin{array}{l}64 \\
\text { (57 to } 72)\end{array}$ & $\begin{array}{l}-0.8 \\
(-4.7 \text { to } 3.2)\end{array}$ & $\begin{array}{l}-5.1(\mathrm{n}=38) \\
(-11.3 \text { to } 1.1)\end{array}$ \\
\hline
\end{tabular}


Abstract S129

\begin{tabular}{llll}
\hline & Leg FFMI & Arm FFMI & Trunk FFMI \\
\hline FEV $_{1}$ & $0.556(p<0.001)$ & $0.429(p=0.002)$ & $0.418(p=0.003)$ \\
METS & $0.652(p<0.001)$ & $0.555(p<0.001)$ & $0.507(p<0.001)$
\end{tabular}

Preferential loss of FFM occurs in adults with CF. Such loss is related to severity of lung disease and physical activity. It can occur in the presence of a normal weight/height ratio. A similar loss of BMC occurs with preservation of FM. A hierarchical pattern of FFM loss of legs > arms > trunk was demonstrated whilst bone mineral loss occurred generally.

Sponsored by the CF Trust, UK and the British Lung Foundation.

\section{TNF $\alpha$ : Its role in respiratory disease}

\section{S130 GENETIC SUSCEPTIBILITY TO OZONE EXPOSURE}

I.A. Yang', O. Holz', R.A. Jorres², H. Magnussen², S.J. Barton', J.A. Cakebread', J.W. Holloway', S.T. Holgate'. 'Asthma Genetics Laboratory, Divisions ofHuman Genetics \& IIR (Respiratory, Cell and Molecular Biology), University of Southampton, UK; ${ }^{2}$ Hospital Grosshansdorf, Center for Pneumology and Thoracic Surgery, Grosshansdorf, Germany

Ozone is a major air pollutant with adverse health effects, yet there is variability in response between individuals. Genetic determinants that modulate ozone-induced lung inflammation have been found in mice specifically inbred to be prone or resistant to ozone exposure.' We hypothesised that polymorphisms in homologous human genes would influence response to ozone.

Methods: 37 participants (12 asthmatic, 25 healthy) who had undergone ozone challenge (intermittent exercise during inhalation of ozone $\geqslant 200 \mathrm{ppb}$ ) were genotyped using ARMS-PCR for tumour necrosis factor- $\alpha$ (TNF), glutathione peroxidase (GPXI), manganese superoxide dismutase (SOD2) and toll-like receptor 4 (TLR4) polymorphisms.

Results: There was no difference in lung function response between asthmatics and healthy participants. Mean change in FEV, with ozone challenge was $-9.0 \%$ baseline in TNF $-308 \mathrm{G} / \mathrm{G}$ individuals, compared to $-0.6 \%$ baseline in TNF $-308 \mathrm{G} / \mathrm{A}$ or $\mathrm{A} / \mathrm{A}$ individuals $195 \% \mathrm{Cl}$ for difference between means -14.5 to $-2.3, p=0.008, t$ test). This difference remained significant even when only including $250 \mathrm{ppb}$ exposures for $3 \mathrm{~h}(\mathrm{p}=0.007$, Mann-Whitney $U$ test, $n=32$ ). No significant differences were detected with GPX1 or SOD2, whereas the TLR4 polymorphism was too infrequent to analyse. No significant interactions between genotypes were observed (General Linear Model, SPSS V111).

Conclusions: This is the first study to extend the genetic linkage findings of ozone exposure in mice to clinical ozone challenges. These results suggest that the TNF locus is a genetic factor for susceptibility to ozone exposure, as it is in the mouse.' TNF haplotyping of larger cohorts and functional analysis of cellular models are required to confirm these findings.

Supported by: Allen+Hanburys/Thoracic Society of Australia and New Zealand Respiratory Research Fellowship

1. Kleeberger SR, et al. Linkage analysis of susceptibility to ozone-induced lung inflammation in inbred mice. Nat Genet 1997; 17:475-8.

\section{S131 POLYMORPHISMS IN THE PROMOTER OF TUMOR NECROSIS FACTOR (TNF) ALPHA GENE IN PATIENTS WITH SILICOSIS AND THE DEVELOPMENT OF PROGRESSIVE MASSIVE FIBROSIS}

Y. Ohtsuka, X. Wang, K. Kimura', T. Ishida, J. Saito, M. Munakata. Department of Pulmonary Medicine, School of Medicine, Fukushima Medical University, Fukushinma, Japan; 'Iwamizawa Rousai Hospital, Hokkaido, Japan

It is well known that there was pronounced individual variation in the severity of silicosis even in the same exposure environments. To explain this, we made our hypothesis that there might be an association between genetic polymorphisms of TNF- $\alpha$ promotor region and lung responses to silica particles in silicotic patients. To examine our hypothesis, we studied the association of TNF $\alpha$ promoter polymorphisms $(-308,-238$ and -376$)$ with the roentgenological severity of silicosis in 124 sex-, smoking-, and exposure history - matched Japanese silicotic patients. Silicotic patients were divided into three groups, $\operatorname{Pr}$ (profusion rate) $1(1 / 0-1 / 1, n=47), \operatorname{Pr} 3(2 / 0-2 / 2, n=36)$, and PMF (progressive massive fibrosis) (4c, $n=43$ ) according to the ILO classification. We also examined 122 healthy controls within the same regional district. TNF- $\alpha$ promoter polymorphisms were determined using PCR-RFLP method. Results showed that frequency of A-308 (GA/AA) genotype is significantly higher in $\operatorname{Pr} 1$ and $\operatorname{Pr} 3$ patients, $(17 \%$ and $22 \%)$ as compared with PMF and controls $10 \%$ and $4 \%)(p<0.005)$. There were no significant differences at the -238 and -376 loci among the groups and controls. There are no linkage disequilibilium among these regions. From these results, the TNF promoter single nucleotide polymorphism (SNP) -308 might affect the development of silicosis.

\section{S132 TNF PROMOTER REGION SINGLE NUCLEOTIDE POLYMORPHISM IN ARDS}

A.L. Lagan, E. Beddow, S. Mumby, G.J. Quinlan, P. Goldstraw, J.D. Christie, P.N. Lanken, A.B. Fisher, R.M. Du Bois, K.I. Welsh, T.W. Evans. Unit of Critical Care, Dept Thoracic Surgery \& Clinical Genomics Group, ILDU Imperial College Faculty of Medicine. Division of Pulmonary Medicine, Department of Medicine, University of Pennsylvania Medical School, Philadelphia, USA

Critical illness in adults frequently predisposes to, or is accompanied by acute lung injury (ALI), which in its most severe manifestation is termed the acute respiratory distress syndrome (ARDS). Therapy for ALI/ARDS is supportive and mortality rates remain high (30-70\%). The incidence of ALI/ARDS in a given patient population is dependent in part upon the nature of the precipitating insult, and by inference upon individual susceptibility. Genetic variation may therefore contribute to the onset of ARDS in at risk populations. Acute inflammation is a major contributing factor to ARDS and TNF is a major mediator of the inflammatory response. We therefore performed a case control study to test the association of a TNF promoter region polymorphism (-857) with ARDS. Patients were consented and DNA extracted from whole blood and stored until time of analysis in sterile water at $-20^{\circ} \mathrm{C}$. Archived controls were used for comparison. The genotype of the biallelic single nucleotide polymorphism was determined by polymerase chain reaction in association with sequence-specific primers incorporating mismatches at the $3^{\prime}$ end.

Patients with established ARDS (15 UK patients, 28 USA patients) were typed for the TNF -857 promoter region polymorphism and compared with a normal control population ( 347 UK controls) and an at risk group of surgical lung resection patients (26 UK patients). A significant increase in the rarer -857 T allele was observed in the ARDS group when compared to controls $(31 \% v 14 \%, p=0.01)$ and the at risk group $(31 \% \vee 4 \%, p=0.006)$. These preliminary results indicate that variation in the expression of TNF a major pro-inflammatory cytokine may contribute to the onset of ARDS. However increased patient numbers and functionality studies will be required before firm conclusions can be drawn.

Work in part funded by the Dunhill Medical Trust and The British Lung Foundation.

\section{S133 AUGMENTATION OF TNF $\alpha$-INDUCED APOPTOSIS OF HUMAN NEUTROPHILS BY THE AMINOPEPTIDASE INHIBITORS BESTATIN AND ACTINONIN}

A. Sobolewski, B.J. Reed, E.R. Chilvers. Department of Respiratory Medicine, University of Cambridge, School of Clinical Medicine, Addenbrookes' and Papworth Hospitals, Hills Road, Cambridge, CB2 2QQ, UK

Neutrophil apoptosis plays an important role in the control of lung inflammation, resulting in cell clearance without a pro-inflammatory response. Therapeutic enhancement of this process therefore represents a major experimental goal. TNF is unique in its ability to induce both neutrophil apoptosis and priming. Aminopeptidase enzymes (AP) are involved in both protein maturation and degradation, and their inhibition has been shown to induce apoptosis in leukaemic cell lines. The aim of this study was to investigate the effect of aminopeptidase inhibition on TNFo-induced apoptosis in human neutrophils. Neutrophils were isolated from human blood using 
a Percoll separation. Cells were pre-incubated with AP inhibitors actinonin and bestatin $(0.001-1 \mathrm{mM}, 30 \mathrm{~min})$ in RPMI $(10 \%$ autologous serum), prior to the addition of TNF $\alpha(100 \mathrm{U} / \mathrm{ml}, 6 \mathrm{~h})$, and apoptosis assessed by morphology. FACS analysis was used to assess membrane TNFR55/75 following TNF $\alpha$ exposure $(100 \mathrm{U} / \mathrm{ml}, 15 \mathrm{~min})$ +/- actinonin/bestatin $(1 \mathrm{mM})$. Shape change and superoxide assays were performed to study neutrophil priming and activation. To elucidate the nature of neutrophil APs, colorimetric enzyme-substrate assays were conducted on cell lysates in the presence of actinonin/bestatin. TNF $\alpha$ alone enhanced the rate of apoptosis $(12 \pm 2 \%)$ compared to control levels $(5 \pm 2 \%)$. Actinonin or bestatin alone had no effect, but in conjunction with TNF $\alpha$, increased apoptosis to $21 \pm 3 \%$ and $20 \pm 2 \%$ respectively. Actinonin and bestatin did not affect neutrophil TNFR55/75 expression, shape change, or agonist induced superoxide anion generation. Enzyme-substrate inhibition profiles suggested the involvement of a basic AP. In conclusion, the AP inhibitors, actinonin and bestatin, augment TNF $\alpha$-induced neutrophil apoptosis, independent of neutrophil priming, activation or altered TNFR55/75 expression. This effect is likely to be via inhibition of a specific aminopeptidase involved in maturation/degradation of a protein in the TNF $\alpha$ signalling cascade.

\section{S134 TREATMENT OF CHRONIC SEVERE ASTHMA WITH A SOLUBLE TNF ALPHA RECEPTOR (ETANERCEPT)}

K.S. Babu', S.H. Arshad', E.J. Bell', S.D. McLoughlin', P.H. Howarth', A.J. Chauhan', S.T. Holgate'. 'Respiratory Cell \& Molecular Biology, Southampton General Hospital, Southampton, UK; ${ }^{2}$ Department of Respiratory Medicine, St. Mary's Hospital, Portsmouth, UK

Tumor Necrosis Factor Alpha (TNF- $\alpha$ ) is a pro-inflammatory cytokine implicated in the pathogenesis of asthma. TNF- $\alpha$ is increased in the airways of asthmatics and is involved in the production of IL-8, RANTES and GM-CSF by airway epithelial cells. Therefore there is a good case for blocking TNF- $\alpha$ as a therapeutic strategy in asthma. This open labelled study evaluated the efficacy of etanercept- a soluble TNF- $\alpha$ receptor (p75) linked to the Fc portion of human $\lg _{1}$ in the treatment of chronic severe asthma.

Methods: 10 patients (18-65 years) with chronic severe asthma on regular oral corticosteroids $(13 \pm 10.1 \mathrm{mg} /$ day), high dose inhaled steroids, long acting inhaled $\beta_{2}$ agonists and/or oral theophyllines were enrolled. They had an FEV of at least $50 \%$ of their predicted and demonstrated a reversibility of at least $9 \%$ with inhaled salbutamol. The study involved administration of $25 \mathrm{mg}$ of etanercept twice weekly for 12 weeks. Lung function measurements, methacholine response, and asthma control questionnaire were completed before and after the study.

Results: Treatment with etanercept resulted in clinically significant improvements in the lung function. FEV improved from $2.21 \mathrm{~L} / \mathrm{sec}$ (1.81-2.52), median (IQR) to $2.68 \mathrm{~L} / \mathrm{sec}(1.77-2.75, \mathrm{p}=0.037)$ and the FEV, /FVC ratio improved from $75.63 \%$ (68.03-81.27) to $82.39 \%(69.64-88.75, p=0.028)$. The mean asthma control symptom score improved from $26(18.25-28.5)$ to $11.5(4-16, p=0.005)$. Airway hyper-responsiveness as measured by methacholine dose response improved from $234.44 \mathrm{ml} / \mathrm{mg}$ to $9.21 \mathrm{ml} / \mathrm{mg} \mathrm{(p=0.021).} \mathrm{All}$ patients successfully withdrew their nebulised $\beta_{2}$ agonist medication from a mean dose of salbutamol $8.25 \mathrm{mg} /$ day

Conclusions: Etanercept improves $\mathrm{FEV}_{1}, \mathrm{FEV}_{1} / \mathrm{FVC}$ ratio, asthma control symptom scores, airway hyperresponsiveness and the use of rescue nebulised bronchodilator medication in patients with chronic severe asthma. Blocking the effects of TNF- $\alpha$ could prove to be an effective and novel therapeutic strategy for the treatment of patients with chronic severe corticosteroid dependent asthma.

This abstract was supported by Wyeth Laboratories, Berks, UK.

\section{Epidemiology and ethnicity in respiratory disease}

\section{S135 DO VARIATIONS IN CHEST WALL DIMENSIONS EXPLAIN ETHNICITY-RELATED DIFFERENCES IN LUNG FUNCTION?}

A.L. Whittaker', A.J. Sutton', C.S. Beardsmore'. 'Departments of Child Health' and 'Epidemiology and Public Health, University of Leicester, UK

Introduction: Differences in lung function between people of varying ethnic origins may affect interpretation of individual results if inappropriate predicted values are used. Our aim was to characterise differences in spirometry in Asian (ethnic origins in the Indian Subcontinent) and white children and relate these to differences in chest dimensions. We hypothesised that Asian children would have smaller values for lung function and the differences would be explained by variations in chest size.

Methods: Children were seen in primary schools for measurements of FVC, $\mathrm{FEV}_{1}, \mathrm{PEF}, \mathrm{MMEF}, \mathrm{FEF}_{50}$, and $\mathrm{FEF}_{25}$, using a spirometer. Chest circumference, chest height, transverse diameter and A-P diameter were measured using an anthropometric tape measure and anthropometer. Relationships between standing height, lung function, chest dimensions, gender, and ethnicity were assessed using multiple linear regression analysis.

Results: Ninety-three white and 201 Asian children were included in the study. The final models explained $78 \%$ of the variability in FVC, $72 \%$ in $\mathrm{FEV}_{1}$ and $45 \%$ in PEF (adjusted $\mathrm{R}^{2}$ values). Standing height was the single most important predictor, however height squared, chest height, gender, and chest volume were also useful predictors for some outcomes. Ethnicity also remained an important predictor for all three measures, particularly FVC and $\mathrm{FEV}_{1}$, having adjusted for all other variables. FVC and FEV were smaller on average in the Asian children by $0.23 \mathrm{~L}(95 \% \mathrm{Cl} 0.17$ to 0.28$)$ and $0.18 \mathrm{~L}(95 \% \mathrm{Cl} 0.13$ to $0.23)$ respectively. The average decrement in PEF in Asian children was $10 \mathrm{~L} \cdot \mathrm{min}^{-1}(95 \% \mathrm{Cl}-0.2$ to 20.6$)$. The influence of chest dimensions (both singly and in combinations to represent the chest volume as a cylinder or a box) on the prediction was examined to see if they explained the ethnic differences in lung function. Only small differences in the regression coefficients for ethnicity were observed when variables related to chest volume were included in the model.

Conclusions: Equations taking account of ethnicity have been generated and can be used for the accurate prediction of spirometry. The influence of ethnicity on lung volumes is not explained by differences in chest dimensions. Further research in genetic and socio-economic factors is required in order to determine what factors are responsible for the ethnic differences in lung function reported here.

\section{S136 NORMATIVE DATA FOR TOTAL SERUM IMMUNOGLOBULIN E MEASUREMENTS IN CHILDREN OF 3 ETHNICITIES}

E.Y. Chan, S.A. McKenzie. Department of Paediatric Respiratory Medicine, Barts and the London NHS Trust, UK

Background: It is about 20 years since total immunoglobulin $\mathrm{E}(\mathrm{lgE})$ measurements were published for children without atopic disease. It is possible that the recent increase in atopic disease is reflected in altered measurement in subjects who have no clinical expression of atopy. If the measurement of $\lg E$ is to be used as a clinical test of atopy, contemporary normative data must be available.

Aim: To measure total serum lgE in healthy children of three ethnicities born and living in an inner city environment.

Method: Subjects were aged 1-12 years of Afro-Caribbean, Bangladeshi, and white British ethnicities with no personal history of atopic disease (asthma, eczema, hayfever, or food allergy). Extra blood $(1 \mathrm{ml})$ for the measurement of total lgE was collected when blood was taken for other purposes or when a surgical procedure was being undertaken

Results: Measurements from 151 boys (median age 5.4 years) and 106 girls (median age 6.0 years) included 127 Bangladeshis, 58 Afro-Caribbeans, and 72 white British children. $\log _{10}$ total $\lg E$ increased with age $\left(\log _{10} \lg E=1.291+0.077^{*}\right.$ age; $\left.p<0.001\right)$ but was not related to gender or ethnicity. The data were significantly (6-fold) higher than previously published measurements. ${ }^{2}$

Conclusion: These contemporary normative data can be used to determine how useful lgE measurements are in separating healthy children from children with atopic illnesses.

1. Von Mutius E. Clin Exp Allergy 1998;28(Suppl 5):45-9.

2. Kjellman N. Clin Allergy 1976;6:51-9.

\section{S137 EFFECT OF ETHNICITY ON ASTHMA PREVALENCE AND HEALTH SERVICE UTILISATION IN THE UK: A SYSTEMATIC REVIEW}

G. Netuveli', A. Sheikh', B. Hurwitz ${ }^{3}$ M. Barnes ${ }^{4}$, M. Fletcher ${ }^{5}$, M. Levy 6 , S.R. Durham ${ }^{2}$. 'Dept of Primary Health Care; ${ }^{2} \mathrm{NHLI}$, ICSTM, London; ${ }^{3}$ Dept of English King's College, London; ${ }^{4}$ National asthma Campaign; ${ }^{5}$ National Respiratory Training Centre, Warwick; 'UK Kenton Bridge Medical Centre, London

Background: Healthcare providers perceive asthma prevalence to be higher in some ethnic minorities than in the white majority UK population but epidemiological studies to date have reached conflicting conclusions. 
Objective: To review systematically the effect of minority ethnic status on asthma prevalence and service utilisation in Britain.

Methods: A systematic review of studies reporting prevalence of and/or hospitalisation for asthma were identified using the standard search strategies. Outcome variables were self reported asthma or wheeze. Only studies on populations living in the UK, reporting data on at least one minority ethnic group and the white majority and on children (below 16 years) were included. Differences in proportions and odds ratios, if reported, were pooled using both fixed and random effects models.

Results: Of the 36 studies identified, five studies reporting data on prevalence and two studies on hospital admission met the inclusion criteria. Meta-analysis of prevalence data was performed on the outcome measures and ethnicity separately using a random effects model. We found no significant difference in asthma or wheeze prevalence between whites and minority ethnic groups in the UK. The two studies reporting hospital admission rates could not be synthesized. Nevertheless, one group (5 to 14 years old) in one of the studies showed a greater odds ratio for Asians for hospitalisation for asthma (OR 2.03, 1.32 to 3.12 ).

Conclusions: There is no difference in the prevalence of asthma or wheeze between minority ethnic groups and the white majority in the UK. The dearth of data currently available does not allow conclusions to be reliably drawn regarding hospital admission rates for asthma.

Acknowledgments: A NHS R\&D National Primary Care Fellowship supports AS. This work has been supported by a grant from the National Asthma Campaign, UK.

\section{S138 MIND THE GAP! AGE AND SEX RELATED CHANGES IN THE EXPRESSION OF ASTHMA, ECZEMA, AND HAYFEVER}

M. Osmano, P.J. Helmso, C. Simpson, M. Taylor'. Depts of Child Health and General Practice and 'Primary Care, University of Aberdeen, UK

Backgroun d: Asthma prevalence is higher in males during childhood reversing to a female predominance during adolescence. The timing of this "gender switch" and its relation to other atopic diseases in whole populations is less clear.

Methods: Prevalences of currently active asthma, eczema, and hayfever were identified from individuals who consulted their GP at least once for one or more of the above in the year April 1998-99. Records were extracted from 47 Scottish Morbidity Recording General Practices (population 252 538).

Results: Changes in the sex distribution were apparent during the adolescent period such that hayfever and asthma became more prominent in females. Females also had a higher prevalence of eczema in childhood that became more prominent in early adulthood. The gender switch for eczema precedes hayfever which in turn precedes that for asthma. See figure.

Conclusion: The similar, although differently phased, patterns in the adolescent gender switch suggests a shared underlying mechanism. Further studies on the relative contributions of sex hormones and socio-cultural influences seem justified.

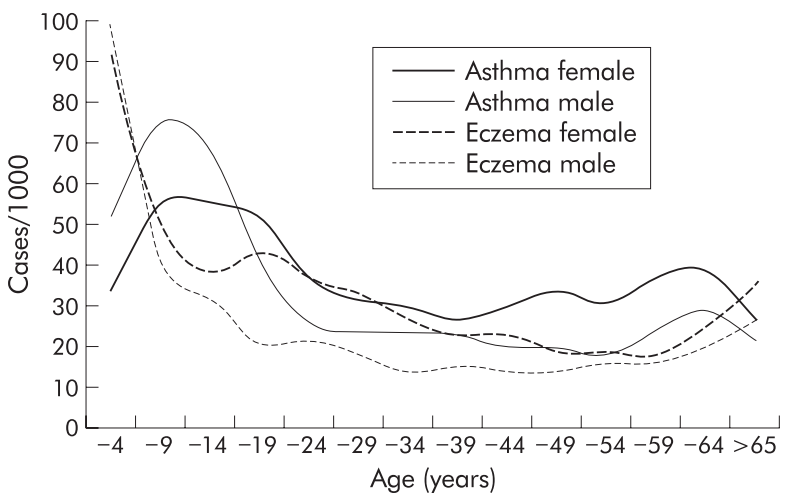

Abstract S138 "Active" asthma and exzema.

\section{S139 DO HOUSING IMPROVEMENTS IMPROVE RESPIRATORY HEALTH?}

M Somerville', M Basham', C Foy², A Barton' for the Torbay Healthy Housing Group. 'Plymouth and South Devon Research and Development Support Unit, University of Plymouth, Tamar Science Park, Plymouth, UK; ${ }^{2}$ Gloucestershire Research and Development Support Unit, UK

Cold damp housing has been associated with poor respiratory health, but few studies have tried to evaluate the effect of improving housing on the occupants' health.' During a community development project in a deprived area of Torquay, local residents surveyed their council-owned homes and reported high levels of damp, mould, and respiratory illness. Torbay Council agreed to improve the houses over a two year period and funding was obtained for evaluation. Of the 142 houses on the estate, 119 agreed to randomisation, which was carried out at a public meeting; 50 houses were selected for improvement in the first year. Measurements of the indoor environment, general and respiratory health were taken at baseline and annually for the next two years in all houses and for all occupants.

At baseline, there were 480 people living in 119 houses. The population profile was young, with $58 \%$ aged 20 and under and $10 \%$ aged 50 and over. Bedroom and living room temperatures improved after renovation (central heating and insulation), but only bedroom temperatures showed a significant difference $(p=0.002)$ between improved and unimproved houses at the end of the first year. Self-reported asthma prevalence in those aged under 18 years declined from $24 \%$ at baseline to $14 \%$ at the end of the study. Frequency of asthma symptoms ${ }^{2}$ reported in the month before each survey also reduced. The difference between those living in improved and unimproved houses at the end of the first year was not significant. Severity, as estimated by BTS asthma steps, remained unchanged in those continuing to report asthma. The study demonstrates the feasibility of evaluating the health effects of housing improvements. Further work is in progress to evaluate the social and economic impact of the renovations.

1. Thompson $\mathrm{H}$, et al. Health effects of housing improvement: systematic review of intervention studies. BM 2001;323:187-90.

2. Steen $N$, et al. Development of a symptom-based outcome measure for asthma. BM 1994;309:1065-8.

\section{S140 FACTORS ASSOCIATED WITH QUALITY OF LIFE IN A COMMUNITY BASED SAMPLE OF YOUNG ADULTS WITH ASTHMA}

W. Chaudhri', J. Knox', D. Jarvis', B.D.W. Harrison², R. Hall ${ }^{3}$, D. Seaton ${ }^{3}$, P. Burney', S. Chinn', C. Luczynska'. 'Dept of Public Health Sciences, Kings College, London, UK; ${ }^{2}$ Norfolk and Norwich Hospital, Brunswick Rd, Norwich, UK; ${ }^{3}$ lpswich Hospital, Heath Road, Ipswich, UK

Quality of life (QOL) in asthma patients provides a measure of the effect of the disease on an individual's everyday life but there is little information on factors associated with QOL in asthmatics in the UK. In $1997 / 1998$ a short questionnaire was sent to 1140 subjects who took part in the East Anglia Respiratory Health Survey I (EARHS I) in 1991. Responders with symptoms suggestive of asthma (waking with shortness of breath $O R$ having an asthma attack in last twelve months OR current use of asthma medication) completed the Marks' QOL Questionnaire 14 domains-breathlessness, mood, social, and concerns). Regression analyses were conducted on the square root transformed QOL score to determine the difference in mean adjusted QOL score (MEAN DIFF) by gender, age-group, $(<35,35-44.9$, $\geqslant 45$ ), social class group, smoking status, and whether symptoms were present in EARHS I. To examine associations within each domain Mann Whitney tests were performed. Of the 983 who responded, 242 subjects aged between 27 and 53 years had symptoms of asthma and information on QOL. Worse QOL was reported by women (MEAN DIFF: 0.19; $95 \% \mathrm{Cl} 0.04,0.33$ ) and those who had asthma in EARHS I (MEAN DIFF: $0.27 ; 95 \% \mathrm{Cl} 0.11,0.43$ ). Worse QOL was observed in social class $V$ and those with undetermined social class (housewives/students). Women had higher scores than men in the mood $(p<0.01)$, social $(p=0.02)$ and concern domains $(p=0.04)$ but not in the breathlessness domain $(p=0.14)$. Compared to those with "new onset asthma" those with asthma at EARHS I had higher scores in the breathlessness $(p<0.01)$, social $(p<0.01)$ and concern ( $p<0.01$ ) domains but not in the mood domain $(p=0.24)$. These findings show that amongst asthmatics, women and those who have had disease for a longer period of time report worse quality of life.

Funded by the National Asthma Campaign. 


\section{Drug therapy in COPD}

\section{S141 BUDESONIDE/FORMOTEROL IN A SINGLE INHALER $\left(\right.$ SYMBICORT $^{\circledR}$ ) REDUCES SEVERE EXACERBATIONS IN PATIENTS WITH MODERATE TO SEVERE COPD}

L.M. Campbell', W. Szafranski' on behalf of the study group. 'Department of Medicine, University of Glasgow, UK; ${ }^{2}$ Department of Lung Diseases, Voivodeship Specialist Hospital, Radom, Poland

The efficacy of budesonide/formoterol in a single inhaler (B/F, Symbi$\mathrm{cort}^{\circledR}$ ) on COPD exacerbations was evaluated in a placebo-controlled, parallel-group, multicentre study. 812 adult patients with established COPD (median 5 years since diagnosis, mean age 64 years, mean baseline $\mathrm{FEV}, 0.99 \mathrm{~L}(36 \%$ predicted)) received two inhalations of either B/F 160/4.5 $\mathrm{ug}$ (total delivered dose 320/9 $\mu \mathrm{g}$ ), budesonide (B) $200 \mu \mathrm{g}$ metered dose, formoterol (F) $4.5 \mu \mathrm{g}$ delivered dose, or placebo (PL) bid for 12 months.

Numbers of severe exacerbations (requiring oral steroid course (OSC) and/or antibiotics and/or hospitalisation) were recorded. Mean severe exacerbation rates were $1.4,1.6,1.8$, and 1.9 exac/ patient/y in the $B / F, B, F$, and PL groups, respectively. Severe exacerbations were reduced by $24 \%(p=0.035), 15 \%(p=0.224)$ and $2 \%$ $(p=0.895) \vee \mathrm{PL}$ with $\mathrm{B} / \mathrm{F}, \mathrm{B}$ and $\mathrm{F} ; \mathrm{B} / \mathrm{F}$ also reduced mean exacerbation rates by $23 \% \vee F(p=0.043)$. The lowest rates of OSC associated with exacerbations were in the $B / F$ and $B$ groups $(0.74$ and 0.76 $\mathrm{OSC} /$ patient/ $\mathrm{y} \vee 1.04$ and 1.07 with $\mathrm{F}$ and $\mathrm{PL}$ ). $\mathrm{B} / \mathrm{F}$ and $\mathrm{B}$ reduced the number of OSC by $31 \%$ and $29 \%$ v PL (both $p<0.05$ ), and B/F $v$ $\mathrm{F}$ by $28 \%(\mathrm{p}<0.05)$.

Thus, budesonide/formoterol in a single inhaler (Symbicort ${ }^{\circledast}$ ) produced statistically and clinically significant reductions in severe exacerbations in patients with moderate to severe COPD and was more effective than either budesonide or formoterol alone.

\section{S142 BUDESONIDE/FORMOTEROL IN A SINGLE INHALER (SYMBICORT ${ }^{\oplus}$ ) PROVIDES EARLY AND SUSTAINED IMPROVEMENT IN LUNG FUNCTION IN MODERATE TO SEVERE COPD}

P. Anderson on behalf of the study group. Dept. Respiratory Medicine, Sheffield Chest Clinic, Sheffield, UK

COPD patients $(n=812$, median 5 y since diagnosis, mean age 64 years, mean baseline $\mathrm{FEV}, 0.99 \mathrm{~L}(36 \%$ predicted)) received 2 inhalations of budesonide/formoterol (B/F, Symbicort ${ }^{\circledR}$ ) 160/4.5 $\mathrm{\mu g}$ (total delivered dose $320 / 9 \mu g$ ), budesonide (B) $200 \mu g$ metered dose, formoterol (F) $4.5 \mu g$ delivered dose or placebo (PL) bid for 12 months. Lung function was assessed by FEV, and PEF.

All treatments significantly improved $F E V_{1}$ vs $\mathrm{PL}$. B/F increased FEV, by $15 \% \vee$ placebo, $9 \% \vee \mathrm{B}$ (both $\mathrm{p}<0.001$ ) and $1 \% \vee \mathrm{F}(\mathrm{ns})$. These improvements were maintained throughout the 12-month study. In the first day, $B / F$ increased morning PEF (mean change from run-in) by $10.9 \mathrm{~L} / \mathrm{min}(\mathrm{PL}$ by $0.3 \mathrm{~L} / \mathrm{min}, \mathrm{B}-0.3 \mathrm{~L} / \mathrm{min} ; \mathrm{F} 6.4 \mathrm{~L} / \mathrm{min} ; \mathrm{B} / \mathrm{F} v \mathrm{PL}$ and $B, p<0.001, B / F \vee F, p=0.081)$. After the first week, $B / F$ increased morning PEF by $15.6 \mathrm{~L} / \mathrm{min}(\mathrm{PL}$ by $1.1 \mathrm{~L} / \mathrm{min}, \mathrm{B} 3.4 \mathrm{~L} / \mathrm{min}, \mathrm{F} 8.8$ $\mathrm{L} / \mathrm{min} ; \mathrm{B} / \mathrm{F} \vee \mathrm{PL}$ and $\mathrm{B}, \mathrm{p}<0.001, \mathrm{~B} / \mathrm{F} v \mathrm{~F}, \mathrm{p}=0.002)$. At 12 months, $\mathrm{B} / \mathrm{F}$ improved morning PEF by $26.4 \mathrm{~L} / \mathrm{min}$ (PL by $2.4 \mathrm{~L} / \mathrm{min}, \mathrm{B} 10.6$ $\mathrm{L} / \mathrm{min}, \mathrm{F} 14.7 \mathrm{~L} / \mathrm{min} ; \mathrm{B} / \mathrm{F} v \mathrm{PL}, \mathrm{B}$, and $\mathrm{F}$ all $\mathrm{p}<0.001)$ and improved evening PEF by $23.1 \mathrm{~L} / \mathrm{min}(\mathrm{PL}$ by $3.0 \mathrm{~L} / \mathrm{min}, \mathrm{B} 8.6 \mathrm{~L} / \mathrm{min}, \mathrm{F} 11.8$ $\mathrm{L} / \mathrm{min} ; \mathrm{B} / \mathrm{F} \vee \mathrm{PL}, \mathrm{B}$ and $\mathrm{F}$ all $\mathrm{p}<0.001)$.

Budesonide/formoterol (Symbicort ${ }^{\circledR}$ ) provides rapid and sustained, clinically relevant improvements in lung function in patients with moderate to severe COPD, with greater improvements in PEF than placebo or either monocomponent.

\section{S143 BUDESONIDE/FORMOTEROL IN A SINGLE INHALER (SYMBICORT ${ }^{\circledR}$ ) PROVIDES SUSTAINED RELIEF FROM SYMPTOMS IN MODERATE TO SEVERE COPD}

L.M. Campbell', W. Szafranski' on behalf of the study group. 'Department of Medicine, University of Glasgow, UK; ${ }^{2}$ Dept. Lung Diseases, Voivodeship Specialist Hospital, Radom, Poland

Symptom relief with budesonide/formoterol $\left(B / F\right.$, Symbicort $\left.{ }^{\circledR}\right)$, budesonide (B), formoterol (F) or placebo (PL) was compared in patients with moderate to severe COPD. 812 patients (mean age 64 years) received 2 inhalations of either $B / F \quad 160 / 4.5 \mathrm{~kg}$ (total delivered dose 320/9 $\mu \mathrm{g}$ ), B $200 \mu \mathrm{mg}$ metered dose, F $4.5 \mu \mathrm{g}$ delivered dose or PL bid for 12 months. Daytime symptom scores of shortness of breath, cough and chest tightness, night-time awakenings due to symptoms (all 0-4 [none to severe], total symptom score (0-16)) and reliever medication use were recorded. $B / F$ decreased individual and total scores after 1 week $v \mathrm{PL}, \mathrm{B}$, and $\mathrm{F}$ (all $\mathrm{p}<0.001$ ). At 12 months, $B / F$ reduced the total scores vs $P L, B$ and $F$ by 1.12 0.84 (both $p<0.001$ ) and $0.41(p=0.043)$. B/F reduced shortness of breath scores by $0.36 \vee \mathrm{PL}$ and $0.26 \vee \mathrm{B}$ (both $\mathrm{p}<0.001$ ), decreased chest tightness scores by 0.21 vs $P L(p<0.001)$ and $0.13 \vee B$ $(p=0.043)$, decreased cough scores by $0.19 \vee P L(p=0.002)$ and 0.22 vs $B(p<0.001)$ and decreased awakening scores by $0.34 \vee P L$ $(p<0.001), 0.20 \vee B(p=0.003)$ and $0.16 \vee F(p=0.019) . B / F$ increased symptom-controlled days by $7 \% \vee P L(p=0.001)$, increased awakening-free nights by $14 \% \vee P L(p<0.001)$ and $10 \% \vee B$ $(p=0.001)$, increased days free from shortness of breath by $12 \% \vee P L$ $(p<0.001)$ and increased days free from chest tightness by $7.5 \%$ v PL $(p=0.015) . B / F$ reduced use of reliever medication by 1.3 and 0.7 inhalations $/ 24 \mathrm{~h} \vee \mathrm{PL}$ and $\mathrm{B}$ (both $\mathrm{p}=0.001$ ), and increased reliever-free days by $8.6 \% \vee \mathrm{PL}(p=0.003)$. Budesonide/formoterol (Symbicort ${ }^{\oplus}$ ) provides early and sustained relief from symptoms in patients with moderate to severe COPD.

\section{S144 THE EFFECT OF 3 MONTHS ORAL CLARITHROMYCIN ON SPIROMETRY, SHUTTLE WALK DISTANCE AND HEALTH STATUS SCORES IN PATIENTS WITH MODERATE TO SEVERE STABLE COPD}

D. Banerjee' 2, O.A. Khair', D. Honeybourne'. 'Department of Respiratory Medicine, City Hospital NHS Trust, Dudley Road, Birmingham, UK. ${ }^{2}$ Department of Respiratory Medicine, Birmingham Heartlands Hospital, Bordesley Green East, Birmingham, UK

Introduction and aims: Macrolides antibiotics are commonly used in the treatment of acute exacerbations of COPD. This study looked at the possible effects of long-term oral clarithromycin on spirometry, shuttle walk distance and health status scores in patients with COPD during the stable clinical state.

Methods: Randomised, double-blind controlled trial of oral clarithromycin 500mg o.d. (CI) vs Placebo (PI) o.d. for 3 months in stable patients with moderate to severe COPD who have not had an exacerbation for 6 weeks. The St George respiratory questionnaire (SGRQ) and Short form-36 (SF-36) were measured. The mean changes in parameter for each group were compared using either a 2 independent group $t$ test or a Mann-Whitney $U$ test where appropriate.

Results: 67 patients were randomised to receive $\mathrm{Cl}(\mathrm{n}=31)$ or $\mathrm{Pl}$ $(n=36) .46(69 \%)$ were male, mean age (SD) was $66.7(7.9)$ years and $27(40 \%)$ were current smokers. Mean (SD) FEV 1 and FEV $1 \%$ predicted were $1.12(0.41) \mathrm{L}$ and 43.2 (11.4) \% respectively. All patients were taking inhaled corticosteroids. Data for 7 patients were unavailable and intention to treat analyses were performed on the $\mathrm{Cl}$ group $(n=26)$ and the $\mathrm{Pl}$ group $(n=34)$. There were no significant differences in spirometry or shuttle walk distance parameters. However, there was a statistically significant improvement in SGRQ symptom score and SF-36 physical functioning in the $\mathrm{Cl}$ group compared to the $\mathrm{Pl}$ group. Differences in the other health status parameters were not statistically significant.

Conclusion: In this study, long term oral clarithromycin improved symptom and physical functioning scores in moderate to severe stable COPD patients

\begin{tabular}{llll}
\hline Abstract S144 & & \\
\hline & $\begin{array}{l}\text { Clarithromycin } \\
\text { mean change } \\
\text { (SD) }\end{array}$ & $\begin{array}{l}\text { Placebo mean } \\
\text { change (SD) }\end{array}$ & $\mathrm{p}$ Value \\
\hline SGRQ symptom & $-7.6(18.6)$ & $2.6(13.7)$ & 0.04 \\
SF-36 physical & $6.7(15.9)$ & $-6.2(19.6)$ & 0.01 \\
functioning & & & \\
\hline
\end{tabular}




\section{S145 EFFECT OF BUDESONIDE/FORMOTEROL ON SEVERE EXACERBATIONS AND LUNG FUNCTION IN MODERATE TO SEVERE COPD}

P.M.A. Calverley on behalf of the Symbicort ${ }^{\circledast}$ study group. University Hospital Aintree, Liverpool, UK

Inhaled corticosteroids (ICS) are recommended in the prevention of COPD exacerbations in patients with an $\mathrm{FEV}_{1}<50 \%$ predicted. Whether long-acting $\beta$-agonist therapy is equally effective as ICS, or the combination superior to the components, is not known. We studied 1022 adults (mean age 64 years; mean $\mathrm{FEV}, 0.99 \mathrm{~L}$ (36\% predicted)) with a history of at least one exacerbation in the previous year. To ensure baseline stability, all received formoterol (F) 9 ug bid and oral prednisolone $30 \mathrm{mg}$ od for two weeks. Patients were then randomised to receive either F $9 \mu \mathrm{g}$, budesonide (B) $400 \mu \mathrm{g}$, budesonide/ formoterol in a single inhaler (B/F, 320/9 $\mu$ g respectively) or an identical placebo (PL), all given bid for 12 months. Severe exacerbations (as episodes requiring oral corticosteroid and/or antibiotics and/or hospitalisation) and lung function on 6 occasions post-randomisation were measured. $B / F$ prolonged time to the first exacerbation $\vee F$ $(p<0.01), B$ and $P L$ (both $p<0.05) ; B / F$ reduced the relative risk of exacerbating by $30 \% \vee \mathrm{F}, 29 \% \vee \mathrm{PL}$ (both $\mathrm{p}<0.01$ ) and $23 \% \vee \mathrm{B}$ $(p<0.05)$. The mean number of exacerbations/patient/ year was $1.38,1.60,1.85$ and 1.80 for $B / F, B, F$, and PL. The time to the first oral steroid course was increased and the number of oral steroid courses was reduced by $\mathrm{B} / \mathrm{F}$ compared with all other treatments: reductions of $45 \% \vee \mathrm{PL}(\mathrm{p}<0.001), 28 \% \vee \mathrm{B}(\mathrm{p}<0.05)$ and $30 \% \vee \mathrm{F}$ $(p<0.05)$. In addition, significantly fewer patients withdrew while taking $B / F(p=0.001 \vee P L$ and $F ; p<0.05 \vee B)$. Post-dose $F E V_{1}$ increased significantly with $B / F: 14 \% \vee P L(p<0.001), 11 \% \vee B(p<0.001)$ and $5 \%$ v $(p<0.01)$.

These data show that combining budesonide and formoterol produces a greater reduction in the severe exacerbation rate than either drug alone, even in moderate to severe COPD, and that this can be achieved with relatively modest doses of the inhaled corticosteroid.

\section{S146 A DOUBLE BLIND DOUBLE CROSS OVER COMPARISON OF HIGH DOSE COMBINED $\beta_{2}$ AGONIST AND ANTICHOLINERGIC BRONCHODILATORS DELIVERED BY NEBULISER OR BY INHALER AND SPACER IN MODERATE TO SEVERE STABLE COPD PATIENTS}

S.C. Johnson', E. Gardener', S.P. Hanley3. 'Physiotherapy department, ${ }^{2}$ Department of Research and Development ${ }^{3}$ Medical Directorate, North Manchester General Hospital, M8 6RL, UK

Introduction: This abstract reports a comparison of high dose salbutamol + ipratropium bromide delivered by inhaler+spacer or by nebuliser in a group of moderate to severe COPD patients. The effects on breathlessness and peak flow are reported.

Methods: Study design: baseline assessment followed by two crossover treatment cycles of 28 days. The two treatment periods in each cycle, given in random order, comprised; (a) 14 days of nebulised $2.5 \mathrm{mg}$ salbutamol and $500 \mathrm{mcg}$ ipratropium bromide or (b) 14 days of Combivent ${ }^{\text {TM }}(100 \mathrm{mcg}$ salbutamol $+20 \mathrm{mcg}$ ipratropium bromide) 6 puffs via Volumaticl spacer. A double dummy technique was used to blind patient and operator. The San Diego Shortness of Breath Questionnaire (SOBQ) was measured at the end of each treatment period. Peak flow (PEF) was recorded twice daily. Visual analogue score (VAS) of breathlessness was recorded each evening. Analysis: where possible analysis was undertaken on an intention to treat basis. Treatment order effect (whether treatment effect differed depending on the order of treatment) was assessed using a graphical technique. A two sample $t$ test was used to detect any between treatment differences.

Results: Fifty patients entered the study, mean age 68 years (SD7.5) FEV 0.8 litres (SD 0.31) $\mathrm{FEV}_{1} \% 34 \%$ (SD 11.7). Analysis revealed a treatment order effect for cycle 1 i.e. those randomised first to active nebuliser had a lower average SOBQ and higher average $\mathrm{PEF}$ for the cycle. This was not seen in cycle 2 . As a consequence, further analyses was confined to cycle 2, $n=36$ (32 male). Missing data, due to hospital admission $(n=2)$ and consent withdrawal $(n=1)$, all during the active nebuliser phase, caused exclusion from the analysis. The differences were not significant between nebuliser $v$ inhaler. They were: $\mathrm{SOBQ}(\mathrm{n}=33) 2.5(95 \% \mathrm{Cl}-1.3$ to 6.4$) \dagger=1.3 \mathrm{p}=0.19$ : $\mathrm{PEF}$ $(n=32) 2.8 \mathrm{l} / \mathrm{min}(95 \% \mathrm{Cl}-3.8$ to 9.3$) t=0.9 \mathrm{p}=0.40$ : VAS $(n=32)$ $-0.1 \mathrm{cms}(95 \% \mathrm{Cl}-4.2$ to 0.2$) \mathrm{t}=-0.7 \mathrm{p}=0.48$.

Conclusions: Although patients often request nebuliser treatment, this study does not support its prescription in stable COPD patients.

\section{Patient and physician viewpoints in COPD}

\section{S147 SURVEY OF ATTITUDE OF 100 PATIENTS WITH COPD TO ARTIFICIAL VENTILATION AND CARDIOPULMONARY RESUSCITATION}

K.A. Gaber, M. Barnett, Y. Blanchant, C.R. McGavin. Chest Clinic, Derriford Hospital, Plymouth, UK

Doctors are being encouraged to discuss and document end of life decisions with vulnerable patients admitted to hospital as part of involving patients in their own management. We wanted to ascertain the views of COPD patients.

Method: Patients with COPD under follow up by Respiratory Nurse Specialists (RNS) and the Chest clinic (of Derriford Hospital) were surveyed. Patients were approached by letter and personally by RNS. Written information about COPD and its clinical management including Non Invasive Ventilation (NIV), Invasive Ventilation (IV), and Cardiopulmonary Resuscitation (CPR) were given and discussed with each patient. Consent was obtained. Patients were asked to fill in Quality Of Life Questionnaire (locally developed at Plymouth University). The following information were obtained; age, sex, spirometry, hospital admissions, or antidepressant usage in the last 12 months and oxygen usage. Patients were asked to consider scenario in which they were admitted to hospital and after standard treatment, failed to improve, continued to deteriorate or developed cardiopulmonary arrest, "Reaching that stage, would you wish to have NIV, IV or CPR?"

Results: 100 patients were surveyed $(41$ were males and 59 females), mean age of $74(48-92)$ years. The mean FEV 1 was 0.78 $(0.41-1.3) \mathrm{I}$ and $\mathrm{FEV} 1 \%$ of $42.7 \%(11-96 \%) .50 \%$ of patients had FEV $1<40 \%$ predicted and $35 \%$ had FEV 1 between 40 and $59 \%$ predicted. 24 patients (24\%) were on LTOT, $8(8 \%)$ used antidepressant and $56(56 \%)$ had been admitted to hospital over the last 12 months. 48 patients (48\%) wanted all treatments (CPR, NIV and IV) and 12 (12\%) wanted non. 19 patients (19\%) said No" for CPR but "Yes" to NIV and IV. 10 patients (10\%) said "No" for CPR and IV but "Yes" for NIV. The remaining 11 patients $(11 \%)$ gave mixed answers. Using ANOVAR analysis and Chi Square test there were no significant statistical differences between the groups. Conclusion; The commonly available measurable parameters of COPD could not predict patients views on CPR, IV, or NIV. These issues should be discussed with vulnerable patients preferably before hospital admission.

\section{S148 CHRONIC OBSTRUCTIVE PULMONARY DISEASE-ALIGNING PATIENT AND PHYSICIAN VIEWS}

I. Murdoch', I. Draffan', M. Karavali', V. Higgins'2. 'AstraZeneca, Macclesfield, United Kingdom; ${ }^{2}$ Adelphi Group Products, Macclesfield, UK

COPD patients suffer many debilitating symptoms which ultimately impair their quality of life. A Respiratory Disease-Specific Programme was conducted in respiratory and primary care clinics in 5 European countries. Primary care physicians (PCPs) and Respiratory Specialists (RSs) ( $n=769$ ) completed patient record forms (PRFs) prospectively, on the type and severity of COPD symptoms and impact of these on patients' lifestyle in terms of ability to undertake activities of daily living (ADL). Similarly, patients returned a self-completion form (SCF), detailing the severity, frequency, and impact of their respiratory symptoms on ADL. A total of 2446 patients were diagnosed with COPD (diagnosis recorded as COPD, emphysema or chronic bronchitis) and $72 \%(n=1768)$ of these returned a SCF.

PRF data showed that the most commonly reported symptoms of patients diagnosed with COPD were breathlessness on exertion $(86 \%)$, daytime cough $(82 \%)$, sputum $(75 \%)$, and nocturnal cough $(67 \%)$. SCF data showed that patients also identified these as key symptoms, recorded as either a moderate or major problem in the previous 6 months for $71 \%, 70 \%, 64 \%$, and $61 \%$ of patients reporting severity respectively.

For these 4 key symptoms and others (wheezing, breathlessness at rest, tightening of chest, and nocturnal wakening) COPD patients regarded these to be more severe than PCPs and RSs. Patients suggested that COPD restricts their ability to undertake ADLs (work/housework, leisure activities and ability to exercise) to a greater degree than that perceived by PCPs $(p<0.01)$ and RSs $(p<0.01)$. 
These data demonstrate that breathlessness, cough and sputum production are frequent and important symptoms in patients with COPD, and that physicians may underestimate the severity and restrictive impact of these symptoms, and others, on COPD patients quality of life.

\begin{tabular}{|llll|}
\hline \multicolumn{3}{l}{ Abstract S149 } & \\
\hline & \multicolumn{3}{l|}{ Prevalence } \\
\cline { 2 - 4 } Symptom & Overall & Smokers & Non-smokers \\
\hline Breathlessness on mild exertion & $29 \%$ & $34 \%$ & $25 \%$ \\
Frequent winter colds & $22 \%$ & $29 \%$ & $20 \%$ \\
Persistent cough & $13 \%$ & $19 \%$ & $9 \%$ \\
Persistent sputum production & $9 \%$ & $13 \%$ & $7 \%$ \\
Any & $49 \%$ & $61 \%$ & $41 \%$ \\
\hline & & & \\
\hline
\end{tabular}

\section{S149 WHAT DOES THE GENERAL PUBLIC KNOW ABOUT COPD?}

D.M.G. Halpin', C. Ferenbach', D. Bellamy ${ }^{3}$, M. Rudolf ${ }^{4}$ on behalf of the BTS COPD consortium. 'Royal Devon \& Exeter Hospital, ${ }^{2}$ St Mary's Hospital, Portsmouth, UK; ${ }^{3}$ James Fisher Medical Centre, Bournemouth and ${ }^{4}$ Ealing Hospital

As part of continuing activities to promote the implementation of key messages contained in the BTS COPD guidelines the BTS COPD consortium undertook market research among the general population to help understand public knowledge about COPD and their attitude to chronic respiratory symptoms. MORI were commissioned to add a number of questions drafted by members of the Consortium to their weekly omnibus survey. A representative sample of 866 adults (age 15 and over) were interviewed in their own homes in 188 locations around the UK. $49 \%$ were lifelong non-smokers, $20 \%$ ex-smokers and $31 \%$ current smokers. Over $90 \%$ of respondents said that they had heard of asthma, Parkinson's disease, cervical cancer, MS and chronic bronchitis. $79 \%$ had heard of emphysema but only $35 \%$ had heard of COPD. A larger proportion of smokers had heard of COPD $(45 \%)$ compared with non-smokers $(24 \%)$. Knowledge about smoking as a causative factor for various conditions was mixed. A majority of respondents $(53 \%)$ thought that cervical cancer was the most common of these conditions. Only just over half of the respondents suggested that breathlessness on exertion or coughing could be the first signs of a serious lung disease. Many respondents had experienced persistent respiratory symptoms, but only just over half had consulted their GP about them. In 2 out of 3 cases this was because they were unconcerned or unaware that the symptoms may be important, and in nearly 1 in 4 cases this was because they thought they would simply be told to stop smoking. See table.

One in three members of the public state that they had heard of COPD, but many more recognise the terms chronic bronchitis and emphysema. Few were aware that COPD is common and is caused by smoking. Many individuals experience persistent respiratory symptoms but nearly half had not reported these to their GP often because they were unaware of their importance.

\section{S150 WHERE DO COPD PATIENTS DIE?}

M. Sridhar', I. Grace', M.R. Partridge'. 'Imperial College of Science, Technology and Medicine, NHLI Division; ${ }^{2}$ Department of Statistics at Charing Cross Hospital, London

Background: We analysed mortality statistics for England and Wales from the Office of National statistics to determine the place of death of patients certified as having died primarily due to COPD ICOAD, chronic bronchitis or emphysema) between the years 1970 to 2000 (at 5 year intervals). Deaths were classified as having occurred in a hospital or other health care institution (nursing home, NHS Mental health Units) or at home (the usual residence of the deceased or other, including residential homes). As a comparison the place of death of patients certified as having died with asthma and all causes were also analysed.

Results: Over the years between 1970 and 2000 there was gradual and significant increase in the proportion of patients with

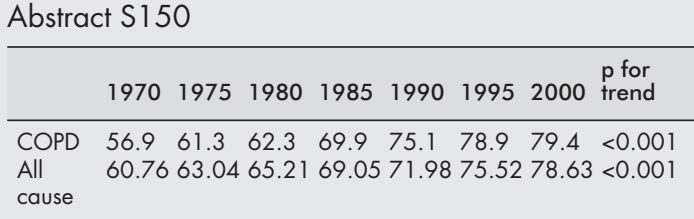

COPD dying in hospitals. Examination of all-cause mortality figures showed a similar trend. See table.

The figures for asthma showed a less striking trend to increase but this increase was not evident in the younger age group (under 40), perhaps reflecting the problems with mis-certification of asthma deaths.

Conclusion: An ever increasing proportion of patients with COPD die in hospital, reflecting a trend in the population in general. The reason for this trend needs further investigation and the observation should inform discussions on resource allocation and end of life care for patients with COPD.

\section{S151 MORTALITY PROJECTIONS FOR OBSTRUCTIVE LUNG DISEASE IN ENGLAND \& WALES}

A.L. Hansell', L. Knorr-Held'2, V. Schmid ${ }^{3}$. 'Dept Epidemiology \& Public Health, Imperial College, London; ${ }^{2}$ Dept of Mathematics and Statistics, Lancaster University, UK; ${ }^{3}$ Dept of Statistics, Ludwig-Maximilians University, Munich, Germany

Obstructive lung disease is a leading cause of mortality and morbidity worldwide and accounts for $20 \%$ of respiratory mortality in the UK (British Thoracic Society. The Burden of Lung Disease. London: British Thoracic Society 2001). Few studies have attempted to project the future burden of disease and none of these related to the UK. Simple extrapolation from current rates is likely to be misleading due to strong cohort and period trends (Lee PN, et al. Thorax 1990;45:657-65).

A recently developed Bayesian method was used to make 10 year mortality projections for chronic obstructive pulmonary disease (COPD) and asthma for those aged 45 years and over in England \& Wales, by extrapolating age, period and cohort trends for 1945-1999. Particular advantages of the Bayesian method used are that a good fit to the data is ensured and statistical uncertainty intervals can be automatically provided. Different smoothing constraints were tested. The final model was selected on the basis of the best predictions for the last 10 years of data (1989-1999). Adjusting for changes in ICD versions made little difference to the accuracy of these predictions. Also, use of a smoking covariate as a period effect did not improve predictions. Cohort covariates were unlikely to have improved predictions as the data hold good information about cohort trends for the oldest cohorts, which experience the largest number of deaths.

Projections suggested a median fall in rates for males of $24 \% 195 \%$ $\mathrm{Cl}:-52 \%$ to $+14 \%$ ) by 2009 on a 1999 baseline and corresponding $2 \%(95 \% \mathrm{Cl}:-40 \%$ to $+65 \%)$ rise in rates for females. By five year age-groups, posterior median projections of rates fell in both sexes, except in older females: for those aged 75-79 years rates rose then fell, while rates rose throughout for aged 80 years and over. Numbers of female deaths were projected to rise to around 14000 per year, equalling numbers of male deaths by the end of the decade. Credible intervals around projections were wide, reflecting marked yearly variations in the numbers of deaths.

\section{Risk factors in allergic disease}

\section{S152 THE EFFECT OF MITE ALLERGEN CONTROL BY THE USE OF ALLERGEN-IMPERMEABLE COVERS IN ADULT ASTHMA: THE SMAC TRIAL}

A. Custovic, L. Forster, E. Matthews, J. Martin, L. Letley, M. Vickers, J. Britton, D. Strachan, P. Howarth, D. Altmann, C. Frost, A. Woodcock and MRC GP research framework. MRC General Practice Research Framework at the MRC Clinical Trials Unit, Stephenson House, 158-160 North Gower Road, London NW1 2ND

There is a considerable controversy about the effectiveness of dust mite allergen avoidance in asthma. We carried out a randomised, parallel 
group, double-blind, placebo controlled trial of dust mite avoidance (allergen-impermeable covers for mattress, pillow, and quilt) in adult asthmatics. At entry, mite-specific lgE was determined, and patients were randomised with minimisation on smoking, pet ownership and mite-specific lgE. Patients and assessors were blind to the patients' dust mite sensitivity status. From 2479 patients who were screened for eligibility, 1150 from 135 general practices were randomised to receive active $(n=574)$ or placebo $(n=576)$ bed covers. PEFR was recorded twice daily during a 4 week run-in period and during months 6 and 12 of the study. In the first 6 months of the trial patients took their usual inhaled steroid therapy. Following this, a controlled reduction of inhaled steroids was attempted until either all inhaled steroid has been discontinued, or until asthma control deteriorated (Months 7-12). Homes were visited at the start in all patients, and revisited at 6 and 12 months in a $10 \%$ random sample to collect mattress dust for measurement of Der $p 1$. Der $p 1$ was significantly lower in the active group at 6 months $(p=0.023)$, but there was no difference between the groups at 12 months. $65.4 \%$ and $65.1 \%$ of patients were mite sensitive in the active and placebo group respectively. A total of 457 active and 459 placebo patients had PEFR data at both baseline and 6 months. PEFR improved significantly in both groups (active 409.7 to $417.7 \mathrm{l} / \mathrm{min}, \mathrm{p}<0.0001$; placebo 419.3 to $428.9 \mathrm{l} / \mathrm{min}, \mathrm{p}<0.0001$ ). After adjusting for baseline differences using analysis of covariance, there was no significant difference between the two groups (difference in means $(95 \% \mathrm{Cl})$, active $v$ placebo: all subjects $-2.11 \quad(-6.55$, 2.32), $p=0.35$; mite sensitive subjects $-1.71(-7.28,3.85) p=0.55)$. There was no difference between the groups in complete cessation of inhaled steroids or the proportionate change in steroid dose during reduction at 12 months, either in all subjects or in mite sensitive subjects only. In conclusion, allergen-impermeable bed covers seem clinically ineffective for routine management of adult asthma in primary care in the UK.

\section{S153 EXPOSURE TO INDOOR AEROALLERGENS AND SUBSEQUENT SENSITISATION AND WHEEZE IN AN ENGLISH BIRTH COHORT}

J.M. Harris, P. Cullinan, P. Mills, S. Moffat, C. White, A.J. Newman Taylor. Occupational \& Environmental Medicine, Faculty of Medicine, Imperial College of Science, Technology and Medicine, London, UK

A prospective cohort study of childhood asthma and allergic disease based in Ashford, Kent has been underway since 1993. A consecutive series of newly-pregnant women were recruited $193 \%$ of those eligible) and 642 babies were born. Eight weeks after birth samples of dust from the living room floor and infant's beds were collected. Levels of indoor aeroallergens (Der pl, house dust mite, and Fel dl, cat fur) were measured.

Each child was visited annually, and at age 5-6 sensitivity to Der $\mathrm{pl}, \mathrm{Fel} d \mathrm{l}$ and mixed grass pollens was measured in 552 (86\%) children using skin prick tests. The prevalence of sensitisation (mean weal diameter $2 \mathrm{~mm}+$ greater than the negative control) to these allergens were $10 \%, 9 \%$ and $9 \%$ respectively; $92(17 \%)$ of the children were atopic. Thirty nine atopic children (7\% of cohort) were wheezing at this age. Rates of sensitisation and atopic, current wheezing by quartiles of exposure categories using measurements from the living room carpet were as shown in the table.

The patterns above suggest that the association between levels of Der $\mathrm{pl}$ and $\mathrm{Fel} d \mathrm{l}$ experienced in early-life and subsequent sensitisation/wheeze has an "inverted u" shape. The reductions in risk at the highest exposure levels may be explained by an immunotolerance or by confounding determinants of sensitisation. Further investigations into these are underway.

\section{S154 ATOPY, ASTHMA, HAYFEVER AND THE RESPONSE TO INTRADERMAL TESTING WITH ENVIRONMENTAL MYCOBACTERIA AMONG CHILDREN IN RURAL CRETE}

C. Zekveld, P. Cullinan, I. Dimitroulis, A. Pedioti', V. Bibaki-Liakou', I. Bibakis', J. Stanford, ${ }^{2}$ A. Newman Taylor. Department of Occupational and Environmental Medicine, Imperial College (NHLI), London; 'AntiTuberculosis Unit, Venezalion Hospital, Heraklion, Crete; ${ }^{2}$ Department of Medical Microbiology, University College Hospital, London

Subclinical infection with environmental mycobacteria (eMB) may exert a strong immuno-regulatory effect. Among 805 children aged between 8 and 18 years, and living in rural Crete, we examined skin responses to intradermal inoculation with four locally prevalent $e M B$ species ( $M$ gordonae, $M$ fortuitum, $M$ intracellulare, and $M$ chelonae) identified by pilot testing with 20 species in three villages. We compared the skin responses between children with or without atopy (as judged by skin prick testing), asthma or hayfever. Virtually all children had received $B C G$ vaccination.

$643(80 \%)$ children had a positive response ( $3 \mathrm{~mm}$ or more) to at least one eMB species; $147(18 \%)$ reacted to all four. $182(23 \%)$ children had a positive skin prick test to one or more local aeroallergens; the prevalences of current wheeze $(4 \%)$ and seasonal rhinitis $(5 \%)$ were much lower.

Intradermal responses to $\mathrm{eMB}$ were unrelated to any of the outcomes in this population. This was true for each or all of the eMB species; and also for stronger $(10 \mathrm{~mm}$ or more) responses. Moreover there was no evidence, among atopic children, of a relationship between eMB skin responses and the presence of absence of allergic symptoms. These findings do not support the suggestion that early infection with environmental mycobacteria is protective in the development of childhood allergic diseases.

\section{S155 DOES VACCINATION INCREASE THE RISK OF DEVELOPING ALLERGIC DISEASE?: A BIRTH COHORT STUDY}

T.M. McKeever, S.A. Lewis, C. Smith, R. Hubbard. Division of Respiratory Medicine, University of Nottingham, UK

Objectives: There has been a rise in allergic disease prevalence in the last couple of decades, and one hypothesis for this increase is the introduction of widespread immunisation against infectious diseases.

Methods: Using the West Midlands General Practice Research Database, we have used a previously established birth cohort to examine the effect of vaccination to diphtheria, polio, pertussis and tetanus (DPPT) or measles, mumps and rubella (MMR) on the incidence of doctor diagnosed asthma and eczema.

Results: In univariate analysis there was an association between vaccination and the development of allergic disease such that vaccination to DPPT increased risk of asthma (HR $14.095 \% \mathrm{Cl} 7.3-$ 26.9) and eczema (HR 9.4 95\% Cl 5.9-14.9) and similar strong effects were seen for vaccination to MMR. However there were significant interactions between vaccination and consulting behaviour, such that the effect of vaccination was limited to those in the lowest level of consulting behaviour, and was no longer significant in subsequent levels of consulting behaviour, suggesting that the initial observed effects were due to ascertainment bias.

Conclusions: Our data suggest that it is unlikely that currently recommended routine vaccinations are a risk factor for asthma or eczema.

\begin{tabular}{|c|c|c|c|c|c|}
\hline $\begin{array}{l}\text { Der } p 1 \\
\text { levels }(\mu \mathrm{g} / \mathrm{g})\end{array}$ & $\begin{array}{l}\mathrm{n}(\%) \\
\text { sensitised }\end{array}$ & $\begin{array}{l}\mathrm{n}(\%) \text { atopic } \\
\text { wheezing }\end{array}$ & $\begin{array}{l}\text { Fel d1 levels } \\
(\mathrm{lg} / \mathrm{g})\end{array}$ & $\begin{array}{l}n(\%) \\
\text { sensitised }\end{array}$ & $\begin{array}{l}n(\%) \text { atopic } \\
\text { wheezing }\end{array}$ \\
\hline $0.02-0.39$ & $10(7 \%)$ & $7(5 \%)$ & $0.01-0.53$ & $8(6 \%)$ & $7(5 \%)$ \\
\hline $0.39-1.28$ & $18(14 \%)$ & $13(10 \%)$ & $0.53-1.76$ & 11 (8\%) & $11(8 \%)$ \\
\hline $1.28-5.66$ & 17 (12\%) & $11(8 \%)$ & $1.76-18$ & $17(12 \%)$ & $11(8 \%)$ \\
\hline $5.66-385$ & $7(5 \%)$ & $7(5 \%)$ & $18-14151$ & $11(8 \%)$ & $10(7 \%)$ \\
\hline
\end{tabular}




\begin{tabular}{|c|c|c|c|c|}
\hline & \multicolumn{2}{|c|}{ Wheeze in the 2 nd year ${ }^{\dagger}(n=98)$} & \multicolumn{2}{|c|}{ Eczema in the $2 n d$ year $^{\dagger}(n=160)$} \\
\hline & \multicolumn{4}{|c|}{ Maternal vitamin E intake } \\
\hline & Diet + supplements & Diet only & Diet + supplements & Diet only \\
\hline $1^{\ddagger}$ & 1.00 & 1.00 & 1.00 & 1.00 \\
\hline $2^{\ddagger}$ & $0.63(0.30-1.34)$ & $0.57(0.27-1.17)$ & $0.77(0.41-1.44)$ & $0.53(0.29-0.99)$ * \\
\hline $3^{\ddagger}$ & $0.56(0.27-1.15)$ & $0.61(0.31-1.23)$ & $0.44(0.23-0.83)^{*}$ & $0.43(0.23-0.78)$ * \\
\hline $4^{\ddagger}$ & $0.55(0.26-1.16)$ & $0.50(0.24-1.07)$ & $0.83(0.45-1.51)$ & $0.53(0.28-0.98)$ * \\
\hline $5 \neq$ & $0.58(0.26-1.19)$ & $0.50(0.23-1.07)$ & $0.41(0.21-0.80)^{*}$ & $0.48(0.25-0.91)$ * \\
\hline $\mathrm{p}$ (trend) & 0.107 & 0.065 & $0.024^{*}$ & $0.030 *$ \\
\hline
\end{tabular}

${ }^{*} p<0.05 ;{ }^{\dagger}$ Logistic regression: odds ratio $(95 \% \mathrm{Cl})$ adjusted for gender, maternal age, maternal atopy, socia class, maternal smoking \& antibiotics; ${ }^{\ddagger}$ Quintiles of intake.

\section{S156 REGULAR OILY FISH CONSUMPTION IS PROTECTIVE AGAINST SYMPTOMATIC ASTHMA}

B. Patel, R. Luben, D. Lomas, N. Wareham. Departments of Public Health and Primary Care, and Department of Medicine, University of Cambridge, Hills Road, Cambridge

Oily fish is rich in $n-3$ polyunsaturated fatty acids (PUFAs). While $n-6$ PUFAs are the main substrate for the production of arachodonic acid (AA), n-3 PUFAs are competitive inhibitors of AA metabolism, and may reduce the production of 4-series leukotrienes and bronchoconstricting prostagladins such as $\mathrm{PGD}_{2}$ from $\mathrm{AA}$.

To assess the association between oily fish consumption and symptomatic asthma, we conducted a nested case control study within the European Prospective Investigation of Cancer (EPIC)-Norfolk cohort. Between 1993 and 1998 all participants completed a baseline health and lifestyle questionnaire (HLQ). Cases were selected by a positive response and controls by a negative response to the question "has your doctor ever told you have asthma?" in the HLQ. Frequency of oily fish consumption was also recorded in the HLQ. In 1998 potential cases and matched controls were asked to complete the East Anglia Respiratory Questionnaire about respiratory symptoms experienced in the previous 12 months.

Complete data were available on 333 cases who reported wheeze in the previous 12 months, and 437 asymptomatic controls. Significantly more controls than cases reported eating oily fish at least twice a week $(12.4 \% \vee 7.5 \%, p=0.03)$. In logistic regression analysis, after adjusting for age, sex, BMl, social class and smoking, regular oily fish consumption ( $=$ twice a week) relative to rare $<$ than once a week) was associated with a reduced odds ratio (OR) for self-reported wheeze (OR 0.5, $\mathrm{p}=0.01)$, wheeze with breathlessness (OR $0.48, p=0.01$ ), awakening with tightness in the chest (OR 0.41 $\mathrm{p}=0.08$ ) and asthma attack (OR $0.57, \mathrm{p}=0.08$ ) in the preceding 12 months. Moreover, in a sub-analysis of 55 controls without diagnosed asthma who were excluded from the main analysis because of self-reported wheeze in the previous 12 months, only $7.3 \%$ reported eating oily fish twice a week or more. Relative to rare consumption, the adjusted $O R$ for wheeze with regular oily fish consumption in this group was $0.54(p=0.28)$.

In conclusion we have shown an association between oily fish consumption and symptomatic wheeze in people with diagnosed asthma and without diagnosed asthma. These data support the hypothesis that regular consumption of oily fish may be protective against symptomatic asthma.

\section{S157 THE EFFECTS OF MATERNAL DIET AND EARLY ANTIBIOTIC USE ON ALLERGIC DISEASE IN THE SECOND YEAR OF LIFE}

S. Fleming, G. McNeill, G. Devereux, A. Seaton. Department of Environmental and Occupational Medicine, University of Aberdeen, UK

The suggestion that maternal and early life influences may be important in the onset of allergic disease is being investigated. A cohort of 2000 pregnant women was identified and their diet assessed by food frequency questionnaire during pregnancy. Allergic disease was assessed in their children at 6, 12 \& 24 months of age. Questionnaire data were available from 1371 children for the 2 nd year of life. Wheeze and eczema were associated with male gender, maternal atopy, social class and maternal smoking but not with maternal dietary antioxidant vitamins. However when the analysis was restricted to atopic mothers only $(n=714)$, an inverse association between maternal vitamin $E$ intake and eczema was found as shown in the table.

There were positive associations between eczema and early antibiotic administration, which remained when the analysis was restricted to antibiotics given for non-skin conditions in the first 6 months and the development of eczema from 7-24 months (OR 1.33, $p=0.038)$. A similar association with wheeze disappeared when restricted to antibiotics given for non-chest conditions. This may be an indication of the effect of early antibiotics on the development of atopy. Vitamin $E$ intake during pregnancy and early introduction of antibiotics may be related to the onset of allergic disease in childhood. 


\section{Poster presentations}

\section{Respiratory muscles}

\section{P1 MULTIDISCIPLINARY MOTOR NEURONE DISEASE CLINICS: DO THEY IMPROVE PATIENT CARE?}

B.V. Prathibha, C. Wylie, N. Pease, F.J. Thomas. Nevill Hall Hospital, Brecon Road, Abergavenny, South Wales, UK

Motor neurone disease is a progressive neurological disorder which results in respiratory failure eventually. Currently the respiratory input into the management of these patients occurs at the terminal stages of their life. In addition, these patients need input from other specialties including palliative care and gastroenterology. We describe here a multidisciplinary approach with a new type of clinic set up so that the patients are seen by these specialists early on in the illness in the hope of improving the quality of care.

Aim: The aim was to set up a one stop clinic providing combined respiratory, neurological, and palliative care advice with input from voluntary organisations (MND association) and social workers and to assess any perceived benefits from the patients and carers point of view.

Design: The clinic is scheduled once a month and the three physicians (respiratory, neurology, and palliative care) sit in the same room with the other organisation representatives in the next room. The patients are offered an appointment in the specialist MND clinic following confirmation of the diagnosis by the neurologist. A special proforma has been designed to include the neurological, respiratory, gastroenterological, and social aspects of care. At each visit, the different variables including spirometry and blood gases are recorded. All patients undergo baseline overnight sleep study. The subject of NIPPV and intubation is introduced during these visits depending on the symptoms. The patients then make informed choices about their treatment. A patient feedback questionnaire has been designed and is given to each new patient.

Results: The clinic was established in November 2001 and to date 11 patients ( 7 males and four females) have been seen. The median age is 63 for men and 62 for women. The median FVC at presentation is $89 \%$ predicted and median $\mathrm{PO}_{2}$ and $\mathrm{PCO}_{2}$ are 11.5 and 4.8 respectively. The decline in the FVC over 9 months is associated with increasing $\mathrm{PCO}_{2}$ and symptoms. Three have been opted for NIPPV for symptom palliation. All patients rated the clinic highly, preferred to come to a single clinic than to three different ones and felt that they had adequate time to discuss all their concerns and have them allayed.

Conclusion: MND patients need specialised input from many professionals and too often this is patchy and unsatisfactory. The organisation of a combined clinic of the type described above has improved patient care and communication between the different specialists and has resulted in the efficient use of time and other limited resources.

\section{P2 OUTCOMES OF ASSISTED VENTILATION IN MOTOR NEURONE DISEASE}

S. Sundaram, I.E. Smith, J.M. Shneerson. Respiratory Support And Sleep Centre, Papworth Hospital, Cambridge, UK

Assisted Ventilation may prolong survival in patients with respiratory failure in motor neurone disease (MND), but there are few published reports.

We performed a retrospective study of all patients referred to our tertiary care centre between 1993 and 2002.

We identified 109 patients; mean age 60 (SD 9.0) years, $74 \%$ of whom were male. Fifty-one per cent had bulbar symptoms. Sixteen patients were transferred from intensive care units elsewhere with tracheostomy ventilation (TV). Fiffy-five patients were offered non-invasive ventilation (NIV), of whom 43 accepted and 12 declined treatment. Thirty seven patients were assessed at our unit and were thought not to require any assisted ventilation. One patient required only a tracheostomy with no assisted ventilation. Nine of the TV patients were weaned to NIV. Patients who still required TV at the time of discharge managed to self ventilate by day, but within three months were once again continuously dependent on it. Among patients offered NIV de novo, 36 had hypercapnia (mean $\mathrm{PaCO}_{2} 8.0 \mathrm{kPa}$ ) and 19 had orthopnoea causing sleep disturbance. The arterial blood gases while self ventilating on air improved in patients treated with NIV. At first follow up, the mean fall in $\mathrm{PaCO}_{2}(95 \% \mathrm{Cl})$ was $2.3 \mathrm{kPa}(1.2$ to 3.4 ; $\mathrm{p}<0.001)$ and the mean rise in $\mathrm{PaO}_{2}$ was $1.1 \mathrm{kPa}(0.30$ to 2.0; $p<0.01)$. Bulbar weakness was as frequent in patients given NIV as those that declined $(p=0.66)$.

There have been 86 deaths. Using the Kaplan-Meier method, the median survival was four months in patients who declined treatment and 11 months in those thought not to need NIV. There was no difference in survival between patients transferred intubated from ICU 12 months) and other subjects who started assisted ventilation (14 months; $\mathrm{p}=0.89$ ).

Many MND patients treated with tracheostomy ventilation can be weaned to NIV. In our cohort bulbar weakness did not preclude NIV. Survival was best for patients using assisted ventilation and worst for those who declined treatment.

\section{P3 OUTCOME OF NON-INVASIVE DOMICILIARY VENTILATION FOR PREVIOUS POLIOMYELITIS: IMPACT OF SCOLIOSIS}

Y. Tai, D. McKeon, I.E. Smith, J.M. Shneerson. Respiratory Support and Sleep Centre, Papworth Hospital, Cambridge, UK

Survivors of poliomyelitis can develop new respiratory disability many years after the acute illness. Scoliosis, muscle weakness, and decreased respiratory drive may all contribute. We examined the effect of scoliosis on survival in 51 patients (20 women) with previous poliomyelitis established on home mechanical ventilation (HMV) in our centre.

Poliomyelitis was contracted at a median age of 6.5 years; $82 \%$ of the patients had a thoracic scoliosis. The mean interval from contracting poliomyelitis to HMV was 46 (SD 8.8) years. Arterial blood gases on room air prior to $\mathrm{HMV}$ showed $\mathrm{PaO}_{2} 7.2 \mathrm{kPa}$ and $\mathrm{PaCO}_{2} 8.6 \mathrm{kPa}$, which significantly improved $(\mathrm{p}<0.05)$ after one month of treatment to $\mathrm{PaO}_{2} 9.5 \mathrm{kPa}$ and $\mathrm{PaCO}_{2} 6.6 \mathrm{kPa}$. The improvement was maintained at a mean follow up of 58 months. The Kaplan-Meir plot for life expectancy from initiation of ventilation is shown in the figure: survival was 7.35 years for subjects without scoliosis (solid line) $v 12.1$ years for subjects with scoliosis (dashed line) $(p=0.048)$.

Comparing subjects with and without scoliosis there were no differences in blood gases or overnight monitoring at presentation or during follow up on $\mathrm{HMV}$; those with scoliosis had a smaller vital capacity $1.18 \mathrm{~L} \vee 1.49 \mathrm{~L}(\mathrm{p}=0.013)$ and were younger when they had contracted poliomyelitis (median age $5 \vee 20$ years; $p=0.003$ ); the time interval from poliomyelitis to HMV was similar for the two groups and so those with scoliosis started ventilation at an earlier age. Recalculating life expectancy from birth there was no difference between the two groups $(p=0.989)$.

This study confirms that HMV can normalise arterial blood gas tensions in patients with previous poliomyelitis and the improvement is maintained. Overall survival is good. Compared to subjects with a scoliosis survival from the initiation of HMV is worse for subjects without a scoliosis largely because they are older when they develop ventilatory failure.

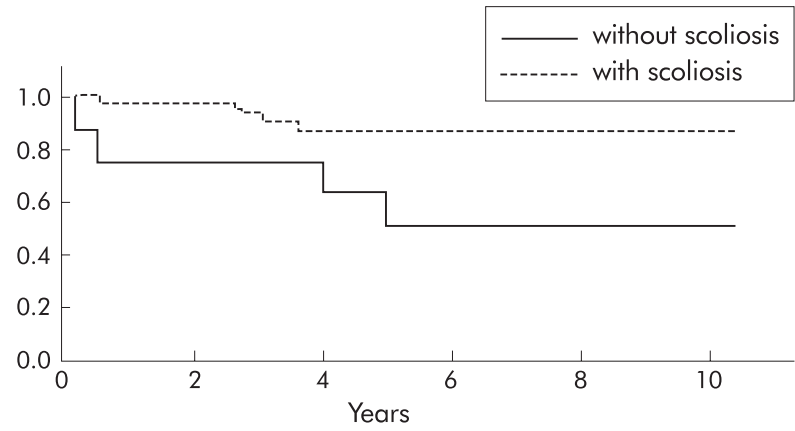

Abstract P3 Kaplan-Meir plot for life expectancy from initiation of ventilation. 


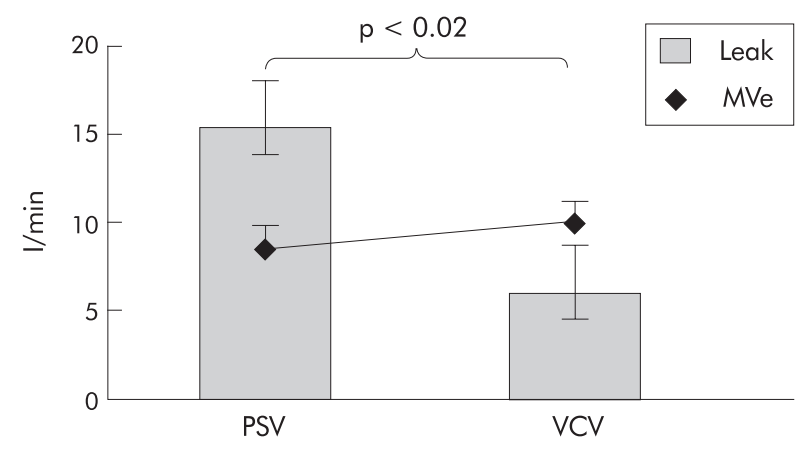

Abstract P4

\section{P4 COMPARISON OF PRESSURE AND VOLUME TARGETED NON-INVASIVE VENTILATION IN CHEST WALL DEFORMITY}

\section{J.M. Tuggey, M.W. Elliott. St James's University Hospital, Leeds, UK}

Introduction: The use of domiciliary non-invasive ventilation (NIV) in chest wall deformity is established and of proven benefit in terms of arterial blood gas tensions, sleep quality and daytime function. Previous studies have compared in the short term, volume, and pressure targeted ventilation. As part of a larger study, we investigated the effect of ventilation mode on minute ventilation and mask leak.

Method: 10 patients (mean age 64; mean FVC 0.55 I) with chest wall deformity, established on home NIV completed a randomised crossover study comparing pressure support and volume ventilation using the Breas PV403 ventilator. Ventilator parameters were set following a daytime titration period according to patient comfort and so that MVe was identical for both PSV and VCV. Patients used the machine at home for four weeks for each modality. At the end of each period, patients were admitted for a sleep study, including measurement of ventilation using a pneumotachometer connected to the ventilator circuit, adjacent to the nasal mask. Delivered tidal volume (VTi), expired tidal volume (VTe), expired minute volume (MVe) and respiratory rate (fR) were measured throughout the night. Minute leak was calculated by $(\mathrm{Vti}-\mathrm{Vte}) \times \mathrm{fR}$.

Results: See figure.

Ventilation was slightly greater in the volume group. There was significantly more leak during PSV than compared to VCV $(16 \mathrm{v} 6 \mathrm{l} / \mathrm{min})$.

Conclusion: Volume cycled ventilation results in less leak without compromising ventilation compared to PSV. Excessive amounts of leak during PSV may result in greater arousals and sleep fragmentation.

$\mathrm{JT}$ is funded by the NHS Northern \& Yorkshire Executive

\section{P5 COMPARISON OF PRESSURE AND VOLUME CYCLED VENTILATION IN KYPHOSCOLIOSIS (A RANDOMISED, SINGLE BLIND, CROSSOVER, PILOT STUDY)}

S.E. Higgins, R. Russell, B.E.A. Lams, A.J. Williams, A.C. Davidson. LaneFox Respiratory Unit, St Thomas' Hospital, London, UK

Introduction: This study compared pressure controlled (PCV) and volume controlled ventilation (VCV) in patients with kyphoscoliosis (KS). Two previous studies have been published with conflicting results. One suggests that some more severe patients were inadequately treated with PCV (Schonhofer B, et al. Eur Respir J 1997;10:184-91). The second study found no differences between the modes but suggested that patient fatigue was reduced using PCV (Ferris G, et al. Am J Phys Med Rehabil 2000;79:24-9).

Patients \& methods: Twelve patients (three female, nine male) with $\mathrm{KS}$ and longstanding chronic respiratory failure (CRF) $\left(\mathrm{PaCO}_{2}\right.$ at enrolment was $5.96+0.73 \mathrm{kPa}$ ) were recruited into the study, (mean age 59 (8.9) years). All patients were receiving domiciliary nocturnal pressure ventilation. We measured the following at base line; $A B G s$, overnight Pulse Oximetry and transcutaneous $\mathrm{CO}_{2}, \mathrm{FVC}, \mathrm{FEV}{ }_{1}, \mathrm{SNIP}$, Psychomotor Vigilance (PVT), OSLER Sleep latency, Epworth Sleepiness Scale, and Actigraphy \& Sleep Diary for 10 days. Each patient was then randomised to one month of PCV or VCV using the BREAS PV403 ventilator. The subjects were blinded to the mode of ventilation. At the end of one month all of the above parameters were remeasured, the mode changed and the parameters remeasured after a further month. At the end of the study the subjects were asked to express a preference for month one or month two.
Abstract P6 Beck, SGRQ and SF-36 following PC or $\mathrm{VC}$ ventilation for four weeks.

\begin{tabular}{lllll}
\hline & Beck & SGRQ total & PF & RL \\
\hline Pre & 6.5 & $39.6(17.1)$ & $31.4(28.7)$ & $28.6(39.3)$ \\
PC & $6.3(4.9)$ & $38.7(16.8)$ & $29.3(31.3)$ & $39.2(45.3)$ \\
VC & $6.8(5.3)$ & $36.6(15.4)$ & $32.1(29)$ & $35.7(47.6)$ \\
& & $6^{*}$ & $74^{*}$ & $73^{*}$ \\
\hline & & SF & EV & GH \\
\hline Pre & & $69.9(30.7)$ & $45.7(24.7)$ & $53.9(25.0)$ \\
PC & & $77.9(25.7)$ & $50.0(21.2)$ & $54.7(22.0)$ \\
VC & & $65.1(26.0)$ & $43.6(27.7)$ & $53.7(21.0)$ \\
Norm & & $82^{*}$ & $61^{*}$ & $63^{*}$ \\
\hline
\end{tabular}

Data mean (SD); comparisons made with Wilcoxon rank test. SF-36 data. ${ }^{*} \mathrm{p}<0.005 \mathrm{v}$ all study scores.

$\mathrm{PF}$, physical function; RL, role limit; SF, social function; EV energy/vitality; $\mathrm{GH}$, general health.

Results: Five subjects were excluded (three due to underlying OSA, one due to an unrelated illness, and one was unable to tolerate VCV). In the remaining seven no significant differences were found between PCV and VCV in most of the physiological variables. The hours of ventilator use was greater during PCV compared with VCV $(8: 22$ v 7:49), but this did not reach statistical significance $(p=0.07)$. Actigraphy revealed a significantly lower sleep fragmentation index in the PCV limb of the study $(32.6 \vee 38.5 ; p=0.04)$. This was supported by the subjects reported sleep quality during PCV $(2.17 v 2.45 ; p=0.01)$. In addition, subjects preferred PCV to VCV $(p<0.0002)$.

Conclusions: Patients with KS and CRF are adequately ventilated with both PCV and VCV modes. However patients prefer PCV, and subjective and objective sleep quality is improved with PCV.

\section{P6 COMPARISON OF PRESSURE $V$ VOLUME CYCLED VENTILATION IN KYPHOSCOLIOSIS A RANDOMISED SINGLE BLIND CROSSOVER STUDY: QUALITY OF LIFE OUTCOME DATA}

R.E.K. Russell, S.E. Higgins, B. Lams, A.J. Williams, A.C. Davidson. Lane Fox Respiratory Unit, St Thomas' Hospital, London, UK

Introduction: Patients with kyphoscoliosis (KS) may require long term ventilatory support as disease progression occurs. The ventilator used may be either volume (VC) or pressure controlled (PC). It is unclear which strategy is most beneficial for patients with KS.

Patients and methods: 12 subjects with KS (nine males, mean age 59 (8)) who were receiving long term ventilatory support were enrolled in a single blind randomised crossover pilot study. Ventilators were provided by the manufacturer (Breas PV403) and were set to provide the same minute volume in either volume or pressure control mode. Subjects completed the St George's Respiratory Questionnaire (SGRQ), the SF-36 health questionnaire, and the Beck depression score pre treatment and after four weeks on the trial ventilator at each setting.

Results: One subject was excluded due to intercurrent illness, three due to obstructive sleep apnoea, and one subject could not tolerate VC. Pulmonary function and QoL data are shown in table 1. There were no significant differences between pre treatment values and those obtained after four weeks on the two ventilator settings. However these subjects scored on all values much greater than healthy normals, indicating significant impairment of QoL. Beck depression scores were within the normal range.

Conclusions: Patients with KS on long term ventilation have significantly impaired QoL and the ventilator strategy used (VC or PC) does not affect this.

\section{P7 ADDUCTOR POLLICIS AND QUADRICEPS STRENGTH IN COPD}

M.G.G. Soliman', W.D-C. Man', D. Nikoletou', M.L. Harris', G.F. Rafferty', N. Mustfa', M.I. Polkey², A.J. Williams', J. Moxham? 'Respiratory Muscle Laboratories, King's College Hospital, UK; ${ }^{2}$ Royal Brompton Hospital, UK; ${ }^{3}$ St Thomas' Hospital, London, UK

Background: Peripheral muscle weakness is common in COPD, but the cause remains controversial. Better understanding of the distribution of muscle weakness may reveal the underlying 


\begin{tabular}{lllll|}
\hline \multicolumn{4}{l}{ Abstract P8 } \\
\hline & FVC \% & MIP & MEP & SNIP $\mathrm{CmH}_{2} \mathrm{O}$ \\
\hline VS & $29.9(25.6)$ & $33.1(17.7)$ & $54.9(34.8)$ & $28.2(14.2)$ \\
NS & $53.6(39.9)$ & $49.2(26.3)$ & $61.6(33.9)$ & $58.2(22.3)$ \\
p Value & 0.01 & 0.03 & 0.6 & $<0.0001$ \\
\hline
\end{tabular}

pathological process; chronic inactivity and disuse atrophy should lead to preferential involvement of the muscles with the greatest decrease in utilisation - that is, the lower limbs - whereas the loss of muscle function should be equally distributed if the underlying pathology is a more generalised process such as inflammation or malnutrition. To address this question, we used the non-volitional technique of supramaximal magnetic nerve stimulation to assess the strength of the adductor pollicis and quadriceps muscles.

Methods: 14 patients with stable COPD (not on oral corticosteroids) and 14 age matched healthy controls were recruited. Twitch adductor pollicis tension (TwAP) and twitch quadriceps tension (TwQ) were determined following supramaximal magnetic stimulation of the ulnar and femoral nerves respectively.

Results: Patients and controls were well matched for age, weight, height, and BMI. Mean FEV and FEV predicted for the COPD patients were 0.861 and $37 \%$ respectively. TwAP was similar in the COPD and healthy elderly groups $(7.04 \vee 7.30 \mathrm{~N} ; p=0.69)$. However TwQ was significantly lower in the COPD group $(68.2$ v $104 \mathrm{~N} ; \mathrm{p}=<0.001)$.

Discussion: In non-weight losing, stable COPD patients, quadriceps strength is significantly reduced compared with age matched healthy controls, whereas the strength of the adductor pollicis is normal. This is indirect evidence that there is not a generalised weakness in stable COPD, and that disuse atrophy is an important contributor towards peripheral muscle weakness.

WDCM is a Clinical Research Training Fellow of the MRC (UK).

\section{P8 THE RELATIONSHIP BETWEEN RESPIRATORY MUSCLE STRENGTH AND NEED FOR VENTILATORY SUPPORT IN POST-POLIO PATIENTS}

M.G.G. Soliman, S.E. Higgins, D.R. El-Kabir, B. Pereira, W.D-C. Man, A.C. Davidson, R.S. Howard, A.J. Williams. The Lane-Fox Respiratory Unit, St Thomas' Hospital, London, UK

Respiratory failure requiring nocturnal ventilatory support is a recognised late complication of previous poliomyelitis and is probably secondary to respiratory muscle weakness. Respiratory muscle strength was measured in 50 post-polio patients to assess the relationship between the need for ventilatory support and the degree of respiratory muscle weakness. Tests included forced vital capacity (FVC), maximum inspiratory (MIP) and expiratory pressures (MEP), and sniff nasal pressure (SNIP).

During the time of testing, 21 patients were using nocturnal ventilatory support (VS) compared with 29 on no support (NS). No significant differences in age, BMl, or time since acute episode, were found between the two groups. Respiratory muscle strength (mean (SD)) are presented in the table.

Of the patients requiring no support, those who had been ventilated during the acute episode had a significantly lower SNIP than those who had never been ventilated (48.8 (17.6) v 65.8 (27.4); $\mathrm{p}=0.04$ ).

Post-polio patients requiring nocturnal ventilatory support have a greater degree of inspiratory muscle weakness. SNIP is the most sensitive non-invasive test of inspiratory muscle weakness, and may prove to be a potentially useful tool in monitoring the progression of respiratory muscle weakness and subsequent ventilatory failure in post-polio patients.

\section{P9 COPD ALTERS THE DIAPHRAGM MOTOR AREA RESPONSE TO TRANSCRANIAL MAGNETIC STIMULATION}

N.S. Hopkinson, T. Sharshar, E. Ross, M.J. Dayer, A.H. Nickol, J. Moxham ${ }^{2}$, M.I. Polkey. Muscle Lab, Royal Brompton Hospital; ${ }^{2}$ King's College Hospital, London, UK

Background and methods: The threshold and stimulus response curve to transcranial magnetic stimulation (TMS) of the diaphragm motor area can provide information about cortical excitability in health and disease. We studied five patients with severe COPD (mean $\mathrm{FEV}, 20.2 \%$ predicted) and eight healthy controls. Stimulation series

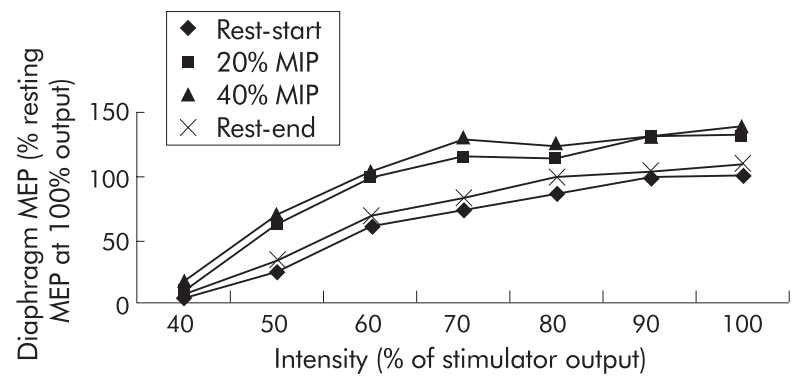

Abstract P9 The stimulus response curves for COPD patients are shown.

were delivered over the vertex using a $110 \mathrm{~mm}$ double cone coil (Magstim 200 mono-pulse) at a variety of intensities in random order and the surface diaphragm motor evoked potential (MEP) recorded. Series were performed at resting end expiration and then, facilitated, during inspiratory manoeuvres at $20 \%$ and $40 \%$ of maximum inspiratory pressure (MIP) and then again at rest. Healthy subjects also performed a run at $60 \%$ MIP. The stimulus response curves for COPD patients are shown in the figure. This group showed a significant increase from rest to $20 \%$ facilitation but no further increase at $40 \%$. By contrast controls showed a stepwise increase in MEP to $40 \%$ with no further increase at $60 \%$.

Conclusion: COPD patients have a reduced cortical reserve perhaps because they are already facilitated at rest by an increased work of breathing.

This study was funded by the Wellcome Trust.

\section{P10 THE EFFECT OF LONG TERM DOMICILIARY NON-INVASIVE POSITIVE PRESSURE VENTILATION (NIPPV) ON RESPIRATORY MUSCLE FUNCTION IN CHRONIC TYPE 2 RESPIRATORY FAILURE}

A.D. Gething, E.M. Williams, A. Phillips, B.V. Prathibha. ${ }^{2}$ School of Applied Science, University of Glamorgan; ${ }^{2}$ Respiratory Department, Nevill Hall Hospital, Abergavenny

In subjects with acute and chronic type 2 respiratory failure (due to kyphoscoliosis, sleep hypoventilation, and chronic obstructive pulmonary disease) application of nasal intermittent positive pressure ventilation (NIPPV) helps to restore arterial blood gases to normal levels, and aid breathing by unloading inspiratory muscles. (1) The effect of NIPPV on inspiratory muscle strength (IMs) and endurance (IMe) was tested. ${ }^{(2)}$

Methods: Two patient groups were studied, group one $(n=6$, age 63 (7) years; mean (SD)) who had just started with NIPPV and group two ( $n=6,66(14)$ years) who had undergone domiciliary ventilation (dNIPPV) for longer than six months. Group one patients were tested twice, once soon after the initiation of NIPPV and again after three months of dNIPPV. Inspiratory muscle strength and endurance were tested by asking the patient to perform three maximal inspiratory manoeuvres (a measure of IMs) followed by repeated submaximal $(80 \%)$ manoeuvres with ever decreasing intermittent rest periods until task failure (a measure of $\mathrm{IMe}$ ). ${ }^{(2)}$ All patients were naïve to the $\mathrm{IMe}$ testing procedure.

Results: Group one patients (dNIPPV $<1$ month) had significantly $(\mathrm{p}<0.05)$ lower $\mathrm{PaO}_{2}$ than group two (dNIPPV >6 month) of 6.7 (2.5) $\checkmark 8.6(1.9) \mathrm{kPa}$ respectively. The $\mathrm{PaCO}_{2}$ was not significantly different at 7.5 (1.3) $\vee 6.4(0.9) \mathrm{kPa}$ respectively. The maximal inspiratory pressure (MIP) a measure of IMs was highest in group two (Groupl 32 (4) $v$ Group 247 (9) $\mathrm{cmH}_{2}$ O) as was sustainable MIP, a measure of IMe (Groupl 102 (50) v Group2 158 (50) time/pressure units). In group 1, three months of dNIPPV improved MIP from 32 (4) to 48 (14) $\mathrm{CmH}_{2} \mathrm{O}$ but had no effect on sustainable MIP.

Conclusions: These data show that using dNIPPV for three months and longer improves inspiratory muscle strength. Improving inspiratory muscle endurance takes longer to achieve and can only be seen in the patients receiving dNIPPV for over six months (Group two). There are no long term benefits of using dNIPPV in terms of increasing inspiratory muscle strength but there are for endurance. How these improvements in endurance relate to general health status have yet to be evaluated.

1. Vitacca M, et al. Chest 2000;1 18:1286.

2. Gething, et al. Thorax 2001;56 (Suppl III): 11 . 


\section{COPD exacerbations: ITU to discharge}

\section{P1 1 DEVELOPMENT OF A RESPIRATORY HIGH DEPENDENCY UNIT: EXPERIENCE FROM THE FIRST 20 MONTHS}

D.M.G. Halpin, A. Jackson, C.D. Sheldon, N.J. Withers, S. Dodds. Royal Devon \& Exeter Hospital, Exeter, Devon, UK

Non-invasive ventilation (NIV) has been used at the Royal Devon \& Exeter Hospital since the early 1990s. Patients receiving NIV were treated on the specialist respiratory ward and as awareness of the benefits spread, NIV became the treatment of choice for patients admitted in hypercapnic respiratory failure secondary to COPD. The number of patients requiring NIV grew steadily and a business case was prepared for an appropriately staffed and equipped respiratory high dependency unit (rHDU). Eventually funding was secured from money allocated to critical care services and the unit opened in November 2000. Here we present an analysis of our experience over the first 20 months.

The unit has four beds and is staffed with a nurse:patient ratio of $1: 2$. It is located in one bay of the respiratory ward. Two hundred and twenty three patients have been admitted, with 33 admitted more than once. The mean age was 64.5 yrs (range 18 to 91 ) and $51 \%$ of patients were male. Most admissions (71\%) occurred between $8 \mathrm{am}$ and $8 \mathrm{pm}$ and were mainly transfers of patients admitted acutely to the Emergency Medical Unit. Fifty one admissions (20\%) were patients transferred out of the ITU and 38 had tracheostomies. The median length of stay was 4.04 days (range 1 to 37 ).

One hundred and nine patients received NIV, with 19 ventilated more than once. The mean age was 69.3 yrs (range 18 to 91 ) and $50 \%$ were male. The median duration of NIV therapy was 25.0 hours (range 0.5 to 505). There were 29 deaths in patients treated with NIV. In most of these it had been agreed in advance that more invasive treatment would not be undertaken. Ten patients were transferred to ITU because of failure to respond to NIV

Twenty six patients received CPAP therapy, with one being treated more than once. The mean age was 70.2 yrs (range 32 to 88 ) and $64 \%$ were male. The median duration of CPAP therapy was 9.5 hours (range 0.5 to 134). There were five deaths and four patients were transferred to ITU because of failure to respond to CPAP.

The opening of the rHDU has allowed significant numbers of patients with respiratory failure to be managed effectively. Many of these patients would previously have been admitted to ITU. Appropriate staffing of the unit has allowed admissions to be accepted throughout the day and night and nearly one in three admissions occurred outside the normal working day. The rHDU has significantly enhanced the service we are able to offer patients.

\section{P12 VARIATION IN CONSULTANT'S PROGNOSTIC ESTIMATES FOR IDENTICAL PATIENTS MAY EXPLAIN VARIATION IN COPD INTENSIVE CARE UNIT (ICU) ADMISSION: SIMULATION STUDY FROM ONE CRITICAL CARE NETWORK}

M.J. Wildman², J. O'Dea, S. Walia, M. Tindall, O. Kostopoulou, Z. Khan. Critical Care Units City Hospital and University Hospital Birmingham; ${ }^{2}$ London School Of Hygiene \& Tropical Medicine, London, UK

Introduction: Anecdotal evidence suggests variation in gatekeeping decisions for COPD patients with respiratory failure considered for ICU. (1) However little is known about the approach of different doctors to the management of identical patients and the causes for the suggested variability. This study exploits the fact that consultants on call will occasionally make ICU admitting decisions on the basis of information conveyed over the phone. Simulation of such phone calls allows many clinicians to make decisions about identical patients in a way that mimics real life.

Methods: One hundred and twenty consultants caring for acute admissions in the Heart of England Critical Care Network were invited to take part. An investigator in the role of a registrar who had just assessed a COPD patient requiring ICU admission phoned consultants who were told that the patient was likely to arrest soon if not intubated. All relevant clinical information about the patient was available from the investigator. Clinicians were asked whether they would admit the patient to ICU and asked to predict ICU, hospital, and 180 day survival following admission. The probability of survival for the patients was subsequently calculated using the SUPPORT model. ${ }^{(2)}$ Doctors considered three patients all of whom had single organ failure, but who varied in characteristics shown in multivariate analysis to be predictive of outcome. ${ }^{(2)}$

Results: Out of 120 clinicians, $98(82 \%)$ took part. Patient one: $88.8 \%$ of doctors admitted patient one, with admitters giving a mean predicted hospital survival of $46.1 \%$ and non-admitters a significantly lower mean predicted hospital survival of $12.7 \% \quad(p<0.00005)$. Patient one's SUPPORT probability of 30 day survival was $89 \%$. Patient two: $64.3 \%$ of clinicians admitted patient two, with admitters giving a mean predicted hospital survival of $37.9 \%$ v $11.8 \%$ for nonadmitters $(\mathrm{p}<0.00005)$. Patient two's SUPPORT probability of 30 day survival was $94 \%$. Patient three: $39.8 \%$ admitted patient three, with admitters giving a mean predicted hospital survival of $28.2 \%$ v $13.0 \%$ for non-admitters $(p<0.00005)$. Patient three's SUPPORT probability of 30 day survival was $68 \%$.

Conclusion: Predicted hospital survival for identical patients following ICU admission differed significantly between clinicians who would admit compared to those who would not. This study suggests that an important source of variability in ICU admission may arise from marked variation in predictions of survival and that objective models of patient outcome might reduce variability and support equity.

1. Wildman M. BM 1998;317:1667.

2. Conners AF Jr, et al. Am J Resp Crit Care Med 1996;154:959-67.

\section{P13 CLINICAL DECISION MAKING: VENTILATING PATIENTS WITH EXACERBATIONS OF CHRONIC OBSTRUCTIVE PULMONARY DISEASE (COPD)}

F.M.R. Perrin', C.W.G. Turton', M. Renshaw'. 'Respiratory Department, ${ }^{2}$ Clinical Effectiveness Unit, Brighton and Sussex University Hospital, Eastern Road, Brighton, UK

Introduction: The decision to institute mechanical ventilation in a patient presenting in acute respiratory failure secondary to an exacerbation of COPD can be difficult and controversial. This study aimed to determine whether there is a real difference in the views held between respiratory physicians, general physicians, and intensivists, whether there are significant differences in practice between individual clinicians and which clinical factors most influence decision making.

Method: A questionnaire was circulated by post to 600 consultants (200 in each specialty) selected randomly and at meetings to 125 SpRs. It included 25 different demographic, chest and comorbid variables that were each scored 0 to 3 according to the perceived relevance of the variable in the decision to embark on a trial of ventilation (where 0 was irrelevant and three contraindicated ventilation). The total score for all the variables was calculated for each clinician (0 to 75$)$

Results: 356 questionnaires were returned; 321 were completed fully and analysed (120 respiratory, 109 general medicine, 92 intensive care). The total scores for the clinicians in each specialty were: respiratory: mean $37.5,95 \% \mathrm{Cl} 358$ to 39.3 , range 15 to 68 ; general medicine: mean $39.5,95 \% \mathrm{Cl} 37.7$ to 41.4 , range 12 to 65 ; intensive care: mean $38.8,95 \% \mathrm{Cl} 36.9$ to 40.8 , range 16 to 64 . The importance placed on the different variables were ordered similarly across specialties. The most influential factors in deciding not to ventilate a patient were documented permanent cognitive dysfunction needing constant supervision, nursing home resident failing all activities of daily living, lung cancer deemed inoperable due to COPD, previous CVA such that patient chair bound or aphasic, and previous difficulty weaning from ventilation. The factors regarded as being least important were documented depression requiring treatment, osteoporosis with vertebral body collapse, continued smoking $>20$ cigarettes/day, plasma albumin $<30 \mathrm{~g} / \mathrm{l}$, and an above knee amputation for peripheral vascular disease.

Conclusions: There were no significant differences in clinical decision making between the specialties but there was wide variation between individual clinicians. Few of the factors investigated would contraindicate ventilation alone.

P14 CARDIOVASCULAR COMORBIDITY IS ASSOCIATED WITH HIGHER MORTALITY IN CHRONIC OBSTRUCTIVE PULMONARY DISEASE (COPD) PATIENTS ADMITTED TO AN INTENSIVE CARE UNIT

K.S. Srinivasan, A. Spiliopopolous, M.K. Sridhar. Staffordshire General Hospital, Stafford, UK

Background: $30-50 \%$ of patients admitted to an Intensive Care Unit (ICU) with respiratory failure due to COPD die in hospital. (1) (2) Studies 
have highlighted the difficulty of predicting mortality in this patient group from standard clinical and other scoring systems. ${ }^{(3)}$ We present results of a retrospective analysis of data examining mortality in an unselected group of COPD patients admitted to the ICU in a district general hospital in England.

Patients and methods: Fifty nine patients 31 male, 28 female; mean age 66.2 years (50 to 86$)$ ) with COPD, who had been admitted with respiratory failure to the ICU at Stafford General Hospital in the last five years were studied. Of these patients, 1718 male, 9 female; mean age 72 years (52 to 86)) died while on ICU and 12 (5 male, 7 female) died within a year of their discharge. Age, smoking history (past or present), usage of oxygen or nebulisers at home, and associated cardiovascular diseases (ischaemic heart disease, heart failure, or hypertension), were studied as predictors of mortality. We do not know the severity of COPD before admission $\left(F E V_{1}\right)$ in these patients. Patients with associated cardiovascular conditions were found to have the highest mortality. Of the 59 patients, 24 had heart disease $(40 \%)$ and the death rate in this group was $54 \%$ (13 of 24 ). Of these nine died during their stay in ICU.

Conclusion: COPD patients with cardiovascular comorbidity suffer a higher mortality following admission to ICU with respiratory failure. This information may have a bearing on prognostication and decision making in COPD patients being considered for invasive ventilation and admissions to ICU.

1. Hill AT, et al. Respir Med 1998;92:156-61.

2. Sonell MG, et al. JAMA 1995;274:1852-7.

3. Kaelin RM, et al. Chest 1987;92:971-8.

\section{P15 REFERRAL AND OUTCOME OF PATIENTS WITH AN EXACERBATION OF COPD REQUIRING ADMISSION TO INTENSIVE CARE}

A. MacDuff', I.S. Grant', S. MacKenzie'2, P.T. Reid'. 'Depts of Respiratory Medicine, and 'Intensive Care, Lothian University Health Trust, Edinburgh

Exacerbations of COPD complicated by acidotic respiratory failure which fail to respond to medical management, including non-invasive ventilation (NIV), are associated with a high mortality rate. When a reversible aetiological factor is not present the decision to refer to ICU is difficult with reservations on outcome and pressure on ICU resources (Thorax 1997:52 (Suppl 5):S1-S28). To examine our own practice, we performed a retrospective audit of admissions to the Intensive Care Units in our Trust. We identified 56 consecutive patients between April 1999 to April 2001 in whom a primary diagnosis of COPD was recorded. Of these, 37 were excluded as having a reversible cause of respiratory failure or an alternative diagnosis.

The median age was 64.5 years (range 49 to 78 ). Nine patients were previously unknown to respiratory physicians. It was the first admission with respiratory failure requiring ventilatory support in five of the nine previously admitted patients. Previous lung function was only available on five patients: median $\mathrm{FEV}, 0.7 \mathrm{l}$ (range 0.35 to 1.7 ). Prior to ICU referral median $\left[\mathrm{H}^{+}\right]=65.25 \mathrm{mmol} / \mathrm{I}(\mathrm{IQR}$ 61.33-77.7), $\mathrm{PaCO}_{2}=12.82 \mathrm{kPa}$ (IQR 8.93-15.6) and $\mathrm{PaO}_{2}=8.2 \mathrm{kPa}$ (IQR 6.29.0). The median duration of assessment prior to ICU admission was 5.5 hours (IQR 2-10.1). Seventeen patients required invasive ventilation, one non-invasive ventilatory support. Two patients died in ICU (one of respiratory failure, one of refractory hypotension). The median duration of ICU stay for study patients was three days (IQR 2-6.23) compared to 2.7 days for all ICU patients at the Royal Infirmary and Western General. Sixteen were successfully discharged to respiratory unit. One patient died in respiratory failure but 15 patients were discharged and 14 were alive at one year. The median hospital stay was 13 days (IQR 9-19.3) (mean stay acute exacerbations of COPD in Lothian Trust $=8.3$ days).

Our data suggest that severe respiratory failure may be the first presentation of COPD to hospital. Limited time and information may be available to make an informed decision on referral to ITU, but length of stay and outcome are comparable to other ICU admissions.

\section{P16 AN AUDIT OF THE USE OF NON INVASIVE VENTILATION IN ACUTE EXACERBATIONS OF COPD}

C.J. Jolley, R. Savine, S. Ellum, O. Gilleard, B.E.A. Lams, A.C. Davidson. Lane-Fox Respiratory Unit, St. Thomas' Hospital, London, UK

Introduction: The benefits of non-invasive ventilation (NIV) in the treatment of acute type 2 respiratory failure secondary to chronic obstructive pulmonary disease (COPD) are well documented (Thorax 2000;55:550-4).
Guidelines for the use of NIV have been published by the British Thoracic Society (BTS) (Thorax 2002;57:192-21). We investigated whether all patients with an acute exacerbation of COPD who fulfil the BTS's criteria for NIV are being referred for this treatment.

Methods: We identified 203 patients who were admitted to St Thomas's Hospital, London, with an acute exacerbation of COPD between $1 / 11 / 2001$ and 10/7/2002 by searching the Accident \& Emergency (A\&E) register, High Dependency Unit (HDU), and Patient At Risk Team databases. The results of arterial blood gas (ABG) analysis on presentation to A\&E were obtained from the patients' $A \& E$ notes. We noted whether the patient was admitted to a general ward, received NIV on the HDU, or was intubated in A\&E.

Results: Fiffy four patients were excluded because their admission ABGs were not available from their A\&E notes, leaving 147 patients' data for analysis. Of 147 patients, 110 were admitted to a general ward, 33/147 received NIV on the High Dependency Unit, and $4 / 147$ patients were intubated in A\&E. The mean (SD) pH of patients admitted to a general ward was $7.38(0.06)$. The mean (SD) pH on admission of patients referred for NIV was 7.27 (0.09). Of 147 patients admitted via A\&E, 18 did not receive NIV despite meeting the BTS criteria on the basis of their arterial blood gas analysis on initial presentation. Their mean (SD) pH was $7.28(0.05)$.

Conclusion: A significant proportion of patients with acute type 2 respiratory failure secondary to COPD who meet the criteria for NIV do not receive it. Strategies are required to improve the pick up rate of patients who would benefit from NIV at the time of their presentation to A\&E.

\section{P17 NON-INVASIVE VENTILATION IN PATIENTS WITH RESPIRATORY FAILURE: EXPERIENCE FROM A DEVELOPING COUNTRY}

F. Hussain, J. Iqbal, R. Haqqee. Aga Khan University Hospital, Karachi, Pakistan

Objective: Non-invasive ventilation (NIV) has been found to be effective in the management of respiratory failure. In developing countries there is severe shortage of ICU beds and NIV service is still in early stages of development. Studies are needed, from developing countries, to determine its effectiveness among patients with different types of respiratory failure. In this study we have evaluated Asian patients with both types of respiratory failure as well as patients with respiratory distress and impending respiratory failure.

Method: The study was conducted at Aga Khan University Hospital Karachi, Pakistan. All patients who were treated with NIV during 1999-2000 were evaluated. Demographic details, history, and physical examination of patients were recorded. Laboratory data included serial arterial blood gas (ABG) analysis, chest radiograph, blood count, and electrolytes. Details of medical treatment, of NIV and their outcome were recorded.

Results: A total of 68 patients (35 males) were evaluated. Their Mean (SD) age was 60 (16.75) (range 16 to 90) yrs. The commonest indication for the use of NIV was Type II respiratory failure due to COPD exacerbation. There was significant improvement of mean $\mathrm{PaCO}_{2}$ within two hours of treatment $(76 \vee 70 \mathrm{~mm} \mathrm{Hg}, \mathrm{p}=0.001)$ and of mean pH at 24 hours $(7.30 \vee 7.36, p<0.001)$. Out of 37 patients, $25(68 \%)$ improved with NIV alone, five (14\%) needed invasive ventilation, and seven $(19 \%)$ were considered unsuitable for invasive ventilation despite deterioration. Mortality was 30\% (1 1 patients). NIV was applied to 17 patients with Type I respiratory failure; pneumonia, asthma, and pulmonary oedema were the common underlying diseases. The mean $\mathrm{PaO}_{2}$ improved within two hours of treatment 153 $v 87 \mathrm{~mm} \mathrm{Hg}, \mathrm{p}=0.003)$. Ten (59\%) patients were successfully treated with NIV alone, six (35\%) needed subsequent invasive ventilation, and one $(6 \%)$ was considered inappropriate for invasive ventilation despite deterioration. Six patients died (mortality 35\%). Neuromuscular diseases and pulmonary oedema were the common causes among 14 patients with respiratory distress (respiratory rate $>30$ along with use of accessory muscles and laboured breathing). NIV was successful in $9(64 \%)$ patients, $3(22 \%)$ needed invasive ventilation, and two (14\%) died. At the time of admission $16(24 \%)$ patients were considered inappropriate for invasive ventilation, based on the nature of medical illnesses, prior physical health, and patient's wishes. In this group $14(88 \%)$ patients had Type II respiratory failure and two $(12 \%)$ had Type I respiratory failure. Overall $8(50 \%)$ out of 16 had a successful outcome with NIV.

Conclusion: NIV can be used effectively in developing countries to treat patients with respiratory failure. It can be applied outside ICU and can obviate the need of invasive mechanical ventilation in responders. It can be used, with good effect, in patients not suitable for invasive mechanical ventilation. 


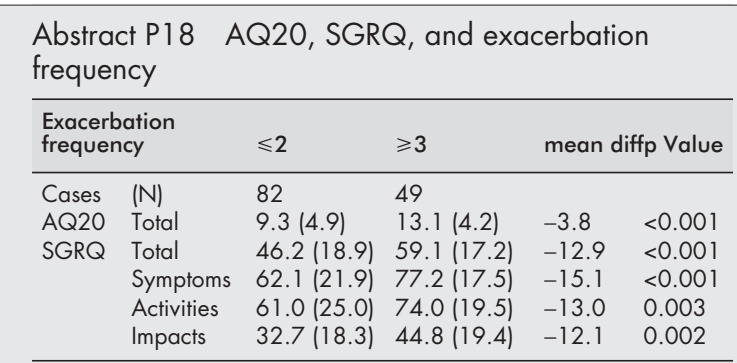

\section{P18 RELATIONSHIP BETWEEN AQ20, SGRQ, AND EXACERBATION FREQUENCY IN PEOPLE WITH COPD IN PRIMARY CARE}

A. Choudhury, C.M. Dawson, H.E. Kilvington, C.J. Griffiths, G.S. Feder, J.A. Wedzicha. Department of General Practice and Academic Respiratory Medicine, St Bartholomew's and Royal London School of Medicine and Dentistry, London, UK

Health related quality of life (HRQoL) is closely related to exacerbation frequency in patients with chronic obstructive pulmonary disease (COPD). The main obstacle to measuring HRQoL in primary care is their size and complex nature. The Airways Questionnaire 20 (AQ20) (Barley EA, et al. Respir Med 1998;92:1207-14) is a short, simple, disease specific questionnaire. Dimensions range from 0 to 20 (worst health).

Patients were recruited from general practices in East London. Patients were aged 40 and above, current or ex-smokers and were prescribed regular inhaled steroids. We asked patients to complete the AQ20 and the St George's Respiratory Questionnaire (SQRQ) simultaneously at interview.

One hundred and thirty one patients (68 male) of median age 66 years (range 48 to 87 ) with COPD were recruited from 12 general practices. Mean (SD) FEV was 1.28 (0.55) I, FEV predicted 50.0 (18.0)\%, mean $\mathrm{FEV}_{1} / \mathrm{FVC}$ ratio was 57.0 (16.0)\%. Patients had a yearly exacerbation rate of 2.4 (2.5). The median AQ20 score was 11 with a range of one to 19 . The median SGRQ total score was 51.0 with a range of 7.7 to 93.3 . The AQ20 was strongly correlated to the SGRQ (Spearman rank correlation); SGRQ symptom (0.548), activities (0.681), impacts (0.742), and total (0.749). $p<0.001$. Other statistically significant findings with $A Q 20$ were predicted FEV $(-0.271)$ and pack years smoked $(0.242) p<0.005$. Patients were divided into those who had less than three COPD exacerbations per year (infrequent exacerbators) and those who had three or more exacerbations per year (frequent exacerbators).

The AQ20 shows a strong relationship to yearly exacerbation rate. The AQ20 is simple to implement and can be used to assess health status for patients with COPD in primary care where time and resources are limited.

\section{P19 USING A PRIVATE SECTOR PARTNERSHIP TO PROVIDE SUPPORTED EARLY DISCHARGE FOR ACUTE EXACERBATIONS OF COPD IN A DISTRICT GENERAL HOSPITAL}

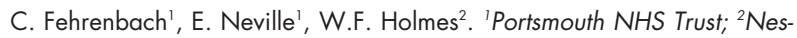
tor Healthcare PLC, UK

Home care is successful in about $25 \%$ patients hospitalised with an exacerbation of COPD, (1) though nursing resources are not always available to provide it. Encouraged by the government's endorsement of partnerships between the NHS and the private sector, ${ }^{(2)}$ a six month home care service was commissioned from Nestor Primecare, for nurse led $24 \mathrm{hr}$ care for selected patients following early hospital discharge. The Trust Respiratory nurses reviewed patients one month after discharge in clinic.

Following medical assessment in hospital (protocol available), the nurse accompanied and transported the patient home, and thereafter provided care over a 10 day period, (mean attendance: five hours). During the day, patients had direct access to the nurses, and at night through a dedicated telephone support line. When thought necessary, readmissions were organised by the nurse, not the patient's general practitioners.

In Portsmouth, about 580000 patients are served by two district general hospitals and three Primary Care Trusts. During the six months to July 2002 there were 276 admissions to the Trust coded J44.1 (exacerbation of COPD). The average hospital stay for this condition locally is eight days. Of these patients, 124 (44\%) (50\% male) received supported discharge within 1-2 days of admission. Fifty four calls for support came from 34 patients but none needed referral to their GP. There were 18 readmissions during the period of home care $(14 \%)$. Seven patients died during readmission, but none of those remaining at home. Patient satisfaction was high, and only one person refused home care.

Conclusion: This 10 day service was successful in a higher proportion of patients than in previous studies, and at a total cost of $£ 85000$.

1. Gravil JH, et al. Lancet 1998;351:1853-8.

2. DoH. NHS Plan. London: Department of Health, 2001.

\section{P20 A REGULAR VISIT TO PATIENTS ADMITTED WITH COPD REDUCES FURTHER HOSPITAL ADMISSIONS}

R. Rowles, R. Bennett, A. Hill, S. Jones, J. Goldman. Heart and Lung Unit, Torbay Hospital, Torquay, TQ2 7AA UK

The Torbay Hospital Outreach Respiratory Team (THORT) has been supporting the early discharge of patients with exacerbations of COPD since December 1999. It was noted that certain patients were having frequent admissions often with minor exacerbations that were then managed at home by THORT. It was proposed that regular maintenance visits by the team on patients identified as having frequent admissions or significant problems with anxiety would reduce these.

The first of these visits was in July 2001. In total 12 patients have been placed on the maintenance list and data has been collected on admissions pre and post the start of visits. For the purpose of this study 10 patients were selected who had complete sets of admission data for 12 months prior to the visits, and had been receiving maintenance visits for at least two months. Total bed days and THORT days were averaged over the previous 12 month period, and for the period since the onset of maintenance visits to give an indication of whether admissions had subsequently reduced.

The results show that in the year prior to the start of visits the average number of bed days and facilitated discharge days under THORT per month for the 10 patients studied was 40.1 . After the introduction of maintenance visits the average number of days was 8.7 per month.

The average number of maintenance visits undertaken per month was 12.8

Although this study has comparable data on a small number of patients, preliminary results suggest that regular maintenance visits to patients with COPD identified as having frequent exacerbations do have a positive impact on their ability to cope at home and avoid admission to hospital. In conclusion directing resources towards providing regular support to appropriate patients with COPD would be seen to be a cost effective way of reducing the use of healthcare resources by COPD patients over the winter months.

\section{P2 1 PRIMARY CARE EXPERIENCE OF PATIENTS ADMITTED TO HOSPITAL WITH AN EXACERBATION OF CHRONIC OBSTRUCTIVE PULMONARY DISEASE}

A. Coe, A.O.C. Johnson. Pontefract General Infirmary, Pontefract, West Yorkshire, UK

Although 'Hospital at Home' schemes for early facilitated discharge of patients with exacerbations of chronic obstructive pulmonary disease (COPD) have become more prevalent, schemes to prevent admission are less widespread. AC was awarded a Health Action Zone fellowship to investigate the role of a respiratory therapist in assessing and triaging potential admissions with exacerbations of COPD into those who require admission, early facilitated discharge (by referral to our Hospital at Home Scheme) or conventional discharge. This survey was done to inform the design of the potential new service.

Fifty three patients (mean age 71) admitted to hospital with exacerbations of COPD were questioned about their contact with primary care prior to admission. There were 26 men and 27 women. Symptoms which prompted admission included increased breathlessness $(92 \%)$, wheeze $(72 \%)$, cough $(55 \%)$, increased sputum volume $(49 \%)$, sputum colour change $(45 \%)$, fever $(38 \%)$. Three patients denied any change in symptoms. Prior to admission, $25 \%$ of the patients had had no contact with primary care services, $25 \%$ had telephone contact only, $34 \%$ were seen at home, and $17 \%$ were seen in the GP surgery. Of the 40 patients who had some contact with primary care, $40 \%$ had contact in the morning, $40 \%$ in the afternoon 
and only $12.5 \%$ out of hours (no data on three patients). Only 8 patients were seen twice with the same exacerbation prior to admission.

When asked why they had bypassed primary care most patients said either that they or a relative thought the situation too urgent for a GP to manage, or that previous experience was that the GP would send them to hospital anyway. The three patients with no change in symptoms had all bypassed primary care and were admitted because of anxious relatives. In contrast to the time of primary care contact, attendance at hospital was mostly out of hours $(51 \%)$ or afternoon $(36 \%)$. About half of the admissions were arranged by GPs, a quarter saw the GP but self presented to A\&E anyway, and a quarter bypassed primary care altogether.

We conclude that many patients and relatives admitted with COPD have little confidence in primary care's ability to deal with exacerbations, especially out of hours. A 24 hour service with specialist training may be able to deal with some patients with exacerbations and prevent unnecessary admission to hospital.

\section{Bronchoscopic and other lung investigations}

\section{P22 BRONCHOSCOPY PRACTICE IN ENGLAND AND WALES, 2002}

J. Pickles, M. Jeffrey, A. Datta, A.A. Jeffrey. Chest Clinic, Northampton General Hospital, Northampton NNI 5BD, UK

Aims: To assess current practice in bronchoscopy preparation in England and Wales.

Methods: Questionnaires were faxed to respiratory consultants listed in The BTS Directory." We looked at the population, number of consultants and bronchoscopies undertaken, topical anaesthetic use, sedative use and how adequate sedation is judged.

Results: There was a response rate of $76 \%$ (344 responses to 452 questionnaires). Median consultant numbers per hospital was three (IQR 4-6), median population served per consultant was 11600 (IQR 90-150 000). The majority of bronchoscopists use lignocaine spray to the throat $(70 \%)$, sometimes with spray to the nose $(43 \%)$, together with gel to the nose $(65 \%)$. The majority use $4 \%$ lignocaine to the vocal cords $(54 \%)$ and $2 \%$ to the bronchi $(71 \%)$. Atropine is used routinely by $13 \%$. Sedation with midazolam $(78 \%)$ or other combinations $(22 \%)$ is routine. The option of sedation is only discussed with the patients by $8.4 \%$ of consultants. Only three operators use formal sedation scores to assess patient level of sedation. Oxygen saturation was the commonest measurement used $(n=98)$ to judge sedation. Otherwise, response to sedation was judged by clinical experience $(n=60)$, patient response $(n=57)$, and conscious level $(n=50)$.

Conclusion: Despite the recent BTS guidelines there is considerable variation in bronchoscopy practice, particularly in sedation practice. Patient level of sedation is not formally assessed and combinations of sedatives and analgesics are used contrary to the recent guidelines on safe sedation practice. ${ }^{(2)}$ Sedation options are not routinely discussed. The wide variations in practice may reflect the lack of consistent evidence based guidance on sedation techniques for bronchoscopy. Further study to determine optimal technique is required.

1. BTS/Astra Zeneca. The directory of respiratory medicine [7th ed]. Hawker Publishing, 2002.

2. Safe sedation practice. Academy of Medical Royal Colleges and their Faculties, 2001.

\section{P23 FLUORESCENCE BRONCHOSCOPY IN PATIENTS WITH ABNORMAL SPUTUM CYTOLOGY}

A.K. Baneriee', P.H. Rabbitts', P.J.M. George2. 'Molecular Oncology Group, University Of Cambridge, UK; ${ }^{2}$ The Middlesex Hospital, London, UK

Introduction: Patients at risk of lung cancer with abnormal sputum cytology or bronchial washings but no other evidence of lung cancer present a management dilemma. Autofluorescence bronchoscopy detects preinvasive endobronchial lesions and carcinomas with greater sensitivity than conventional bronchoscopy. We present a series of patients with abnormal sputum cytology or bronchial wash cytology investigated further with autofluorescence bronchoscopy.
Abstract P23 Bronchoscopy results and outcomes for the study patients

\begin{tabular}{|c|c|c|}
\hline n Abnormality & Bronchoscopy & Outcome \\
\hline $\begin{array}{l}54 \text { Sputum SQC } \\
1 \text { Bronch wash } \\
\text { SQC }\end{array}$ & No abnormality & $\begin{array}{l}\text { Disease free } 7-24 \mathrm{mths} \\
\text { Disease free } 42 \mathrm{mths}\end{array}$ \\
\hline $\begin{array}{l}31 \text { Sputum SQC } \\
2 \text { Bronch wash } \\
\text { SQC }\end{array}$ & $\begin{array}{l}\text { Abnormal } \\
\text { fluorescence }\end{array}$ & $\begin{array}{l}S Q C \text { in areas of abnormal } \\
\text { fluorescence }\end{array}$ \\
\hline 1 Sputum SQC & $\begin{array}{l}\text { Abnormal } \\
\text { fluorescence }\end{array}$ & $\begin{array}{l}\text { CIS in area of abnormal } \\
\text { fluorescence (12 mth follow } \\
\text { up) }\end{array}$ \\
\hline 1 Sputum SQC & $\begin{array}{l}\text { Abnormal } \\
\text { fluorescence }\end{array}$ & $\begin{array}{l}\text { Normal histology (false +ve) } \\
\text { Disease free at } 48 \mathrm{mths}\end{array}$ \\
\hline
\end{tabular}

Methods: Patients selected had no clinical or radiological evidence of invasive carcinoma and no bronchoscopic abnormality within the preceding two months. The visible bronchial tree was inspected with white light and autofluorescence using the Storz bronchoscope. Biopsies were taken of all areas appearing abnormal bronchoscopically.

Results: Ten patients were studied, eight males with a mean age of 66.5yrs (range 51 to $79 \mathrm{yrs}$ ). All were smokers, mean exposure 47 pack years (range 18.5 to 79). The table shows the bronchoscopy results and outcomes for the study patients.

Conclusions: In this group of patients, no abnormality detectable at fluorescence bronchoscopy suggests a good outcome, with no evidence of carcinoma at up to 42 months. Abnormal fluorescence may reveal the presence of radiologically occult carcinoma, or high grade preinvasive lesion, but may also be a false positive finding. High grade preinvasive lesions may exfoliate cells that resemble squamous carcinoma cells. Fluorescence bronchoscopy may provide useful information in this difficult group of patients. This study is limited by the small numbers and relatively short duration of follow up, but suggests that a larger study should be undertaken.

\section{P24 ENDOBRONCHIAL MIMICS OF LUNG CANCER: A CASE REPORT AND REVIEW OF BRONCHOSCOPY DATA BASE}

S. Lohani, P.R. Chadwick, G.R. Armstrong, B.R. O'Driscoll, S.C.O. Taggart. Respiratory Medicine Department, Hope Hospital, Salford M6 8HD, UK

Case report: A 72 year old ex-smoker and retired fireman with previous asbestos exposure, presented with a six week history of cough, breathlessness, and wheeze. Clinical examination revealed a small basal effusion. CXR examination confirmed the clinical findings. ESR was $122 \mathrm{~mm} /$ hour. CT scanning revealed a loculated effusion and loss of volume in right lower lobe (RLL) with associated distal consolidation. Initial and subsequent bronchoscopies demonstrated an endobronchial RLL "tumour" but biopsies were negative for cancer. The patient was informed that cancer seemed the most likely cause of his symptoms. However, ultrasound guided aspiration of the fluid revealed an empyema which grew Actinomyces israelii. This was subsequently demonstrated to be present in both previous biopsy specimens. The patient made a full clinical and bronchoscopic recovery after six weeks of penicillin treatment.

Review of bronchoscopy data base: Over the past 10 years, our team has performed 4199 bronchoscopies of which 1122 (27\%) were found to have lesions suspicious of lung cancer. Of these 1004 $(89.5 \%)$ have had cancer confirmed by histology or cytology and 101 (9.0\%) have been treated as cancer although the biopsies were negative. Of patients with suspicious bronchoscopies, 17 (1.5\%) had a specific non-malignant diagnosis (see table).

Conclusions: Endobronchial mimics of lung cancer account for $>1 \%$ of suspicious bronchial lesions. We recommend caution in informing patients of cancer until either histological confirmation is obtained or other causes are excluded. 


\begin{tabular}{llll} 
Abstract P24 & & & \\
\hline Diagnosis & No & Diagnosis & No \\
\hline Benign polyps & 3 & Tracheopathica & 1 \\
Active tuberculosis & 3 & Hyalinising granuloma & 1 \\
Carcinoid & 2 & Sarcoid & 1 \\
Leiomyoma & 1 & Healed tuberculosis & 1 \\
Bronchial adenoma & 1 & Organised blood clot & 1 \\
Vasculitis & 1 & Actinomycosis & 1 \\
\hline
\end{tabular}

\section{P25 BRONCHOALVEOLAR LAVAGE, NON-INVASIVE INVESTIGATIONS AND RADIOLOGY: IMPACT ON TREATMENT IN PATIENTS WITH HAEMATOLOGICAL MALIGNANCIES AND PULMONARY INFILTRATES}

A.R. Benjamin, E.F. Bowen. Respiratory Medicine Unit, Hammersmith Hospital, London W12 ONN, UK

Pulmonary infiltrates are a frequent complication in immunosuppressed patients with haematological malignancies requiring early diagnosis with prompt appropriate treatment. We investigated the diagnostic yield of bronchoalveolar lavage (BAL) and non-invasive sampling (NIS) in this population over a 12 month period and evaluated their impact on treatment modification. We compared high resolution computed tomography (HRCT) findings with these results. Twenty five bronchoscopies (FOBs) (21 patients) were performed during this period. Seventeen out of 21 patients were post bone marrow transplant and $4 / 21$, on high dose chemotherapy. Pre FOB, 16/25 cases were neutropenic and/or lymphopenic, 15/25 were thrombocytopenic, $22 / 25$ were pyrexial, and $22 / 25$ were on empirical antibiotics. All were hypoxic and required supplemental oxygen periprocedure. Post $F O B, 1 / 21$ patient required admission to the intensive care unit.

Blood cultures, sputum cultures, and nasopharyngeal aspirates were positive in $3 / 41,4 / 21$, and $1 / 3$ samples respectively and treatment was modified in $2 / 25,2 / 25$, and $1 / 25$ cases respectively. Overall, NIS was positive in $8 / 25(32 \%)$ cases with subsequent treatment modification in $5 / 25(20 \%)$ cases. BAL was positive in $10 / 25$ $(40 \%)$ cases. (7/25 bacterial, $2 / 25$ viral, $1 / 25$ PCP) and treatment was modified in $8 / 25(32 \%)$ cases. Where NIS was positive, BAL confirmed the diagnosis only once and in one case revealed another organism that changed management further.

In 2/25 cases, chest radiograph (CXR) was not done prior to HRCT. CXR was abnormal in $16 / 25$ cases, 13 of which proceeded to HRCT with subsequent treatment modification in 5/13 cases. In 7/25 cases, CXR was normal of which all had abnormal HRCTs with treatment modification in 1/7 case. Overall, HRCT led to treatment modification in 6/22 cases, in which BAL confirmed the suspected aetiology in $2 / 22$ cases

This data indicates that the sequential use of NIS and BAL gives the highest diagnostic yield of pulmonary infiltrates. At our institution, HRCT was not sensitive enough to allow for its confident use as a diagnostic tool in place of BAL. Although FOB is a high risk procedure in this population, this data supports BAL as a safe and useful investigation.

\section{P26 CT GUIDED LUNG BIOPSIES: DO THEY PROVIDE THE DIAGNOSIS?}

P.L. Williams, R.K. Rajakulasingam. Department of Respiratory Medicine, Homerton University Hospital, London, UK

When requesting $\mathrm{CT}$ guided lung biopsies we are frequently asked "Will the patient tolerate a pneumothorax?". With this in mind we performed an audit of this technique to assess the success rate of the procedure, frequency of complications, and the sensitivity and specificity for diagnosing lung cancer.

Using the CT record book to identify cases we recorded details of all $(n=68)$ patients recorded as having had "lung biopsy" over the previous four year period. We used patient case notes, the lung cancer database, and the computer based histology records and CT reports to record, where possible, the indication, histology obtained, whether or not further investigative procedures had been required, any documented complications, and the final diagnosis.

Of the 68 patients recorded as "lung biopsy", 1\% actually had pleural biopsy and $4 \%$ lung aspirate. Of these there were no complications and the procedure provided the diagnosis. Sixty four patients were scheduled for the procedure: percutaneous core needle biopsy under CT guidance, following infiltration with subcutaneous lignocaine. 1-"multiple" passes were made as required/tolerated. Each patient had a postprocedure CT check for pneumothorax. Of the 64, $6 \%$ were cancelled due to radiological improvement and $6 \%$ abandoned due to technical difficulties, leaving 56.

The indication for biopsy was suspected lung cancer in $91 \%$. We wanted to know whether the procedure provided the final diagnosis or if further measures needed. Of the 46 patients in whom adequate information was available, the histological sample from biopsy was successful in providing the final diagnosis in $71 \%$. Histology was obtained but further investigations were needed in $26 \%$. No histology was obtained in $4 \%$. Of the 56 patients who underwent any procedure pneumothorax preventing biopsy occurred in just $2 \%$. Smaller pneumothoraces occurred in $13 \%$, and the remaining $85 \%$ experienced no complications.

The sensitivity of the procedure for diagnosing lung cancer was $90 \%$, specificity $100 \%$, and false negative rate $8 \%$. These and the complication rate compared favourably with other published studies of lung biopsy. In a DGH this procedure is still useful and we have demonstrated a relatively low complication rate.

\section{P27 GENERAL PRACTICE OPEN ACCESS SPIROMETRY: WHO WAS REFERRED IN 2001?}

A.H. Kendrick, D. Smith, M.R. Hetzel, J.R. Catterall, G. Laszlo. Department of Respiratory Medicine, Bristol Royal Infirmary, Bristol, UK

We provide an Open Access spirometry service to primary care. Patients attend from 09:30 to 11:30 Mon to Fri. A technologist obtains a history, spirometry pre and post $\beta_{2}$-agonist via spacer and pulse oximetry. A report and recommendations are sent to the GP.

Aim: To review the throughput of this service in 2001

Methods: The records of all patients attending the service were reviewed. Data are given as median (range).

Results: 706 patients attended, with 55 having further studies after a trial of steroids. Age was 54.7 yr (10 to 91), 366 were female and body mass index (BMI) was $27.4 \mathrm{~kg} \cdot \mathrm{m}^{-2}$ (14.9 to 52.6 ). $198 \mathrm{had}$ a $\mathrm{BMI} \geqslant 30 \mathrm{~kg} \cdot \mathrm{m}^{-2}$. History: 274 were smokers, 438 had cough, 368 had wheeze, and 397 had sputum production. MRC dyspnoea grade ( $\mathrm{n}=696$ ) was Grade 1-110, Grade 2-230, Grade 3-223, Grade 4-116, and Grade $5-17$. One hundred and ninety six patients had no medication, 49 were on antibiotics, 261 on a $\beta_{2}$ agonist, 24 on an anti-muscarinic, 177 on oral/inhaled steroids, 32 on a $\beta$ blocker, 57 on a blood pressure tablet, and 203 patients were on other therapies. Lung Function: FEV \% predicted was 84.7 (15 to 143), FVC\%predicted was 89.1 (14.7 to 144) and FEV \%FVC was 74.9 (23.9 to 100). Sixty one studies showed submaximal/variable efforts. One hundred and fifteen patients had normal spirometry, 68 reversible airflow obstruction, 438 irreversible airflow obstruction, and 24 a restrictive defect. For reversibility, absolute change in $\mathrm{FEV}_{1}$ was 0.4 / 10.2 to 1.45 ) and $\%$ change was $29.8 \%$ (15.4 to 271). Nine out of 55 steroid trials showed a positive response with the absolute change in $\mathrm{FEV}_{1}$ (post $\beta_{2}$ agonist) of $0.45 /(0.2$ to 0.8 ) and $\%$ change of $29.8 \%(15.1$ to 64.0). Pulse oximetry ( $n=676$ ) showed 34 patients had an $\mathrm{O}_{2}$ saturation $\leqslant 92 \%$. Recommendations: 25 patients with airflow obstruction to change from $\beta$ blockers, 145 patients for a trial of inhaled steroids with repeat spirometry, 40 to be prescribed a $\beta_{2}$ agonist, 184 to be referred for further investigations-LTOT ( $n=34)$, ?occupational lung disease $(n=36)$, ?ElA $(n=27)$, excessive dyspnoea for spirometry $(n=63)$, and restrictive defect $(n=24)$.

Conclusion: This service (1) accurately assesses simple lung function, (2) identifies groups of patients requiring further investigation or a change in therapy, and (3) provides recommendations to assist the primary care physicians to manage their patients.

\section{P28 SERIAL PEAK FLOW MEASUREMENTS FOR THE DIAGNOSIS OF OCCUPATIONAL ASTHMA IMPROVING THE QUALITY}

V. Huggins', W. Anees', C. Pantin², P.S. Burge'. 'Occupational Lung Disease unit, Birmingham Heartlands Hospital, UK; ${ }^{2}$ Department of Respiratory Medicine, North Staffordshire Hospital, Stoke-on-Trent, UK

Serial measurements of peak expiratory flow (PEF) are usually the most appropriate first step in the investigation of occupational asthma.

Different centres have reported widely different success in obtaining records of sufficient data quantity for diagnosis. We have investigated different methods of instruction and determined the return rate and quality of the resulting record for the diagnosis of occupational asthma using predefined criteria. 
Methods: Three instruction methods have been investigated: 159 were instructed by post (postal group), 86 were personally instructed by a PEF specialist (personal group), and 40 were instructed by others-for example, GPs, occupational health physicians, or nurses.

Results: The postal return rate was $56 \%$ and the personal return rate $85 \%$, adequate data quantity was similar in the postal and personal groups (54.8\% and 58.8\% respectively). Pre-existing records plotted from graph charts were only adequate in $23 \%$, compared with pre-existing records plotted from occupational forms (61\% adequate). Failure of the record to contain consecutive periods of $\geqslant 3$ workdays was the most common reason for inadequate data quantity.

Conclusion: The quality and return rate of PEFs for diagnosing occupational asthma is better when patients have been given specific instructions from a PEF specialist and recording is on a dedicated form

\section{P29 DIFFERENCES IN INDICES OF PEAK EXPIRATORY FLOW VARIABILITY BETWEEN WORKERS WITH OCCUPATIONAL ASTHMA AND IRRITANT (GRAIN DUST) EXPOSED HEALTHY SUBJECTS}

W. Anees', D. Blainey², V. Huggins', K. Robertson', P.S. Burge'. 'Occupational lung disease unit, Birmingham Heartlands Hospital, Birmingham, UK; ${ }^{2}$ Broomfield hospital, Chelmsford, Essex, UK

Introduction and Aims: Serial peak expiratory flow (PEF) records have been recommended as a first line investigation in workers suspected as having occupational asthma. It is unclear, however, to what extent they can differentiate between workers with occupational asthma and healthy workers exposed to irritant agents, and which index of PEF variability is best at doing so.

Methods: Indices of PEF variability were compared in three groups of subjects. (1) Forty healthy grain exposed farmers and dockers. (2) Forty two consecutive subjects with independently confirmed occupational asthma. (3) Forty eight non-occupational asthmatics.

Results: The index of PEF variability that best separated the occupational asthmatic workers from the others was the difference in mean PEF between rest and work periods. The upper $95 \%$ confidence limit of this index for the grain workers was $2.8 \%$ of predicted PEF 116 $\mathrm{L} / \mathrm{min})$, for non-occupational asthmatics $3.3 \%$ predicted PEF $(15$ $\mathrm{L} / \mathrm{min})$. Sensitivity for diagnosing occupational asthma using this index was $70 \%$. Only $40 \%$ of workers with confirmed occupational asthma had a PEF diurnal variability $>17 \%$ of predicted, the upper limit for grain workers. An increase in diurnal variation on work days of $>7 \%$ (the upper $95 \%$ limit for non-occupational asthmatics) had a sensitivity of only $27 \%$ for the diagnosis of occupational asthma. The difference between maximum PEF on work days and minimum PEF on rest days was poor at separating occupational asthmatic workers from those with non-occupational asthma.

Conclusion: Difference in mean PEF between work and rest days is the best simple index for differentiating subjects with occupational asthma from those with non-occupational asthma or irritant exposed healthy subjects.

\section{P30 REPEATABILITY OF CHLORIDE LEVELS IN EXHALED BREATH CONDENSATE (EBC)}

A. Zacharasiewicz, N. Wilson, A.M. Li, CH. Lex, J. Hooper, A. Bush. Department of Paediatric Respiratory Medicine, Royal Brompton Hospital, UK

Background: Reproducibility of measurements of EBC is controversial (Effros, et al. Am J Respir Crit Care Med 2002:165:663-9). It has been suggested that there is a wide variation in dilution of the collected sample, which would make interpretation of measurements difficult.

Aim: to assess the repeatability of Sodium [Na] and Chloride [Cl] measurements in EBC in healthy adults and in asthmatic and cystic fibrosis (CF) children.

Methods: EBC were collected for 10 minutes using a Condenser (Ecoscreen (Jaeger) and wearing a nose clip. [Na] and $[\mathrm{Cl}]$ were measured with a CIBA Corning M $644 \mathrm{NaCl}$ Analyser. Samples of five healthy adults were collected five times within one day with a 20 minute interval each. To measure period repeatability, the collection was repeated on another day within eight weeks. Technical repeatability was measured by aliquoting each sample into two tubes. Within day repeatability was also assessed in seven asthmatic and eight CF children from two collections within two hours. Repeatability was calculated according to Chinn (Chinn S. Thorax 1991;46:4546).

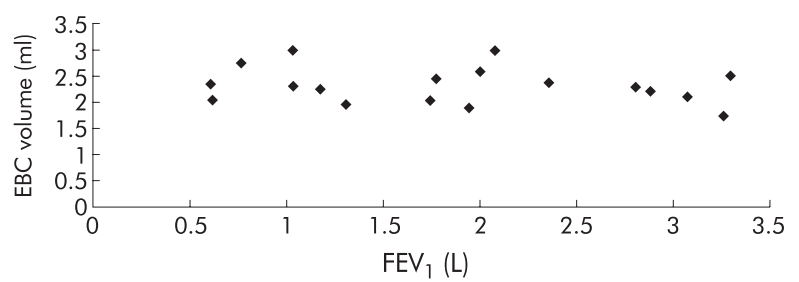

Abstract P3 1 FEV $v$ EBC volume produced.

Results: Na could not be detected in $>30 \% E B C$, so was not analysed further. For normals, the mean (SD) [Cl] was $4(0.95) \mathrm{mmol} / \mathrm{L}$; the technical repeatability within one sample, calculated by the single determination range was $\pm 1.96 \mathrm{mmol} / \mathrm{L}$, and the within subject day repeatability assessed as the $95 \%$ range for change within a day was $\pm 1.16 \mathrm{mmol} / \mathrm{L}$, the within period (eight weeks) range of change was $\pm 2.18 \mathrm{mmol} / \mathrm{L}$. For asthmatics, the mean [Cl] was $4.4(0.9) \mathrm{mmol} / \mathrm{L}$ with a range of change of \pm 2.98 , in $C F$ patients the mean $[\mathrm{Cl}]$ was 5 (1.5) $\mathrm{mmol} / \mathrm{L}$ and $95 \%$ range for change was $\pm 3.38 \mathrm{mmol} / \mathrm{L}$.

Conclusion: The variability of measured levels is similar for within test (paired estimates of the same sample), within day and between visits, for both normals and children with asthma and CF. Thus our data suggest that the major source of variability of $\left[\mathrm{Cl}^{-}\right]$can be explained by limitation of the measurement assay method used, rather than as an effect of intrinsic variability in EBC collection per se. The wide use of EBC is most likely dependant on the development of highly sensitive and reproducible assays, rather than further refinements of the collection technique.

\section{P3 1 DOES AIRFLOW OBSTRUCTION OR INHALATION OF SALBUTAMOL INCREASE THE VOLUME OF EXHALED BREATH CONDENSATE COLLECTED IN STABLE COPD} AND ASTHMA?

S. Manney', J.G. Ayres', J. Baker'. 'Air Pollution Laboratory, Heartlands Research Institute, Birmingham Heartlands \& Solihull NHS Trust, Bordesley Green East, Birmingham, B9 5SS, UK; ${ }^{2}$ Division of Environmental Health \& Risk Management, University of Birmingham, Edgbaston, Birmingham, B15 2T, UK

Introduction: Exhaled breath condensate (EBC) collection, although widely accepted as a method for measuring molecules in exhaled breath, is not well characterised methodologically. Factors such as airflow obstruction, bronchodilator therapy, or respiratory rate may affect the volume of EBC volume achieved. The main aims of this study were to examine whether EBC volume collected could be increased post salbutamol inhalation, and whether EBC volume collected was directly linked to airflow obstruction as assessed by FEV

Methods: Eighteen volunteers were studied (10 COPD, eight asthmatics). Each completed six collections over three days. On each occasion, subjects were asked to refrain from taking short acting bronchodilators for six hours before the study. Two collections were made on each day (breathing via mouthpiece and two way valve into two Teflon tubes in ice for 15 minutes), pre and post inhalation of $200 \mu \mathrm{g}$ salbutamol. Spirometry was completed on all volunteers at the end of both collections. On each of the three days, volunteers completed the same protocol for reproducibility of volumes.

Results: There was no correlation between airflow obstruction and EBC volume collected.

There was no significant difference in $E B C$ volume pre and post bronchodilator when considering all 18 patients $(2.26 \mathrm{ml}(\mathrm{SD})(0.35)$ pre; $2.31 \mathrm{ml}(0.35)$ post) $(p=0.46)$. For patients with COPD the respective volumes were $2.21 \mathrm{ml}(0.30)$ and $2.35 \mathrm{ml}(0.32)$ (NS) while for asthma they were $2.33 \mathrm{ml}(0.42)$ and $2.22 \mathrm{ml}(0.37)$ $(p=0.04)$.

Conclusion: EBC volume was not related to the degree of airflow obstruction and bronchodilator inhalation did not increase EBC volume.

\section{P32 A NOVEL DEVICE FOR THE PRECISE MEASUREMENT OF RESPIRATORY HEAT AND MOISTURE LOSS}

J.B. McCafferty', P.K. Kew², A. Haston², J.A. Innes'. 'Respiratory Unit, Western General Hospital, Edinburgh; ${ }^{2}$ Department of Mechanical and Chemical Engineering, Heriot-Watt University, Edinburgh

Background: It is proposed that respiratory heat and moisture loss (RHML) are altered by airway inflammation and that measurements of 
RHML may yield a useful non-invasive marker of such inflammation. Simple exhaled temperature and humidity measurements are however insufficient to characterise RHML as heat and moisture loss are affected by respiratory pattern and inspirate temperature and humidity. To precisely measure RHML in normal subjects and patients with airway disease we have developed a novel device which can control inspired air temperature, humidity, minute ventilation, and tidal volume.

Methods: The device is a compact trolley mounted air conditioning module delivering air at up to $1500 \mathrm{ml} / \mathrm{s}$ with a controllable temperature $\left(3^{\circ} \mathrm{C}\right.$ to $\left.40^{\circ} \mathrm{C}\right)$ and moisture content $(5$ to $40 \mathrm{~g} / \mathrm{kg}$ dry air). Air is delivered to the inspiratory side of a 2 way valve by means of a flow past configuration. Temperature and humidity sensors located on inspiratory and expiratory sides of the breathing valve allow accurate, continuous recording of the thermodynamic state of air entering and leaving the respiratory tract. A computer linked to an ultrasonic flowmeter in the expiratory limb is used to generate auditory and visual feedback cues to help subjects control respiratory rate and flow respectively, allowing standardisation of ventilatory pattern between subjects. RHML at steady state is calculated as the product of mass flowrate and "enthalpy" difference between inspired and expired air. Six normal volunteers breathed inspirate at $7^{\circ} \mathrm{C}$ at a target minute ventilation of $15 \mathrm{l} / \mathrm{min}$ with either a slow deep pattern (rate 10/min, tidal volume $1455(101) \mathrm{ml}(\mathrm{SD}))$ or rapid shallow pattern $(30 / \mathrm{min}$, tidal volume $525(36) \mathrm{ml}$ ).

Results: Mean respiratory heat loss was 15.7 (1.4) Watts during rapid shallow breathing and increased to 18.3 (1.3) Watts during the slow deep pattern $(p=0.009)$. At the same tidal volumes respiratory water loss was measured as $334(30) \mathrm{\mu l} / \mathrm{min}$ and $408(46) \mathrm{\mu l} / \mathrm{min}$ respectively $(p=0.008)$.

Conclusions: This new measurement device was sufficiently sensitive to detect a significant increase in RHML with increasing tidal volume at fixed minute ventilation in normal subjects. When normal ranges are defined it will be used to measure RHML under matching conditions in patients with COPD, asthma, and cystic fibrosis.

\section{P33 EFFECT OF VARYING RESPIRATORY PATTERN ON EXHALED BREATH CONDENSATE COLLECTION}

S. Tate, J. McCafferty, J.A. Innes, A.P. Greening. Respiratory Medicine Unit, Western General Hospital, Edinburgh

Background: Exhaled breath condensate $(E B C)$ has been proposed as a non-invasive means of measuring pulmonary inflammation. At present little is known about how the respiratory pattern during collection influences the properties of the EBC sample. We hypothesised that variations in respiratory pattern would have significant effects on volume and concentration of EBC.

Methods: Ten control subjects had EBC collected three times for six minute periods each on the same day. For the three collections subjects were instructed to follow constant respiratory rates (RR) for the six minutes $(10,20$, and 30 breaths per minute in random order), at a constant flow rate $1500 \mathrm{mls} / \mathrm{s}$ ) resulting in three different tidal volumes $(\mathrm{Vt}),(1500,750$, and $500 \mathrm{mls})$ but constant total respired volume for the three collections $(90 \mathrm{~L})$. A custom built air conditioning unit was used which allowed inspired air temperature and humidity to be kept constant. The expiratory limb of the unit housed heat and humidity sensors which allowed determination of heat (W) and moisture loss (mg/g dry air) from the respiratory tract. The system was linked to a flow meter and computer with an audiovisual cue so that the subjects could maintain the necessary RR and flow rate. A commercial breath condenser system (Jaeger) was fixed to the expiratory port to allow collection of EBC. Total volume achieved and solute concentration (nitrite) were measured for each collection.

Results: A strong correlation was found between EBC volume $(\mu \mathrm{l})$ and $\mathrm{Vt}_{t}(\mathrm{r}=0.73, \mathrm{p}<0.0001)$. However nitrite concentration was unaffected by $V_{t}(r=0.04, p=0.83)$ or total volume collected $(r=-0.1$, $p=0.62$ ). There was a strong correlation between moisture loss from the respirator tract and $\mathrm{Vt}(\mathrm{r}=0.7, \mathrm{p}=0.0001)$. Calculated total water loss in six minutes related to actual EBC volume collected gave a measure of efficiency of collection. This calculated efficiency was also related to $\mathrm{V}_{t}(\mathrm{r}=0.5, \mathrm{p}=0.018)$.

Conclusion: Collected EBC volume is enhanced significantly at higher tidal volumes. This appears a function of increased moisture loss with higher $\mathrm{Vt}$ but also increased condenser efficiency. Increased yield of EBC volume does not result in dilution of non-volatile solutes in the sample. The influence of other variables such as flow rate and inspired temperature on EBC collection need to be examined.

\section{COPD: Assessment and treatment}

\section{P34 MECHANISMS OF BRONCHIAL HYPER-RESPONSIVENESS IN COPD}

P.P. Walker, P.M.A. Calverley. Department of Medicine, University Hospital Aintree, Liverpool, UK

Bronchial hyper-reactivity (BHR) is a hallmark feature of asthma and a common, though not fundamental, feature of COPD. In asthma the response represents narrowing of the airway lumen due to contraction of airway smooth muscle (ASM). In COPD there is a relationship between BHR and airflow obstruction and we hypothesise that responsiveness is related less to changes in ASM and resistance and more to increase in hyperinflation. Hence an increase in residual volume (RV) will reduce the ability of the airway-parenchymal interface to overcome narrowing of the airway lumen.

We studied 10 subjects with mild to moderate COPD-baseline $\mathrm{FEV}_{1} 1.59 \mathrm{I}$ (SD) (0.45I), $\mathrm{FEV}, \%$ predicted $55 \%$ (16\%), $\mathrm{FEV}_{1} / \mathrm{FVC}$ ratio $0.49(0.1)$ - who underwent standard methacholine challenge testing. At baseline subjects had moderate increases in airway resistance measured by body plethysomnography (Raw 3.04 - predicted 1.86), moderate increases in resistance ( $\mathrm{R} 5 \mathrm{~Hz} 0.67$ - predicted 0.32 ) and impedance $(\mathrm{Z} 5 \mathrm{~Hz} 0.77$ - predicted 0.32$)$ measured by IOS and moderate reduction in inspiratory capacity (2.531 - predicted 2.91I). Median PC20 was 0.54 (range 0.1 to $>16$ ) and all but one subject achieved a PC20 at $<2 \mathrm{mg} / \mathrm{ml}$.

After challenge mean $\mathrm{FEV}_{1}$ fell by $34 \%$ to 1.051 ( $\left.\mathrm{p}<0.01\right)$, mean FVC fell by $38 \%$ to 2.081 ( $p<0.01)$, mean SVC by $30 \%$ from 3.741 to $2.611(p<0.04)$, and IC by $38 \%$ to 1.601 ( $p<0.01)$. TLC, when measured, was constant therefore these changes represent significant increases in RV. Airway resistance by body plethysomnography (Raw) increased overall $(3.04$ v 12.16$)$ but showed minimal change in three subjects while measurement in five other subjects was technically difficult. IOS measurements showed an overall modest increase in impedance $(0.77 \vee 1.01 ; \mathrm{p}<0.01)$ which was due to a fall in reactance $(-0.35 \vee-0.55 ; \mathrm{p}<0.04)$. There was no significant change in resistance $(0.67 \vee 0.83 ; p<0.12)$.

In individuals with COPD who are subject to bronchial challenge assessment of changes in $\mathrm{FEV}_{1}$ does not give a true measure of change in respiratory system resistance. BHR is likely to be determined less by ASM contraction and more by increases in hyperinflation, which in turn alter the ability of the lung to overcome airway narrowing.

\section{P35 CHRONIC BRONCHITIS, SMOKING AND SOCIOECONOMIC STATUS IN THE NHANES III DATASET}

M. Sridhar', G. Netuveli', A. Sheikh'. 'NHLI Division of Faculty of Medicine; ${ }^{2}$ Department of Primary Health Care \& General Practice, Charing Cross Hospital, UK; Imperial College of Science, Technology \& Medicine, London, UK

Background: While some studies have suggested that differences in the prevalence of chronic bronchitis in the various socioeconomic groups is due to differences in smoking habits, others suggest an explanation unrelated to smoking.

Methods: We have examined the relation between the prevalence of chronic bronchitis, smoking and socioeconomic status (SES) in a subset of the extracted from the US NHANES III data set representing a population of 115 million adults between the ages of 30 and 75 years. A measure of SES was constructed using household income and education. Current smoking status, duration of smoking, and intensity of smoking were also noted. Symptom identified bronchitis (SIB) and doctor diagnosed bronchitis (DDB) were used as outcome measures. Data was analysed using logistic regression analysis with appropriate population weighting.

Results: SIB was significantly related to age above 70 years, African and Hispanic ethnicity, upper SES, and smoking. DDB was related to age, being male, African and Hispanic ethnicity, SES, and smoking. Of these, being male, ethnicity, and higher SES were protective. In SIB, within each stratum of SES, the only consistently significant variable was current smoking status. No significant interaction between smoking and SES was detected. When DDB was used as outcome measure, the current smoking status was significantly associated only in the upper middle SES strata. For both outcomes, stratification according to current smoking status revealed SES to be significant in current smokers only. 
Conclusions: Socioeconomic status had a significant influence on the prevalence of SIB that was independent of cigarette smoking. The protective effect of higher socioeconomic status was most significant for current smokers. This effect seemed to be independent of smoking behaviour in the different SES strata and merits further scrutiny.

Acknowledgements: An NHS R\&D National Primary Care Fellowship supports AS. GN is supported by a grant from the National Asthma Campaign, UK.

\section{P36 MEDICAL RESEARCH COUNCIL DYSPNOEA SCALE IS A MEASURE OF FUNCTIONAL CAPACITY AND GLOBAL PERCEIVED HEALTH STATUS}

C. McAlpine', W. Cochrane ${ }^{1,2}$, B. Watson'2, M. Heatley', S. Dogan', G. Dovey-Pearce', G. Afolabi'. 'Northumbria Healthcare NHS Trust, Northumbria, UK; ${ }^{2}$ Northumbria University, Northumbria, UK

Introduction: The Medical Research Council Dyspnoea scale (MRCD) has been used to categorise disability in patients with COPD, though it remains unclear what the scale measures.

Participants: All 218 participants had stable COPD. Sixty per cent reported MRCD scale grade 3, 29\% grade 4 , and $11 \%$ grade 5 . Forty four per cent were male and mean $(95 \% \mathrm{Cl})$ age was 68.8 (67.8 to 69.7) years.

Methods: Participants were graded on the MRCD scale on the basis of self report information. Incremental shuttle walking test (ISWT) distance, spirometric, and anthropometric measurements were recorded. Self report data were recorded to assess: perceived health status (Chronic Respiratory Disease Questionnaire (CRDQ)); personal cognitions of illness (Illness Perceptions Questionnaire); affect (Hospital Anxiety and Depression Scale); and self efficacy (COPD Self Efficacy Scale). $\chi^{2}$ And Oneway and Kruskal Wallis ANOVA analysis were carried out to assess differences in respondent characteristics across MRCD grade.

Results: Mean percent predicted FEV 1 was $9.93 \%$ higher people with grade 3 than those with grade $5(F=4.33, p=0.01)$. Resting oxygen saturation was significantly higher in people with grade 3 compared with those with grades 4 and $5(F=9.11, p<0.001)$. People with grade 3 had a mean ISWT of 235.0 m compared with 153.0 and $117.7 \mathrm{~m}$ for grades 4 and 5 respectively $(F=20.05$, $p<0.001)$. On the CRDQ, mastery, and emotional functioning were significantly higher, and fatigue and dyspnoea were significantly lower, in people with grade 3 compared with those with grade 4 and $5(p<0.02)$. Anxiety was significantly higher in people with grade 3 compared with those with grade $4\left(\chi^{2}=6.13, p=0.04\right)$ and people with grade 5 had a higher perception of treatment control compared with those with grade $4\left(\chi^{2}=8.24, p=0.016\right)$. Of people with grade $3,31.8 \%$ regarded themselves as being in good health, compared with $16.7 \%$ with grade 4 , and only $4.5 \%$ with grade $5\left(\chi^{2}=10.38\right.$, $p=0.006$ ). There were no significant differences across MRCD grade in body mass index, age, depression, self efficacy, or gender.

Conclusion: Only the ISWT and patients' Global Perceived Health showed significant differences across all three grades of MRCD scale. The MRCD scale reflects patients' functional capacity but also their global perceived health status.

\section{P37 THE INFLUENCE OF BMI ON LUNG CT DENSITOMETRY IN EMPHYSEMA}

D.G. Parr', M. Needham', B. Stoel', J. Stolk', R.A. Stockley'. 'Lung Investigation Unit, University Hospital, Birmingham, UK; ${ }^{2}$ Division of Image Processing, Leiden University Medical Centre, Leiden, the Netherlands.

Lung CT densitometry correlates well with pathological and physiological measures of emphysema and is the most sensitive measure for detecting disease progression in alpha- 1 antitrypsin deficiency (AATD) (Dowson, et al. Am J Respir Crit Care Med 200 1;164:18059). Long term reproducibility is subject to errors arising from limitations in the reconstruction algorithm, with changes in denser tissues affecting lung density measurements by altering beam hardening effects. Emphysematous lung is of similar density to air, and therefore changes in air densitometry reflect influences on lung densitometry. We looked at the effect of body mass index (BMI) on measured values for air density within the patient. Using a lung phantom, we then measured the effects on lung densitometry of an increase in chest wall thickness as would occur with increasing BMI.

Methods: We performed voxel densitometry of tracheal air on single slice inspiratory high resolution CT images at the level of the aortic arch in 41 patients with AATD (PiZ) and related the results to BMI. In addition, a thoracic phantom containing fixed, whole dog lung

\begin{tabular}{|c|c|c|c|c|}
\hline Densitometry method & $\begin{array}{l}\text { Slice } 1 \\
\text { thin dog }\end{array}$ & $\begin{array}{l}\text { Slice } 1 \text { fat } \\
\text { dog }\end{array}$ & $\begin{array}{l}\text { t Slice } 2 \\
\text { thin dog }\end{array}$ & $\begin{array}{l}\text { Slice } 2 \text { fat } \\
\text { dog }\end{array}$ \\
\hline Relative area $(-910 \mathrm{HU})$ & 30.2 & 23.421 & 38.706 & 30.007 \\
\hline $\begin{array}{l}\text { 15th Percentile point } \\
(\mathrm{HU})\end{array}$ & -926.0 & -920.3 & -932.1 & -929.5 \\
\hline Mean lung density $(\mathrm{HU})$ & -856.8 & -847.6 & -851.8 & -841.6 \\
\hline
\end{tabular}

(KCARE, $\mathrm{KCH}$, London) was imaged before and after attaching to the outer surface two flexible water filled containers of total volume $3.5 \mathrm{~L}$ (Durex,UK) simulating increased chest wall mass. Lung densitometry was performed on two images taken from each series using the PULMO-CMS software (B Stoel, Leiden University). Three techniques were used; relative area at a threshold of $-910 \mathrm{HU}$, the 15th percentile point and the mean lung density.

Results and conclusions: Tracheal air density measurements correlated well with BMI (Spearman's rho $=0.55, \mathrm{p}<0.001$ ). Lung phantom densitometry was influenced by chest wall thickness as shown in the table.

A change in BMI over time will alter measured lung density. The effect of an increase in $\mathrm{BMI}$ could therefore be to reduce apparent emphysema severity

\section{P38 CT SCANNING IN SUBJECTS WITH COPD AND THEIR RELATIVES}

P.P. Walker', J. Griffiths', J. Curtis', E. Thwaite', P.M.A. Calverley' 'Department of Respiratory Medicine; ${ }^{2}$ Department of Radiology, University Hospital Aintree, Liverpool, UK

Participants in a prospective COPD genetics study underwent thoracic HRCT scanning. Ninety two scans were performed in 71 subjects with COPD and 21 smoking siblings. We documented the frequency of lung nodules and other abnormalities and assessed their follow up to identify possible tumours. We also correlated spirometry and presence of visually scored emphysema on CT and compared the presence of bronchiectesis with clinical symptoms. Mean FEV , was $1.46 \mathrm{l}, \%$ predicted $F E V, 50 \%$. COPD severity by GOLD criteria: grade $1=9(10 \%)$, grade $2 A=20(22 \%)$, grade $2 B=30(33 \%)$, grade $3=$ $12(13 \%)$, no COPD $=21(23 \%)$.

Twenty one (23\%) subjects had lung nodules $-4 / 21$ in siblings without COPD. No malignancies have to date been confirmed. Ten (11\%) subjects required investigation for other abnormalities including lobar collapse and asbestosis. Thirty nine additional radiology investigations have been completed so far (29 CT). Twenty one (23\%) subjects had bronchiectesis on CT scan. In this population 42/92 subjects had chronic bronchitis clinically but only $6 / 21$ subjects with bronchiectesis had symptoms of chronic bronchitis - no positive correlation.

The worse the spirometry the more likely emphysema was to be present but $33 \%$ of smokers with normal spirometry had CT evidence of emphysema. Pulmonary nodules were common and hence resource implications high. Spirometry was imprecise in identifying mildmoderate emphysema but severe obstruction correlated well with CT findings. In subjects without a recent infection bronchiectesis is frequently present without symptoms.

\begin{tabular}{lll}
\hline Abstract P38 & & \\
\hline GOLD criteria & No CT emphysema & CT emphysema \\
\hline Normal & 14 & 7 \\
GOLD 1 & 5 & 4 \\
GOLD 2A & 5 & 15 \\
GOLD 2B & 2 & 28 \\
GOLD 3 & 0 & 12 \\
\hline
\end{tabular}




\section{P39 MULTIDIMENSIONAL (IDS) ASSESSMENT OF COPD IN THE COMMUNITY: CLINICAL IMPACT IS UNDERESTIMATED BY CURRENT GUIDELINES}

A.D. Lawrence, N.P. Keaney. Chest Clinic, Sunderland Royal Hospital, Sunderland, Tyne \& Wear, UK

The staging of the severity of COPD is based on the degree of airflow limitation. Specific FEV 1 cut points are used by the BTS, ATS, and ERS Guidelines. GOLD mentions the impact of COPD, and the imperfect relationship between impaired spirometric values and symptomatology, COPD also has systemic consequences. The IDS system of classifying COPD incorporates airflow limitation (impairment), dyspnoea (disability) and nutritional depletion (BMl-systemic involvement).

Our community database provided information on 401 patients (203 female) attending COPD clinics in eight general practices in Sunderland. Airflow limitation was classified according to standard BTS, ATS, ERS, and GOLD criteria using pre and post bronchodilator $\mathrm{FEV}_{1}$, and compared with IDS

Use of post bronchodilator FEV $\mathrm{FV}_{1}$ does not perturb the IDS classification. All guidelines significantly underestimate the impact of COPD with their methods of classifying severity from spirometric impairment alone.

\begin{tabular}{ll} 
Abstract P39 & Table 1 \\
\hline & IDS criteria \\
\hline Stage I & $\mathrm{FEV}_{1} \geqslant 50 \%, \mathrm{mild} / \mathrm{mod}$ dyspnoea, no nutritional \\
& depletion \\
Stage II & $\mathrm{FEV}_{1} 35-49 \%, \mathrm{mild} / \mathrm{mod}$ dyspnoea, no nutritional \\
& depletion \\
Stage III & FEV $<35 \%$, severe dyspnoea, nutritional \\
& depletion \\
\hline
\end{tabular}

\section{P40 A COMPARISON OF INTENSIVE, MDI-DELIVERED AND NEBULISED BRONCHODILATOR THERAPY IN SEVERE COPD}

T.K. Rogers, A. Venners, H. Drayton, P. Camplin, G. Segust, R. McCook. Chest Clinic, Doncaster Royal Infirmary, Armthorpe Road, Doncaster, S. Yorks, DN2 5LT, UK

Background: We have investigated whether nebulised therapy provides superior bronchodilation to a combination of high-dose anticholinergic and long acting $\beta_{2}$ agonist therapy, delivered via MDI and large volume spacer, in a well characterised group of stable patients with severe COPD ( $\mathrm{FEV}_{1}<40 \%$ predicted).

Methods: We undertook a comparison of three regimes of four times daily nebulised therapy (salbutamol $5 \mathrm{mg}$ /ipratropium bromide $500 \mathrm{\mu g} /$ Combivent 1 nebule) each used for a week in randomised order, against combined therapy with salmeterol $50 \mu \mathrm{gg}$ twice daily and ipratropium bromide $160 \mathrm{\mu g}$ four times daily via MDI and large volume spacer, in an open label, sequential, cross over study. The outcome measure was self recorded peak flow rates, measured in the morning, pretreatment (am), afternoon (pm) and night time (nocte).

Results: Seventy three patients were enrolled, until the required number of 42 subjects with adequate data was obtained. Each of the three nebulised regimens was highly significantly, and probably clinically, superior to the MDI therapy, but there was no significant difference between them (ANOVA and paired $t$ tests), see table.

Conclusions: Overall in this group of patients with severe COPD, nebulised therapy produced greater improvement in peak expiratory flow rate than could be achieved with intensive MDI-based therapy.

\begin{tabular}{lllll}
\multicolumn{4}{l}{ Abstract P40 } \\
\hline $\begin{array}{l}\text { Time of } \\
\text { day }\end{array}$ & $\begin{array}{l}\text { MDI Ipra/ } \\
\text { Salmeterol }\end{array}$ & $\begin{array}{l}\text { Nebulised } \\
\text { Salbutamol }\end{array}$ & $\begin{array}{l}\text { Nebulised } \\
\text { Ipratropium }\end{array}$ & $\begin{array}{l}\text { Nebulised } \\
\text { Combivent }\end{array}$ \\
\hline am & $189(10)$ & $223(13)$ & $224(12)$ & $227(12)$ \\
pm & $201(11)$ & $227(13)$ & $229(12)$ & $234(13)$ \\
nocte & $204(11)$ & $229(13)$ & $226(12)$ & $231(13)$ \\
\hline
\end{tabular}

\section{P4 1 ECONOMIC IMPLICATIONS OF REDUCTIONS IN COPD REHOSPITALISATION DUE TO TREATMENT WITH INHALED CORTICOSTEROIDS (ICS)}

M.D. Spencer. GlaxoSmithKline, Greenford, UK

Background: A study in an Ontario observational database has demonstrated reductions in the rate of COPD rehospitalisation and death of $24 \%$ and $29 \%$ respectively using ICS (Sin, et al. Am J Respir Crit Care Med 2001;164:580-4). Based on Hospital Episode Statistics there were 78908 admissions for COPD in the year 2000/1 in England \& Wales, with an average length of stay of 9.1 days, Sin and Tu's data suggest over 15000 of these would have been rehospitalisations (assumes current ICS usage 50\%). This equates to a hospitalisation cost of over $£ 155.8 \mathrm{~m}$ with over $£ 31 \mathrm{~m}$ for rehospitalisations.

Methods: The economic implications of reductions in exacerbations have been assessed by modelling these risk reductions on a hypothetical population of 1000 patients, accounting for uncertainty in parameters by second order "Monte Carlo" simulation techniques. The implications of a hypothetical increase in the use of ICS by patients with an initial hospitalisation from $50 \%$ to $100 \%$ in England \& Wales are then considered.

Results: For the hypothetical cohort of 1000 patients a comparison with and without ICS produces a reduction in hospital days of 622 $(95 \% \mathrm{Cl} 494$ to 760$)$ equivalent to a cost saving of $£ 135.6 \mathrm{~K} 195 \% \mathrm{Cl}$ $95 \mathrm{~K}$ to $189 \mathrm{~K})$. Applying this to an increase in ICS use from $50 \%$ to $100 \%$ for all patients with an initial hospitalisation, would result in a reduction in hospital days of $19751(95 \% \mathrm{Cl} 15799$ to 24256$)$ equivalent to a cost saving of $£ 4.29 \mathrm{~m}(95 \% \mathrm{Cl} £ 3.08 \mathrm{~m}$ to $5.74 \mathrm{~m})$, thus offsetting a considerable portion of the drug purchase cost.

Conclusion: These results suggest that, additional to the obvious clinical benefits of avoiding COPD hospitalisation and mortality, valuable NHS resources may also be freed. In addition the financial cost (over $£ 4 \mathrm{~m}$ ) of these hospital days may undervalue these resources during periods of high hospital bed occupancy.

\section{P42 THE ASSOCIATION OF SURVIVAL WITH THE USE OF INHALED CORTICOSTEROIDS (ICS) SEEN IN OBSERVATIONAL STUDIES OF COPD CANNOT BE EXPLAINED BY UNOBSERVED MARKERS OF DISEASE SEVERITY (FEV $\%$ PREDICTED, HEALTH STATUS, BODY MASS INDEX)}

M.D. Spencer. GlaxoSmithKline, Greenford, UK

Background: Recent COPD studies performed in observational databases have shown benefits of ICS and ICS + LABA in terms of reductions in mortality and of hospitalisation (Sin, et al. 2001; Soriano, et al. 2002). A criticism of such studies, is the lack of randomisation and hence potential bias caused by the confounding of unobserved factors. This study investigates the relationship of a number of potential markers of disease severity in COPD to ICS prescription, and hence the potential for confounding.

Methods: The Health Survey for England 95, 96, and 97 (96 only for health status) forms the population for this study. Respondents with

\begin{tabular}{|c|c|c|c|c|c|c|c|c|c|c|}
\hline \multirow[t]{3}{*}{ Abstract P39 } & \multicolumn{10}{|c|}{ Table 2} \\
\hline & \multicolumn{2}{|l|}{ IDS } & \multicolumn{2}{|l|}{ GOLD } & \multicolumn{2}{|l|}{ ATS } & \multicolumn{2}{|l|}{ BTS } & \multicolumn{2}{|l|}{ ERS } \\
\hline & Pre $\%$ & Pst \% & Pre \% & Pst \% & Pre \% & Pst \% & Pre \% & Pst \% & Pre \% & Pst $\%$ \\
\hline Normal & - & 1 & - & 8 & - & 9 & 6 & 25 & - & 10 \\
\hline Mild stage I & 37 & 37 & 15 & 21 & 73 & 71 & 51 & 41 & 39 & 40 \\
\hline Moderate stage II & 16 & 5 & 5823 & 5118 & 18 & 14 & 26 & 25 & 34 & 30 \\
\hline Severe stage III & 57 & 57 & 4 & 2 & 9 & 6 & 17 & 9 & 27 & 20 \\
\hline
\end{tabular}




\begin{tabular}{|c|c|c|c|c|}
\hline \multirow[b]{3}{*}{$\begin{array}{l}\text { ICS } \\
\text { No ICS }\end{array}$} & \multicolumn{4}{|c|}{ Mean (Median) by ICS prescription group } \\
\hline & \multicolumn{2}{|c|}{$\mathrm{FEV}_{1} \%$ pred BMI } & \multirow{2}{*}{$\begin{array}{l}\text { EQ-5D } \\
0.66(0.69) \\
0.78(0.79)\end{array}$} & \multirow{2}{*}{$\begin{array}{l}\text { SF-6D } \\
0.66(0.65) \\
0.74(0.78)\end{array}$} \\
\hline & $\begin{array}{l}47(47) \\
55(57)\end{array}$ & $\begin{array}{l}26(25) \\
26(25)\end{array}$ & & \\
\hline
\end{tabular}

an $\mathrm{FEV}, \mathrm{FVC}$ ratio $<70 \%$ and an $\mathrm{FEV}, \%$ predicted of $<70 \%$ were selected for analysis. Mean age $=65.9$ and $42.2 \%$ were women. The relationship between disease severity and ICS prescription in COPD was investigated and the statistical significance tested using the independent $t$ test

Results: No statistically significant relationship was found between ICS prescription and $B M I(p=0.518)$, whilst in the case of $F E V, \%$ predicted and of health status measured by both the EQ-5D and by the SF-6D patients taking ICS had on average worse scores than those not taking ICS (all $p<0.001$ ). See table.

Conclusions were unchanged when the population was limited to only those patients receiving at least one respiratory medicine.

Conclusions: In respondents to The Health Survey for England with objective airflow obstruction there was no association between ICS prescription and $\mathrm{FEV}, \mathrm{BMI}$ or health status that could explain benefits seen in other studies. The benefits of ICS seen in observational studies are unlikely to be explained by these differences between those taking and not taking ICS.

\section{P43 AUDIT OF LTOT PRESCRIPTIONS IN BLAENAU GWENT}

B. Perreira, S. Venn, B.V. Prathibha. Nevill Hall Hospital, Brecon Road, Abergavenny, South Wales, UK

Introduction: The ex-mining area of Blaenau Gwent has a high incidence of occupational and smoking related lung diseases. Provision of high quality clinical care to those individuals with severe disease includes the appropriate prescription of LTOT along with education to facilitate compliance. This audit was conducted with the aim of establishing whether the prescription and use of long term oxygen therapy in Blaenau Gwent complies with established guidelines.

Method: Audit criteria and standards were set using guidelines given in the Royal College of Physicians report Domiciliary Oxygen therapy services. Patients in Blaenau Gwent using oxygen concentrators in June 2000 were identified from Health Authority records. Criteria were measured from a review of hospital notes and by using a short postal questionnaire.

Results: Fifty patients were identified as using oxygen concentrators in Blaenau Gwent in June 2000. Notes were obtained for 48 of these patients $(96 \%)$ and questionnaires were returned from 44 patients $(88 \%)$. Thirty five $(73 \%)$ of the patients were under the care of a respiratory physician. Only $15(31.2 \%)$ had documented evidence of complete adherence to the guidelines prior to LTOT prescription (all under the care of a respiratory physician), although $39(81.3 \%)$ of the patients had blood gases recorded. Thirty three (69\%) had documented evidence of prescription for 15 hours a day, although the information given to patients was not recorded in the majority of cases. Of the respondents to the questionnaire, $38(86.4 \%)$ reported using their oxygen for long periods of time rather than intermittently and $34(77.3 \%)$ reported daily use of 15 hours or more. Thirty six $(81.8 \%)$ understood to use the oxygen correctly and $22(50 \%)$ were able to give a written explanation for their need of oxygen therapy.

Conclusion: The majority of patients who are prescribed LTOT report using it appropriately, although fewer understand the reason for treatment. However, it is of concern that appropriate assessment prior to LTOT prescription can only be confirmed in $31 \%$ cases and a chest physician had not seen $27 \%$ of the patients receiving LTOT. Following this audit a specially designed nurse led LTOT clinic has been started with referral to the chest physician. A patient information booklet has been introduced along with a blood gases record. A further audit will be conducted in one year to assess the impact of the clinic on the quality of care that patients using LTOT receive.

\section{P44 WEIGHT CHANGE IN CHRONIC OBSTRUCTIVE PULMONARY DISEASE (COPD): NOT JUST A PROBLEM OF UNDERNUTRITION}

C.E. Weekes', N.T. Bateman ${ }^{2} .{ }^{1}$ Departments of Nutrition \& Dietetics; ${ }^{2}$ Respiratory Medicine, Guy's and St Thomas' Hospital NHS Trust, London, UK

Weight loss is frequently reported in patients with COPD and is associated with increased morbidity and mortality. In contrast, for the past 30 years the general population has become more overweight and therefore at increased risk for heart disease and diabetes. While reviewing patients for a nutrition intervention study it was noted that many patients presenting to chest clinic were overweight. The aims of this study were to establish the number of outpatients with COPD who were overweight, obese, or at risk as a result of weight loss.

Two hundred and seventy six consecutive patients with COPD (143 male; 133 female) were reviewed by the dietitian during a routine visit to the chest clinics at Guy's and St Thomas' Hospitals. Weight, height, and history of weight change were recorded, together with smoking status, presence of oedema, and steroid use. Medical records were reviewed to establish the length of chest related hospital stays and mortality. Mean age was 67.3 (10.3) years and body mass index (BMI) $26.2(6.7) \mathrm{kg} / \mathrm{m}^{2}$. Patients were categorised as underweight, acceptable, overweight, or obese (BMI <20.0, 20.0 - 24.9, 25.0 29.9 or $\geqslant 30.0 \mathrm{~kg} / \mathrm{m}^{2}$ ). Weight change was considered significant if it exceeded $5 \%$ in either direction.

Approximately half the patients were overweight $(n=80(29 \%))$ or obese $(n=61(22 \%))$ while one in six $(n=43(16 \%))$ were underweight. Sixty one (22\%) patients reported significant weight loss and $53(19 \%)$ reported weight gain. Oedema was noted in $26(9 \%)$ patients.

Recent weight loss was associated with chest infection in $25(9 \%)$ patients, gastrointestinal symptoms in nine $(3 \%)$, and social reasons in five $(2 \%)$, while $22(8 \%)$ patients reported gradual weight loss over one to two years. Twenty two $(8 \%)$ patients with significant weight loss had $\mathrm{BMI} \geqslant 20.0 \mathrm{~kg} / \mathrm{m}^{2}$, and $15(5 \%)$ had $\mathrm{BMI} \geqslant 25.0 \mathrm{~kg} / \mathrm{m}^{2}$.

Recent weight gain was associated with smoking cessation in 17 $(6 \%)$ patients, oedema in six $(2 \%)$ and recent oral steroid use in two $(<1 \%)$. The remaining $28(10 \%)$ reported a gradual increase over several years.

There was a non-significant trend for increasing $\mathrm{BMI}$ to be associated with shorter length of hospital stay (5.9 (12.7) days for underweight to $2.9(8.4)$ for obese) but neither BMI nor weight change were associated with mortality.

The majority of publications on COPD are on the undernourished, yet it would appear from this study that about half the outpatients with COPD seen in this Trust were overweight or obese. The effect of being overweight on patients with COPD deserves further study.

\section{P45 BRONCHOSCOPIC LUNG VOLUME REDUCTION (BLVR) WITH ENDOBRONCHIAL VALVE IMPLANTS}

T.P. Toma, N.S. Hopkinson, N. Shen, J. Hillier, P. Goldstraw, C. Morgan, D.M. Hansell, M.I. Polkey, D.M. Geddes. Royal Brompton Hospital, Imperial College, London, UK

Background: Surgical lung volume reduction (LVRS) can palliate symptoms in selected patients with advanced emphysema. We hypothesised that a similar effect could be achieved by blocking segmental bronchi leading to areas of bullous emphysema.

Aim: In this study we investigated the safety and efficacy of BLVR using the Emphasys $®$ valve implant and delivery system, in patients unsuitable for surgical LVRS.

Methods: Three male patients have undergone so far unilateral BLVR under general anaesthesia. Three valves were placed in each patient in the most affected lobe as evaluated on ventilation/perfusion scans and CT scans.

Results: See table. BVR was effective in shrinking emphysematous lung and expanding a collapsed lobe in one patient. The effect was

\begin{tabular}{|c|c|c|c|}
\hline $\mathrm{FEV}_{1}(\mathrm{I})$ & $\begin{array}{l}\text { Shuttle } \\
\text { distance }(\mathrm{m})\end{array}$ & $\begin{array}{l}\text { Residual } \\
\text { ) volume (I) }\end{array}$ & $\begin{array}{l}\text { Diffusion } \\
\text { capacity (\% } \\
\text { predicted) }\end{array}$ \\
\hline Before procedure $0.82(0.26)$ & $246(120)$ & $6.72(1.37)$ & $29.5(9.4)$ \\
\hline After 7 days & $336(49)$ & $5.66(1.18)$ & $38(8.8)$ \\
\hline
\end{tabular}


sustained at one month follow up. Post procedure complications included: one pneumothorax at day 36 which resolved without drainage, and one COPD exacerbation at day 40 treated with antibiotics and steroids. None of the implants became dislodged.

Conclusion: This pilot study suggests that lung volume reduction can be achieved in humans with flexible bronchoscopy and specific valve implants. The implants are safe and easy to place and have the potential for extending indications and reducing morbidity, mortality and costs in patients with severe emphysema.

\section{Sleep assessment and treatment}

\section{P46 AN ATTEMPT TO ASSESS THE DURATION OF PROBABLE MORBIDITY PRIOR TO THE DIAGNOSIS OF OBSTRUCTIVE SLEEP APNOEA SYNDROME (OSAS)}

R. Ghiassi, M.R. Partridge. The Sleep Laboratory, Imperial College of Science, Technology and Medicine, NHLI Division at Charing Cross Hospital, London, UK

Recent work from Canada has suggested that hospital admissions and physician costs in the two years prior to diagnosis of OSAS was significantly higher in those with OSAS than in a control group.' In the year prior to diagnosis it has also been shown that prescribed medication costs were significantly higher; medication being needed for hypertension, ischaemic heart disease, and congestive heart failure. ${ }^{2}$ The rate of road traffic crashes and occupational accidents is also higher amongst those with untreated OSAS.

To gain an insight into the duration of possible prediagnosis morbidity, we administered a questionnaire to 117 consecutive OSAS patients being treated with CPAP who attended this laboratory for review. Of these $107 / 117(91.5 \%)$ reported that prior to diagnosis someone had complained about loud snoring and responders recorded that first mention of this had been a median of 12 years prior to diagnosis (range 2 to 47). Also 91/117 (77.8\%) reported witnessed apnoea prior to diagnosis and this had been observed a median eight years prior to diagnosis (range 1 to 49). Ninety seven out of $117(82.9 \%)$ reported sleepiness in the day time now or in the past and this had been present for a median of seven years (range 0.5 to 62 years). Seventy eight respondents were in employment and $37.2 \%$ of these reported having had two or more jobs in the last five years. Of the respondents, 85 were drivers and 21 of these $(24.7 \%)$ reported having had a road traffic accident in the previous five years with five respondents having had two and one having had four such crashes. Overall these results suggest the likelihood of significant prediagnosis morbidity and greater public and primary care awareness of OSAS is needed.

1. Kryger M H, et al. Sleep 1996;19:S111-16.

2. Otake K, et al. Thorax 2002;57:417-22.

\section{P47 URINARY SYMPTOMS DO NOT CORRELATE WITH SEVERITY OF OBSTRUCTIVE SLEEP APNOEA OR COMPLIANCE WITH CPAP}

K.E. Lewis', A.J. Watkins², L. Seale', I.E. Bartle', P. Ebden'. 'Prince Philip Hospital, Dafen, Llanelli, Wales, SA 14 8QF, UK

Introduction: Studies have found increased urinary disturbance in patients with obstructive sleep apnoea (OSA). The reason remains unclear and may be due to in part to prostatic symptoms in middle aged men. We examined whether higher urinary symptom scores prior to treatment are correlated with more respiratory disturbance in OSA, and if more symptoms reduce compliance by interfering with machine use.

Methods: Seventy four consecutive males with OSA, mean (SD) age of 55.2 (9.0) years, mean BMI 34.5 (5.9), mean Epworth Sleepiness Score 15.4 (5.2), mean AHI 28.1 (22.8) per hour and mean 5\% dip rate of 31 (21.7) per hour were prospectively studied. They completed the International Prostate Symptom Score and American Urological Questionnaire prior to CPAP therapy. Machine clock timers were hidden and machine on time was checked at one month. Correlations between the various subscores for urinary symptoms and measures of respiratory disturbance and machine on time were assessed using Spearman's rho.
Results: Our patients had a mean Total Urinary Score of 5.72 (4.27), range 0 to 19. The correlation between Urinary Obstructive Scores and $\mathrm{AHI}$ was rho $-0.229(\mathrm{p}=0.121)$. The correlation between Urinary Irritative Scores and AHI was rho $-0.100(p=0.506)$. The correlation between Urinary Total Scores and AHI was rho -0.197 $(p=0.185)$

Machine on-time was correlated to Urinary Obstructive Scores with rho $-0.221(p=0.301)$ to Urinary Irritative Scores with rho -0.030 $(p=0.798)$ and Total Urinary Scores rho $0.032(p=0.790)$.

Conclusion: Patients with OSA have Urinary Scores similar to the normal population as measured by standard urology tools, and any increased urinary disturbance, prior to CPAP is not significantly correlated with either the respiratory disturbance or reduced compliance with CPAP.

\section{P48 APOLIPOPROTEIN E: A ROLE IN SLEEP DISORDERED BREATHING?}

R.L. Riha, P. Brander, M. Vennelle, N.J. Douglas. Dept of Medicine, University of Edinburgh, Royal Infirmary, Edinburgh

Apolipoprotein E e4 (Apo E e4) is an important risk factor for the development of early onset Alzheimer's disease, as well as being an independent risk factor for cardiovascular disease. A recent study by Kadotani et al' demonstrated a correlation of Apo E e4 with a higher apnoea/hypopnoea index in a population with sleep disordered breathing (SDB). The aim of our study was to examine the distribution of Apo $E$ alleles and genotype in patients in the UK with SDB compared to controls.

Method: A case control study was undertaken from 1997-2002. One hundred consecutive patients with SDB were recruited randomly from the clinic register. Each case was matched to a sibling. All cases and controls were asked to complete a self administered questionnaire and underwent clinical examination. All subjects underwent routine PSG or home monitoring. Studies were scored manually. All subjects had $20 \mathrm{ml}$ of blood taken. Genotyping was performed on DNA extracted from peripheral blood lymphocytes using PCR-RFLP with polymorphisms for the three Apo E alleles determined according to published techniques.

Results: Results for 38 matched pairs are presented. Male:female ratio was $46: 30$. Mean age did not differ significantly between index patients and siblings (50.5 (SD)(8) v 60 (10)) nor did BMI (27 (2) v 28 (4)). AHI was significantly higher for cases (41) (IQR 27-52) v 13 (IQR 10-20). There was no significant difference in the distribution of either alleles or genotypes of Apo E between cases and controls (genotypes shown). See table.

The $\mathrm{K}$ statistic for alleles between cases and controls was 0.35 $(p<0.0001)$ and for genotypes was $0.32(p=0.002)$. Allelic frequency in the population was equivalent to that of the UK population: E2 (0.13); E3 (0.71); E4 (0.16).

Discussion: There is no difference in distribution of Apo $E$ alleles and genotypes between subjects with SDB and their siblings. Our results are supported by two other studies looking at $A p o E$ in relation to SDB. ${ }^{2}{ }^{3}$ Further samples will be genotyped both for Apo $\mathrm{E}$ and other candidate genes in this ongoing study.

Acknowledgements: nursing staff \& technicians of the Scottish National Sleep Laboratory; Dr N McArdle; Genetics Core, Wellcome Trust Clinical Research Facility, Edinburgh.

Supported by: Helen Bearpark Scholarship; AFUW fund; ERS LTT fellowship.

1. Kadotani, et al. JAMA 2001:285:2888-90.

2. Saarelainen, et al. Clin Gen 1998:53:147-8.

3. Foley, et al. JAMA 2001:286:1447-8.

\begin{tabular}{|lllll}
\hline \multicolumn{2}{l}{ Abstract P48 } \\
\hline & E3/E3 & E2/E3 & E3/E4 & E2/E2 \\
\hline Cases & 16 & 7 & 13 & 1 \\
Controls & 22 & 5 & 10 & 2 \\
\hline
\end{tabular}




\begin{tabular}{llll} 
Abstract P49 & & \\
\hline Groups & & $\begin{array}{c}\text { Mean age } \\
\text { (SEM) }\end{array}$ & $\begin{array}{l}\text { Mean Epworth } \\
\text { Score (SEM) }\end{array}$ \\
\hline Healthy volunteers & $\mathrm{N}=17,7$ male & 42 yrs $(2.6)$ & $4(0.5)$ \\
Treated narcolepsy & $N=9,5$ male & 41 yrs $(6.5)$ & $9(1.5)$ \\
Treated OSA & $N=16$, all male & 53 yrs (2.0) & $5(0.6)$ \\
Untreated OSA & $N=10,9$ male & 48 yrs (4.0) & $13(0.9)$ \\
\hline
\end{tabular}

P49 REACTION TIME (RT) TESTS IN OBSTRUCTIVE SLEEP APNOEA (OSA) AND NARCOLEPSY PATIENTS: THE PSYCHOMOTOR VIGILANCE TASK (PVT) IS A BETTER DISCRIMINATOR THAN THE SIMPLE UNPREPARED REACTION TIME (SURT)

D.C. Randall, S.N. Pilsworth, M.A. King, J.M. Shneerson, I.E. Smith. Papworth Hospital Sleep Centre, Cambridge, UK

RT tests are commonly used to assess performance of patients with sleep disorders and healthy subjects who undergo sleep deprivation but it is unclear which is most suitable for use in the clinical setting as an additional tool for assessing the effectiveness of treatment. The aim of this study was to compare the performance of four groups: healthy volunteers, treated narcolepsy patients, OSA patients treated with $\mathrm{nCPAP}$, and untreated OSA patients (desaturation index = 15, Epworth Sleepiness Scale score $=10$ ) on two different tests of sustained attention used in our lab: the PVT and the SURT.

Methods: Subjects completed the 10 min tasks in the afternoon, in random order. Subject demographics are presented in the table.

Results: One way ANOVA showed that there was a significant difference between the four groups on PVT slope (time on task decrement) $\left(F_{(3,48)}=6.3, p<0.005\right)$. Bonferroni post hoc tests revealed that both narcolepsy and untreated OSA patients showed more reaction fatigue - that is, an increase of reaction time over time-on this task than did healthy volunteers $(p<0.01)$. Mann-Whitney tests also revealed significant differences between the groups on SURT mean RT and number of gaps. These were due to narcolepsy patients having slower reaction times and a greater number of gaps (RT $>1 \mathrm{~s}$ ) (for both, $\mathrm{p}<0.05$ ) than both healthy volunteers and treated OSA patients. There were no significant differences between the untreated OSA patients and the other groups on this task.

Discussion: Despite treatment, narcolepsy patients showed impairments on both tasks of sustained attention. Unlike the PVT, the SURT did not distinguish between untreated patients with OSA and healthy volunteers or those on nCPAP and may not be appropriate to follow treatment effects.

\section{P50 ANALYSIS OF SLEEP STAGE USING A NEURAL NETWORK: COMPARISON TO MANUAL SCORING IN PATIENTS WITH OBSTRUCTIVE SLEEP APNOEA (OSA)}

F. Buchanan, N. Wiltshire, J.R. Catterall A.H. Kendrick. Sleep Unit, Dept Respiratory Medicine, Bristol Royal Infirmary, Bristol, UK

Sleep is a continuum, often with short 2-3s events. Traditional scoring using Rechtschaffen \& Kales (R\&K) uses multiple channels and assigns one of seven sleep stages to each 30s epoch with further analysis for arousals. Neural net analysis uses $1 \mathrm{~s}$ epochs and analyses sleep using one EEG channel, assigning a probability of a sleep stage to each epoch.

Aim: To compare R\&K scoring to neural net in patients with OSA.

Methods: Patients underwent one night's full polysomnography (SleepLab 1000e, Jaeger). A single scorer manually scored the study using R\&K and American Sleep Disorders Association arousals criteria. Data files were converted into European Data Format (EDF) and analysed using a neural network (Bio Sleep v4.0, Oxford BioSignals) using a single EEG channel. Median filter was set to $15 \mathrm{~s}$ and a threshold of 0.25 was used to score arousals, which had a minimum duration of $3 \mathrm{~s}$. Data are median (range).

Results: Twenty two patients (two female) were compared. Median Apnoea index was $2.45(0$ to 67.1$)$ and Apnoea-hypopnoea index was 16.6 (0.7 to 93.2). The conversion to EDF took 40 mins and analysis 15 mins, as against a median of 120 mins using R\&K. The EEG analysis is summarised below (see table).

Conclusion: The differences observed between R\&K and Neural $\mathrm{Net}$ reflect the shorter epoch analysis time of 30 s against 1s. Neural

\begin{tabular}{llll} 
Abstract P50 & & & \\
\hline & R\&K & Neural net & $\mathrm{p}$ Value \\
\hline Analysed time (hr) & $6.95(1.9-8.4)$ & $6.98(1.9-8.3)$ & $\mathrm{NS}$ \\
Total sleep time (hr) & $5.10(1.4-7.4)$ & $4.87(1.4-6.8)$ & $\mathrm{NS}$ \\
Sleep efficiency (\%) & $73.9(37.2-92.6)$ & $77.6(45.6-94.5)$ & $\mathrm{NS}$ \\
Number awakenings & $25.5(6-58)$ & $38(9-102)$ & $\mathrm{p}<0.05$ \\
Time awake (min) & $65.0(17-218)$ & $82.3(22.3-245)$ & $\mathrm{NS}$ \\
Deep sleep (min) & $58.7(0-231)$ & $37.0(0-106.2)$ & $\mathrm{p}<0.05$ \\
Arousals/hr & $20.7(5-86.1)$ & $14.4(2-41.0)$ & $\mathrm{p}<0.05$ \\
Arousal duration (s) & $5.75(4.7-6.5)$ & $6.0(5.0-7.0)$ & $\mathrm{p}<0.05$
\end{tabular}

R\&K, Rechtschaffen \& Kales.

network analysis provides (1) a simple computerised scoring of overnight sleep studies; (2) a more sensitive analysis of the sleep continuum, and (3) reduces the overall time of analysis of sleep studies as compared to R\&K.

\section{P51 COMPARISON OF R\&K AND BIOSLEEP SYSTEMS FOR ANALYSIS OF OBSTRUCTIVE SLEEP APNOEA}

F. Diamantea, P.P. Walker, S. Lowe, D. Barr, T. Mckown, P.M.A. Calverley. Department of Medicine, University Hospital Aintree, Liverpool, UK

At present the gold standard in the objective assessment of sleep apnoea is still polysomnography with data analysis using the Rechtschaffen \& Kales (R\&K) guidelines for 20-30 second sleep epochs. This is time consuming, expensive and relates poorly to symptoms. An attractive alternative based on neural net technology is the BioSleep system. It is a single channel method, which provides second by second measurements and classifies sleep as awake, light or deep using probability assessment rather than the traditional 6-7 point staging system. This may provide better analysis of microarousals and also shortens technician analysis time to less than five minutes.

We compared BioSleep to R\&K analysis using the same data set from 28 patients with moderate or severe OSA (Mean AHI $=52.4$ (SD 18.4)) during split night polysomnography in which therapeutic CPAP was administered for half the night. BioSleep $(B)$ was as good as R\&K (R) at detecting differences between CPAP and air in terms of arousals (B: $44 \vee 80 ; p<0.001, R: 51 \vee 79 ; p<0.02)$, arousal index (B: $12 v$ $22 ; \mathrm{p}<0001$, R: $18 \vee 29 ; \mathrm{p}<0.005)$, deep sleep $\%$ time (B: $30 \% \vee 8 \%$; $\mathrm{p}<0.0001, \mathrm{R}: 24 \%$ v $7 \% ; \mathrm{p}<0.0001)$ and light sleep $\%$ time (B: $70 \%$ $\vee 92 \% ; p<0.001, R: 76 \%$ v 93\%; $p<0.0002)$. BioSleep found greater differences in number of awakenings (B: $24 v 36 ; p<0.02, R: 2.8 v$ 3.2; $p=0.53)$ and total time awake (B: $0.9 \mathrm{hrs} v 1.27 \mathrm{hrs} ; \mathrm{p}<0.05, \mathrm{R}$ : $0.46 \mathrm{hrs} \vee 0.35 \mathrm{hrs} ; \mathrm{p}=0.15)$ but was inferior at detecting differences in sleep onset latency ( $B: 0.12 \vee 0.14 ; p=0.80, R: 0.26 \vee 0.10$; $p<0.001)$. BioSleep gave a more detailed picture of sleep architecture that was clearly different with therapy. Direct comparison showed that BioSleep predictably scored more awakenings and less total sleep time and sleep maintenance efficiency but there were no significant differences in \% light or deep sleep, number of arousals or arousal index.

BioSleep is a method of sleep analysis comparable to R\&K for several important parameters. While not replacing R\&K for investigation of REM related and complex sleep disorders BioSleep provides additional information compared to R\&K while shortening analysis time and this makes it an attractive analysis method for more widespread use.

\section{P52 THE EFFECT OF RECORDING SITE IN ACOUSTIC ANALYSIS OF SNORING}

M.J. Drinnan', A.M. MacDonald', M. Reda², J.A. Wilson², G.J. Gibson². Departments of 'Medical Physics; ${ }^{2} E N T$ surgery; ${ }^{3}$ Respiratory Medicine Freeman Hospital, Newcastle upon Tyne, UK

Introduction: Several groups have investigated acoustic analysis of snoring, in order to investigate the mechanism of snoring, to distinguish obstructive sleep apnoea from non-apnoeic snoring and to predict the outcome of corrective surgery. However, the recording site has varied, and it is not known how this affects the acoustic parameters measured.

Methods: We studied 47 subjects prior to laser-assisted palatoplasty for severe snoring. All underwent preoperative polysomnography and patients with $\mathrm{AHI}>25 / \mathrm{h}$ were excluded. 
Abstract P52

\begin{tabular}{llll}
\hline Parameter & External mic & Throat mic & Difference \\
\hline Centre frequency & $562 \mathrm{~Hz}$ & $351 \mathrm{~Hz}$ & $-211 \mathrm{~Hz}(\mathrm{p}=0.0005)$ \\
SD frequency & $875 \mathrm{~Hz}$ & $304 \mathrm{~Hz}$ & $-571 \mathrm{~Hz}\left(\mathrm{p}=2 \times 10^{-18}\right)$ \\
Peak factor ratio & 2.36 & 2.37 & $-0.01(\mathrm{~ns})$ \\
\hline
\end{tabular}

Sound was recorded continuously from a throat microphone and an external microphone $1 \mathrm{~m}$ above the patient's head. We analysed all snores, defined as sound level peaks during sleep, lasting 0.1 to 3 seconds, and at least $45 \mathrm{dBA}$ in amplitude measured $1 \mathrm{~m}$ above the patient's head, and calculated three acoustic parameters for each snore: centre frequency, SD frequency (a measure of spread about the centre frequency) (Clin Otolaryngol 1996;21:1 19-23), and peak factor ratio (the ratio of peak to RMS sound energy) (Clin Otolaryngol $1999 ; 240: 130-3)$. For each subject we calculated the mean value for each of the three parameters, for the external and throat microphones separately.

Results: We analysed an average of 1018 snores per subject. The mean values for each acoustic parameter are given in the table.

Discussion: Clearly, the recording site has a profound influence on the acoustic qualities of snoring sounds. In particular, frequencydomain indices (centre and SD frequency) are affected, implying the throat microphone preferentially attenuates the higher frequencies.

Conclusion: When performing acoustic analysis of snoring sounds, it is essential that the recording site is selected with care.

Acknowledgements: We wish to than the British Lung Foundation for their financial support of this work.

\section{P53 EFFECTS OF SIMULATED OBSTRUCTIVE APNOEA ON THE CAROTID BARORECEPTOR: VASCULAR RESISTANCE REFLEX}

C.M. Bowker, V.L. Cooper, S. Davis, S.B. Pearson, M.W. Elliott, R. Hainsworth. Leeds University Teaching Hospitals, Leeds, UK

Obstructive sleep apnoea may lead to hypertension. This study was designed to determine whether the changes in inspiratory pressure and the asphyxia that occurs in this condition can change the gain and/or setting of the carotid baroreflex to maintain arterial pressure at a higher level.

In eight healthy subjects (aged 21-62) we changed the stimulus to carotid baroreceptors using a neck chamber and graded pressures of -40 to $+60 \mathrm{mmHg}$ and assessed vascular resistance responses in the forearm from changes in the blood pressure (Finapres) divided by brachial flow velocity (Doppler ultrasound). Stimulus response curves were defined during (a) sham (no additional stimulus), (b) inspiratory resistance $(\sim 10 \mathrm{mmHg})$, (c) breathing asphyxic gas $\left(12 \% \mathrm{O}_{2}, 5 \%\right.$ $\mathrm{CO}_{2}$ ), and (d) resistance and asphyxia. Sigmoid functions were applied to the curves and the maximal differential (equivalent to peak gain) and the corresponding carotid pressure (equivalent to "set point") were determined.

The sham test had no effect on either the gain or the "set point". Inspiratory resistance alone had no effect on blood pressure. However it reduced the gain from $-3.0(0.6)$ to $-2.1(0.4)$ units $(p<0.5)$ but the curve was not displaced. Asphyxia alone increased blood pressure $(+7.0$ (1.1) mmHg, $p<0.0005)$ and displaced the curve to higher pressures by +16.8 (2.1) $\mathrm{mmHg}(\mathrm{p}<0.0005)$ but had no effect on gain. The combination of resistance and asphyxia both reduced gain and displaced the curve to higher pressures.

These results show that inspiratory resistance decreases the gain of the baroreceptor reflex and in combination with asphyxia also shifts the curve to higher blood pressure levels. If these changes were sustained, they would provide a mechanism to link hypertension with obstructive sleep apnoea.

\section{P54 PREVALENCE OF SLEEP DISORDERED BREATHING IN PATIENTS WITH CONGESTIVE HEART FAILURE}

A. Rashid, J. Chambers, A.J. Williams. Sleep Disorders Centre and Dept of Cardiology, St. Thomas' Hospital, London, UK

Introduction: Despite recent advances in medical and surgical therapy, congestive heart failure (CHF) remains a common and serious condition. CHF has been associated with sleep disordered breathing (SDB) in 51\% patients (Javaheri, et al. Circulation 1998;97:2154-9), including Cheyne-Stokes respiration (CSR) in $40 \%$, and obstructive sleep apnea (OSA) in $11 \%$. SDB is associated with frequent arousals, leading to a persistent activation of the sympathetic nervous system and elevation of catecholamines with deleterious effects on left ventricular function. Heart rate variability (HRV) is considered to be a surrogate for arousals and serves as an independent prognostic indicator for cardiovascular events (Narkiewicz et al. Auton Neurosci $2001 ; 90: 89-94)$.

Methods: Patients suffering from CHF were selected on the basis of echocardiographic findings of ejection fraction (EF) less than $35 \%$. We contacted 72 patients by letter, out of whom 36 patients (32 male and four female) agreed to participate in the study. Patients were sent portable pulse oximeters with a digital probe to wear overnight. The oximeters returned were studied for episodes of desaturations, and an oxygen desaturation index (ODI) was calculated. Desaturations were defined as dips in oxygen saturation greater than $3 \%$ and SDB was defined as an ODI greater than five.

Results: The overall prevalence of SDB in CHF patients was found to be $47.2 \%(17 / 36)$ and the mean ODI amongst these patients was $22.2 / \mathrm{hr}$. The prevalence in patients with an EF less than or equal to $25 \%$ was $53.8 \%(7 / 13)$ with a mean ODI of $26.2 / \mathrm{hr}$, whereas in patients with an EF between $25 \%$ and $35 \%$, it was $43.5 \%(10 / 23)$ with an ODI of $19.5 / \mathrm{hr}$. Increased heart rate variability was noted but was not found to correlate with SDB in our study. Twenty four out of 36 patients had increased HRV associated with concomitant dips in oxygen saturation in only 13. The remaining 11 patients having no SDB as defined here and raising the question as to additional burden of "subclinical" respiratory disturbance.

Conclusion: Our study confirms that nearly half the patients affected by $\mathrm{CHF}$, even in stable condition, have severe nocturnal respiratory disturbances, which increase with increasing severity of CHF.

\section{P55 IS MORE CPAP BETTER IN CLINICAL PRACTICE IN THE MEDIUM/LONG TERM?}

E. Morrish, S.N. Pilsworth, M.A. King, J.M. Shneerson, I.E. Smith. Papworth Hospital, Cambridge, UK

Background: In a trial setting, hours of continuous positive airway pressure (CPAP) use by obstructive sleep apnoea (OSA) patients is correlated with the fall in subjective sleepiness at one month. ${ }^{(1)}$ Our aim was to examine this relationship in a clinical setting after a longer follow up.

Methods: A retrospective review of patients who (a) were diagnosed with OSA according to the criteria as in ${ }^{(1)}$ (oxygen desaturation $>4 \%$ index (DI) $\geqslant 10 / \mathrm{hr}$ and Epworth Sleepiness Scale (ESS) score $\geqslant 10$ ); and (b) were started on CPAP in the year 2000 and continued to use it for an average of $\geqslant 1$ hour each night after $\geqslant 100$ days. Correlation was assessed using Kendall's tau_b coefficient.

Results: One hundred and three subjects ( 82 male) met the study criteria (table). Hours of CPAP use per night and change in ESS were not significantly correlated $(r=-0.094 ; p=0.170)$.

Discussion: In a month long trial setting only $4 \%$ of subjects withdrew from using CPAP. (1) In our experience approximately 15-20\% discontinue CPAP in the long term. Those who use CPAP very little and gain no benefit will increase the correlation between hours of CPAP use and change in ESS early on. After a longer period of time these people have stopped using CPAP. The hours of use of long term compliers with CPAP may be more dependent on intrinsic sleep requirement, the benefits gained from decreased snoring and the level of belief in a decreased risk from cardiovascular events rather than a simple relationship with perceived change in daytime sleepiness.

1. Stradling JR, et al. Sleep 2000;23(suppl 4):S150-3

\section{P56 TREATMENT OF SLEEP APNOEA/HYPOPNOEA SYNDROME (SAHS): A RANDOMISED TRIAL OF STANDARD CARE VERSUS ENHANCED NURSE INTERVENTION IN A DISTRICT GENERAL HOSPITAL}

S. Palmer', S. Selvaraj', C. Dunn', L.M. Osman², J. Cairns ${ }^{3}$, D. Franklin ${ }^{4}$, G. Hulks', D.J. Godden'. 'Highlands and Islands Health Research Institute; ${ }^{2}$ University of Aberdeen; ${ }^{3}$ Health Economics Research Unit; ${ }^{4}$ Highland Acute Hospitals NHS Trust

Background: Patients commencing CPAP therapy in Inverness (DGH) undergo a hospital titration study, telephone nurse review at four days 
Abstract P55 Study group baseline demography and outcome measures

\begin{tabular}{llll}
\hline $\begin{array}{l}\text { Baseline } \\
\text { demography }\end{array}$ & $\begin{array}{l}\text { Median }(5 \text { th, 95th } \\
\text { percentiles) }\end{array}$ & Outcome measures & $\begin{array}{l}\text { Median }(5 \text { th, 95th } \\
\text { percentiles) }\end{array}$ \\
\hline Age (years) & $52.5(29.2,67.3)$ & CPAP use (hrs $/$ night) & $6(2.2,8.5)$ \\
Weight (kg) & $111(76,158.1)$ & Length of use (days) & $523(231.6,802)$ \\
BMI & $36.2(27.5,53.5)$ & ESS & $6(0,16)$ \\
DI (dips/hr) & $40(10,60)$ & Change in ESS & $-10(-20.8,-1)$ \\
ESS & $17(10,23)$ & & \\
\hline
\end{tabular}

and consultant review at six weeks (standard care). A study carried out at the Scottish National Sleep Laboratory found that enhanced nurse intervention after diagnosis improved outcome after CPAP therapy (Hoy CJ, et al. Am J Respir Crit Care Med 1999;159: 10961100 ). Our randomised trial compared impact of standard care versus an enhanced nurse intervention on symptoms and quality of life in patients treated in the DGH setting.

Methods: Seventy consecutive SAHS patients were randomised to receive standard care (SC, $n=35$ ) or standard care plus a specialist nurse home visit at seven days $(\mathrm{HV}, \mathrm{n}=35)$. Patients completed a symptom score, Epworth score, Hospital Anxiety and Depression Score (HADS), and SF-36 at baseline and three months after initial review. CPAP hours of use were recorded.

Results: CPAP therapy resulted in significant improvements in symptoms, Epworth, HADS (table), and in the energy/vitality and mental component summary scores of the SF-36 in both groups. There was no difference between groups in any outcome measure or in hours of use of CPAP.

\begin{tabular}{|c|c|c|c|c|c|}
\hline \multicolumn{6}{|c|}{ Abstract P56 } \\
\hline & \multicolumn{2}{|l|}{$\mathrm{SC}$} & \multicolumn{2}{|l|}{ HV } & \multirow{2}{*}{$\begin{array}{l}-P \text { for dif } \\
\text { SC } \vee \mathrm{HV}\end{array}$} \\
\hline & Base & 3 months & Base & 3 months & \\
\hline Symptoms & 28.4 & $12.8^{*}$ & 27.4 & $12.9^{*}$ & 0.65 \\
\hline Epworth & 15.3 & $7.8^{*}$ & 13.8 & $7.5^{*}$ & 0.76 \\
\hline HAD Anx & 8.2 & $5.9^{*}$ & 8.4 & $5.9^{*}$ & 0.59 \\
\hline HAD Dep & 7.2 & $3.9^{*}$ & 7.1 & $4.6^{*}$ & 0.45 \\
\hline
\end{tabular}

Data are mean values; *Differs from baseline $(p<0.01)$.

Conclusion: CPAP led to marked improvement in patients with SAHS treated in the DGH setting. There was no difference between our two groups, both obtaining a similar magnitude of improvement to that reported in the enhanced care group from the National Sleep Laboratory.

Funded by the William Sutherland Trust.

\section{P57 CONTINUOUS POSITIVE AIRWAYS PRESSURE (CPAP) TREATMENT CAN REDUCE ANXIETY AND DEPRESSION IN OBSTRUCTIVE SLEEP APNOEA}

K.E. Lewis', A.J. Watkins'2, L. Seale', I.E. Bartle', P. Ebden'. 'Prince Philip Hospital, Dafen, Llanelli, Wales SA 14 8QF; ${ }^{2}$ EBMS, University of Wales Swansea, Swansea, Wales SA2 8PP, UK

Introduction: We tested the hypothesis that CPAP treatment will improve anxiety and depression scores and if effects are correlated to machine use.

Methods: Eighty consecutive patients (74 male), age 51.2 (9.7) (mean (SD)) years, mean BMI 35.5 (5.7), mean Epworth Sleepiness Score 14.4 (5.2), mean AHI 25.1 (22.8), and mean 5\% dip-rate of 31 (21.7) per hour, were prospectively studied. They completed the Hospital Anxiety and Depression Score at autotitration and after approximately one month of treatment with CPAP. Clock timers were hidden and machine "on time" was recorded at one month as a surrogate marker for machine use.

One sample $t$ tests assessed if change in anxiety and depression were statistically significantly different from zero. Pearson's correlation assessed the relationship between "on time" and changes in HAD scores.

Results: Patients were followed for a mean of 33.7 (13.4) days. Data was available on 70 patients. No patients reported life events or medication changes over this period. The mean anxiety score fell from $7.8(3.7)$ to $6.3(4.2)$ and the mean value for depression fell from 6.0 (3.7) to 4.9 (3.4) post treatment. Test statistics are -4.03 and -4.11 for hypotheses of zero change in anxiety and depression, respectively; $\mathrm{p}<0.001$ in both cases. Pearson's correlations between change in anxiety and depression with machine use are $r=-0.297$ $(p=0.012)$ and $r=-0.382(p=0.001)$, respectively.

Conclusion: OSA patients have similar anxiety and depression scores to the normal population but there were statistically significant improvements in both scores after only a short period of treatment. We conclude that CPAP has a graded effect, with the greatest benefits observed in those using their machines the most.

\section{Lung cancer}

\section{P58 OUTCOME OF A CODED $\times$ RAY SYSTEM TO FACILITATE REFERRAL OF CASES OF SUSPECTED LUNG CANCER}

J. Hughes, C. Garvey, C. Smyth, M.J. Ledson, M.J. Walshaw. Liverpool Lung Cancer Unit, Royal Liverpool University Hospital, Liverpool, UK

Chest radiography is an important investigation in patients with persistent chest symptoms, and can be an early indication of malignant disease. This is of particular importance when the $x$ ray abnormality is an incidental finding, for example on a preoperative routine film. In order to ensure the rapid investigation of patients with suspected lung cancer, we have introduced a coded $x$ ray reporting system for chest $x$ ray taken in Liverpool, including those performed in the community. When lung cancer is suspected, the reporting radiologist codes the report accordingly and the result is faxed to the relevant clinician, with a suggestion that the patient is referred to the rapid access service. In addition, all reports are electronically sent to the lung cancer specialist nurse, who monitors the referrals and ensures that the clinician acts on the report. We have audited the outcome of the first 477 patients in whom the radiologist flagged as suspected lung cancer using this coding system. Two hundred and fifty six were GP requests; nine of these were subsequently admitted to hospital, 215 were referred to the rapid access outpatient service, 24 were referred to a chest outpatient clinic, and eight refused further investigation. Within the secondary care sector, 206 were taken: 41 were admitted from the A\&E department, one was a clinic attendee, and 164 were inpatients of whom 74 were discharged to attend the rapid access outpatient service. Eight of the latter group were surgical patients in whom the finding of a suspicious $x$ ray lesion was incidental. In the remaining 15 cases, further investigation was not deemed necessary by the supervising clinician. Mean time from chest $x$ ray to attendance at the rapid access outpatient clinic was 14 days, and mean time from referral to first appointment was 8.5 days. Overall 268 patients $(56 \%)$ were subsequently diagnosed with lung cancer. Thus, we have shown that this $x$ ray coding system allows the rapid access of patients with suspected lung cancer to the relevant service. Furthermore, processing the reports through the dedicated lung cancer nurse acts as a safety net and ensures that cases are not missed by busy clinicians. We recommend the use of such coding systems to clinicians charged with providing rapid access lung cancer services.

\section{P59 AVOIDING LATE PRESENTATION OF LUNG CANCER: A PROACTIVE APPROACH TO MANAGEMENT OF ABNORMAL CHEST RADIOGRAPH REPORTS}

J. Brown, P. Murphy, A. Bramwell, J. Wide, J. Corless, J. Hendry. St.Helens and Knowsley Hospitals NHS Trust, Whiston Hospital, Warrington Road, Prescot, Merseyside L35 5DR, UK

Late presentation with lung cancer may preclude radical treatment System breakdowns may result in abnormal chest radiograph (CXR) 
reports not reaching the necessary clinician or being acted upon. In January 2000 we introduced a more pro-active approach to the handling of CXR reports that raise the possibility of lung cancer via a "failsafe" system. In partnership with our radiologists a coding system was used for CXR's requested both from primary and secondary care. CXR's with features strongly suggestive of a bronchial neoplasm were coded "TCXX" by the radiologist. Where neoplasia could not be excluded, a repeat film after 4-6 weeks was advised and a "TPXX" coding was given. All abnormal reports were immediately forwarded to the lung cancer team. Typically we would contact the patients' GP by telephone to discuss the report and requested an urgent referral from the GP. We later audited all new patients attending our rapid access lung cancer clinic over a one year period to assess the impact of this system.

Results: The codes were initially not applied to all abnormal CXRs, especially by trainee and locum radiologists. Of 228 new patients seen, $102(45 \%)$ had CXRs coded TCXX/TPXX and $126(55 \%)$ had abnormal CXRs with no coding. The percentage of patients later confirmed to have lung cancer were: TCXX (71\%), TPXX (23\%), and no code $(63 \%)$. The mean (range) time from day of CXR to clinic attendance was 8.5 (1 to 21) days in the TCXX group and 16 (1 to 162) days in the no code group $(p<0.001)$. A survey of 66 local GPs found that $86 \%$ felt that the failsafe system was helpful and should be continved. A recent re-audit has found that $69 \%$ of abnormal CXR's now receive the appropriate code.

Conclusions: A "failsafe" chest radiograph system is an effective tool that helps to ensure that patients with lung cancer are seen promptly and do not "slip through the net".

\section{P60 COMPULSORY REFERRAL FOLLOWING ABNORMAL CHEST $x$ RAY VERSUS CHOICE}

S.M. Jones', J. Hammond' ${ }^{2}$ A. Dunn ${ }^{2}$, A. Healey², I. Ryland', J. Curtis' ${ }^{2}$, C.J. Warburton', J.E. Earis'. 'Aintree Chest Centre; ' ${ }^{2}$ epartment of Radiology, University Hospital Aintree, Liverpool, UK

The British Thoracic Society recommends that General Practitioners should immediately refer patients to a respiratory physician if a radiology report suggests the possible diagnosis of lung cancer (Thorax 1998:53 (Suppl 1):S1-8). Some Units see such patients directly from the $x$ ray department while others (including ours) rely on the patients GP to refer patients to the rapid access clinic.

An audit of 2369 reports from GP requested chest $x$ ray, dating from 1 st January to 28th February 2002 was conducted. In total, 63 reports were suspicious of malignancy. Of these, 40 advised referral to a chest physician, 16 advised computerised tomography (CT) scan, and seven did not give any advice. Of the 63 positive reports 48 were referred to a chest clinic (31 to the rapid access lung clinic and 16 to another chest clinic). Four patients were already under regular chest follow up and six were under the care of other physicians. Three patients had CT scan undertaken by the Consultant Radiologist and no further action deemed necessary. One patient was admitted via the Casualty Department because of symptoms and one elderly lady in a nursing home subsequently died without being seen. The time to being seen in a clinic ranged from zero to 49 days with a median of eight days.

We have found that only five patients were not referred to outpatients of whom three were investigated with CT directly by the $x$ ray department. One of the remaining two patients presented with symptoms within two days to casualty and only one was missed (this was an elderly lady in a nursing home). Thus we have found that most patients with abnormal $x$ rays are referred in a timely fashion but suggest that the $x$ ray report should specifically suggest rapid access clinic referral. This system preserves both primary care and patient choice and prevents inappropriate referrals.

\section{P61 EXPERIENCE FROM THE FIRST 24 MONTHS OF A FAST TRACK LUNG CANCER CLINIC}

L. Dobson, D.M.G. Halpin, C.D. Sheldon, N.J.Withers. Royal Devon \& Exeter Hospital, Exeter, Devon, UK

In May 2000 a dedicated Fast Track Lung Cancer Clinic was introduced at the Royal Devon \& Exeter Hospital to facilitate referrals via the newly established two week wait (TWW) for suspected tumours. The clinic commenced as a weekly, Consultant provided service with the capability to see up to five urgent referrals weekly. General Pactitioners were informed about the new clinic by means of a letter, which also included local guidelines for referral via the "two week wait criteria". Here we present an analysis of our experience over the first 24 months of this initiative.

A total of 378 new referrals were seen, with $180(47.6 \%)$ of these being referred by the two week wait. Other referrals were via urgent GP letter (95), non-urgent GP letter (73), and Consultant referral (30). Of the TWW referrals seen, 169 (93.9\%) were appropriate according to local guidelines and $100 \%$ of these referrals were seen within two weeks. Over the 24 month period the number of referrals via the TWW route has increased from 36 ( 1 st six months of audit) to 64 (final six months).

Of the 378 patients seen in the FTLCC, $148(39.1 \%)$ have been diagnosed with primary lung cancer or mesothelioma. A further 11 have been diagnosed with secondary cancer. Eighty eight $(48.8 \%)$ of the TWW referrals had a diagnosis of primary lung cancer. Since the introduction of the TWW and the FTLCC there has been a sizeable decrease in the number of patients diagnosed as lung cancer following an emergency admission.

The establishment of a FTLCC has enabled us to fully achieve the TWW target. Referrals via the TWW are increasing and, in the main, are appropriate. The FTLCC may have reduced the number of patients admitted acutely with newly diagnosed lung cancer, but further follow up data will be required before this hypothesis can be confirmed.

\section{P62 APPROPRIATENESS OF REFERRAL PATTERNS UNDER THE TWO WEEK RULE FOR LUNG CANCER}

R.A. Heinink, H. Moudgil. Princess Royal Hospital NHS Trust, Apley Castle, Telford, UK

The Calman-Hine proposals for the management of lung cancer have forged the "two week rule" as the expected standard practice. Although many Trusts are able to meet these targets, less is known about the appropriateness of the actual General Practitioner (GP) referral patterns. This is particularly relevant against a background of direct or partial booking schemes for hospital appointments increasingly being made available to GPs. At this Trust, over a 12 month period to April 2001, there were 126 requests as possible lung cancer categorised under the two-week rule; 124 (98\%) of these had been received centrally by the next working day and $121(96 \%)$ had been offered outpatient appointments within the two weeks. At least $113(90 \%)$ of these patients met at least one of the preset published criteria for respiratory referral. Four of the 13 inappropriate referrals were from one GP practice. Of the 126 referrals, for 92 the criteria were based on radiological abnormalities alone with similar numbers (both $n=46$ ) specifically prompted by the radiologists or on the GPs own initiative. Chest film changes accompanied 15 patients with haemoptysis and one had stridor. Haemoptysis alone was reported in six. Collectively, these two week rule referrals made up $48(36 \%)$ of the 132 patients with diagnosed malignant disease presenting to the chest. A further 35 were diagnosed having been admitted acutely, 22 were detected at follow up clinics (including post admission), 15 were referred as routine whether by their GPs $(n=9)$ or via other consultants $(n=6)$, similarly nine others urgently outside two week rules by GPs $(n=6)$ or via other consultants $(n=3)$, and one patient remained with a non-respiratory physician throughout. Two others are undefined. Although we cannot presently comment on the patterns of referral of those outside the two week rule, this audit provides an insight into the appropriateness of two week referrals encountered here. It recognises the heterogeneous modes of presentation and referral but as expected singles out abnormal radiology as the main determinant. Importantly it also defines a large number $(n=24)$ still electively referred outside the two week rule by both GPs and hospital teams. It concludes (1) that the majority $(90 \%)$ referred under the scheme met the appropriate criteria for referral, (2) that only 48 (38\%) of these were then found to have malignant disease, and (3) that there are areas for improvement advising referral guidelines and timescales both within primary and secondary care.

\section{P63 HAEMOPTYSIS IN PATIENTS WITH A NORMAL CHEST X RAY: CURRENT PRACTICE OF UK CHEST PHYSICIANS BASED ON A POSTAL SURVEY}

N. McAndrew', J. Woolley', D.R. Baldwin', A.L. Burton ${ }^{3}$. 'Wrexham Maelor Hospital, Wrexham, LL13 7TD; ${ }^{2}$ Nottingham City Hospital, Nottingham NG5 1PB; ${ }^{3}$ Royal Preston Hospital, Preston PR2 9HT, UK

Do patients who present with an isolated episode of haemoptysis and a normal chest $x$ ray (CXR) need further investigation? If so, should this be with bronchoscopy $(\mathrm{Br})$, computerised tomographic scan of the tho$\operatorname{rax}(\mathrm{CT})$ or both? We sent a postal questionnaire to 610 UK chest 


\begin{tabular}{|c|c|c|c|c|c|c|c|c|}
\hline \multicolumn{9}{|c|}{ Abstract P63 } \\
\hline & No $1 \mathrm{x}$ & Ix & $\begin{array}{l}\text { Repeat CXR(s) } \\
\text { alone }\end{array}$ & CT alone & Bralone & CT \& Br & $\begin{array}{l}\text { Repeat CXR(s) } \\
\& \mathrm{Br}\end{array}$ & $\begin{array}{l}\text { Repeat CXR(s), } \\
\text { CT \& Br }\end{array}$ \\
\hline $35 \mathrm{NS}$ & $72 \%$ & $28 \%$ & $18 \%$ & $2 \%$ & $3 \%$ & $1 \%$ & $1 \%$ & $1 \%$ \\
\hline $59 S$ & $13 \%$ & $87 \%$ & $15 \%$ & $3 \%$ & $30 \%$ & $27 \%$ & $10 \%$ & $1 \%$ \\
\hline $70 \mathrm{~s}$ & $2 \%$ & $98 \%$ & $9 \%$ & $5 \%$ & $32 \%$ & $38 \%$ & $9 \%$ & $3 \%$ \\
\hline $65 \mathrm{~S}$ & $1 \%$ & $99 \%$ & $2 \%$ & $3 \%$ & $32 \%$ & $51 \%$ & $7 \%$ & $4 \%$ \\
\hline 7ONS & $7 \%$ & $93 \%$ & $19 \%$ & $8 \%$ & $25 \%$ & $30 \%$ & $7 \%$ & $3 \%$ \\
\hline
\end{tabular}

physicians posing five clinical scenarios. The scenarios described patients with isolated haemoptysis and a normal CXR: a 35 year old never smoker (35NS); a 59 year old smoker with acute bronchitis (59S); a 70 year old smoker who had a violent coughing fit (70S); a 65 year old smoker with haemoptysis "out of the blue" (65S); a 70 year old never smoked (70NS). We received 291 replies $(48 \%)$ (table).

Older patients and smokers are more likely to be investigated $\left(p<0.0001,35 N S \vee 70 N S ; p<0.01,70 S \vee 70 N S ; \chi^{2}\right)$. A minority would not investigate minor haemoptysis occurring during acute bronchitis. CXR surveillance is more popular than CT alone $\left(p<0.001 ; \chi^{2}\right)$. For smokers nearly $1 / 3$ of respondents would rely on bronchoscopy alone. The most popular strategy employs both bronchoscopy and CT, $(p<0.025, \mathrm{Br}$ alone $v \mathrm{CT} \& \mathrm{Br})$. The reliance on bronchoscopy alone is interesting as there is some evidence to suggest CT has greater sensitivity for picking up lesions not visible on CXR (ER) 2001;18(Suppl 33):P2595). CXR surveillance may have little benefit to the patient because it is less sensitive than either CT or bronchoscopy and because it will inevitably delay diagnosis. This survey has illustrated a lack of consensus in a difficult area of respiratory medicine.

\section{P64 THE EFFECTIVENESS OF A LUNG CANCER MULTI DISCIPLINARY TEAM (MDT): A DISTRICT GENERAL HOSPITAL (DGH) EXPERIENCE}

J.T. Samuel, S. Abubakar, A. David, K.J. Taylor, T.J. Charles. Glan Clwyd District General Hospital \& The North Wales Cancer Center, Rhyl, Denbighshire LL18 5UJ, UK

Introduction: A number of strategies have been developed to combat lung cancer in the United Kingdom starting with the Calman Hine Report in 1995. The MDT approach incorporating various medical and paramedical specialities has been uniformly advocated to provide efficient and effective delivery of care. The effectiveness of the MDT approach has not been evaluated.

Aim: To assess the effectiveness of a lung cancer MDT in the management of lung cancer.

Methods: We audited the management of patients with lung cancer from Glan Clwyd DGH over a one year period before and after MDT was instituted at the North Wales Cancer Centre.

Results: See table.

Conclusions: In our audit, there were no significant differences in the diagnosis or treatment of patients after the formal MDT approach was adopted. More non-invasive investigations were performed and data collection was more extensive and structured. Further larger audits on the effectiveness of the MDT approach in lung cancer are required to determine if it indeed provides more effective delivery of care than previously practised.

\begin{tabular}{lll} 
Abstract P64 & & \\
\hline & Pre MDT & Post MDT \\
\hline Patient number & 104 & 107 \\
Emergency admissions & $42 \%$ & $29 \%$ \\
Diagnosis in 2 weeks & $66 \%$ & $64 \%$ \\
Histology & $74 \%$ & $68 \%$ \\
CT thorax & $79 \%$ & $62 \%$ \\
Surgical rate & $9 \%$ & $5 \%$ \\
Chemotherapy/radiotherapy & $53 \%$ & $54 \%$ \\
\hline
\end{tabular}

\section{P65 ANALYSIS OF HISTOLOGY RESULTS TO IMPROVE PROTOCOLS FOR THE DIAGNOSIS OF LUNG CANCER}

S.M. Jones, P.P. Walker, I. Rylands, M. Babores, J.E. Earis, C.J. Warburton. Aintree Chest Centre, University Hospital Aintree, Liverpool, UK

There is still considerable variation in schematics for the management of pulmonary malignancies. This is particularly true for pleural disease where evidence is lacking. We looked at all 1012 cytological or histological assessments performed, in 654 patients referred between January 2000 and January 2001 with suspected pulmonary malignancy to produce local recommendations.

Pleural fluid cytology was analysed for diagnosis in 202 patients, of whom 48 had 2-4 samples taken. Twenty of $264(9 \%)$ samples showed malignancy. In the 48 cases where a second sample was analysed only $3 / 48$ were positive. In all three the initial result had been "suspicious or suggestive" of malignancy. Thirty eight sets of pleural biopsies were performed in 34 patients and $7 / 38(18 \%)$ showed malignancy. Twenty one patients with pleural effusions had a bronchoscopy. This was unhelpful in 18/21 irrespective of whether initial pleural cytology was reported as suspicious (7) or not (11). The $3 / 21$ patients with diagnostic bronchoscopic abnormalities all had central abnormalities on CT.

At bronchoscopy where no lesion was visible 154 patients had a blind wash, brush biopsy or both. Eight of $154(5 \%)$ had positive cytology and the addition of a brush biopsy increased the yield from $2 \%(2 / 92)$ to $11 \%(6 / 62)$. In 104 patients where a visible lesion was biopsied, an additional wash, brush biopsy or both increased the diagnostic yield by $6 \%$ - positive biopsy in 44 (42\%), wash only in two $(2 \%)$, brush only in three $(3 \%)$, and wash and brush only in one $(1 \%)$. Thirteen patients with a visible lesion and negative histology had a second bronchoscopy and 4/13 (31\%) produced positive histology.

We recommend (1) Diagnostic rate for blind pleural procedures is small but significant. If initial pleural cytology is not "suspicious" further aspiration without biopsy is unhelpful. (2) Bronchoscopy should not be performed for undiagnosed effusions in the absence of central CT abnormalities. (3) If blind samples are taken at bronchoscopy then a wash and a brush should be performed. (4) Wash, brush biopsy, and bronchial biopsy should be performed for all visible endobronchial lesions. (5) Repeat bronchoscopy is a reasonable option in some patients although histology is obtained in $<50 \%$.

\section{P66 RADICAL TREATMENT FOR NSCLC IN NORTHERN AND YORKSHIRE REGION}

E.T. Peel, C. Bennett. North Tyneside General Hospital, Northern \& Yorkshire Cancer Registry Information Service, UK

The casenotes of 400 consecutive patients with histologically confirmed non-small cell lung cancer (NSCLC) diagnosed in 1998 and registered with Northern and Yorkshire Cancer Registry Information Service (NYCRIS) database, were audited. The aim of the study was to determine the proportion of NSCLC patients receiving radical treatment (surgery or radical radiotherapy - dose $\geqslant 50 G y$ ) and how they were assessed.

Data is presented on 368 patients (92\%) in the table.

In patients not receiving radical treatment, the commonest reasons for those decisions were: advanced lung cancer $(54 \%)$, died $(18 \%)$, refused $(13 \%)$, other disease (12\%). The open and close rate was $8 \%$.

We conclude that staging and performance status are not well documented, although the majority of patients have significant comorbid illness, the main determinant of suitability for radical treatment is the extent of the disease. 


\begin{tabular}{|llllllll|}
\hline Abstract P66 & Median & $\begin{array}{l}\text { \% Not staged } \\
\text { explicitly }\end{array}$ & $\begin{array}{l}\text { \% PS } \\
\text { recorded } \\
\text { age }\end{array}$ & $\begin{array}{l}\text { Median } \\
\text { FEV }\end{array}$ & $\begin{array}{l}\text { \% Co-morbid } \\
\text { illness }\end{array}$ \\
\hline Surgery alone & 36 & 64 & 81 & 3 & 1.8 & 75 \\
Surgery + radical DXT & 7 & 62 & 100 & 0 & 2.6 & 43 \\
Surgery + palliative DXT & 3 & 75 & 100 & 33 & 1.1 & 66 \\
Radical DXT alone & 21 & 69 & 86 & 19 & 1.7 & 71 \\
Palliative DXT & 164 & 70 & 80 & 15 & 1.5 & 67 \\
No surgery or DXT & 137 & 72 & 90 & 12 & 1.5 & 69 \\
\hline
\end{tabular}

\section{P67 LUNG CANCER IN WOMEN THROUGH THE 1990s}

A. Bastin, D. Eraut, A. Davison, A.S. Haque, A. Hutchings, A. Lamont, C. Trask. Southend Associate University Teaching Hospital, Southend on Sea, Essex SSO ORY, UK

Lung cancer incidence is rising in females $(F)$ and falling in males (M) in the UK. Smoking in young females increased in the early $90 \mathrm{~s}$. We have analysed the pattern of lung cancer in 2127 new cases (1421 $\mathrm{M}(67 \%)+706 \mathrm{~F}(33 \%))$ presenting over a 10 year period from 1990 to 1999 from the Southend Lung Cancer Study. This includes every case in a well defined population of 325000 . The mean age of $F$ was $70.8 \mathrm{yrs}(S D 10.3)$ and $M 71.7 \mathrm{yrs}$ (SD 9.7) ( $p<0.04))$. There was a higher percentage of $F$ never smokers $(8.5 \%)$ compared with $M$ $(1.9 \%)(p<0.001)$. The proportion of never smokers was greater in elderly $\mathrm{F}$ compared to younger $\mathrm{F}(\mathrm{p}=0.019)$. There was a higher percentage of $M$ ex-smokers $(60.2 \%)$ compared with $F(51.1 \%)$ $(p<0.001)$. There were $39.6 \%$ of $F$ current smokers compared to $37.9 \% M(p=0.47)$. The proportion of current smokers falls with increasing age at diagnosis in both $M$ and $F$ (test for trend $p<0.001$ ).

We found a lack of evidence that the proportion of $F$ to $M$ had increased from 1990 to 1999 overall (test for trend $\mathrm{p}=0.3$ ) nor in under 65 s alone $(p=0.151)$. In patients with confirmed squamous, adeno, or small cell histology (total 1342), there was an overall difference $(p=0.001)$ in the proportion of histological types between $M$ and F: squamous cell carcinoma $54 \% M v 41 \% \mathrm{~F}$; adenocarcinoma $23 \%$ $M v 28 \% F$; small cell carcinoma $23 \% M v 31 \% F\left(\chi^{2} p<0.001\right)$. Never smoking $F$ were more likely to have adenocarcinoma than $F$ who smoked $\left(\chi^{2} \quad p<0.001\right)$. We found no evidence that the proportion of adenocarcinoma in smokers and never smokers changed over time in $M$ and $F$ (test for trend $p=0.67)$. The proportion for $M(10.5 \%)$ and $F$ $(8.9 \%)$ having either radical radiotherapy or surgery differed by $1.6 \%$ (95\% Cl $-1.1 \%$ to $4.2 \%)\left(\chi^{2} \mathrm{p}=0.26\right)$.

Conclusion: In comparing $M$ and $F$ with lung cancer through the 90 s we have found: (I) $F$ to be slightly younger than $M$; (ii) differences in histological types, with adenocarcinoma and small cell being more common in $\mathrm{F}$ and squamous cell carcinoma in $\mathrm{M}$; (iii) more $\mathrm{F}$ never smokers; (iv) older $F$ were more likely to be never smokers than younger $\mathrm{F}$; (v) F never smokers were more likely to have adenocarcinoma than F smokers; (vi) no difference in the proportion of men and women presenting over time, and (vii) no evidence of a gender bias in those receiving "curative" treatment.

\section{P68 LUNG CANCER IN OCTOGENARIANS, THE ROLE OF THE SURGEON}

I.R. Ramnarine, S. Thomas, A.N.A. Jilaihawi, D. Prakash. The Department of Thoracic Surgery, Hairmyres Hospital, East Kilbride, Glasgow, G75 8RG, UK

Objective: To analyse the pattern of management of patients in their eighties referred to the surgeon with clinical evidence of lung cancer.

Methods: A retrospective series of 6280 year old patients (42 males) with a clinical diagnosis of lung cancer referred to the surgeon over a 15 year period. The surgical management of these patients was reviewed.

Results: Pathological confirmation of tumour type was in 54 patients $(87 \%)$ with 39 lung cancers (28 squamous cell, eight adenocarcinoma, one large cell, and one small cell carcinoma), seven diffuse malignant mesotheliomas, three metastases, and five other tumour types. Of the lung cancer patients, 10 had tumours that were unresectable (mean survival 8.4 months) and 29 had resectable lesions. Of the latter group, eleven patients had comorbid disease making them inoperable (mean survival 6.4 months). Eighteen patients had lung tumour resection. There were four pneumonectomies, 13 lobectomies, and one bilobectomy. Ten patients had stage 1, five had stage two, and three had stage $3 a$ lung pathology. Operative mortality was $11 \%$ (two patients). One, two, and three year survivals were $72 \%, 64 \%$, and $23 \%$ respectively. Four of the nine late deaths were from tumour spread and there are seven disease free survivors.

Conclusion: Age should not be a discriminating factor in determining operability in lung cancer patients. The surgeon plays an important role in the management of octogenarians with lung cancer offering a wide range of services from minor diagnostic procedures to definitive surgery.

\section{P69 COPYING CORRESPONDENCE TO CHEST CLINIC PATIENTS: A SURVEY OF PATIENT VIEWS}

S. Lohani, P.J. Sullivan, R. O'Driscoll, S.C.O. Taggart. Respiratory Department, Hope Hospital, Salford, M6 8HD, UK

Introduction: As part of the NHS plan to improve communication between health professionals and patients by 2004, it is expected that all future patients will receive copies of correspondence. It is likely that this directive will have important effects on the way in which providers deliver their service in addition to changing the way they relate to their patients.

Methods: From mid April to mid June 2002, we surveyed all patients attending our general respiratory clinics in addition to those visiting our dedicated Lung Cancer Clinic. Patients were asked to complete a self administered questionnaire with tick box format, designed to identify which patients wished to receive all or some copies of their correspondence and which patients preferred to receive no copies. The patient's wishes were then recorded on their correspondence and letters were forwarded to the patients if required. Patients were requested to discuss any errors in their medications at their next clinic visit. Our medical secretaries recorded any incidents arising out of the introduction of this system. Patient preferences for receiving copies of correspondence at general respiratory and specialist lung cancer clinics (table).

A significantly greater proportion of patients attending our dedicated Lung Cancer Clinic preferred to receive no copies of correspondence than their general respiratory counterparts $(27 \% \vee 14 \%$, $p=0.013$ using Fisher's exact test). Overall, $84 \%$ of our patients expressed a preference to receive copies of correspondence. Throughout the study period, no incidents were recorded bar one patient who wished to point out a medication error

Conclusions: Based on our two month experience, we recommend caution for those planning to introduce copies of correspondence to all patients attending clinics as a small proportion would not wish to receive this. This proportion rises to almost a third for those patients attending Lung Cancer Clinics.

Abstract P69 Patient preferences for receiving copies of correspondence at general respiratory and specialist lung cancer clinics

\begin{tabular}{llll}
\hline $\begin{array}{l}\text { Preference for } \\
\text { correspondence }\end{array}$ & $\begin{array}{l}\text { General } \\
\text { respiratory }\end{array}$ & Lung cancer & Total \\
\hline All or some copies & $273(86 \%)$ & $45(73 \%)$ & $318(84 \%)$ \\
No copies & $44(14 \%)$ & $17(27 \%)$ & $61(16 \%)$ \\
Total & 317 & 62 & 379 \\
\hline
\end{tabular}




\section{Lung injury, inflammation, and infection}

\section{P70 EPIDEMIOLOGY OF LUNG FUNCTION AND IMMUNOLOGY IN PIGEON BREEDERS: FIVE YEAR FOLLOW UP}

T. Ismail, F. Boyd, C. McSharry, P. Lynch, C. Lynch, G. Boyd. Department of Respiratory Medicine and Immunology, North Glasgow University Hospitals NHS Trust, UK

Background: The immunopathogenesis of pigeon fanciers' allergic alveolitis is unresolved. Most studies rely on subjects presenting at clinics and proper epidemiological evaluation is lacking. Changes in lung function, immunology, and associated symptoms among a cohort of pigeon fanciers were assessed.

Methods: Forty pigeon fanciers had serial lung function, serum antibody to inhaled avian antigens and symptoms monitored for five years.

Results: Between 1997 and 2002 there was a significant reduction in FEV1 $(T=-2.87, p=0.007)$ and $F E V_{1} / F V C(T=-3.68$, $p=0.001)$. Twenty one subjects were seropositive in 1997 and a further four subjects showed evidence of new sensitisation in 2002 and there was no significant increase in the paired mean titre. The \% predicted FEV, correlated inversely with serum antibody titre $(r=-0.380$, $p=0.019)$ and peripheral blood CD8 lymphocyte proportion $(r=$ $-0.319, p<0.05)$. The antibody titre also correlated inversely with the $C D 4: C D 8$ ratio $(r=-0.325, p=0.014)$. Eleven subjects had symptoms of extrinsic allergic alveolitis in 2002 compared to 13 subjects in 1997.

Conclusion: Serial lung function in statistically determined cohort of pigeon fanciers seems to deteriorate significantly depending on the extent of the humoral antibody response to the inhaled antigens. These changes are associated with underlying immune dysfunction involving the imbalance of T-helper and T-cytotoxic lymphocytes. Subclinical inflammatory changes are common among pigeon fanciers and may be predictive of disease progression.

\section{P71 SERIAL LUNG FUNCTION FOLLOWING PERIPHERAL BLOOD STEM CELL TRANSPLANTATION}

L. Warren, L. Cormican, M. Gillion, D. Wilson, R. Freeman, S. Schey', H. Milburn. Departments of Respiratory Medicine; 'Haematology, Guy's Hospital, St. Thomas Street, London, SE 1 TEH, UK

Background: Pulmonary function tests (PFTs) are an established tool in the prediction of respiratory complications following allogenic bone marrow transplantation (BMT) for haematological malignancy. Most of these patients are now managed with allogenic and autologous peripheral blood stem cell transplants (PBSCT), a procedure less toxic than BMT and thought to result in fewer pulmonary complications.

Methods: In this observational study we have followed serial pulmonary function tests in 70 patients up to $81 / 2$ years following PBSCT for multiple myeloma $(n=35)$ and leukemia $(n=35,43 \% A M L$, $6 \% \mathrm{ALL}, 14 \% \mathrm{CML}, 37 \% \mathrm{CLL}$ ) to determine whether there were any

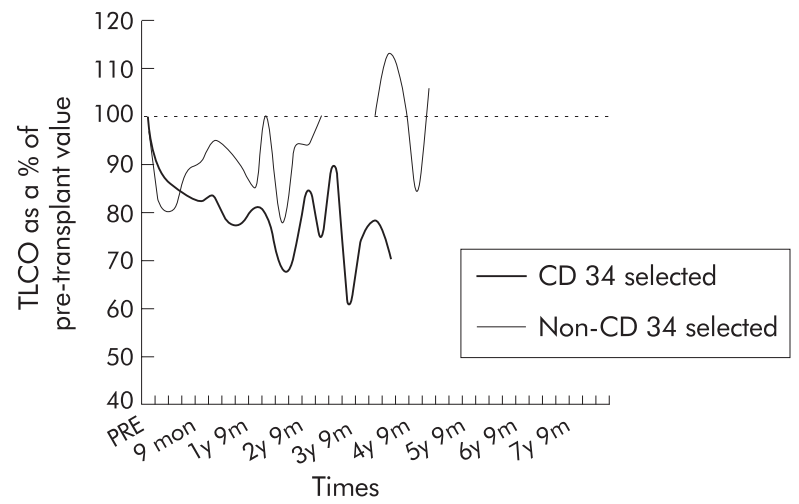

Abstract P71 Mean TLCO of CD34 and non-CD34 selected groups. groups which were particularly susceptible to respiratory complications. The selection of CD34+ cells was performed in 22 cases during the harvesting of peripheral blood stem cells prior to transplantation.

Results: Over the duration of the study there was a notable decrease in TLCO (figure) and an increase in RV (non-significant) in those who received CD34 selected, in comparison to non-CD34 selected grafts.

There was deterioration in lung function data (FEV, and FVC) in the AML population in comparison to the combined PBSCT population.

Conclusion: Even though PBSCT is associated with fewer pulmonary complications, certain subgroups of patients are more prone to changes in lung function over time. This justifies the performance of serial lung function in these patients.

\section{P72 LUNG REPAIR BY HAEMATOPOIETIC STEM CELLS (HSC) AFTER BONE MARROW TRANSPLANT (BMT)}

S.M. Janes, T. Hunt, M. Brittan, R. Jeffery, S. Forbes, K. Hodivala-Dilke, M. Alison, N. Wright, R. Poulsom, M.J.D. Griffiths. Cancer Research UK, London WC2A 3PX, UK

Introduction: Recent data suggest that HSC contribute to repopulation of the pulmonary parenchyma after BMT (Krause DS, et al. Cell 2001;105:369-77; Kotton DN, et al. Development $2001 ; 128: 5181-8)$. The aim of this study was to define in mice following BMT the phenotype and time course of the appearance of donor-derived cells in the pulmonary parenchyma.

Methods: Six week old female recipient mice were irradiated with 10 Gray as a split dose two hours apart to ablate their BM and then received male wild type whole BM by tail vein injection. Four mice were sacrificed at weekly intervals for six weeks and then at eight and 10 months. Lung sections were hybridised with FITC-labelled $Y$ chromosome paint (Star-FISH, Cambio, Cambridge, UK) to detect cells of donor origin. For the mouse tissue we combined in situ hybridisation for the $\mathrm{Y}$ chromosome with immunohistochemistry for specific markers for macrophages, myofibroblasts, endothelial and epithelial cells.

Results: $Y+$ cells were widespread in the lung parenchyma a week after BMT, engraftment peaked between four and six weeks, and persisted for at least 43 weeks (figure). $Y+$ cells were absent from the airway epithelium and the endothelium of venules and arterioles. $Y+$ cells stained positively with epithelial cell markers $\mathrm{Tl} \alpha$, Lectin lycopersicum esculentum, and CAM5.2.

Conclusions: Donor-derived HSC $(Y+)$ cell's morphology and staining suggests that they become part of the alveolar epithelium. The role of $\mathrm{BM}$ derived stem cells in lung repair is unknown but may be a novel target for manipulation or a means of delivering gene therapy to the distal lung.

$\mathrm{SJ}$ is supported by a training fellowship from the MRC UK.

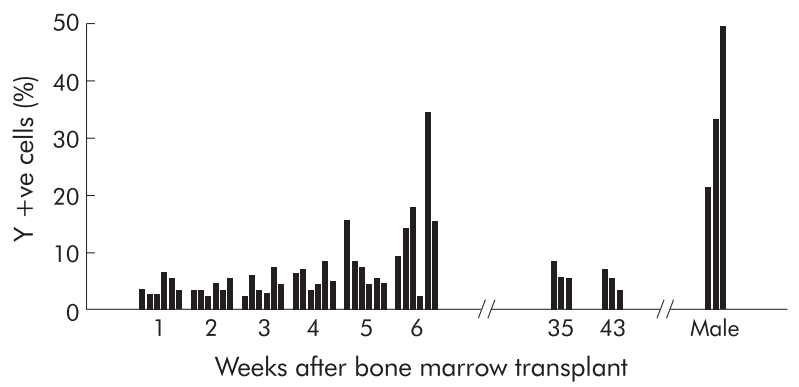

Abstract P72

\section{P73 PERIPHERAL BLOOD CHANGES AND CRYPTOGENIC FIBROSING ALVEOLITIS. POSSIBLE MARKERS OF DISEASE?}

V.A. Varney, D.T. Salisbury, H. Parnell. Department of Respiratory Medicine, St Helier Hospital, Wrythe Lane, Carshalton, Surrey SM5 IAA, UK

Background: Cryptogenic fibrosing alveolitis (CFA) is a disease associated with activated alveolar macrophages at broncho alveolar lavage. In the peripheral blood, autoantibodies and elevation of the ESR are common but not diagnostic. The prevalence of CFA has increased in the last decade providing more patients for studies.

We have observed a consistent feature (not yet described) of elevation in peripheral blood monocytes $(M \varnothing)$, mean red cell volume (MCV), and serum gamma glutamyl transferase (GGT). In all cases 


\begin{tabular}{|c|c|c|c|}
\hline \multicolumn{4}{|l|}{ Abstract P73 } \\
\hline $\begin{array}{l}\text { Parameter } \\
\text { (normal range } \\
\text { units) }\end{array}$ & $\begin{array}{l}\text { Control mean } \\
\text { (SD) }\end{array}$ & $\begin{array}{l}\text { CFA mean } \\
\text { (SD) }\end{array}$ & $\begin{array}{l}\mathrm{p} \text { Value } \\
\text { unpaired T test }\end{array}$ \\
\hline $\begin{array}{l}\text { MCV } \\
\text { (84-98) FL }\end{array}$ & $\begin{array}{l}89(4.20) \\
n=160\end{array}$ & $\begin{array}{l}94(6.1) \\
\mathrm{n}=91\end{array}$ & $p<0.001$ \\
\hline $\begin{array}{l}M \varnothing \\
(<0.6) 10^{9} / /\end{array}$ & $\begin{array}{l}0.35(1.1) \\
n=160\end{array}$ & $\begin{array}{l}0.68(0.24) \\
n=91\end{array}$ & $p<0.001$ \\
\hline $\begin{array}{l}\text { GTT } \\
(7-40) \cup / I\end{array}$ & $\begin{array}{l}29(22) \\
n=150\end{array}$ & $\begin{array}{l}40.2(31.7) \\
n=61\end{array}$ & $p<0.02$ \\
\hline
\end{tabular}

CFA, Cryptogenic fibrosing alveolitis; $M \varnothing$, peripheral blood monocytes; MCV, mean red cell volume; GGT, serum gamma glutamyl transferase.

alcohol intake and drugs were excluded as a cause. Full liver function tests were otherwise normal along with vitamin B 12 and folate levels. A bone marrow was examined in three cases confirmed a true macrocytosis without any other myelodysplastic features.

Methods: Ninety one patients (age 41-86 years) presenting with a new diagnosis of CFA had their baseline haematology and biochemistry studied. The results were compared with an age and gender matched reference range obtained from the same laboratory of non-CFA general population controls. The abnormalities at baseline were noted to be persistent throughout the patients treatments.

Results: See table.

Conclusion: Ninety one CFA patients showed a statistical increase in peripheral blood monocytes, MCV, and serum GGT. This observation requires further investigations and may represent a systemic disease with cytokine or other mediator effects on the bone marrow responsible for these changes.

\section{P74 PULMONARY VASCULAR ENDOTHELIAL GROWTH FACTOR (VEGF) RECEPTOR EXPRESSION IN ACUTE RESPIRATORY DISTRESS SYNDROME (ARDS)}

A.R.L. Medford', L. Armstrong', N.B.N. Ibrahim², K.M. Uppington', A.B. Millar'. 'Lung Research Group, University of Bristol Division of Medicine, Southmead Hospital, Bristol, UK; ${ }^{2}$ Department of Pathology, Frenchay Hospital, Bristol, UK

Previous work in our laboratory suggested a role for VEGF (an angiogenic and permeability factor) in the pathogenesis of ARDS (Thickett DR, et al. Am J Resp Crit Care Med 2001;164:1601-5). VEGF receptors (VEGFR 1, VEGFR2) are thought to be predominantly expressed in vascular endothelium and monocytes. Modulatory VEGF neuropilin co-receptors (NP1 and NP2) expression occurs in a variety of human tissues. The expression of these receptors in human normal and ARDS lung has not been extensively investigated. We hypothesised that there would be expression of these receptors on both sides of the alveolar-capillary membrane with significant upregulation in ARDS lung consistent with VEGF having a significant role. Immunohistochemistry was performed on post-mortem human lung sections from normal subjects and patients with late ARDS $(n=4)$ using semiquantitative densitometric analysis via Histometrix software. RT-PCR was performed on cultured human type II alveolar cells (AE2), alveolar macrophages (AMs) and peripheral blood monocytes (PBMs) from normal subjects and ARDS patients. In normal lung, VEGF, VEGFR1 and VEGFR2 protein were expressed in vascular endothelium, alveolar epithelium, and AMs. NP1 and two were confined to the alveolar side. In late ARDS lung, there was significant upregulation of VEGF, VEGFR1, VEGFR2, NP1 but not NP2. NP1 and 2 expression was also evident on the vascular side of the alveolar-capillary membrane. RT-PCR confirmed VEGFR1, VEGFR2, and NP1 mRNA expression in $A M s$ and $A E 2$ cells. PBMs expressed only VEGFR 1 mRNA. These data confirm the presence of VEGF receptors and co-receptors on the alveolar side of human lung in addition to the known vascular endothelial expression of VEGFR 1 and 2 . There is significant upregulation of these receptors in late ARDS consistent with VEGF having a significant role in ARDS on both sides of the alveolarcapillary membrane. The type 2 alveolar epithelial cell and alveolar macrophage appear to be additional targets for VEGF in human lung.

\section{P75 VASCULAR ENDOTHELIAL GROWTH FACTOR ISOFORM $\left(V^{\prime} G_{165} b_{1}, V\right.$ GF $_{165}$ AND VEGF $\left._{188}\right)$ EXPRESSION IN HUMAN AND MURINE INJURED LUNG}

A.R.L. Medford', L. Armstrong', S. Godinho', D.O. Bates', S.J. Harper ${ }^{2}$ A.B. Millar'. 'Lung Research Group, University of Bristol Division of Medicine, Southmead Hospital, Bristol, UK; ${ }^{2}$ Microvascular Research Laboratory, Department of Physiology, University of Bristol, Southwell Street, Bristol, UK

Previous work in our laboratory suggested a role for VEGF in the pathogenesis of ARDS (Thickett DR, et al. Am J Rep Crit Care Med $2001 ; 164: 1601-5)$. However, VEGF is compartmentalised in normal human lung (Kaner RJ, et al. Mol Med 2001;7:240-6). More recently, VEGF ${ }_{165} b$ has been identified as an inhibitory splice variant found in a variety of human tissues including lung (Bates DO, et al. Cancer Res 2002;62:4123-31). There is mounting evidence that the VEGF isoforms have different biological roles. We hypothesised that the $V E_{165}{ }_{165}$ bould be significantly expressed in normal lung and that the distribution of the VEGF ${ }_{155} b_{1} V E G F_{165}$, and VEGF ${ }_{10}$ isoforms would be altered in acute respiratory distress syndrome (ARDS). Total RNA was extracted from cultured "normal" human type 2 alveolar epithelial cells (AE2, $n=4)$, as well as alveolar macrophages (AMs, $n=11$ ) from normal subjects, at risk and ARDS patients. RNA was also extracted from C57BL/6 mice after lipopolysaccharide (LPS) induced lung injury $(n=9)$ and sham controls $(n=2)$. RT-PCR with VEGF isoform-specific primers was performed to assess VEGF ${ }_{165} \mathrm{~b}, \mathrm{VEGF}_{165}$ and VEGF ${ }_{189}$ mRNA expression. VEGF ${ }_{165} \mathrm{~b}$ was expressed in all samples $(n=26)$. In injured murine lung, VEGF ${ }_{165}$ and VEGF ${ }_{189}$ were both expressed compared to only VEGF ${ }_{165}$ in normal murine lung. Seventy five per cent $(n=6)$ of samples expressing only VEGF ${ }_{165}$ were from normal lung. $75 \%(n=12)$ of samples expressing both VEGF and $\mathrm{VEGF}_{189}$ were from injured lung. Expression of VEGF splice variants appears to be influenced by lung injury whereas the inhibitory splice variant is endogenously expressed in the lung. This may partially account for the high levels of compartmentalised VEGF in normal human lung. The relative expression of the inhibitory and the active isoforms may determine the functional role, in particular the pathogenic role of VEGF in ARDS. Further research is required to define the exact relative expression of these isoforms.

\section{P76 AN AUDIT OF THE MANAGEMENT OF COMMUNITY ACQUIRED PNEUMONIA IN SOUTHAMPTON GENERAL HOSPITAL}

S.K. West, S. King. Respiratory Department, Southampton General Hospital (SGH), UK

Introduction: The publication of the British Thoracic Society (BTS) guidelines into community acquired pneumonia (CAP) (Thorax 2001:56(Suppl IV):iv1-iv64) prompted a review of clinical practices at Southampton General Hospital (SGH). The aim was to assess the present use of the core prognostic features outlined in the guidelines (confusion, urea, respiratory rate, and blood pressure) and their relation to management decisions.

Method: The patient group was all those admitted to SGH between September and November 2001 (inclusive) given the diagnosis of pneumonia on their discharge summary. Patients were then excluded according to the BTS exclusion criteria. The data was collected from the patients' notes; specifically initial investigations, core prognostic features, severity of the pneumonia, antibiotic prescription, and patient outcome. The results were then compared with the guidelines and national statistics.

Results: Seventy three notes were obtained from 113 requested, of which 38 met the inclusion criteria. The main reason for exclusion was a recent hospital stay. The average age was 70 years and the length of stay nine days. Only seven patients had any documented assessment of severity, three of which were said to be severe. Subsequent use of information within the notes indicated that $45 \%$ could be classified as severe, $42 \%$ non-severe, and $13 \%$ mild. Measurement of core prognostic features varied, with blood pressure measured in $97 \%$ of cases, urea $82 \%$, respiratory rate $74 \%$, and mental test score $50 \%$. Other problems with note keeping included minimal inclusion of radiographic results in the notes. The majority of antibiotic choices were appropriate in type but with $70 \%$ of first line antibiotics being given intravenously despite only $45 \%$ classifiable as severe. The overall death rate was $23.6 \%$ with three unrelated deaths. Two patients were subsequently changed to palliative management and four deaths were attributed directly to CAP. Thus the death rate from CAP was $10.5 \%$ with $89 \%$ of these initially graded as severe. $53 \%$ of all those graded as severe died. 
Conclusions: There is presently minimal use of any formal severity assessment and a possible over reliance on intravenous antibiotics. This audit may provide a background on which to investigate the benefits of severity assessment forms in the care of all those admitted locally with possible CAP.

\section{P77 STAPHYLOCOCCUS AUREUS CELL WALL DEFICIENT BACTERIA ARE HIGHLY RESISTANT TO CELL WALL ACTIVE ANTIBIOTICS AND HAVE AN ALTERED PROFILE TO OTHER CLASSES OF ANTIBIOTIC}

C. Elmer, P. Cook , F. Nattress, P. Cheung ${ }^{2}$, T. Fawcett ${ }^{3} .{ }^{1}$ University Hospital of North Durham; ${ }^{2}$ Centre for Comparative Public Health, University of Durham; ${ }^{3}$ School Of Biological and Biomedical Sciences, University of Durham, UK

Background: Bacteria may lose all or part of their cell walls under certain environmental conditions, including the presence of cell wall-active antibiotics. Cell wall deficient bacteria (CWDB) are hard to detect by light microscopy or culture, but can proliferate in vivo and on specialised media. We investigated whether cell wall deficiency confers stable resistance to penicillin and its affect on the response to other antibiotics.

Method: Staphylococcus aureus cells were cultured in the presence of sublethal levels of penicillin $G$ on various media, including one optimal for CWDB. Minimum inhibitory concentration (MIC) estimations were performed after three step increases in penicillin concentration and on cells that had been passaged in the absence of penicillin. Cells were examined by Gram staining and electron microscopy. Different strains of CWDB were subjected to disc diffusion tests for a range of other antibiotics.

Results: CWDB have a different colony morphology, stain Gram negative and have indistinct margins and altered cell morphology on electron microscopy. The MIC for penicillin increased following serial passages, particularly on CWDB optimal media $(32$ units $/ \mathrm{ml}$, compared with 1 unit/ml on DST medium, after 12 passages). After seven passages without penicillin the cell wall was regained, but penicillin resistance was maintained. CWDB were resistant to other cell wall active antibiotics. They also exhibited altered profiles in comparison to the wild type cells for erythromycin, trimethoprim, tetracycline, novobiocin, and nitrofurantoin.

Conclusion: In the presence of sublethal levels of antibiotics and media optimal for CWDB, S aureus rapidly develops a high degree of stable penicillin resistance. We propose that loss of the cell wall, though rarely demonstrated in clinical microbiology laboratories, is an important cause of antimicrobial resistance to cell wall active antibiotics. Surprisingly these cells show significant alterations in sensitivity to other classes of antibiotics too. This could have profound implications for antibiotic therapy.

\section{P78 IgA1 PROTEASE FROM NON-TYPABLE HAEMOPHILUS INFLUENZAE CLEAVES TWO SITES IN HUMAN IgA1 HINGE REGION}

D. Mistry, S. He, R.A. Stockley. Lung Investigation Unit, Queen Elizabeth Hospital, Birmingham, UK

Bacterial $\lg \mathrm{A} 1$ proteases are thought to be important virulence factors in respiratory tract infections. This group of proteolytic enzymes specifically cleave one of several post-proline peptide bonds within the hinge region of human immunoglobulin $\mathrm{A} 1$.

We have partially purified an IgA 1 protease with a different cleavage specificity, from a clinical isolate of non typable Haemophilus influenzae (NTHI), by anion exchange chromatography. Proteolytic assays were carried out with human $\lg \mathrm{A} 1, \lg \mathrm{A} 2$, and serum albumin. PCR of the NTHI genome was carried out with $\lg A l$ protease sequence-specific primers to identify the gene(s) coding for the $\lg \mathrm{A} 1$ protease(s) producing this cleavage pattern.

The protease specifically cleaved human $\lg \mathrm{A} 1$ and did not cleave human $\lg \mathrm{A} 2$ or serum albumin. However, the $\lg \mathrm{A} 1$ protease cleaved more than one site within the hinge region of human $\operatorname{lgA} 1$. PCR amplification produced one lgAl protease gene (iga) product. The PCR products contained homologous sequences to other iga genes of the serine-type $\lg \mathrm{A} 1$ proteases and interspersed between these sequences were new deletions and insertions. The results indicate that the NTHI contains one iga gene sequence that encodes one $\lg \mathrm{Al}$ protease, which cleaves more than one peptide bond in the $\lg A 1$ heavy chain. The iga gene sequence may produce the unique cleavage specificity of the NTHI lgAl protease and further work will require the identification of the residues essential for the $\lg \mathrm{A} l$ protease activity, to allow the design of specific inhibitors to this important class of proteolytic enzymes.

\section{P79 ELEVATED NASAL NITRIC OXIDE CORRELATES WITH REDUCED NASAL MUCOCILIARY CLEARANCE IN BRONCHIECTASIS}

A. Shoemark, S. Kharitonov, P. Barnes, R. Wilson. Host Defence Unit, Royal Brompton and Harefield NHS Trust and National Heart and Lung Institute, Imperial College of Science Technology and Medicine, London, UK

Effective mucociliary clearance (MCC) is an important first line host defence against infection. Nitric oxide (NO) is also thought to aid in host defence through its antibacterial properties and by increasing ciliary beat (Runer et al. 1998). NO is upregulated in the presence of infection and inflammation (Kharitonov et al. 1995). The aim of this study was to establish the relationship between nasal MCC and NO in patients with bronchiectasis, a disease associated with impaired host defence and inflammation.

Nasal MCC and NO were measured in 30 non-smoking subjects with stable bronchiectasis confirmed by CT scan, age 25-82 yrs mean 57, male (11), and eight control subjects with no respiratory problems. Eight patients regularly used nasal corticosteroids. Subjects with cystic fibrosis or primary ciliary dyskinesia were excluded as nasal NO is known to be low. NO was measured directly from the nostril during a breath hold using a chemiluminescence analyzer (LR2000 Logan research ltd. Rochester, UK). Three patients could not hold their breath for long enough for the test to be performed correctly. Nasal MCC time was measured by the saccharin test.

In bronchiectasis subjects without nasal corticosteroids, nasal NO was significantly $(p<0.05)$ elevated in patients $(n=6)$ with nasal MCC $>60$ minutes, mean 658ppb (SD 186), compared to patients $(n=13)$ with nasal MCC $=60$ minutes, mean $402 \mathrm{ppb}(180)$, and controls, mean $418 \mathrm{ppb}$ (136). There was a correlation between nasal MCC and $\mathrm{NO}$ in patients with clearance $=60$ minutes. $r=0.6, p<0.05$. Although no significant difference in NO was found between subjects with no nasal corticosteroids and patients regularly taking nasal corticosteroids, in subjects regularly taking nasal corticosteroids there was no correlation between nasal MCC and NO.

In conclusion nasal NO is elevated in bronchiectasis patients with delayed nasal mucociliary clearance. This finding may be due to increased inflammation in the upper respiratory tract. Delayed clearance occurs despite any increase in ciliary beat that might occur with increased NO.

\section{P80 STUDY OF EXHALED NITRIC OXIDE IN STABLE BRONCHIECTASIS}

L.J. Ozerovitch, A. Shoemark, S. Kharitonov, P. Barnes, R. Wilson. Host Defence Unit, Royal Brompton and Harefield NHS Trust and National Heart and Lung Institute, Imperial College of Science Technology and Medicine, London, UK

We have previously shown that nitric oxide (NO) is elevated in some patients with stable bronchiectasis, but in others levels are normal. Exhaled NO levels are high along with systemic markers of inflammation (CRP and peripheral blood neutrophil count) during an exacerbation and fall after antibiotic therapy. These results suggest that NO is a marker of lung inflammation. The present study was conducted to compare those patients with elevated $\mathrm{NO}$ when stable to those with normal levels.

Twenty three patients with bronchiectasis shown on CT scan underwent a protocol of investigation which included full lung function tests, sputum examinations, blood investigations, ciliary studies, sweat test, shuttle walking test, and St George's Respiratory Questionnaire (SGRQ).

There was no relationship between $\mathrm{NO}$ and walking distance nor any component of the SGRQ. There was also no relationship between $\mathrm{NO}$ and extent of bronchiectasis on CT scan, blood inflammatory markers, sputum bacteriology, sputum eosinophil count, nor any lung function parameter. There were correlations between gas transfer $(\%$ predicted) and walking distance ( $p<0.05$ ); walking distance and CRP $(p<0.05)$; gas transfer (\% predicted) and Total SGRQ $(p<0.05)$; walking distance and Total SGRQ and also the Activities component $(p<0.004)$; and Total SGRQ and FEV1 (\% predicted) $(p<0.05)$.

In conclusion we do not know why some patients with stable bronchiectasis have elevated exhaled NO. We are continuing to study more patients using the same protocol, and also carrying out a more detailed analysis of the CT scans-for example, airway wall thickness - and a long term study to see if elevated NO influences the subsequent clinical course. 


\section{Clinical asthma and the nurse's role}

\section{P81 CHILDHOOD ASTHMA IN THE HIGHLANDS OF SCOTLAND: MORBIDITY AND SCHOOL ABSENCE}

J.B. Austin', S. Selvaraj', G. Russell3. 'Highland Primary Care NHS Trust; ${ }^{2}$ Highlands and Islands Health Research Institute; ${ }^{3}$ University of Aberdeen, UK

Background: The prevalence of childhood asthma in Scotland is one of the highest in the world. Allergic diseases may cause significant morbidity. The aims of this study were to describe the prevalence of asthma, eczema, and hay fever in the Highlands of Scotland and in the Shetland Isles and to examine factors in relation to quality of life and social deprivation.

Methods: A total population survey of 12 year old children using a parent completed questionnaire.

Results: $86.3 \%(2658 / 3080)$ returned questionnaires. Of the 2549 questionnaires analysed, $476(18.7 \%)$ reported asthma, 362 (14.2\%) wheeze in last 12 months, 508 (19.9\%) hay fever, and 555 $(21.8 \%)$ eczema. Of the children reporting asthma or wheeze, $35.4 \%$ (229/647) had missed school because of asthma or wheeze, $38.0 \%$ $(246 / 647)$ had missed physical education. Of subjects with lifetime wheeze, $62.5 \%$ (354/566) reported sleep disturbance. Deprivation measured by DEPCAT scores was associated with maternal smoking and bronchitis in the child but not with allergic diseases.

Conclusion: Compared with previous studies, the prevalence of asthma is unchanged but eczema has increased in Highland adolescents. Allergic disease has a significant impact on school attendance and physical activity. Deprivation is associated with maternal smoking and bronchitis in the child but not with allergic diseases. The impact of allergic diseases in rural areas may be different from urban areas.

Acknowledgement: This study was funded by Chest, Heart and Stroke Scotland.

\section{P82 CAN ASTHMA LIAISON NURSES REDUCE UNSCHEDULED CARE IN A DEPRIVED MULTIETHNIC POPULATION? ELECTRA: THE EAST LONDON CONTROLLED TRIAL FOR HIGH-RISK ASTHMA}

C. Griffiths, G. Foster, G. Feder, H. Tate, S. Eldridge, N. Barnes', A. Livingstone, T. Coats', for The Electra Group. Dept General Practice and Primary Care, Barts and the Royal London Hospital, London, El 4NS; 'The London Hospital, London E I, UK

Introduction: Evaluations of specialist nurses have focused on education in secondary care rather than liaison with primary care, and have not been set in multiethnic populations.

Design: Cluster randomised control trial comparing liaison nurse intervention versus best usual practice.

Setting: Forty four general practices in Tower Hamlets, east London Participants: 324 adults and children with asthma recruited after hospital admission or accident and emergency attendance.

Intervention: Intervention practices received two educational visits from liaison nurses to promote care of high risk patients. Participants from intervention practices received structured self management education from a liaison nurse. Control practices received one liaison nurse visit to discuss standard asthma guidelines. Participants from control practices received an inhaler technique check.

Main outcome measures: Participants free of unscheduled care; time to first unscheduled contact.

Results: Fifty per cent of participants were south Asian, 34\% white, and $16 \%$ other ethnicities. Primary outcome data was available for $319 / 324(98 \%)$ participants. Intervention by specialist nurse increased time to reattendance with an exacerbation (hazard ratio $1.17(1.01-1.36) p<0.05)$ and percentage of participants not attending with exacerbations $(p=0.056)$. Time to re-attendance was increased for white participants $(1.33(1.08-1.56) \mathrm{p}=0.006)$ but not south Asians (1.18 (0.96-1.45) $p=0.12$ )

Conclusions: Asthma liaison nurse intervention reduced unscheduled care in a deprived multiethnic population; white participants benefited but south Asians did not. Interventions are needed that improve asthma morbidity in non-white people with asthma.

Funded by the National Asthma Campaign.

\section{P83 DEVELOPMENT OF CENTRALISED ASTHMA NURSE SPECIALIST FOLLOW UP SERVICE FOR PATIENTS WHO ATTEND OUT OF HOURS FOR ASTHMA}

C. Calder, M. Reilly, L.M.Osman, A. Douglas, J.G. Douglas. Chest Clinic, Aberdeen Royal Infirmary, Aberdeen, AB25 2ZN, UK

Background: Local audit of patients attending out of hours $(\mathrm{OOH})$ for asthma highlighted gaps in follow up in primary care. One possible solution was asthma nurse specialists (ANSPs) based in $\mathrm{OOH}$ centre, providing a centralised service for reviewing these patients.

Aim: Improvement in primary care management of patients attending OOHS for asthma.

Design: Twenty four practices in two Local Health Care Cooperatives matched by size and deprivation category were randomised to 12 practices whose patients would participate in ANSP service and 12 practices whose asthma care remained the same. Patients, aged 5-60, were recruited over 14 months.

Method: Two part time ANSPs with advanced asthma qualifications were employed. Patients were identified from $\mathrm{OOH}^{\prime} \mathrm{s}$ cooperative and Accident \& Emergency daily. The Intervention group was sent a flyer followed by an asthma assessment telephone call by the nurses offering an appointment for a review at the Primary Resource Centre or at one rural practice between 09:00 to 18:00. A letter of invitation was sent if no telephone or if unable to contact. Prior to review and telephone call the nurses read primary care notes and repeat asthma prescriptions for each patient. The review, taking approximately 45-60 minutes, included asthma education, medication advice, and self management plans. Following review and or telephone call a summary liaison form was sent to the GP.

Results: Telephone assessment 177/237 (77\%), contact time was median 9 (2-29) days. Attended review 62/237 (26\%), contact time was median 18 (8-72) days. Refused review 81/237 (34\%), 48 $(20 \%)$ because had hospital/GP review already. Thirty eight out of $237(16 \%)$ defaulted review appointment. Unable to contact 56 (24\%) patients. Nurse management recommendations advised for $46 / 62(74 \%)$ of those reviewed, of which $16(35 \%)$ recommendations were acted on in the practice.

Conclusion: There was a poor response from patients to centralised review. This type of service may not be appropriate for $\mathrm{OOH}^{\prime}$ attenders

\section{P84 WHAT TREATMENT FACTORS INFLUENCE READMISSION AFTER A HOSPITAL ADMISSION FOR ACUTE ASTHMA?}

W. Rea, G. Perkins, A. Abhyankar, D.R. Thickett. Queen Elizabeth Hospital, Birmingham B15 2TH, UK

The most severe forms of asthma attack are the life threatening forms which result in hospitalisation. Once patients have been stabilised as an inpatient, the clinician is faced with the question of what to do with the inhaled therapy of patients admitted with acute exacerbations of asthma? The purpose of this study was to see if discharge medication influenced subsequent readmission rate.

Methods: All patients with a coding diagnosis of asthma were traced through hospital computer system. In 2000 there were 357 patients so labelled. Audit was carried out on 100 patients' notes. Patients with a greater than 20 pack year smoking history or a diagnosis of COPD anywhere in the notes were excluded unless asthma had been diagnosed by a consultant chest physician. Subsequent to the case note audit, the patients were sent a short questionnaire at the end of 2001, inquiring about admissions to hospitals other than QEH. Steroid use and casualty attendance. Questionnaire letters have been received back from 54 out of 100 .

Results: The BTS asthma step of the admitted patients correlated well with the risk of subsequent readmission $\left(r^{2}=0.89, p=0.01\right)$. Patients with step 0 or step 1 asthma treatment had a low asthma readmission rate in the subsequent year $(6 \%)$. Overall readmission rate was $41 \%$ in the year following discharge. Of those readmissions, $25 \%$ were to a different trust. Treatment by a respiratory specialist did not decrease the rate of readmission but did result in slightly longer hospital stays ( 1.2 days $p=0.05$ Mann Whitney $U$ test).

Median dose of inhaled corticosteroid dose was $400 \mu \mathrm{g}$ BDP upon admission and $800 \mathrm{\mu g}$ equivalent upon discharge $(p=0.009)$. There was no difference in the median steroid dose of patients admitted subsequently $(805 \mu \mathrm{g})$ compared to those who were not admitted $(800 \mu \mathrm{g}, p=0.9)$. The time to first readmission after the index admission in 2000 was significantly longer in patients treated with long acting $\beta$ agonists (105 days) compared to the group untreated 
with LAB (27.5 days, $\mathrm{p}=0.02$ Mann Whitney $U$ test). Of patients discharged from hospital, $38 \%$ have subsequently been prescribed a long acting $\beta$ agonist.

Conclusions: The level of treatment received by patients seems to reflect their risk of hospitalisation in this cohort. Readmission rates are high and admission to more than one hospital is common in Birmingham. Specialist care has little effect upon crude readmission rates. The routine use of LAB following hospital discharge needs evaluating in a clinical trial.

\section{P85 PSYCHIATRIC MORBIDITY IN DIFFICULT ASTHMA}

E. Conway', C. Kelly', J. Gamble ${ }^{3}$, L. Heaney ${ }^{2}$. Departments of 'Mental Health; ${ }^{2}$ Medicine, Queen's University Belfast; ${ }^{3}$ Regional Respiratory Centre, Belfast City Hospital Belfast BT9 7BL, UK

Introduction: Near fatal/fatal asthma are associated with psychiatric morbidity (PM). Studies have demonstrated PM in asthmatics of varying severity using questionnaire screening tools, but it is unclear how this relates to ICD 10 psychiatric diagnosis (gold standard). The aim of this study was to: (1) examine psychiatrist diagnosed morbidity in a population of difficult asthmatics (persisting symptoms/frequent exacerbations despite high dose inhaled steroids/long acting $\beta$ agonist, and (2) relate ICD 10 diagnosis to response to the Hospital Anxiety Depression Questionnaire (HAD), a commonly used screening tool, with defined normal values.

Methods: Sequential new referrals to a difficult asthma clinic completed HAD questionnaire and were invited to attend for psychiatric interview as part of a systematic evaluation protocol. Psychiatric interview was performed, by an experienced medical liaison psychiatrist, blinded to all clinical information. After interview, an ICDI0 diagnosis was recorded and treatment instituted as appropriate.

Results: Seventy eight patients were recruited (seven refused psychiatric assessment but were otherwise protocol compliant, five were non-compliant with the protocol). Of the remaining 66 subjects, $33(50 \%)$ had an ICD 10 psychiatric diagnosis; only seven (10\%) were receiving current treatment. Depressive illness (F32) was common (20 $(30 \%))$ followed by generalised anxiety disorder (F41) (5 (8\%)) plus a variety of other conditions. Anxiety (13.1 (0.82) v $8.5(0.7))$ and depression (10.1 (0.71) v $4.8(0.47))$ scores were significantly higher in subjects with an ICD10 diagnosis $(p<0.001)$. The positive (PPV) and negative predictive value (NPV) for abnormal HADS scores for all psychiatric diagnoses were $60 \%$ and $86 \%$ respectively and for depressive illness, PPV 76\%, NPV 96\%.

Conclusion: There is a high prevalence of psychiatric morbidity, particularly depression, in difficult asthmatics, based on psychiatric interview, most undiagnosed at referral. HADS score has poor overall positive predictive value for psychiatric illness but normal depression score virtually excludes the commonest condition, depressive illness. We are currently examining the predictive role of undiagnosed psychiatric disorder in asthma outcome and optimal screening instruments in this population

\section{P86 PSYCHOSOCIAL FACTORS IN ADULTS AT RISK OF ADVERSE ASTHMA OUTCOMES: RELATIONSHIPS WITH SYMPTOM CONTROL AND QUALITY OF LIFE}

J.R. Smith', M. Koutantii ${ }^{1}$, S. Mildenhall ${ }^{2}$, B.D.W. Harrison ${ }^{2}$, M. Noble ${ }^{3}$ 'University Of East Anglia; ${ }^{2}$ Norfolk \& Norwich University Hospital; ${ }^{3}$ Acle Medical Centre, UK

Background: Despite effective treatments, a proportion of asthmatics suffer from poorly controlled disease and consequent reduced quality of life (QoL), hospital admissions, near fatal, and fatal asthma attacks. This study assesses psychosocial characteristics of adults at risk of such adverse asthma outcomes and examines relationships with symptom control and QoL.

Methods: Ninety two adults with severe asthma (on BTS Step 4/5 treatment and/or with previous admissions for asthma) who exhibited poor compliance (failure to attend clinics or comply with asthma management in other ways) were recruited via hospitals and GP practices in Norfolk and Suffolk. Cross-sectional socio-demographic/socioeconomic data and self report measures of symptom control, QoL, psychiatric morbidity, perceived control over asthma, coping, and aspects of self management were collected via interviews in patients' homes.

Results: In common with those experiencing fatal and near fatal asthma, these patients represent a socioeconomically disadvantaged group (for example, 60\% not working, 63\% receiving free prescriptions) with reduced QoL (mean 1.17 on a 0 (very good) to 2 (very poor) scale), high levels of psychiatric morbidity (for example, $36 \%$ experiencing moderate-severe anxiety, 33\% reaching cut off for psychiatric caseness), and inadequate self management (for example, $82 \%$ not monitoring asthma, 39\% smoking, $61 \%$ owning pets). High psychiatric morbidity, low perceived control over asthma and various indicators of low socioeconomic status were significantly correlated with poor symptom control and reduced QoL (all $p<0.001$ ). Being overweight, use of coping strategies which involve focussing on asthma (both $\mathrm{p}<0.05$ ) or hiding asthma and, interestingly, high compliance (both $p<0.001$ ) were also associated with poorer asthma related QoL.

Conclusions: Various mechanisms may explain associations between psychosocial characteristics, symptom control, and QoL in at risk asthmatics and the direction of relationships is likely to be two way. Further psychological research to improve understanding in this area and an intervention study which attempts to address psychosocial issues in improving outcomes for at risk asthmatics are underway.

\section{P87 RELATIONSHIP BETWEEN AIRWAY OBSTRUCTION AND SYMPTOM CONTROL IN PATIENTS WITH DIFFICULT ASTHMA}

S. Aburuz', J. McElnay ', J. Millership', J. Gamble ${ }^{3}$, L. Heaney ${ }^{2.3}$. 'School of Pharmacy; 'Department of Medicine, Queen's University Belfast; ${ }^{3}$ Regional Respiratory Centre, Belfast City Hospital, Belfast

Introduction: Several studies have demonstrated a poor relationship between symptom control and airways obstruction in patients with asthma. We examined this relationship in a population of difficult asthmatics (persisting asthma symptoms/frequent exacerbations requiring systemic steroids despite maintenance high dose inhaled corticosteroids (ICS) and a long acting $\beta_{2}$ agonist) attending a hospital outpatient clinic.

Methods: $\mathrm{FEV}_{1} \%$ and asthma control scores (ACSs) (Juniper et al. 2001) were measured at the first clinic visit and at a follow up visit after a variable period of evaluation and treatment (9 (6.2) months).

Results: Fifty seven patients (37 females; median age 40 years; range 19-72 years; median ICS dose at presentation $2000 \mu$; range 1000-6400 jg BDP equivalent; median rescue steroid courses 12 months pre-referral $=5$ ). Patients had poor control at the initial visit (mean ACS 4.1 (1.3); FEV \% 66.3 (22.7)). At the initial visit, FEV \% was correlated with limitation of activity $(p=0.003)$, shortness of breath $(p=0.018)$, wheezing $(p=0.025)$ and ACS $(p=0.018) . F E V \%$ was significantly improved at the follow up visit $(75.3$ (22.6), $\mathrm{p}<0.0001)$ with an associated improvement in ACS $(2.8$ (1.3), $p<0.0001)$. However, at the follow up, there was no correlation between $\mathrm{FEV}_{1} \%$ and any measured index of asthma control. At the initial visit, 25 patients had severe obstruction (FEV $\%<60 \%$ ) and had significantly poorer ACSs (mean $4.6 \vee 3.7, p=0.003$ ). At the follow up, 15 patients had $\mathrm{FEV}_{1} \%<60 \%$ but no difference in $\mathrm{ACSs}(2.7 \mathrm{v}$ $2.8, \mathrm{p}=0.9)$. Best $\mathrm{FEV}, \%$ was significantly less in this latter group $(62$ (21) v 96\% (16\%), $p<0.0001)$ consistent with fixed airflow obstruction. When this group was excluded, $\mathrm{FEV}, \%$ at follow up, was significantly correlated with night waking $(p=0.02)$, wheezing $(p=0.05)$, and ACS $(p=0.036)$.

Conclusion: $\mathrm{FEV}, \%$ correlates well with asthma symptoms in difficult asthma patients with poor control but not when control improves. This loss of relationship is due to subjects with fixed airflow obstruction where good subjective control does not exclude the presence of significant obstruction.

1. Juniper E, et al. Resp Med 2001;95:319-23.

\section{P88 UTILITY OF HISTORY IN THE DIAGNOSIS OF CHRONIC COUGH}

J.C. Ojoo, S.A. Mulrennan, J.A. Kastelik, R. Thompson, A.E. Redington, A.H. Morice. Academic Department of Medicine, University of Hull, Castle Hill Hospital Cottingham, UK

Background: Patients referred to specialist cough clinics often have a history of prolonged period of cough, multiple investigations, and unsuccessful trials of therapy. It may be that symptoms in these patients are atypical making diagnosis of underlying condition difficult.

Aim: To determine presenting symptoms of patients with chronic cough in relation to their diagnosis.

Methods: In a prospective study based on a therapeutic rather than investigative protocol, consecutive patients with a history of cough for over eight weeks referred to the Hull Cough Clinic were enrolled. 
Assessment was by structured history, physical examination, chest radiograph, spirometry, and reversibility to nebulised salbutamol. From this information a diagnosis was made and patient had an eight week therapeutic trial. Further therapeutic trials were carried out depending on response to treatment and the likely diagnoses. Investigations were carried out in cases of failed therapeutic trials and to exclude specific pathology (in this study lung cancer, localised bronchiectasis and interstitial lung disease).

Results: One hundred and eleven (73 female) patients mean (SD) age 56 yr (12.8) were recruited. Main initial diagnoses were gastrooesophageal reflux disease (GER) $52(46.9 \%)$, asthma $23(20.7 \%)$, and rhinitis $19(17.1 \%)$. Sixty three patients have had at least two clinic visits. Of those discharged so far, median (range) duration of cough was $8.2 \mathrm{yr}(0.25$ to 64$)$ and mean duration of follow up was 14 weeks (2.4 clinic visits). Twenty four out of $63(38 \%)$ patients were discharged at the second and 10/23 (43\%) at their third visit. The main underlying diagnoses at discharge were GER $(25.7 \%)$, asthma $(22.9 \%)$, and rhinitis $(11.4 \%)$. Twenty per cent required investigations to arrive at the diagnosis and exclude other pathology, the rest were managed successfully on the therapeutic protocol. Symptoms associated with diagnosis of asthma were dyspnoea ( $p=0.007)$, wheeze $(p=0.04)$ and choking $(p=0.03)$, GER were heartburn $(p=0.01)$ and sour taste in the mouth $(p=0.06)$ and for rhinitis were postnasal drip $(p=0.05)$ and breathlessness $(0.03)$.

Conclusion: In patients in whom we have made a diagnosis and are satisfied with treatment, the presenting complex of symptoms is indicative of the underlying cause of their cough. This finding highlights the importance of the history taking in the assessment of patients with chronic cough.

\section{P89 FACTORS ASSOCIATED WITH POST BRONCHODILATOR FEV 1 IN ADULTS WITH ASTHMA}

M.A. Berry, R.H. Green, A.J. Wardlaw, I.D. Pavord. Glenfield Hospital, Groby Road, Leicester, LE3 9QP, UK

Fixed airflow obstruction occurs in a minority of asthmatic patients but is a predictor of overall mortality. Smoking has been identified as a factor associated with airflow limitation in asthma but evidence implicating other factors has been conflicting. In one study ten Brinke et al (Am J Respir Crit Care Med 2001;164:744-8) identified elevated induced sputum eosinophil count as an independant risk factor for persistent airflow limitation in a homogeneous population of severe asthmatics. We have investigated factors associated with the post bronchodilator $F E V$, in a heterogeneous population of 249 adults with a clinical diagnosis of asthma of variable severity and evidence of airflow variability and/or airway hyper-responsiveness. Patients had either never smoked or were ex-smokers with less than five pack years smoking history. Patients underwent methacholine challenge testing, pre and post bronchodilator spirometry, skin, and radioallergosorbent testing to commonly encountered aeroallergens and induced sputum analysis. Multiple independent linear regression analysis was used to identify any independent predictors of post bronchodilator $\mathrm{FEV}, \%$ of predicted. Duration of symptoms, atopy, dose of methacholine required to cause a $20 \%$ fall in $\mathrm{FEV}_{1}\left(\mathrm{PC}_{20}\right)$, induced sputum eosinophil count and induced sputum neutrophil count were not significant independent predictors of post bronchodilator $\mathrm{FEV}, \quad \mathrm{r}^{2}=0.060$, $p=0.162$ ). Patients with post bronchodilator $\mathrm{FEV}_{\text {, }}$ of less than $80 \%$ predicted had significantly higher induced sputum eosinophil counts (geometric mean 3.95\%) than those with $\mathrm{FEV}_{1}$ above $80 \%$ predicted (geometric mean $1.88 \%$, mean fold difference $2.10,95 \%$ confidence interval 1.19 to $3.72, p=0.011$ ). Measures of current airway inflammation and responsiveness do not independently predict persistent airflow limitation, as measured by post bronchodilator $\mathrm{FEV}, \%$ predicted in a heterogeneous population of non-smoking adult asthmatics. Although sputum eosinophil counts are higher in patients with persistent airflow limitation, sputum eosinophilia is not predictive of airflow limitation and cannot be implicated as causative.

\section{P90 EMITS: IMPACT OF A CHANGE IN TRANSPORT POLICY ON RESPIRATORY HEALTH IN OXFORD}

S.J. MacNeill', M. Barnes', R. Pitman'2, P. Cullinan'. 'Occupational \& Environmental Medicine, Faculty of Medicine, Imperial College of Science, Technology and Medicine, 16 Manresa Rd, London SW3 6LR, UK; ${ }^{2} \mathrm{Com}-$ munity Services, Oxford City Council, Ramsay House, 10 St Ebbe's St, Oxford OXI 1PT, UK

EMITS (Environmental Monitoring of an Integrated Transport Strategy) was established to examine the effects of a change in transport policy on public health as well as on other aspects of life in Oxford. The Oxford Transport Strategy (OTS) was implemented in June 1999 and involved many changes, focused primarily on the city center where all traffic was barred from some streets and private vehicles from others.

Between 1998 and 2000, 1386 children aged 6 to 10 were recruited from seven Oxford schools. Schools were visited two to three times a year in different seasons for five day periods. On each day of each visit, research nurses measured the childrens' peak expiratory flow and distributed questionnaires enquiring about respiratory symptoms on the previous day. Parents were also sent questionnaires enquiring about the medical history of the child and the presence of pollutants and irritants in the home. Regression analyses of daily peak flows among all children showed that lung function improved significantly by 5.87 units (std err $=0.71, p<0.001$ ) post-OTS after controlling for potential confounders. Similarly, the odds of wheeze decreased post-OTS (OR=0.84, 95\% $\mathrm{Cl} 0.77$ to 0.92$)$.

While it is not clear at this stage that these improvements can be attributed solely to the transport strategy, they suggest that traffic management can result in valuable improvements in public health.

\section{P91 HARIG: ASTHMA CARE IN THE HUNTINGDON PCT 2002}

D. Lightfood, J. McAllister, for the HARIG Group. Hinchingbrooke NHS Trust, Huntingdon; Huntingdon PCT, UK

Introduction: Asthma has considerable impact in terms of morbidity and health care costs. The $A C E^{(1)}$ survey suggests that over $30 \%$ of patients who report feeling well to their health professional suffer daily asthma symptoms. The HARIG study set out to raise asthma awareness within the Huntingdon PCT and use the results to develop a strategy for asthma care.

Method: A shortened version of the "Impact of asthma" questionnaire was used for the study. This was freely distributed in all GP surgeries and given to patients who attended to request repeat scripts and for review. A freepost address was supplied for return of questionnaires.

Results: PCT population was estimated at 147000 patients. 493 responses received, with asthma prevalence estimated at $5 \%$, returns approximately $6.7 \%$ of asthma population. Female:male split was $60 \%: 40 \%$. Average duration of asthma was $>5$ years, with a predominance of sufferers in the 18-65 year age group. Thirty seven per cent of patients reported daily asthma symptoms, with this figure higher than $50 \%$ in some GP practices. More than $30 \%$ of patients experienced significant night time waking. There was considerable variance in use of reliever medication with $32 \%$ of patients reporting use at least once per day but this figure was as high as $67 \%$ in one practice. $45 \%$ of respondents reported that asthma has at least a moderate effect on their lives, but this did not always correlate with the reporting of other symptoms.

Conclusion and discussion: Amongst the HARIG questionnaire respondents there is considerable unmet need for asthma symptom control. The results broadly mirror those of the ACE survey with around one third of respondents suffering continued symptoms. There may however have been some selection bias in those who responded to the survey and the results may not be a representative sample of the whole PCT population. Within the Huntingdon PCT there may be further opportunity for standardisation of care and sharing of good practice between GP surgeries.

This project has been supported with a project grant from GlaxoSmithKline Pharmaceuticals PLC.

1. Jones KG et al. Int J Clin Pract 2002;56:89-93.

\section{P92 IMPULSE OSCILLOMETRY IN SUBJECTS WITH SEVERE ASTHMA}

W. Anees, S. Manney, J. Mukhopadhyay, D. Petkova, J.G. Ayres. Severe and Brittle Asthma Unit, Birmingham Heartlands Hospital, Birmingham B9 5SS, UK

Introduction: Many subjects with severe asthma describe a worsening of symptoms on lying flat. This is felt to be due to an increase in vagal tone.

Aims: (1) To describe airway resistance and capacitative reactance measurements as assessed by impulse oscillometry (IOS) in subjects with severe asthma and to relate them to spirometric parameters. (2) To see whether IOS parameters are affected by posture. (3) To determine whether IOS could identify subjects who clinically had vocal cord dysfunction. 
Methods: Forty one subjects with severe asthma attending a tertiary referral centre underwent spirometry and IOS whilst sitting using an MS-IOS digital instrument (Jaeger AG). IOS was repeated on lying flat. Subjects were clinically assessed (by JGA) as to whether they had a significant component of vocal cord dysfunction.

Results: Eighty per cent were female, mean age 43.6 years with an $\mathrm{FEV}_{1} 69.5 \%$ predicted. Mean total airway resistance (R5) was increased at $203 \%$ predicted with $73 \%$ of subjects having abnormally high values ( $>150 \%$ predicted). Proximal airway resistance (R20) was a mean of $141 \%$ predicted being $>150 \%$ predicted in $32 \%$. An increase in R20 occurred on lying down ( $p=0.03)$ with a fall in distal capacitative reactance (X5) ( $p=0.05)$ for the group as a whole. Although total airway resistance (R5) did not increase significantly $(p=0.18)$, increases in R5 on lying down did correlate with body mass index ( $r=0.47, p=0.003)$. R5 was inversely correlated with $F E V_{1}$ and peak expiratory flow $(r=-0.42)$ but R20 did not. X5 also correlated with $\mathrm{FEV}_{1}(\mathrm{r}=0.52)$. IOS parameters or change in IOS parameters could not identify subjects who clinically had vocal cord dysfunction.

Conclusion: Increased airway resistance using IOS was demonstrated in some patients with severe asthma and increased significantly on lying down, possibly due to an increase in vagal tone. It was not helpful for identifying subjects thought to have vocal cord dysfunction.

\section{Pulmonary vascular biology/pulmonary hypertension}

\section{P93 THE EFFECTS OF ACUTE HYPOXIA ON PROLIFERATION AND P38 MAP KINASE ACTIVITY IN HUMAN FIBROBLASTS FROM THE PULMONARY AND SYSTEMIC CIRCULATIONS}

H. Mortimer, A. Peacock, P. Scott', D. Welsh. Scottish Pulmonary Vascular Unit, Western Infirmary; 'Institute Biomedical and Life Sciences, University of Glasgow, UK

Introduction: We have previously shown that proliferation and p38 MAP kinase phosphorylation in pulmonary artery fibroblasts is increased by hypoxia in animal models (Welsh, et al. Am J Resp Crit Care Med 1998 and 2001). We now wish to know whether this is true in man. In this study, we have examined the effect of acute hypoxia on human pulmonary and mammary artery (systemic) fibroblast proliferation and p38 MAP kinase activity.

Methods: Fibroblasts were harvested from human pulmonary artery (HPAF) and mammary artery (HMF) obtained from cardiothoracic surgery and utilised between passages $3-10$. Cells were quiesced for 24 hours then stimulated by acute hypoxia for 24 hours $\left(\mathrm{PO}_{2}=20 \mathrm{mmHg}\right)$ with or without $0,0.1,1,3,5$, and $10 \%$ serum. Fibroblast proliferation was measured by $\left[^{3} \mathrm{H}\right]$ thymidine uptake and p38 MAP kinase activity was measured by Western Blotting analysis.

Results: $\left[{ }^{3} \mathrm{H}\right]$ Thymidine incorporation was increased by acute hypoxia $(p<0.01)$ in the HPAF cells, but not in the HMF cells. Acute hypoxia also gave rise to 38 MAP kinase activation in the HPAF cells and to a lesser extent in the HMF cells (results not shown).

Conclusion: Acute hypoxia stimulated the proliferation of HPAF cells but not HMF cells. The increase in p38 MAP kinase activity to hypoxia may suggest a role in the regulation of cell cycle associated events. These results are consistent with our animal findings showing that these effects transcend species and may be important in man.

Chest, Heart and Stroke (Scotland) and British Heart Foundation.

A

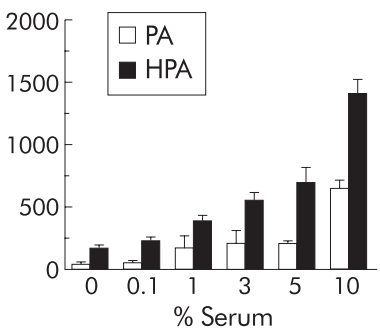

Abstract P93 (A) HPAF. (B) HMF.
P94 ERYTHROCYTE SUPEROXIDE DISMUTASE ACTIVITY IN INFANTS WITH PERSISTENT PULMONARY HYPERTENSION AND CONGENITAL DIAPHRAGMATIC HERNIA

G.M. Walker, K.F. Kasem, C.F. Davis, M.R. Maclean. University of Glasgow, Glasgow, UK

Introduction: The antioxidant enzyme system is the primary intracellular defence system of the lung against oxygen toxicity. Endogenous SOD is the major mammalian antioxidant enzyme and catalyses the dismutation of superoxide anion to hydrogen peroxide. Reduced SOD activity has been demonstrated in animal models of Congenital Diaphragmatic Hernia $(\mathrm{CDH})^{\prime}$ and in post mortem specimens from infants with persistent pulmonary hypertension of the newborn (PPHN). ${ }^{2}$ Erythrocyte SOD activity from live infants with these disorders has not been reported.

Methods: Blood was sampled from seven infants with PPHN and five with $\mathrm{CDH}$. All were near term and mechanically ventilated. Following centrifugation and plasma removal, erythrocytes were stored at $-20^{\circ} \mathrm{C}$ until analysis. SOD activity was calculated spectrophotometrically using the auto-oxidation of pyrogallol. ${ }^{3}$ Haemoglobin concentration was calculated using a standard Drabkin's reagent. Differences in median values were calculated using the Mann-Whitney test.

Results: Median erythrocyte SOD activity of infants with PPHN was $1374.15 \mathrm{\mu} / \mathrm{g} \mathrm{Hb}$ (range 1114 to 1791). Erythrocyte SOD activity of infants with $\mathrm{CDH}$ was significantly lower (median $=1213.5$, range 1016 to $1490.5, p=0.01$ ).

Conclusion: Infants with $\mathrm{CDH}$ have lower erythrocyte SOD activity compared to similarly ventilated patients. This gives further evidence to explain the clinical fragility and poor response to inhaled nitric oxide seen in $\mathrm{CDH}$.

1. Kapur P, et al. J Pediatr Surg 1999;34:354-6.

2. Asikainen TM, et al. Pediatr Pulmonol $2001 ; 32: 193-200$.

3. Marklund S, et al. Eur J Biochem 1974;47:411-15.

\section{P95 SUPEROXIDE AND SUPEROXIDE DISMUTASE IN PULMONARY HYPOXIC VASOCONSTRICTION}

P.H. Milliken, R.M. Wadsworth. University of Strathclyde, UK

Superoxide is known to cause vascular damage and can consume protective NO that maintains a low pulmonary pressure. There is evidence suggesting that hypoxia upregulates $\mathrm{NADH} / \mathrm{NADPH}$ oxidase and xanthine oxidase in the smooth muscle and endothelial cells of the pulmonary artery, increasing superoxide production. Superoxide dismutase (SOD) is widely distributed in the cells of the vasculature, destroying superoxide, however, the role of SOD in hypoxic vasoconstriction is unknown. ' Therefore, the effect of endogenous SOD inhibition and superoxide generation on hypoxic pulmonary vasoconstriction was investigated.

Male Sprague-Dawley rats were anaesthetised and the trachea and pulmonary artery cannulated. The lungs were ventilated with $20 \% \mathrm{O}_{2}$ (normoxia), and perfused with $30 \mathrm{ml}$ of modified Krebs solution. U46619 give preconstriction before a 10min hypoxic challenge $\left.15 \% \mathrm{CO}_{2}, 95 \% \mathrm{~N}_{2}\right)$ The lungs were then returned to normoxia for 10 mins before changing the perfusate to one containing either $1 \mathrm{mM}$ DETCA (high affinity $\mathrm{Cu}^{2+}$ chelator that inhibits endogenous SOD) or $10 \mu$ M LY83583 (superoxide generator). The lungs were allowed to equilibrate for 30 mins, before repeating the hypoxic and normoxic challenges. The NO donor SNAP $\left(1.7 \times 10^{-8}-1.7 \times 10^{.5}\right.$ moles) was used after hypoxia in the LY83583 experiments.

Hypoxia induced a sustained monophasic increase in pulmonary pressure, that was reproducible $(1.8(0.2) \mathrm{mmHg} 1$ st challenge and 1.7 (0.3) $\mathrm{mmHg} 2$ nd challenge, $n=5)$. LY83583 augmented the hypoxic vasoconstriction (3.5 (1.0) $\mathrm{mmHg}, \mathrm{n}=7$ ), and attenuated the SNAP induced vasodilation. These results suggest that superoxide can support or enhance hypoxic vasoconstriction, perhaps by consuming endogenous NO. However, there was a significant reduction of hypoxic vasoconstriction in the presence of DETCA $(1.0(0.3) \mathrm{mmHg}$, $n=7, p<0.05)$. Overall, these results suggest that hypoxic vasoconstriction can be modified by endogenous superoxide, however this interaction may be dependent upon the site of superoxide generation and the concentration of superoxide, as there is likely to be a greater amount of superoxide present in the experiments involving LY83583.

$\mathrm{PH}$ Milliken is funded by the British Heart Foundation (FS/ 2001056)

1. Demiryürek AT, et al. Pharmacol Ther 1999;84:355-65. 


\section{P96 MICE OVER EXPRESSING THE 5-HT TRANSPORTER: A NEW MODEL FOR PULMONARY HYPERTENSION?}

I. Morecroft, L. Loughlin, J. MacKenzie, A. Harmar', M.R. Maclean. Institute of Biomedical and Life Sciences, University of Glasgow, Glasgow, G12 8QQ; 'Dept Neuroscience, Edinburgh University, Edinburgh, EH9 9JZ, UK

The 5-hydroxytryptamine transporter (5HTT) may play a key role in pulmonary vascular remodelling in primary pulmonary arterial hypertension (PAH) and secondary hypoxia-related PAH. Attenuated hypoxic pulmonary hypertension has been reported in mice lacking the $5 \mathrm{HTT}$ transporter gene and there is $5 \mathrm{HTT}$ over-expression in patients with PAH (Eddahibi et al. J Clin Invest 2000;105:1555-62; Eddahibi $S$ et al. J Clin Invest 200 1; 108:1 141-50). Here we examine the development of $\mathrm{PAH}$ in mice over expressing the $5 \mathrm{HTT}$ gene $(5 \mathrm{HTT}+$ mice) and C57BL/ $6 \times \mathrm{CBA}$ wild type mice (WT) mice (female, 1 year old) exposed to two wks chronic hypoxia. Right ventricular (RV):total ventricular (TV) ratio was used as an index of pulmonary hypertension. In WT mice, RV/TV ratio was not altered after two wks hypoxia (0.205 (0.005) cf. $0.238(0.012), n=6-7)$. In 5HTT+ mice, however, RV/TV ratio was increased $(\mathrm{p}<0.001, \mathrm{n}=7)$ from 0.194 $(0.009)$ to $0.297(0.018)$. The number of remodelled small $(<80 \mu \mathrm{m}$ i.d.) vessels (assessed histologically) increased ( $p<0.001, n=6$ ) from $2.61(0.7 \%)$, (control hypoxic) to $7.8(0.5 \%)(5 \mathrm{HTT}+$ hypoxic). ET-1, $\mathrm{NA}$, and $5 \mathrm{HT}$ induced contraction was measured using wire myography in small pulmonary arteries ( $200 \mu \mathrm{m}$ i.d.) and were, in WT mice vessels $\left(p E C_{50} s: 9.8(0.3)>9.0(0.2)>6.9(0.2)\right.$ respectively). NA constriction was markedly reduced in the $5 \mathrm{HTT}+$ mice and the potencies of ET-1 and 5HT were reduced (pEC ${ }_{50} s: 8.4(0.3)$ and 5.6 (0.3) respectively). In the $5 \mathrm{HTT}+$ mice exposed to hypoxia however, there was a marked restoration of the contractile response to $\mathrm{NA}\left(\mathrm{pEC} \mathrm{C}_{50}\right.$ : $8.6(0.1))$ and an increase in the response to ET-1 and $5 \mathrm{HT}$ ( $p \mathrm{EC}_{50} \mathrm{~s}$ : $8.6(0.3)$ and $6.9(0.4)$ respectively). The maximum response to ET-1 was increased by $\sim 40 \%$. In WT mice lung membranes, the $5 \mathrm{HTT}$ ligand ${ }^{3}[\mathrm{H}]-\beta-\mathrm{ClT}$ bound with a $B_{\text {max }}$ of $1.068(0.022) \mathrm{fmolmg}^{-1}$ and $a \mathrm{~K}_{\mathrm{D}}$ of $0.869(0.054) \mathrm{nM}(n=3)$. Non-specific binding was assessed using cold fluoxetine. Binding was markedly increased in the $5 \mathrm{HTT}+$ mice, with a $B_{\text {ax }}$ of $13.32(0.25) \mathrm{fmolmg}^{-1}$ and a $\mathrm{K}_{\mathrm{D}}$ value of $3.745(0.033)$ $\mathrm{nM}(\mathrm{n}=3)$. The results indicate that PAH is accelerated in $5 \mathrm{HTT}+$ mice and this is associated with remodelling, increased lung 5HTT and increased contractile responses to $5 \mathrm{HT}, \mathrm{ET}-1$ and restoration of NA-induced contraction. $5 \mathrm{HTT}+$ mice may provide a novel model of genetically susceptible PAH.

\section{P97 HUMAN UROTENSIN-II ACTS AS A PULMONARY VASOCONSTRICTOR IN AN IN VIVO RABBIT MODEL OF PULMONARY HYPERTENSION SECONDARY TO LEFT VENTRICULAR DYSFUNCTION}

G.A. Deuchar', M.R. Maclean², M.N. Hicks'. 'Department of Medical Cardiology, Glasgow Royal Infirmary; ${ }^{2}$ Institute of Biomedical and Life Sciences, University of Glasgow, Glasgow G12 8QQ, UK

The recently cloned peptide, human Urotensin-II (hU-II) has been shown to be the most potent vasoconstrictor identified to date, being an order of magnitude more potent than endothelin-1. In the pulmonary circulation, hU-II has been shown to result in a greater vasoconstrictor effect in isolated main pulmonary arteries from rats with pulmonary hypertension (PHT) secondary to chronic hypoxia. However, in isolated human pulmonary arteries, a vasoconstrictor response to hU-Il was only uncovered following nitric oxide synthase (NOS) inhibition (Maclean, et al. Br J Pharmacol 2000;130:201-4). Here we investigated the pulmonary and systemic effects of hU-II alone and in the presence of the NOS inhibitor L-NAME in a previously described in vivo rabbit model of PHT secondary to LVD (Deuchar, et al. Cardiovasc Res 1998;38:500-7). Briefly, eight weeks following coronary artery ligation or sham operation, ejection fraction (EF) was assessed using echocardiography and experiments were carried out in closed chest anaesthetised rabbits. Pulmonary arterial pressure (PAP) and systemic aortic pressure (AOP) were measured directly before and after cumulative administration of hU-II (0.001-5.0 nmoles. $\left.\mathrm{kg}^{-1}\right)$ either alone or following the infusion of L-NAME (30 umoles. min $\left.^{-1}\right)$. Coronary ligated rabbits had reduced EF (45.2 (1.0\%) $v 73.0(0.9 \%), p<0.001)$, elevated basal PAP $(16.8(0.4) \mathrm{mmHg} v$ $13.6(0.2) \mathrm{mmHg}, p<0.001)$ and evidence of right ventricular hypertrophy as measured by right ventricular/final body weight ratio $(0.69$ $(0.03) \mathrm{g} / \mathrm{kg} \vee 0.48(0.01) \mathrm{g} / \mathrm{kg}$ final body weight, $p<0.001)$. A small significant pulmonary pressor effect was demonstrated to hU-Il alone, with the maximum response being greater in rabbits with PHT $(2.6$
(0.5) $\mathrm{mmHg}, \mathrm{n}=8, v 1.0(0.3) \mathrm{mmHg}$ in controls, $n=8, p<0.05)$. Following NOS inhibition, there was a trend towards the maximum pulmonary pressor effect being increased in both groups (8.5 (2.6) $\mathrm{mmHg}, \mathrm{n}=12$, and $4.8(1.5) \mathrm{mmHg}, \mathrm{n}=13$, in rabbits with PHT and controls respectively). In contrast, it was shown that hU-II had no effect on systemic AOP in any of the experimental groups. In conclusion, hU-II appears to act as a selective pulmonary vasoconstrictor with no effect on AOP.

\section{P98 THE PROSTACYCLIN ANALOGUE CICAPROST, INDUCES HETEROLOGOUS DESENSITISATION IN PULMONARY ARTERY SMOOTH MUSCLE CELLS VIA PROTEIN KINASE A DEPENDENT INHIBITION OF ADENYLYL CYCLASE}

A. Sobolewski, K.B. Jourdan, P.D. Upton, N.W. Morrell. Department of Respiratory Medicine, University of Cambridge, Addenbrooke's and Papworth Hospitals, Cambridge, CB2 2QQ, UK

Prostacyclin is the major arachidonic metabolite produced in vascular cells and is a powerful vasodilator of the pulmonary vasculature. Long term infusion of prostacyclin analogues is an effective treatment for primary and secondary pulmonary hypertension, but due to desensitisation of the agonist response, increased doses are required to maintain efficacy. The aim of this study was to investigate the mechanisms of prostacyclin desesnsitisation using the prostacyclin analogue cicaprost in rat pulmonary artery smooth muscle cells (PASMC) PASMC were isolated from precapillary pulmonary arteries of WKY. For timecourse studies confluent, serum-starved PASMC, were pre-incubated with cicaprost $(63 \mathrm{nM}$, for $15 \mathrm{~min}$ to $12 \mathrm{~h})$, washed and stimulated with cicaprost $(63 \mathrm{nM}, 15 \mathrm{~min})$. In further studies other conventional Gso-adenylyl cyclase coupled agonists (ISO, $1 \mu M$ ), adrenomedullin (ADM, 100nM), bradykinin (BRAD, 1 $\mu M)$ ' forskolin

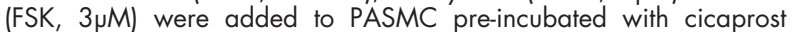
$(63 \mathrm{nM}, 6 \mathrm{~h})$. For resensitisation experiments cicaprost pre-incubated PASMC $(63 \mathrm{nM}, 3 \mathrm{~h})$ were placed in serum-free media for 3 to $24 \mathrm{~h}$ prior to cicaprost addition ( $63 \mathrm{nM}, 15 \mathrm{~min})$. For inhibitor studies a one hour pre-incubation with the PKA inhibitor, H89 $(1 \mu M)$ or the PKC inhibitor, bisindolylmaleimide (BIS, $100 \mathrm{nM})$ was introduced prior to cicaprost pre-incubation $(6 \mathrm{~h}, 63 \mathrm{nM})$. Experiments were terminated with $0.1 \mathrm{M} \mathrm{HCl}$ and assayed for cAMP activity using an ELISA kit. Desensitisation of the CAMP response occurred after 6 hours pre-incubation with cicaprost $(n=3, p<0.05)$. Desensitisation also occurred with ISO, BRAD, ADM in addition to FSK $(n=3, p<0.05)$. The desensitisation effect was reversible, with cAMP returning to control levels 12 hours after removal of the agonist $(n=3, p<0.05)$. PKA inhibition abrogated cicaprost desensitisation $(80(5 \%))$, whereas the PKC inhibition reversed the effect to a lesser extent (59 (3\%)). In conclusion, chronic treatment of PASMC with cicaprost, induced heterologous, reversible desensitisation by inhibition of adenylyl cyclase activity. Our data suggest that the desensitisation by cicaprost is mediated predominantly by PKA, and to a lesser extent, PKC inhibitable isoforms of adenylyl cyclase.

\section{P99 BONE MORPHOGENETIC PROTEIN-4 INHIBITS HUMAN LUNG FIBROBLAST PROLIFERATION VIA EFFECTS ON} CELL CYCLE REGULATORS

T.K. Jeffery, P.D. Upton, R.C. Trembath', N.W. Morrell. Respiratory Medicine Unit, Department of Medicine, University of Cambridge, School of Clinical Medicine, Addenbrooke's Hospital, Cambridge, UK; 'Division of Medical Genetics, University of Leicester, Leicester, UK

Introduction: Mutations in the bone morphogenetic protein type II receptor (BMPR2) have been identified in patients with primary pulmonary hypertension. Given that BMPR2 mutations may inhibit BMP-effects on cell proliferation we examined the effect of BMP-4 on the cell cycle and signal transduction via Smad-1 and p38 MAPK pathways in human lung fibroblasts. Since this is the first time that pulmonary fibroblasts have been used to examine BMP-4 responses we also characterised BMPR2 receptor expression, ligand binding, and the effect of BMP-4 on fibroblast growth.

Methods: Human fetal lung fibroblasts (HFL-1) were used. BMPR2 expression was determined using RT-PCR. Ligand binding studies were carried out by constructing competition curves for ${ }^{125}$-BMP4 against BMPs 2, 4, and 7, and TGF $\beta 1$. To determine the effect of BMP-4 on the cell cycle, propidium iodide stained cells were analysed utilising a fluoresence activated cell sorter (FACS). Western analysis using antibodies directed towards phospho-p38 ${ }^{\text {MAPK }}$, phospho-Smad-1 and the cell cycle proteins, p21, p27, cyclin D, and cyclin dependent kinase-2 (cdk2) was performed. 
Results: BMPR2 mRNA was expressed in HFLs and binding experiments demonstrated that BMP-2 and BMP-4 competed equipotently for ${ }^{125}$-BMP-4 binding, BMP-7 was weakly competitive and TGF $\beta$ did not compete. BMP-4 dose-dependently $(1-100 \mathrm{ng} / \mathrm{ml})$ inhibited ${ }^{3} \mathrm{H}-$ thymidine incorporation as well as proliferation of HFLs. FACS analysis demonstrated that BMP-4 $(50 \mathrm{ng} / \mathrm{ml})$ inhibited cells from exiting the G0/G1 phase of the cell cycle. BMP-4 up regulated the expression of inhibitors of the G0/G1 phase of the cell cycle, p21, and p27, with a corresponding down regulation in expression of the positive regulators, cyclin D and cdk2. Phospho-Smad-1 and phospho-p38 ${ }^{\text {MAPK }}$ were rapidly induced in response to BMP-4.

Conclusion: BMP-4 leads to cell cycle arrest in HFL-1 cells via induction of $\mathrm{p} 21 / \mathrm{p} 27$ and inhibition of cyclin D/cdk2. The relative contribution of $\mathrm{p} 38^{\mathrm{MAPR}}$ and Smad-1 on BMP-4 induced cell cycle arrest remains to be determined.

Funded by: British Heart Foundation and NHMRC of Australia (TK Jeffery).

\section{P100 THE RESPONSE OF FIBROTIC AND NON-FIBROTIC PATIENTS WITH SCLERODERMA ASSOCIATED PULMONARY ARTEIAL HYPERTENSION (SSCPAH) TO CONTINUOUS AMBULATORY ILOPROST THERAPY: IS A SIMILAR THERAPEUTIC APPROACH JUSTIFIED?}

D. Mukerjee, B. Coleiro, C. Elliott, C. Knight, C.M. Black, J.G. Coghlan. Departments of Rheumatology and Cardiology, Royal Free Hospital, Pond Street, London NW3 2QG, UK

Background: It is now accepted that patients with non-fibrotic $\mathrm{SScPAH}$ respond to epoprostenol therapy in a similar fashion to Primary Pulmonary Hypertension (PPH). We have observed that PHT underlying $\mathrm{SSc}$-associated fibrosis is not predominantly hypoxiadriven and postulated that the mechanisms underlying fibrosis/nonfibrosis-associated PAH in SSc may be similar and thus responsive to the same therapeutic strategy.

Methods: Forty seven patients were initiated on continuous ambulatory iloprost therapy between 1996-2001 as they met the funding criteria for this therapy. Functional impairment was quantified using the Six Minute Walk Test (SMWT).

Findings: Forty seven $S S c$ patients $(M: F=7: 40)$ with a $m R A P=10$ $\mathrm{mmHg}, \mathrm{mPAP}=42 \mathrm{mmHg}$, mean $\mathrm{SVO} 2=59 \%$, and mean $\mathrm{Cl}=1.8$ were started on ambulatory iloprost. There was no significant difference in haemodynamic parameters between fibrotics $(n=20) /$ non-fibrotics $(n=27)$. Initial mean $S M W T=175 m$ fibrotics $/ 215 m$ non-fibrotics, a mean improvement of $29 \%$ in the fibrotics $(226 \mathrm{~m})$ and $36 \%$ in non-fibrotics $(291 \mathrm{~m})$ at 12 weeks. This change was clinically significant in both groups: gain of $52 \mathrm{~m}$ in fibrotics/ $78 \mathrm{~m}$ non-fibrotics. At 24 weeks this benefit was maintained $(272 \mathrm{~m}$ in fibrotics $/ 321 \mathrm{~m}$ in non-fibrotics) with a mean gain of 28 metres in both groups. Six month survival was $81 \%$, with $23(49 \%)$ patients surviving $>1$ year of therapy (14 fibrotics/9 non-fibrotics).

Conclusion: In a SSc patient cohort similar in structure in terms of initial hemodynamic findings, treated with ambulatory iloprost, the response to therapy in terms of functional benefit is similar in fibrotic and non-fibrotic patients. This justifies inclusion of SSc patients with pulmonary fibrosis associated PHT in trials of newer therapies.

\section{P101 ELEVATED PULMONARY: AORTIC RATIO IN PATIENTS WITH PULMONARY ARTERIAL HYPERTENSION ASSOCIATED WITH SYSTEMIC SCLEROSIS}

F. Guarasci, D. Manuel, M. Akil, C. Davies, D. Fishwick, E.J.R. Van Beek, D.G. Kiely. Pulmonary Vascular Disease Unit, Dept of Radiology and Dept of Rheumatology, Royal Hallamshire Hospital, Glossop Road, Sheffield, UK

Pulmonary arterial hypertension (PAH) occurs in approximately $15 \%$ of patients with systemic sclerosis (SS) and is associated with a poor prognosis. A number of screening regimes using non-invasive techniques are currently used to identify patients for cardiac catheterisation. We have examined whether the pulmonary:aortic $(\mathrm{P}: \mathrm{A})$ ratio measured using $\mathrm{CT}$ may be a useful non-invasive predictor of PAH in these patients.

Twenty one patients (three male), mean age 60 yrs (range 45-70 yrs) with SS and suspected pulmonary hypertension were studied. Patients underwent HRCT and CTPA in addition to right heart catheterisation. CT scans were interpreted by investigators blinded to the results of the other investigations. The main pulmonary artery and aortic diameter were measured transversely at the same level and the ratio calculated.
Of the 21 patients studied, 19 had PAH at rest (MPAP at least $25 \mathrm{mmHg}$ ). There was a significant correlation between MPAP measured at $\mathrm{RHC}$ and $\mathrm{P}: \mathrm{A}$ ratio, $r=0.50, p=0.02$. All 16 patients with an elevated P:A ratio $>1$ had PAH. Patients with a P:A ratio $>1$ compared to patients with a P:A ratio $<1$ had a significantly greater MPAP: 43 (13) v $26(14) \mathrm{mmHg}(95 \% \mathrm{Cl} 2$ to $31 \mathrm{mmHg}, p=0.02)$. Using a P:A ratio of $>1$ to identify patients with $\mathrm{PAH}$ at cardiac catheterisation had a PPV of 1.0 and a NPV of 0.4

In conclusion, an elevated pulmonary to aortic ratio measured using $\mathrm{CT}$ scanning techniques predicts the presence of $\mathrm{PAH}$ in patients with systemic sclerosis. In these patients undergoing CT scanning of the thorax an elevated ratio should alert the physician to the possibility of pulmonary hypertension.

\section{P102 THE HAEMODYNAMIC EFFECTS OF "PULSED" INHALED NITRIC OXIDE IN PATIENTS WITH PULMONARY HYPERTENSION}

T. Siddons, T.W. Higenbottam, F. Guarasci, I. Armstrong, K. McCormack D.G. Kiely. Pulmonary Vascular Disease Unit, Royal Hallamshire Hospital, Glossop Road, Sheffield, UK

We examined the acute haemodynamic effects of pulsed inhaled nitric oxide (iNO) in patients with pulmonary hypertension. Patients with pulmonary arterial hypertension $(n=35)$, chronic thromboembolic pulmonary hypertension $(n=17)$, and hypoxic lung disease $(n=7)$ were challenged acutely with pulsed iNO at a dose of $1.6 \times 10^{-7}$ moles per breath during right heart catheterisation. The iNO was delivered in pulses via nasal cannulae using a breath activated device for five minutes. Pulsed iNO significantly reduced mean pulmonary artery pressure (MPAP): 45.0 (14) v 47.2 (13) mm Hg, pulmonary vascular resistance (PVR): 785 (432) v 878 (456) dynes.s.cm-5, and increased CO: $4.4(1.6) \vee 4.2(1.5) \mathrm{l} / \mathrm{min}(\mathrm{p}<0.001)$. There was no significant change in mean arterial pressure (MAP): 92 (16) v 91 (14) $\mathrm{mmHg}$ but a significant $(p<0.05)$ fall in systemic vascular resistance (SVR): 1696 (560) v 1784 (568) dynes.s.cm-5. We identified four response types (1) Non responder $=$ reduction in PVR $<20 \%$. (2) Pressure response type $=$ reduction in mPAP and PVR $>20 \%$. (3) Flow response type $=$ reduction in PVR $>20 \%$ with a reduction in $\mathrm{MPAP}<10 \%$ and a clinically significant increase in CO. (4) Resistance response type = reduction in PVR $>20 \%$ with reduction in mPAP between $10-20 \%$ and a non clinically significant increase in $\mathrm{CO}$ (see table).

Pulsed iNO appears to be an effective acute pulmonary vasodilator. Further work needs to be performed to examine whether these response types predict outcome from therapy or mortality.

\begin{tabular}{|c|c|c|c|c|c|c|c|}
\hline \multicolumn{8}{|c|}{ Abstract P102 } \\
\hline \multirow[b]{2}{*}{$n$} & \multirow[b]{2}{*}{ Response type } & \multicolumn{2}{|c|}{ MPAP $(\mathrm{mmHg})$} & \multicolumn{2}{|c|}{$\mathrm{CO}(\mathrm{L} / \mathrm{min})$} & \multicolumn{2}{|c|}{$\begin{array}{l}\text { PVR } \\
\text { (dynes.s.cm-5) }\end{array}$} \\
\hline & & Base & $\begin{array}{l}\text { During } \\
\text { iNO }\end{array}$ & Base & $\begin{array}{l}\text { During } \\
\text { iNO }\end{array}$ & Base & $\begin{array}{l}\text { During } \\
\text { iNO }\end{array}$ \\
\hline 36 & Non-responder & 48.4 & 48.5 & 4.3 & 4.4 & 11.3 & 11.1 \\
\hline 9 & Pressure & 43.3 & 33.7 & 4.3 & 4.6 & 8.8 & 6.3 \\
\hline 8 & Flow & 49.5 & 47.8 & 3.7 & 4.8 & 12.3 & 9.0 \\
\hline 6 & Resistance & 42.8 & 37.2 & 3.9 & 4.2 & 10.2 & 7.7 \\
\hline
\end{tabular}

\section{P103 COMPASSIONATE TREATMENT OF PULMONARY HYPERTENSION WITH LONG TERM INHALED NITRIC OXIDE AND ORAL SILDENAFIL (VIAGRA ${ }^{\circledR}$ )}

T. Siddons, T.W. Higenbottam, K. Amsha, I. Armstrong, K. McCormack, D.G. Kiely. Pulmonary Vascular Disease Unit, Royal Hallamshire Hospital, Glossop Road, Sheffield, UK

Despite recent encouraging reports of trials of analogues of prostacyclin and antagonists of the endothelin-1 receptors, many patients with pulmonary hypertension $(\mathrm{PH})$, still receive no treatment as a result of the expense and/or difficulty in administering these therapies. We examined the effects of long term pulsed inhaled nitric oxide (iNO) or oral sildenafil (Viagra ${ }^{\circledast}$ ) in eleven disabled patients with $\mathrm{PH}$ associated with other disease processes and in two patients with primary $\mathrm{PH}$, who did not receive funding for prostaglandin therapy. Patients were offered compassionate treatment with either pulsed iNO or sildenafil, one patient receiving first pulsed iNO then sildenafil after a wash out period. 
Sildenafil at doses of 25 to $50 \mathrm{mg}$ three times per day resulted in a significant improvement in shuttle walking test 274 (189) m v 201 (116) $\mathrm{m}$ at baseline $(\mathrm{p}=0.045)$. Two patients discontinued therapy due to deterioration, but six patients continue on sildenafil with a mean duration of therapy of 16 months (range 4-22). In contrast, all patients given pulsed iNO for 12 hours overnight deteriorated, with no patient continuing on therapy for longer than 11 months (mean six months, range $2-11$.

In conclusion, although long term nocturnal therapy with pulsed iNO appears to be an ineffective treatment for pulmonary hypertension, sildenafil appears to be an effective and well tolerated form of therapy in this heterogeneous patient population.

\section{P104 FLOW VELOCITY PROFILES AND HAEMODYNAMICS IN PULMONARY HYPERTENSION: A PILOT STUDY}

T. Saba, J. Foster', M. Cockburn', M. Cowan' 'A. Peacock. Scottish Pulmonary Vascular Unit; 'Department of Radiology, Western Infirmary, Glasgow, Scotland

Standard assessment of the pulmonary circulation including mean pulmonary artery pressure (MPAP), cardiac output (CO), and pulmonary vascular resistance (PVR) predict prognosis in pulmonary hypertension (PHT) but do not always predict the severity of exercise intolerance. We wondered whether flow velocity profiles, which are likely to reflect the haemodynamic response to exercise, would help to explain the differences in symptoms between patients with similar resting haemodynamics.

Method: We used cardiac triggered cine MRI to measure mean velocity (MV) and peak velocity (PV) in the right pulmonary artery in three subjects with PHT and one normal subject (see table 1) within two days of cardiac catheterisation and six minute walk testing $(6 \mathrm{mwt})$. Studies were performed at rest (R) and after three minutes of straight leg raising exercise (EX).

Results: See figures 1 and 2 .

Conclusions: Resting pulmonary haemodynamics may not predict exercise tolerance in PHT. Exercise MRI is feasible in this group of patients and flow velocity profiles obtained in this way may help to explain the differences in symptoms between patients with similar pulmonary haemodynamics.

\begin{tabular}{|lllllll|}
\hline \multicolumn{2}{l}{ Abstract P104 } \\
\hline \multicolumn{1}{l}{ Diagnosis } & Age & $\begin{array}{l}\text { MPAP } \\
\mathrm{mmHg}\end{array}$ & CO I/min & PVR & 6 mwt (m) \\
\hline 1 & PPH & 59 & 47 & 3.7 & 12.7 & 438 \\
2 & PPH & 66 & 40 & 2.6 & 15.4 & 210 \\
3 & PortoPHT & 31 & 41 & 6.3 & 6.5 & 260 \\
4 & Normal & 48 & 16 & 3.4 & 4.7 & 550 \\
\hline
\end{tabular}

\begin{tabular}{clllll}
\hline $\mathbf{2}$ & $\mathrm{R}$ & $\mathrm{EX}$ & $\mathbf{3}$ & $\mathrm{R}$ & $\mathrm{EX}$ \\
\hline $\mathrm{MVcm} / \mathrm{s}$ & 4.0 & 4.5 & 10.2 & 10.6 \\
\hline $\mathrm{PV} \mathrm{cm} / \mathrm{s}$ & 21.7 & 20.5 & 28.8 & 26.0 \\
\hline
\end{tabular} MV R PV

Abstract P104 Figure 1 Flow velocity profiles in subjects. Two and three showed flattening with exercise.

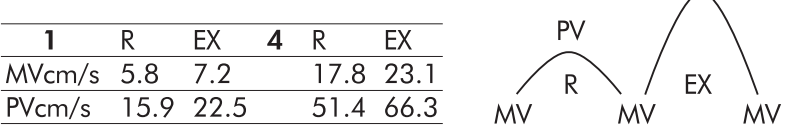

Abstract P104 Figure 2 Flow velocity profiles in subjects one and four, showing peaking with exercise.

\section{P105 HAEMODYNAMICS DURING EXERCISE ARE A BETTER MEASURE OF VASODILATOR RESPONSE IN HUMAN SUBJECTS WITH PULMONARY HYPERTENSION}

R. Syyed, A.J. Peacock. Scottish Pulmonary Vascular Unit, Western Infirmary, Glasgow, UK

Patients with pulmonary hypertension (PHT) are deemed "nonresponders" (NR) if they show no response to vasodilators at rest. We therefore decided to investigate the effects of vasodilators on pulmonary haemodynamics during exercise.

Methods: We investigated four patients, (two female, two male) with PHT to determine pressure and flow changes over a range of flows. Flow was changed by straight leg raising. A micromanometer tipped continuous pulmonary artery pressure (PAP) catheter was inserted. All four were non-responders to a vasodilator challenge (defined as a reduction of $>20 \%$ in pulmonary vascular resistance). Resting pressure was measured and then three mins of supine alternate straight leg raising was performed, while the subjects inhaled air or nitric oxide (NO, 40-80 ppm) and oxygen $\left(\mathrm{O}_{2}, 15 \mathrm{~L}\right.$ $\mathrm{min}$ ). Cardiac Output (CO) was measured by non-invasive impedence cardiography. Subject data was pooled using the method described by Poon (J Appl Physiol 1998;64:854-9). The best-fit line for Pressure Flow (P-Q) plots was determined by linear regression. An adjusted two paired student $t$ test was used to compare the line gradients.

Results: We found that although total pulmonary vascular resistance (as defined as mean PAP/ CO) showed no change at rest, the slope of the $\mathrm{P}-\mathrm{Q}$ plots decreased with vasodilators during exercise $(p<0.0005)$ (see figure).

Conclusion: In each of these four subjects, whilst there was no vasodilator response at rest, there was an improving relationship between pressure and flow during exercise whilst receiving the vasodilators $\mathrm{NO}$ and $\mathrm{O}_{2}$. In patients with $\mathrm{PHT}$, the assessment of vasodilator response may be better performed during exercise than at rest.

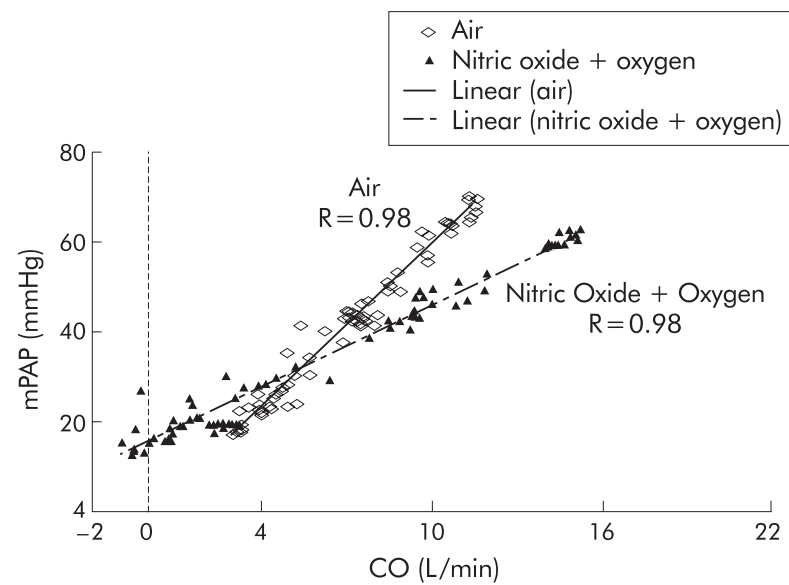

Abstract P105 Pressure flow changes during exercise.

\section{P106 FUNCTIONAL AND HAEMODYNAMIC RESPONSE AFTER PULMONARY THROMOBENDARTERECTOMY FOR THROMBOEMBOLIC PULMONARY HYPERTENSION}

F. Reichenberger, J. Parameshwar, D. Hodgkins, J. Dunning, J. Pepke-Zaba. Papworth Hospital, Papworth Everard, Cambridge, UK

Pulmonary Thrombo-Endarterectomy (PTE) is the treatment of choice in pulmonary hypertension due to proximal chronic thromboembolism. We evaluated the midterm functional and haemodynamic outcome until one year after PTE. The functional capacity was assessed using the six minute walking distance (6MWD). Haemodynamic parameters were obtained during right heart catheterisation with Swan Ganz catheter and cardiac output with thermodilution method. Until March 2002, 88 patients underwent PTE (44 male, 43 female, mean age 52 (18-81) years) at Papworth Hospital. Fifty eight patients completed a three months follow up, and 35 patients 12 months follow up. Two patients were followed up by other centres and two patients did not attend follow up visits. Preoperative functional and haemodynamic data show a severely reduced 6MWD and cardiac index with 


\begin{tabular}{lllll} 
Abstract P106 & & & & \\
\hline & $\begin{array}{l}\text { 6MWD } \\
\text { (metres) }\end{array}$ & $\begin{array}{l}\text { RAP } \\
\text { (mmHg) }\end{array}$ & $\begin{array}{l}\text { mPAP } \\
\text { (mmHg) }\end{array}$ & $\begin{array}{l}\text { Cardiac } \\
\text { Index } \\
(1 / m i n / q m)\end{array}$ \\
\hline preop (88) & $238 \pm 115$ & $10 \pm 6$ & $48 \pm 11$ & $1.7 \pm 0.5$ \\
3 mo postop (58) & $386 \pm 124^{*}$ & $3 \pm 4^{*}$ & $24 \pm 9^{*}$ & $2.5 \pm 0.7^{*}$ \\
1 year postop (35) & $382 \pm 123$ & $3 \pm 3$ & $22 \pm 10$ & $2.4 \pm 0.5$ \\
\hline$t$ test: $p<0.001$.
\end{tabular}

increased right atrial pressure (RAP) and mean pulmonary artery pressure (mPAP). Three months after surgery there was a significant increase in 6MWD and cardiac index, and a decrease in RAP and mPAP. These changes were statistically significant ( $t$ test $p<0.0001)$. The functional and haemodynamic improvement sustained also 12 months postoperatively (see table). None of the patients died between three months and 12 months follow up.

Conclusion: Patients have a significant and sustained functional and haemodynamic improvement after PTE with normalisation of haemodynamic parameters in the majority of patients. The main improvement is achieved within three months after surgery.

\section{Cystic fibrosis: Inflammatory consequences of chronic infection}

\section{P107 NEUTROPHIL APOPTOSIS AND BACTERIAL INFECTION IN CYSTIC FIBROSIS}

A.P. Watt', J. Courtney', J. Moore 2, J.S. Elborn', M. Ennis'. 'Respiratory Research Group, Centre for Infection, Inflammation and Repair, The Queen's University of Belfast; ${ }^{2}$ Department of Bacteriology, Belfast City Hospital, Belfast, UK.

In cystic fibrosis (CF), impaired mucociliary clearance leads to chronic endobronchial bacterial infection and inflammation, mediated by neutrophils. Pseudomonas aeruginosa infection is associated with an exaggerated inflammatory response and colonisation with Burkholderia cepacia is often accompanied by progressive pulmonary deterioration. Apoptosis of inflammatory cells is considered an essential requirement for the resolution of an inflammatory response. It was hypothesised that the number of neutrophils undergoing apoptosis would alter with the agent of infection in CF lungs. The aim of this study was to assess the relationship between levels of neutrophil apoptosis and sputum microbiology in matched clinically stable CF patients. In this preliminary study 29 patients were recruited: six (4M) with no Gram negative infection, 10 (4M) colonised with $P$ aeruginosa, $9(4 \mathrm{M})$ with $B$ cepacia infection and $4(3 \mathrm{M})$ with other Gram negative infections such as Stenotrophomonas maltophilia. Sputum was induced as previously described (Kelly, et al. 2002). Cells were recovered from sputum plugs. Apoptosis was investigated by staining sputum cells with propidium iodide (PI) and annexin V (AV), and subsequent flow cytometric analysis. Non-parametric statistic analyses were used throughout. The \% of necrotic granulocytes $\left(\mathrm{AV}-\mathrm{PI}^{+}\right)$was significantly higher in the $P$ aeruginosa group $(17.1$ $(2.5 \%), p=0.008)$ and the $B$ cepacia group (13.9 (1.3\%), $p=0.008$ ) compared to those with no Gram negative infection (7.5 (1.6\%)). B cepacia patients also had a significantly higher $\%$ of secondary necrotic cells $\left(\mathrm{AV}^{+} \mathrm{Pl} \mathrm{I}^{+}\right)$than those with no Gram negative infection (13.6 (2.3\%), $5.8(1.9 \%), p=0.02)$ whilst those with no Gram negative infection had a significantly higher $\%$ of cells which were neither apoptotic nor necrotic $\left(\mathrm{AV}^{-} \mathrm{PI}^{-}\right)$than the $B$ cepacia group 177.6 $(5.2 \%), 58.3(4.4 \%), p=0.02)$. These preliminary results indicate that neutrophil apoptosis is associated with the type of organism colonising the CF lung. The greater number of granulocytes in cell death pathways from patients infected with $P$ aeruginosa or $B$ cepacia suggests that this may be a mechanism of greater inflammation and subsequent lung injury in CF.

\section{P108 A COMPARISON OF INFLAMMATORY MARKER LEVELS IN CLINICALLY STABLE CYSTIC FIBROSIS PATIENTS WITH CHRONIC INFECTION BY UNIQUE AND EPIDEMIC STRAINS OF PSEUDOMONAS AERUGINOSA}

A.M. Jones', L. Martin², R. Bright-Thomas', M.E. Dodd', S. Elborn ${ }^{3}$, A.K. Webb'. 'Manchester Adult CF Centre; ${ }^{2}$ School of Pharmacy, Queen's University, Belfast; ${ }^{3}$ Northern Ireland Adult CF Unit, Belfast

Introduction: There are an increasing number of reports of Pseudomonas aeruginosa cross infection outbreaks at cystic fibrosis (CF) centres. The clinical significance of acquisition of an epidemic strain for patients who already harbour $P$ aeruginosa is unclear. If epidemic strains are more virulent they may be more likely to provoke an enhanced host inflammatory response. We compared levels of inflammatory markers in clinically stable CF patients who harbour epidemic and unique strains of $P$ aeruginosa.

Methods: Patients at the Manchester Adult CF Centre were invited to participate if they were clinically stable and had received a course of intravenous antibiotics 4-6 weeks earlier. Patients were grouped upon the basis of bacterial fingerprinting results into those who harbour epidemic and unique $P$ aeruginosa strains; patients co-infected with Burkholderia cepacia complex were excluded. The following were measured: total white cell (WCC) and neutrophil blood counts; sputum neutrophil elastase (NE), interleukin (IL)-8, tumour necrosis factor (TNF) $\alpha$; plasma IL-6, NE/ $\alpha_{1}$-antitrypsin complexes; serum C-reactive protein (CRP); and urine TNFrl. The groups were compared using $t$ tests and Mann Whitney $U$ tests.

Results: The two groups (unique $v$ epidemic) ( $n=20$ patients both groups) were well matched for mean age (28.5 $v 28.0$ years), percentage predicted (\%) FEV $(44.4 \vee 45.7)$, \%FVC (60.1 v 67.0) and BMI $(20.8 \vee 20.4)$. The two groups had similar mean (SD) WCC $(10.3(2.8) \vee 11.5(3.3))$ and neutrophil $(7.4(2.8) \vee 7.6(3.1))$ counts. There were no significant group differences in median (range) levels of sputum NE (22.0 (10.5 to 38.6$)$ v $17.3(7.1$ to 173.5$) \mathrm{\mu g} / \mathrm{ml})$, IL-8 (13449 (5521 to 56514) v 9989 (3782 to 155500$) \mathrm{pg} / \mathrm{ml}$ ), and TNF $\alpha(11$ (0 to 169) $\vee 9$ (0 to 278$) \mathrm{pg} / \mathrm{ml})$, plasma lL-6 (5.8 (1.3 to $25.7)$ v $6.4(1.2$ to 38.8$) \mathrm{pg} / \mathrm{ml})$ and $\mathrm{NE} / \alpha_{1}$-antitrypsin complexes (246 (109 to 2115$)$ v 229 (118 to 533) ng/ml), serum CRP (8 (1 to 29) v 8 (1 to 71$) \mathrm{mg} / \mathrm{L})$ and urine TNFr1 (1 167 (286 to 3531) v988 (286 to 5082$) \mathrm{pg} / \mathrm{ml}$ ).

Conclusions: There are no differences in levels of inflammatory markers between clinically stable adult CF patients who harbour epidemic and unique strains of $P$ aeruginosa.

\section{P109 A PSEUDOMONAS AERUGINOSA EXOTOXIN, PYOCYANIN, IMPAIRS PHAGOCYTOSIS AND CLEARANCE OF APOPTOTIC CELLS}

S.M. Bianchi, L.R. Usher, D.H. Dockrell, G.W. Taylor, M.K.B. Whyte. Respiratory Medicine Unit, University of Sheffield, Sheffield, UK

Pseudomonas aeruginosa infection in patients with cystic fibrosis and bronchiectasis is characterised by a profound neutrophilic inflammation, with neutrophil products (for example, elastases) implicated in tissue injury. We have shown that pyocyanin, a pseudomonas exotoxin, accelerates neutrophil apoptosis (programmed cell death) potentially promoting bacterial persistence (Usher, et al. J Immunol 2002;168:1861-8). We hypothesised that accelerated neutrophil death may result in further tissue injury if apoptotic cell clearance was impaired. We therefore studied the effects of pyocyanin on the ingestion of apoptotic neutrophils (APMN) by monocyte-derived macrophages (MDM). All data are expressed as controls versus 24 hour pyocyanin pretreatment. We have observed a time $(100(0 \%) \vee 22.7$ $(7.1 \%), p<0.001)$ and concentration dependent reduction in MDM ingestion of APMN in the presence of pyocyanin. We have shown that the reduced interaction is not due to loss of viability (23.2 (2.5) v 22.7 (1.2) cells $/ \times 400$ field, $p=0.440$ ) or induction of MDM apoptosis by physiological concentrations of pyocyanin $(0.6(0.15 \%) \vee 0.93$ $(0.41 \%), p=0.108)$. Similarly we have demonstrated no loss in MDM function as assessed by basal and lipopolysaccharide induced MDM cytokine production. The impairment of phagocytosis has been shown to be specific to APMN as MDM phagocytosis of opsonised latex bead is not impaired $(33.3(4.2 \%) \vee 29.7(2.3 \%), p=0.312)$. Also we have determined that the pyocyanin-induced defect is specific to the MDM-APMN killed by constitutive ageing or following exposure to pyocyanin are ingested similarly by healthy MDM. Using flow cytometry we have shown that pyocyanin is capable of inducing high levels of reactive oxygen species (ROS) within the MDM-however it 
does not appear that this mechanism is responsible for the defect as alternative sources of ROS (for example, hydrogen peroxide) do not result in similar impairment of phagocytosis, and antioxidants are unable to recover phagocytic capacity. We conclude that pyocyanin reduces MDM phagocytosis of APMN in vitro. This represents a novel mechanism by which microorganisms may contribute to immune evasion, bacterial persistence, ongoing inflammation, and lung injury.

\section{P110 BURKHOLDERIA CEPACIA COMPLEX: A MULTILOCUS SEQUENCE TYPING (MLST) SCHEME BASED APPROACH}

K.M. Thickett' 2, E. Mahenthiralingam ${ }^{3}$, D. Honeybourne' 2, C.G. Dowson². 'Adult Cystic Fibrosis Unit, Department of Respiratory Medicine, Heartlands Hospital, Bordesley Green East, Birmingham, B9 5SS, UK; ${ }^{2}$ Department of Biological Sciences, University of Warwick, Coventry, CV7 4AL, UK; ${ }^{3}$ Cardiff School of Biosciences, Main Building, Museum Avenue, PO Box 915, Cardiff University, Cardiff, Wales, UK

The accurate identification of Burkholderia cepacia complex in cystic fibrosis (CF) is challenging. A polyphasic taxonomic approach is often employed in the study of $B$ cepacia complex, including total protein electrophoresis, fatty acid analysis, biochemical tests, PCR amplification of rDNA, random PCR amplification (RAPD), pulse-field gel electrophoresis, and ribotype analysis. However, molecular diagnostic probes based upon the polymerase chain reaction (PCR) provide a rapid and frequently highly discriminatory means of identifying microbial infection. Recently recA sequence analysis has helped to unravel the phylogenetic relationships within the $B$ cepacia complex and has formed the basis for a rapid PCR based identification of B.cepacia complex genomovars.' Multi locus sequence typing (MLST) is a powerful, portable and rapid means of undertaking identification, typing and population genetic analysis and enables population based analyses not possible by pulse field gel electrophoresis (PFGE) or other macrorestriction profiling and allows the establishment of a world wide data base for the unambiguous identification of different strains. We have currently developed six loci for use in an MLST scheme for $B$ cepacia complex including $\operatorname{Rec} A$, recA, beta subunit of ATP synthase atpd, poly-hydroxyalkoanoate polymerase phaC, glutamate synthetase (NADPH) large chain precursor, gltB, DNA gyrase $\beta$ subunit, gyrB and GTP binding protein lepA. Southern blotting of whole chromosomes and restriction digests of total chromosomal DNA from $B$ cepacia complex, (B cepacia genomovars I and III, Burkholderia multivorans, Burkholderia stabilis, and Burkholderia vietnamiensis) has been used to confirm the presence of loci as single copies. We describe preliminary phylogenetic relationships from these loci using isolates widely distributed across a recA phylogenetic tree.

1. Mahenthiralingam E, et al. J Clin Micro 2000;38:3165-73.

\section{P111 STRAIN DEPENDENT SYSTEMIC INVASION AND INFLAMMATION IN A MURINE MODEL OF ACUTE PULMONARY BURKHOLDERIA CEPACIA INFECTION}

J.C. Davies' 2, R. Shaw', T. Lloyd', D.M. Geddes', E.W.F.W. Alton'. 'Dept of Gene Therapy, Imperial College; ${ }^{2}$ Dept of Paediatric Respiratory Medicine, Royal Brompton Hospital, London, UK

Burkholderia cepacia infection in CF may be asymptomatic, adversely affect lung function or invade systemically leading to the potentially fatal "cepacia syndrome". Certain genomovars appear more detrimental than others, possibly related to invasiveness and pro-inflammatory activity: cepacia syndrome has been reported with GII and GIII organisms, and GIII infected lung transplant recipients have increased mortality. To explore this further, we tested a variety of $B$ cepacia strains in a murine model of acute pulmonary infection. $\mathrm{C} 57 \mathrm{Bl} / 6$ mice (6/group) were infected by nasal sniffing with $10^{\circ} \mathrm{CF}$ organisms including: 18822 \& 13010 (Glla\&b), ET12 (GIII), and 18870 \& 14294 (GIVa\&b) (BCCM/LMG, Belgium). Forty eight hours later blood was obtained by abdominal venesection, the spleen was removed, and a BAL performed. Homogenised spleens were cultured in a semi-quantitative fashion on cepacia-specific medium. Growth was scored as 0 (negative), 1 (scanty colonies), 2 (multiple colonies), or 3 (confluent growth). Consistently high levels of systemic invasion were seen in animals infected with GIII and GII strains (scores of 3 in every animal). In contrast, the GIV strains produced less frequent and lower levels of invasion (mean (SEM) score GIVa 0.8 (0.4), GIVb 1.7 (0.2); $p<0.05$ compared with other infected groups). BAL neutrophil counts were higher in all infected groups than in PBS controls $(p<0.05$ or less), the highest levels being in groups GIII and Gllb. ET12 was significantly more pro-inflammatory than all the other strains, when the cytokines TNF $\alpha$, IL-6, and IL-1 $\beta$ were measured both in BAL and serum. Although the GIl strains were more invasive than the GIV, this was not reflected by differences in these ctyokines. This animal model confirms a differential host response to various $B$ cepacia organisms, which appears to be related both to genomovar and strain. Strains from GII and GIII, those associated with cepacia syndrome in patients, were highly invasive. Further, the GIII organism elicited the greatest inflammatory response, both locally and systemically, suggesting a mechanism for the reported poor outcome after lung transplantation. Further studies, both in animal models and in the clinical setting are warranted, to improve attempts to identify patients most at risk of disease.

\section{P1 12 VARIABILITY IN PRO-INFLAMMATORY ACTIVITY OF PURIFIED LIPOPOLYSACCHARIDE (LPS) FROM SELECTED BURKHOLDERIA CEPACIA GENOMOVARS; THE ROLE OF LPS STRUCTURAL DIFFERENCES AND CHEMOTYPE}

A. De Soyza' 2, R. Demarco De Hormaeche', P. Corris'. 'Lung Biology and Transplantation group, University of Newcastle upon Tyne; ${ }^{2}$ Freeman Hospital and Department of Microbiology and Immunology, University of Newcastle upon Tyne, UK

Gram negative bacterial infections are a major cause of morbidity in adult cystic fibrosis patient. As compared to those infected with Ps aeruginosa alone the outcomes of Burkholderia cepacia infected patients are poorer. There also appears to be variability in outcomes within the B cepacia infected population (Frangolais, et al. Am J Respir Crit Care Med 1999). The most striking disease pattern is Cepacia Syndrome a fatal pneumonitis-sepsis syndrome. There are many host and pathogen factors which may contribute to this variability. The designation of closely related but distinct species, Genomovars I to VII, within the $B$ cepacia complex may offer some insights into this variable pattern. Genomovars III, II, and V are the most prevalent in CF. We have shown poor outcomes in Genomovar III infected patients post-transplant (De Soyza, et al. Lancet 2001). As lipopolysaccharide (LPS) is a major mediator of sepsis we have investigated the pro-inflammatory potential of $B$ cepacia LPS.

Methods: We selected three strains of $B$ cepacia after pilot work suggested widely varying biological activity. These strains, GII (LMG 14273 and 13010) and GIII (LMG12614, an ET12 clone), were grown overnight and harvested. The bacteria were sonicated and treated with DNAse and proteinase K. LPS extraction using hot phenol was undertaken with final DNA/ protein contamination approx $5 \%$. The purified LPS (1-100ng) were added to differentiated U937 macrophages and the tissue culture supernatants were collected at 0 and $24 \mathrm{hrs}$. Time course experiments were also undertaken up to $48 \mathrm{hrs}$. The supernatants were tested for septic shock related cytokines IL-1, IL-6 and TNF using ELISA. We silver stained 16\% urea/SDS-PAGE gels to assess LPS structure.

Results: A dose-response was seen. The greatest difference was noted between the purified LPS at a dose of 100ng for TNF induction where LMG 12614 (ET12) was twice as effective at induction as compared to LMG 14273 (GII). LMG 13010 (GII) was as effective as the GIII, LMG12614. The gels confirmed that all were of rough LPS chemotype. The two high cytokine inducers LMG12614 and 13010 (GIII and GII) appeared to have the same LPS banding patterns; rough LPS with two distinct bands whilst LMG 14273 a distinct LPS pattern.

Conclusions: The observed differences between $B$ cepacia complex strains LPS may in part explain the variable clinical outcomes. We identified differences between genomovars demonstrating that within the two most common genomovars there is a wide spectrum of activity. One GII strain appears to have the same LPS as the ET-12 GIII strain which may explain why some GIl patients can develop Cepacia Syndrome. The chemotype of the LPS e.g. rough or smooth may be less important than the structural motifs within each LPS.

\section{P1 13 LIPOPOLYSACCHARIDE STRUCTURAL PROFILES ARE NOT ALTERED IN GENOMOVAR III BURKHOLDERIA CEPACIA QUORUM SENSING KNOCKOUTS}

A. De Soyza, R. Demarco De Hormaeche, P. Corris. Lung Biology and Transplantation group, University of Newcastle, Freeman Hospital and Department of Microbiology and Immunology, University of Newcastle upon Tyne, UK

Background: Attempts at creating vaccines using isolated bacterial antigens have been relatively unsuccessful for many important pathogens such as Pseudomonas. Gram negative bacteria form biofilms in 
cystic fibrosis lungs that appear to protect bacteria within the biofilm from antibiotics even at bactericidal levels. The maturation of biofilms is dependent on quorum sensing systems. Quorum sensing mutants produce greatly attenuated infections in animal models and may offer the possibility of an attenuated strain (perhaps with additional mutations in virulence genes) for use as a biological vaccine. Recent data has shown that quorum sensing also controls virulence related genes for proteases and iron binding siderophores. The effect of quorum sensing on a major bacterial pro-inflammatory moiety, lipopolysaccharide (LPS) is less clear. Work with Pseudomonas aeruginosa has demonstrated that migA a lipid A glycosyltransferase, has a quorum sensing box upstream. There is no data in the Cepacia field assessing the effect of quorum sensing knockouts on LPS structure. Two Burkholderia cepacia quorum sensing mutants, $\mathrm{HI} 11-\mathrm{l}$ and $\mathrm{HI} 11-\mathrm{R}$ have been created that are defective in the acylhomoserine lactone $(\mathrm{AHL})$ synthase cepl and the AHL receptor cepR respectively. The effect of these mutations are to impair biofilm formation. These strains therefore may be potentially considered for investigation as starting points for biological vaccines. The effect of these mutations on LPS is however unclear.

Methods: The wild type parent genomovar III, strain $\mathrm{HI} 11$ and the two mutants were kindly gifted (Dr B Huber, University Munich). These were grown in Luria Broth (LB) with appropriate selective antibiotics over 24 hours at $37^{\circ} \mathrm{C}$. The organism were then centrifuged and resuspended to a standard optical density at $600 \mathrm{~nm}$ of 0.2 . SDS-PAGE was conducted with subsequent silver staining to assess any changes in LPS profiles.

Results: Silver stained SDS-PAGE gels revealed the LPS of all three strains were of smooth chemotype with multiple bands suggesting variable $O$ side chain lengths. There were however no differences between the strains in terms of number or pattern of the bands seen on the gels suggesting the $O$ chains of the LPS were not affected by the quorum sensing mutations. There appeared to be no differences in the LPS core. As opposed to data from Pseudomonas spp quorum sensing B cepacia mutants with smooth LPS do appear to have a change in LPS structure under the above growth conditions.

Conclusions: If quorum sensing mutants are to be considered as starting points for biological vaccines it may be important to create quorum sensing mutants in a wild type genomovar III strain with a LPS that has a low inflammatory potential.

\section{Cystic fibrosis: Treatments and outcomes}

\section{P114 COMPARISON OF THE HALOLITE ${ }^{\circledR}$ ADAPTIVE AEROSOL DELIVERY (AAD ${ }^{\text {TM}}$ ) SYSTEM WITH A HIGH OUTPUT NEBULISER SYSTEM IN CYSTIC FIBROSIS PATIENTS}

R.J. Marsden', S.P. Conway ${ }^{2}$, M.E. Dodd ${ }^{3}$, F.P. Edenborough ${ }^{4}$, A.S. Rigby ${ }^{5}$, C.J. Taylor ${ }^{5}$, P.H. Weller'. 'Profile Therapeutics, UK; ${ }^{2}$ St. James' Hospital, Leeds; ${ }^{3}$ Wythenshawe Hospital, Manchester; ${ }^{4}$ Northern General Hospital, Sheffield; ${ }^{5}$ Sheffield Children's Hospital, Sheffield; ${ }^{6}$ The Birmingham Childrens' Hospital, Birmingham, UK

The Halolite AAD system (AAD) has been developed to improve inhaled medication delivery by giving feedback during and at the end of treatment. It will not deliver aerosol during nose breathing, talking, or if there is a poor mouthpiece lip seal. A multicentre randomised parallel study (MAL $25-70$ ) comparing AAD with conventional high efficiency nebulisers (NEB) was conducted at CF centres in Australia, Canada, USA, and Europe. Patients established on DNase and inhaled antibiotics were randomised to AAD or NEB within seven days following an exacerbation requiring oral or IV antibiotics. Patients used the study device to take their inhaled medications for 182 days. The primary outcome variable was change in FEV, from day $0-182$. Secondary outcome variables were days antibiotic use, exacerbation frequency, time to first exacerbation, safety, adherence to prescribed regimen, and compliance with device (by overt electronic monitoring in $20 \%$ of the sample). Patients completed a questionnaire to assess device acceptability.

259 patients were randomised (133 AAD, 126 NEB) median age 17 years, median FEV, $56 \%$ predicted. Preliminary analysis of mean change in $\%$ predicted FEV, from baseline to day 182 was $-3.5 \%$ for AAD and $-2.4 \%$ for NEB. Difference in means $=1.1 \%$ confidence interval $=-4.2 \%$ to $6.3 \%, p=0.7$. There was no statistically significant difference between $A A D$ and NEB for the secondary outcome variables except for a higher incidence of chest tightness per 1000 days $(0.97$ for $A A D$ and 0.32 for NEB $(p<0.05))$. Completed questionnaires showed that compared to their previous nebuliser $69 \%$ of $A A D$ patients felt that they received more dose compared to $30.4 \%$ for NEB; $56 \%$ of AAD patients felt better compared to $30 \%$ for NEB; $82 \%$ of $A A D$ patients preferred the trial device compared to $37 \%$ for NEB. These differences were significantly different $(p<0.0001)$. Fiffy one per cent of prescribed doses were taken beyond $90 \%$ of the programmed dose for AAD compared with $26 \%$ of prescribed doses where the compressor was run for $>6$ minutes for NEB $(p<0.006)$.

Conclusions: There were no differences in the efficacy outcome variables between the devices. A higher incidence of chest tightness for $A A D$ may be indicative of more successful delivery of antibiotic to the lung. Patient acceptability of the device was better for AAD than for NEB. Patients take more doses to an acceptable level using AAD.

\section{P1 15 COMPLIANCE IN CYSTIC FIBROSIS PATIENTS USING TWO DIFFERENT AEROSOL DELIVERY SYSTEMS}

S.P. Conway', M.E. Dodd', R.J. Marsden³, P.H. Weller'. 'St. James' Hospital, Leeds, UK; ${ }^{2}$ Wythenshawe Hospital, Manchester, UK; ${ }^{3}$ Profile Therapeutics, UK; ${ }^{4}$ The Birmingham Childrens' Hospital, Birmingham, UK

CF patients may use conventional high output nebulisers (NEB) for up to an hour a day to aerosolise therapies aimed at preserving lung function. Poor techniques such as nose breathing, talking and inadequate lip seal compromise the time spent breathing correctly on the mouthpiece' and hence the amount of drug delivered to the lungs. Halolite (AAD) has been designed to generate aerosol only whilst patients breathe correctly through the mouthpiece. The system provides feedback to the patient during treatment and signals once the pre-programmed dose has been successfully delivered. The aim of this study was to compare compliance with AAD to compliance with NEB.

Patient Logging System (PLS) monitoring technology was used in a device study comparing AAD with NEB to overtly monitor 50 of 259 patients (from study MAL 25-70) over 182 days. "Adherence" (doses started/by the number of doses prescribed) and "compliance" (the \% of doses taken correctly) were recorded. For AAD a correctly taken dose was defined as delivery of $>90 \%$ of the pre-programmed dose, and for the NEB when the Porta-Neb ${ }^{\circledast}$ PLS compressor was run for $>6$ minutes. "True compliance" is adherence $\mathrm{X}$ compliance. FEV , was recorded on day 0 and 28 . The use of oral or IV antibiotics was recorded on day 0 and 28 . The sample included 30 AAD and 20 NEB patients. Provisional data shows that adherence was $62 \%$ for AAD and $47 \%$ for NEB ( $>>0.05)$. Compliance was $84 \%$ for AAD and $43 \%$ for NEB. "True compliance" was significantly higher for AAD $(51 \%)$ compared to NEB $(27 \%)(p=0.006)$. For NEB there was no correlation between true compliance and \% change in $\mathrm{FEV}$, after 28 days, neither was there a correlation between true compliance and \% of change in FEV, for the subgroup of patients who did not need oral or IV antibiotics for an exacerbation $(n=14)$. For AAD there was no correlation between true compliance and $\mathrm{FEV}_{1}$, however for the subgroup of patients who did not need oral or IV antibiotics for an exacerbation there was a correlation between \% change in $F E V$, and true compliance for AAD ( $n=17)(R=0.53)(p<0.03)$

Conclusion: These data show that the AAD system increases true compliance and may contribute to the maintenance of $\mathrm{FEV}_{1}$.

1. Denyer J, et al. Does inhalation duty cycle in-vitro predict inhaled dose during domiciliary nebulizer use? Drug delivery to the lungs VIII 1997.

\section{P1 16 PORTACATH COMPLICATIONS IN CYSTIC FIBROSIS PATIENTS}

M. Suresh, W. Lenney, G. Hooper, C. Mayer, J. Forrest, A. Walsh. Academic Dept. of Child Health, North Staffs. Hospitals Trust, Staffs, UK

Introduction: Cystic fibrosis (CF) patients requiring repeated intravenous antibiotic courses eventually benefit from insertion of permanent vascular catheters such as a Portacath. There is little information on complications arising from such procedures, which need to be taken into consideration when discussing the relative merit with patients and parents.

Patients: Over a 12 year period out of a population of 78 patients 18 (six male, 12 female) have received 30 Portacaths. Ten patients were $<18$ years old, 11 have only needed one Portacath to date, three have required two, two have needed three, and two have received four Portacaths.

Results: Average length of use: 36.7 months. Longest surviving Portacath: 8 years 10 months. Reasons for failure: Catheter 
disconnection one, systemic/infective complications three, lung collapse one, leakage four, blockage with pain six. Short survival of the first Portacath is a marker for failure with subsequent Portacaths.

Discussion: Complications are relative frequent and unpredictable. Our findings are similar to others in CF and oncology patients. Needle-phobia is a common experience in CF Clinics but the use of Portacaths needs full discussion with families and should not be entered into lightly.

\section{P1 17 ANTIBIOTIC RELATED RENAL IMPAIRMENT IN ADULT CF PATIENTS}

M. Al-Aloul, B. Govin, P.A. Stockton, M.J. Ledson, M.J. Walshaw. Regional Adult CF Unit, The Cardiothoracic Centre, Liverpool, UK

Colonisation with multiresistant Pseudomonas aeruginosa $(\mathrm{Pa})$ in $\mathrm{CF}$ results in repeated use of a limited number of antibiotics to which the organisms are sensitive, increasing the risk of toxic effects. In Liverpool, we have a multiresistant strain colonising many CF patients that is only sensitive to aminoglycosides and polymyxins, antibiotics that are known to cause renal damage that may be associated with excess levels and also cumulative lifetime dose. To assess the impact of repeated dosing with these antibiotics, we have compared renal function in those colonised with multiresistant $\mathrm{Pa}$ with a group of $\mathrm{CF}$ patients with similar clinical parameters but who are colonised by sensitive Pa strains. Overall, 52 patients (32 multiresistant) were studied (mean age (range): multiresistant 23.6 years (15 to 42) $v$ sensitive 24.9 (18 to 37): FEV \% predicted: 61.3 (16 to 95$)$ v 61.8 (17 to 115); BMl: 21.6 (18 to 28) v 20.6 (14 to 27); CF related diabetes: 9 $\checkmark 4$ (all $P=N S)$. During exacerbations, all patients had aminoglycoside levels measured and adjusted after the 4 th dose as per protocol and no episodes of acute toxicity were noted. Renal function was measured by estimation of serum urea and electrolytes and 24 hour urinary creatinine clearance when patients were in a stable clinical state. Patients produced satisfactory 24 hour urine collections, demonstrated by sufficient urine volume and total urinary creatinine excretion. All patients had serum creatinine and urea levels within the normal range, but those colonised with multiresistant strains had a lower creatinine clearance (mean $75 \mathrm{mls} / \mathrm{min}$ (range 11 to 115) $\mathrm{v}$ $101 \mathrm{mls} / \mathrm{min}(28$ to 171$)),(\mathrm{p}<0.002)$, and had received more IV aminoglycoside courses (mean 23 per patient (range 0 to 100)) $v(8$ $(0$ to 30$)),(p<0.007))$, and more IV colomycin courses (22 (0 to 80$)$ $\checkmark 7.4(0$ to 50$)),(p<0.006))$. Also, the total number of IV courses correlated with worsening renal function $(r=0.39, p<0.001)$. We conclude that repeated dosing with these potentially nephrotoxic drugs has damaged the renal function in those patients colonised by multiresistant $\mathrm{Pa}$ strains, and in some patients we can now no longer use these important antibiotics. This study illustrates the need to prevent colonisation by such strains if possible in CF patients. This is best achieved by measures designed to prevent cross infection, including suitable patient segregation.

\section{P1 18 VARIATION IN REPORTED INTAKE OF PANCREATIC ENZYME REPLACEMENT THERAPY IN ADULTS WITH CYSTIC FIBROSIS}

S. Thornton, S. Kelly, F. Edenborough. Adult Cystic Fibrosis Unit, Northern General Hospital, UK

Background: Pancreatic Enzyme Replacement Therapy (PERT), the number of enzymes taken with food, is assessed as part of the annual review. In this centre both the doctor and dietitian review independently, however it is the amount each patient reports to the doctor which is recorded on the UK National Cystic Fibrosis (CF) database.

Aim: To investigate whether reported intakes of PERT capsules differ when assessed by dietitian, doctor, or using a food diary.

Methodology: Patients attending their annual review at the CF Centre between July 2000 to May 2002 were asked the number of PERT capsules they consumed with meals, snacks, nutritional supplements, drinks etc each day. This is the recall method and is recorded by the dietitian (in conjunction with diet history) and the doctor on the same day. Patients are also requested to complete at least a three day food diary (two weekdays and one weekend day). They record the quantities and types of food, fluid and PERT consumed on those days. The numbers of PERT recorded by each method were compared.

Results: There was significantly less PERT intake reported using the recall method by dietitian, $3.6 \pm 10.3$ enzymes ( $p=0.04, t$ Test) compared to the database documented value (doctor).

The number of enzymes recorded on the food diaries was also lower than the database documented value (doctor), $3.8 \pm 9.1$ enzymes but was not significant $(0.07,+$ Test). In addition, there is considerable variation in the number of PERT capsules reported using each method (table).

Conclusion: The actual over reporting of enzyme intake for the UK CF database is slight overall, however for an individual patient the report can vary by over $100 \%$ difference. It is also shown that $40 \%$ of patients are more than $25 \%$ inaccurate in their reporting. This may have implications for their individual enzyme assessment and monitoring. In light of this evidence we suggest that consideration is made to the method of assessment used.

\section{P119 INTRAVENOUS ANTIBIOTICS INCREASE EXHALED NITRIC OXIDE IN CHILDREN WITH CYSTIC FIBROSIS}

A. Jaffé, G. Slade, J. Rae, A. Laverty. Portex Respiratory Unit, Great Ormond Street Hospital for Children \& Institute of Child Health, London, UK

Introduction: Data on the effect of intravenous (IV) antibiotics on exhaled nitric oxide (NO) in $\mathrm{CF}$ and on correlation of $\mathrm{NO}$ with lung function are conflicting because of lack of standardisation for the measurement of $\mathrm{NO}$.

Aims: To assess the effect of IV antibiotics on exhaled NO in CF children and to correlate NO with lung function. Methods: Exhaled $\mathrm{NO}$ was measured on line during a slow exhalation according to ATS guidelines using an exhalation flow of $50 \mathrm{~mL} / \mathrm{s}$ (NiOX, Aerocrine,) in CF patients admitted for IV antibiotics. Pulmonary function was measured according to ATS guidelines (Masterscreen, Jaeger). Measurements before and after treatment were compared by Wilcoxon Signed Ranks Test. Spearman correlation tests were used to assess correlation. A value of $p<0.05$ was considered significant.

Results: Fourteen CF subjects (10 female), median age (range) 12.1 years $(5.9$ to 16.0$)$ were studied. Genotypes were as follows: $\Delta \mathrm{F} 508 / \Delta \mathrm{F} 508, \mathrm{n}=10 ; \Delta \mathrm{F} 508 / \mathrm{N} 1303 \mathrm{~K}, \mathrm{n}=1 ; \Delta \mathrm{F} 508 /-, \mathrm{n}=3$. Reasons for admission were: infective exacerbation, $n=4$; routine quarterly antibiotics, $n=10$. Cough swab or sputum culture on admission: Pseudomonas aeruginosa, $\mathrm{n}=6$; Staphylococcus aureus, $\mathrm{n}=6$ (one had coliforms and one had Stenotrophomonas maltophilia in addition); no growth, $\mathrm{n}=2$. Median (range) length of treatment was $8.5(6-15)$ days. There was a significant improvement in mean (SEM) $\% F E V$, from $60.0(6.3)$ to $68.0(5.4)(p=0.02)$ and \%FVC from 66.3 (5.5) to 75.1 (4.9) ( $p=0.003)$. There was a significant increase in NO following treatment (median (range): pre; $5.8 \mathrm{ppb}$ (2.0 to 14.3), post; $9.2 \mathrm{ppb}(0.8$ to 25.1$), \mathrm{p}=0.02)$. There was no correlation between $\mathrm{NO}$ and \%FEV1 or \%FVC.

Discussion: $\mathrm{NO}$ is raised in lung diseases with an inflammatory component, however this is not true for CF, and is further supported by these results. Various hypotheses have been proposed to account for this. These include: entrapment in sputum; degradation by superoxide production from activated neutrophils; NO reductase in $P$ aeruginosa; reduced inducible NO synthase expression in epithelial cells. Our results support these hypotheses as it is likely that antibiotic treatment results in a reduction in $P$ aeruginosa colony forming units, neutrophil activation and airway sputum volume.

Conclusion: IV antibiotics increase exhaled NO in CF. However $\mathrm{NO}$ does not correlate with lung function and is not a useful marker of lung disease in CF.

\begin{tabular}{|llllll|}
\hline Abstract P1 18 & \multicolumn{7}{l|}{} \\
\hline$\%$ difference from database (doctor) & $<25 \%$ & $26-50 \%$ & $51-75 \%$ & $76-100 \%$ & $>100 \%$ \\
$\%$ reported to dietitian & 61 & 30 & 2 & 7 & 2 \\
$\%$ reported on food diary & 60 & 30 & 5 & 5 & 0 \\
\hline
\end{tabular}




\section{P120 FACTORS DETERMINING DIAPHRAGM STRENGTH IN CHILDREN AND YOUNG ADULTS WITH CYSTIC FIBROSIS}

N. Hart ${ }^{13} 5$, P. Tounian' ${ }^{2}$, M.I. Polkey ${ }^{3}$, A. Clement' ${ }^{1}$, J. Moxham ${ }^{4}$, F. Lofaso ${ }^{5}$, B. Fauroux'. 'Paediatric Pulmonary Department; ${ }^{2}$ Gastroenterology Department, Armand Trousseau Hospital, Paris, France; Respiratory Muscle Laboratories, ${ }^{3}$ Royal Brompton Hospital; ${ }^{4}$ Kings College Hospital, London, UK; ${ }^{5}$ Department Of Clinical Physiology, Raymond Poincare Hospital, Garches, France

Respiratory failure is the major cause of death in patients with cystic fibrosis (CF). Although the diaphragm is the most important muscle of inspiration, it is unclear as to whether there are any alterations in diaphragm strength in patients with cystic fibrosis (CF). In the current study, we hypothesised that diaphragm strength would be determined by airflow obstruction and pulmonary hyperinflation, gas exchange impairment, inspiratory muscle load, and nutritional status of the patient. 20 patients with CF were studied (mean age 15.1 (2.8) years). We measured twitch transdiaphragmatic pressure (Tw Pdi) in response to bilateral anterior magnetic phrenic nerve stimulation to quantify diaphragm strength; forced expiratory volume in $1 \mathrm{sec}\left(\mathrm{FEV}_{1}\right)$ and functional residual capacity (\% Pred FRC) as estimations of airflow obstruction and hyperinflation; and diaphragm pressure time index $\left(\mathrm{PTI}_{\mathrm{di}}\right)$ as an indicator of diaphragm load. Nutritional status was evaluated using body mass index adjusted for age and sex (BMI z-score), fat mass (FM), fat-free mass (FFM) and arm-muscle circumference $(\mathrm{AMC})$. These were determined from measurements of height, body weight, mid-arm circumference (AC), and skinfold thickness (SK) at four different sites (biceps, triceps, subscapular, and suprailiac). FM and FFM were calculated from SK and weight. AMC was calculated from the formula $A C-(0.134$ * biceps $S K / 2+$ triceps $S K / 2)$. FM, FFM, and $A M C$ were expressed as percent predicted for statural age $\left(\%\right.$ Pred $\left._{\mathrm{SA}}\right)$. Results are expressed as mean (SD). Tw Pdi was 24.3 (5.5) $\mathrm{CmH}_{2} \mathrm{O} ; \%$ Pred $\mathrm{FEV}_{1}$ was 44.5 (21.4); \% Pred $\mathrm{FRC}_{\mathrm{pl}}$ was 159.8 (40.9); $\mathrm{PaO}_{2}$ was 9.5 (1.5) kPa; $\mathrm{PaCO}_{2}$ was $5.5(0.6) \mathrm{kPa}$; and PTI was 0.05 (0.03). Univariate regression analysis demonstrated Tw Pdi correlated with \% Pred $\mathrm{FEV}_{1}(\mathrm{p}=0.001 ; \mathrm{r}=+0.68) ; \%$ Pred $\mathrm{FRC}_{\mathrm{pl}}$ $(p=0.005 ; r=-0.65) ; \mathrm{PaO}_{2}(\mathrm{p}=0.001 ; \mathrm{r}=+0.68) ; \mathrm{PaCO}_{2}(\mathrm{p}=0.03 ; \mathrm{r}$ $=-0.50) ; B M I$-score $(p=0.003 ; r=+0.63) ; \% \operatorname{Pred}_{\mathrm{SA}}$ AMC $(r=$ $+0.45 ; p=0.04) ;$ and $\% \operatorname{Pred}_{S A} F F M(r=+0.47 ; p=0.04)$. There were no correlations between Tw Pdi and \% $\operatorname{Pred}_{S A} F M(p=0.1)$ and $\mathrm{PTI}_{\mathrm{di}}$ $(p=0.2)$. In conclusion, in children and young adults with $C F$, diaphragm strength falls as airways obstruction and hyperinflation increases, gas exchange impairment worsens, and nutritional status declines. However, load does not have effect of diaphragm strength.

Dr N Hart was supported by the Scadding-Morriston Davies Joint Respiratory Medicine Fellowship, a European Respiratory Society Long Term Fellowship and a grant from the Association Française Contre Les Myopathies.

\section{P121 INCREASED ENERGY EXPENDITURE DUE TO COUGHING IN ADULTS WITH CYSTIC FIBROSIS}

A.A. lonescu, R. Colasanti', L.S. Nixon, C.E. Bolton, M. Williams', D.J. Shale. Section of Respiratory Medicine University of Wales College of Medicine; 'School of Applied Sciences University of Glamorgan, Cardiff, UK

Patients with Cystic Fibrosis (CF) have increased resting energy expenditure (REE). We hypothesised that frequent coughing, occurring spontaneously and during physiotherapy in most patients, adds to the REE and overall energy expenditure (EE), which may contribute to weight loss.

We measured breath by breath REE (K4b, Cosmed) in 12 clinically stable patients and 10 healthy subjects. A steady state baseline was recorded and each subject coughed voluntarily three times after breathing in to TLC. A further 10 patients had REE measured on repeated occasions by the same method. During 45, 10 minute recordings of REE 144 spontaneous coughs occurred. The REE before coughing occurred and after each coughing episode was recorded. The mean recovery time (VE, I/min back to baseline) after the three voluntary coughs (41 seconds) was used to calculate the EE after spontaneous coughing as the area under the curve after each cough by locally designed software. We also compared the REE after spontaneous coughs to the baseline REE, because the duration of the baseline record was variable (mean 106, maximum 371, minimum 8 seconds). There was no difference in EE after three coughs (ratio to baseline), between patients and healthy subjects ( $p>0.05)$. The FEV (patients) was inversely related to EE after three coughs, $r=-0.0 .58$, $p=0.04$. Recovery time was similar to the healthy subjects. Spontaneous coughs in patients increased the EE by a mean $(95 \% \mathrm{CI}) 7.9$ (2.7 to 13.3$) \%$ compared with baseline, mean $(95 \%$ CI) 32.63 (30.51 to $34.75)$, and $30.66(28.59$ to 32.73$) \mathrm{kcal} / \mathrm{kg} / \mathrm{min}, \mathrm{p}<0.01$. The REE (area under curve over time) with spontaneous coughs was greater than REE recorded when spontaneous coughs were removed from the 10 minutes measurement; mean (95\% Cl) 28.0 (24.7 to 31.3) and 21.5 (18.6 to 24.3), respectively, $p=0.016$.

Cough increased the REE in adults with CF. For accuracy REE measurements in CF should include any spontaneous coughs that occur. Due to the frequency of coughing, the energy costs are likely to increase the negative energy balance in some patients.

Sponsored by the Cystic Fibrosis Trust UK.

\section{P122 SEQUENTIAL SINGLE LUNG TRANSPLANTATION FOR SEPTIC LUNG DISEASE; A SINGLE CENTRE COMPARISON BETWEEN CF AND NON-CF BRONCHIECTASIS}

A. De Soyza', K. Gould 2, G. Parry', J.H. Dark', P.A. Corris' ${ }^{3}$. 'Lung Biology and Transplantation Group; ${ }^{2}$ Department of Microbiology; ${ }^{3}$ Cardiothoracic Transplantation, Freeman Hospital, University of Newcastle, Newcastle upon Tyne, UK

Background: The outcomes for transplantation for septic lung conditions including Cystic Fibrosis (CF) and non-CF bronchiectasis have been comparable to those for non-septic lung conditions despite the additional risks associated with these conditions. Given the current organ donor shortage it is important to compare outcomes for each pre-transplant outcome within a single centre to allow appropriate allocation of organs. It is recognised that certain pre-transplant indications such as Pulmonary Fibrosis and Pulmonary Hypertension are associated with poor transplant outcomes. We have not previously compared the outcomes of Cystic Fibrosis and non-CF Bronchiectasis at this centre.

Methods: We identified all patients receiving lung transplants at this centre for septic lung diseases through our transplant database. A survival table was constructed and the two groups were compared using the log rank (Mantel-Cox) test for real survival. Pre-transplant recipient characteristics were also compared including pre-transplant body mass index, pre-transplant FEV1, need for non-invasive ventilation and median time post transplant.

Results: We have transplanted $96 \mathrm{CF}$ patients and 26 non-CF Bronchiectatics (NCFBr) over 10 years. Median age at transplantation CF group 25.4 yrs (range 16 to 49.5 ) and $\mathrm{NCFBr}$ median $48 \mathrm{yrs}$ (range 25 to 56 ) $p<0.05$. Survival was comparable between groups at one year CF $81 \%, \mathrm{NCFBr}-76.5 \% \mathrm{p}=0.46$. At five years survival was $70 \%$ for $\mathrm{CF}$ and $72 \%$ for $\mathrm{NCFBr} p=\mathrm{NS}$. Pre-transplant FEV $1 \%$ predicted was similar between groups median in the $\mathrm{CF}$ group $=19.1 \%$ (range 8 to $35 \%$ ) and NCFBr 18 (9 to $49 \%$ ) p=NS. In each group early deaths were predominantly related to sepsis: The early deaths were due to sepsis in the CF group (9/96) and also in the $\mathrm{NCFBr}$ group $13 \%(4 / 29)$ Fishers exact test $\mathrm{p}=0.3$. Median pre-transplant Body mass index (BMI $-\mathrm{kg} / \mathrm{m})$ was 17.8 (range 12 to 24) for $\mathrm{CF}$ and $\mathrm{NCFBr}=23$ (range 16 to 32), Mann Whitney, $p<0.01$. No patient in the Bronchiectasis group required non-invasive ventilation pre-transplant whilst 11/96 CF patients had NIV pre-transplant Fisher exact test $p=0.11$. Similarly there were 12/96 CF patients infected with Burkholderia cepacia complex organisms whilst no $\mathrm{NCFBr}$ were infected with this organism $p=0.11$ pre-transplant Fisher exact test $p=0.11$.

Conclusions: Cystic Fibrosis patients had poorer pre-transplant nutrition and were more likely to be infected with $B$ cepacia complex. There were however no statistical differences in post transplant survival noted. We conclude that despite these features CF patients are as likely to benefit from pulmonary transplantation than older non-CF bronchiectasis patients.

\section{P123 RECOMBINANT SENDAI VIRUS-MEDIATED CFTR CDNA TRANSFER}

S. Ferrari ${ }^{1-4}$, R. Farley ${ }^{1-4}$, F. Munkonge ${ }^{1-4}$, U. Griesenbach ${ }^{1-4}$, S. Smith ${ }^{1-4}$ J. You ${ }^{2}$, A. lida ${ }^{2}$, B. Wainwright ${ }^{3}$, D. Geddes ${ }^{1-4}$, M. Hasegawa ${ }^{2}$, E. Alton ${ }^{1-4}$. $^{\prime}$ 'Department of Gene Therapy, NHLI, London, UK; ${ }^{2}$ DNAVEC Research Inc, Japan; ${ }^{3}$ University of Queensland, Brisbane, Australia; ${ }^{4}$ UK CF Gene Therapy Consortium, Edinburgh-London-Oxford, UK

To assess applicability for Cystic Fibrosis (CF), recombinant Sendai virus (SeV) carrying the CFTR gene was tested on different models of airway epithelial cells. In vitro CFTR activity was assessed at two days 
post-infection in murine mammary epithelial cells (C127 -/-) using a radioactive iodide efflux assay after challenge with forskolin/IBMX (F/I). Cells infected with a SeV vector carrying a mutated CFTR sequence (SeV-mCFTR) were used as controls whilst T84 were used as positive controls and results expressed as $\Delta k \cdot \mathrm{min}^{-1}$ (s.e.m). T84 cells showed maximal efflux rate $2 \mathrm{~min}$ after $\mathrm{F} / \mathrm{I}$ addition $(0.24(0.02)$, $\mathrm{n}=30$ ) compared to $\mathrm{Cl} 27$-/- values $(0.004$ (0.004), $\mathrm{n}=11)$, $p<0.001$. All three SeV-CFTR clones tested showed a characteristic delayed peak three minutes after F/I addition (SeV-CFTR 23-3 (0.34 (0.04), $n=12)$; SeV-CFTR 23-4 (0.26 (0.03), $n=6)$; SeV-CFTR 1-3 $(0.16(0.05), n=12)$. As for untreated $\mathrm{Cl} 27-/-$ cells, treatment with SeV-mCFTR did not lead to any change in efflux $(-0.01(0.02), n=12)$. In vivo experiments were carried out in G551D CF transgenic mice by administration of SeV-CFTR 23-3 and 23-4 (5 × 10 pfu, n=9) through nasal perfusion and CFTR activity analysed at day $2,7,14$, and 28. SeV-mCFTR $(n=9)$ was used as control. Two days post-infection, NPD, values in animals treated with SeV-CFTR $23-3 / 23-4 \quad(-0.11$ (0.97) mV) were significantly $(p<0.05)$ higher (that is, towards non-CF values) than those observed in animals treated with SeV-mCFTR $(-3.25(0.73) \mathrm{mV})$. NPD in animals treated with SeV-CFTR 23-3/23-4 remained significantly $(p<0.05)$ higher at day $7(0.20(0.73) \mathrm{mV}$ as opposed to $-1.55(1.23) \mathrm{mV}$ in controls). Baseline values at day seven for animals treated with SeV-mCFTR $(30.8$ (3.6) $\mathrm{mV}$ ) were not different to untreated CF mice, but in mice treated with SeV-CFTR 23-3/23-4 they were not different from those observed in wild type mice 112.1 (1.5) $\mathrm{mV}, \mathrm{p}<0.01$ compared to controls). By day 28, baseline and low chloride values had reversed to typical G551D CF mouse values. In conclusion we have shown that $\mathrm{SeV}$ can mediate CFTR gene transfer both in vitro and in vivo.

\section{P124 GENE SILENCING THROUGH RNA INTERFERENCE AS PUTATIVE THERAPY FOR CF}

U. Griesenbach' ${ }^{4}$, S. Escudero-Garcia' ${ }^{4}$, R. Farley' ${ }^{4}$, S, Cheng ${ }^{2}$, D.M. Geddes' 4 , N.J. 'Caplen ${ }^{3}$, E.W.W.W. Alton' ${ }^{4}$. 'Department of Gene Therapy, National Heart and Lung Institute, Faculty of Medicine, Imperial College, London, UK; ${ }^{2}$ Genzyme Corporation, Framingham, USA; sMedical Genetics Branch, NHGRI, National Institute of Health, Bethesda, USA; ${ }^{4}$ United Kingdom CF Gene Therapy Consortium, UK

Gene silencing through dsRNA-mediated RNA interference (RNAi) has been described in C elegans and Drosophila. We, and others, have recently described $\mathrm{RNAi}$ in mammalian cells mediated by small dsRNAs of 21-23 nucleotides (nts) termed short interfering RNAs (siRNAs) (Caplen NJ, PNAs 2002). Here, we provide preliminary proof of principle that gene silencing through RNAi can be achieved in lung in vivo. Balb/C mice (female 6-8 weeks) were simultaneously transfected with pcDNA3CAT $(80 \mathrm{\mu g} / \mathrm{mouse})$ and 22 nts siRNA corresponding to CAT $(40 \mu \mathrm{g} / \mathrm{mouse})$ or an irrelevant control siRNA, each complexed with the cationic lipid GL67 ( $n=12$ in both groups). Forty eight hours after transfection the lungs were harvested and CAT activity assayed. CAT expression was reduced by $90 \%$ in animals treated with CAT siRNA, when compared to controls (control siRNA: 736.9 (437) pg CAT/mg protein, CAT siRNA: 80.1 (31.4) pg $\mathrm{CAT} / \mathrm{mg}$ protein, $\mathrm{p}<0.001$. A potential confounding factor is the co-transfection of the plasmid and siRNA, allowing for a nonposttranscriptional silencing mechanism of action. To address this, we compared the silencing of the green fluorescent protein (eGFP) using a siRNA against eGFP either in cells co-transfected with eGFP plasmid and siRNA or cells stably expressing a destabilised version of eGFP (eGFPd2) transfected with siRNA alone. The degree of silencing in both cases exceeded $90 \%$. We have recently shown that, in contrast to the liver, uptake of DNA into the nucleus of airway epithelial cells is extremely inefficient in vivo. However, cytoplasmic transfection can be readily achieved. RNAi has been shown to primarily act within the cytoplasmic compartment and so may offer a major advantage over conventional antisense strategies that have been proposed for several disorders. Although the pathophysiology in CF is not completely understood, several proteins may provide good targets for gene silencing, including the epithelial sodium channel (EnaC), as well as several pro-inflammatory cytokines, such as IL-8 and chaperones, which retain delta F508 CFTR within the endoplasmic reticulum.

\section{P125 INFECTION OF THE MURINE LUNG WITH NON-TRANSMISSIBLE RECOMBINANT SENDAI VIRUS EXPRESSING THE SECRETED PROTEIN INTERLEUKIN 10}

U. Griesenbach', T. Shiraki-lida' 2 , S. Ferrari' , H.L. Gautrey', X. Hou' ${ }^{2}$ A. lida $^{2}$, D.M. Geddes', M. Hasegawa', E.W.F.W. Alton'. 'Department of Gene Therapy, Faculty of Medicine, Imperial College London, London, UK; ${ }^{2}$ DNAVEC Research Inc., Tsukuba, Ibaraki, Japan

We have previously shown that a transmission-competent recombinant Sendai virus carrying mouse interleukin 10 cDNA (SeV-IL 10) infects the airways efficiently. A second generation, and for human use potentially safer $\mathrm{SeV}$ has recently been generated, in which the gene for the membrane fusion protein ( $F$ protein) has been deleted from the viral genome (SeV/ $\Delta \mathrm{F}$ ) (Li et al. J Virol 2000;74:6564-9). The F protein is essential for virus entry into the cell and is supplied in trans during vector production. However, following infection and virus replication of the vector genome in vivo the virus cannot re-infect neighbouring cells. Here, we assessed the efficiency of F-defective $\mathrm{SeV}(\mathrm{SeV}-\mathrm{IL} 10 / \Delta \mathrm{F})$ in lungs of mice and in primate trachea. The lungs of $\mathrm{C} 57 \mathrm{BI} / 6$ mice were transfected by placing $\mathrm{SeV}$-IL $10 / \Delta \mathrm{F}$ or a SeVLacŽ $\Delta \mathrm{F}$ control virus $\left(7 \times 10^{4}\right.$ and $\left.7 \times 10^{7} \mathrm{CIU} / \mathrm{mouse}\right)$ as a single 100 $\mu$ bolus into the nasal cavity and the solution was sniffed into the lungs. Lung tissue and serum was harvested 2, 7, and 14 days after infection. IL10 production was measured in lung homogenate and serum using standard ELISA. In lung homogenate expression was highest two days after infection and was 2 and 4 logs above control levels for $7 \times 10^{4}$ and $7 \times 10^{7} \mathrm{CIU} /$ mouse, respectively. At day seven expression in mice transfected with the higher dose had dropped to $1.3 \%$ of the day two levels, but only to $13 \%$ in the lower dose. At day 14 the lower dose still showed low but significant IL 10 expression, whereas the higher dose was not longer different from control levels. High levels of serum IL 10 were detected two days after infection with $7 \times 10^{7} \mathrm{ClU} / \mathrm{mouse}$, but not in animals infected with $7 \times 10^{4}$ $\mathrm{CIU} / \mathrm{mouse}$, and were still significantly increased seven days after infection (SeV-IL 10/DF: 3500 (492) and 75 (18) pg/ml at day two and seven respectively, SeV-LacŽ $\Delta F$ : 5.5 (5.4) and $0 \mathrm{pg} / \mathrm{ml}$ at day two and seven respectively, $n=6, p<0.05)$. When compared to the previously used transmission-competent, first generation $\mathrm{SeV}$ virus (historical data), there was no difference in IL10 expression in lung homogenate or serum. We also transfected fresh and mucus depleted segments of primate trachea with $\mathrm{SeV}-\mathrm{IL} 10 / \Delta \mathrm{F}$ or a SeV-LacŽ $\Delta \mathrm{F}$ control virus $\left(10^{6}\right.$ and $\left.10^{7} \mathrm{CIU}\right)$ ex vivo $(n=1)$ and preliminary results indicated efficient transfetion of fresh and mucus-depleted tissue

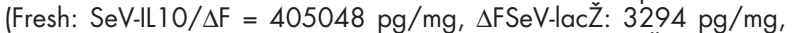

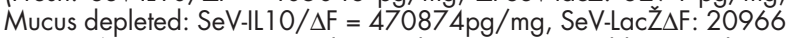
pg IL $10 / \mathrm{mg}$ protein). In conclusion, the non-transmissible second generation SeV-IL 10/ $\Delta$ F infects airway epithelium as efficiently as the first generation virus. Preliminary results indicate that the virus titre at least in part determines the decline of transgene expression and that SeVIL 10/ $\mathrm{F}$ infects primate trachea efficiently.

\section{Rehabilitation, symptoms, and smoking}

\section{P126 PILOT DATA: PULMONARY REHABILITATION IN RESTRICTIVE LUNG DISEASE}

K. Dix, C. Daly, R. Garrod', M. Howard, C. Rayner. St George's Hospital NHS Trust; 'School of Physiotherapy St George's Hospital and Kingston University, UK

Introduction: Pulmonary rehabilitation is a proven therapy for patients with obstructive pulmonary disease, however little is known concerning the effects in patients with restrictive disease. This study reports on data from an seven week, $\times 2$ weekly, "real life" programme of exercise and education in which patients with both restrictive and obstructive disease were admitted.

Methods: Outcome measures were baseline spirometry. Exercise tolerance using Shuttle Walk Test (SWT), health related quality of life using St George's Hospital Respiratory Disease Questionnaire (SGRQ), mood state using Hospital Anxiety and Depression Scale (HAD).

Results: Sixty seven patients completed the course. Of these patients, 57 had baseline spirometry from which 48 were classified as obstructive; mean $\mathrm{FEV}_{1}, 1.09$ (0.4), FVC 2.5 (0.7) I and 9 restrictive, mean FEV, 1.42 (0.4), FVC 1.8 (0.5) I

Both obstructive and restrictive patients showed a statistically significant effect of rehabilitation on SWT; mean difference (SD) 47.8 (86.9) $(p=0.0005), 91.1$ (64.1) m ( $p=0.002)$ respectively. However, the difference between the groups (43.3) $\mathrm{m}$ was not significant $(p=0.16)$. There were statistically significant improvements in SGRQ $(p=<0.001)$ and depression $(p=<0.001)$ for the obstructive group and a trend in response for the restrictive group.

Conclusion: Low power means we cannot rule out the possibility of type II error for difference in SWT between the groups. Data from an uncontrolled "real life "study indicates that patients with restrictive disease may show greater improvements in exercise tolerance than 
obstructive disease. This report highlights the need for baseline spirometry in the assessment and thorough evaluation of pulmonary rehabilitation in differing populations.

\section{P127 OXYGEN COST SCORE FOLLOWING 4 STEP COPD PROGRAMME AND COMMUNITY BASED PULMONARY REHABILITATION}

K. Holt, S. Dryden, I. Houghton, I. Eccleston. Wyre Primary Care Trust, Lancashire, UK

Since January 2000 Wyre PCT has been implementing a 4 step COPD Health Improvement Programme (HImP) to ensure correct diagnosis and optimal, guidelines led, therapy. The programme, developed in collaboration with Blackpool Victoria Hospital Chest Clinic, involves spirometry assessment by a mobile spirometry service visiting all General Practices with further assessment and patient management by COPD trained Practice Nurses. Following diagnosis (step 1), inhaled steroid trial (step2), anticholinergic trial (step 3), and long acting $\beta 2$ trial (step 4), community based pulmonary rehabilitation (PR) is now (since July 2001) offered to those patients motivated to attend. PR, led by a Physiotherapist and a Respiratory Nurse, is offered at two sites in the PCT; a Local Authority Sports Centre and a Village Community Centre. Patients attend for two hours twice a week for eight weeks and are then invited to attend one hour a week for ongoing supervised exercise. The Oxygen Cost Score (OCS) is used to assess breathlessness by functional ability at each stage of the HImP and PR programme.

Data from 55 COPD patients (10mild, 21 moderate, and 30 severe using BTS criteria for severity) were used to examine dyspnoea, measured by OCS; before entering the 4 step programme (stage 1), on completion of the 4 step programme and before entering PR (stage 2 ), on completion of the eight week PR programme (stage 3).

The mean OCS and mean improvement (MI) after stage 2 and 3 is shown in the table.

\begin{tabular}{|c|c|c|c|c|c|}
\hline & Stage 1 & Stage 2 & MI & Stage 3 & MI \\
\hline Mild & 3.25 & 4.5 & 1.25 & 4.75 & 0.25 \\
\hline Moderate & 4.35 & 4.4 & 0.5 & 5.35 & 0.95 \\
\hline Severe & 3.66 & 3.83 & 0.17 & 4.73 & 0.9 \\
\hline
\end{tabular}

Conclusions: These preliminary data suggest that COPD patients demonstrate an improvement in functional ability following a four-step community based HImP with further improvement gained by attending community PR. Patients with moderate and severe COPD appear to demonstrate greater improvement from PR than from the 4 step programme. The number of mild COPD patients referred for PR are too small to be meaningful.

\section{P128 BREATHLESSNESS AND ANXIETY MANAGEMENT COURSE FOR PATIENTS WITH COPD UNSUITABLE FOR CONVENTIONAL PULMONARY REHABILITATION}

K. Pye, J. Batten, L. Davies. Aintree Chest Centre, University Hospital Aintree, UK

Pulmonary rehabilitation is known to improve health status, symptoms, and exercise tolerance in patients with COPD. Particularly in secondary care, many COPD patients are unsuitable for conventional PR due to concomitant disease or extremely limited mobility. This breathlessness and anxiety management course, run by COPD nurse specialists, was designed specifically for these patients. All are receiving optimal medical therapy and are clinically stable at entry and have been deemed unsuitable for conventional PR. Patients attend once a week for 2 hrs over a six week period. Individual assessment is made and anxiety management, breathing control, relaxation techniques, partially supervised physical exercise, and support and practical advice tailored as appropriate. There were 41 patients referred to the course, but only 19 attended for initial assessment (12 not motivated, three transport problems, three repeated COPD exacerbations, four misc). Assessment at visit one and final visit consists of resting pulse and respiratory rate, $6 \mathrm{MWD}$ (with pre and post Borg scores), SGRQ,
$H A D$, and MRC dyspnoea scale. Patients who exacerbated were allowed to continue if well enough, those patients who did not complete the course were not reassessed. Mean (SD) age 69 (7) years, 11 (58\%) female, FEV, 1.03 (0.48) litres, \% pred FEV1 36.9 (1 1.9), $\mathrm{pH} 7.39$ (0.04), $\mathrm{pO}_{2} 10.4$ (2.3) kPa, $\mathrm{pCO}_{2} 4.8$ (0.7) kPa (on air).

Nine patients did not complete the course (six repeated COPD exacerbations, two worsening of pre-existing arthritis, one developed lung cancer).

There was no significant difference in age, sex, lung function, or exercise tolerance between those patients who completed the course and those who did not. There was a significant improvement in resting respiratory rate (18.4 to 15.6, $\mathrm{p}<0.05)$, change in Borg score after 6MWD (4 to $3.1, p<0.05$ ), HAD depression score (7.5 to 5.7 , $\mathrm{p}<0.05)$ and the Activity (82.5 to 73.3, $\mathrm{p}<0.01)$ and Impacts $(60.9$ to $51.06, p<0.05)$ score of the SGRQ. Although the $6 \mathrm{MWD}$ was not significantly improved in this small group there was a trend to improvement (202 to $280 \mathrm{~m})$.

Individually tailored programs are time consuming and drop out rate is high. However, those that do complete the course benefit in terms of health status and symptoms. Although numbers are small these results are encouraging and merit further study.

\section{P129 DIFFERENT EXERCISE RESPONSES IN SUBJECTS WITH IDIOPATHIC HYPERVENTILATION}

S. Jack, C.J. Warburton. Aintree Chest Centre, University Hospital Aintree, Liverpool L9 7AL, UK

Hyperventilation may occur as an acute and a chronic phenomenon. In addition, it may occur in isolation (idiopathic) or may co-exist with other disease processes. There are very little data on homogeneity within any of these subgroups.

We have studied 40 subjects referred through our chest clinics with symptoms suggestive of idiopathic hyperventilation $(\mathrm{HH})$ and normal lung function. IH was confirmed by resting arterial hypocapnia and sustained hypocapnia during ramp-incremental exercise. Further analysis of the ventilatory response to exercise revealed that 11 of the 40 subjects demonstrated acute upon chronic hyperventilation at exercise onset (defined as RER > 1.0), six of the 40 subjects demonstrated acute upon chronic hyperventilation at rest which continued during early exercise. Twenty three of the 40 subjects did not demonstrate acute hyperventilation in addition to their chronic hyperventilatory state.

There were no differences between the three groups in the Hospital Anxiety and Depression scores, Nijmegen Questionnaires, and St George's Respiratory Questionnaires or in maximally achieved parameters during cycle ergometry and in breath-hold tolerance both on 21 and $100 \% \mathrm{O}_{2}$. Resting respiratory rate was higher (mean (SD) $33.7(6.7 \mathrm{br} / \mathrm{min})$ in the chronic group compared to the those demonstrating acute hyperventilation in addition to their chronic hyperventilation (mean (SD) $27.7(8.3))(p<0.02)$. There was a significant correlation between anxiety, resting $\mathrm{PETCO}_{2}$ and $\mathrm{BHT}$ s and between $\mathrm{PETCO}_{2}$ at rest and at maximal work rate achieved in the chronic group $(p<0.05)$. Resting RR was significantly correlated with resting VE and resting $\mathrm{PETCO}_{2}$ also in the chronic group. These relationships were not evident in the two groups which demonstrated acute and chronic hyperventilation. There was a significant correlation in these groups between anxiety and resting heart rate, which may be explained by anticipatory anxiety.

These data demonstrate that within this population of seemingly homogenous subjects with $\mathrm{IH}$, there are different responses to exercise. This does not appear to alter the overall symptomatology of the groups or their other physiological indices. Whether the different responses to exercise relate to chronicity of symptoms needs further study.

\section{P130 THE DAILY EXPERIENCES OF PATIENTS LIVING AND COPING WITH LONG TERM OXYGEN THERAPY (LTOT)}

M. Logue, J.G. Daly, R. Sharkey. Altnagelvin Hospital Derry N Ireland

Aim: To describe experiences of patients who had been using oxygen at home for at least one year.

Patients: Five women and two men (mean age 58yrs) were identified from a hospital register of known oxygen dependant patients.

Inclusion criteria: diagnosis of chronic respiratory disease, using oxygen $>16 \mathrm{hrs}$ daily and able to communicate verbally.

Design and methods: A phenomenological method was used to describe the daily problems encountered by patients living with LTOT 
and how they coped. Unstructured audio-taped interviews lasting approx 60 minutes were conducted in the patients' home. Interviews were directed by the patients. Tapes were transcribed verbatim, checked for accuracy, and analysed using Colaizzi's method.

Main Findings: Themes which emerged included that of feeling unprepared, living life on a lead, feeling stigmatised, and no longer your own person.

Feeling unprepared related uncertainty about the purpose of treatment, and impact on lifestyle. Descriptions ranged from nervousness to fear about the equipment and oxygen as well as a sense of being left to "get on with it" unsupported. All were unprepared for the timing of the introduction and felt disappointed that it had come "too soon in life". Living life on a lead referred to restrictions caused by physical attachment to the equipment, lack of spontaneity, and pre-planning activities around oxygen. Feeling stigmatised related to a sense shame or embarrassment felt if seen in public with cumbersome cylinders and visible tubing. Coping descriptions referred to "camouflage" to conceal equipment outside, "brazening it out", or "just going without". No longer your own person related to descriptions of reduced autonomy, changes in status and role, and reliance on family or others. Coping descriptions related to social support and spiritual belief.

Conclusions: The patients in this small study would have benefited from the support of a respiratory nurse to provide counselling and support prior to the introduction of LTOT as well as follow up. Sense of stigma prevented some patients from using oxygen for outdoor activities. In the light of the ongoing review of domiciliary oxygen services, the experiences of these patients may have a bearing on the process of assessment and counselling prior to provision of LTOT and lightweight oxygen systems.

\section{P131 A SURVEY OF THE PHARMACOLOGICAL MANAGEMENT OF THE SYMPTOM OF DYSPNOEA}

L.J. Alloway', C.L. Davis'2, P. Hockey'. 'St Barnabas Hospice, Worthing; ${ }^{2}$ Countess Mountbatten House, Southampton; ${ }^{3}$ Southampton University Hospitals NHS Trust, Southampton, UK

Background: The symptom of dyspnoea is an increasing clinical problem, with rising incidence and longevity of patients with respiratory diseases. The current evidence base for treatment is sparse and often contradictory.

Aims: To determine current clinical practice in the symptomatic pharmacological management of dyspnoea, when disease related treatments are optimised, in patients with malignant and nonmalignant diseases. To compare practice in two groups: respiratory and palliative medicine consultants.

Methods: Postal survey to consultant members of British Thoracic Society and Association of Palliative Medicine, with letter from the "Respiratory Centre" accompanying the anonymous survey. Data was analysed using nonparametric statistics to compare the results from the two groups.

Results: 896 surveys were sent. Of these $46 \%$ were returned: $37 \%$ useable $(32 \%$ respiratory (R), $45 \%$ palliative medicine (PM)), $13 \%$ respondents did not specify which specialist register they are henceforth excluded from analysis.

Strong opioids are most frequently used first line for the symptomatic treatment of dyspnoea in both groups, followed by Benzodiazepines. $\chi^{2}$ Test was significant $(p<0.0001)$ showing opioids used more frequently by PM and weak opioids were more frequently $(p<0.003)$ prescribed by R physicians.
The majority of both groups ( $\mathrm{R} 72 \%, \mathrm{PM} 61 \%$ ) agreed that there is not enough evidence available to support the use of their choice of drug.

PM physicians used significantly more: Lorazepam ( $<<0.0001)$ Cannabinoids $(p<0.0001)$, Levomepromazine $(p<0.0001)$, and nebulised saline $(p<0.0001)$. Oxygen was used more frequently by $R$ ( $p<0.002)$

PM physicians were more likely $(p<0.02)$ to prescribe the same drugs for non-malignant as for malignant diagnoses. $R$ physicians were significantly $(p<0.03)$ more concerned about respiratory depression with opioids in patients with non-malignant disease, compared to PM.

Conclusions: The data shows there are significant differences in the prescribing practices of $P M$ and $R$ physicians. It highlights concerns regarding lack of evidence base for practice and over $95 \%$ of the total sample felt more research was needed in this area.

Supported by an Educational Grant from Link Pharmaceuticals Ltd.

\section{P132 USE OF THE PULMONARY FUNCTIONAL STATUS AND DYSPNOEA QUESTIONNAIRE AS AN OUTCOME MEASURE IN MULTIDISCIPLINARY PULMONARY REHABILITATION}

W.D. Edwards, S.J. Miller, T.L. Griffiths. Cardiff and Vale NHS Trust and The Section of Respiratory Medicine, University of Wales College of Medicine, Llandough Hospital, Penarth Vale of Glamorgan, CF64 2XX, UK

Background: The Pulmonary Functional Status and Dyspnoea Questionnaire (PFSDQ) was developed as a self completed measure of changes in the ability to perform activities of daily living and the associated intensity of dyspnoea. It has been validated in patients with COPD. (Lareau, et al. Heart Lung 1994;23:242-50). A prime goal of pulmonary rehabilitation is to improve patients' independence and quality of daily life. Hence, we sought to determine whether this questionnaire might be an appropriate outcome measure for use in multidisciplinary pulmonary rehabilitation programmes.

Method: Fifty one patients with chronic respiratory disease attending multidisciplinary pulmonary rehabilitation completed the PFSDQ at the start and end of their programme. In completing the PFSDQ, patients rated each of 78 commonly performed activities on a 0 (best) -10 (worst) scale to indicate the level of change from pre-morbid activity level and the dyspnoea associated with each activity. Patients also scored their overall experience of dyspnoea on "most days" in the last year, "today" and during "most day to day" activities.

Results: See table.

Conclusion: The PFSDQ is sensitive to the effects of multidisciplinary pulmonary rehabilitation with the majority of scores changing significantly. It may, therefore, represent a useful additional outcome measure, based as it is, on the activities of daily living.

\section{P133 HIGH LEVELS OF CIGARETTE SMOKING IN AN HIV INFECTED POPULATION-TIME TO FORGET OPPORTUNISTIC INFECTIONS AND CONSIDER LIFESTYLE DISEASES?}

I. Levy, C. Smith, E. Kaya, C.A. Sabin, M.A. Johnson, M.C.I. Lipman. Royal Free Hospital and School of Medicine, London NW3, UK

Antiretroviral (ARV) therapy has transformed the management of HIV disease. Improved immunity has led to reduced direct HIV-related morbidity and mortality; and a new focus on risk reduction for chronic

\begin{tabular}{|llll|}
\hline Abstract P132 & & & \\
\hline & $\begin{array}{l}\text { Before rehab } \\
\text { Mean (SD) }\end{array}$ & $\begin{array}{l}\text { After rehab } \\
\text { Mean (SD) }\end{array}$ & Significance \\
\hline Activity level & $249.0(151.7)$ & $187.8(154.3)$ & 0.000 \\
Total score for all activities & $9.6(13.8)$ & $7.3(12.9)$ & 0.004 \\
Mean score per activity & & & \\
Dyspnoea score & $194.3(131.7)$ & $161.1(124.9)$ & 0.007 \\
Total score for all activities & $5.6(6.6)$ & $4.9(6.8)$ & 0.3 \\
Mean score per activity & $7.2(6.5)$ & $8.3(7.4)$ & 0.5 \\
Number of episodes of severe dyspnoea per month & $5.7(2.2)$ & $4.9(2.2)$ & 0.002 \\
Score "most days" & $5.2(2.0)$ & $4.2(2.2)$ & 0.002 \\
Score "today" & $5.5(2.0)$ & $4.6(2.1)$ & 0.001 \\
Score in "day to day activity" & & & \\
\hline & & & \\
\hline
\end{tabular}


conditions such as cardiovascular disease. This is especially important as many of the ARVs induce significant metabolic complications such as hyperlipidemia. We sought to characterise smoking habits within an HIV infected population, using a questionnaire-based evaluation linked to our prospective HIV database. Over a six week survey period, 394 ambulant subjects were enrolled $185 \%$ male, median age 34 - IQR 29-39, 30\% previous clinical AIDS diagnosis, 73\% using ARVS). Forty five per cent were current smokers and $24 \%$ ex-smokers. The current smokers were heavy users with a median pack year consumption of 15, IQR $6-25$, and $75 \%$ reporting the first cigarette of the day within one hour of waking. They had a significant increased self reported incidence of chest infections compared to ex-smokers and non-smokers. Of the current smokers, $74 \%$ had tried to quit. Nicotine replacement and no cessation aids were the two most frequently used methods ( $44 \%$ each). The majority of ex-smokers stopped without specific cessation aids (69\%). Cardiovascular risk factors were common, with elevated blood lipids reported in $20 \%$. $30 \%$ of individuals had a family history of heart disease, and up to $10 \%$ drank more alcohol than the current recommended upper limits. Our study, the largest undertaken in Britain so far, reveals high levels of cigarette smoking within individuals who demonstrate considerable risk factors for smoking related disease. Smoking cessation work should target this at risk population.

\section{P134 THE EFFECT OF SCHOOL TUTOR GROUP SMOKING PREVALENCE ON THE RISK OF INCIDENT SMOKING IN SECONDARY SCHOOL CHILDREN: A LONGITUDINAL STUDY}

A.W.P. Molyneux', S.A. Lewis', M. Antoniak', W. Browne ${ }^{3}$, A. McNeill', R.J. Madeley ${ }^{2}$, C.A. Godfrey ${ }^{5}$, R. Britton'. 'City Hospital; ' ${ }^{2}$ University Hospital Nottingham; ${ }^{3}$ Institute of Education; ${ }^{4}$ St George's Hospital, London; ${ }^{5}$ Centre for Health Economics, University of York, UK

Rationale: Peer smoking is associated with starting smoking in childhood, but this effect may be biased by smokers' selection of smoking peers and their overestimation of smoking among their peers compared to non-smokers. We have prospectively investigated the effect of tutor group smoking prevalence (an objective, unbiased measure of peer smoking) on the uptake of smoking in Nottinghamshire school children.

Methods: A questionnaire survey of past and current smoking behaviour, parental, and sibling smoking histories was performed in pupils in Years 9 and 10 (aged 13 to 15) in 10 secondary schools in Nottinghamshire, UK in 2000, and repeated in 2001. Data were linked to identify all children who started smoking between the two surveys (incident smokers). We calculated the prevalence of current smokers in each pupils school tutor group in 2000. The independent determinants of incident smoking were analysed by multiple logistic regression and multilevel modelling.

Results: We obtained paired data on 2109 pupils in 2001 (73\% follow up). Of the 1766 non-current smokers in 2000, 267 (15\%) were incident smokers by 2001 . Tutor group smoking prevalence was a significant risk factors for incident smoking after adjusting for female sex, parental and sibling smoking; the risk of incident smoking was independently greater for those in the highest quartile $v$ lowest quartile of tutor group smoking $(19 \%$ v $12 \%$ respectively, adjusted odds ratio $1.78,95 \%$ Confidence Intervals 1.20 to 2.65$)$. Multilevel modelling showed a negligible effect of schools.

Conclusions: Tutor group smoking prevalence is an important, independent, and unbiased determinant of incident smoking in teenagers.

Funded by the Wellcome Trust.

\section{P135 A SURVEY OF SMOKING ATTITUDES AND BEHAVIOURS OF YOUNG PEOPLE ACCESSING A YOUTH PROJECT IN A DEPRIVED AREA OF NOTTINGHAM}

E.L. Jones', N, Romilly2 , A. Challenger ${ }^{3}$, R. Burton², E. Broadfield', S.A. Lewis', J.R. Britton'. 'Division of Respiratory Medicine, City Hospital, University of Nottingham; ${ }^{2}$ The Zone Youth Project, Aspley, Nottingham; ${ }^{3} \mathrm{New}$ Leaf Smoking Cessation Service, Nottingham, UK

Rationale: Cigarette smoking is the single most important avoidable cause of mortality and morbidity in the developed world and although overall smoking prevalence is falling, young people in socioeconomically deprived areas in the UK continue to take up smoking at an alarming rate.
Methods: We conducted a questionnaire survey of 11 to 20 year olds The Zone Youth Project, a voluntary sector project in one of Nottingham's most deprived areas, over a month period at all drop-in cafes, dance sessions, schools outreach, and sexual health sessions. A measurement of exhaled carbon monoxide (CO) was performed in consenting individuals.

Results: 264 valid questionnaires were returned and $75 \%$ of respondents consented to exhaled $\mathrm{CO}$ measurement. The median age of respondents was 14.0 (11-21) with $42 \%$ male. $49 \%$ were self reported current, regular smokers. Amongst the smokers the median $\mathrm{CO}$ reading was $8 \mathrm{ppm}(1-32)$ median Fagerstrom score 3.0 (0-7) and median number of cigarettes smoked per day 10. Most smokers obtained their cigarettes from newsagents, friends, or "faghouses" (contraband) and $94 \%$ came from households with at least one smoking adult. Of those who smoked, $65 \%$ would like to quit smoking and $85 \%$ had made previous unsuccessful attempts, giving up for an average of 0-3 weeks. $84 \%$ said that they would like the chance to use some kind of NRT with a preference for gum amongst the girls and patches amongst the boys. Family support and willpower rated highest amongst non-pharmacological aids with one to one support from a counsellor also scoring highly.

Conclusions: Our survey confirms the expected high prevalence of smoking in this group but the proportion of those young smokers who would like to quit and who have previously tried and failed was higher than expected. Views expressed will guide the design of specific smoking cessation services in this group.

Funded by Cancer Research UK.

\section{P136 SMOKING CESSATION SERVICES FOR TEENAGE SMOKERS: QUALITATIVE AND PILOT INTERVENTION STUDIES}

A.W.P. Molyneux', S.A. Lewis', T.J. Coleman², A. McNeill³, R.J. Madeley², C.A. Godfrey4, J.R. Britton'. 'City Hospital; '2University Hospital Nottingham; ${ }^{3}$ St George's Hospital, London; ${ }^{4}$ Centre for Health Economics, University of York, UK

Rationale: Most adult smokers started smoking as adolescents. Half of adolescent smokers would like to stop smoking and $70 \%$ have tried unsuccessfully to give up. There is little provision of smoking cessation services for teenagers. We carried out a study in secondary schools to investigate teenage smokers' views of how smoking cessation help should be provided to them, and developed and piloted a prototype school-based smoking cessation intervention based upon these findings.

Qualitative Study Methods: We invited teenage smokers laged 13 to 16 , previously identified by a questionnaire survey), from six secondary schools in Nottinghamshire, UK, to attend focus groups to discuss smoking behaviours, knowledge, and attitudes to smoking cessation, delivery of smoking cessation services to teenage smokers, and barriers and motivators to use of such services. Group discussions were recorded and transcribed. Transcripts were coded, sorted and indexed using a framework analysis by three researchers. Emergent themes are presented.

Results: 135 smokers participated in 25 groups. Teenage smokers would prefer help with cessation that is non-judgmental, confidential, and delivered by trained counsellors to individuals or small friendship groups, at school in school time, with the opportunity to use Nicotine Replacement Therapy.

Pilot Study Methods: We conducted an uncontrolled pilot study of a prototype cessation intervention in three schools. We invited smokers (aged 14 to 16, identified by a survey) to attend the intervention alone or in small friendship groups, receiving six weekly smoking cessation counselling sessions delivered by a trained counsellor, with a six week course of Nicotine Replacement Therapy (NRT) given as appropriate under medical supervision. Self reported abstinence was validated by an exhaled carbon monoxide of $\leqslant 5 \mathrm{ppm}$. Results 158 smokers were screened and 155 enrolled, 91 (59\%) female, mean age 15, smoking mean 10 cigarettes per day. One hundred and forty nine $(96.1 \%)$ participants set a quit date, $138(89 \%)$ used some NRT, of whom 47 used a six week course. At the final visit 21 (14\%) were abstinent and 82 participants $(53 \%)$ attended.

Conclusions: This study suggests that this school based smoking cessation intervention is acceptable to teenage smokers, that it is feasible and associated with reasonable cessation rates.

Funded by the Wellcome Trust. 


\section{P137 AN ASSESSMENT OF SMOKING IN ACUTE MEDICAL INPATIENTS}

E. Poon, C.W. Lau, L.Y.C. Yam, C.W. Lam'. Pamela Youde Nethersole Eastern Hospital; 'Ruttonjee Hospital, HK

Aim: To investigate the epidemiology and smoking habits of hospital inpatients and to assess the willingness of smokers to quit.

Study population: Inpatients of acute medical wards of the Pamela Youde Nethersole Eastern Hospital, Hong Kong.

Method: A questionnaire survey was prospectively administered by nursing staff on admission of all patients to the acute medical wards between 15th April and 22nd April 2002. Data collected included age, gender, smoking status/history, and an assessment of willingness to quit. Patients who could not provide such information were excluded.

Results: There were 446 admissions to six acute medical wards over the one week period. Out of the $418(93.7 \%)$ questionnaires returned, there were 208 males $(49.8 \%)$. Mean ages were 66.2 (17.5) years (males) and 69.7 (18.0) years (females). 171 (41.1\%) patients had ever smoked (135 males). Males smoked more than females $(29.8$ (22.7) $\vee 15.9$ (14.2) pack years $(p<0.0001))$ and started smoking at a younger age $(35.8$ (17.4) v 45.5 (16.3) years $(p=0.008)) .57(13.6 \%)$ current smokers (47 males) had smoked 25.4 (22.4) pack years. Of these, $35(61.4 \%)$ had ever thought of quitting, $33(57.9 \%)$ acknowledged a willingness to quit, and $26(45.6 \%)$ accepted help towards quitting. Neither current age, age at which smoking commenced, or overall consumption in pack years was related to attitude towards smoking cessation, although those who had smoked less tended to be more willing to quit. Males had thought more about and were significantly more willing to quit than females however, an equal proportion of both sexes accepted help with cessation. $114(27.3 \%)$ ex-smokers (89 males) had quit for 12.5 (11.4) years, having smoked 28.5 (21.7) pack years.

Conclusions: $41.1 \%$ of medical inpatients surveyed had ever smoked. $13.6 \%$ were current active smokers and of these $45.6 \%$ agreed to intervention with help in smoking cessation. The hospital inpatient setting provides a good opportunity to assess smoking status with a view to assisting smokers to quit.

\section{The therapy of asthma}

\section{P138 BODIPY-TMR-CGP 12177: AN IRREVERSIBLE FLUORESCENT PROBE FOR THE HUMAN $\beta_{2}$-ADRENOCEPTOR WITH AGONIST PROPERTIES}

J.G. Baker, I.P. Hall, S.J. Hill. Institute of Cell Signalling, Medical School, Queen's Medical Centre, Nottingham NG7 2UH, UK

Current methods to study native receptors in cells from patients are thwarted by the need for large numbers of cells. Fluorescent techniques offer an opportunity to work at the single cell level. BODIPY-TMR-CGP12177 (BOD-CGP) is a fluorescent analogue of the hydrophilic $\beta$-adrenoceptor $\left(\beta_{2}-A R\right)$ antagonist CGP 12177. It can stimulate cAMP accumulation ( $\left.E C_{50}=28.2(4.0) \mathrm{nM}, \mathrm{n}=3\right)$ and gene transcription $\left(\mathrm{EC}_{50}=25.3(3.6) \mathrm{nM} ; \mathrm{E}_{\mathrm{MAX}}=47 \%\right.$ of the maximum isoprenaline response) in CHO-K 1 cells expressing the human $\beta_{2}-\mathrm{AR}$ and a cyclic AMP response element reporter gene ( $\mathrm{CHO}-\beta_{2}$ cells $\left.s\right)$. Studies using ${ }^{3} \mathrm{H}-\mathrm{CGP}-12177$ indicated that BOD-CGP was able to displace the specific binding of this radioligand to the human $\beta_{2}-A R$ with an apparent $K_{D}$ of 15.8 (3.0) $n M, n=4$. Pretreatment with BOD-CGP $(100 \mathrm{nM})$ reduced the maximal specific binding of ${ }^{3} \mathrm{H}-\mathrm{CGP} 12177$ by $50 \%$, even after the fluorescent ligand had been washed out two hour previously. This provides strong evidence that BOD-CGP is effectively irreversible over this time period. Visualisation of the binding of the fluorescent BOD-CGP to single living cells $\left(\mathrm{CHO}-\beta_{2}\right)$ using confocal microscopy demonstrated clear membrane labelling at concentratons of 30nM BOD-CGP and above. Analysis of the total pixel intensities of each image enabled an estimate of the $\mathrm{K}_{\mathrm{D}}$ for BOD-CGP to be determined from saturation analysis $(27.6(6.4) \mathrm{nM} ; \mathrm{n}=4)$. The binding of 50nM BOD-CGP to living cells was also inhibited by increasing concentrations of the $\beta_{2}$-antagonist ICI $118551\left(I C_{50}=4.30(0.86) \mathrm{nM}\right.$; $\mathrm{n}=4)$ and CGP $12177(0.76(0.40) \mathrm{nM} ; \mathrm{n}=3)$. Simultaneous imaging of the binding of this red fluorescent ligand (BOD-CGP) to a $\beta_{2}$-AR-Green Fluorescent Protein fusion protein in CHO-K1 cells indicated that BOD-CGP did not induce receptor internalisation. A similar observation was made with the low efficacy agonist salbutamol, whereas the full agonist isoprenaline caused substantial receptor internalisation. In conclusion, we have fully characterised a novel fluorescent ligand for the human $\beta_{2}$-AR and shown that it can be used to visualise receptors in single living cells. The irreversible nature of this ligand should make it readily applicable to the study of $\beta_{2}$-ARs in acutely isolated native human cells in health and disease.

JGB is a Wellcome Trust Clinical Training Fellow.

\section{P139 DO SELECTIVE TOPICAL $\beta$ ANTAGONISTS FOR} GLAUCOMA HAVE LESS RESPIRATORY SIDE EFFECTS?

J.A. Nightingale', J.F. Kirwan ${ }^{2} .{ }^{1}$ Royal Brompton and Harefield NHS Trust; ${ }^{2}$ Institute of Ophthalmology

Topical $\beta$ antagonists are prescribed for glaucoma in approximately 500000 people in the UK. We have previously shown that prescription of these drugs is associated with an excess incidence of airways obstruction. (1) Selective topical $\beta$ antagonists are marketed as being less likely to induce airways obstruction than non-selective topical $\beta$ antagonists. The aim of our study was to determine whether selective topical $\beta$ antagonists are associated with a smaller risk of developing airways obstruction than non-selective topical $\beta$ antagonists.

Data obtained from the Mediplus ${ }^{\circledR}$ UK primary care database were used to perform a historical cohort study to determine one year incidence of airways obstruction in subjects following treatment with topical $\beta$ antagonists for glaucoma for the period 1993-1998. Cases were defined as having received a first prescription of a drug specifically used in the management of airways obstruction. Only subjects with no history of airways obstruction were included. Analysis was performed using proportional hazard modeling to minimise potential confounding.

For selective topical $\beta$ antagonists 12 of 324 treated subjects developed airways obstruction, compared with 112 of 9094 controls (adjusted hazard rate 3.0 (95\% confidence interval 1.6 to 5.4$)$ ). For non-selective topical $\beta$ antagonists the corresponding figures were 69 of 2321 subjects compared with the same control group ladjusted hazard rate 2.2 (95\% confidence interval 1.6 to 3.0$)$ ). There was no significant difference between groups $(p=0.5)$, both being associated with a significantly increased risk of airways obstruction.

We conclude that selective topical beta antagonists do not appear to have less respiratory side effects than non-selective topical $\beta$ antagonists in the treatment of glaucoma.

1. JF Kirwan, et al. BM 2002:(in press).

\section{P140 SMALL AIRWAY RETICULAR BASEMENT MEMBRANE (RBM) THICKENING IN CLINICALLY STABLE LUNG TRANSPLANT RECIPIENTS (SLTR) IS NOT AFFECTED BY THREE MONTHS TREATMENT WITH INHALED CORTICOSTEROIDS (ICS)}

C. Ward', A. De Soyza, A. Fisher, G. Pritchard, P. Corris. Lung biology and transplantation group, university of Newcastle Upon Tyne; 'The Freeman Hospital, NE7 7DN, UK

Introduction: Recent publications have demonstrated potentially pathological changes in clinically stable lung transplant recipients (SLTR), with frank airway remodelling demonstrated in allograft recipients with established BOS. In asthma Rbm thickening has been demonstrated at an early stage and it is suggested that ICS treatment reduces this. There is no such data regarding $\mathrm{Rbm}$ thickening in small airways sampled at transbronchial biopsy (TBB) of lung allografts.

Hypotheses: Rbm thickening exists in small airways of lung allograft recipients. ICS treatment may decrease small airway $\mathrm{Rbm}$ thickening.

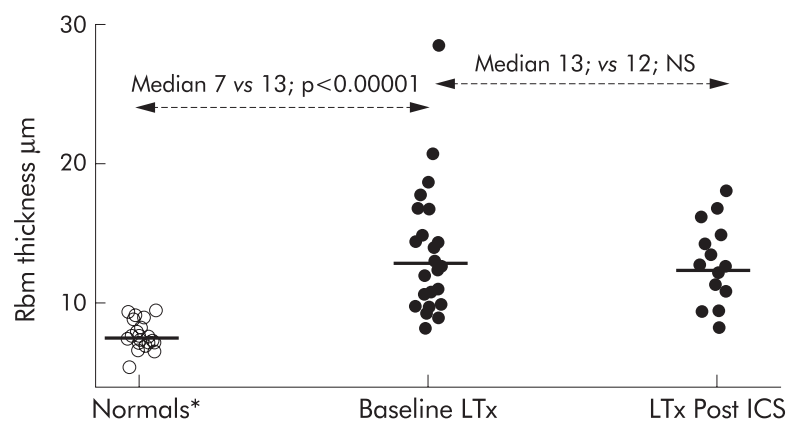

Abstract P140 *Normal large airway Rbm (Ward, et al. Am J Respir Crit Care Med 2001;164:1718-21). 
Methods: Thirty one SLTR $>3$ months post transplantation, randomised to three months $400 \mu \mathrm{g}$ CFC BDP bd or a formulation

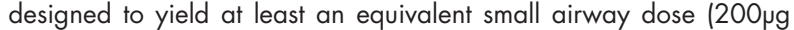
HFS BDP bd Autohaler). Bronchoscopy BAL and TBB pre and post ICS. TBB were fixed in $10 \%$ buffered formalin, embedded in paraffin and $\mathrm{H}+\mathrm{E}$ stained. Assessment of airway Rbm thickness was carried out at Image analysis from serial stepwise sections by a blind experienced observer, exceeding ERS criteria (>1 mm Rbm always scored). Ethical considerations required the use of a normal range for RBM thickness in large airway biopsies (Ward, et al. Am J Respir Crit Care Med $2001 ; 164: 1718-21)$. Rbm is thought to be systematically thicker in large airways. See figure.

Conclusion: Small airway Rbm thickening exists even in SLTR, but its significance with regard to the subsequent profound remodelling in BOS (1) is not known. three months of moderate ICS did not affect $\mathrm{Rbm}$ thickening. Further longitudinal studies, including the effect of anti-remodelling strategies are possible.

\section{P141 RAPID EFFECTS OF SINGLE DOSE FLUTICASONE PROPIONATE ON ALLERGEN-INDUCED EARLY ASTHMATIC RESPONSES IN MAN}

R.I. Ketchell, M.I. Allenby, M.W. Jensen, G.W. Clarke, B.J. O'Connor. Department of Respiratory Medicine and Allergy, GKT School of Medicine, King's College, Bessemer Road, London, SE5 9PJ, UK

Background: There is no evidence to date to suggest that inhaled glucocorticosteroids (GCS) affect the early asthmatic response (EAR) following allergen inhalation in sensitised asthmatic subjects. This is perhaps surprising as we have previously demonstrated that single dose inhaled fluticasone propionate (FP) attenuates airway responsiveness to the mast cell stimulus adenosine $5^{\prime}$-monophosphate (AMP) within two hours (Ketchell, et al. J Allergy Clin Immunol 2002:(in press)).

Objective: The aim of this study was to assess the effect of inhaled FP on allergen-induced airway narrowing.

Methods: In a randomised, double-blind placebo controlled, crossover study in mild steroid-naive asthmatics, 12 subjects with a known EAR to inhaled allergen, underwent two constant-dose allergen challenges separated by 3-4 weeks. Each challenge was preceded two hrs earlier by a single dose of inhaled FP 1000 pg or matched placebo via an accuhaler ${ }^{\mathrm{TM}}$. The EAR was measured as the $\%$ change in FEV from baseline at $5,10,15,20,30,45,60,90$, and $120 \mathrm{~min}$ utes following allergen challenge and the area under the curve during the first two hours $\left(A \cup C_{0-2 h r}\right)$.

Results: FP 1000ug significantly attenuated the EAR compared to placebo with a 45.1 (12.2) \% reduction in $A \cup C_{0-2 h r}(p=0.04)$. A reduction in $\mathrm{FEV}_{1}$, was observed at each time interval, although differences were not significant until the 90 minute time point $(p=0.01)$. The mean maximal \% fall in $\mathrm{FEV}_{1}$ was observed at the 15 min time interval and was $18.9 \%$ following a single dose of FP $1000 \mu \mathrm{g}$ and $21.6 \%$ following placebo (ns)

Conclusions: This study confirms rapid anti-inflammatory effects of inhaled FP in man. The time interval of protection suggests an effect on mast cell mediator responses such as increased microvascular permeability and/or mucosal blood flow rather than on mast cell degranulation and immediate smooth muscle responses. Inhaled GCS may provide additional beneficial topical effects in the management of acute allergic asthma.

\section{P142 FOUR MONTH ADJUSTABLE OR FIXED BD DOSING WITH BUDESONIDE/FORMOTEROL IN A SINGLE INHALER REDUCES SYMPTOM SEVERITY}

P. Ind', J. Haughney', D. Price', J-P. Rosen ${ }^{3}$, J. Kennelly' ${ }^{3}$ ' 'Hammersmith Hospital, UK; ${ }^{2}$ University of Aberdeen, UK; ${ }^{3}$ AstraZeneca UK, Luton, UK

$\operatorname{Aim}(\mathbf{s})$ : To evaluate the efficacy of budesonide/formoterol combination (B/F; Symbicort Turbuhaler) administered as either adjustable or fixed bd dosing.

Methods: Patients (mean age 48 years) were randomised to adjustable maintenance $(n=782$; $B / F$ two inhalations bd for four weeks, thereafter $1-4$ inhalations bd depending on asthma symptoms for 12 weeks) or fixed $B / F$ dosing ( $n=771$; two inhalations bd for 16 weeks). Primary efficacy variables were reduction in symptom severity (according to GINA definitions) and total exacerbations.

Results: Changes in symptom severity are shown in the table. Ninety four per cent of patients (both groups) reported no exacerbations. The average number of daily inhalations was $15 \%$ lower in the adjustable dosing group.

Conclusions: Patients treated with both adjustable and fixed-dose $B / F$ demonstrated a reduction in symptom severity as shown by the marked shift from moderate to mild intermittent. Overall there was a $46 \%$ reduction in patients graded as moderate persistent and a doubling of patients categorised as mild intermittent. A reduced overall daily dose was observed in the adjustable arm.

\section{P143 SYMBICORT USED IN A GUIDED SELF MANAGEMENT PLAN PROVIDES ADDITIONAL ENABLEMENT TO ASTHMA PATIENTS COMPARED WITH FIXED DOSING}

J. Haughney', D. Price', J-P. Rosen², K. Morrison². 'University of Aberdeen, UK; ${ }^{2}$ AstraZeneca UK, Luton, UK

Aims: To assess the effect on patient enablement of a guided self management plan compared with fixed dosing in asthma patients prescribed Symbicort.

Methods: After a four week run-in on budesonide/formoterol two inhalations bid, patients received adjustable dosing $(n=124)$ or fixed dosing $(n=104)$ for 12 weeks. Patients completed a validated Patient Enablement Instrument ${ }^{\prime}$ (PEI) within eight weeks of the last clinic visit. The PEI has six questions ("As a result of your treatment do you feel you are: Q1: able to cope with life; Q2: able to understand your illness; Q3: able to cope with your illness; Q4: able to keep yourself healthy; Q5: confident about your health; Q6: able to help yourself"). Patients' responses are scored 0 ("same or less" or "not applicable") to two ("much better"). A mean difference in total score of $\geqslant 0.8^{2}$ between groups, or an individual's total score of $=6$ is considered to reflect a relevant treatment benefit. ${ }^{3}$

Results: The baseline and demographic characteristics of the two groups were well matched. The total PEl scores were (mean (SD)) 6.24 (3.73) and 5.44 (3.84) in the adjustable and fixed dosing groups, respectively (difference $0.8(95 \% \mathrm{Cl}-0.2$ to 1.8$), p=0.12)$. A statistically significantly greater proportion of patients receiving adjustable dosing had a score of $\geqslant 6$ compared with fixed dosing $157 \% \vee 43 \%$, $p=0.04)$. There were no significant differences between responses to Q1, Q3, Q5, and Q6

Conclusions: Guided self management with Symbicort provides a greater level of patient enablement than fixed dosing.

1. Howie JG, et al. Fam Pract 1998;15:165-71.

2. Dowell J, et al. Br J Gen Pract 2001;51:200-5.

3. Howie JG, et al. Br J Gen Pract 1995;45:121-6.

\begin{tabular}{|c|c|c|c|c|c|c|}
\hline & \multicolumn{6}{|c|}{ Symptom severity } \\
\hline & \multicolumn{2}{|c|}{ Screening (week 0) } & \multicolumn{2}{|c|}{ Baseline (week 4) } & \multicolumn{2}{|c|}{$\begin{array}{l}\text { Endpoint (week } 16 \text { or } \\
\text { time of withdrawal) }\end{array}$} \\
\hline & $\mathrm{A}^{*}$ & $\mathrm{~F}^{* *}$ & A & $\mathrm{F}$ & A & $\mathrm{F}$ \\
\hline $\begin{array}{l}\text { Number of patients } \\
\text { Severity status (\%) }\end{array}$ & 775 & 766 & 775 & 766 & 754 & 737 \\
\hline Severe persistent & 6 & 6 & 4 & 4 & 4 & 4 \\
\hline Moderate persistent & 45 & 47 & 34 & 33 & 25 & 25 \\
\hline Mild persistent & 31 & 29 & 32 & 31 & 31 & 30 \\
\hline Mild intermittent & 18 & 19 & 30 & 33 & 40 & 40 \\
\hline
\end{tabular}




\section{P144 AIRWAY DRUG DELIVERY: SIZE MATTERS-BIGGER IS INDEED BETTER}

O.S. Usmani, M.F. Biddiscombe, P.J. Barnes. Department of Thoracic Medicine, National Heart and Lung Institute, Imperial College School of Medicine, London, UK

Current inhaler devices are inefficient as the dose delivered is a polydisperse distribution of drug particle sizes, and only $20 \%$ reaches the lungs. Aerosol particle size influences drug deposition and in vitro models conclude 2-6um as optimal. Monodisperse aerosols are the appropriate research tools to investigate particle size effects, as the dose is within a narrow size distribution. We hypothesised that engineering such aerosols of salbutamol would identify the ideal bronchodilator particle size and improve inhaled therapeutic drug delivery.

We previously described our use of a spinning top aerosol generator to produce such aerosols, and reported larger particles were significantly more potent bronchodilators in asthmatics-6 $6 \mathrm{~m} \sim 3 \mu \mathrm{m}$ $>1.5 \mu \mathrm{m}$, at $1 / 10^{\text {th }}$ standard $\mathrm{MDI}$ doses. The $6 \mu \mathrm{m}$ particles achieved equivalence with $200 \mu \mathrm{g}$ MI salbutamol. No adverse effects were observed. We then hypothesised the differences were a result of the deposition characteristics of each particle size, in that larger particles were better matched to their target site of action.

We therefore undertook to assess lung and extrathoracic deposition patterns using 2D planar imaging and ${ }^{99 \mathrm{~m} T c-l a b e l l e d ~ m o n o d i s p e r s e}$ salbutamol (30 pg dose) aerosols, in 12 asthmatic subjects with simultaneous measures of clinical efficacy. The smaller particles achieved greater total lung deposition; $1.5 \mu \mathrm{m}(56 \%), 3 \mu \mathrm{m}(50 \%)$, 6um (46\%), however the larger particles were more efficacious $6 \mu \mathrm{m} \sim 3 \mu \mathrm{m}>1.5 \mu \mathrm{m}$ $\left(F E V_{1}, p<0.05\right)$. We quantified regional deposition in the lungs and found more of the larger particles in the central+intermediate zone; $6 \mu \mathrm{m}(75 \%), 3 \mu \mathrm{m}(66 \%), 1.5 \mu \mathrm{m}(56 \%)$. The penetration index confirmed greater peripheral lung penetration of the smaller particles;

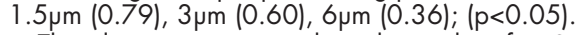

The data support our hypothesis that for $\beta_{2}$-agonists, regional airway targeting to the conducting airways is more important than alveolar deposition. Particles in the higher part of the respirable range achieve the greatest clinical response and we advocate small doses, when correctly delivered, may improve the therapeutic index. We now aim to investigate the effects of inspiratory flow and respiratory disease severity on aerosol deposition. With resurgence of interest in inhalation drug delivery as a means of systemic therapy, we must take the opportunity to research basic aerosol science to enhance the efficiency of clinical airway drug delivery.

\section{P145 THE MEASUREMENT OF THE SUBMICROMETER SIZE DISTRIBUTION OF METERED DOSE INHALERS (MDI)}

M. Crampton', R.P. Kinnersley', J.G. Ayres'2. 'Divison of Environmental Health \& Risk Management, University of Birmingham, Birmingham, B 15 2TT; ${ }^{2}$ Department of Respiratory Medicine, Birmingham Heartlands Hospital, Birmingham, B9 5SS, UK

Recent studies indicate that toxicity of materials in ultrafine aerosol form is greater than that of the same material in coarser aerosol form. At the same time it has been observed that the HFA formulation of beclomethasone (Qvar) is more potent compared to the CFC product. A pertinent difference between the two is that the former yields smaller particles.

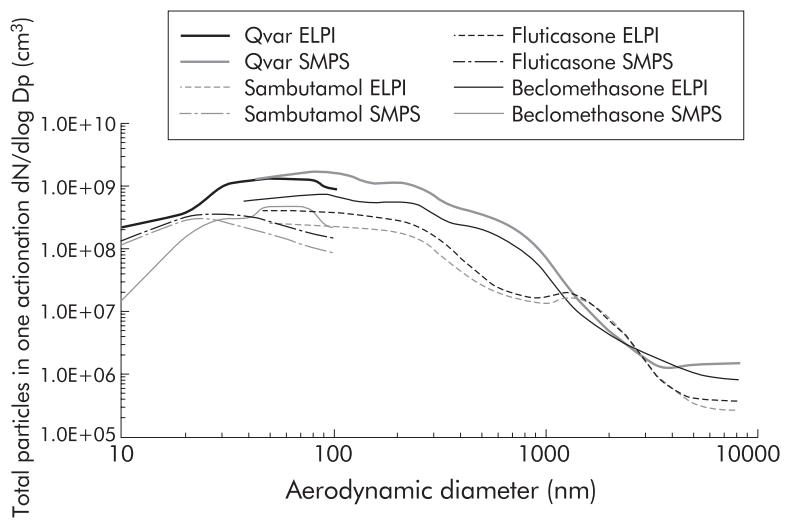

Abstract P145 Size distribution of pMDls (number weighted).
The size distribution by number of a range of $\mathrm{pMDls}$ (including Qvar [3M], Salbutamol [GSK], HFA-Fluticasone [GSK], Beclomethasone [GSK], Budesonide [AstraZeneca]) was measured to determine the proportion of particles in the sub-micrometer range. Measurements were made by Electrical Low Pressure Impactor (size range $30 \mathrm{~nm}-10 \mu \mathrm{m}$ ) and a Scanning Mobility Particle Sizer (size range 10-100nm). All devices tested yielded high numbers of fine and ultrafine particles, the number being greater for HFA than for CFC propelled MDIs $1 \%<100 \mathrm{~nm}$, HFA-Qvar:76\%, HFA-Salbutamol:75\%, HFA-Fluticasone:76\%, CFC-Belcomethasone:65\%, CFC-Budesonide:57\%). We suggest that the number of fine and ultrafine particles, the concentration of drug per particle, the molecular properties and the hydroscopic properties of the drug, may all influence MDI potencies. See figure.

This work was supported by an academic grant from 3M Pharmaceuticals.

\section{P146 PD20 TO METHACHOLINE IS PREDICTED BY AIRWAY INFLAMMATION AND REMODELLING: A SYSTEMATIC LONGITUDINAL, STEROID INTERVENTION STUDY OF AIRWAY BIOPSY (Ebb) AND BAL PARAMETERS}

C. Ward' D. Reid ${ }^{2}$, M. Pais², B. Orsida², B. Feltis ${ }^{2}$, R. Bish², D. Johns ${ }^{2}$, E. Haydn Walters'. 'Lung Biology and Transplantation Group, University of Newcastle upon Tyne and The Freeman Hospital, UK; ${ }^{2}$ University of Tasmania and Monash University, Australia

Introduction: We have recently shown that PD20 may be predicted from a model including terms measuring airways remodelling; (Reticular basement membrane (Rbm) thickening) and inflammation quantified at BAL.' In this study we have refined our paradigm, including information on large airway biopsy (ebb) inflammation and the effect of inhaled corticosteroid treatment.

Hypothesis: Ebb provides complimentary inflammatory indices to BAL, which supports a link between inflammation, airway remodelling and PD20

Methods: A double blind, randomised, placebo-controlled, parallel group study of inhaled fluticasone propionate (up to 12 months

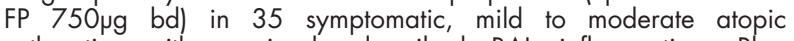
asthmatics with previously described $\mathrm{BAL}$ inflammation, $\mathrm{Rbm}$ thickening and PD20.' Quantification of ebb inflammation by immunohistochemistry/image analysis. ' Matrix analysis of individual univariate correlations, with subsequent "best subsets" multiple regression.

Results: Cross sectional, multiple regression analysis explained $56 \%$ of the variability in BHR, 23\% related to log EG2 (eosinophil) counts, $19 \%$ to $\log$ Rbm thickness, and $14 \%$ to BAL epithelial cells improving our previous model (overall $40 \%$; 1). Following three months FP ebb inflammatory cell counts fell significantly, with no further FP effect, for example, baseline median ebb EG2 count $8 / \mathrm{mm}$ basement membrane $(\mathrm{bm})$, (range 0-32), three month; $2 / \mathrm{mm} \mathrm{bm}$ (0-22; $<<0.01 v$ baseline), 12 month median $2 / \mathrm{mm} \mathrm{bm}(0-72$, NS $v$ 3 months). Changes in ebb inflammation preceded an effect on $\mathrm{Rbm}$ thickness and the maximal effect on BHR (@12 months FP, 1).

Conclusion: Ebb and BAL are complimentary, and further support the view that PD20 to methacholine reflects airway remodelling and inflammation in asthmatic subjects. A lack of specificity for any one part of asthma pathophysiology may represent a strength of BHR testing

Chris Ward is an ERS research fellow.

1. Ward, et al. Thorax 2002;57:309-16.

\section{P147 RELATIONSHIP BETWEEN ADHERENCE TO PRESCRIBED REGIMENS AND ASTHMA CONTROL IN PATIENTS WITH DIFFICULT ASTHMA}

S. Aburuz', J. McElnay', J. Millership ', J. Gamble ${ }^{3}$, L. Heaney ${ }^{2}{ }^{3}$. 'School of Pharmacy; 'Department of Medicine, Queen's University Belfast; ${ }^{3}$ Regional Respiratory Centre, Belfast City Hospital, Belfast

Introduction: Non-adherence with prescribed therapy is a major problem in the management of chronic illness. The aim of the present study was to examine the relationship between asthma control and adherence to oral therapy (prednisolone (P) and theophylline (T)) and high dose inhaled steroids (HDIS) in a population of difficult asthmatics (persisting asthma symptoms/frequent exacerbations requiring systemic steroids despite maintenance high dose inhaled corticosteroids plus a long acting $\beta_{2}$ agonist)

Method: A range of parameters was used to assess adherence and asthma control in patients, with difficult to control asthma, attending a 
hospital outpatient clinic. Plasma P was measured by HPLC. Plasma T was measured by fluorescence polarization immunoassay. Urinary Cortisol (C) was measured by HPLC and corrected for creatinine (UCC ratio). Non-adherence was recorded if (a) Prednisolone: no plasma $P$ detectable; (b) Theophylline: no plasma T detectable. Adherence to HDIS was recorded if morning UCC ratio was suppressed below 8.0 $\mathrm{nmol} \mathrm{C} / \mathrm{mmol}$ creatinine. Asthma symptom control scores (ASCSs) were recorded using the Juniper Asthma Control Questionnaire. (I)

Results: Eleven $(25 \%)$ patients of 44 patients taking $T$ and 14 $(56 \%)$ of 25 patients taking $P$ were non-adherent. Of 43 patients tak-

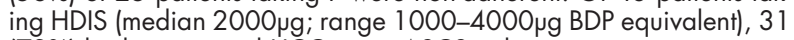
$(72 \%)$ had suppressed UCC ratio. ASCS, wheezing a morning symptoms were significantly lower $(p<0.05)$ in patients with UCC suppression. There was no relationship between $\mathrm{FEV}_{1} \%$ and suppression of urinary cortisol. Asthma control did not vary with adherence with theophylline or prednisolone.

Discussion: There was a high prevalence of non-adherence with prescribed oral therapy in patients with difficult asthma. Asthma control was related to the presence of urinary cortisol suppression in patients receiving HDIS, which may reflect non-adherence with inhaled therapy. Failure to suppress urinary cortisol can be used as a marker of possible non-adherence to HDIS in this population.

1. Juniper E, et al. Resp Med 2001;95:319-23.

\section{P148 EARLY ANTIBIOTIC PRESCRIPTION AND ALLERGIC DISEASE IN UK ADULTS}

P. Cullinan, J.M. Harris, P. Mills, S. Moffat, C. White, F. Figg, A. Moon, A.J. Newman Taylor. Occupational \& Environmental Medicine, Faculty of Medicine, Imperial College of Science, Technology and Medicine, London, UK

To investigate any association between treatment with antibiotics in early life and subsequent atopic or allergic disease, we studied a population of adults in southeast England. 1063 men and women (93\% of those eligible), parents of a birth cohort based in Ashford, Kent, agreed to participate. Allergic diseases were defined by responses to a questionnaire; atopy was measured by responses to skin prick tests with common aerollergens. Prescription information for the first five years of life was successfully obtained from the general practice records of $746(70 \%)$ subjects. There were no important differences in the rates of atopy or allergic disease between those whose records were or were not available for examination.

Thirty six percent of adults were atopic; $14 \%$ reported a history of asthma and $29 \%$ hay fever. $564(76 \%)$ had at least one antibiotic prescription, with a median of three. The median age at first prescription was 1.5 years. Older subjects were more likely not to have a prescription record, indicating a temporal increase in prescription patterns. There was a clear association with family size, whereby adults with fewer siblings were significantly more likely to receive at least five prescriptions by the age of five years $(p<0.001)$. There was no evidence of any association between atopy and total antibiotic prescriptions in total (adjusted OR 1.01, 95\% confidence interval (0.97 to 1.05 ) per prescription), a pattern which did not alter at any age or by antibiotic class. Asthmatics - with or without atopy-were more likely to have received an antibiotic prescription by the age of five years (adjusted OR 1.08 (1.03 to 1.13) per prescription). This relationship increased with age at prescription ladjusted OR 1.02 per prescription by age $1 ; 1.18$ age $1-2 ; 1.14$ age $2-3 ; 1.23$ age $3-4$; 1.32 age 4-5). A similar but less marked pattern was observed for hay fever, again with or without evidence of pollen sensitisation. These findings do not rule out a positive association between antibiotic use and subsequent allergic disease; but are more probably explained by a protopathic bias.

\section{P149 IS A TWO WEEK TRIAL OF ORAL PREDNISOLONE PREDICTIVE OF TARGET LUNG FUNCTION IN PAEDIATRIC ASTHMA?}

C. Lex, D.N.R. Payne, N.M. Wilson, A.M. Li, A. Zacharasiewicz, A. Bush. Department of Paediatric Respiratory Medicine, Royal Brompton Hospital, London, UK

The optimum length of a steroid reversibility trial in children is not known. We have used a two week trial of oral prednisolone to determine target lung function for subsequent asthma therapy. The aim of this study was to determine whether in fact on subsequent visits some children actually exceeded this "target lung function".

Methods: Twenty five severe asthmatic children (median age 13 (range 6-16) years were studied. Severe asthma was defined as persistent symptoms despite treatment with $\geqslant 1600 \mu \mathrm{g} / \mathrm{d}$ of inhaled budesonide or equivalent, long-acting $\beta_{2}$-agonist \pm regular steroids. $\mathrm{FEV}$, was measured following high-dose systemic steroids (oral prednisolone $40 \mathrm{mg} /$ day for 14 days) and compared with the highest $\mathrm{FEV}$, obtained in subsequent visits during the following year. Results: The mean (SD) FEV as a percentage of the predicted value following the formal steroid trial was 74,28 $\pm 19,66$ (range 38-103). A total of $8(32 \%)$ children actually obtained an increase of $>10 \%$ above their "target" $F E V$, during the following year at routine clinic spirometry. There were no important changes in prescribed asthma medications (for example, cyclosporine, methotrexate, s.c. terbutaline), which might have accounted for this in these eight children. In $17 / 25(68 \%)$ children FEV measured in subsequent visits remained stable or declined. The mean age of both patient groups was equal, and it was not possible to predict which children would exceed "target lung function" from baseline characteristics or post-steroid FEV

Conclusions: A two weeks course of prednisolone is not necessarily predictive of target lung function. Further studies are needed to find out whether a higher dose or longer duration of the steroid trial is more predictive, or whether adding an acute inhalation of $\beta_{2}$-agonist at the end of the trial may increase predictive power.

\section{Tuberculosis}

\section{P150 TUBERCULOSIS INCIDENT AND OUTBREAK SURVEILLANCE IN ENGLAND AND WALES: REPORTS FROM JANUARY 1999 TO JUNE 2002}

A. Story, J. Watson. Communicable Disease Surveillance Centre, Respiratory Division, Tuberculosis Section, 61 Colindale Avenue, London, NW9 $5 E Q$, UK

We are currently developing a central information resource on tuberculosis incidents and outbreaks that can be used to inform future management. The aims of this surveillance initiative are to gain insight into the occurrence and distribution of tuberculosis incidents and outbreaks; monitor and evaluate the effectiveness of control measures and inform policy; network relevant parties as an accessible information resource for one another; and provide a means of recording examples of best practice and lessons learned.

In order to more fully understand the terms and specification required for this new aspect of tuberculosis surveillance we have retrospectively collated tuberculosis related incidents and outbreaks reported to CDSC from January 1999 to June 2002.

A total of 47 reports associated with actual and potential tuberculosis transmission involving health workers and institutions have been received, 33 of which relate to health and allied professionals posing an infection risk. Similarly, 29 reports relating to education workers and institutions, including one outbreak, which involved extensive contact investigation have been received. There are four reported instances of tuberculosis among prisoners and one incident involving a prison guard. A total of 22 instances of tuberculosis presenting a potential public health threat to airline passengers have been reported, 18 involving passengers and four airline employees.

Incidents and outbreaks reported to CDSC to date represent only a sample of the total episodes. It is planned that the development of a more formal system to capture, collate, and report on tuberculosis incidents and outbreaks will enable a more accurate assessment of their frequency, distribution and public health impact to be made.

\section{P151 TRENDS IN TUBERCULOSIS CASE FATALITY IN ENGLAND AND WALES 1988-2000}

A.R. Martineau', P.D.O. Davies'. 'Department of Respiratory Medicine, Newham General Hospital, London E13 8SL, UK; ${ }^{2}$ Aintree Chest Centre, University Hospital Aintree, Liverpool L9 7AL, UK

To determine trends in tuberculosis (TB) case fatality in England and Wales according to age and disease site we analysed published notification and mortality data for TB from 1988-2000 (see table overleaf). In contrast to 1974-1987, (1) case fatality for TB of all sites and age groups combined fell over this period despite increasing incidence of disease. Declining case fatality is likely to be due to changes in TB epidemiology: younger patients with higher rates of extra-pulmonary disease and lower case fatality rates accounted for an increasing proportion of TB cases over the study period. Improvements in TB notification rate, under diagnosis of TB at post mortem and improved case management may also have contributed to the decreasing case fatality rates observed.

1. Nisar M, et al. Thorax 1991;46:438-40. 


\begin{tabular}{|c|c|c|c|c|c|c|c|}
\hline Year & Respiratory & CNS & Other sites & Age 0-34 & Age $35-74$ & Age $>74$ & Total \\
\hline 1988 & 9.55 & 17.8 & 7.21 & 0.42 & 10.10 & 36.94 & 9.26 \\
\hline 1989 & 8.03 & 42.3 & 6.9 & 0.69 & 8.15 & 34.95 & 8.16 \\
\hline 1990 & 7.94 & 16 & 5.21 & 0.69 & 7.80 & 29.97 & 7.49 \\
\hline 1991 & 8.45 & 8.2 & 5.48 & 0.57 & 7.81 & 40.6 & 7.76 \\
\hline 1992 & 7.35 & 16.85 & 5.99 & 0.67 & 7.61 & 31.14 & 7.21 \\
\hline 1993 & 7.14 & 9.09 & 6.04 & 0.71 & 7.75 & 30.02 & 7.14 \\
\hline 1994 & 7.74 & 13 & 6.07 & 0.77 & 8.39 & 28.84 & 7.48 \\
\hline 1995 & 8.56 & 9.18 & 5.91 & 1.03 & 8.47 & 32.33 & 7.97 \\
\hline 1996 & 7.46 & 12.39 & 6.37 & 0.56 & 7.85 & 30.87 & 7.36 \\
\hline 1997 & 6.52 & 11.02 & 6.15 & 0.57 & 6.96 & 29.08 & 6.57 \\
\hline 1998 & 6.36 & 9.16 & 6.18 & 0.89 & 6.76 & 30.43 & 6.44 \\
\hline 1999 & 6.87 & 4.92 & 4.47 & 0.59 & 6.13 & 30.68 & 6.23 \\
\hline 2000 & 5.78 & 7.45 & 4.81 & 0.60 & 5.64 & 29.46 & 5.63 \\
\hline
\end{tabular}

\section{P152 ERRORS IN THE NOTIFICATION OF TUBERCULOSIS}

C. Seehra', F.A. Drobniewski', P.G. More ${ }^{2}$, D.C.S. Hutchison ${ }^{3}$. 'Lambeth, Southwark \& Lewisham Health Authority (LSLHA); ${ }^{2}$ PHLS Mycobacterium Reference Unit, Dulwich; ${ }^{3}$ Respiratory Medicine, King's College Hospital, London, UK

Introduction: In the UK, clinical disease with Mycobacterium tuberculosis (MTB) is notifiable by law, whether cases are MTB culture positive or culture negative treated as MTB. Notification initiates contact tracing and provides epidemiological data. Two main sources of error exist: (1) Under notification (failure to notify cases of MTB infection). (2) Over notification (notification of cases not due to MTB infection). Both errors can occur and we have examined local procedures, with emphasis on over notification.

Methods: We have studied notified patients treated at King's College Hospital (KCH) and resident in LSLHA. Over notification in 1996 to 2000 inclusive was defined for this audit by comparison of notified cases with microbiologically confirmed cases. Under notification (limited to 1999) was detected by search of $\mathrm{KCH}$ Pharmacy data base for patients prescribed anti-tuberculosis chemotherapy, but not notified as TB.

Results: In 1996 to 2000, 279 cases were notified as tuberculosis, $229(82 \%)$ being MTB culture positive. Forty one culture negative cases were notified, 32 being clinically probable MTB and nine probably not. Nine cases were culture positive with non-MTB mycobacteria. Thus 18 notified patients definitely or probably did not have MTB infection, an over notification rate of $18 / 279$ or $6.5 \%(\mathrm{Cl} 4$ to 10$)$. $18 / 50$ or $36 \%(\mathrm{Cl} 23$ to 51$)$ of all notified cases who were not MTB culture positive were erroneously notified as MTB.

In 1999, 45 cases were notified. The Pharmacy data base identified 11 unnotified MTB cases, giving an under notification rate of $11 / 45$ or $24.4 \%(\mathrm{Cl} 13$ to 40$)$. Four notified patients did not have MTB infection, giving an over-notification rate of $4 / 45$ or $8.8 \%(\mathrm{Cl} 2$ to 21 ).

Conclusion: Combining over and under notification rates for 1999 yields a net under notification rate of $7 / 45$ or $15.6 \%(\mathrm{Cl} 7$ to 29$)$. The microbiological data base should lead to notification of all MTB positive cases. Culture negative cases contribute an important error and must be verified by other means, the Pharmacy data base proving to be the most successful. Denotification procedures must be more vigorously pursued.

\section{P153 UPDATE ON AN OUTBREAK OF ISONIAZID MONO-RESISTANT TUBERCULOSIS IN NORTH LONDON}

M. Ruddy', A.P. Davies², M.D. Yates', S. Yates², S. Balasegaram³, B. Patel $^{2}$, S. Lozewicz ${ }^{2}$, S. Sen ${ }^{4}$, M. Bahl' ${ }^{5}$, E. James ${ }^{6}$, M. Lipman 7 , Y. Drabu', J. Watson ${ }^{3}, \mathrm{M}$. Piper ${ }^{8}, \mathrm{~F}$. Dronniewski ${ }^{1}, \mathrm{H}$ Maguire $^{3}$, on behalf of a Londonwide Incident Committee. 'PHLS Mycobacterium Reference Unit, King's College Hospital (Dulwich); ${ }^{2}$ North Middlesex University Hospital Trust; ${ }^{3}$ PHLS Communicable Disease Surveillance Centre; ${ }^{4}$ Barnet, Enfield \& Haringey Health Authority; ${ }^{5}$ Camden \& Islington Health Authority; ${ }^{6}$ Barnet \& Chase Farm Hospital Trust; ${ }^{7}$ Royal Free Hospital Trust; ${ }^{8}$ Department of Health Prison Policy Unit

Since January 2000 an outbreak of isoniazid mono-resistant Mycobacterium tuberculosis has been investigated. Typing of suspected isolates has been performed at the PHLS Mycobacterial Reference Unit using a new rapid PCR-based method (RAPET), with confirmation by RFLP IS6110 typing showing a distinctive 15 band pattern. Case finding has been performed by initial retrospective and continuing prospective analysis of isoniazid mono-resistant isolates from the source and neighbouring hospitals, and all isoniazid monoresistant isolates in London from January 1999 onwards, along with comparison of the fingerprint to RFLP IS6 110 databases. The earliest case detected was a Nigerian student resident in London in 1995. Epidemiological links were established by questionnaire in face to face interviews. Outbreak control is coordinated by an Incident Control Committee at the PHLS Communicable Disease Surveillance Centre. To date 97 patients' isolates have demonstrated the RAPET pattern. Eighty eight microbiologically confirmed cases have been identified in London, with 14 clinically proven cases in their contacts and nine epidemiologically linked cases outside London. Contact tracing so far has suggested a higher than predicted transmission rate (11\%). Thirty contacts have been placed on chemoprophylaxis. The epidemic curve suggests that the peak of the outbreak may not yet have been reached. A wide range of racial and social groups have been involved in the outbreak with 17 of the cases associated with prisons including one prison staff member. The investigation has revealed the first documented case of transmission in a UK prison. Nosocomial transmission has occurred involving staff and patients. The involvement of IVDUs and sex workers along with documented compliance problems in $38 \%$ of cases has made control of the outbreak difficult

\section{P154 MISSED DIAGNOSIS OF TUBERCULOSIS IN THE ACCIDENT AND EMERGENCY DEPARTMENT}

M. Bhattacharyya, A. Choudhury, G.E. Packe. Newham Chest Clinic, Shrewsbury Road, Forest Gate, London E7 8QP, UK

In our district many patients present with illness first to the accident \& emergency department (A\&E) at our hospital rather than to primary care. This is a result of several factors such as poor understanding of the primary care system in the UK, and poor access to primary care. The aim of this study was to ascertain how many patients with tuberculosis in our district present to the A\&E department, and how well they are diagnosed at presentation.

Of the 243 notified cases of tuberculosis (TB) in Newham during 1999, 121 (50\%) patients were seen at $A \& E$ prior to notification. Of these 121 patients, 62 had pulmonary disease; their median age was 32 years (range 1 to 88 years). Fifty one patients attended the A\&E department more than once (total 211 visits). The number of attendances: number of patients, was as follows: 1 attendance: 70 patients, $2: 30,3: 9,4: 8,5: 3,6: 1$. Of the 171 visits for which $A \& E$ patient information cards were traced: $143(84 \%)$ were for TB related episodes, $12(7 \%)$ were episodes unrelated to TB, and $16(9 \%)$ did not wait after being triaged.

Records for the TB related episodes (143 visits) were divided into those in whom TB was suspected when seen in the A\&E department (37 visits), and those in whom TB was not suspected (106 visits). All patients where TB was suspected had one or more symptoms suggestive of the diagnosis (cough, haemoptysis, night sweats, breathlessness, lymphadenopathy, and fever). However, in the 106 A\&E visits where TB was not suspected, 61 (58\%) cases had one or more symptoms suggestive of TB.

Of the 37 visits at which TB was suspected, the number of cases and the department of the clinician who raised the suspicion were: 29 A\&E clinician, five general medicine, two surgical, one gynaecology. Of the cases that were unsuspected, the majority of cases were seen only by A\&E clinicians. In all cases where presenting symptoms may 
have suggested TB (98 visits), 19 (19\%) patients had a chest $x$ ray performed and nine $(9 \%)$ had sputum taken for acid-fast bacilli. Those patients that were not followed up later presented to A\&E or another hospital department where the correct diagnosis was eventually made.

Many TB patients attended A\&E where the diagnosis was unsuspected. In our district, with a high incidence of TB, A\&E staff need better education about the diagnosis of TB.

\section{P155 LONDON ACCIDENT AND EMERGENCY (A\&E) DEPARTMENTS: AN OPPORTUNITY TO DIAGNOSE TUBERCULOSIS (TB) EARLY?}

A. Smith, A. Goodburn, A. Story, R. Miller, G. Scott, H. Booth. TB Clinic, Middlesex Hospital, University College London Hospitals (UCLH), UK

TB is a major health problem in London, accounting for more than $40 \%$ of the national total. North Central London Sector has seen the highest rate of increase in TB since 1998 (155\%), and is also the epicentre of an ongoing outbreak of isoniazid resistant TB. Early case finding remains an essential part of TB control.

Aims: (1) To identify the rate of usage of $A \& E$ to access healthcare in our TB patient population. (2) To identify whether A\&E attendances present an opportunity to diagnose TB early.

Methods: TB notifications between the period from January 2001 to March 2002 (15 months), were cross referenced against A\&E and microbiology records for the six month period prior to each notification.

Results: There were 171 TB notifications during this time. Of these, $48(28 \%)$ patients were seen in $A \& E$ a total of 69 times prior to their diagnosis. Thirty two patients had pulmonary TB (19 smear positive) and 10 were HIV positive (one previously undiagnosed). Thirty five patients were admitted from A\&E of which 27 were diagnosed as a direct result of their admission. Four patients were discharged from A\&E with TB clinic follow up. Seventeen (eight admitted) of the 48 patients did not have TB diagnosed or considered at the time of their A\&E attendance(s).

Conclusions: $A$ third of our patients with subsequent TB diagnoses had attended A\&E within the six month period preceding notification. This may reflect the difficulties that this patient population has in accessing healthcare by other means. It also serves to emphasise the key role A\&E staff have to play in TB case finding among this population.

Thirty five out of 48 patients with TB were admitted, suggesting that these patients were systemically unwell on presentation, and emphasising the importance of liaison between acute general medical and TB services. In 17 out of 48 cases the diagnosis of TB was either not made or considered, representing a potential missed opportunity to diagnose TB early.

\section{P156 PERFORMANCE STATUS ON ADMISSION TO HOSPITAL: A SIMPLE PREDICTOR OF DEATH IN PATIENTS WITH PULMONARY TUBERCULOSIS IN SOUTH AFRICA}

S. De Valliere', M. Nthangheni' ${ }^{2}$ R.D. Barker'. 'Department of Medicine, Medical University of Southern Africa-Polokwane Campus, Pietersburg, South Africa; ${ }^{2}$ Statistics and Operations Research, University of the North, Private Bag X1 106, Sovenga 0727, South Africa; ${ }^{3}$ King's College Hospital, Bessemer Road, Camberwell, London, UK

Background: Simple measures are required to identify patients most at risk of dying from tuberculosis. Performance status has been used in studies of cancer patients but we are not aware that it has been tested as a predictor of mortality among patients with tuberculosis.
Methods: During a period of three months all adults lage $\geqslant 15$ years) admitted to one of six South African hospitals and put on anti-tuberculous treatment for suspected or confirmed pulmonary tuberculosis were eligible. At the time treatment was initiated performance status was recorded by the doctor or the nurse in charge. The vital status of the patient (alive or dead) was determined two months after initiation of treatment (table).

Results: 347 patients were admitted to hospital and started on TB treatment during the study period. For nine patients performance status was not obtained and so analysis has been restricted to the remaining 338 patients.

Discussion: Performance status at the time of diagnosis appears to be a powerful predictor of mortality in this population.

Supported by TB Alert.

\section{P157 TUBERCULOSIS AND HIV SEROPREVALENCE IN LAMBETH, SOUTHWARK AND LEWISHAM, AN AREA OF SOUTH LONDON}

M. Melzer', A. Warley², H. Milburn², D. O'Sullivan ${ }^{3}$, R.D. Barker ${ }^{4}$, D. Shelton $^{5}$, F. Drobniewski ${ }^{5}$, G.L. French' ${ }^{2}$ 'Department of Infection and Tropical Medicine, Northwick Park Hospital, Watford Rd, Harrow, Middlesex HAl 3UJ: '2Department of Infection and Respiratory Medicine, Guy's and St Thomas' Hospital, Lambeth Palace Rd, London SE I 7GD; ${ }^{3}$ Lambeth, Southwark and Lewisham Health authority, 1 Lower Marsh St., Lambeth, London SE $17 N T$; ${ }^{4}$ Department of Respiratory Medicine, King's College Hospital, Denmark Hill, London SE5 9RS; ${ }^{5}$ Mycobacterium Reference Unit, Dulwich Hospital, East Dulwich Grove, London SE22 8QF, UK

Since the mid 1980s the number of cases of TB notified within the UK has continued to rise although the contribution of HIV to this rise remains unclear. A 12 month prospective cohort study was conducted at chest and HIV clinics in four hospitals in Lambeth, Southwark, and Lewisham (LSL), an area of South London, to determine the proportion of patients with culture-proven TB infected with HIV. Secondary aims were to determine the proportion of patients with TB and undiagnosed HIV at first presentation to chest clinics, to determine the proportion of patients presenting with TB as an AIDS defining illness (ADI) and to identify risk factors for co-infection with TB and HIV. In chest clinics, demographic data and left over blood from patients aged 16 or over with culture-proven TB was collected, anonymised, and HIV tested. In HIV clinics, demographic data on patients with TB already known to be HIV seropositive was also obtained. Twenty one patients $13 \%$, $95 \% \mathrm{Cl} 8 \%$ to $19 \%$ ) of 159 with culture-proven TB were infected with HIV. Four $(3 \%)$ of 133 patients at first presentation to chest clinics had undiagnosed HIV; two were subsequently diagnosed. Of the 21 patients with TB and HIV nine $(43 \%)$ presented with TB as an ADI. Patients with TB and HIV were significantly more likely to be aged between 35-55 years compared to HIV seronegative patients (12/21 $(57 \%)$ v $38 / 138(28 \%), p=0.006)$. None of the patients from the Indian Subcontinent were HIV seropositive (0/21 v 25/138 (18\%), $p=0.047)$. At the present time, universal HIV testing of patients with culture-proven TB in chest clinics within the UK is unlikely to significantly reduce the number of patients with undiagnosed HIV.

\section{P158 OUTCOME OF HIV ASSOCIATED TUBERCULOSIS IN THE ERA OF HIGHLY ACTIVE ANTIRETROVIRAL THERAPY (HAART)}

K. Dheda, F. Lampe, M. Lipman, M. Johnson. Department of HIV and Thoracic Medicine, Royal Free Hospital, \& School of Medicine, London, UK

Background: The benefit of HAART on outcome in TB/HIV co-infected patients is unclear due to concerns about drug adherence, absorption,

\begin{tabular}{|c|c|c|c|c|c|c|c|}
\hline \multicolumn{8}{|l|}{ Abstract P156 } \\
\hline \multirow[b]{2}{*}{ Performance status } & \multicolumn{2}{|c|}{ alive } & \multicolumn{2}{|c|}{ died } & \multirow[b]{2}{*}{ Total $n$} & \multirow[b]{2}{*}{ RR } & \multirow[b]{2}{*}{$95 \% \mathrm{Cl}$} \\
\hline & $\mathrm{n}$ & $\%$ & $\mathrm{n}$ & $\%$ & & & \\
\hline 0 & 109 & $95 \%$ & 6 & $5 \%$ & 115 & 1 & - \\
\hline 1 & 58 & $95 \%$ & 3 & $5 \%$ & 61 & 0.94 & (0.24 to 3.64 ) \\
\hline 2 & 74 & $88 \%$ & 10 & $12 \%$ & 84 & 2.28 & (0.86 to 6.02$)$ \\
\hline 3 & 20 & $66 \%$ & 10 & $33 \%$ & 30 & 6.39 & (2.52 to 16.18 ) \\
\hline 4 & 23 & $48 \%$ & 25 & $52 \%$ & 48 & 9.98 & (4.18 to 21.95 ) \\
\hline Total & 286 & $84 \%$ & 54 & $16 \%$ & 338 & & \\
\hline
\end{tabular}


overlapping toxicities, drug interactions, and paradoxical reactions. We therefore investigated the effect of HAART on survival in co-infected patients.

Methods: Ninety two HIV/TB co-infected patients treated at a London HIV clinic were retrospectively identified. Patients starting TB treatment prior to 1 January $1996(n=36)$ were compared to those starting TB treatment after 1 January $1996(n=56)$, when HAART was introduced. HAART was included as a time-updated covariate in a Cox regression model with adjustment for other prognostic factors.

Results: Compared to patients treated for TB prior to 1996, those treated during or after 1996 were older (median age $35 \vee 30$ ), and were more likely to be women $(43 \%$ v $17 \%)$, Black African $163 \%$ v $44 \%)$, and in the heterosexual risk group (68\% v $47 \%$ ). Baseline CD4 count tended to be lower for patients in the post-1996 compared to the pre-1996 group $\left(73 \%\right.$ versus $\left.61 \%<200 / \mathrm{mm}^{3}\right)$. TB treatment with Rifabutin was much more common in the post-1996 group $134 \% v$ $6 \%$ ). During a median of 2.6 years of follow up there were 29 deaths. Three years after initiation of TB treatment, survival was $63 \%$ in the pre-1996 group compared to $79 \%$ in the post-1996 group $(p=0.19$, log rank test over whole follow up period). This difference in survival was significant after adjustment for baseline CD4 count (HR for post1996 versus pre-1996: $0.42 ; 95 \% \mathrm{Cl} 0.18$ to $0.96, p=0.039$ ). In a Cox model that included HAART use as time-updated variable, and adjusted for baseline CD4 count, extrapulmonary TB, previous AIDS and calendar period (pre or post 1996), the use of HAART resulted in a marked reduction in risk of death (HR: $0.26 ; 95 \% \mathrm{Cl} 0.10$ to 0.72 , $\mathrm{p}=0.009$ ) and explained the effect of calendar period. Initial CD4 count $<200 / \mathrm{mm}^{3}$ (HR: $3.4 ; 95 \% \mathrm{Cl} 1.3$ to $9.3, \mathrm{p}=0.015$ ) and a prior history of AIDS (HR: 2.3; $95 \% \mathrm{Cl} 1.1$ to $5.1, \mathrm{p}=0.032$ ) were also independently associated with risk of death.

Conclusion: Use of HAART in patients with TB/HIV co-infection results in a substantial reduction in risk of death.

\section{P159 TUBERCULOSIS: A CHEAP DISEASE?}

S. Berkovitz, R. Hurd, M. Mandani, D. Adler, N. Johnson. Whittington Hospital, London; Camden and Islington Community NHS Trust, UK

Tuberculosis is an increasing problem in some parts of London. Despite a lack of evidence, it is often regarded as a cheap disease to treat. To test this hypothesis, we describe the first comprehensive cost analysis of a dedicated adult tuberculosis service in a busy district general hospital.

All relevant care data was obtained for patients notified with tuberculosis or given chemoprophylaxis in the year 2000, up to their discharge from hospital care. Computerised information was obtained from hospital and tuberculosis office databases in addition to paper based tuberculosis record cards and medical notes. Mean costs for inpatient ( $£ 170$ per bed night) and outpatient ( $£ 67$ per appointment) episodes were provided by the Trust finance department and specific drug costs the hospital pharmacy.

Ninety seven patients were notified with tuberculosis and 23 patients given chemoprophylaxis. Mean age at notification was 37 years (median 29, range 13-91). Of the notified patients, 46 were admitted to hospital at some stage, 11 on two occasions. Mean length of stay was eight days (median one day, range 1-83). Two patients died in hospital. Completion rates for therapy were $89 \%$. A summary of the cost analysis is shown in the table.

Tuberculosis is not a cheap disease to treat. If control of tuberculosis is not taken seriously, it poses a considerable health economic problem.

\begin{tabular}{|lr|}
\hline $\begin{array}{l}\text { Abstract P159 } \\
\text { analysis }\end{array}$ & A summary of the cost \\
\hline Cost category & Cost (£) \\
\hline Inpatient & 126932 \\
Respiratory outpatient & 24322 \\
Other outpatient & 7362 \\
Surgical procedures & 13862 \\
Radiology & 72605 \\
Medication & 26710 \\
TB nurse service & 75000 \\
Other & 6438 \\
TOTAL & 353231 \\
Cost per patient treated & 3592 \\
\hline
\end{tabular}

\section{P160 TUBERCULOSIS AND HEALTH BELIEFS IN THE BANGLADESHI COMMUNITY OF EAST LONDON}

V. White', S. Hillier'2, J. Moore-Gillon'. 'Depts of Respiratory Medicine; ${ }^{2}$ Human Science and Medical Ethics, Bart's and the London School of Medicine \& Dentistry, ECIA 7BE, UK

We investigated the understanding and recognition of symptoms of $T B$, any associated stigma, use of alternative practitioners and attitudes to medication amongst Bangladeshi patients with TB in Tower Hamlets, east London. Forty three newly diagnosed subjects were approached and 41 took part. Twenty six were male and 15 female, aged 20-85 (median 36). 19 had pulmonary and 22 extra pulmonary TB. Each underwent two interviews, of c. $1 \mathrm{~h}$ duration, the first within a few days of diagnosis, the second after 1-3 m treatment. Interviews were semi-structured, for analysis with NVivo software after transcription.

Strikingly, only two subjects admitted to knowing that TB could infect organs other than the lungs. Twenty four of the 41 associated cough, haemoptysis, and weight loss with TB, but the significance of fevers and night sweats was largely unrecognised. Most were afraid to discuss their diagnosis outside their close family, with 31 subjects believing there was significant stigma associated with TB and five stating that TB affected a sufferer's prospects of marriage. While most (27) expressed no concerns about medication, the others were unhappy with size and/or number of tablets and duration of treatment. Only six admitted to pluralistic health practices: while adhering to standard therapy, they consulted religious leaders and took herbal remedies.

Seven subjects admitted to being not literate in any language. Adherence, assessed semi-objectively (including tablet counts and urine checks) was not related to literacy, proficiency in English, nor educational attainment, and the least compliant patient was UK university educated. Fear of TB, the desire for cure and respect for medical staff were the most commonly expressed reasons for adherence.

Our findings of lack of awareness of TB symptoms and of substantial stigma must give rise to concerns both about delays in presentation for medical care and regarding contact tracing. Culturally appropriate health education initiatives may help address these problems. We keep in mind, however, that our findings might not necessarily have been significantly different had we studied UK born white TB patients.

VLCW is supported by Bart's Special Trustees and Spitalfields Community Trust.

\section{P161 NO BOOSTER EFFECT IN BCG IMMUNISED TUBERCULOSIS CONTACTS UNDERGOING REPEAT HEAF TESTING}

A.R. Martineau, M. Chaudery, J. Jeyanathan, G.E. Packe. Newham Chest Clinic, Shrewsbury Road, Forest Gate, London E7 8QP, UK

Individuals who undergo repeat tuberculin testing may exhibit increasing reaction size in the absence of new infection. This phenomenon is known as boosting. Boosting is thought to be commoner in BCG vaccinated individuals compared to non-vaccinated individuals.' BTS guidelines only advise repeat tuberculin testing in non-BCG vaccinated TB contacts whose initial tuberculin (Heaf) test is negative. ${ }^{2}$

We performed a retrospective analysis of TB contacts to establish if there was an excess of increased Heaf reaction on repeat Heaf testing in $B C G$ vaccinated individuals. One hundred and forty one TB contacts, with an initially negative Heaf test (grade $0-1$ ), had repeat tuberculin testing at our clinic $(74$ female; age range 18 days to 60 years). Sixteen had neither history of BCG nor BCG scar; 119 had either $(n=19)$ or both $(n=100)$; six had no recorded details of $B C G$

\begin{tabular}{lcc} 
Abstract P161 & & \\
\hline Heaf grade increase increase & $\begin{array}{c}\text { No Heaf grade } \\
\text { incer }\end{array}$ \\
\hline Previous BCG & 27 & 92 \\
No previous BCG & 7 & 9 \\
\hline
\end{tabular}


status and were excluded from the analysis. In 34 cases, the grade of the 2 nd Heaf test was unequivocally higher than the grade of the $1 \mathrm{st}$ Heaf test. In the remainder (101 cases) it was not. 27/119 (22.7\%) of those with previous BCG demonstrated an increase in Heaf grade, compared with $7 / 16(43.8 \%)$ of those with no evidence of previous BCG (table).
These results do not support the contention that boosting is more common in BCG vaccinated subjects.

1. Menzies D. Am J Respir Crit Care Med 1999:159;15-21.

2. Joint Tuberculosis Committee of the British Thoracic Society. Thorax 2000;55:887-901. 